\title{
Die semantische Entwicklung von Intensivierern
}

\author{
Dissertation
}

zur Erlangung des philosophischen Doktorgrades

an der Philosophischen Fakultät der Georg-August-Universität Göttingen

vorgelegt von Olga Laitenberger, geb. Karpova aus Ščjolkovo (Щёлково), Russland 
Gutachter

1. Prof. Dr. Michael Job

2. Prof. Dr. Regine Eckardt

Tag der mündlichen Prüfung: 29.01.2016 


\section{Inhaltsverzeichnis}

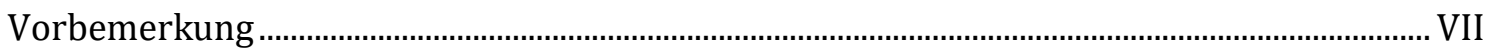

1. Gegenstand und Ziele der Arbeit ......................................................................................................

2. Datenerhebung und Forschungsmethoden....................................................................................

2.1 Deutsche Intensivierer: Probleme und Datengrundlage....................................................... 3

2.1.1 Biedermanns Liste.......................................................................................................

2.1.2 Die COSMAS-Liste ...........................................................................................................

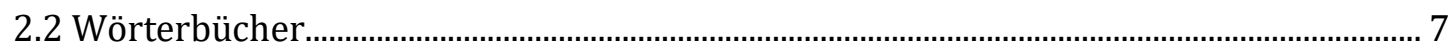

2.3 Textkorpora ……................................................................................................................. 7

2.4 Russische Grad-Adjektive und -Adverbien .......................................................................... 9

2.5 Materialauswertung ...................................................................................................... 9

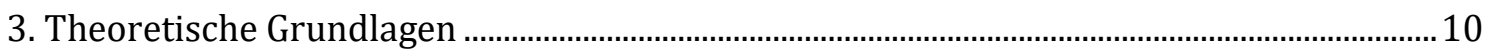

3.1 Zum Begriff der Intensivierung ……………………......................................................10

3.2 Intensivierer in der bisherigen Forschung ....................................................................11

3.3 Intensivierungsmittel: Klassifizierung ……….................................................................13

3.4 Intensivierbare Ausdrücke ...........................................................................................16

3.4.1 Adjektive und Adverbien ........................................................................................

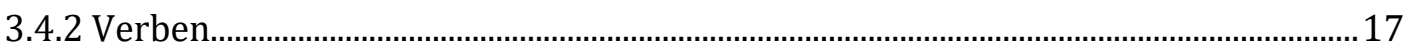

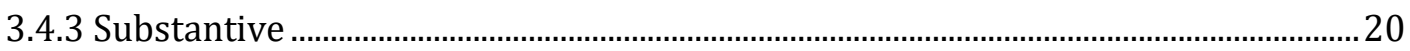

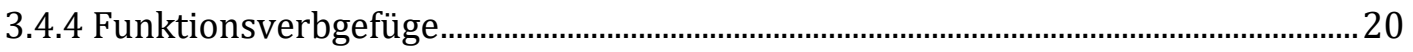

3.5 Natur der Intensivierung: Semantische Felder ...............................................................22

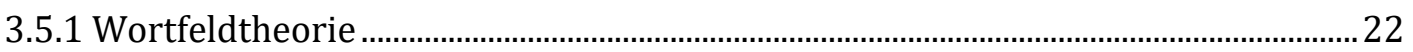

3.5.2 Deutsche Grad-Adjektive und -Adverbien und semantische Felder..........................25

3.5.3 Russische Grad-Adjektive und -Adverbien und semantische Felder .......................28

3.6 Natur der Intensivierung: Mechanismen...........................................................................28

3.6.1 Metapher und Metonymie: allgemeine Vorstellungen ...............................................29

3.6.2 Metapher und Metonymie in der Adjektiv- und Adverbiallexik.................................32

3.6.3 Intensivierung und Grammatikalisierung ..................................................................... 41

3.6.3.1 Zum Begriff der Grammatikalisierung...................................................................... 41

3.6.3.2 Mechanismen und Parameter der Grammatikalisierung in Bezug auf deutsche Grad-Adjektive und -Adverbien.................................................................................. 44

3.6.3.3 Zum Grad der Grammatikalisierung: semantic bleaching........................................46

3.6.3.4 Semantisch-pragmatische Veränderungen im Rahmen der Grammatikalisierung: Traugotts tendencies …………………………………...... 49

3.6.3.5 Semantisch-pragmatische Veränderungen im Rahmen der Grammatikalisierung: subjectification 
3.6.3.6 Bedeutungswandel im Rahmen der Grammatikalisierung: Metapher.

3.6.3.7 Semantisch-pragmatische Veränderungen im Rahmen der Grammatikalisierung: Invited Inferencing Theory of Semantic Change und Metonymie

3.6.3.8 Zur Entstehung von Grad-Adjektiven und -Adverbien.............................................56

4. Zur Entstehung von deutschen Grad-Adjektiven und Grad-Adverbien ..................................68

4.1 Wortgruppe 'Furcht erregend' ........................................................................................68

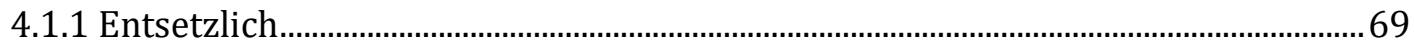

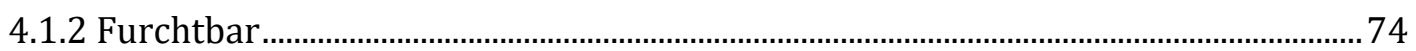

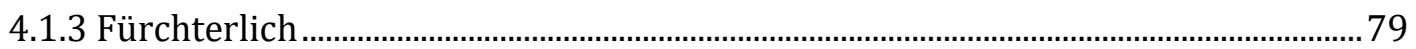

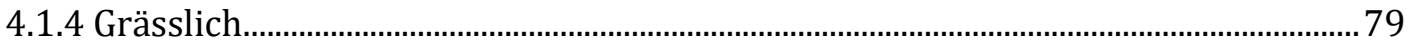

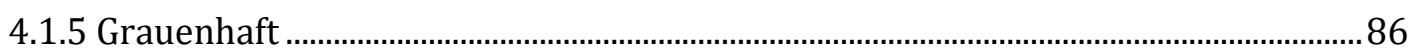

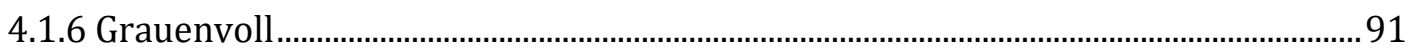

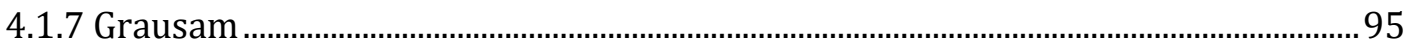

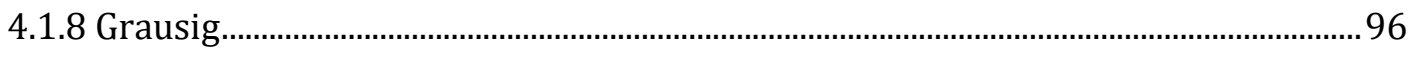

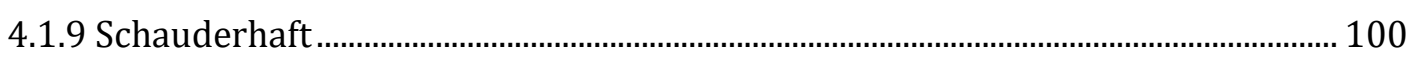

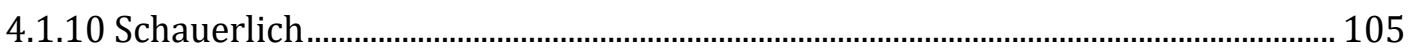

4.1.11 Schaurig .................................................................................................................. 109

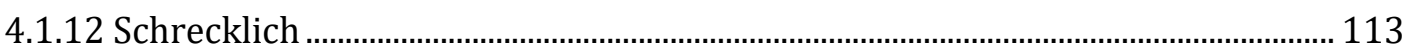

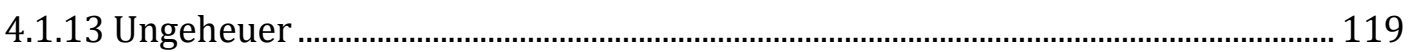

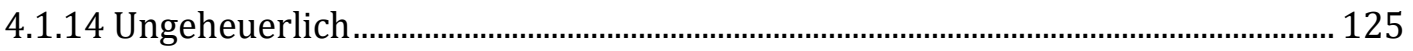

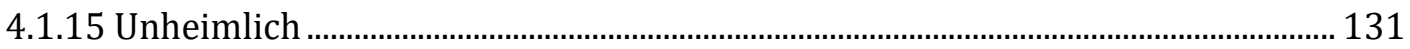

4.2 Wortgruppe 'Übel wollend / verursachend' ............................................................... 136

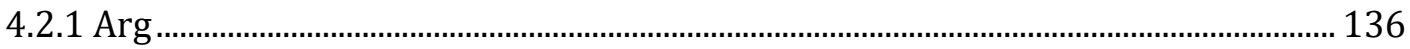

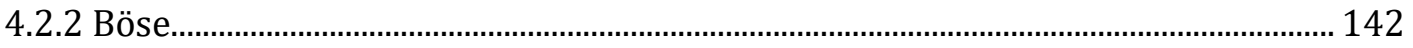

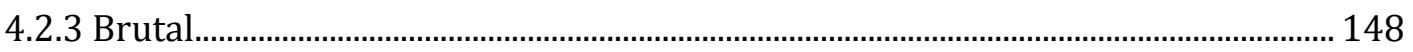

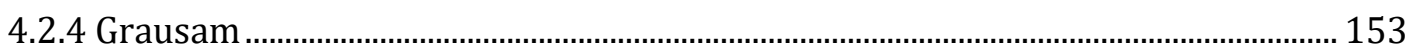

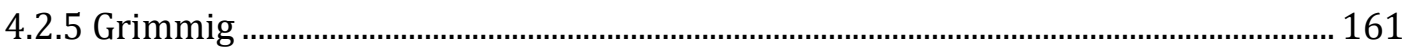

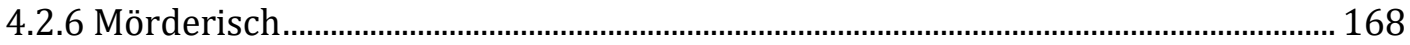

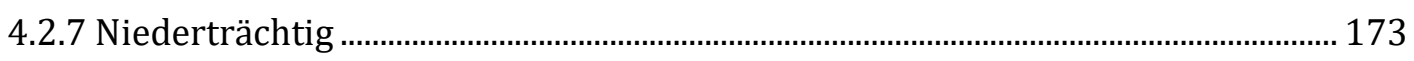

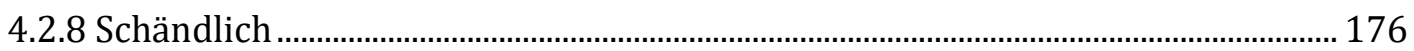

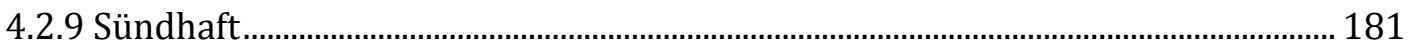

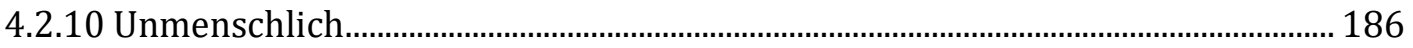

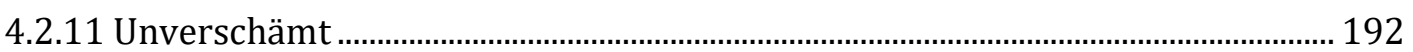

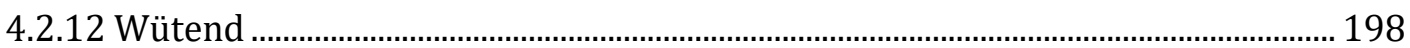

4.3 Wortgruppe 'Sich wie ein Tier verhaltend' ........................................................................ 203

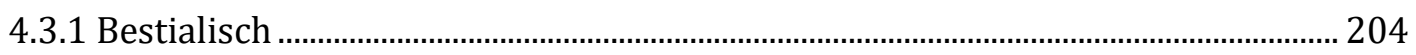

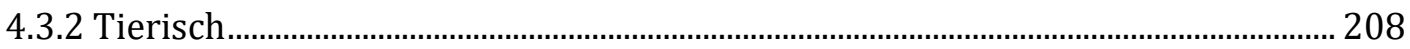




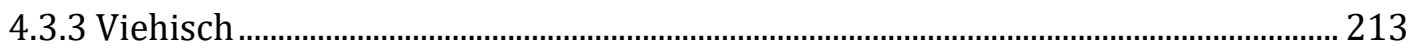

4.4 Wortgruppe 'Geistig gestört',.......................................................................................... 218

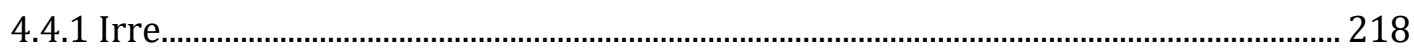

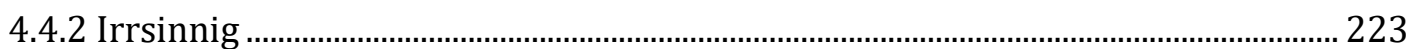

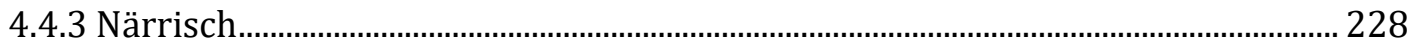

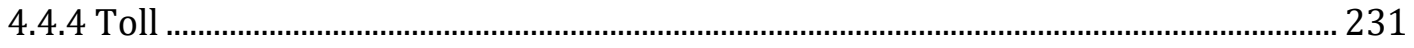

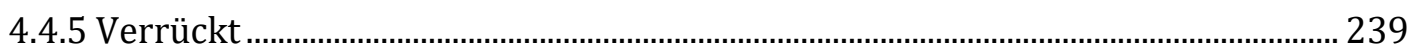

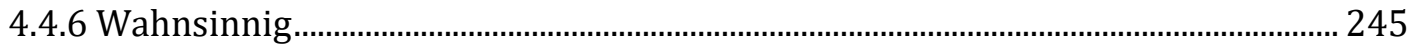

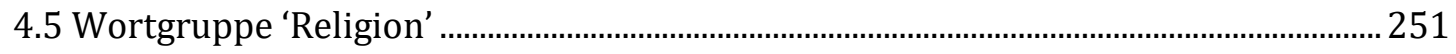

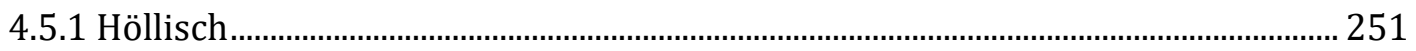

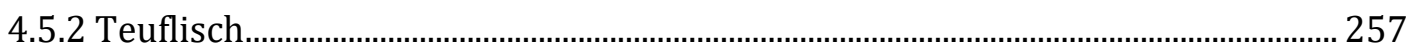

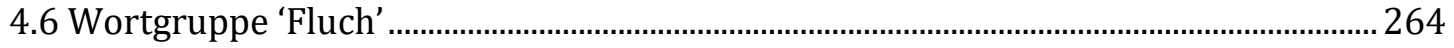

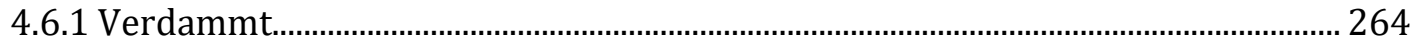

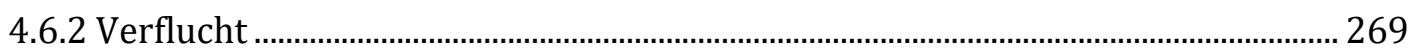

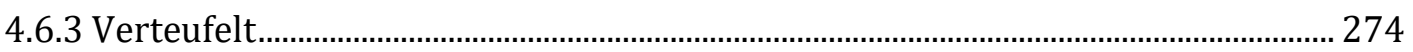

5. Zur Entstehung von russ. Gradadverbien ........................................................................... 279

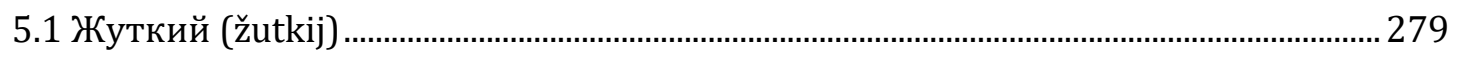

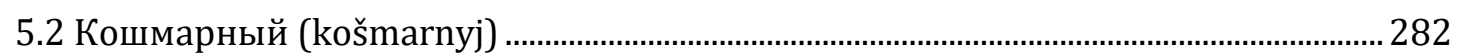

5.3 Страшный (strašnyj) ...................................................................................................... 286

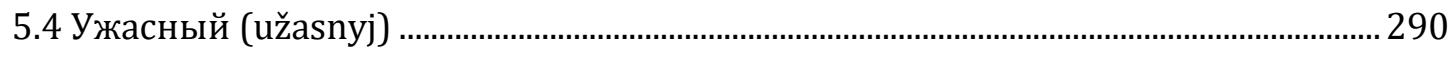

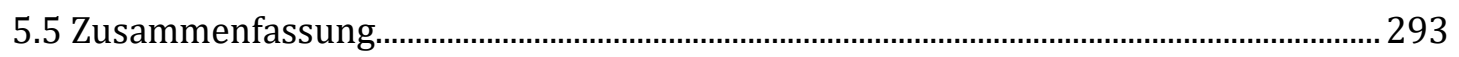

6. Relevanz der deutschen Wortfelder für das Russische........................................................... 295

6.1 Wortgruppe 'Übel wollend / verursachend' ....................................................................... 295

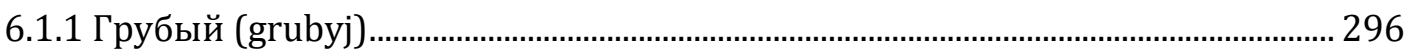

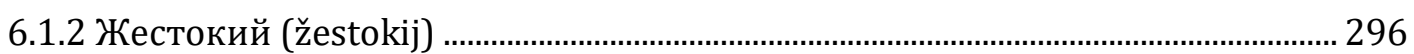

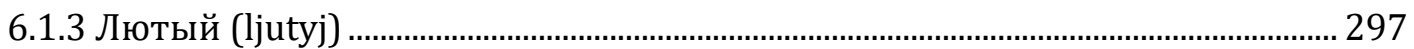

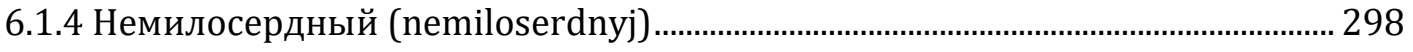

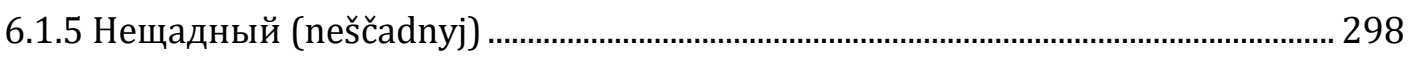

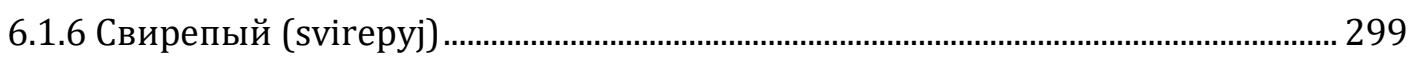

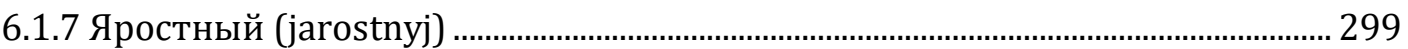

6.2 Wortgruppe 'Sich wie ein Tier verhaltend' ....................................................................... 300

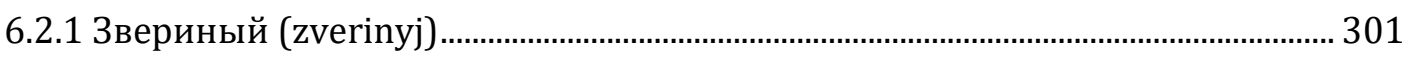

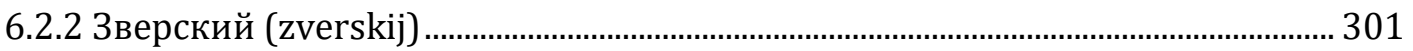

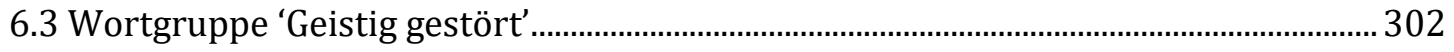

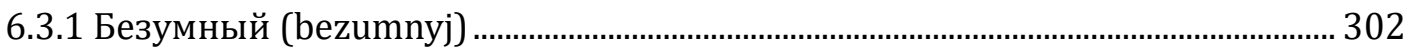

6.3.2 Бешеный (bešenyj) ..................................................................................................... 303

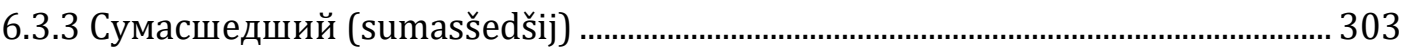




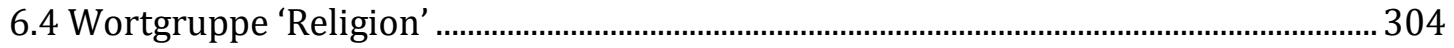

6.4.1 Адский (adskij) ....................................................................................................... 305

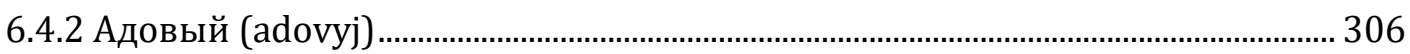

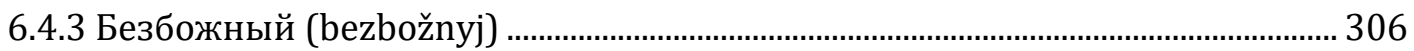

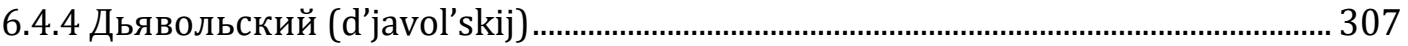

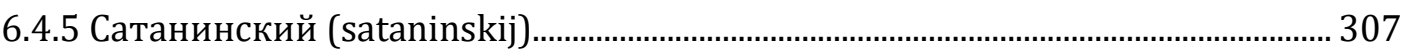

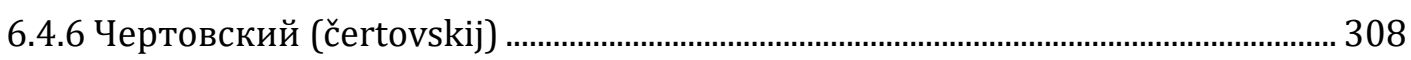

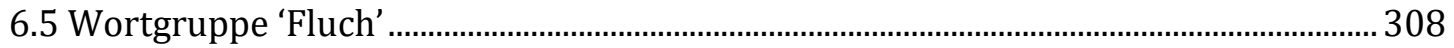

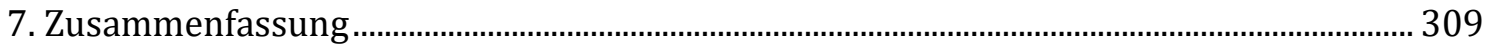

7.1 Resümee - Summary - Резюме...................................................................................... 312

8. Literatur- und Abkürzungsverzeichnis................................................................................. 314 


\section{Vorbemerkung}

Auf das Phänomen der Intensivierung wurde ich bei einer Untersuchung russischer Adjektive aufmerksam, als ich 2009 meine Diplomarbeit schrieb und an der Zusammenstellung einer Datenbank des semantischen Wandels in der russischen adjektivischen und adverbialen Lexik arbeitete. Um die Natur dieses Phänomens besser zu verstehen und dessen Relevanz für die deutsche Sprache, die ich zu der Zeit studierte und für die ich mich besonders interessierte, festzustellen, forschte ich zunächst 2011-2012 im Rahmen eines Forschungsaufenthalts im Slavischen Seminar der Universität Göttingen. Die Literatur- und Korpusrecherche hat umfangreiches Material ergeben und nahegelegt, dass Intensivierung im Deutschen nicht nur eine sehr verbreitete Erscheinung ist, sondern auch im Vergleich zum Russischen eine vielfältigere und in gewissem Maße komplexere Erscheinung darstellt. Die Wortpalette, die im Deutschen für den Ausdruck der Intensivierung gebraucht wird, ist viel breiter als im Russischen. Dabei werden nicht nur Adjektive und Adverbien intensiviert, sondern auch zahlreiche Funktionsverbgefüge. Ich widmete diesem faszinierenden Phänomen die vorliegende Arbeit, die im Januar 2016 im Fach Allgemeine Sprachwissenschaft der Philosophischen Fakultät der Universität Göttingen als Dissertation angenommen wurde.

An dieser Stelle muss ich darauf hinweisen, dass einige Links zu den Belegen, die ich zur Exemplifizierung verschiedenen Internet-Quellen entnommen habe, nicht mehr abrufbar sind. Deshalb sind sie in Übereinstimmung mit vorherrschenden Zitierpraktiken mit dem Datum der letzten Konsultation vor der Disputation versehen worden. Das Ersetzen durch neuere Links wäre einerseits sehr zeitaufwendig gewesen und wäre vielfach auch ins Leere gegangen; andererseits wären neuere Links vermutlich ebenfalls nicht von Dauer. Internet-Quellen erweisen sich dabei zwar in der Recherche-Arbeit als sehr hilfreich, aber ihre Volatilität stellt ein Problem dar, das gegenwärtig nicht prinzipienbasiert gelöst werden kann.

Meinem „Doktorvater", Professor MichaEL JoB, möchte ich meinen herzlichen Dank für seine Betreuung, die stetige und engagierte Unterstützung, Hilfsbereitschaft sowie für seine technische Hilfe bei der Vorbereitung meiner Arbeit für die Veröffentlichung aussprechen. Durch sein Engagement konnte ich mich meiner neugeborenen Tochter FIONA widmen und unsere gemeinsame Zeit genießen.

Meiner zweiten Betreuerin, Frau Professorin REGINE ECKARDT (jetzt Universität Konstanz) danke ich sehr für ihre Unterstützung und vielfältigen Rat.

Für Hilfe bei der Materialrecherche danke ich Herrn Professor REICHMANN und Frau Professorin LoBEnSTEIN-REICHMANN, die mir das Archiv des Frühneuhochdeutschen Korpus zugänglich gemacht haben.

Ebenso danke ich Dr. VOLKER HARM für die Diskussion meines Materials, die wertvollen Ratschläge und den Zugang zu dem Archiv des Deutschen Wörterbuchs in Göttingen. 
Mein tiefempfundener Dank gilt auch der GRADUIERTENSCHULE FÜR GEISTESWISSENSCHAFTEN GÖTTINGEN für die finanzielle Unterstützung meiner Arbeit in unterschiedlichen Phasen ihrer Entstehung.

Ganz besonders hervorheben möchte ich schließlich meinen Ehemann OSKAR LAITENBERGER und meine Eltern für ihre stetige moralische Unterstützung und Hilfe.

Göttingen, im August 2017 


\section{Gegenstand und Ziele der Arbeit}

Den Gegenstand der vorliegenden Arbeit bilden Grad-Adjektive und -Adverbien, die eine hohe Ausprägung eines durch das Bezugswort ausgedrückten Begriffs bezeichnen. Im Fokus der Untersuchung steht der Entwicklungsgang von Intensivierern aus dem negativen in den positiven Bereich bzw. Grad-Adverbien und -Adjektive "negativen Bedeutungsgehalts“, wie sie in Werner (1960: 244) bezeichnet werden ${ }^{1}$. Es sind Lexeme, die ursprünglich "eine negative Empfindung“ bezeichnen und von daher mit negativ konnotierten Bezugswörtern kollokieren, anschließend jedoch „als bloße Verstärkungen“ auftreten, „die in dieser Funktion nun auch vor Ausdrücke „positiven“ Gehalts treten“ können (Werner 1960: 244). Diese Intensivierer sind einstellungsbezogen, d.h. sie demonstrieren die subjektive Einstellung des Sprechers, vgl. die folgenden Beispiele:

(1) furchtbares Tier (,Furcht erregendes Tier') vs. furchtbare Freude (,sehr große Freude');

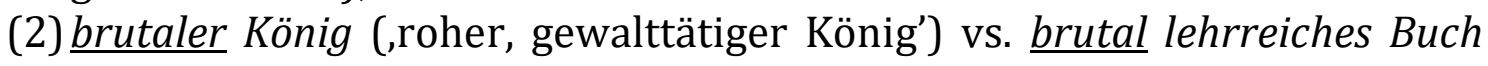
(,sehr lehrreiches Buch');

(3) verrückter Mensch (,geistig kranker Mensch') vs. verrückt schönes Wetter (,sehr schönes Wetter');

(4) unverschämte Manieren (,sich über die Grenzen des Taktes und des Anstandes hinwegsetzende Manieren') vs. unverschämt leckere Speisen (,sehr leckere Speisen').

Aus den Zusammensetzungen in (1) - (4) geht hervor, dass die Lexeme furchtbar, brutal, verrückt und unverschämt sich in der Ausgangbedeutung auf verschiedene negative Eigenschaften von Personen, Tieren und des menschlichen Verhaltens beziehen und keinen Zusammenhang mit der Intensivierung aufweisen. Die Gradbedeutung, die sich dagegen in den Verwendungen furchtbare Freude, brutal lehrreich, verrückt schönes Wetter und unverschämt leckere Speisen erkennen lässt, hat den Bezug zur Ausgangsbedeutung komplett verloren. Infolge dieser Bedeutungsentleerung entdecken die Lexeme neue Kookkurrenzmöglichkeiten und können in Zusammensetzungen mit positiv konnotierten Prädikaten auftreten.

Im Rahmen meiner Arbeit verfolge ich das Ziel, die Entwicklung der Gradbedeutung im Deutschen aus einer diachronen Perspektive zu beschreiben und die Schritte, die für diesen Prozess ausschlaggebend sind, anhand von zahlreichen Belegen zu exemplifizieren. Außerdem wird die Relevanz des Phänomens in anderen Sprachen, vor allem im Russischen, überprüft. Dieses Ziel lässt sich durch das Lösen der folgenden Aufgaben erreichen:

Erstens sollen die Mechanismen, die der Intensivierung in der Adjektiv- und Adverbiallexik zugrunde liegen, genauer untersucht werden. In diesem Zusammenhang werde ich der Frage nachgehen, welche kognitiven Prozesse zu der Verblassung der Ausgangsbedeutung und zu der Entstehung der reinen Gradbedeutung führen. Insbesondere werde ich meine Aufmerksamkeit auf die Rolle von Metapher, Metonymie und Grammatikalisierung richten. Ich plädiere dafür, dass metaphorische und metonymische Sprachmittel gelegentlich auf ein früheres Stadium des

${ }^{1}$ Hofmann (1930: 105) bietet die Bezeichnung „Steigerungsadverbia mit traurigem Affektinhalt“. 
Entwicklungsgangs eines Intensivierers einwirken, jedoch für die Erklärung des gesamten Bedeutungswandels, wie es in (1) - (4) demonstriert wurde, nicht ausreichend sind. Ich werde dagegen demonstrieren, dass die Herausbildung von Intensivierung viele Ähnlichkeiten mit dem Prozess der Grammatikalisierung aufweist.

Zweitens werde ich die Stufen des Entwicklungsgangs darstellen, welche ein Lexem bei der Herausbildung der Gradbedeutung einschlägt. Meine These besteht darin, dass es eine begrenzte Anzahl sowie eine bestimmte Reihenfolge von Stufen gibt, die für jeden Intensivierer gilt, der eine hohe Ausprägung eines Begriffs zum Ausdruck bringt. Kurz zusammengefasst kann man die Stufen folgendermaßen präsentieren:

- Auftreten des Lexems in der Ausgangsbedeutung;

- Auftreten des Lexems in mehrdeutigen Kontexten. Die Gradbedeutung erscheint als Nebenbedeutung durch das Ableiten einer kontextabhängigen Inferenz. Der Bezug auf die Ausgangsbedeutung ist nicht beseitigt;

- Konventionalisierung der Gradbedeutung; das Auftreten des Intensivierers in negativ konnotierten Kontexten, der Bezug auf die Ausgangsbedeutung ist damit nicht beseitigt;

- Auftreten der Gradbedeutung in positiv konnotierten Kontexten, der Bezug auf die Ausgangsbedeutung ist beseitigt.

Drittens soll das Phänomen bzw. dessen Mechanismus auf Regularitäten überprüft werden. Es wird demonstriert, dass die untersuchten Grad-Adjektive und -Adverbien bestimmte Wortgruppen je nach der Ausgangsbedeutung bilden können, wobei Lexeme innerhalb einer Gruppe einen ähnlichen Entwicklungsgang der Intensitätsbedeutung einschlagen. Im Rahmen der vorliegenden Arbeit werden folgende deutsche Wörter aus 6 Gruppen untersucht:

- 'Furcht erregend' (entsetzlich, furchtbar, fürchterlich, grässlich, grauenhaft, grauenvoll, grausig, schauderhaft, schauerlich, schaurig, schrecklich, ungeheuer, ungeheuerlich, unheimlich);

- 'Übel wollend / verursachend' (arg, böse, brutal, grausam, grimmig, niederträchtig, mörderisch, schändlich, unmenschlich, unverschämt, wütend);

- 'Sich wie ein Tier verhaltend' (bestialisch, tierisch, viehisch);

- 'Geistig gestört' (irre, irrsinnig, närrisch, toll, verrückt, wahnsinnig);

- 'Religion' (höllisch, teuflisch);

- 'Fluch' (verdammt, verflucht, verteufelt).

Die Lexeme grauenhaft, grauenvoll, grausig sowie böse, niederträchtig, schändlich und anschließend närrisch können keine positiv konnotierten Bezugswörter intensivieren, fungieren jedoch in der Gegenwartssprache als Grad-Adjektive- bzw. -Adverbien und sind mit der entsprechenden Bezeichnung im DUDEN (2012) versehen. Ich habe sie in die Liste der zu untersuchenden Wörter eingefügt, um die Analyse zu vervollständigen. Darüber hinaus werden insgesamt 40 deutsche Lexeme untersucht.

Der typologische Teil dieser Arbeit schließt die durchgeführte Untersuchung ab. Anhand des russischen Materials werde ich demonstrieren, dass die angesprochenen Entwicklungsstufen der Gradbedeutung, die für die deutschen Intensivierer 
entworfen wurden, auch für russische Grad-Adjektive und -Adverbien gelten. Außerdem werde ich meine Aufmerksamkeit auf die typologische Relevanz der Wortfelder, die ich als Basis für die Entwicklung von Gradbedeutung ansehe, richten.

Es gibt kaum Publikationen, die diesem Problem gewidmet sind. In den Arbeiten von Hofmann (1930) und Hentschel (1998) werden zwar Gruppen von Intensivierern angesprochen, jedoch mit nur wenigen Belegen exemplifiziert, die nicht genügend Stoff für eine ausführliche Analyse der Problematik bieten. In meiner Arbeit werde ich demonstrieren, dass die Wortfelder, die im Deutschen zur Entstehung von Grad-Adjektiven und -Adverbien führen, auch für das Russische relevant sind.

\section{Datenerhebung und Forschungsmethoden}

Das von mir verfolgte Ziel, eine möglichst umfassende und präzise Beschreibung der Entstehungsgeschichte von Intensivierern im gegenwärtigen Deutsch zu liefern sowie das Auffinden ihrer Äquivalente im Russischen zu ermöglichen, erfordert eine sehr präzise Materialauswertung. Von daher wurde meine besondere Aufmerksamkeit auf die folgenden Aspekte der Datenerhebung gelenkt: die Liste der zu untersuchenden Lexeme und das Belegmaterial.

\subsection{Deutsche Intensivierer: Probleme und Datengrundlage}

Die Liste der zu untersuchenden deutschen Lexeme wurde von mir in drei Etappen zusammengestellt. Zunächst arbeitete ich mit der von Biedermann erstellten Liste (siehe Abschnitt 2.1.1), dann mit der DeReWo Liste (siehe Abschnitt 2.1.2.). Anschließend habe ich auf der Basis des Materials die Liste der für meine Untersuchung relevanten Lexeme zusammengestellt.

\subsubsection{Biedermanns Liste}

Als Basis für meine Untersuchung habe ich die Liste von Biedermann (1969: 133174) genommen. Biedermanns Dissertation stellt die erste grundlegende Arbeit dar, die ein umfassendes Verzeichnis von Grad-Adjektiven und -Adverbien des gegenwärtigen Deutsch sowie zahlreiches Belegmaterial und die Angaben über die Häufigkeit des Gebrauchs bietet. Es sollen jedoch einige Probleme erörtert werden, auf die ich während der Bearbeitung des übernommenen Materials gestoßen bin. Diese verdienstvolle Liste konnte ich allerdings für meine Studie aus zwei wesentlichen Gründen nicht ohne einige erforderliche Veränderungen bzw. Überarbeitungen nicht übernehmen.

Erstens benötigt die Liste eine sorgfältige Überprüfung hinsichtlich ihrer Aktualität. Biedermanns Dissertation wurde im Jahre 1969 abgeschlossen. Obgleich Biedermann den Versuch unternimmt, in seiner Arbeit „alle aktuellen Ga [...] zu erfassen", soll die relativ große Zeitspanne, die zwischen dem Erscheinen seiner Dissertation und meiner Arbeit liegt, zur Kenntnis genommen werden. Daraus ergeben sich wichtige Fragen bzw. wie vollständig Biedermanns Liste ist und inwiefern sie dem heutigen Sprachzustand entspricht. Mit dieser Problemstellung möchte ich die Vorteile der modernen Technologien ansprechen. Die Entwicklung von digita- 
lisierten Textkorpora wie COSMAS und DTA hat heutzutage nicht nur eine präzisere Suche nach den nötigen Belegen ermöglicht, sondern auch den Umfang des zu untersuchenden Materials wesentlich vergrößert. Ein solches Herangehen an die Bearbeitung des Stoffs war zu Biedermanns Zeit nicht möglich. Die gelegentlichen Lücken in dem Lexemverzeichnis und auch Belegmängel, die in seinem Material auftreten, sollen deshalb in meiner Arbeit behoben werden.

Ein weiteres Problem, das die Aktualität von Biedermanns Material und damit seinen Anspruch auf dessen Vollständigkeit in Bezug auf die heutige deutsche Sprache in Frage stellt, bezieht sich auf den „stete[n] Wechsel“ (Fettig 1934: 2) von Grad-Adjektiven und -Adverbien, die oft „zur Modeerscheinung werden und nach entsprechend kurzer Zeit wieder verschwinden“ (Biedermann 1969: 127). „Die meisten Gradadverbien sind nur eine gewisse Zeit im Gebrauch, dann werden sie durch neue Gradadverbien ersetzt" (Fettig 1934: 2) ${ }^{2}$. Dies geschieht durch den „ständigen[n] Synonymenschub“, welcher „die modernen Sprachen ohne Unterbrechung mit neuen Kraftausdrücken“ „bereichert“ (Werner 1960: 244). Die Tendenz von Intensivierern zum Wechsel lässt sich mit dem Verlust ihrer Expressivität verbinden. Denn Grad-Adverbien und -Adjektive stellen „übertreibende“ bzw. „hyperbolische[] Steigerungen" (Hofmann 1930: 3-10) dar. Das Verblassen des durch ihre Verwendung erzielten „Affekts“ (Biedermann 1969: 126, siehe auch Borst 1902: 1) wird durch den „Hang zu hyperbolischer Ausdrucksweise“ (Borst 1902: 2) erklärt und als „Gesetz der Abschwächung“ (Sperber 1914, 30-31) oder „exhyperbolische Tendenz" (Berz 1953: 19) bezeichnet. Sperber (1914: 31) verweist darauf, dass ,je mehr das neue Wort vordringt, je häufiger es also gesprochen wird, um so mehr verschwinden die Affekte, die ursprünglich an ihm hafteten, und wenn es endlich so weit ist, daß es das alte Wort ganz verdrängt hat, ist es selbst so farblos geworden, daß es seinerseits wieder im Kampf mit einem neu auftretenden Affektträger den Kürzeren ziehen muss". Er exemplifiziert diese Annahme am Beispiel von den Adjektiven schrecklich, entsetzlich und ungeheuer, die „ursprünglich stark affektbetont waren“ (Sperber 1914: 31). Der häufige Gebrauch hat den allmählichen Verlust der emotionalen Komponente des Lexems zu Folge (Biedermann 1969: 126-127, siehe auch Hofmann 1930: 105ff.), sodass „ständig neue Mittel und neue Plereme zur Intensivierung herangezogen werden" (Biedermann 1969: 127). Das Ersetzen der Intensivierer durch neue Formen wird in der Literatur als „renewal“ bezeichnet:

„Intensifiers are especially subject to renewal, presumably because of their markedly emotional function“. (Hopper, Traugott 2003: 122). „....] newly innovated forms compete with older ones because they are felt to be more expressive than what was available before. This competition allows, even encourages, the recession or loss of older forms" (Hopper, Traugott 2003: 124).

Darüber hinaus kann geschlossen werden, dass im heutigen Deutsch sich neue intensivierende Lexeme entwickelt haben können, die in Biedermanns Liste nicht

\footnotetext{
${ }^{2}$ Siehe auch Stoffel 1901: 2, Borst 1902: 157-158, Hofmann 1930: 105, Fettig 1934: 3, Kühner 1934: 1, Berz 1953: 19, Kirchner 1955: 117.
} 
vorkommen. Andererseits können Lexeme, die früher eine intensivierende Funktion hatten, diese inzwischen verloren haben. In diesem Zusammenhang ist eine Aktualisierung des lexikalischen Materials vonnöten.

Das letztere Problem zielt auf die Auswahl von Lexemen, die für meine Untersuchung relevant sind. Biedermann bietet eine ausgiebige Auswahl an Grad-Adjektiven und -Adverbien. Ein Blick auf die entsprechenden lexikographischen Beschreibungen im DUDEN (2012) zwingt jedoch zu der Feststellung, dass manche Lexeme dort keine intensivierende Funktion aufweisen, z.B. beträchtlich hoch (Biedermann 1969: 135), weitgehend genauer (Biedermann 1969: 147), faszinierend weiß (Biedermann 1969: 172) u.a. Dagegen werden die Verwendungen wie eine ungewöhnlich schöne Frau (DUDEN 2012, s.v. ungewöhnlich), unwahrscheinliches Glück (DUDEN 2012, s.v. unwahrscheinlich) und eine unglaubliche Menge (DUDEN 2012, s.v. unglaublich) im DUDEN (2012) als Intensivierer behandelt, obwohl sie im Vergleich zu furchtbar ('Angst erregend' -> 'in hohem Grad'), arg ('böse' -> 'in hohem Grad') oder sehr ('schmerzhaft' -> 'in hohem Grad') ebenfalls keinen besonders großen Sprung in der Bedeutungsentwicklung gemacht zu haben scheinen. Der Unterschied zwischen ungewöhnlich gut und furchtbar gut liegt vor allem daran, dass furchtbar die mit dem ursprünglichen Gebrauch verbundenen negativen Konnotationen verloren hat und damit eine bemerkenswerte Ausbleichung des semantischen Gehalts aufweist. Bei ungewöhnlich bleibt dagegen der Zusammenhang mit der Ausgangsbedeutung „vom Üblichen, Gewohnten, Erwarteten abweichend“ erhalten. Die Verwendung ungewöhnlich gut kann kontextabhängig eine weitere, auf Intensität bezogene Interpretation durch „Inferenzprozeduren“ (Portz 2009: 149150) erwerben, demonstriert jedoch keinen Verlust der ursprünglichen Bedeutung. In diesem Zusammenhang entsteht die Frage, welchen Kriterien ein Lexem entsprechen soll, um im DUDEN (2012) als Intensivierer aufgenommen zu werden. Die Antwort ${ }^{3}$ des DUDENS verweist auf die Häufigkeit des Auftretens eines Lexems in der bestimmten Bedeutung.

Der DUDEN, im Gegensatz zu der vor mehr als 40 Jahren veröffentlichten Arbeit von Biedermann, richtet sich auf den gegenwärtigen Sprachzustand und bietet damit zuverlässige Informationen über die Bedeutungspalette der Lexeme. Deshalb werde ich die Wörter, die im DUDEN - im Gegensatz zu Biedermann - nicht als Gradadverbien vorkommen, in meiner Arbeit nicht berücksichtigen.

\subsubsection{Die COSMAS-Liste}

Wie ich in 2.1.1 dargelegt habe, benötigt die Biedermann-Liste eine Aktualisierung. Seit der Veröffentlichung von Biedermanns Dissertation sind bis heute keine Arbeiten erschienen, die das Lexemverzeichnis gezielt auf den neuesten Stand gebracht hätten. Von daher soll solch ein Verzeichnis manuell erstellt werden. Eine allgemeine Liste der nach Häufigkeit sortierten Adjektive und Adverbien findet sich in dem Häufigkeitswörterbuch gesprochener Sprache von Ruoff (1989). Jedoch, wie Ruoff (1989: 9) in dem Kapitel „Voraussetzungen“ angibt, hat die Arbeit an dem Projekt bzw. das Sammeln der Tonbandaufnahmen und ihre Auswertung im Jahre 1955 begonnen und bis in den Anfang der achtziger Jahre gedauert. Von

\footnotetext{
$3<$ http://www.duden.de/ueber_duden/wie-kommt-ein-wort-in-den-duden>
} 
daher entspricht diese Liste auch nicht der auf Aktualität zielenden Voraussetzung. Außerdem bleibt sie auf die gesprochene Sprache beschränkt und schließt damit die Besonderheiten der geschriebenen Sprache aus.

Ich wandte mich für die Erfüllung des gestellten Zwecks an das Institut für Deutsche Sprache (IDS) in Mannheim und bekam vier aktuelle korpusbasierte Wortlisten mit jeweils 30.000, 40.000, 100.000 und 250.000 Einheiten zu DeReKo ${ }^{4}$. Die Schwierigkeit bei der Arbeit mit diesem Material besteht nun darin, dass die Liste nicht nach Wortarten geordnet ist. Darüber hinaus sind weder die Kategorien „Adjektiv“ und "Adverb“ noch eine Charakterisierung „qualitativ" darin ausgezeichnet. Mit diesen Kriterien musste ich selbst an die Liste herangehen und die von mir gewünschten Einträge manuell herausfiltern.

Es soll an dieser Stelle kurz der Umfang der Liste und des deutschen Wortschatzes angesprochen werden. Nach den Angaben von DUDEN „setzt man den Wortschatz der deutschen Gegenwartssprache auf zwischen 300000 und 500000 Wörter (Grundformen) an. Der aktive Wortschatz eines deutschen Durchschnittssprechers wird heute auf 12000 bis 16000 Wörter (davon etwa 3500 Fremdwörter) geschätzt. Ohne Schwierigkeiten verstanden werden mindestens 50000 Wörter"5. Das Dudenkorpus enthält dabei ca. 9 Millionen Wörter. „Die enorme Diskrepanz zum Umfang eines Wörterbuchs erklärt sich dadurch, dass die meisten Wörter im Dudenkorpus nur ein- bis wenige Male vorkommen und deshalb keine Aufnahmekandidaten für ein Wörterbuch sind“6. Die von mir ausgewählte Liste mit 250.000 Wörtern soll von daher den heutigen Sprachgebrauch widerspiegeln können und damit eine solide Grundlage für meine Untersuchung bilden.

Ich werde nun kurz die Schritte erläutern, die ich bei der Bearbeitung der IDSWortliste durchgeführt habe. Zunächst wendete ich mich an meine früheren Kollegen aus der linguistischen Abteilung in der Firma ABBYY ${ }^{7}$. Mit ihrer Hilfe wurden alle Substantive und Verben aus der Liste entfernt. Um eine lediglich aus Adjektiven und Adverbien bestehende Liste zusammenzustellen, habe ich manuell alle Wörter, die zu anderen Wortkategorien gehören, entfernt. Beim nächsten Schritt wählte ich aus der entstandenen Liste von Adjektiven und Adverbien die für meine Untersuchung geeigneten Lexeme aus. Dafür habe ich eine Reihe von Kriterien aufgestellt, denen die für mich relevanten Wörter entsprechen sollen:

- qualitativ sein

- mehrdeutig sein (gemäß DUDEN 2012)

- Gradbedeutung aufweisen (gemäß DUDEN 2012)

- negativ konnotierte Ausgangsbedeutung haben

- positiv konnotierte Gradbedeutung haben

In Bezug auf das letzte Kriterium habe ich im Laufe meiner Recherche festgestellt, dass sich im Korpus sehr oft Belege finden, die den Gebrauch eines Intensivierers

\footnotetext{
${ }^{4}$ DeReKo = das Deutsche Referenzkorpus <http://www1.ids-mannheim.de/kl/projekte/korpora/>

5 16.09.2014 <http://www.duden.de/sprachwissen/sprachratgeber/zum-umfang-des-deutschen-wortschatzes>

${ }_{6}$ 16.09.2014 < http://www.duden.de/sprachwissen/sprachratgeber/zum-umfang-des-deutschen-wortschatzes>

${ }^{7}$ ABBYY GmbH < www.abbyy.de> ist ein Hersteller von Software für Textverarbeitung, Dokumentenerfassung sowie von Wörterbüchern.
} 
mit einem positiv konnotierten Kookkurrenzpartner demonstrieren, wobei der Gebrauch in DUDEN (2012) nicht erwähnt wird und das Auftreten des Lexems nur in Verbindung mit negativ konnotierten Bezugswörtern veranschaulicht. Darüber hinaus habe ich bei der Bearbeitung der IDS-Liste auf das letzte Kriterium verzichtet und erst während der Untersuchung die unnötigen Lexeme gestrichen. Insgesamt wurden für meinen Zweck 40 Lexeme ausgewählt.

\subsection{Wörterbücher}

Das in der vorliegenden Arbeit angestrebte Ziel, die Entwicklungsgeschichte von Intensivierungsmitteln zu verfolgen, benötigt zunächst eine zuverlässige Grundlage, die den Einstieg in die Problematik ermöglichen kann. Diese Basis bieten historische Wörterbücher, die das Bedeutungsspektrum eines Lexems sowie dessen Gebrauch auf verschiedenen Sprachstufen bezeugen.

Es soll jedoch darauf hingewiesen werden, dass aufgrund von aktuell laufenden Arbeiten nicht alle Informationen über die zu untersuchenden Lexeme zugänglich sind. Viele ergiebige Quellen, beispielsweise AWB, BMZ und FWB, die ein umfassendes Material mit zahlreichen Belegen bieten, befinden sich in der Erstellungsphase; deshalb finden sich die Angaben lediglich über die Lexeme in den schon erschienenen Bänden. Andere Quellen, z.B. DWB, werden neu bearbeitet, wobei die neu erschienen Artikel gelegentlich wesentliche Unterschiede zu der ersten Fassung aufweisen. Von daher muss man zur Kenntnis nehmen, dass die älteren Angaben weniger informativ als die neuen sein können. Die fehlenden Informationen über die Lexeme werden an den entsprechenden Stellen erörtert. Ich habe die mangelhaften Informationen teilweise ausfindig machen können, indem ich mich an die Arbeitsstelle von FWB an Herrn Prof. Dr. Reichmann und Frau Prof. Dr. Lobenstein-Reichmann sowie an die Arbeitsstelle des DWB an Herrn Dr. Harm gewendet und mit deren Erlaubnis eine Recherche in den Archiven durchgeführt habe.

Ein weiteres Problem bei der Verwendung von Wörterbüchern bezieht sich auf die Einheitlichkeit der in ihnen enthaltenen Informationen. Wie Os (1989: 5) mit Recht anmerkt, „fehlt" häufig „eine einheitliche Erfassung der sprachlichen Erscheinung 'Intensivierung'". Die Analyse hat ergeben, dass in vielen Fällen Belege mit der intensivierenden Funktion des Lexems irrtümlich in eine andere Bedeutung eingeordnet werden. Gelegentlich verzichten Lexikographen auf Definitionen und exemplifizieren den Gebrauch nur mit Belegen. In diesem Zusammenhang sollen die aus Wörterbüchern entnommenen Informationen durch die in Textkorpora enthaltenen Belege ergänzt werden.

\subsection{Textkorpora}

Textkorpora stellen eine solide Materialbasis für die durchgeführte Untersuchung zur Verfügung. Sie ergänzen den teilweise fehlenden Stoff in den konsultierten Wörterbüchern und bieten die Möglichkeit, die Wortgeschichte möglichst präzise zu rekonstruieren. Den Kern der Materialgrundlage bilden systematisch aufgebaute Textsammlungen unterschiedlicher Größe und Herkunft, die eine ausgiebige Anzahl von Belegen für die entsprechenden Zeitstufen zur Verfügung stellen: 
Die Mittelhochdeutsche Begriffsdatenbank (MHDBDB) der Universität Salzburg umfasst 650 Texte, bietet damit "den Zugriff auf die wichtigsten Werke der mittelhochdeutschen Dichtung"8 und hat das Ziel, „die größte elektronische Sammlung deutscher Texte aus dem Mittelalter" ${ }^{\text {“9 }}$ aufzubauen. Die in dem Korpus enthaltenen Texte beziehen sich auf den Zeitraum 1050 - 1350.

Das Bonner Frühneuhochdeutschkorpus (FnhdC) besteht aus 40 Quellen und enthält das Material für den Zeitraum 1350 - 1700. Das 400.000 Seiten umfassende Korpus der frühnhd. Sprache wurde von der Arbeitsstelle des Frühneuhochdeutschen Wörterbuchs zusammengestellt.

Das Deutsche Textarchiv (DTA) besteht aus ca. 1630 Werken und umfasst den Zeitraum 1600 - 1900. DTA „enthält Texte unterschiedlicher Disziplinen und Textsorten, welche mit dem Ziel zusammengestellt wurden, das gesamte Spektrum der deutschen Sprache zu erfassen und somit ein ausgewogenes historisches Referenzkorpus in deutscher Sprache zu schaffen"10. DTA unterscheidet sich von den anderen vergleichbaren Projekten „durch die sorgfältige Auswahl der Texte und Ausgaben, die sehr hohe Erfassungsgenauigkeit, die strukturelle und linguistische Erschließung der Textdaten sowie die Verlässlichkeit der Metadaten"11;

Das Projekt Gutenberg-DE „enthält mehr als 7000 Texte von über 1400 Autoren"12 und stellt damit „die größte elektronische Volltextsammlung deutschsprachiger Literatur"13 dar.

Das Projekt Digitales Wörterbuch der deutschen Sprache (DWDS), welches das DWDS-Kernkorpus sowie weitere in die DWDS-Abfrageplattform eingebundene Korpora zur Verfügung stellt, befindet sich im Aufbau. Das Portal stellt ein „zeitlich und nach Textsorten (Belletristik, Gebrauchsliteratur, Wissenschaft, Journalistische Prosa) ausgewogenes Korpus des gesamten 20. Jahrhunderts" ${ }^{14}$ dar und bietet über 1,8 Millionen Belege aus 15 Korpora;

Das Projekt Corpus Search, Management and Analysis System (COSMAS) des Instituts für deutsche Sprache in Mannheim bietet die Möglichkeit für die Recherche in 231 Korpora mit insgesamt 29,2 Mrd. laufenden Wortformen bzw. 72,9 Mio. Buchseiten. COSMAS ermöglicht die Suche in dem historischen Archiv (ab der zweiten Hälfte des 17. Jh. bis 1962) sowie in dem Archiv der geschriebenen Sprache (ab dem 18. Jh. bis heute) ${ }^{15}$.

Meine Untersuchung stützt sich hauptsächlich auf die oben genannten Korpora, jedoch übernehme ich in seltenen Fällen auch Belege aus dem Internet, u.a. $<w w w . g o o g l e .-b o o k s . d e>$. Das Internet bietet mehrere Milliarden Textseiten und stellt damit ein riesiges und konstant wachsendes Textarchiv dar. „Die leichte $\mathrm{Zu}$ gänglichkeit eines fast unbeschränkt grossen Korpus, seine elektronische Form und die Automatisierungsmöglichkeiten für die Belegerfassung machen das WWW

\footnotetext{
$8<$ http://mhdbdb.sbg.ac.at:8000/info.de.html >

$9<$ http://mhdbdb.sbg.ac.at:8000/info.de.html>

10 12.06.2015: <http://www.deutschestextarchiv.de/doku/ueberblick>

11 12.06.2015: <http://www.deutschestextarchiv.de/doku/ueberblick >

12 12.06.2015: <http://gutenberg.spiegel.de/>

13 12.06.2015: <http://gutenberg.spiegel.de/>

$1412.06 .2015<\mathrm{http}: / /$ www.dwds.de/ressourcen/korpora/>

15 12.06.2015 < http://www.ids-mannheim.de/cosmas2/projekt/referenz/archive.html>
} 
zu einer idealen Quelle für die Wortschatzerforschung" (Bickel 2006: 81-82). In meiner Arbeit benutze ich diese Quelle, um einen fragwürdigen Gebrauch zu überprüfen bzw. festzustellen. Das Internet mag zwar Bedenken an seiner Zuverlässigkeit hervorrufen, jedoch, wie Bickel in seiner Arbeit überzeugend darlegt, stellt das Internet „ein äusserst brauchbares Korpus“ dar (Bickel 2006: 80). „Die Grösse und Vielfalt des Korpus garantierte seine Stabilität auch während der enormen Wachstumsphase" (Bickel 2006: 80). Da für meine Untersuchung die Tatsache relevant ist, dass ein gewisser Gebrauch stattgefunden hat, z.B. das Auftreten eines Gradadverbs in positiv konnotierten Kontexten, erfüllt das Internet als Korpus seinen Zweck als Forschungsdatenbasis.

\subsection{Russische Grad-Adjektive und -Adverbien}

Die Liste der russischen Grad-Adjektive und -Adverbien wurde auf eine ähnliche Weise zusammengestellt wie die deutsche. Zunächst habe ich mit einer Häufigkeitsliste der adjektivischen und adverbialen Lexik gearbeitet. Dafür verwendete ich das auf den aktuellen Angaben des russischen Korpus basierende Häufigkeitswörterbuch des russischen Wortschatzes (Ljaševskaja, Šarov 2009). Mit Hilfe dieses Wörterbuchs sortierte ich die Lexeme aus, welche im Russischen eine intensivierende Funktion aufweisen. Für die Auswahl waren die Lexeme relevant, die eine negativ konnotierte Ausgangsbedeutung haben. Die Liste der Wörter habe ich dann durch Synonymwörterbücher ergänzt. Anschließend habe ich die ausgewählten Wörter (insgesamt 28 Lexeme) je nach der Ausgangsbedeutung in Wortfelder verteilt.

Das meiste Belegmaterial habe ich im Korpus der russischen Sprache ${ }^{16}(\mathrm{RC})$ gesammelt. In einzelnen Fällen habe ich den Sprachgebrauch im Internet überprüft. Alle Belege sind mit der entsprechenden Quellenangabe und einer Übersetzung versehen.

\subsection{Materialauswertung}

Das in der vorliegenden Arbeit präsentierte Material stellt eine Auswahl aus den oben bezeichneten Quellen bzw. Wörterbüchern und Textkorpora dar. Da im Fokus der Forschung die Untersuchung des Entwicklungsgangs von Grad-Adjektiven und -Adverbien liegt, wurden die Quellen deshalb auf diese Wortgruppe gezielt befragt.

Alle Belege sind mit den Angaben über die Fundstellen versehen. Verwendungen, die von mir konstruiert worden sind, bleiben unmarkiert.

$16<$ www.ruscorpora.ru> 


\section{Theoretische Grundlagen}

\subsection{Zum Begriff der Intensivierung}

Intensivierung hat in den bisherigen Studien viel Aufmerksamkeit erzielt. In der Literatur finden sich zahlreiche Bezeichnungen der Erscheinung wie „Intensivierung“ (Os 1989: 1, Peters 1993: 1, Kirschbaum 2002a: 1), „Steigerung, Verstärkung, Grad, Graduierung, grading, gradation, scaling“ (Os 1989: 1), „intensification“ (Bolinger 1972: 11, Allerton 15). Die ersten Arbeiten, die gezielt der Untersuchung von Grad-Adjektiven und -Adverbien dienen, bieten folgende Definitionen, z.B. in Kip (1901: 145):

Unter Steigerungs- oder Intensitätsadverbien „verstehen wir solche Adverbien, bzw. adverbial gebrauchte Wörter anderer Kategorien und adverbial gebrauchte Redensarten, durch welche Verbal- oder Nominalbegriffe gesteigert oder intensiviert werden, ohne dass dabei ihre Bedeutung wesentlich geändert wird“.

Fettig (1934: 1) formuliert diese Definition um und verweist u.a. auf Adjektive als mögliche Kookkurrenzpartner von Gradadverbien:

„Als Gradadverbien bezeichnen wir die Adverbien, die den durch Adjektiv, Adverb oder Verb ausgedrückten Begriff in bezug auf seinen Grad näher bestimmen."

Das Phänomen kann sich jedoch nicht nur auf die Verstärkung, sondern auch auf die Abschwächung eines Begriffs beziehen, vgl. die Definition von Os (1989: 2):

„'Intensivierung' ist die funktional-semantische Kategorie der Verstärkung und der Abschwächung intensivierbarer sprachlicher Ausdrücke."

Dementsprechend werden Grad-Adjektive und -Adverbien als Verstärkungswörter (Müller 1899: 8), Steigerungsadverbien (Kip 1900: 145, Borst 1902: 26), Intensiva bzw. Re-striktiva (Fettig 1934: 1), Intensitätsadverbien (Kip 1900: 145), Intensivadverbien (Kühner 1934: 1, Kirchner 1955: 6) und Intensivierungsmittel- oder Operatoren (Os 1989: 2) sowie als intensifiers (Bolinger 1972), adverbs of degree (Jonson 1967) und reinforcers (Paradis 2000) bezeichnet:

(5) „I use the term intensifier for any device that scales a quality, whether up or down or somewhere between the two" (Bolinger 1972,17);

(6) „'Intensivierungsmittel' (oder 'Intensivierungsoperatoren') sind sprachliche Elemente unterschiedlicher grammatischer Kategorien welche die Funktion der Ausdrucksverstärkung bzw. -abschwächung haben“ (Os 1989: 2).

Intensivierer, auch Intensivierungsoperatoren genannt, treten mit intensivierbaren Ausdrücken auf, die auch als Intensivierungsoperanden (Os 1989: 2) bezeichnet werden. Die letzten sind Prädikate, die in den Skopusbereich eines Intensivierungsmittels gelangen und „(einfache) oder komplexe sprachliche Einheiten, in denen Verstärkung bzw. Abschwächung vorliegt" (Os 1989: 2), darstellen. Aus der Zusammenstellung von Intensivierungsoperatoren- und Operanden entstehen Intensivausdrücke. Sie verstehen sich „als Syntagmen, in denen syntaktisch zulässige 
Kollokationen von Intensivierungsmitteln und intensivierbaren Ausdrücken vorkommen" (Os 1989: 2). Die Beziehung zwischen einem Intensivierungsmittel und einem „Kern“ (Biedermann 1969: 42) wird in Os (1989: 9) auch als modifier + head, operator + operand, operator + nucleus, Modifikator + Modificandum, Bestimmungswort + Kern bezeichnet.

Somit steht im Fokus der Intensivausdrücke eine Skala mit zwei Extremen (Leech 1974: 108), auf der die durch Intensivierungsmittel modifizierten Begriffe auf bestimmten Positionen eingeordnet werden. Die Position auf der Skala hängt von der Art des Intensivierers bzw. des durch ihn ausgedrückten Grads ab. Die Klassifizierung von Intensivierungsmitteln nach dem Grad wird unten in 3.3 dargestellt.

Als Voraussetzung für die Erfüllung der Funktion von Grad-Adjektiven und -Adverbien gilt die Graduierbarkeit bzw. gradability von deren Bezugswörtern (vgl. Paradis 2000: 241). Darüber hinaus ist „[i]hre Verwendbarkeit [...] davon anhängig, ob der head, den sie modifizieren sollen, graduierbar und damit intensivierbar ist" (Peters 1993: 9). Als Bezugswörter von Grad-Adjektiven und -Adverbien können Adjektive, Adverbien, Verben, Substantive und Funktionsverbgefüge auftreten. Ihre Graduierbarkeit wird unten in 3.4 diskutiert.

\subsection{Intensivierer in der bisherigen Forschung}

Die bisherigen Arbeiten umfassen verschiedene Aspekte des Phänomens: Katalogisieren der Lexeme, Beschreibung der einzelnen Wortgeschichten in bestimmten Zeitstufen und Sprachen, Analyse der Herkunft nach verschiedenen Kriterien, Gruppierung von Lexemen nach der Gradausprägung sowie der Ausgangsbedeutung und Untersuchung von zugrunde liegenden Mechanismen.

Ein Überblick über die auf das Deutsche bezogenen Veröffentlichungen findet sich in Biedermann (1969). Es wird dort u.a. darauf hingewiesen, dass für die deutsche Sprache „hauptsächlich“ Arbeiten „für ältere Perioden der Sprachgeschichte“ (Biedermann 1969: 2) vorliegen. Diese Aufsätze bieten ergiebiges Material zur Entwicklung einzelner Wörter. Jedoch weist Biedermann (1969: 4) darauf hin, dass „generelle Tendenzen und Vergleiche innerhalb der Klasse der Ga nur kaum möglich sind“.

Die Analyse von Intensivierern in verschiedenen Zeitstufen ihrer Herausbildung steht im Mittelpunkt der Studien von Kip (1901), Leuschner (1918) und Fritz (1934). Die Arbeit von Kip (1901) widmet sich der geistlichen Dichtung des 11. und 12. Jahrhunderts und stellt eine „Vorarbeit“ zu einem „Specialwörterbuch für das 11. und 12. Jahrhundert" (Kip 1901: 144) dar. Der Autor untersucht die Herausbildung von ca. 60 Lexemen „nach syntaktischen, morphologischen und semantischen Kriterien" (Biedermann 1969: 2). Da die Forschung das Erstellen eines Wörterbuchs bezweckt, bietet Kip eine große Menge von Belegen für jedes berücksichtigte Lexem. Leuschner (1918: 3) kritisiert jedoch das mangelhafte Herangehen des Autors an die Analyse des Materials, in dem „das Ahd. in ihr fast garnicht berücksichtigt wird und fast alle grösseren Dichtungen weltlichen Inhalts [...] nicht herangezogen sind“.

Leuschner (1918) bietet eine ausführliche Analyse von Gradadverbien im Ahd. und Mhd., basierend auf Statistiken und reichlichem Belegmaterial. Laut Bieder- 
mann (1969: 3) untersucht Leuschner jedoch lediglich „10 unmotiviert ausgewählte“ Lexeme. Dabei stehen die Wortbeschreibungen „isoliert und beziehungslos nebeneinander [...], sodass keine Querverbindungen zwischen den einzelnen Ga möglich sind" (Biedermann 1969: 3). Eine weitere frequenzorientierte Studie von Fritz (1934) bezieht sich ebenso auf das Mittelhochdeutsche und bietet umfangreicheres Material im Vergleich zu Leuschner. Jedoch sind die Wortartikel „unterschiedlich aufgebaut, das Belegmaterial ,inkonsequent berücksichtigt", sodass die Schlußfolgerungen „nur schwer möglich“ (Biedermann 1969: 3) sind.

Ein kurzer Überblick über die Arten der Wortverstärkung im frühen Nhd. findet sich in Müller (1899). Von besonderem Interesse für meine Arbeit erscheint die Analyse von kontextuellen Beschränkungen von einzelnen Grad-Adjektiven und Adverbien, die nach dem mittelhochdeutschen Gebrauch verloren gehen und das Auftreten von ursprünglich negativ konnotierten Lexemen mit positiv konnotierten Bezugswörtern demonstrieren, z.B. schrecklich / höllisch / diebisch gefreut (Müller 1899: 7-8). Das Gleiche gilt auch für die Verbindungen von ursprünglich positiv konnotierten Lexemen mit negativ konnotierten Bezugswörtern, z.B. hübsch kalt.

Einen ergiebigen Überblick über die Wortgeschichte einzelner Gradadverbien bietet der Aufsatz von Biener (1940). Neben der Aufgabe, , alle in der wissenschaftlichen Literatur an vielen Stellen verstreuten Belege zu erfassen“, verfolgt er das Ziel, die Herausbildung von 14 Intensivierern in "chronologischer Ordnung" zu exemplifizieren (Biener 1940: 166). Die Studie demonstriert den Gebrauch der Wörter in verschiedenen Zeitspannen vom Ahd. bis zum Nhd., bietet jedoch keine Erklärung, welche Mechanismen dem Bedeutungswandel zugrunde liegen.

Die Mechanismen, die bei der Entwicklung von Gradadverbien im Deutschen mitwirken, werden in den Arbeiten von Os (1989), Hentschel (1998), Paradis (2000, 2008), Kirschbaum (2002a, b) und Ghesquière, Davidse (2011) untersucht. Die Autoren präsentieren verschiedene Blickwinkel auf die Problematik, indem sie Gradadverbien im Rahmen der kognitiven Theorie der Metapher und Metonymie sowie der Grammatikalisierungstheorie behandeln. Ihre Standpunkte werden in den folgenden Kapitelabschnitten ausführlich erörtert.

Es gibt eine große Menge von Arbeiten, die sich der Intensivierung im Englischen sowie in anderen Sprachen widmen. Die Dissertation von Spitzbardt (1954) bietet eine ausführliche Liste englischer Steigerungsadverbien. Eine detaillierte Übersicht der Werke, die das Phänomen in verschiedenen Sprachstufen untersuchen, findet sich beispielsweise in Peters (1993). In Bezug auf die vorliegende Arbeit von besonderem Interesse sind die Studien, die sich mit theoretischen Fragen bzw. mit der Untersuchung der Natur von Gradadverbien befassen. So bieten Stoffel (1901), Borst (1902), Fettig (1934), Biedermann (1969) und Bolinger (1972) nicht nur zahlreiche Belege, sondern auch verschiedene Klassifizierungen des Stoffs, die in dem nächsten Kapitelabschnitt präsentiert werden. In Peters (1993) wird neben einer ausführlichen Untersuchung der Geschichte von englischen Gradadverbien außerdem der Gebrauch der Lexeme auf allen Zeitabschnitten ihrer Entwicklung sowie die Erklärung von zugrunde liegenden Mechanismen dargestellt. 
Eine kookkurrenzbezogene Studie wird in Greenbaum (1970) präsentiert. Greenbaum richtete sein Augenmerk auf die Untersuchung von Kollokationsmöglichkeiten von 8 Intensivierern (certainly, really, badly, (very) much, greatly, entirely, utterly, completely) sowie die Beschränkungen ihres Auftretens in bestimmten Kontexten mittels verschiedener Tests und Informanten. Diese Arbeit bringt zum Ausdruck, dass keine festen Aussagen über die Beziehung zwischen bestimmten Gradadverbien und deren Bezugswörtern bzw. Verben gemacht werden können. Denn „for some intensifiers, e.g. utterly, the collocational range of verbs can be defined semantically. For others, there appears to be a collocational range of verbs that are semantically homogenous, but nevertheless some verbs that are apparently in the same semantic group are unacceptable as collocates" (Greenbaum 1970: 83). Ein Beispiel für eine solche Generalisierung mit einer Ausnahme bietet das Adverb deeply, welches mit hate, dislike, admire, love und value auftreten kann, jedoch nicht mit like. In diesem Zusammenhang erscheint eine ausführliche Beschreibung von Kookkurrenzmöglichkeiten von Intensivierern für meine Arbeit nicht notwendig. Bei der Analyse von Kollokationen steht somit die Möglichkeit des Auftretens eines Grad-Adjektivs bzw. -Adverbs in negativ sowie positiv konnotierten Zusammensetzungen im Zentrum meines Interesses.

Aus der oben eingeführten Übersicht lässt sich schließen, dass ungeachtet der großen Menge an Literatur, die der Intensivierung gewidmet ist und verschiedene Aspekte des Phänomens berücksichtigt, es bis heute keine Arbeiten gibt, die eine ausführliche Analyse der Herausbildung von deutschen Grad-Adjektiven und -Adverbien in den Fokus stellen und die Erklärung von Mechanismen der Entwicklung dieser lexikalischen Klasse anstreben.

\subsection{Intensivierungsmittel: Klassifizierung}

In 3.1 wurde angesprochen, dass Steigerungsadverbien dem Ausdruck des Grads eines Begriffs dienen. Da der Grad auf unterschiedlichen Positionen einer Skala liegen kann, handelt es sich um verschiedene Typen von Intensivierern. Seit dem Anfang des 20. Jahrhunderts haben sich mehrere Forscher um das Problem der Unterscheidung von Intensivierungsmitteln bemüht und zahlreiche Klassifizierungen hinsichtlich deren Orientierung auf einer Skala entwickelt.

Stoffel (1901) bietet eine zweiteilige Differenzierung zwischen“ „intensives" bzw. „intensive adverbs" und „down-toners" bzw. „down-toning adverbs“. Die erste Wortgruppe der Gradadverbien bezeichnet ,a high or very high degree of a quality" (Stoffel 1901: 129) und wird mit dem Beispiel der Lexeme full, pure, very, right, quite, so und as exemplifiziert. In Bezug auf die Herkunft der Wörter fügt Stoffel hinzu: „most of the adverbs derived from adjectives expressing absolute qualities“" (Stoffel 1901: 1). Die zweite Gruppe bringt „, a moderate, slight, or just perceptible degree of a quality" (Stoffel 1901: 129) zum Ausdruck und lässt sich durch die Lexeme rather und pretty veranschaulichen. Jedoch stellt Stoffel in den Fokus seiner Arbeit den Entwicklungsgang sowie den gegenwärtigen Gebrauch von Lexemen und geht somit auf keine ausführliche Diskussion hinsichtlich der Klassifizierung ein.

Auf eine zweiteilige Gliederung der Intensivierer stützen sich weitere Werke der ersten Hälfte des 20. Jahrhunderts, z.B. die von Borst (1902) und Fettig (1934), 
welche sich der Untersuchung von Steigerungsadverbien bzw. Intensiva und Abschwächungsadverbien bzw. Restriktiva im Englischen widmen.

Quirk et al. (1972: 259-261) bieten dagegen eine dreiteilige Gliederung von Gradadverbien. Neben downtoners unterscheiden sie emphasizers und amplifiers. Emphasizers „have a general heightening effect" (z.B. a certain winner, a definite loss, plain nonsense), amplifiers "scale upwards from an assumed norm“ (z.B. a complete fool, utter folly, an extreme enemy) und downtoners ",have a lowering effect, usually scaling downwards from an assumed norm"17.

Eine detaillierte Einteilung von Gradadverbien in mehrere Klassen je nach der "Graduationsstufe“ wurde in Biedermann (1969, 131 ff.) präsentiert. „Es sind dies sechs Klassen, sowie eine siebte, deren Elemente als Ga ${ }^{18}$ auf mehreren Stufen möglich sind" (Biedermann 1969: 131). Er unterscheidet damit die folgenden Kategorien:

- hohe Graduationsstufe, die „nach unten hin von der gemäßigten [...] und nach oben hin von der absoluten Graduation [...] abgegrenzt" wird, „die meisten“ Gradadverbien "umfasst" und damit „die bedeutendste Stufe“ ist, z.B. sehr, stark, furchtbar (Biedermann 1969: 133);

- absolute Graduationsstufe, die sich auf die Lexeme bezieht, welche „eine nicht mehr zu steigernde und nicht mehr zu übertreffende Intensität der betreffenden Kerne“ bezeichnen, z.B. völlig, total (Biedermann 1969: 174);

- gemäßigte Graduationsstufe, die „nach oben hin abgegrenzt von der hohen [...], nach unten hin von der schwachen [...] Graduation" wird, z.B. ziemlich, einigermaßen (Biedermann 1969: 182);

- schwache Graduationsstufe, „die zwischen der gemäßigten [...] und der minimalen [...] Graduation steht", z.B. etwas, bißchen (Biedermann 1969: 186);

- minimale Graduationsstufe, die noch einen weniger ausgeprägter Intensitätsgrad der Sachverhalte als die schwache Graduationsstufe zum Ausdruck bringt, z.B. wenig, kaum (Biedermann 1969: 189);

- negative Graduationsstufe ist „die Klasse mit der geringsten Intensität“ und im Wort nicht ausgeprägt (Biedermann 1969: 191).

Bolinger (1972: 17) hat Biedermanns Klassifikation auf vier Klassen reduziert, sodass jede Klasse einem bestimmten Bereich auf einer Intensivierungsskala entspricht:

- „boosters: upper part of a scale, looking up“, z.B. perfect, terrible;

- "compromisers: middle of the scale, often trying to look both ways at once“, z.B. rather, fairly;

- „diminishers: lower part of the scale, looking down“, z.B. indifferent, little;

- „minimizers: lower end of the scale“, z.B. a bit, an iota.

\footnotetext{
${ }^{17}$ Quirk et al. (1979: 260) verweisen darauf, dass amplifiers je nach dem Kontext als emphasizers verwendet werden können, vgl. total. In total destruction wird total als amplifier gebraucht und in dem Sinne „distruction of everything" verstanden. In der Zusammensetzung total nonsense fungiert total als emphasizer und intensiviert das Bezugswort nonsense.

18 Gradadverbien
} 
Wie es in Peters (1993: 4) zu recht angemerkt wurde, ist für die Klassifizierungen von Biedermann und Bolinger „eine feinere Einteilung der down-toners" ausschlaggebend während die intensives im Gegensatz „undifferenziert bleiben“. Dieser Mangel wird in CGEL (1985: 589ff.) behoben, in dem eine detailliertere Gliederung angeboten wird. Zunächst wird die Intensivierungsskala in zwei Bereiche geteilt bzw. amplifiers „upwards from an assumed norm" und downtoners „,downwards from an assumed norm" (CGEL 1985: 590) angesetzt. Der Gruppe amplifiers gehören maximizers („,an denote the upper extreme of the scale“, z.B. completely, extremely, fully) und boosters ("denote a high degree, a high point on the scale“, z.B. greatly, highly, terribly) (CGEL 1985: 590). Die Gruppe downtoners schließt approximators (,serve to express an approximation to the force of the verb, while indicating that the verb concerned expresses more than is relevant", z.B. almost, nearly), compromisers (,have only a slight lowering effect and tend [...] to call in question the appropriateness of the verb concerned“, z.B. rather, sufficiently, more or less), diminishers („scale downwards and roughly mean 'to a small extent'“, z.B. partly, somewhat) und minimizers („,are negative maximizers, 'not to any extent'“, z.B. hardly, scarcely) ein.

In Bäcklund (1973) findet sich eine weitere Klassifikation, die sich nicht nur nach Stufen gliedert, sondern auch auf andere semantische Kriterien gerichtet ist. Diese Klassifizierung sieht die folgenden zehn Kategorien vor, vgl. auch die aus Bäcklund (1973) und Os (1989: 99) übernommenen englischen und deutschen Beispiele:

- „Adverbs expressing the complete or partial absence of the concepts denoted by their heads", z.B. almost, nearly; vgl. fast, nahezu (Bäcklund 1973: 19);

- „Adverbs expressing the minimum degree or the degree just above the nonpresence of the concepts denoted by their heads", z.B. barely, hardly; vgl. kaum, gerade (Bäcklund 1973: 37);

- „Adverbs expressing a low degree“, z.B. a little, a bit, slightly; vgl. ein bißchen, ein wenig, etwas (Bäcklund 1973: 47);

- „Adverbs expressing a low degree of a positive idea“, z.B. little; vgl. wenig (Bäcklund 1973: 67);

- „Adverbs expressing a moderate degree“, z.B. quite, pretty; vgl. ziemlich, ganz (Bäcklund 1973: 69);

- „Adverbs expressing an increasing degree“, z.B. increasingly; vgl. zunehmend (Bäcklund 1973: 156);

- „Adverbs expressing a high degree“, z.B. bloody, awfully, highly; vgl. sehr, besonders, schrecklich, überaus (Bäcklund 1973: 158);

- „Adverbs expressing the highest degree“, z.B. wholly, completely; vgl. total, völlig, wirklich (Bäcklund 1973: 194);

- „Adverbs meeting the demand of a subsequent verbal“, z.B. enough, sufficiently; vgl. genug, genügend (Bäcklund 1973: 238);

- „PRO-forms in the function of adverbs of degree“, z.B. this, that; vgl. so, derart (Bäcklund 1973: 241). 
Während die oben genannten Klassifizierungen an englischem Material entwickelt wurden, arbeitete Os (1989) eine auf dem Deutschen basierende achtstufige Gliederung der Intensivierer (Os 1989: 99) aus. Sie beruht auf der Klassifikation von Biedermann (1969) und CGEL (1985): die absolute Stufe, die approximative Stufe, die höchste Stufe, die hohe Stufe, die gemäßigte Stufe, die schwache Stufe, die minimale Stufe, die negative Stufe.

Meine Arbeit widmet sich Intensivierern der hohen Stufe gemäß der Klassifikation von Biedermann (1969) und Os (1989). In Peters (1993) werden die Lexeme auch boosters genannt, gemäß der Aufteilung von Bolinger.

\subsection{Intensivierbare Ausdrücke}

\subsubsection{Adjektive und Adverbien}

Graduierbarkeit gehört zu den Eigenschaften der Adjektiv- und Adverbiallexik und steht in Zusammenhang mit deren Intensivierbarkeit. Bierwisch (1987a: 130) postuliert, dass „Graduierung durch [...] Vergleichen“ „konstituiert wird“. Darauf verweist auch Warren (1984: 93): „The gradability of an adjective is reflected in its ability to express comparison [...] and in its ability to be intensified [...] or attenuated [...]. Traditionally, gradability is considered a criterion of adjectivehood".

Laut Motsch (1971: 31ff.) und Lyons (1977: 271) stehen graduierbare Wörter im Gegensatz zu nichtgraduierbaren und bilden damit eine binäre Opposition. Die Opposition dieser Art ist "the most common feature of gradable words" (Vermeire 1979: 20) und "one of the most important principles governing the structure of languages" (Lyons 1977: 271). Bierwisch (1987b: 15) unterscheidet ebenso zwischen „nicht-graduierbaren“ und "graduierbaren“ Adjektiven, die er dementsprechend als „relativ“ (z.B. lang / kurz) und „absolut“ (z.B. rund, verheiratet) bezeichnet, vgl.:

„der wesentliche Faktor, der den Charakter der relativen Adjektive bestimmt" ist „die Graduierbarkeit der durch sie wiedergegebenen Bedingungen oder Eigenschaften. [...] Adjektive sind absolut, wenn die durch sie ausgedrückte Bedingung nicht graduiert werden kann."

Graduierbarkeit lässt Begriffe auf einer Skala einordnen und begünstigt von daher das Auftreten von antonymischen Paaren, z.B. gro $\beta$ vs. klein, teuer vs. günstig. Jedoch, wie Vermeire (1979: 20) angemerkt hat, sind nicht alle binäre Paare graduierbar und nicht alle graduierbaren Wörter bilden binäre Paare. Da für die vorliegende Arbeit eine grobe Qualifizierung ausreichend ist, werde ich ferner auf die Einzelheiten der angesprochenen Problematik nicht eingehen.

Zifonun (1997: 46) unterscheidet zwischen absoluten und relativen Adjektiven. Absolute Adjektive (z.B. rot, verheiratet) haben keine Komparationsstufe, sind nicht graduierbar und, wie (Lyons 1963: 62) anmerkt, „divide the universe of discourse absolutely without admitting degrees of more and less". Relative Adjektive (z.B. schön / hässlich) sind „durch eine Dimension oder durch den Wert auf einer entsprechenden Skala gekennzeichnet“ (Trost 2006: 12), sind „bound up with comparison“ (Lyons 1963: 62), i.e. ,allow comparison with respect to some relative and more subjective norm" (Vermeire 1979: 21). Absolute Adjektive bilden komplementäre Gegensatzpaare, relative Adjektive dagegen antonymische (Lyons 1977: 
279, vgl. auch Os 1989: 56). Vermeire (1979: 22) weist darauf hin, dass diese Klassifizierung problematisch ist, insofern es absolute Adjektive gibt, die in bestimmten Kontexten Graduierbarkeit aufweisen können, vgl. *deader vs. absolutely dead, longer vs. *absolutely long. Daraus schließt er Folgendes: „the opposition absolute/relative is itself not a complementary pair but rather a relative, antonymous pair so that a strict dichotomization between gradable and non-gradable words becomes problematic" (Vermeire 1979: 22).

Diese Ansicht wird auch von Isitt (1984: 14) geteilt. Sie verweist darauf, dass „(w)hat is gradable for one speaker is not necessarily so for another". Denn Wörter, die "absolute conditions" bezeichnen und von daher nicht graduierbar sind (z.B. true, basic, colourless, square) gelegentlich in den Kontexten gebraucht werden, wo sie doch Graduierbarkeit aufweisen, z.B. very true, fairly basic, rather colourless, square enough (Isitt 1984: 14). Das lässt sich auch in dem von mir untersuchten Material feststellen, z.B. in der Zusammensetzung furchtbar still, wobei 'furchtbar' in Bezug auf still als „Furcht erregend“ und „besonders stark ausgeprägt" verstanden werden kann.

In diesem Zusammenhang unterscheidet Vermeire (1979: 23) drei Typen von Adjektiven in Bezug auf ihre Graduierbarkeit:

- „fully relative words which are inherently gradable“ und „have an implicit scale from which degrees can be measured" (z.B.good, bad);

- „fully absolute words which are inherently nongradable“ und "have no implicit scale" (z.B. dead, triangular);

- „basically absolute words which however can be relativized“ (z.B. full, empty).

Os (1989: 56) hat die Klassifikation von Vermeire im Grunde übernommen, indem er zwischen antonymischen Paaren, nicht intensivierbaren komplementären Paaren und intensivierbaren komplementären Paaren unterscheidet.

Wie es Peters (1993: 3) festgestellt hat, gehören boosters, und damit die in der vorliegenden Arbeit zu untersuchenden Wörter zu dem ersten Typ von Lexemen. Sie werden außerdem als scale words (Leech, Svartvik 2013: 115) bezeichnet. Diese Gruppe beinhaltet Lexeme, die antonymische Gegensatzpaare bilden und gelegentlich durch andere Adjektive ergänzt werden können. „Gemeinsam mit ihnen bilden sie eine Skala, deren Positionen miteinander vergleichbar sind. Die Vergleichbarkeit bietet die Grundlage für die Intensivierbarkeit" (Peters 1993: 10).

In Bezug auf die Intensivierbarkeit von Adverbien verweisen Os (1989: 79) und Peters (1993: 10) darauf, dass intensivierbare Adjektive auch in adverbialer Verwendung intensivierbar sind. Die Grundlage für deren Intensivierbarkeit „ist wiederum die Möglichkeit des Vergleichs" (Peters 1993: 10).

\subsubsection{Verben}

Nach Os (1989: 71) ist „(d)er Intensivierbarkeit von Verben [...] in der Literatur weit weniger Aufmerksamkeit gewidmet worden als der von Adjektiven." Die wenigen Studien, die sich mit diesem Thema beschäftigt haben, bieten keine generellen Aussagen über die Fragestellung, verweisen jedoch auf die damit verbundenen 
Probleme. Bolinger (1972: 164) berichtet, dass „[a] very few verbs are not intensifiable either inherently or extensively“. In Os' (1989: 71) Paraphrase heißt das, dass „die überwiegende Mehrzahl von Verben in irgendeiner Weise intensivierbar ist“, wobei zwischen „inhärenter" und „extensiver“ Intensivierung unterschieden werden soll, vgl. die aus Os (1989: 72) übernommenen Belege:

(7) Flüstre nicht so. Ich kann dich kaum verstehen.

(7') 'Sprich nicht so sehr leise.'

(8) Flüstre nicht so. Ich kann die anderen kaum verstehen.

(8') 'Störe mich nicht mit deinem Flüstern.'

In den Belegen (7) - (8) wird die Bedeutung des Verbs flüstern durch den Kontext bzw. durch den Folgesatz bestimmt. Der Beleg (7) demonstriert inhärente Intensivierung, indem die Bedeutung des Verbs „sehr leise sprechen“ durch den Folgesatz modifiziert wird. In (8) dagegen wird diese Bedeutung laut Os durch den Folgesatz verhindert, sodass das Flüstern als "Störfaktor" angesehen wird (Os 1989: 72).

Peters (1993: 11) verweist darauf, dass „in Zweifelsfällen“, wenn einem Verb entweder eine qualitativ modifizierende oder intensivierende Bedeutung zugeschrieben werden kann, die Bedeutung des Verbs die entscheidende Rolle spielt. In diesem Zusammenhang wird die Aufmerksamkeit der Forscher auf die Bedeutung dieser Klasse gerichtet und das Kollokieren dieser Klassen mit Grad-Adjektiven und -Adverbien überprüft. In Peters (1993: 11-17) findet sich eine kurze Übersicht von verbalen Klassifikationen, die die grundlegenden Ansätze von Kenny (1963), Vendler (1967), Leech (1987) und CGEL (1985) berücksichtigt. Ich werde diese Klassifikationen in der vorliegenden Arbeit nicht darstellen, da sie in der im weiteren angesprochenen Klassifizierung von CGEL zusammengefasst werden.

Eine traditionelle Kategorisierung des Verbwortschatzes ist in DUDEN (1995: 90) dargestellt und bietet den Unterschied zwischen Tätigkeits-, Vorgangs- und Zustandsverben. CGEL (1985: 201) bietet eine umfangreichere Klassifikation, die den Verbwortschatz je nach situation type einordnet. Darüber hinaus bezieht sich jedes Verb auf eine bestimmte Situation, „die für den Gebrauch des Verbs kennzeichnend ist" (Peters 1993: 12). Es handelt sich um zwei große Klassen bzw. stative situation types und dynamic situation types, die in weitere Unterklassen eingeteilt werden. Beispielsweise gehören der Klasse stative situation types die folgenden Unterklassen an: quality, state, intellectual states und states of emotion or attitude. Peters (1993: 18) stellt fest, dass stative Verben „eben aufgrund ihrer Bedeutung für das Anlegen einer Gradskala empfänglich sind. Sie sind insofern den Adjektiven sehr ähnlich“. Kortmann plädiert (1985: 29) dafür, dass stative Verben nur atelischer Natur sein können bzw. keinen Endpunkt voraussetzten. In diesem Zusammenhang nimmt Peters (1993: 18) eine „Affinität zwischen nicht zielgerichteten Situationstypen bzw. den dazu gehörenden Verben und der Graduierbarkeit durch boosters" an. Es liegt jedoch nicht genügend Evidenz dafür vor, dass Verben telischer Natur nicht mit Gradadverbien auftreten können, denn telische Verben, die verschiedene dynamische Situationen bezeichnen, werden von maximizers und minimizers modifiziert, z.B. recover completely/hardly (Peters 1993: 17).

Die oben genannten Behauptungen über die Kookkurrenzmöglichkeiten von Verben mit Gradadverbien basieren teilweise auf der Studie von Greenbaum 
(1970), der die Beziehungen zwischen den verbalen Klassen und bestimmten Intensivierern festzustellen versuchte. Es wurde u.a. nahegelegt, dass verbs of activity nahezu keine Kollokationen mit degree intensifiers aufweisen, jedoch mit manner adjuncts bzw. Adverbien der Art und Weise. Stative Verben wie z.B. intend, wish und like, die in Greenbaums Terminologie als attitudinal verbs bezeichnet werden, stellen typische Kookkurrenzpartner von Gradadverbien dar, so Greenbaum (1970: 83ff.).

Ein weiteres Beschreibungsmodell des deutschen verbalen Wortschatzes bieten Ballmer \& Brennenstuhl (1986). Sie gruppieren Verben in „Modelle“ bzw. Verbkategorien, die jeweils aus einer Menge von Verbschemaklassen bestehen (Ballmer, Brennstuhl 1986: 56). Die Besonderheit dieser Klassifikation besteht darin, dass jede Kategorie einen vollen Prozess beschreibt bzw. den Vor-, Nach- und Endzustand berücksichtigt und damit auf eine Veränderung hinweist. So kann ein Auto „im Laufe der Zeit nacheinander '(noch) nicht vom Rost angegriffen sein', 'anfangen zu rosten', 'leicht angerostet sein', 'rostig sein', 'verrosten', '(völlig) verrostet sein'“ (Os 1989: 76). In diesem Zusammenhang hebt Os die Kategorien 'spezieller Vorgänge und Prozesse' und 'Bearbeitung' hervor, die, seiner Meinung nach, zu „Verwandlungs- bzw. Veränderungshandlungen gehören. [...] Bei Veränderung bzw. Verwandlung ist die Rede von einem Vorzustand und einem Nachzustand, so daß es möglich ist, Vergleiche anzustellen oder das Ausmaß, den Grad der Veränderung festzustellen“ (Os 1989: 74). Von daher äußert Os die Vermutung, dass die Verben aus diesen zwei Kategorien intensivierbar sind, vgl. die aus Os (1989: 74) übernommenen Belege:

(9) Der Zustand hat sich weitgehend verbessert.

(10) Die Lage hat sich sehr beruhigt.

Die oben genannten Thesen können auch in Bezug auf die in meiner Arbeit zu untersuchenden Gradadverbien angewandt werden. So legte die Untersuchung meines Materials nahe, dass Lexeme, die dem Ausdruck eines hohen Grads dienen, mit Tätigkeits- und Vorgangsverben kollokieren können, vgl.:

(11) Er hat furchtbar geschrien.

(12) Es hat schrecklich gedonnert.

(13) Der König hat sein Volk grausam unterdrückt.

(14) Ich schäme mich / freue mich unheimlich.

(15) Er hat sich arg geärgert.

Wie oben schon erwähnt, verweisen Verben, die mit Gradadverbien kollokieren können, auf eine Veränderung bzw. Verwandlung des Subjekts bzw. Objekts und charakterisieren somit den Grad dieser Veränderung bzw. Verwandlung, vgl.:

(16) Er hat mich furchtbar enttäuscht.

(16') 'sehr enttäuscht'

(17) Mein Kopf tut mir tierisch/verflucht weh.

(17') 'tut sehr weh' 


\subsubsection{Substantive}

Spitzbardt (1954: 25) verweist darauf, dass dem Phänomen der Intensivierung Substantive unterliegen, die „einen Vorgang, Zustand oder eine Qualität ausdrücken".

Os (1989: 77) berichtet, dass Substantive „sowohl aufgrund von Intensitäts- als auch aufgrund von Extensitätsmerkmalen intensiviert werden“ können. „In dieser Hinsicht unterscheiden sie sich nicht von Adjektiven und Verben. " Das kann bei der Behandlung von Substantiven adjektivischer oder verbaler Herkunft berücksichtigt werden. Eine Skala kann außerdem angelegt werden, wenn „die semantische Relation zwischen Subjekt und Prädikat eine Eigenschaftszuweisung ist" (Os 1989: 78), vgl. das aus Os (1989: 78) stammende Beispiel:

(18)a. Er ist ein Trottel.

b. ${ }^{*}$ Er ist Trottel.

(19)a. Er ist ein Pianist.

b. Er ist Pianist.

Das Wort Trottel in (18a) ist inhärent intensivierbar, sodass die dadurch auszudrückende wertende Komponente in verschiedenem Maße ausgeprägt sein kann, vgl. ein furchtbarer Trottel, ein absoluter Trottel. Das Wort Pianist in (19a) wird dagegen klassifizierend verwendet und verweist auf den Beruf einer Person. Die Bedeutung von Pianist in (19b) wird zusätzlich eine wertende Komponente erfahren, die den Pianisten als sehr virtuos bezeichnet. Darüber hinaus erhält „das Substantiv [...] ein sekundäres Bedeutungsmerkmal [...], wodurch Intensivierung ermöglicht wird“ (Os 1989: 79).

\subsubsection{Funktionsverbgefüge}

Gradadverbien können außer Verben und Adjektive auch Funktionsverbgefüge intensivieren. Unter Funktionsverbgefügen (ferner FVG) versteht man Ausdrücke, die aus einem deverbalen Nomen und einem bedeutungsarmen Verb bestehen, z.B. einem Irrtum unterliegen, zur Verfügung haben, zum Abschluss bringen (Eisenberg 2006: 9). Der verbale Teil eines FVGs wird als Funktionsverb bzw. FV bezeichnet. Das Substantiv in dem nominalen Teil kann in einer Präpositionalgruppe (z.B. zur Anwendung kommen), als Substantiv im Akkusativ (z.B. von etw. Kenntnis nehmen), im Dativ (z.B. j-n einer Prüfung unterziehen), im Genitiv (z.B. einer genaueren Untersuchung bedürfen) oder im Nominativ (z.B. keine Übereinstimmung besteht) auftreten (Helbig 1979: 273-275).

Funktionsverbgefüge unterscheiden sich von den üblichen Substantiv-VerbKollokationen dadurch, dass ,in diesem Bereich ein leichter Phraseologisierungsaspekt vorhanden" ist (Burger 2007: 55). Von daher werden sie nicht nur in den Grammatiken der deutschen Sprache (z.B. Eisenberg 2006, Helbig, Buscha 2005, Duden 2009), sondern auch in der Phraseologie behandelt (z.B. Pilz 1981, Fleischer 1982, Burger 2007 u. a.). Bis heute gibt es keine klare Definition ihres Status, denn „Funktionsverbgefüge' ist mit sicherheit keine grammatische Kategorie" (Eisenberg 2006: 309). Laut Fleischer (1982: 139) gehören FVG zu „Phraseoschablonen“ bzw. zu einem „Grenzbereich der Phraseologie zur Syntax“ während Pilz 
(1981: 89) nicht alle FVG zu Phraseologismen zuordnet, sondern lediglich diejenigen, die „eine entscheidende Bedeutungsnuancierung oder -differenzierung leisten, die vom einfachen, nicht nominalisierten Verb abweicht". Darüber hinaus befinden sich Funktionsverbgefüge in einem Kontinuum zwischen "freien Wortketten“ und "nagelfesten Verbindungen“" (Pottelberge 2001: 14).

Funktionsverbgefüge spielen eine erhebliche Rolle bei der Modifikation durch Gradadverbien. Durch das Auftreten in Verbindung mit einem FVG lässt sich eine Änderung der Skopuskonstituente eines Intensivierungsmittels feststellen, vgl. die folgenden Belege:

(20) Er steht unter furchtbarem Druck.

(21) Er steht furchtbar unter Druck.

In (20) intensiviert furchtbar das Substantiv Druck. In (21) gelangt dagegen nicht das Prädikat stehen, sondern das ganze Funktionsverbgefüge unter Druck stehen in den Skopusbereich des Intensivierers. Eine ähnliche Situation lässt sich in (22) (23) feststellen, vgl.:

(22) Er ist in arge Schwierigkeiten geraten.

(23) Er ist arg in Schwierigkeiten geraten.

In (22) fungiert arg als Gradadjektiv und modifiziert das Substantiv Schwierigkeiten. In (23) gehört zu dem Bezugsbereich von arg nicht das Verb geraten, sondern das Funktionsverbgefüge in Schwierigkeiten geraten.

In der Literatur wird ein Adverb als ein „Beiwort zum Verb“ (DUDEN 1995: $355^{19}$ ) dargestellt. Darüber hinaus fokussieren Adverbien auf das unmittelbar folgende Verb (Os 1989: 16). Das Belegmaterial demonstriert jedoch eindeutig, dass der Skopus des Lexems sich erweitern kann und damit Funktionsverbgefüge einschließen, vgl. (24) - (26):

(24) Er geht mir furchtbar auf die Nerven.

(25) Der Herzog kam in furchtbare Wut. <06.07.2015: http://gutenberg.spiegel.de/buch/geschichten-aus-schweren-zeiten-6611/8>

(26) Das Spiel kann furchtbar in die Hose gehen.

Der Bedeutungsaufbau von Funktionsverbgefügen ist dadurch gekennzeichnet, dass die Bedeutung des Nomens die lexikalische Gesamtbedeutung der Fügung impliziert, sodass ein FVG „in den meisten Fällen durch das entsprechende Vollverb substituiert werden" kann (Helbig 1979: 276). So wird z.B. das FVG j-m aufdie Nerven gehen in (24) durch nerven paraphrasiert. Helbig, Buscha (2005: 68) verweisen darauf, dass „das FVG in der Bedeutung weitgehend" auch „einem Adjektiv“ entsprechen kann, welches „den gleichen Stamm“ hat wie das Nomen im FVG. Von daher wird das FVG in Wut kommen in (25) durch wütend werden paraphrasiert. Die Bedeutung der Wortverbindung in die Hose gehen in (26) ist dagegen aus der Bedeutung der Bestandteile nicht erkennbar, weil er in hohem Grade idiomatisiert ist. Die Bedeutung dieses Phraseologismus' wird in DUDEN (2012) mit misslingen umschrieben.

${ }^{19}$ In DUDEN (2009: 569) wird diese These korrigiert, indem ein Adverb als Modifikator von Adjektiven, Adverbien, Substantiven und ganzen Sätzen dargestellt wird. 
Die vorliegende Untersuchung legt die Annahme nahe, dass nicht alle Funktionsverbgefüge in den Skopus von Gradadverbien geraten können. Die Analyse der Belege führt zu dem Schluss, dass durch ein Intensivierungsmittel nur solche Funktionsverbgefüge modifizierbar sind, die „skalierbare Größen darstellen“ bzw. entweder ein intensivierbares Nomen enthalten oder eine durch einen intensivierbaren Begriff auszudrückende Paraphrase erlauben (Os 1989: 82). Darüber hinaus steht die Intensivierbarkeit eines FVGs in einem Zusammenhang mit der Intensivierbarkeit des Nomens, vgl.:

(27) arg in Bedrängnis geraten

(27') 'in sehr große Bedrängnis geraten'

(28) irrsinnig unter Druck stehen

(28') 'unter sehr großem Druck stehen'

(29) fürchterlich ins Schwitzen kommen

(29') 'anfangen, sehr stark zu schwitzen'

Wenn im Skopus eines Gradadverbs eine idiomatische Wortverbindung steht, deren Bedeutung nicht aus der Bedeutung des Nomens abgeleitet werden kann, soll man die Intensivierbarkeit der Paraphrase berücksichtigen, vgl.:

(30) sich furchtbar in die Länge ziehen

(30') 'sich sehr verzögern'

(31) wahnsinnig unter die Haut gehen

(31') 'sehr starke Empfindungen auslösen'.

\subsection{Natur der Intensivierung: Semantische Felder}

\subsubsection{Wortfeldtheorie}

Das systematische Herangehen an die Analyse von Grad-Adjektiven und -Adverbien in Bezug auf ihre Ausgangsbedeutung ist in der Literatur bekannt und wurde beispielsweise in den Arbeiten von Hofmann (1930), Biedermann (1969), Hentschel (1998), Peters (1998) und Kirschbaum (2002) präsentiert. In diesen Werken werden Intensivierer nicht chaotisch und einzeln, sondern gruppenweise behandelt bzw. in semantische Felder klassifiziert. Das Aufteilen von Lexemen in Wortgruppen stützt sich hauptsächlich auf die Ideen von Trier $(1931,1932,1934)$ bzw. seine Wortfeldtheorie.

Auf „gewisse Systeme zusammengehöriger Bedeutungen“, die nur „als Ganzes verständlich“ sind und „aus deren Organisation erst die semasiologische Stellung der einzelnen Ausdrücke vollkommen verständlich wird“, wurde schon z.B. von Meyer (1910a: 356) hingewiesen und anhand des Beispiels von militärischen Rangbezeichnungen (Meyer 1910b) demonstriert. Darüber hinaus versteht er unter einem Bedeutungssystem "die Zusammenordnung einer begrenzten Anzahl von Ausdrücken unter einem individuellen Gesichtspunkt" (Meyer 1910a: 359).

Die Notwendigkeit, den Bedeutungswandel innerhalb von lexikalischen Gruppen zu untersuchen, wird in Sperber (1923) hervorgehoben. Er verweist auf semantische Verschiebungen bei Affektträgern bzw. „mit einem starken Gefühlston verbundenen“ Wörtern (Sperber 1923: 39), die „nicht an einer scharf umgrenzten 
Einzelvorstellung“ haften, sondern sich „auf ganze Vorstellungskomplexe“ erstrecken (Sperber 1923: 64). Damit wird zum Ausdruck gebracht, dass im Fall des Bedeutungswandels „nicht nur ein Wort davon betroffen“ wird, sondern mehrere, die zu demselben Vorstellungskomplex gehören (Sperber 1923: 64-65).

Trier (1931: 11) erwähnt in diesem Zusammenhang die Oppositionslehre von Saussure (1922), der sich über das Zeichensystem mit der oppositiven Bestimmung von dessen Elementen geäußert und auf die Feststellung des Sinngehalts eines Worts durch "seine gleichzeitigen Nachbarn im selben Begriffsfeld“ sowie auf die Notwendigkeit der Wortforschung in Bezug „auf die gleichzeitigen (synchronen) Gruppenbildungen" verwiesen hat.

Trier (1932a: 418) merkt an, dass Gunter Ipsen „als erster von Feld gesprochen“ hat, indem er den Begriff „Bedeutungsfeld“ (Ipsen 1924: 225) einführte:

„die Eigenwörter stehn in einer Sprache nie allein, sondern sind eingeordnet in Bedeutungsgruppen; damit ist nicht eine etymologische Gruppe gemeint, am wenigsten um chimärische 'Wurzeln' aufgereihte Wörter, sondern solche, deren gegenständlicher Sinngehalt mit andern Sinngehalten verknüpft ist. Diese Verknüpfung aber ist nicht als Aneinanderreihung an einem Assoziationsfaden gemeint, sondern so, daß die ganze Gruppe ein 'Bedeutungsfeld' absteckt, das in sich gegliedert ist $[\ldots]^{\prime \prime}$.

Dieser Auffassung schließt sich Trier an, indem er behauptet (1931: 1), dass „kein ausgesprochenes Wort [...] im Bewußtsein des Sprechers und Hörers so vereinzelt“ dasteht. Denn es „taucht eine Fülle anderer Worte auf, die dem ausgesprochenen begrifflich enger oder ferner benachbart sind. Es sind seine Begriffsverwandten. Sie bilden unter sich und mit dem ausgesprochenen Wort ein gegliedertes Ganzes, ein Gefüge, das man Wortfeld oder sprachliches Zeichenfeld nennen kann" (Trier 1931: 1). Den ganzen Wortschatz sieht Trier (1932a: 419) als eine „aus übergeordneten Großfelder[n]“ bestehende Einheit.

Trier (1931: 1) unterscheidet zwischen dem Wortfeld und dem Begriffsfeld, wobei das Wortfeld „die äußere, zeichenhafte Seite der begrifflichen Aufteilung“ des Begriffsfelds bzw. des „Begriffskomplex[es]“, „Begriffsblock[s]“, „Begriffsbezirk[s]" ist. Er bezeichnet das Wortfeld als „Wortmantel" und „Wortdecke“ des Begriffsfelds, wobei die das Feld bildenden Wörter es "mosaikartig“ aufteilen. Dabei entstehen in dem Begriffsblock keine Lücken: „[e]s gibt keine blinden Flecke im Feld, sondern nur jeweils so oder so geartete geschlossene und totale Aufteilungen eines Bereiches" (Trier 1934: 192). Die Bedeutung eines Worts befindet sich im Zusammenhang mit dem ganzen Feld bzw. mit den zum Feld gehörenden Wörtern: „wenn das einzelne Wortzeichen verstanden werden soll, und es WIRD verstanden IM MASSE der Gegenwärtigkeit des Feldes. Es 'bedeutet' nur in diesem Ganzen und KRAFT dieses Ganzen. [...] Das Wort folgt hier dem allgemeinen Wesen aller Zeichen. Zu diesem Wesen gehört es, daß der Bezeichnungsinhalt und Umfang eines Zeichens sich richtet nach der Stellung, die das Zeichen innerhalb der Gesamtheit der übrigen ihm inhaltlich benachbarten Zeichen einnimmt" (1931: 6). 
In Bezug auf historische Wortuntersuchungen verweist Trier (1931: 22) darauf, dass eine Wortgeschichte durch eine Wortfeldgeschichte bzw. „Begriffsgeschichte" ersetzt werden soll, da „man die Einzelbegriffe eines Feldes nur in der sprachlichen Aufteilung des Feldes hat". Am Beispiel von Intellektualwörtern hat Trier (1932b sowie 1932a: 421-423) demonstriert, dass bei dem Bedeutungswandel in einem Wort das ganze Feld in den Wandel involviert wird. In der Wortfeldgeschichte handelt es sich „um einen Kampf der Wörter [...], bei dem die einen siegen und die anderen [...] untergehn, während in den gemeinten Inhalten und im Gefüge der Sprache im Grunde schließlich doch alles beim alten bleibt" (Trier 1932a: 423). Von daher ist die Feldgliederung in historischer Perspektive mit der Umgliederung des Begriffsfeldes (Trier 1932a: 423) verbunden. Um ein Feld diachronisch zu erforschen, soll man die „Geschichte der Feldeinteilungen“ (Trier 1931: 22) verfolgen und „eine sprungweise von Querschnitt zu Querschnitt fortgehende, stets und immer von neuem das Gesamtfeld ins Auge fassende zeitlich rückwärts und vorwärts vergleichende Beschreibung" (Trier 1931: 13) erzielen. Inwiefern die Feldgeschichte möglichst nahe der tatsächlichen Entwicklung wird, hängt von der „Dichtigkeit der angelegten Querschnitte" (Trier 1931: 13) ab.

Obgleich Triers Wortfeldtheorie lebhafte Diskussion und Kritik erfahren hat ${ }^{20}$, die beispielsweise die Lückenlosigkeit der Wortfelder oder die Bestimmung des Inhalts eines Lexems durch seine Nachbarn in Frage stellte, „bleibt der Kern der Theorie Triers, der paradigmatische lexikalische Systemgedanke, allerdings unberührt" (Lee, Reichmann 1973: 24-25). In Bezug auf Grad-Adjektive und -Adverbien soll lediglich darauf hingewiesen werden, dass die Analyse von Wortgruppen sich als sehr sinnvoll und produktiv erwiesen hat. Im weiteren Verlauf meiner Arbeit werde ich demonstrieren, dass die Lexeme, die zu derselben Bedeutungsgruppe gehören, einen ähnlichen Entwicklungsgang aufweisen. Diese Tatsache lässt Verallgemeinerungen über den gesamten Prozess der Entstehung von Gradbedeutung sowie Voraussagen über die weitere Entwicklung von Lexemen zu.

Ein ergiebiger Versuch, Triers Wortfeldtheorie in Bezug auf Grad-Adverbien und-Adjektive anzuwenden und damit „wenigstens hie und da die Gründe der verschiedenen Ausbreitung und der Bevorzugung einzelner Bedeutungsfelder (BF) zu erfassen“, wurde in Hofmann (1930) unternommen. Dabei wird „[a]uf einem Bedeutungsfeld [...] vieles zusammenstehen, was etymologisch nicht zusammengehört, aber doch synonym oder bedeutungsverwandt ist" (Hofmann 1930: 106).

Hofmann (1930: 106ff.) unterteilt alle Bedeutungsfelder je nach der Ausgangsbedeutung des Gradadverbs in zwei „Gebiete“: das objektive Gebiet (dazu gehören Größenbezeichnungen und Ausdrücke der Totalität, Menge, Größe usw.) und das subjektive affektische Gebiet, „bei dem psychologische Voraussetzungen (Stimmung und Gefühl) vorherrschen" (Spitzbardt 1954: 278). Die Intensivierer, die ich in der vorliegenden Abhandlung untersuche, gehören dem letzteren Gebiet in dieser Klassifikation an.

\footnotetext{
${ }^{20}$ Eine Übersicht der an die Wortfeldtheorie gerichteten Kritik findet sich beispielsweise in Lee, Reichmann (1973: 17-24) sowie in Biedermann (1969: 66-68) und Lehrer (1974: 16-19).
} 
Hofmanns Arbeit ist insbesondere dadurch ergiebig, dass er eine große Vielfalt von Intensivierern aus verschiedenen indogermanischen Sprachen einer begrenzten Anzahl von Bedeutungsfeldern zugeordnet hat und demonstriert, dass "fast jede Sprache auf jedem Felde vertreten“ 21 ist, „aber die Zusammensetzung wird doch oft verschieden sein: auf dem einen Feld wuchert die eine Sprache, auf dem anderen hat sie spärlich Fuß gefaßt". Dabei stehen "die beliebtesten Steigerungsadverbien der einzelnen Sprachen [...] auf ganz verschiedenen Feldern“ (Hofmann 1930: 106).

\subsubsection{Deutsche Grad-Adjektive und -Adverbien und semantische Felder}

In der vorliegenden Arbeit biete ich die Analyse von 40 Intensiverer aus 6 Wortgruppen unterschiedlichen Umfangs:

- 'Furcht erregend' (entsetzlich, furchtbar, fürchterlich, grässlich, grauenhaft, grauenvoll, grausig, schauderhaft, schauerlich, schaurig, schrecklich, ungeheuer, ungeheuerlich, unheimlich);

- 'Übel wollend/verursachend' (arg, böse, brutal, grausam, grimmig, mörderisch, niederträchtig, schändlich, sündhaft, unmenschlich, unverschämt, wütend);

- 'Sich wie ein Tier verhaltend' (bestialisch, tierisch, viehisch);

- 'Geistig gestört' (irre, irrsinnig, närrisch, toll, verrückt, wahnsinnig);

- 'Religion' (höllisch, teuflisch);

- 'Fluch' (verdammt, verflucht, verteufelt).

Die Kernidee Triers bzw. Hofmanns, Lexeme nicht isoliert, sondern im Zusammenhang mit ihren Begriffsverwandten zu untersuchen, habe ich für meine Untersuchung übernommen. Die Lexeme, die im Rahmen meiner Arbeit analysiert worden sind, sehe ich als Glieder der entsprechenden Wortfelder an. In diesem Zusammenhang wurden alle zu behandelnden Lexeme den entsprechenden Wortfeldern zugeordnet.

Ich habe mich bei der Analyse der Entwicklungsgänge der Gradbedeutung lediglich auf die festgesetzte Wortliste beschränken und deshalb auf die komplette Untersuchung von einzelnen Querschnitten bzw. auf den Aspekt der Feldumgliederung in jeder Zeitstufe verzichten müssen. Dies geschah hauptsächlich aus zwei Gründen. Erstens habe ich mich aus zeitlichen Gründen auf die Herausbildung der für den aktuellen Sprachgebrauch relevanten Intensitätsbedeutungen konzentriert und dieses Ziel im Laufe meiner Arbeit verfolgt. Die Wörter, die in den vorherigen Zeitstufen die dem zu untersuchenden Feld entsprechende Bedeutung aufwiesen, in der Gegenwartssprache jedoch keine Gradbedeutung demonstrieren können, gerieten von daher nicht in den Fokus meiner Arbeit. Die Analyse von Feldumgliederungen bzw. von „verlorenen“ Wörtern bildet damit eine Nebenaufgabe, die im Rahmen meiner Dissertation aus zeitlichen Gründen nicht durchge-

\footnotetext{
${ }^{21}$ Damit meint Hofmann balto-slawische Sprachen.
} 
führt werden konnte. Diese Beschränkung soll jedoch die Ergebnisse meiner Untersuchung nicht beeinträchtigen, denn eine ausführliche Analyse des Entwicklungsgangs der Intensivierung auf allen Sprachstufen und anhand mehrerer Lexeme stellt die Hauptaufgabe der vorliegenden Arbeit dar und soll den Mechanismus dieses Phänomens veranschaulichen.

Bei der Klassifizierung von Wörtern orientierte ich mich an der Klassifikation von Biedermann (1969) sowie der von Dornseiff (2004). Biedermann (1969: 97ff.) bietet eine Aufteilung der Lexeme nach dem Motivationssem und Herkunftssem und trägt damit „dem linguistischen Bestreben nach möglichst differenzierter Klassenbildung und nach Darlegung auch feinerer struktureller Zusammenhänge" (Biedermann 1969: 98) Rechnung. Da die regionale Herkunft sowie die Zugehörigkeit eines Lexems zu der Normal- oder Umgangssprache usw. für meine Fragestellung nicht relevant sind, werden diese Parameter im weiteren nicht berücksichtigt. Die Klassifikation nach Motivationssemen enthält 37 Subklassen, wobei „andere Einteilungen möglich“ „und die Übergänge und Abgrenzungen häufig fließend und mehr oder weniger klar" (Biedermann 1969: 131) sind.

Nach der Aktualisierung von Biedermanns Materials habe ich weitere Intensivierer entdeckt, die eine entsprechende Klassifizierung benötigen. In diesem $\mathrm{Zu}$ sammenhang benutzte ich das Wörterbuch von Dornseiff (2004). Dornseiff bietet eine Aufteilung der Lexeme in 20 Kategorien, wobei diese Kategorien in weitere Sachgruppen unterteilt werden. Das Verteilen der Wörter kann meistens klar und eindeutig durchgeführt werden, wie es beispielsweise in Bezug auf die Gruppe „Furcht, Schrecken“ (Dornseiff 2004: 188ff.) der Fall war. Jedoch, wie es schon oben in dem Zitat von Biedermann angesprochen wurde, sind die Grenzen zwischen den Wortgruppen sehr oft vage und fließend. Von daher gerieten manche von mir untersuchten Lexeme, die auf der intuitiven Ebene zu demselben Wortfeld gehören, bei Dornseiff in verschiedene Bedeutungsgruppen, jedoch zu der gleichen Kategorie. Von daher habe ich die durch die Wörter ausgedrückten Begriffe in der Einleitung zu jedem Kapitelabschnitt angegeben.

Beispielsweise bringen die Prädikate des menschlichen Charakters bzw. Verhaltens (arg, böse, brutal, grausam, grimmig, mörderisch, niederträchtig, schändlich, unmenschlich, unverschämt und wütend), die Dornseiffs Kategorie „Fühlen, Affekte, Charaktereigenschaften“ (Dornseiff 2004: 170-195) angehören, die Begriffe „Unlust verursachen“, „Übel wollen“ und „Zorn“ zum Ausdruck. Es erscheint jedoch als sinnvoll, die Wörter als eine ganze Gruppe zu behandeln, wie es in Biedermann auch gemacht wurde. In seiner Klassifizierung haben die diesem Wortfeld angehörenden Lexeme das Motivationssem 'ungut'. Jedoch „kann die Motivation der vorliegenden Klasse nur notdürftig paraphrasiert werden. Es wird darunter alles verstanden, was tadelnswert, böse, unangenehm, beklagenswert ist" (Biedermann $1969,165)$. Die Berechtigung dieses Wortfelds, wie es in dem entsprechenden Kapitel weiter unten demonstriert wird, erweist sich durch die Ähnlichkeit des Entwicklungsgangs, den die Mitglieder der Gruppe eingeschlagen haben.

Die Wörter irre, irrsinnig, närrisch, toll, verrückt und wahnsinnig entsprechen Dornseiffs Kategorie 11 „Das Denken“ (Dornseiff 2004: 198-217) und bringen die 
Begriffe „dumm“, „verrückt“ und „Unlogik“ zum Ausdruck. In Biedermanns Klassifizierung (1969: 161) treten sie jedoch wiederum zusammen mit dem Motivationssem 'Wahn' auf.

Ein mit dem Aufteilen der Lexeme aufgetretenes Problem bringt uns zu der Kritik an Triers Wortfeldtheorie, die ein Wortfeld als ein Ganzes mit klaren Grenzen ansieht. Lehrer (1974: 10ff.) hat angedeutet und auf dem Material von englischen Koch- und Lautwörtern demonstriert, dass die Wörter in einer Gruppe zwar miteinander verbunden sind, jedoch nicht den gleichen Status haben, sondern sich im Zentrum oder an der Peripherie befinden können. „Basic [...] words [...] determine the important semantic contrasts in a field" (Lehrer 1974: 10). Damit spricht Lehrer implizit die Schwierigkeit einer klaren Abgrenzung eines Felds und der eindeutigen Entscheidung über die Zugehörigkeit eines Lexems zu einem Feld an.

Auf der Basis meines Materials kann ich das angesprochene Problem am Beispiel der Gruppe „Von irre bis wahnsinnig“ veranschaulichen. Die zu dem Feld gehörenden Wörter irre, irrsinnig, toll, wahnsinnig und verrückt verweisen direkt auf eine geistig-seelische Störung einer Person. Dabei soll angemerkt werden, dass toll sowie wahnsinnig laut DUDEN (2012) in dieser Bedeutung als veraltet gekennzeichnet sind 22 . Wahnsinnig findet sich in der Umgangssprache, und verrückt wird lediglich salopp gebraucht. Was das Adjektiv närrisch betrifft, kann es keinen $\mathrm{Zu}$ sammenhang mit einer geistigen Krankheit aufweisen. Jedoch bezieht es sich auf Personen und deren Handlungen, die wenig Vernunft und damit eine gewisse mentale Störung demonstrieren. Darüber hinaus kann das Lexem als Glied der besprochenen Gruppe im Rahmen des vorgestellten Ziels behandelt werden.

Es soll anschließend darauf hingewiesen werden, dass unter der Ausgangsbedeutung eines Lexems die Bedeutung verstanden wird, die als Basis für die Entwicklung der Gradbedeutung gedient hat. Diese Rolle wird nicht immer der ursprünglichen Bedeutung eines Worts zugewiesen. Es lässt sich am Beispiel von verrückt demonstrieren. Verrückt stellte zunächst die Ableitung von verrücken im Sinne „wegschieben“, „versetzten“, „wegziehen“, „seinen Platz verlassen“23 dar und wandelte erst später zu der Bedeutung „thöricht, verwirrt, irrig“ (DWB, s.v. verrücken), aus der sich die Gradbedeutung entwickelt hat. Es ist von daher wichtig, die Wortfelder so zu gestalten, dass die sie bildenden Lexeme den gemeinsamen Grundbegriff in Bezug auf die Intensivierung haben. In manchen Fällen lohnt es sich deshalb, zuerst die Geschichte eines Wortes zu untersuchen und erst danach die Klassifizierung durchzuführen bzw. zu korrigieren.

Im Laufe meiner Arbeit habe ich außerdem festgestellt, dass eine eindeutige Identifizierung der Ausgangsbedeutung in manchen Fällen problematisch zu sein scheint. Beispielsweise bei der Analyse des Belegmaterials von grimmig habe ich zwei mögliche Sinnrichtungen ermitteln können, die als Basis für die Herausbildung von Intensivierung dienen könnten - Bedeutung des Schreckens und Bedeutung des rohen, gewalttätigen Verhaltens. Ich habe dieses Problem gelöst, indem ich beide Wege der Entwicklung von Gradbedeutung in dem Kapitelabschnitt 4.2 „Übel wollend / verursachend“ beschrieben habe.

\footnotetext{
22 Wahnsinnig wird in der Medizin als veraltet, sonst salopp gebraucht, so DUDEN (2012, s.v. wahnsinnig).

${ }^{23}$ Angaben aus den Belegzetteln der FWBD.
} 


\subsubsection{Russische Grad-Adjektive und -Adverbien und semantische Felder}

Die Problematik der Relevanz von semantischen Feldern in Bezug auf Grad-Adjektive und -Adverbien wurde in der bisherigen Forschung kaum anhand des Materials anderer Sprachen, z.B. des Russischen, untersucht. Hentschel (1998) bietet einzelne Belege, die den Gebrauch von Intensivierern aus den Feldern 'Menge, Fülle', 'Stärke', 'Größe' 'Gewicht' und 'Wahrheit' veranschaulichen. Außerdem findet sich dort ein Überblick auf "schreckliche Intensivierung“ (Hentschel 1998: 124) in verschiedenen indogermanischen und nicht-indogermanischen Sprachen. Es wird u.a. demonstriert, dass das 'Schreckliche' nicht nur im Deutschen, sondern in einer sehr großen Anzahl verwandter wie nicht-verwandter Sprachen zur emphatischen Verstärkung benutzt wird (Hentschel 1998: 131).

In Karpova (2013) demonstriere ich die Relevanz von vier Wortfeldern ('Wahnsinn', 'Phantasie', 'Schrecken', 'Größe') für fünf Sprachen aus drei Sprachgruppen: Russisch, Italienisch, Spanisch, Französisch und Englisch. Die ausführliche Entwicklungsgeschichte von englischen Grad-Adjektiven und -Adverbien wird in Peters (1993) je nach der „primären Bedeutung“ dargestellt. Abgesehen von den erwähnten Arbeiten sind mir gegenwärtig keine weiteren Publikationen bekannt, die sich mit der angesprochenen Problematik beschäftigen.

Eine Übersicht über einzelne Grad-Adjektive und -Adverbien aus synchronischer Sicht findet sich in Rachilina et al. (2009 und 2010). Der Versuch, Intensivierer aus bestimmten semantischen Bereichen im Russischen festzustellen, wurde von mir im Jahre 2011 unternommen ${ }^{24}$ und in Karpova et al. (2011a) angesprochen ${ }^{25}$. In Karpova (2012) demonstriere ich einzelne semantische Bereiche, die im Deutschen und Russischen dem Ausdruck der Intensivierung dienen, sowie kulturell spezifische Wortfelder, die für das Russische nicht relevant sind, im Deutschen jedoch verwendet werden.

Das Auffinden von gemeinsamen und unterschiedlichen Wortfeldern bei dem Vergleich der Sprachen stellt eine anspruchsvolle Aufgabe dar. Dadurch wird demonstriert, dass bestimmte Konzepte den Begriff der Intensivierung entwickeln können. Zugleich erscheinen manche Konzepte als kulturell spezifisch und stellen damit eine sprachliche Besonderheit dar. Die Suche nach kulturell spezifischen Wortfeldern, die als Quellen für die Entwicklung der Gradbedeutung dienen können, ist ein vielversprechendes Gebiet, das aber im Rahmen dieser Arbeit leider nicht erforscht werden konnte.

\subsection{Natur der Intensivierung: Mechanismen}

Das Problem der Natur von Intensivierungen wurde bis jetzt in der Literatur nur sporadisch behandelt. Dabei liefern die meisten Werke verschiedene Überlegun-

\footnotetext{
${ }^{24}$ Im Jahre 2011 arbeitete ich an der Erstellung einer Datenbank des Bedeutungswandels in der russischen Adjektiv- und Adverbiallexik.

${ }^{25}$ In der Datenbank kann man mit Hilfe von Abfragen Bedeutungswandel aus bestimmten semantischen Bereichen untersuchen.
} 
gen zum Thema und bieten damit keine einheitliche Meinung über die Mechanismen, die die Gradbedeutung in Erscheinung treten lassen. In diesem Zusammenhang verfolge ich in meiner Arbeit das Ziel, den Entwicklungsgang der Intensivierung möglichst präzise zu beschreiben.

Vorher soll eine Bemerkung über die Herkunft der Intensivierer gemacht werden. In den vorliegenden Studien werden Grad-Adjektive und -Adverbien semantisch mit den Qualitäts-Adjektiven und -Adverbien verknüpft. Biedermann zufolge „,beweisen“",[a]lle historischen Untersuchungen [...], daß die Ga aus den Qa entstanden sind" (Biedermann 1969: 115). Diese Annahme wird außerdem von Traugott bestätigt: „[m] ost intensifiers derive from content words" (Traugott 1982: 251), siehe auch Paradis $(2000,2008: 337)$ und Ghesquière, Davidse (2011: 252). In diesem Zusammenhang berichtet Biedermann, dass der Übergang von Qualitätsadverbien zu Gradadverbien „[...] das [ist], was man gemeinhin mit Bedeutungswandel bezeichnet" (Biedermann 1969: 122). Darüber hinaus können Qualitäts-Adverbien und -Adjektive sich durch bestimmte Mechanismen der semantischen Verschiebung in Intensivierer verwandeln. Die Frage, die nun im Rahmen meiner Arbeit beantwortet werden soll, bezieht sich auf die kognitiven Prozesse, die einen solchen Übergang ermöglichen.

Die auf die Entwicklung von Intensivierern bezogenen Studien kann man grob in zwei Gruppen aufteilen. Die erste Gruppe verweist auf die metaphorische bzw. metonymische Natur des Phänomens (z.B. Paul 1909: 96, Biedermann 1969, Kirschbaum 2002a, b, Portz 2009), während die zweite Gruppe es im Rahmen von Grammatikalisierungsprozessen behandelt (z.B. Hofmann 1930, Peters 1993, Heine, Kuteva 2000: 50-51, 302; Hopper, Traugott 2003: 122-123; Rachilina et al. 2010, Paradis 2000, Paradis 2008, Vandewinkel, Davidse 2008). Es soll jedoch dabei darauf hingewiesen werden, dass die Einwirkung von Metapher und Metonymie auch bei Grammatikalisierungsprozessen beschrieben wird (siehe z.B. Paradis 2000, Paradis 2008, Gehweiler 2010). Darauf werde ich in den Kapitelabschnitten 3.6.3.6 und 3.6.3.7 detailliert eingehen.

\subsubsection{Metapher und Metonymie: allgemeine Vorstellungen}

Metapher und Metonymie gehören zu den klassischen Mitteln des semantischen Wandels. Seit der Antike werden sie im Rahmen der Rhetorik als Redefiguren betrachtet, die als Substitute eines sprachlichen Ausdrucks für das poetisch-rhetorische und stilistische Schmücken der Rede gebraucht werden.

Die Bedeutunger des 19. Jahrhunderts, wie z.B. Paul (1880), Bréal (1900) und Meillet (1912), entwickelten in Verbindung mit diesen Tropen eine logisch-rhetorische Klassifikation, die den semantischen Wandel als eine Bedeutungsverengung, -erweiterung oder -übertragung darstellt. In Ullmann (1967: 189) wird diese Kategorisierung als „das logische Grundgerüst“ bezeichnet und folgendermaßen skizziert: „[f]ür das logische Verhältnis der neu entstandenen Bedeutung zur älteren gibt es nur drei Möglichkeiten: Die neue Bedeutung kann enger, weiter oder mit der alten umfangsgleich sein." Während die Bedeutungsverengung und -erweiterung als Metonymie bzw. Synekdoche betrachtet werden können, wird die Bedeutungsübertragung im Rahmen eines metaphorischen Prozesses durchgeführt. 
Die Grundvorstellung von Metapher und Metonymie beruht auf den Assoziationsprinzipien Similarität (oder Ähnlichkeit) bzw. Analogie und Kontiguität bzw. Berührung. Der Unterschied zwischen den beiden Mechanismen wird in der traditionellen Literatur folgendermaßen skizziert:

„[...] the difference between metonyms and metaphors is that in the case of metonyms conventional and novel referents are somehow contiguous, whereas in the case of metaphors conventional and novel referents are similar in some respect or respects" (Warren 1992: 64).

Die Basis für die Metaphernforschung hat Aristoteles, „der erste Theoretiker der Metapher" (Rolf 2005: 21), in seiner Vergleichstheorie gebildet. Gemäß dieser Theorie wird ein sprachlicher Ausdruck durch einen metaphorischen Ausdruck ersetzt. Darüber hinaus stellt die Metapher ein „elliptisches Gleichnis“ dar (Rolf 2005: 22).

Das in der Antike zugrunde liegende Prinzip der Analogie wurde in die traditionellen Beschreibungen der Metapher übernommen und findet sich beispielsweise in der traditionellen Theorie des Bedeutungswandels von Ullmann (1967: 204). Laut Ullmann kann die Metapher aufgrund der wesensmäßigen oder gefühlsmäßigen Ähnlichkeit erfolgen. Die wesensmäßige Similarität wird durch die Beispiele 'Tischbein' und 'Flußarm' exemplifiziert (Ullmann 1967: 207-208). Dabei wird z.B. zwischen der Ähnlichkeit der äußeren Gestalt (Kopf eines Menschen / von Kohl oder Salat) und der Gleichheit der Funktion (Fuß eines Tisches / eines Berges) unterschieden (Paul 1920: 95-96). Bei dem zweiten Typ stützt sich die Similarität „ausschließlich auf den analogen Eindruck und den vergleichbaren Affektgehalt der beiden Sinne“ (Ullmann 1967: 208), z.B. „die Übertragungen von Sinneswahrnehmungen auf Geistig-Seelisches ('Bitterkeit')“.

Im Fall der Metonymie „werden Namen aufgrund von Sinnverbindungen übertragen; es handelt sich hier jedoch nicht um eine Ähnlichkeitsbeziehung, sondern um ein räumliches, zeitliches oder kausales Verhältnis. Das Assoziationsfeld eines Wortes ist nicht auf ähnliche Sinne beschränkt, sondern kann alles, was sonst damit verbunden ist, einschließen [...]: z.B. der Erzeuger für das Erzeugnis, das Gefäß für den Inhalt" (Ullmann 1967: 214-215). Als Beispiel führt Ullmann (1967: 214) das Wort 'Stadt' ein, welches in Bezug auf eine größere Siedlung oder deren Bewohner gebraucht werden kann. Warren (1992) bietet ein systematisches Herangehen an die Beschreibung der Kontiguitätsbeziehungen und stellt eine Klassifizierung der metonymischen Verbindungen mit Material von englischen Adjektiven auf.

Im Rahmen der kognitiven Linguistik wird die seit der Antike bekannte und in der neueren Literatur übernommene Dichotomie zwischen Similarität und Kontiguität präziser formuliert. Die ersten Schritte in dieser Richtung wurden in Lakoff, Johnson (1980) and Lakoff (1987) unternommen. Darüber hinaus werden Metapher und Metonymie als Produkte der menschlichen Denkprozesse bzw. Konzeptualisierungsmechanismen und damit als unabdingbare Bestandteile der Alltagssprache angesehen. Diese Prozesse stellen somit „a relationship between concepts and not merely words" (Kövecses, Radden 1998: 38) dar, sind jedoch unterschiedlicher Natur und haben verschiedene Zwecke: 
„Metaphor is principally a way of conceiving of one thing in terms of another, and its primary function is understanding. Metonymy, on the other hand, has primarily a referential function, that is, it allows to use one entity to stand for another" (Lakoff, Johnson 1980: 36).

Die neueren Definitionen von Metapher und Metonymie stützen sich auf die Begriffe domain ${ }^{26}$ und ICM. Unter einer Domäne versteht man einen konzeptuellen Bereich bzw. „a semantic structure that functions as the base for at least one concept profile“ (Croft 1993: 339). Dabei ist „the base [...] that aspect of knowledge which is necessarily presupposed in conceptualizing the profile" (Croft 1993: 338). Darüber hinaus wird ein Objekt in Bezug auf die vorhandenen Vorstellungen über dieses Objekt definiert, vgl. die Prädikate [CIRCLE] und [ARC] (Langacker 1987: 183-184, siehe auch Croft 1993: 338). Ein Bogen wird beispielsweise immer in Bezug auf einen Kreis definiert. Darüber hinaus dient ein Kreis als Basis für einen Bogen, der dementsprechend ein Profil darstellt.

Der von Lakoff verwendete Begriff ICM (=idealised cognitive model) stellt eine übergeordnete Domäne dar (siehe auch Barcelona 2003: 211) und bezieht sich auf kognitiv strukturierte Gesamtheiten, die das menschliche Wissen über die Welt organisieren bzw. systematisieren. ICMs können aus verschiedenen auf ein Konzept bezogenen Domänen bestehen und werden in der Literatur auch durch die Begriffe domain matrix (Croft 1993: 340), frame Taylor (1989: 87)27, abstract domain (Langacker 1987: 150) sowie u.U. scene, schema oder script ${ }^{28}$ ersetzt (Langacker 1987: 150). Als Beispiel einer ICM bietet Lakoff (1987: 74) das Konzept 'Mutter', welches aus den Domänen „, birth model“, , genetic model“ , „, nurturance model“, „, marital model" und "genealogical model" besteht.

Der Unterschied zwischen der Metapher und der Metonymie lässt sich demnach wie folgt erklären:

- Lakoff \& Johnson (1987: 114): „Metaphor mapping involves a source domain and a target domain [...]. The mapping is typically partial. It maps the structure in the source domain onto a corresponding structure in the target domain. A metonymic mapping occurs within a single conceptual domain, which is structured by an ICM."

- Croft (1993: 348): „Metaphor is a mapping between two domains that are not part of the same matrix [...]. In metonymy, on the other hand, the mapping occurs only within a domain matrix".

Aus diesen Definitionen folgt, dass die Metapher eine Verbindung bzw. das Projizieren zwischen der Ursprungs- und Zieldomäne herstellt mit dem Zweck, einen Sachverhalt durch einen anderen zu erklären oder zu verstehen, während die Metonymie innerhalb einer Domäne bzw. Domänenmatrix, ICM oder Frame fungiert und einen Verweis von einem Sachverhalt auf einen anderen ermöglicht. In Crofts

\footnotetext{
${ }^{26}$ In der russischen Literatur findet man die Bezeichnung taksonomičeskij klass ('taxonomische Klasse'), z.B. in Padučeva (2004).

27 "knowledge network linking the multiple domains associated with a given linguistic form" Taylor (1989: 87).

28 "temporal sequencing and causal relations which link events and states within certain action frames" Taylor (1989: 87).
} 
Terminologie (1993: 348) stellt die Metapher damit „domain mapping“ (vgl. 32-33) und die Metonymie „domain highlighting“ (vgl. 34-35) dar, indem die Domäne hervorgehoben wird, die ansonsten eine sekundäre Rolle spielt:

(32) She's in the living room.

(33) She's in a good mood. (Croft 1993: 345)

(34) Proust spent most of his time in bed.

(35) Proust is tough to read. (Croft 1993: 348)

Die Belege (32) - (33) demonstrieren die metaphorische Verbindung der Konzepte SPACE und EMOTIONS. Der Gebrauch von in in (33) lässt erkennen, dass die konzeptuelle Struktur der auf Raum bezogenen Domäne auf das Zielkonzept bzw. die Domäne der Gefühle angewendet wird, sodass die gleiche bzw. ähnliche Struktur der beiden Entitäten ausschlaggebend ist. Darüber hinaus wird das Befinden einer Person in einem Raum auf das Befinden dieser Person in einem emotionalen $\mathrm{Zu}$ stand metaphorisch projiziert, i.e. Gefühle werden als Raum konzeptualisiert. In (34) - (35) entsteht dagegen eine metonymische Beziehung zwischen den Konzepten PRODUCER und PRODUCT (vgl. Lakoff, Johnson 1980: 38) bzw. dem Schriftsteller und seinen literarischen Werken. Obgleich die Bedeutungsverschiebung in den behandelten Fällen jeweils zwischen zwei Konzepten erfolgt, handelt es sich dabei jedoch um verschiedene Mechanismen. Der semantische Wandel in (32) - (33) findet zwischen zwei Domänen statt, die verschiedenen frames bzw. ICMs [RAUM] und [EMOTIONEN] gehören. Die in (34) - (35) ausgedrückten Konzepte stellen dagegen die Bestandteile einer ICM dar bzw. gehören zum demselben frame PROUST, welches die Konzepte [WRITER] und [LITERAL WORKS] enthält, zwischen denen der Wandel des Fokus erfolgt.

In Barcelona (2002) wird der Versuch unternommen, die von Lakoff geprägte Definition der Metapher und Metonymie sowie die der Domäne präziser zu formulieren. Barcelona schlägt vor, auf den Begriff domain matrix zu verzichten und den Begriff Subdomäne einzuführen. In diesem Fall werden die Begriffe domain und ICM gleichgestellt. Die Metonymie stellt somit eine Verbindung her zwischen den Subdomänen, die einer Domäne bzw. ICM untergeordnet sind, vgl.:

(36) Washington is insensitive to the needs of the people. (Barcelona 2003: 215)

In Bezug auf (36) handelt es sich um die Domäne [Hauptstadt der USA], die die folgenden Subdomänen einschließt: [ORT], [die sich in diesem Ort befindende Behörde] und [die diese Behörde vertretenden Menschen]. Mittels Metonymie kann jede von diesen Domänen aktiviert bzw. highlighted werden.

\subsubsection{Metapher und Metonymie in der Adjektiv- und Adverbiallexik}

Der die Klasse der Adjektive und Adverbien betreffende semantische Wandel hängt in direktem Zusammenhang ab von deren Bezugswörtern bzw. Substantiven, Verben, Adjektiven und Adverbien. Paradis (2000: 234) vertritt die Auffassung, dass " $(\mathrm{t})$ he noun and the adjective exert semantic pressure on one another. The properties of the noun constrain the interpretation of the adjective, and the properties of the adjective assign a perspective in which the noun is to be viewed". Eine ähnliche Sichtweise wird von Kustova geteilt (2004: 27), der zufolge die Änderung 
eines Bezugssubstantivs zur Änderung der Bedeutung eines Eigenschaftsworts führen kann sowie umgekehrt. In Bezug auf die angesprochenen Definitionen von Metapher und Metonymie lässt sich schließen, dass die Verbindung eines Eigenschaftsworts mit einem zu einer anderen Domänenmatrix oder einer anderen ICM gehörenden Bezugswort die metaphorische Verschiebung der Bedeutung des Adjektivs bzw. Adverbs zu Folge hat. Die Verwendung eines Eigenschaftsworts mit einem Bezugswort aus derselben Domänenmatrix bzw. ICM kann dagegen zu einem metonymischen Wandel der Bedeutung führen. Dies kann anhand der folgenden Schemata veranschaulicht werden, vgl.:

(37) Metapher in der Adjektiv- und Adverbiallexik:

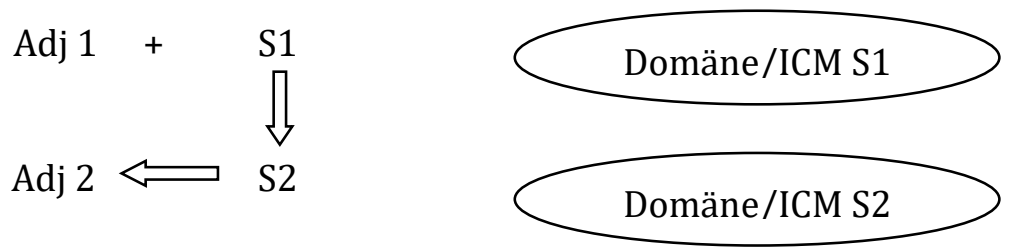

(38)Metonymie in der Adjektiv- und Adverbiallexik:
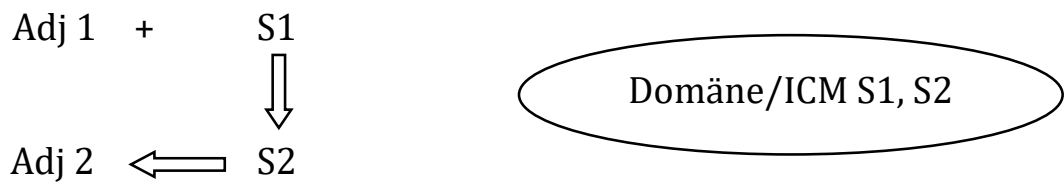

Wie aus der Skizze in (37) folgt, verbindet die Metapher zwei Prädikate S1 und S2 aus verschiedenen Domänen bzw. ICMs und dient als Impuls für die Veränderung der Bedeutung des Adjektivs. In (38) wird dagegen demonstriert, dass die Metonymie auf Objekte S1 und S2 referiert, die derselben Domänenmatrix bzw. ICM gehören. Dadurch bleibt die in dem Adjektiv auszudrückende Eigenschaft unverändert, es verändert sich lediglich der Blickwinkel auf diese Eigenschaft. Diese Ansicht kann anhand der folgenden Belege exemplifiziert werden, vgl. die metaphorische Übertragung in (39) - (40):

(39) An den Wänden standen hohe Regale, alle gefüllt mit staubbedeckten leeren Flaschen. (DWDS: Moers, Walter, Die $131 / 2$ Leben des Käpt'n Blaubär, Frankfurt a.M.: Eichborn 1999, S. 367)

(40) Er habe mit Präsident [...] ausführliche Gespräche über die Bedürfnisse Ägyptens geführt [...], ohne leere Versprechungen zu geben und ohne die Menschen zu enttäuschen. (DWDS: Archiv der Gegenwart, 44, 1974)

In (39) bezieht sich das Adjektiv leer auf die Eigenschaft eines physischen Objekts bzw. eines Behälters. In (40) dagegen handelt es sich um die Eigenschaft eines abstrakten Konzepts bzw. Ergebnis menschlicher sprachlicher Tätigkeit. Bei dem Übergang der Bedeutung von leer aus der Ursprungsdomäne in die Zieldomäne wird die Versprechung als Behälter konzeptualisiert und deren Füllung als die Realisierung des Versprochenen dargestellt. Dementsprechend wird das Fehlen der Füllung im Behälter mit dem Fehlen des Versprochenen metaphorisch verbunden. Die in diesen Bedeutungswandel herangezogenen Domänen gehören nicht zu derselben Domänenmatrix bzw. ICM., sodass man die Bedeutungsverschiebung als Metapher bezeichnen kann. 
Der Mechanismus der Metonymie wird in (41) - (42) exemplifiziert, vgl.:

(41)Ich bin froh, dass wir im Halbfinale standen. Damit haben wir unser Ziel erreicht. (C.II: BRZ13/JAN.02022 Braunschweiger Zeitung, 07.01.2013)

(42)Bei schönem Wetter grilliert man in den Gärten, frohe Gesichter, fröhliches Lachen in entspannter Atmosphäre. (C.II: A98/JUL.49179 St. Galler Tagblatt, 25.07.1998)

In (41) bezieht sich froh auf eine „von Freude erfüllte“ Person (DUDEN 2012, s.v. froh), in (42) dagegen auf das Freude demonstrierende Gesicht. Durch Metonymie werden in der Domänenmatrix bzw. ICM [FREUDE] verschiedene Aspekte der auf den entsprechenden emotionalen Zustand bezogenen Situation hervorgehoben bzw. highlighted: [TRÄGER] und [ERSCHEINUNGSFORM].

Die Frage, inwiefern der semantische Wandel in der Adjektiv- und Adverbiallexik systematisch ist und welche Domänen bzw. Domänenmatrizen mittels der Metapher und der Metonymie verbunden werden können, wurde bis heute in geringem Maße untersucht. Die Beziehungen zwischen einem Adjektiv und einem Nomen, die mittels Metonymie innerhalb einer Domäne in den Fokus geraten, werden in Aarts, Calbert (1979: 79) behandelt und als predicational relators (PRs) ${ }^{29}$ bezeichnet ${ }^{30}$. Ein PR stellt einen Zusammenhang her zwischen den Konzepten, die jeweils durch das Adjektiv und das Bezugswort ausgedrückt werden. Die von den Autoren zur Verfügung gestellte Liste enthält 13 PRs. In Bezug auf das in der vorliegenden Arbeit untersuchte Material sind die folgenden 7 PRs aus dieser Liste von besonders großem Interesse (Aarts, Calbert 1979: 86-90) ${ }^{31}$ :

- PR1 'HAVE' „N has A as a temporal or permanent property“, z.B. heavy table;

- PR2 'EXPERIENCE' „N experiences the mental state (or corporeal condition) A“, z.B. angry man;

- PR3 'MANIFEST' „N manifests that some person[s] experience[s] (experienced) the mental state state (or corporeal condition) A“, z.B. angry letter;

- PR4 'TIME-WHEN',N is the time when some person[s] experience[s] (experienced) the mental state (or corporeal condition) A“, z.B. happy days;

- PR5 'PLACE-WHERE': „N is the place where some person[s] experience[s] (experienced) the mental state (or corporeal condition) A“, z.B. happy home;

- PR6 'CAUSE': „N causes some person[s] to experience the mental state (or corporeal condition) A", z.B. sad event;

\footnotetext{
${ }^{29}$ Warren (1984: 21), die sich ebenso mit den Beziehungen in den Adjektivischen Konstruktionen beschäftigt, ersetzt den Begriff durch „connecting link“ bzw. „relation“.

30 Predicational relators stellen in Aarts, Calbert (1979) neben den nicht-prädikativen Bedeutungselementen bzw. features Bestandteile der lexikalischen Struktur von Adjektiven dar.

${ }^{31}$ Die Liste von PRs von Aarts, Calbert enthält außerdem Intensivierung (PR8 „degree - of").
} 
- PR7 'MANNER-IN-WHICH': „A is the manner in which the action $\mathrm{N}$ (or the action associated with $\mathrm{N}$ ) is performed", z.B. rapid calculations.

Aarts und Calbert unterscheiden zwischen direct modification wie in PR1 und PR2 und indirect modification (Aarts, Calbert 1979: 82) wie in PR3 bis PR7. Sie verweisen darauf, dass "the predicational value of an adjective may change according to the noun it co-occurs with, while its non-predicational meaning remains constant" (Aarts, Calbert 1979: 83). Darüber hinaus plädieren die Autoren dafür, dass durch die Änderung von PRs bzw. den Wechsel von direct modification zu indirect modification kein Bedeutungswandel in dem Adjektiv stattfindet. Warren (1992: 65) dagegen bezeichnet die Fälle von indirect modification als Metonymie und stellt sie als Beispiele von Kontiguität dar. Dieses Herangehen passt in den Rahmen der dargestellten Theorie der kognitiven Metonymie, indem die PRs als Beziehungen zwischen dem Adjektiv und seinem Bezugswort innerhalb eines Frames bzw. einer ICM angesehen werden, vgl. in diesem Zusammenhang Rachilina et al. (2010: 415 - 424), Karpova (2012).

Die in Aarts und Calbert behandelten PRs, oder, in Warrens Terminologie (1992: 21) ausgedrückt, connecting links finden sich in dem von mir analysierten Material, vgl.:

(43) Ich bin froh, dass wir im Halbfinale standen. Damit haben wir unser Ziel erreicht. (C.II: BRZ13/JAN.02022 Braunschweiger Zeitung, 07.01.2013)

(44) Bei schönem Wetter grilliert man in den Gärten, frohe Gesichter, fröhliches Lachen in entspannter Atmosphäre. (C.II: A98/JUL.49179 St. Galler Tagblatt, 25.07.1998)

(45) Die frohe Nachricht erfuhr die Familie gestern durch die Wolfsburger Nachrichten. „Das ist sehr schön“, freute sich die Schwiegertochter [...]. (C.II: BRZ12/JUN.14898 Braunschweiger Zeitung, 28.06.2012)

(46) Im Hinterwasen verbrachte er zusammen mit zwei Brüdern eine frohe und glückliche Jugendzeit. (C.II: A00/NOV.82074 St. Galler Tagblatt, 30.11.2000)

(47) An den vielen Geräten spielten die Kinder froh und ausgelassen. (C.II: RHZ06/JUL.12992 Rhein-Zeitung, 14.07.2006)

Durch die Metonymie werden in der Domänenmatrix bzw. ICM [FREUDE] die folgenden Aspekte der auf den entsprechenden emotionalen Zustand bezogenen Situation hervorgehoben bzw. highlighted: der Träger des Gefühls in (43), die Erscheinungsform in (44), der Grund des zu empfindenden Gefühls in (45), die mit dem Gefühl verbundene Zeitspanne in (46) und anschließend die mit dem Gefühl durchgeführte Tätigkeit (47).

Die in dem Kapitelabschnitt 4.2 untersuchte Gruppe „Übel wollend / verursachend", zu der verschiedene Lexeme des menschlichen Verhaltens gehören (z.B. böse, brutal, grausam, wütend), präsentiert am meisten die Metonymie der Typen MANIFEST in (48) - (49) und MANNER-IN-WHICH in (50), vgl.:

(48) Errette mich / Herr / von den bo̊fen Menfchen / behüte mich für den freveln Leuten / die bo̊fes gedencken in jhrem Hertzen / vnd tåglich Krieg erregen. (DTA: Arndt, Johann: Vom wahren Christenthumb. Bd. 2. Magdeburg, 1610) 
(49) „Wer seid Ihr?“ frägt der Richter mit ziemlich brutalem Ton. (C.II: HK3/B01.00002 Schiller: Der Verbrecher aus verlorener Ehre, Erstdruck: 1786, 2000 [S. 33])

(50) du bist ja besoffen! wütend stiesz sie ihn zurück (DWB: s.v. wüten)

Kirschbaum (2002a) weist der Metapher und der Metonymie die führende Rolle bei der Herausbildung von Intensivierern zu bzw. verweist darauf, dass die Entwicklung von Grad-Adjektiven und -Adverbien lediglich durch das Einsetzen von diesen zwei kognitiven Mechanismen erfolgt. „Die Übertragung räumlicher Strukturen“ stellt demzufolge "ein grundlegendes metaphorisches Muster zur Gewinnung von Intensivierern" dar (Kirschbaum 2002a: 72). Räumliche Dimensionen wie HÖHE (hoch, höchst), TIEFE (tief, zutiefst, abgrundtief), GRÖSSE (gigantisch, riesig, kolossal), ENTFERNUNG (weit, weitaus, bei weitem, weithin), GEWICHT (leicht, schwer) und KRAFT (kräftig, gewaltig, mächtig) dienen als Ausgangsbereiche bzw. sources, während hoher Grad als Zielbereich bzw. target angesehen wird (Kirschbaum 2002a: 72-73), vgl. die aus Kirschbaum $(2002 a$ : 68, 90) übernommenen Belege:

(51) Karl erzählt mir von einem hoch interessanten Artikel.

(52) Tief betrübt schlichen die Spieler in die Kabine.

(53) Ich bin schwer erkältet.

(54) Er macht einen leicht müden Eindruck.

(55) Als Hexen verkleidet tanzten sie um das Walpurgis-Feuer und hatten dabei riesig viel Spaß.

Kirschbaum zufolge wird bei dem metaphorischen Wandel ein Abschnitt einer Differenzskala auf die Skala des Grads projiziert. Dies scheint auf die Bereiche HÖHE und TIEFE zuzutreffen. In Bezug auf hoch soll jedoch angemerkt werden, dass das Lexem ursprünglich die Höhe eines Objekts bzw. Dinge, „die in bedeutender weise ansteigen“, bezeichnete (DWB, s.v. hoch). Das Befinden eines Objekts „in einer bedeutenden erhöhung" (DWB, s.v. hoch) demonstriert den Übergang von einer vertikalen Ausdehnung zu dem höchsten Punkt dieser Ausdehnung und stellt damit eine Art end-point metonymy dar (Rachilina et al. 2010: 421-422, Karpova 2011b: 301), vgl. eine ähnliche Übertragung in der englischen Präposition over bzw. fly over the hill vs. live over the hill in Brugman, Lakoff (1988). Diese metonymische Bildung, die das Positionieren von Objekten auf einem bestimmten Punkt einer Skala bezeichnet, dient ferner als Ausgangspunkt für metaphorische Übertragungen dieser Position in Bezug auf verschiedene abstrakte Sachverhalte, z.B. Wert von Zahlen und Preisen (hohe Summe), Rangordnung bzw. Hierarchie (ein hoher Offizier), Endpunkt einer qualitativen Skala (hohe Ansprüche). In diesem Zusammenhang wird hoch auch als eine Maßbezeichnung für Sachverhalte auf einer Gradskala gebraucht (hohe Empfindlichkeit). Diese Erklärung des Bedeutungswandels gilt auch für tief, vgl. tiefes Meer - tiefe Wolken - tiefe Temperaturen.

Die Herausbildung der Gradbedeutung in anderen semantischen Bereichen wie GRÖSSE oder GEWICHT scheint jedoch komplexerer Natur zu sein, sodass der Weg von schwerer Koffer zu sich schwer freuen und von riesiges Haus zu riesig interessant anhand lediglich klassischer metaphorischer Verbindungen kaum erklärt werden kann. So bezieht sich das Adjektiv schwer laut DWB zunächst auf drückend auf den 
Körper wirkende Objekte in Bezug auf ihr Gewicht, z.B. schwere Last. Dieser Gebrauch wird dann metaphorisch auf abstrakte Sachverhalte übertragen, „die man ja auch als schwere lasten bezeichnet" (DWB, s.v. schwer I.1.b), z.B. schwere Arbeit ${ }^{32}$. Eine „vom großen Gewicht" und daher große körperliche Anstrengung erfordernde Last wird damit als „einen hohen Schwierigkeitsgrad aufweisend [e]“ (DUDEN 2012, s.v. schwer) und von daher große geistige o.ä. Anstrengung erfordernde Arbeit konzeptualisiert. Dieser Übergang demonstriert die metaphorische Verschiebung der Bedeutung aus dem Bereich „physikalische Eigenschaft“ in den Bereich „mentale Eigenschaft“. In der Ausgangsdomäne empfindet die Person als Gefühlsträger Unbehagen durch den Kontakt mit einem physikalischen Objekt. Das Gefühl kann vermieden werden, indem die Person das Objekt abstellt bzw. herunternimmt. Diese semantische Struktur wird in die Zieldomäne übertragen: Der Gefühlsträger empfindet Unbehagen durch das Ausüben einer mentalen Tätigkeit. Das Unbehagen wird vermieden, indem die Person mit der Tätigkeit aufhört.

Eine solche Darstellung der Metapher ist jedoch in Bezug auf Kontexte wie schwere Not und schwere Enttäuschung nicht ausreichend. Zunächst handelt es sich um einen negative Emotionen hervorrufenden Sachverhalt und den metaphorischen Übergang der Bedeutung von schwer in den emotionalen Bereich. Die metaphorische Verschiebung erfolgt durch das Kopzeptualisieren eines physikalischen Objekts von großem Gewicht mit einer „einen hohen Schwierigkeitsgrad aufweisend[en], schwierig[en], nicht leicht zu bewältigen[en]“ (DUDEN 2012, s.v. schwer) Situation. Zugleich wird dem Adjektiv intensivierende Bedeutung hinzugefügt, so auch DWB (s.v. schwer).

Paul (1909: 92-93) erklärt derartiges Auftreten von Intensivierung anhand des ähnlich gelagerten Beispiels von arg durch die Verbindung des Lexems mit einem „syntaktisch angeknüpften Worte“, in dem ein „Teil seines Bedeutungsinhaltes [...] noch einmal ausgedrückt wird“, und verweist in diesem Zusammenhang auf weitere betroffenen Lexeme wie furchtbar, schrecklich, entsetzlich, ungeheuer, schmählich, höllisch, verdammt und sehr. Er nimmt an, dass die Kombination des Lexems mit den Bezugswörtern, „die an sich etwas Böses, Unangenehmes bezeichnen“ (Paul 1909: 92), zu der Verstärkung des Gebrauchs führt. Dieses Phänomen wurde schon früh in Borst (1902: 10-13) angesprochen. Er verweist (Borst 1902: 10-11) darauf, dass „die Wiederholung entweder ein und desselben Begriffs oder begrifflich verwandter Synonyma“ „ein sehr beliebtes Steigerungsmittel ist“. Kirchner (1955: 116) exemplifiziert diesen Mechanismus der Verstärkung durch mehrere Belege und bezeichnet ihn als „asydentische Nebeneinanderstellung" von Gradadverbien. Bolinger (1972: 290) nennt das Phänomen semantic repetition. Repetition bzw. accumulation of sense erfolgt durch arithmetic boosting, wobei ,the elements are added together", z.B. monstrously bad (Bolinger 1972: 291). Andere in der Literatur verwendeten Begriffe sind semantische Dopplung (Steiner 2010: 179, vgl. auch Spitzbardt 1954: 13-14) und Stapelung (Jahr 2000: 91), wobei die letzte in Os (1989: 112) als „kumulative Intensivierung“ bezeichnet wird.

Semantic repetition betrifft jedoch nicht alle negativ konnotierten Lexeme. Paradis (1997: 54) verweist in diesem Zusammenhang auf „extreme adjectives“ bzw.

\footnotetext{
32 Eine ausführliche Darstellung von metaphorischen Übertragungen solcher Art findet sich am Beispiel des russischen Äquivalents tjažjolyj іп Кустова (2004: 279-307).
} 
„implicit superlatives“. Diese Wörter „express a superlative degree of a certain feature [...] and can occupy the [...] negative extreme of a scale“. Pusch (1981: 36) nennt solche Adjektive „superlativisch“. Os verweist darauf, dass diese Lexeme „intensivierend wirken“ (1989: 120) können, weil sie „eine sehr starke positiv- oder negativ-evaluative Bedeutung haben und [...] bezeichnen von sich aus schon einen extrem hohen Grad der Intensivierung" (1989: 186). Darüber hinaus führt die Verbindung von einem „implicit superlative“ bzw. superlativischen Adjektiv mit einem skalierbaren bzw. einen Extremwert aufweisenden Bezugswort zu der Intensivierung des Begriffs, wie es z.B. in der Zusammensetzung schwere Not der Fall ist.

Es ist bemerkenswert, dass die Intensivierung in den Verwendungen wie schwere Not zunächst als eine Nebenbedeutung neben der metaphorisch gebildeten Bedeutung auftritt, die währenddessen die Hauptrolle zu spielen pflegt. Darüber hinaus ist das Auftreten der Gradbedeutung mit der Mehrdeutigkeit des Gebrauchs verbunden. Nachdem die intensivierende Bedeutung konventionalisiert wurde, erscheint sie herausgebildet in Kontexten wie:

(56) Harald Juhnke: Der Entertainer empfing 1997 schwer betrunken Kamerateams von Sat I und RTL zu Interviews. (Züricher Tagesanzeiger, 28.08.1999, S. 65 , zit. durch Kirschbaum 2002: 102)

(56') 'sehr betrunken'

(57) Triumpf der Superreichen. Gewonnen haben bei der Testwahl in Iowa erwartungsgemäss jene beiden Kandidaten, die über das meiste Geld verfügen: der eine, George W. Bush, weil er im Wahlkampf bisher nicht weniger als 37 Millionen Dollar gesammelt hat, der andere, Steve Forbes, weil er privat schwer reich ist. (Züricher Tagesanzeiger, 16.08.1999, S. 5, zit. durch Kirschbaum 2002: 103)

(57') 'sehr reich'

Die Belege (56) - (57) demonstrieren den eindeutigen Gebrauch von schwer in einer intensivierenden Funktion. Dabei bietet die Verwendung keinen Vergleich des betrunkenen Zustands von Personen oder des Reichtums mit einem Objekt von großem Gewicht.

Die Herausbildung der Gradbedeutung stellt damit einen komplexeren Prozess dar, als ihn Kirschbaum (2002) beschrieben hat. Dieser Prozess setzt neben der Metapher das Ableiten einer Implikatur voraus und beginnt demnach mit einem ambigen Gebrauch des Lexems, der sich anschließend zu der eindeutig intensivierenden Verwendung entwickelt.

Kirschbaum (2002a: 131-135) führt ferner an, dass die Entwicklung von Intensivierern außerdem nach dem metonymischen Muster „WIRKUNG steht für GRAD“ erfolgen kann. Als sources gelten z.B. das oben kurz angesprochene Wortfeld „Erregung von Angst und Schrecken“ (z.B. furchtbar, entsetzlich, grausam) sowie "Erregung von Abscheu“ (z.B. abscheulich, ekelhaft, widerlich), „Anormaler Geisteszustand" (z.B. wahnsinnig, irrsinnig, verrückt), Erstaunen (z.B. erstaunlich, unerwartet, überraschend) ${ }^{33}$ u.a. „Der Operand drückt eine Eigenschaft aus, die als Ursache für eine bestimmte Wirkung - ausgedrückt durch den Operator - fungiert,

33 Die Liste aller Ausgangsbereiche für das metonymische Muster findet man in Kirschbaum (2002a: 201). 
wobei der Grad indirekt über die Wirkung erschlossen wird“ (Kirschbaum 2002b: 209), vgl. die aus Kirschbaum (2002a) übernommenen Belege mit Paraphrasen:

(58) Eben damals bekam Julus von seinem Vater Briefe und in denselbigen einen scharfen Verweis, daß er so ärgerlich lebe und so schrecklich viel Gelds verschwende; (Simplicissimus, Continuatio, Kapitel 7, 512, zit. durch Kirschbaum 2002a: 140)

(58') 'so viel Geld, dass man sich erschrickt'

(59) „[...] der Vertrag mit ihrer englischen Gesellschaft verbietet solche Auftritte!“. Frankenfeld weiter: „es wäre irrsinnig teuer gewesen, sie für eine Viertelstunde aus diesem Vertrag loszukaufen". Enttäuscht waren auch mehrere hundert „Emma“-Fans in Düsseldorf, wo der Star gestern seine erste Pressekonferenz geben sollte. (BILDZEITUNG JULI 1967, Nr. 156, S. 1, zit. durch Kirschbaum 2002a: 160)

(59') 'so teuer, daß man irrsinnig wird.'

(60) Ich verdiene lächerlich wenig. (Kirschbaum 2002a: 75)

$(60$ ') 'so wenig, dass es lächerlich ist'

In Bezug auf die Belege (58) - (60) kann Kirschbaums metonymisches Muster „WIRKUNG steht für GRAD“ nicht als klassische Metonymie bezeichnet werden, da es sich von dem traditionellen, am Anfang dieses Kapitelabschnitts skizzierten Schema deutlich unterscheidet. Die Verwendungen in (58) - (60) demonstrieren das Verlassen der ursprünglichen Domäne: die Person in (58) empfindet keinen echten Schreck, in (59) gibt es keinen Bezug auf den anormalen geistig-seelischen Zustand und in (60) verursacht der Kleinverdienst kein echtes Lachen. Durch den Gebrauch mit schrecklich, irrsinnig und lächerlich findet zugleich kein metaphorischer Vergleich mit Angstgefühlen, dem geistigen Zustand und dem Lachen statt, sondern wird der hohe Grad der angesprochenen Sachverhalte hervorgehoben, vgl. die Umformulierungen:

(58") 'sehr viel Geld'

(59") 'sehr teuer'

(60") 'sehr wenig'.

Gemäß der klassischen Vorstellung wird die Metonymie als eine „stand-for“-Beziehung (Kövecses, Radden 1998: 38) bezeichnet bzw. „shift of reference“" (Croft 1993: 349), „whereby the name of one entity $\mathrm{e}^{1}$ is used to refer to another entity $\mathrm{e}^{2}$ which is contiguous to $\mathrm{e}^{1 \text { “ }}$ (Taylor 1989: 122). Diese Möglichkeit, ein Prädikat durch einen Referenten zu ersetzten, wird der Wirkung von referring function (Nunberg 1978: $156)^{34}$ zugewiesen. Diese Funktion begünstigt beispielsweise die Metonymie BESTELLUNG steht für KUNDE in (61):

(61) The ham sandwich is sitting at table 20. (Nunberg 1979: 149)

Aus (61) folgt, dass referring function "the possibility of establishing connections between entities which co-occur within a given conceptual structure" (Taylor 1989: 123-124) bietet. Dirven (2003: 82) verweist sogar darauf, dass „Metonymy [...] can

\footnotetext{
34 "that function that the hearer (correctly) selects from among an indefinitely large number of functions that take the demonstratum as arguments" (Nunberg 1978: 156)
} 
associate all kinds of elements which have a 'natural' link to each other"35. Darüber hinaus stellt die Metonymie eine Art Substitution dar, vgl. in diesem Zusammenhang die metonymisch bedingte Ursache-Wirkung-Beziehung in den Kollokationen fröhliche Kinder und fröhliche Nachricht. Dieses Modell demonstriert die Verschiebung der Bedeutung von der Ursache eines Gefühls (Nachricht) auf einen Gefühlsträger (Kinder). Die Bedeutung von fröhlich bezieht sich weiterhin auf die Domäne der Freude. Dies ist in (58) - (60) jedoch nicht der Fall, da die Bedeutung der Adverbien in die Domäne [HOHER GRAD] übergeht. Darüber hinaus stellt dieser Bedeutungswandel keine Substitution dar und kann von daher nicht als klassische Metonymie bezeichnet werden.

Es soll jedoch darauf hingewiesen werden, dass Kirschbaums Idee, das Auftreten von Gradbedeutung als Metonymie darzustellen, nicht neu ist. Es finden sich mehrere Artikel in der Literatur, die die klassische Definition von Metonymie erweitern und deren Mechanismus als Grundlage für pragmatic inferencing betrachten. Pragmatic inferencing pflegt bei der Entstehung der Gradbedeutung mitzuwirken und mehrdeutigen Gebrauch auszulösen. Panther, Thornburg (2003: 8) bezeichnen die Metonymie als "natural inference schemas, i.e. easily activatable associations among concepts that can be used for inferential purposes". Barcelona (2003b: 97) geht davon aus, dass „(m)etonymy [...] seems to constitute the very skeleton of pragmatic inferencing". Er untermauert diese Annahme folgendermaßen (Barcelona 2003b: 97): ,the domain, concept or frame presented as source is normally a mental activator of the domain, concept or frame presented as target". Barcelona vermeidet es jedoch, pragmatic inferencing als Metonymie zu bezeichnen und fügt deshalb hinzu (2003b: 97-98): „These general pragmatic principles, studied particularly by scholars working in a relevance theoretic framework, cannot be reduced to metonymy. [...] There are other important aspects of pragmatic inferencing that cannot simply be accounted for in terms of metonymic connections but rather in terms of pragmatic principles and rules necessary for a complete understanding of pragmatic inferencing processes".

Die Verbindung von pragmatic inferencing und der Metonymie wird außerdem in den auf Grammatikalisierung bezogenen Werken hervorgehoben, z.B. in Traugott (1988), Traugott, König (1991), Traugott, Dasher (2002) und Hopper, Traugott (2003). Wie es in Diewald (1997: 54) kurz zusammengefasst wird, „verweist das sprachliche Zeichen also auf den außersprachlichen Kontext (genauer, auf einen bestimmten Aspekt des außersprachlichen Kontextes), dessen Teil es ist. [...] Aus der Perspektive des Hörers (bzw. Lesers), stellt diese Art der Metonymie eine Anweisung dar, dasjenige Element des außersprachlichen Kontextes zu finden, auf das der sprachliche Ausdruck verweist, ohne es „wörtlich“ zu nennen“. Zugleich verweisen Heine, Claudi, Hünnemeyer (1991a) sowie Traugott, König (1991), Traugott, Dasher (2002) und Hopper, Traugott (2003) darauf, dass metonymische Prozesse oft neben den metaphorischen beteiligt sind: „easily comprehended metaphors are consistent with typical associations; both exploit pragmatic meaning; both enrich meaning" (Traugott, Dasher 2002: 29).

35 Taylor (1989: 123) merkt jedoch an, dass sich nicht alle Verbindungen zwischen den Referenten als produktiv in der Sprache erweisen, z.B. *Mary was delicious, indem Mary für den von ihr gemachten Cheesecake steht, vgl. diese Idee auch auch in Kövecses \& Radden (1998: 48). 
Abschließend möchte ich eine kurze Zusammenfassung geben. Kirschbaums Theorie, die Grad-Adjektive und -Adverbien mithilfe von entweder metaphorischen oder metonymischen Mustern 'X steht für Grad' zu behandeln, setzt das Phänomen in sehr enge Rahmen. Die Analyse meines Materials legt nahe, dass die Entwicklung von Intensivierern viel komplexer zu sein scheint und die Mitwirkung auch von anderen Mechanismen voraussetzt. Meine Annahme basiert auf der ausführlichen Untersuchung von Lexemen in historischer Perspektive, wogegen Kirschbaum lediglich eine synchrone Studie präsentiert. Ich schließe mich demnach Peters (1993) an, der die Grammatikalisierungstheorie für die Analyse von Intensivierern in Erwägung zieht.

\subsubsection{Intensivierung und Grammatikalisierung}

\subsubsection{Zum Begriff der Grammatikalisierung}

Der Begriff „Grammatikalisierung" wurde von Meillet (1912) eingeführt und folgendermaßen charakterisiert (Meillet 1912: 385, Übersetzung von Campbell, Janda (2000a: 95)):

„[Besides analogy,] another process consists in the change of an autonomous word into the role of a grammatical element.... Th[is]...process...[, involving] the attribution of grammatical character to a formerly independent word...[, is one of] only [two] ways by means of which new grammatical constructs are formed".

Die klassischen Studien über Grammatikalisierung basieren auf der von Meillet vorgeschlagenen Bezeichnung und behandeln das Phänomen als den „Übergang einer lexikalischen, autonomen Form zu einer grammatischen, unselbständigen Form“ (Diewald 1997: 11). Campbell, Janda (2000b: 114) skizzieren „the prototypic (or core) definition most familiar today" folgendermaßen:

some linguistic element > more grammatical.

Da die von mir zu untersuchenden Grad-Adjektiven und -Adverbien keine Entwicklung zu einem grammatischen Element, z.B. einem gebundenen Morphem, demonstrieren, entsteht die Frage, inwiefern der Begriff Grammatikalisierung in Bezug auf diese lexikalische Klasse anwendbar ist und ob Intensivierer im Rahmen der Grammatikalisierungstheorie analysiert werden dürfen. In diesem Zusammenhang erscheint die Anmerkung von Narrog, Heine (2011: 2) besonders hilfreich, die auf vielfältige Modifizierungen der traditionellen Definition des Phänomens sowie verschiedene Herangehensweisen zu dessen Untersuchung hinweist. In der letzten Zeit haben sich mehrere Studien entwickelt, die auf pragmatisch-semantische Aspekte der Grammatikalisierung fokussieren. Sie stellen die Anfangsphase der Grammatikalisierung ins Zentrum ihrer Aufmerksamkeit und heben die Rolle des Bedeutungswandels bzw. der Metapher und der Metonymie hervor, analysieren die Entwicklung von einstellungsorientierten Bedeutungen im Rahmen von subjectification und intersubjectification, integrieren neue Interpretationen der Implikaturtheorie und unterscheiden verschiedene Grammatikalisierungsgrade, die an dem Verlust der ursprünglichen Bedeutung eines Lexems gemessen werden. Defour (2010: 161) verweist darauf, dass „grammaticalization can [...] cause lin- 
guistic elements to lose semantic meaning and to gain pragmatic strength - evolving from propositional meanings, over text-structuring to increasingly interpersonal functions according to a unidirectional cline".

Diese Anmerkung von Defour lässt sich auf die Entwicklung von Grad-Adjektiven und -Adverbien anwenden. Denn diese Lexeme bringen nicht nur die Intensität der Sachverhalte zum Ausdruck, sondern demonstrieren den Verlust des Zusammenhangs mit der propositionalen Bedeutung und den Übergang in den Bereich persönlicher Evaluierungen, vgl. die Definition von Paradis (2000: 238):

„The development of the adjectives into reinforces represents a typical case of grammaticalization when defined as the process whereby lexical items in constrained contexts undergo reanalysis and come to serve increasingly pragmatic functions".

Unter diesem Gesichtspunkt werden Grad-Adjektive und -Adverbien im Rahmen der Grammatikalisierungstheorie in mehreren Aufsätzen behandelt, z.B. sehr, mächtig u.a. in Biener (1940: 167), awfully / horribly nice in Traugott (1982: 251), dreadful coward, total crap, utter nonsense u.a. in Paradis (2000), furchtbar gut sowie awfully, fearfully, frightfully, terribly in Heine, Kuteva (2002: 50-51), pure in Vandewinkel, Davidse (2008).

Die Rolle von Grammatikalisierungsprozessen bzw. Grammatikalisierungsmechanismen in der Adjektiv- und Adverbiallexik beschränkt sich jedoch nicht auf Desemantisierung und die Entwicklung von Gradbedeutung. Gehweiler (2010) demonstriert, wie die 'privative adjectives' 36 lauter und eitel mit der Ausgangsbedeutung 'rein, unvermischt' sich zu 'diminishing intensifiers' bzw. 'downtoners' und anschließend zu „substanz- und pluralbezogene[n] quantifizierende[n] Determinativ[en]" (Zifonun 1997: 1965) herausgebildet haben (vgl. auch die ausführliche Untersuchung von lauter in Eckardt 2002: 314-366). Die Adjektive bloß (Min-Jae 2005: 128-136) und wohl (Min-Jae 2005: 157-173), von denen bloß ebenso wie lauter und eitel zu der Gruppe von privative adjectives gehört, entwickelten sich zunächst zu Gradpartikeln und dann zu Modalpartikeln.

Grammatikalisierung liegt der Herausbildung vom 'proximity Adverb' fast (Eckardt 2010: 66ff.) und 'pragmatic marker'37 well (Defour 2010) zugrunde. Der Grammatikalisierung von Modalpartikeln, die ihre Herkunft ebenso aus qualitativen Adjektiven und Adverbien beziehen (siehe z.B. Diewald 1997: 75, Autenrieth 2002, Eckardt 2010: 56), widmen sich die Doktorarbeit sowie der Aufsatz von Autenrieth $(2002,2005)$, die eine synchrone und diachrone Studie der Lexeme eben, echt, halt, schlicht, einfach und glatt präsentiert, die Doktorarbeit von Min-Jae (2005), in der u.a. die Wortgeschichten von eben, halt, bloß und wohl behandelt werden. Besonders ausschlaggebend für die angesprochenen Adjektive neben der semantischen und z.T. phonologischen ${ }^{38}$ Abnutzung ist die Herausbildung von

\footnotetext{
36 Unter 'privative adjectives' versteht Gehweiler (2010: 297) "adjectives whose meaning is characterized by the absence of a certain quality or attribute".

${ }^{37}$ Defour (2010: 158) verweist auf andere Bezeichnungen, die den Begriff pragmatic marker in der Literatur ersetzen: discourse marker, discourse particles, discourse operator.

${ }^{38}$ Phonologische Abnutzung ist für echt und halt charakteristisch.
} 
neuen Kategorien wie z.B. Steigerungspartikel und Modalpartikel, „die im Vergleich zum Adjektiv Verlust an Flexionsmerkmalen und somit auch morphologische Dekategorisierungsphänomene aufweist" (Autenrieth 2002: 141).

Der Entwicklung von Adjektiven zu Intensivierern und anschließend Modalpartikeln lässt sich außerdem im Russischen feststellen. Rachilina et al. (2010) verweisen auf die Entwicklung von rovnyj mit der Ausgangsbedeutung 'glatt', 'eben', 'gerade' zu einer Fokuspartikel bzw. Gradpartikel ${ }^{39}$ wie in (62) und einer Vergleichspartikel wie in (63). Einen ähnlichen Entwicklungsweg hat das Adjektiv čistyj mit der Ausgangsbedeutung 'rein', 'sauber' eingeschlagen (siehe Švedova 2015) wie in (64) - (65):

(62) Rovno èto ja i chotel skazat'.

(62') 'Genau / gerade das wollte ich sagen'40.

(63) Sijaet cvetok i serebritsa, i osveščaet vsjo vokrug, rovno ogonjok. (ruscorpora.ru: Tramvaj: V noč na Ivana-Kupalu, 1990)

(63') ,Leuchtet die Blume und glänzt silbern und beleuchtet alles ringsherum wie ein Flämmchen'

(64) Byvaet, čto s druz'jami vyezžaem, čisto otdochnut', - soznalsja on [... ] [ruscorpora.ru: Рыбный день (1997) / / «Столица», 1997.05.27]

(64') Es kommt vor, dass wir mit Freunden einen Ausflug machen, einfach sich ausruhen - gab er zu.'

(65) Och, kak u tebja tut teplo.., nu čisto v bane (http://rusgram.narod.ru/16891705.html А. Н. Толстой)

(65') 'Ach, wie warm es hier bei dir ist... nun wie in der Sauna.'

Die Adjektive polnyj mit der Ausgangsbedeutung 'voll' 'gefüllt' und dovol'nyj mit der ursprünglichen Bedeutung 'zufrieden' können neben der Gradbedeutung (z.B. polnaja erunda 'voller Unsinn', dovol'no krupnyj 'ziemlich groß') auch prohibitive Bedeutung (Polno!, Dovol'no! 'genug') erwerben. Der Gebrauch in der prohibitiven Bedeutung ist auch für das Adjektiv zdorovyj mit dem ursprünglichen Sinn 'gesund' ausschlaggebend (zdorov spat'! 'genug schlafen!') und tritt neben der Verwendung des Lexems als Intensivierer (zdorovo udarit'sja 'sich heftig stoßen') auf. Das Adjektiv prostoj mit der Ausgangsbedeutung 'einfach' wird als Modalpartikel mit der Funktion der Steigerung (prosto neverojatno! 'einfach unglaublich'), Fokus wie in (66) sowie Gegenüberstellung wie in (67) verwendet:

(66) Prosto èto ty ne sdelaeš.

(66') ,Das schaffst du nicht'.

(67) Ty ne zabolel, prosto ustal.

(67') ,Du bist nicht krank, du bist einfach/nur müde'.

Die angeführten Beispiele sollen veranschaulichen, dass Grammatikalisierung graduell unterschiedlich ausgeprägt sein kann, insofern sie die Entwicklung eines Le-

\footnotetext{
${ }^{39}$ Fokuspartikel und Gradpartikel werden gleichgestellt. <http://hypermedia.ids-mannheim.de/call/public / sysgram.ansicht?v_id=408>

40 In Bezug auf das angeführte Beispiel (62) soll angemerkt werden, dass die Adjektive genau und gerade ebenso in dieser Funktion verwendet werden können.
} 
xems in unterschiedlichem Grad zu beeinflussen scheint. Lexeme mit intensivierender Bedeutung belegen hauptsächlich den Verlust der propositionalen Bedeutung und die Herausbildung der evaluativen Funktion. In diesem Zusammenhang „sind Gradadverbien in höherem Maße grammatische Elemente als ihre noch mit voller lexikalischer Bedeutung ausgestatteten Quellen“" (Peters 1993: 327). Modalpartikeln sind im Gegensatz zu Gradadverbien noch grammatikalisiertere sprachliche Einheiten, da sie die Entwicklung neuer Kategorien aufweisen. Von daher, wie Peters (1993: 277) mit Recht anmerkt, wird „Grammatikalisierung [...] nunmehr als die Entwicklung eines sprachlichen Zeichens von (mehr) lexikalischer Funktion zu (mehr) grammatischer Funktion verstanden".

\subsubsection{Mechanismen und Parameter der Grammatikalisierung in Bezug auf deutsche Grad-Adjektive und -Adverbien}

Der Grammatikalisierung liegen vier wichtige Mechanismen zugrunde, vgl. Heine, Kuteva (2002: 2) ${ }^{41}$ :

- desemantization bzw. „semantic bleaching“, i.e. „loss in meaning content“;

- extension bzw. „context generalization“, i.e. „use in new contexts“;

- decategorialization, i.e. „loss in morphosyntactic properties characteristic of lexical or other less grammaticalized forms";

- erosion bzw. „phonetic reduction“, i.e. „loss in phonetic substance“.

Die Intensivierer, die im Rahmen dieser Arbeit untersucht werden, demonstrieren keinen Kategorienwechsel, zeigen jedoch andere Grammatikalisierungsmerkmale, wie z.B. Ausbleichen des ursprünglichen semantischen Gehalts bzw. semantic bleaching, das Erwerben einer evaluativen Bedeutung bzw. subjectification, das Auftreten der intensivierenden Bedeutung durch pragmatic inferencing und semantic reanalysis sowie die Erweiterung der Skopuskonstituente. Auf diese Aspekte werde ich im weiteren ausführlich eingehen.

Für die Analyse eines Lexems in Bezug auf Grammatikalisierung wurde in der letzten Dekade des 20. Jh. eine Reihe von Kriterien entwickelt. In diesem Zusammenhang erscheinen die Arbeiten von Lehmann (1982/2002), Traugott (1988) und Sweetser (1988) als besonders anregend.

Lehmann (2002) hebt drei Parameter hervor, die jeweils paradigmatische und syntagmatische Aspekte berücksichtigen, vgl. Tabelle 1. Ich werde die angeführten Parameter in Bezug auf die untersuchten Grad-Adjektive und -Adverbien interpretieren. Eine ähnliche Analyse wurde bereits von Peters (1993: 277-280) hinsichtlich der englischen boosters durchgeführt und liegt deshalb der Darstellung meines Materials zugrunde.

\footnotetext{
${ }^{41}$ Andere Mechanismen, die mit dem früheren Stadium der Grammatikalisierung verbunden sind, wie pragmatic enrichment, invited inferencing und semantic reanalysis, werden unten in den Kapitelabschnitten 3.6.3.7 und 3.6.3.9 besprochen.
} 
Tabelle 1. The parameters of grammaticalization (Lehmann 2002: 110)

$\begin{array}{lll} & \text { paradigmatic } & \text { syntagmatic } \\ \text { weight } & \text { integrity } & \text { structural scope } \\ \text { cohesion } & \text { paradigmaticity } & \text { bondedness } \\ \text { variability } & \text { paradigmatic variabi- } & \text { syntagmatic variability } \\ & \text { lity }\end{array}$

Unter Integrität (integrity) versteht Lehmann (2009: 9.4.) den konstitutionellen Umfang eines Sprachzeichens, „die Komplexität seines Significans und seines Significatums“. Bei Grammatikalisierung nimmt Integrität „durch einander entsprechende phonologische und semantische Prozesse“ (Lehmann 2009: 9.4.) zu. Darüber hinaus kommt es zu einer phonologischen „Abschleifung“ und Desemantisierung bzw. Bedeutungsentleerung. Die deutschen Grad-Adjektive und -Adverbien erfahren keine phonologische, jedoch semantische „Erosion“ (Lehmann 2009: 9.4.). Die morphologische sowie phonologische Form wird beibehalten. Zugleich geht der Zusammenhang mit der ursprünglichen lexikalischen Bedeutung verloren, sodass ausschließlich die Bedeutung der Intensität zum Ausdruck gebracht wird.

Die Paradigmatizität (paradigmaticity) eines Sprachzeichens ist „der Grad, zu dem es in ein Paradigma eingegliedert ist, und die formale und funktionelle Homogenität und Geschlossenheit dieses Paradigmas" (Lehmann 2009: 9.4.). Im Prozess der Grammatikalisierung nimmt sie zu. Peters zufolge (1993: 278), gehört Adjektiv- und Adverbiallexik, die als Quelle für Grad-Adjektive und -Adverbien dient, zu einer relativ großen Klasse, was sich in dem geringen Grad an deren Paradigmatizität widerspiegelt. Durch die Entwicklung zu boosters erfolgt keine große Zunahme auf diesem Parameter, denn die Lexeme bilden immer noch „kein geschlossenes und stark strukturiertes Paradigma" (Peters 1993: 278). Lehmanns Klassifikation setzt jedoch die Möglichkeit voraus, zwei Elemente auf einer Skala zu vergleichen. In diesem Zusammenhang demonstrieren, wie Peters (1993: 278) überzeugend darstellt, Grad-Adjektive und -Adverbien als Gesamtheit im Vergleich zu ihren „noch mit voller lexikalischer Bedeutung ausgestatteten“ Quellen eine in höherem Maße grammatikalisierte Gruppe. „Die Paradigmatizität der Gradkategorien muß wegen der größeren funktionalen Festlegung der Klasse bzw. ihrer Elemente höher angesetzt werden als diejenige von adverbialen Kategorien wie "Quantität", „Art und Weise“ oder „räumliche Relation“" (Peters 1993: 278).

Die Wählbarkeit bzw. paradigmatische Variabilität (variability) bezeichnet den „Grad, zu dem es gegenüber anderen Mitgliedern des Paradigmas frei wählbar, im Kontext gegen sie austauschbar und schließlich überhaupt weglaßbar ist. Sie nimmt bei Grammatikalisierung ab in einem Prozeß, den man Obligatorisierung nennen kann" (Lehmann 2009: 9.4.). Peters (1993: 328) verweist darauf, dass „,innerhalb der Kategorie „boosters“ (abgesehen von registerbedingten Faktoren) keine Wahlmöglichkeit [...] besteht, wohl aber innerhalb des Gesamtsystems der Gradadverbien".

Mit Skopus (structural scopus) bezeichnet Lehmann (2009: 9.4.) die „syntaktische Ebene [...], auf der das Zeichen operiert. Der Skopus nimmt bei Grammatikalisierung ab in einem Prozeß, der Kondensierung genannt worden ist". Allerdings lässt sich hinsichtlich der Grad-Adjektive und -Adverbien keine Kondensierung 
feststellen. Im Gegenteil, man kann von der Erweiterung der Skopuskonstituente reden, weil Intensivierer über Funktionsverbgefüge Skopus haben können, vgl.:

(68) Er ist in arge Schwierigkeiten geraten.

(69) Er ist arg in Schwierigkeiten geraten.

Die syntagmatische Variabilität bzw. Stellungsfreiheit (syntagmatic variability) eines Sprachzeichens stellt die „Umstellbarkeit im Syntagma“ dar und nimmt bei Grammatikalisierung in dem Prozess der Fixierung (Lehmann 2009: 9.4.) ab. Für Intensivierer ist dieser Parameter irrelevant, weil die Lexeme auf allen Stadien ihrer Entwicklung vor dem Bezugswort bzw. der Skopuskonstituente stehen, worauf auch Peters (1993: 278) hinweist.

Die syntagmatische Kohäsion bzw. Fügungsenge (syntagmatic cohesion, bondedness) stellt morphologische Gebundenheit des sprachlichen Zeichens dar, d.h. „die Intimität der Verbindung, die es mit seiner Kokonstituente, seinem späteren Träger, eingeht" (Lehmann 2009: 9.4.). Sie nimmt durch Grammatikalisierung zu; der zugehörige Prozess heißt Koaleszenz. Dieser Prozess wurde im Material der von mir behandelten Lexeme nicht beobachtet, doch sieht man ihn am Beispiel des Adverbs bitter, welches neben der Entwicklung der Gradbedeutung (z.B. bitter nötig haben) auch eine morphologische Verschmelzung erfahren hat, vgl. bitterschwer.

Aus dem oben Gesagten lässt sich der Schluss ziehen, dass die deutschen GradAdjektive und -Adverbien hauptsächlich an den paradigmatischen Parametern gemessen werden können, wobei sich eine eher sehr kleine Verbindung zu den syntagmatischen Parametern feststellen lässt.

\subsubsection{Zum Grad der Grammatikalisierung: semantic bleaching}

Das Problem des Grammatikalisierungsgrads bei Grad-Adjektiven und -Adverbien wurde zum erstenmal in Bolinger (1972) angesprochen. Er demonstriert eine Unterteilung von Gradadverbien in „more or less grammaticalized“ (1972: 22). Bolinger (1972: 22-23, 59-60, 174-175) unterscheidet zwischen zwei Kategorien bzw. (relatively) grammatisized (z.B. so, much, too) und (relatively) ungrammatisized (z.B. terribly, absurdly, really). Darüber hinaus wird gemäß seiner Begrifflichkeit die für meine Fragestellung relevante Kategorie boosters als weniger grammatikalisiert bezeichnet. Jedoch gibt Bolinger keine präzise Definition der festgelegten Kategorien und bietet somit keine Parameter, die für die Klassifizierung der Lexeme verwendet werden konnten.

Die von Lehmann (1982/2002) vorgeschlagene Klassifizierung liefert einen Leitfaden für die Untersuchung des Grammatikalisierungsgrades eines Lexems. In Bezug auf Grad-Adjektive und -Adverbien lässt sich schließen, dass semantische Abnutzung bzw. semantic bleaching eines der wichtigsten Kriterien für eine solche Analyse darstellt. Laut Eckardt (2006: 44) findet dieses Phänomen die erste Erwähnung augenscheinlich in der Arbeit von Georg von der Gabelentz (1891: 238):

„Was erst neu und selten war, wird dann alltäglich, und damit verliert es an Kraft, verblasst, rückt schliesslich wohl gar in die Reihe jener abstracten Bestandtheile der Rede, die es hatte verbessernd und verstärkend ergänzen sollen“. 
Hofmann (1930: 104ff.) vergleicht Desemantisierung mit dem Abschleifen einer „Scheidemünze“, wenn „die sogenannten Steigerungsadverbia allmählich ihren Eigenwert und ursprünglichen Sinn verlieren und sich im Gebrauch abschleifen“.

In der Literatur wird semantische Abnutzung durch zahlreiche Begriffe ${ }^{42}$ bezeichnet, vgl. bleaching (Heine, Claudi, Hünnemeyer 1991a: 40), semantic depletion (Weinreich 1963: 180f., Lehmann 1982: 127), subduction (Guillaume 1964), desemanticization (Lehmann 1982/2002: 144/112; Heine, Reh 1984: 36ff.), semantic weakening (Guimier 1985: 158), generalization or weakening of semantic content (Bybee, Pagliuca 1985: 59-60), fleshing out of meaning (Sweetser 1988: 392), dematerialization (Hewson 1997: 343), Abschwächung (Sperber 1914: 31), Abschleifung (Leuschner 1918: 47, Hofmann 1930: 104), Abschwächung und Verdunkelung (Biener 1940: 167) Desemantisierung (Nübling 2010: 227), semantische Ausbleichung (Diewald 1997: 227), semantisches Verbleichen (Portz 2009: 151). All diese Begriffe beziehen sich auf den „Verlust an Bedeutung bzw. an semantischem Inhalt" (Diewald 1997: 227).

Das Kriterium bleaching liegt mehreren Klassifizierungen von Gradadverbien zugrunde, die den Grammatikalisierungsgrad von betroffenen Lexemen nach diesem Parameter messen. Eine Unterteilung von Gradadjektiven in Bezug auf deren semantische Abnutzung findet sich in Vermeire (1979: 46ff.). Er teilt Intensivierer in drei Gruppen ein, die jedoch keine festen Grenzen haben, sodass „one has to be content with some arbitrary limits of practical utility" (Vermeire 1979: 45): primary intensifiers, secondary intensifiers und tertiary intensifiers. Tertiary intensifiers (z.B. furiously concentrate, love passionately) werden auch „manner adverbs with inherent intensity feature" (Vermeire 1979: 47) genannt. Sie weisen neben der lexikalischen Bedeutung eine intensivierende Funktion auf. Vermeire verweist darauf, dass diese Lexeme in Bolingers Kategorie (relatively) ungrammatisized passen würden. Sie stellen eine relativ große Klasse dar und bieten eine Quelle für neue Intensivierer. Es erscheint jedoch als problematisch, diese Klasse abzugrenzen, weil, wie Bolinger (1972: 23) feststellt, „virtually any adverb modifying an adjective tends to have or to develop an intensifying meaning".

Secondary intensifiers (z.B. terribly, absolutely, utterly, greatly) kombinieren ebenso die intensivierende Funktion mit der semantischen Komponente. Jedoch hat die Intensivierung die führende Rolle übernommen und die Ausgangsbedeutung in den Hintergrund geschoben. Diese Gruppe entspricht ebenfalls Bolingers Kategorie (relatively) ungrammatisized Intensivierer, jedoch mit einem gewissen Unterschied. Vermeire (1979: 50) merkt an, dass ein secondary intensifier im Vergleich zu tertiary intensifier einer stärkeren semantischen Abnutzung unterliegen kann, wie es in awfully lucky und perfectly ridiculous der Fall ist. Dadurch überschreitet es "the limits of its logical applicability and assumes, by catachresis, the function of a general intensifier", sodass neben der Verwendung terribly bad die Kollokation terribly good möglich wird (Peltola 1969: 38) ${ }^{43}$.

\footnotetext{
${ }^{42}$ Die folgende Liste basiert auf den Angaben von Heine, Claudi, Hünnemeyer (1991b: 155 -156), wurde jedoch wesentlich überarbeitet bzw. ergänzt.

43 zit. durch Vermeire 1979: 50.
} 
Primary intensifiers (z.B. very, much, quite, somewhat) weisen einen kompletten Verlust der lexikalischen Bedeutung auf und bringen lediglich den Grad der Intensivierung zum Ausdruck. Das ist eine stabile Klasse, im Gegensatz zu tertiary intensifiers, die eine Modeerscheinung darstellen können und stets dem Prozess von renewal unterliegen, so Vermeire (1979: 48).

Secondary intensifieres stellen damit eine Zwischenstufe zwischen tertiary und primary intensifiers und können durch den Verlust des Zusammenhangs mit der Ausgangsbedeutung in die obere Klasse von primary intensifiers gelangen.

Wie die Untersuchung der deutschen Gradadverbien ergeben hat, haben die meisten Lexeme die letzte Stufe erreicht bzw. den Verlust des Bezugs auf die Ausgangsbedeutung erfahren, z.B. arg gut, furchtbar interessant und verdammt klug. Darüber hinaus kann man davon ausgehen, dass diese Lexeme der Definition von primary intensifiers entsprechen. Es ist jedoch unklar, welche weiteren Bedingungen nach Vermeire ein Intensivierer erfüllen soll, um zu dieser Klasse gezählt werden zu können, z.B. kompletter Verlust der ursprünglichen Bedeutung, wie es bei sehr ${ }^{44}$ der Fall ist.

Ghesquière (2010: 281-282) unterscheidet lediglich zwischen „bleached“ und „non-bleached“ Intensivierern. Bleached intensifiers (z.B. very in a very nice man) "have lost most of their original meaning", während non-bleached intensifiers (z.B. lovely in lovely long legs) "have not bleached to the same degree as true intensifiers and still have an inherent evaluative meaning".

Die in meiner Arbeit untersuchten Grad-Adjektive und -Adverbien demonstrieren den Verlust des Bezugs auf die ursprüngliche Bedeutung und gehören damit zu bleached intensifiers in der Ghesquièreschen Klassifizierung. Es ist festzuhalten, dass die meisten deutschen Grad-Adjektive und -Adverbien, im Gegensatz zu dem Gradadverb sehr, den Zusammenhang mit der Ausgangsbedeutung nicht vollständig verloren haben. Sie können vorwiegend sowohl in der intensivierenden als auch in nicht-intensivierender Bedeutung benutzt werden, vgl. die Belege (70) (73).

(70) Ich kann mir, auch aufgrund meiner eigenen Erfahrungen als Arzt, furchtbare Situationen vorstellen, in denen ich mir beides für mich selber wünschte oder auch bereit wäre, es für meine Frau, selbst für meine Kinder zu tun. (DWDS: Die Zeit, 17.07.2014)

(70') 'Furcht erregende Situationen'

(71) Im Raabtal wächst der Mais fürchterlich gut. (C.II: K97/APR.31476 Kleine Ztg., 26.04.1997)

(71') 'sehr gut'

(72) Was sie nicht wußte, war, daß der Schriftsteller Thackeray, [...] dem sie ihren Roman Jane Eyre widmete, eine wahnsinnige Frau hatte. (DWDS: Schwanitz, D.B., Frankfurt a.M.: Eichborn 1999, S. 246)

(72') 'geistig gestörte Frau'

\footnotetext{
${ }^{44}$ Das Gradadverb sehr entwickelte sich aus den in allen westgermanischen Dialekten bezeugten Entsprechungen, deren Ursprungsbedeutung „schmerzhaft“ war, so Leuschner (1918: 26-32) und Biener (1940: 195).
} 
(73) Bei den Jobs in Floridas Epcot Center begeisterte die Teilnehmer „die wahnsinnig nette internationale Atmosphäre in den Camps, wo sie untergebracht sind“". (DWDS: Die Zeit 05.01.1996)

(73') 'sehr nette'

\subsubsection{Semantisch-pragmatische Veränderungen im Rahmen der Gramma- tikalisierung: Traugotts tendencies}

Traugott (1982, 1988, 1989, 1990, 1995), Sweetser (1988) und Traugott, König (1991) wenden ihre Aufmerksamkeit semantisch-pragmatischen Veränderungen im Rahmen der Grammatikalisierung zu und konzentrieren sich damit auf das Phänomen der Bedeutungsentleerung sowie „[die] Funktion von Elementen im Text und in der Sprechsituation" (Peters 1993: 280). Traugott (1988: 407) vertritt die Ansicht, dass Bedeutungsentleerung erst in den späteren Stadien von Grammatikalisierung in Erscheinung tritt, während die frühere Etappe mit anderen semantisch-pragmatischen Prozessen wie pragmatic enrichment bzw. strengthening verbunden ist.

In diesem Zusammenhang erwähnt sie drei funktional-semantische Komponenten, die in dem linguistischen System zu unterscheiden sind: propositionale, textuelle und expressive Komponente. „The propositional component [...] involves the resources of the language for making it possible to talk about something. [...] The textual component has to do with the resources available for creating a cohesive discourse. [...] The expressive component [...] bears on the resources a language has for expressing personal attitudes to what is being talked about, to the text itself, and to others in the speech situation" (Traugott 1982: 248). Traugott (1982: 248) betont die entscheidende Rolle dieser Komponenten bei Grammatikalisierungsprozessen und vertritt die Ansicht, dass ein zu grammatikalisierendes Lexem den Bedeutungswandel entweder in Bezug auf eine Komponente oder bei dem Übergang von einer Komponente zu einer anderen erfährt. Die von Traugott (1988: 408-410) postulierten „tendencies“ demonstrieren diesen Vorgang von der propositionalen auf die expressive Ebene, vgl.:

\section{(74) Tendency I}

Meanings based in the external described situation > meanings based in the internal (evaluative / perceptual / cognitive) situation

\section{Tendency II}

Meanings based in the described situation > meanings based in the metalinguistic situation

\section{Tendency III}

Meanings tend to become increasingly based in the speaker's subjective belief-state / attitude toward the situation.

Die erste Tendenz ist laut Traugott (1990: 499-500) mit dem Auftreten der Phänomene Pejoration und Melioration sowie mit metaphorischem Bedeutungswandel verbunden. Durch die Metapher geht die auf externe Situationen bezogene Bedeutung in den Bereich persönlicher Einstellungen bzw. die evaluative Sphäre über. Als Beispiel führt Traugott den im Altenglischen stattgefundenen Übergang der 
räumlichen Präposition æfter in die zeitliche Präposition ein, der mittels der Metapher SPACE IS TIME erfolgt war (Traugott 1988: 409). Damit drückt Traugott aus, dass die Metapher ein wichtiger Bestandteil des früheren Stadiums eines Grammatikalisierungsprozesses ist, vgl. auch Hopper, Traugott (2003: 85) und Bybee, Pagliuca (1985: 75).

Die zweite semantisch-pragmatische Tendenz von Traugott bezieht sich auf die Entstehung von Satzverknüpfern sowie die Bedeutungsübertragung auf der metalinguistischen Ebene, wie es z. B in æfter der Fall ist, als das Lexem sich von der zeitlichen Präposition zu einem „temporal connective“ (Traugott 1988: 409) entwickelt hat.

Traugott (1990: 499) unterstreicht, dass die erste Tendenz in die dritte übergehen kann, i.e. ohne dabei die zweite in Anspruch zu nehmen. Dies lässt sich bei den in meiner Arbeit untersuchten deutschen Grad-Adjektiven und -Adverbien feststellen, denn sie üben keine textverknüpfenden Funktionen aus.

Die Tendenz III gilt für die in meiner Arbeit behandelten Grad-Adjektive und Adverbien, die anschließend endgültig den Zusammenhang mit der primären Bedeutung verlieren und Einstellungsausdrücke darstellen. Als subjektiv verwendete sprachliche Einheiten, „they provide ways for speakers to guide the hearer in interpretation" (Traugott 1995: 45). Darüber hinaus bringen Intensivierer neben dem Grad die persönliche Einstellung des Sprechers bzw. Schreibers zu einem Sachverhalt zum Ausdruck, vgl.:

(75) „Es war irrsinnig windig!“ Deshalb auch die vielen Doppelfehler. (C.II: P92/JUL.20631 Die Presse, 13.07.1992)

(76) „Mit dem Leopold arbeite ich nämlich irrsinnig gern zusammen, bei ihm habe ich stets das Gefühl, daß ich in guten Händen bin!" (C.II: X96/MAR.03619 Oberösterreichische Nachr., 28.03.1996)

In (75) und (76) tritt irsinnig als Intensivierer auf, ohne die primäre bzw. mit dem geistigen Zustand eines Menschen verbundene Bedeutung anklingen zu lassen. Das Lexem demonstriert lediglich den Ausprägungsgrad der Sachverhalte und den einstellungsbezogenen Gebrauch. Die von mir vorgenommene korpusbasierte Analyse ließ außerdem erkennen, dass irrsinnig in der intensivierenden Funktion sich zunächst auf negative Sachverhalte bezieht und anschließend mit positiven Kookkurrenzpartnern kollokieren kann. Dieses Phänomen signalisiert nicht nur den Prozess der Desemantisierung, sondern auch die Erweiterung des einstellungsbezogenen Ausdruckspotenzials des Lexems.

\subsubsection{Semantisch-pragmatische Veränderungen im Rahmen der Gramma- tikalisierung: subjectification}

Die in (74) dargestellten drei „tendencies“ veranschaulichen den Übergang einer propositionalen Bedeutung in den Bereich subjektiver Evaluationen bzw. von „less personal to more personal“ (Traugott 1982: 253). Traugott verbindet diesen Übergang mit dem in Seiler (1983: 85) angesprochenen Phänomen „self-orientation“ bzw. subjectification, den sie als "pivotal“ (Traugott 1990: 500) und „major factor in semantic change" (Traugott 1990: 501) bezeichnet und folgendermaßen definiert (Traugott 1995: 31): 
„a pragmatic-semantic process whereby 'meanings become increasingly based in the speaker's subjective belief-state / attitude toward the proposition', in other words, toward what the speaker is talking about".

Subjectification wird in Traugott (1995: 47) als ein irreversibler ${ }^{45}$ Prozess im Rahmen eines Grammatikalisierungsvorgangs dargestellt und von Kranich (2010: 101) als „a subtype of grammaticalization“ bezeichnet.

Traugott (1995: 45) weist darauf hin, dass dieser Prozess in früheren Entwicklungsstadien der Grammatikalisierung im Rahmen der Tendenz I beginnt und sich im Rahmen der dritten Tendenz fortsetzt, indem er die betroffenen Lexeme graduell „more subjective“ macht, vgl.:

„subjectification in grammaticalization is, broadly speaking, the development of a grammatically identifiable expression of speaker belief or speaker attitude to what is said. It is a gradient phenomenon, whereby forms and constructions that at first express primarily concrete, lexical, and objective meanings come through repeated use in local syntactic contexts to serve increasingly abstract, pragmatic, interpersonal, and speaker-based functions" (Traugott 1995: 32).

Darüber hinaus tritt subjectification auf der Stufe von „primary grammaticalization“ auf, denn es „requires prior strengthening of pragmatic inferences that arise in very specific linguistics contexts prior to their semanticization and development into grammatical structures" (Traugott 2010: 41).

Die Rolle von subjectification bei der Herausbildung von englischen Grad-Adjektiven und -Adverbien wurde in Peters (1993), Paradis (2000) und Vandewinkel, Davidse (2008: 262) hervorgehoben. Peters zufolge (1993: 328), „verbleibt“ bei einem herausgebildeten Intensivierer „lediglich die „subjektive“ Komponente des ursprünglichen Inhalts".

Subjectification ist am Material aller in meiner Arbeit behandelten Intensivierer feststellbar. Aus den Verwendungen (75) - (76) geht hervor, dass die ursprüngliche propositionale Bedeutung, die den seelisch-geistigen Zustand einer Person charakterisiert, einen Übergang zu der subjektiven Bezeichnung eines Sachverhalts erfährt. Die Gradbedeutung bringt lediglich die Bedeutung des hohen Grades sowie die persönliche Einstellung zum Ausdruck.

\subsubsection{Bedeutungswandel im Rahmen der Grammatikalisierung: Metapher}

Mehrere Studien heben die Rolle der Metapher bei Grammatikalisierungsprozessen hervor, z.B. Claudi, Heine (1986), Sweetser (1988), Heine, Claudi, Hünnemeyer (1991a: 45ff.), Lichtenberk (1991), Bybee, Perkins, Pagliuca (1994: 283-285). Darüber hinaus werden metaphorische Übertragungen als ein der Grammatikalisierung grundlegender Mechanismus angesehen, vgl. Bybee, Pagliuca (1985: 75):

\footnotetext{
45 Traugott unterteilt Grammatikalisierung in zwei Phasen: „primary grammaticalization“ bzw. "the shift from lexical/constructional to grammatical“ und „secondary grammaticalization” bzw. "the development of already grammatical material into more grammatical elements" (Traugott 2010: 40-41). Nach Kranich (2010: 112) ist die „primary grammaticalization“ mit subjectification verbunden, während für „secondary grammaticalization“ ein Gegenprozess bzw. objectification ausschlaggebend ist. Unter objectification ist ,the loss of subjective meanings and the acquisition of more objective meanings" zu verstehen. Die Entwicklung von Grad-Adjektiven und -Adverbien ist mit „primary grammaticalization“ und daher mit subjectification verbunden.
} 
„grammatical evolution is driven by [...] metaphorical extensions that lead to the increased use of certain items. The metaphorical extensions are cognitively based, and are similar across languages".

Die Rolle von der Metapher bei Grammatikalisierung besteht demnach darin, die Bedeutung aus einem konkreteren Bereich in einen abstrakteren zu übertragen (siehe Bybee, Pagliuca 1985: 72). Dabei erfolgt der semantische Wandel „from concepts which are close to human experience to those that are more difficult to define in terms of human cognition" (Claudi, Heine 1986: 327-328). Bybee, Pagliuca (1985: 72) vertreten die Ansicht, dass der durch die Metapher initiierte Desemantisierungsprozess „is a prerequisite to grammaticalization because grammatical functions in themselves are necessarily abstract".

Die Wirkung der Metapher bei der Grammatikalisierung lässt sich am Beispiel zahlreicher Muster demonstrieren, vgl. z.B. die Entwicklung von Körperteilen in räumliche Konzepte und von räumlichen Konzepten in zeitliche, z.B. back 'body part' -> 'behind' nach dem metaphorischen Muster SPACE IS AN OBJECT und ferner durch die Metaphorisierung in das zeitliche Konzept 'in the past' nach dem Muster SPACE IS TIME (Heine, Claudi, Hünnemeyer 1991: 157-160). Die Übertragung eines räumlichen Konzepts in ein zeitliches Konzept lässt sich außerdem in der Entwicklung von go als Bewegungswerb zum Futurauxiliar veranschaulichen (Traugott 1988: 408).

Nach Traugott (1988: 410) ist die Rolle von metaphorischen Prozessen bei Grammatikalisierung zwar erheblich, scheint jedoch „to be more complex and to involve additional factors". In diesem Zusammenhang verbindet sie den Mechanismus von der Metapher mit semantic-pragmatic tendencies, welche das Auftreten von persönlichen Einstellungen bzw. subjectification sowie von textverknüpfenden Funktionen voraussetzten.

Die Wirkung der Metapher zu Beginn der Entwicklung von Gradbedeutung lässt sich am Material einiger der behandelten Grad-Adjektiven und -Adverbien feststellen und wird in (77) - (78) anhand des Adjektivs irrsinnig exemplarisch gezeigt, vgl.:

(77) Die Franzofen bezeichnen den Ingenieur Sauvage als den eigentlichen Erfinder der Schiffsfchraube, doch ift erwiefen, daß er fich lediglich mit dem Problem derfelben befchäftigte, fpäter irrfinnig wurd und 1857 im Parifer Armenhaufe ftarb. (DTA: Schweiger-Lerchenfeld, Amand von, 1900)

(78) Die Mutter, die noch immer nicht genug Atem finden konnte, fing in die vorgehaltene Hand mit einem irrsinnigen Ausdruck der Augen dumpf zu husten an. (C.II: HK3/A40.00003 Kafka: Die Verwandlung, Entstanden: 1912)

In (77) bezieht sich irrsinnig auf eine externe Situation, wobei das Lexem in der primären Bedeutung verwendet wird, die den geistig-seelischen Zustand einer Person bezeichnet. In (78) handelt es sich um eine metaphorische Übertragung des Sinnes. Als Basis für die semantische Verschiebung dient das Aussehen einer gesunden Person, welches mit dem Aussehen einer erkrankten Person verglichen wird. Die übertragene Bedeutung bezieht sich nicht mehr auf eine externe, sondern eine interne Situation. Der Sprecher bzw. Schreiber verleiht der Bedeutung durch das metaphorische Projizieren seine subjektive Wahrnehmung bzw. Bewertung, 
die das Resultat eines kognitiven Prozesses darstellt, sodass der Sinn des Lexems auf die expressive Ebene verschoben wird. Darüber hinaus wandelt sich die Beschreibung eines objektiven Sachverhalts in (77) zu der subjektiven Einstellung in (78).

Wie jedoch in Eckardt (2006: 55 sowie 2010: 66) zu Recht angemerkt wird, stellt die Metapher keine obligatorische Voraussetzung für die Grammatikalisierung dar, denn „we do find a considerable number of instances of grammaticalization that are not plausibly metaphoric". Ich schließe mich dieser Auffassung an, weil nicht alle von mir untersuchten Grad-Adjektive und -Adverbien ihre Bedeutung durch metaphorische Prozesse erworben haben. Anders ausgedrückt, hat die Metapher in dem Entwicklungsgang nicht bei der Herausbildung aller Grad-Adverbien und Adjektive mitgewirkt, z.B. bei den Gradadverbien des Schreckens.

\subsubsection{Semantisch-pragmatische Veränderungen im Rahmen der Gramma- tikalisierung: Invited Inferencing Theory of Semantic Change und Me- tonymie}

Traugott richtet ihre Aufmerksamkeit u.a. auf Prozesse, die bei dem Übergang einer Bedeutung von einer semantisch-pragmatischen Tendenz zu der anderen mitwirken. Sie führt (Traugott 1988: 407) u.a. ins Feld, dass Desemantisierung bzw. bleaching erst in späteren Grammatikalisierungsstadien auftritt, während die Anfangsphase des Phänomens dagegen mit strengthening des semantischen Gehalts verbunden ist (vgl. auch Hopper, Traugott 2003: 81, Traugott, König 1991: 190ff.). Strengthening of informativeness (Traugott 1988: 411) berücksichtigt das Auftreten von konversationellen Inferenzen in bestimmten Kontexten und steht im Mittelpunkt der von Traugott, Dasher (2002: 34-40) entwickelten Theorie IITSC (Invited Inferencing Theory of Semantic Change). Diese Theorie befasst sich mit dem durch pragmatische Faktoren bedingten Bedeutungswandel und fokussiert auf die graduelle Entwicklung von „coded meanings“ from „utterance meanings“, i.e. Semantisierung von kontextabhängigen Interpretationen, oder, in der Terminologie von Traugott und Dasher (2002: 35) ausgedrückt, „,conventionalizing of pragmatic meanings and their reanalysis as semantic meanings" (vgl. auch Hopper, Traugott 2003: 82).

Der IITSC liegen bestimmte theoretische Ansätze zugrunde. Sie beruht vor allem auf dem von Grice (1975) geprägten Begriff conversational implicature bzw. auf der neo-Grice'schen Theorie der Implikaturen, zu der Horn (1984) und Levinson (2000) weiterführende Beiträge geleistet haben. Levinson (2000) unterscheidet drei Stufen des durch pragmatic inferencing erfolgten Bedeutungswandels:

- particularized conversational implicature (utterance-token meaning, PCI),

- generalized conversational implicature (utterance-type meaning, GCI),

- coded meaning. 
Laut seinem Schema handelt es sich zunächst um das Auftreten von partikularisierten ${ }^{46}$ Implikaturen, die unter spezifischen Umständen bzw. in einem spezifischen Kontext gelten. Diese Implikaturen können den Status generalisierter Implikaturen erreichen, i.e. Implikaturen, die typischerweise gezogen werden. Anschließend kann eine generalisierte Implikatur konventionalisiert werden und als ein fester Bedeutungsteil eines Lexems fungieren.

Diese Idee von Levinson wurde von Traugott, Dasher (2002) im Wesentlichen übernommen, jedoch terminologisch verändert. Zunächst ist zu bemerken, dass sie den Begriff conversational implicature durch invited inference ersetzt haben. Dieser Begriff wurde aus Geis, Zwicky (1971) übernommen, erfährt jedoch bei den Autoren eine weitere Auslegung (Traugott, Dasher 2002: 5). Invited inferences werden in Geis, Zwicky (1971: 565) als „a special class of „implications“ und „clearly distinct from „conversational implicatures““ dargestellt. Diese Beschränkung wird jedoch in Traugott, Dasher (2002) aufgehoben. Außerdem heben die Autoren eine aktive Beziehung zwischen den Interaktionspartnern hervor: „the speaker / writer (SP/W) evokes implicatures and invites the addressee / reader (AD/R) to infer them" (Traugott, Dasher 2002: 5). Darüber hinaus werden Levinsons PCIs als invited inferences (IINs) und GCIs als generalized invited inferences (GIINs) bezeichnet.

Die von Traugott und Dasher ausgearbeitete Theorie IITSC besagt, dass konversationelle Implikaturen bzw. invited inferences durch einen graduellen Prozess zu einem festen Bedeutungsteil eines Lexems werden können. Sie setzt folgende drei Entwicklungsschritte voraus (Traugott, Dasher 2002: 38), vgl. das Bild 1:

utterance-type meaning (conventionalizing of IINs as GIINs)

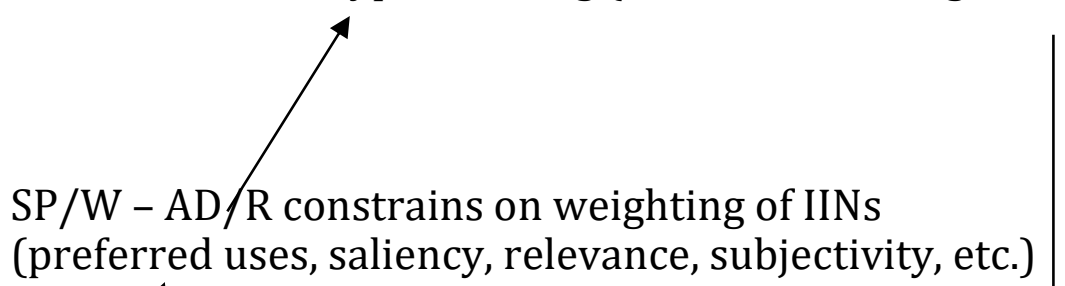

utterance-token meaning:

SP/W exploits IINs innovatively

in associative stream of speech

Stage I coded meaning

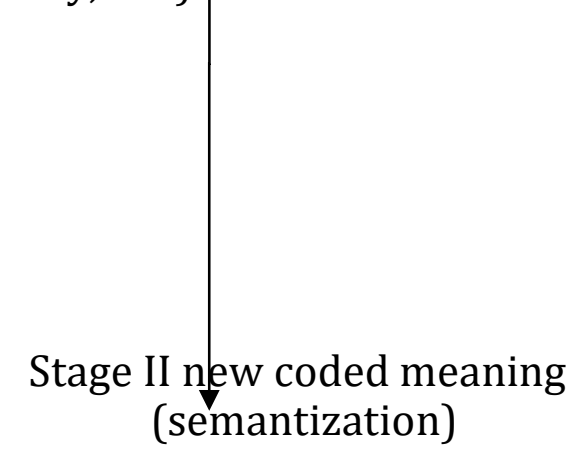

Bild 1. Das Modell Invited Inferencing Theory of Semantic Change (IITSC)

Aus dem angeführten Schema lässt sich schließen, dass eine kodierte bzw. feste Bedeutung eines Lexems als Ausgangspunkt für die Entstehung einer konversationellen Implikatur bzw. invited inference (IIN) in einem spezifischen Kontext gilt.

\footnotetext{
${ }^{46}$ „An implicature $i$ from utterance $U$ is particularized iff $U$ implicates $i$ only by virtue of specific contextual assumptions that would not invariably or even normally obtain“ (Levinson 2000: 16).
} 
Diese als eine Neuerung eingeführte konversationelle Implikatur kann durch stetige Wiederholung konventionalisiert werden und den Status von generalized invited inference (GIIN) erwerben. Wie es Eckardt (2006: 61-62) mit Recht anmerkt, „the item in question, from a certain point on, tends to be used more and more in sentences where the pragmatic inference is more salient, more relevant, much more the real information contribution to discourse than the literal meaning of the sentence". Anschließend unterliegt GIIN dem Prozess der Semantisierung und wird zu einer weiteren Bedeutung des Lexems. Darüber hinaus, wie Diewald (1999: 42) es ausdrückt, sind „[n] eue Bedeutungen [...] also gar nicht völlig neu, überraschend oder originell: sie haben lange Zeit als konversationelle Implikaturen bestanden".

Traugott, Dasher (2002: 19) verbinden das Auftreten von invited inferences bzw. „rich interpretations" mit der Anwendung der zweiten Grice'schen Konversationsmaxime der Quantität (Grice 1975: 45): „[d]o not make your contribution more informative than is required“, "supermaxim" der Qualität ,[ $\mathrm{t}$ ]ry to make your contribution one that is true" (Grice 1975: 46), Levinsons Principle of Informativeness: „[r]ead as much into an utterance as is consistent with what you know about the world“ (Levinson 1983: 146-147) bzw. „read into an utterance more information than it actually contains" (Levinson 1983: 146) sowie Horns R-Heuristic (1984: 13): „[s]ay no more than you must“. Darüber hinaus bieten sie die folgende Umformulierung der Prinzipien: „[s]ay / write no more than you must, and mean more thereby“ (Traugott, Dasher 2002: 19), vgl. auch Hopper, Traugott (2003: 79). Traugott, Dasher (2002: 19) nehmen an, dass diese Prinzipien „evoke[] utterance meanings beyond what is said" und dadurch pragmatische Anreicherung bzw. Bedeutungswandel motivieren.

Traugott (1988) stellt fest, dass strengthening of informativeness keinen Platz in der heutigen Klassifizierung des Bedeutungswandels eingenommen hat. In diesem Zusammenhang schlägt sie vor, dieses Phänomen als Metonymie zu erklären: „I suggest that strengthening of informativeness is a type of metonymy" (Traugott 1988: 411, vgl. auch Traugott, König 1991: 210-211). Der Begriff der Metonymie wird hier also erweitert, insofern als, Traugott zufolge, die Metonymie nicht nur eine Beziehung zwischen zwei zu demselben konzeptuellen Bereich gehörenden Zeichen herstellt, wie es in der klassischen Theorie dargestellt wird, sondern zwischen einem Zeichen und dem außersprachlichen Kontext. Wie Diewald (1997: 54) mit Recht anmerkt, „verweist das sprachliche Zeichen also auf den außersprachlichen Kontext (genauer, auf einen bestimmten Aspekt des außersprachlichen Kontextes), dessen Teil es ist".

Als Begründung für diese Entscheidung schlagen Traugott, König (1991: 211) das dem Phänomen zugrunde liegende Assoziierungsprinzip vor, welches eine Äußerung mit daraus auftretenden Inferenzen verbindet: „We propose extending the notion of metonymy from traditional concrete and overt contexts to cognitive and covert contexts, specially the pragmatic contexts of conversational and conventional inference. The contiguity involved is based in the discourse world“. Ferner behaupten sie (Traugott, König 1991: 212): „Metonymic change involves specifying one meaning in terms of another that is present, even only covertly, in the con- 
text. [...] the metonymic change is from less to more informative, that is, in the direction of explicit coding of relevance and informativeness that earlier was only covertly implied; in other words, it is a case of pragmatic strengthening".

Eine ähnliche Entscheidung treffen Traugott, Dasher (2002: 29) in Bezug auf subjectification, indem sie das Phänomen als eine Unterklasse der Metonymie bzw. Synekdoche bezeichnen.

Paradis (2008: 318) schließt sich der dargestellten Sichtweise an, indem sie den Übergang der propositionalen adjektivischen und adverbialen Bedeutung in den Bereich des Grades durch „implication by means of construals of metonymization between senses“ erklärt. Sie vertritt die Ansicht (Paradis 2008: 331), dass „metonymization is a conventional way of manipulating senses in context".

Eckardt (2006) schlägt vor, pragmatic inferencing und subjectification getrennt von der Metonymie zu behandeln. Sie bezieht sich auf den Aufsatz von Kövecses, Radden (1998) sowie den von Nunberg (1995), denen zufolge Metonymie lediglich „factual links between entities" (Eckardt 2006: 64) als Basis in Anspruch nimmt. Das Auftreten einer metonymischen Verbindung zwischen Konzepten kann demzufolge nicht in „certain communicative situations“ (Eckardt 2006: 64) entstehen, was bei pragmatic inferencing und subjectification dagegen der Fall ist, denn die aus dem Kontext abgeleiteten Inferenzen verweisen auf neue Referenten, während klassische Metonymie lediglich bestimmte Teile eines ICMs highlighten kann. „Traditional examples of metonymy are commonly based on links between ontologically comparatively simple objects like humans, artifacts, events, or at most simple properties" bzw. „on links between independently existent concepts" (Eckardt 2006: 65-66).

\subsubsection{Zur Entstehung von Grad-Adjektiven und -Adverbien}

Das Modell IITSC liegt der von Paradis (2000) durchgeführten diachronen und synchronen Analyse von 10 englischen Grad-Adjektiven (absolute, complete, perfect, total, utter, awful, dreadful, horrible, terrible und extreme) zugrunde. Sie beschreibt den Übergang der Lexeme mit der ursprünglich propositionalen Bedeutung (z.B. an awful sight) in den Bereich des Grades (an awful mess) durch das Einsetzen von implication $^{47}$ (Paradis 2000: 238, 2008: 318). Paradis zufolge (2000: 237) wird der Bedeutungswandel dadurch begünstigt, dass Adjektive bzw. Adverbien die Eigenschaft gradability besitzen, i.e. ,are conzeptualized according to an extreme point on a scale“. „The negative superlative property that characterizes these adjectives is recruited for reinforcement" (Paradis 2000: 236). Dieser Teil der Bedeutung bleibt im Hintergrund, wenn ein Lexem in der propositionalen Bedeutung verwendet wird, wird jedoch "prominent" durch „context-induced associative reasoning" (Paradis 2000: 253), wenn das Lexem in der intensivierenden Bedeutung auftritt. Die Herausbildung der Gradbedeutung wird mit dem Verblassen des ursprünglichen Sinnes des Lexems abgeschlossen und mit pragmatic strengthening bzw. subjectification verbunden (Paradis 2000: 237).

\footnotetext{
47 Paradis verwendet den Begriff implication als Synonym zu Traugotts Begriff pragmatic inferencing (siehe Paradis 2000: 253).
} 
Das von Paradis $(2000,2008)$ dargestellte Schema der Herausbildung von GradAdjektiven und -Adverbien skizziert den Mechanismus der Entwicklung von Gradbedeutung und lässt sich auf das von mir untersuchte Material anwenden. Jedoch benötigt es einige Kommentare und Korrekturen.

Zunächst ist festzustellen, dass Paradis eine vergleichsweise vage Erklärung dafür bietet, wie ein Lexem eine intensivierende Bedeutung erwirbt. Sie nimmt an, dass das Auftreten der intensivierenden Bedeutung durch pragmatic inferencing erfolgt und verweist in diesem Zusammenhang auf die gradability der potentiellen Intensivierer. Es bleibt aber die Frage offen, unter welchen Umständen bzw. in welchen Kontexten der Bedeutungswandel stattfinden kann. Paradis (2000: 241; 2008: 335-336) merkt zutreffend an, dass ein Lexem eine intensivierende Interpretation in Verbindung mit graduierbaren Bezugswörtern erwirbt. Diese Voraussetzung scheint jedoch nicht ausreichend zu sein. Ghesquière, Davidse (2011) unternehmen den Versuch, dieses Problem zu lösen, indem sie ihre Aufmerksamkeit auf eine ausführliche Untersuchung von Kookkurrenzmöglichkeiten der Lexeme complete, whole und particular richten. Sie sind der Ansicht, dass „the different collocational histories of the individual adjectives are an integral part of the change. The initial shift takes place in collocational environments that overlap with those of the source uses" (Ghesquière, Davidse 2011: 275). Daraus lasst sich schließen, dass die Verwendungen, die eine durch pragmatic inferencing gewonnene und auf Intensität bezogene Interpretation erlauben, zugleich den Zusammenhang mit der ursprünglichen Bedeutung des Lexems zeigen. Darüber hinaus löse das Auftreten der Gradbedeutung die Ambiguität im Gebrauch aus.

Das Modell IITSC besagt, dass das Auftreten von invited inferences in spezifischen Kontexten erfolgt. Solche Kontexte werden in Evans, Wilkins (1998: 5) als bridging contexts und in Diewald (1999: 44, Diewald 2006: 4) als critical contexts bezeichnet ${ }^{48}$. Heine (2002: 84) weist darauf hin, dass bridging contexts nicht direkt zu der Entstehung einer neuen lexikalischen Bedeutung führen. Diese Rolle wird dagegen switch contexts (Heine 2002: 85) bzw. isolating contexts (Diewald 2006: 5) zugeschrieben.

Diewald (1999: 44) und Heine (2002: 85-86) bieten ein vierstufiges Szenario für die Entwicklung einer Bedeutung aus einer konversationellen Implikatur:

Stufe I bezieht sich auf den Gebrauch eines Lexems in der Ausgangsbedeutung. Auf der Stufe II tritt das Lexem in bridging contexts bzw. critical contexts auf, welche neben der primären Bedeutung eine Inferenz ableiten lassen. Auf der Stufe III findet sich das Lexem in switch contexts bzw. isolating contexts, welche die konventionalisierte Inferenz bzw. Zielbedeutung in den Vordergrund und die Ausgangs-

\footnotetext{
48 In Eckardt (2011) wird der Begriff precarious contexts eingeführt. Er ist mit bridging bzw. critical contexts verwandt, insofern er mit dem Auftreten von neuen Interpretationen verbunden ist, zeigt jedoch auch Unterschiede. Precarious contexts treten auf, wenn der Gebrauch "the limits of the conservative grammar system" überschritten hat bzw. die Verwendung eines Lexems in einem „risky way“ demonstriert. Die Verwendung in precarious contexts kann in Bezug auf die Ausgangsbedeutung kaum interpretiert werden und bekommt deshalb eine weitere Bedeutung. Bei bridging und critical contexts geht der Bezug zur Ausgangsbedeutung nicht verloren.
} 
bedeutung in den Hintergrund verschieben. Auf der Stufe IV wird die Zielbedeutung in Kontexten gebraucht, die den Bezug auf die Ausgangsbedeutung ausschließen.

Diewald (1999: 42) verbindet die Ambiguität aufweisenden critical contexts mit „funktionaler Überlastung bzw. extremer Undurchsichtigkeit“ der Verwendungen, demzufolge „ein Reanalyseprozeß dieser opaken Konstruktion in Gang kommt", vgl auch Timberlake (1977: 148), der die Mehrdeutigkeit des Gebrauchs als grundlegend für einen Reanalyseprozeß postuliert. Der durch pragmatic overload bedingte Reanalyseprozess wird in Eckardt (2006: 367-368) als semantic reanalysis bezeichnet und als "the heart of most instances of grammaticalization“ (Eckardt 2010: 56) dargestellt:

„The process of semantic reorganisation of a sentence whereby the salient overall conveyed information remains the same, but is composed in a different manner".

Semantic reanalysis stellt damit "redistribution of conceptual content" (Eckardt 2010: 60) dar, wobei ",an old message is restructured in a new way“ (Eckardt 2011: 35 ). Dieser Prozess wird als „an independent mode of semantic change, next to other, better described changes like metaphoric extension, metonymy, narrowing or broadening" verstanden (Eckardt 2011: 43). Ferner verweist Eckardt (2006: 372) darauf, dass der durch semantic reanalysis bedingte semantische Wandel durch drei Entwicklungsstadien abläuft:

„First, there is a PRE-stage, a phase of pragmatic enrichment, of phrasalization of a given construction, and conventionalization of implicatures. The development then reaches the turning point where reanalysis takes place. After reanalysis has taken place, there is a POST-stage where a new item, plus its new meaning, has been acquired and speakers gradually explore its new possibilities [...]".

Es ist hervorzuheben, dass die von Eckardt postulierte POST-Phase der Bedeutungsentwicklung in Timberlake (1977) sowie auch in Andersen (2001) actualization genannt wird. Actualization basiert auf ,innovation“ (Timberlake 1977: 168) bzw. „innovative usage“ (Andersen 2001: 234) und setzt voraus, dass die kontextuellen Beschränkungen, die für ein Lexem vor der Reanalyse ausschlaggebend waren, nach der Reanalyse behoben sind (Timberlake 1977: 156).

Das von Eckardt vorgeschlagene Konzept bietet eine zielführende Ergänzung zu den oben dargestellten Invited Inferencing Theory of Semantic Change von Traugott, Dasher (2002) sowie der Beschreibung des Bedeutungsablaufs von Diewald (1999, 2006) und Heine (2002). Die Rolle von semantic reanalysis wird in den der Grammatikalisierung gewidmeten Studien besonders hervorgehoben. Hopper, Traugott (2003: 39) werten folgendermaßen: „[r]eanalysis is the most important mechanism for grammaticalization". Eckardt (2006: 380) bietet dafür, wie das Einsetzen von semantic reanalysis erfolgt, diese Erklärung:

„The speaker wants to convey a certain piece of information with a given sentence. The information arises for the hearer, as the sum of literal content plus pragmatic inferences. If the contribution of the pragmatic inferences is 
particularly high ('costly'), the hearer will reanalyse some of the pragmatic content as semantic content".

Damit zielt Eckardt darauf ab, dass pragmatic enrichment und generalized invited inferences den semantischen Gehalt des Lexems so bereichern, dass anschließend pragmatic overload des Kontexts entsteht. Hörer bzw. Leser „feel inclined to redistribute the semantic labour and reconstruct former conventional implicatures as new parts of literal content" (Eckardt 2006: 380). Diesen Prozess führt Eckardt auf das Principle of Avoiding Pragmatic Overload ${ }^{49}$ zurück. Sie merkt an (Eckardt 2010: 73), dass dieses Prinzip dem Prozess von semantic reanalysis zugrunde liegen kann.

Der von Diewald (1999) sowie Heine (2002) vorgeschlagene Ablauf der Bedeutungsentwicklung sowie der von Eckardt (2006) beschriebene Prozess von semantic reanalysis lässt sich im Material aller von mir untersuchten Grad-Adjektive und -Adverbien feststellen. Die Korpusanalyse der behandelten Lexeme legt nahe, dass die Herausbildung der Gradbedeutung in die folgenden Etappen unterteilt werden kann:

Etappe I: Verwendung eines Adjektivs bzw. Adverbs in der Ausgangsbedeutung.

Etappe II: Bereicherung bzw. pragmatic enrichment und semantic strengthening des ursprünglichen semantischen Gehalts durch das Auftreten der intensivierenden Bedeutung infolge pragmatic inferencing. Das Lexem wird in bridging contexts gebraucht, welche mehrere Interpretationen erlauben.

Etappe III: Die Mehrdeutigkeit des Gebrauchs eines Lexems hat pragmatic overload sowie semantic reanalysis zur Folge. Die Gradbedeutung wird konventionalisiert und in switch contexts verwendet. Das Lexem findet sich in Kombination mit negativ konnotierten Bezugswörtern, sodass der Bezug auf die Ausgangsbedeutung nicht beseitigt ist.

Etappe IV: Die konventionalisierte, einstellungsbezogene Bedeutung unterliegt dem Prozess der Semantisierung und actualization, wird in positiv konnotierten Wortverbindungen gebraucht und verliert den Zusammenhang mit der Ausgangsbedeutung.

\subsubsection{Zur Entstehung der Gradbedeutung bei fürchterlich}

Die in 3.6.3.8. dargestellten Entwicklungsschritte werden nun am Beispiel des Lexems fürchterlich veranschaulicht.

\subsection{Lexikographische Angaben}

Nach den Angaben von Kluge (s.v.) wurde die Form fürchterlich aus dem Substantiv Furcht abgeleitet. DWB verweist jedoch auf die Ableitung des Lexems aus dem Verb

\footnotetext{
${ }^{49}$ Eckardt verweist auf syntactic reanalysis bzw. transparency principle von Lightfoot (1979), die als „classical precursor" (Eckardt 2006: 383) für semantic reanalysis bzw. principle of avoiding pragmatic overload gelten und als "semantic counterpart of Lightfoot's principle" (Eckardt 2010: 74) verstanden werden können.
} 
fürchten. Dabei hat das Lexem keinen „direkten Zusammenhang mit ahd. for(a)htlîch, mhd. vorht(e)lich" (DWB, s.v.), vgl. auch Pfeifer s.v.. Obwohl Kluge keine Informationen zu der Entstehungszeit des Lexems liefert, führen Pfeifer und die Beleglage in DWB das Auftreten des Worts auf die erste Hälfte des 17. Jh. bzw. auf das späte Frühneuhochdeutsche zurück.

1. fürchterlich in FWB (s.v.) „schrecklich; furchtgebietend“; „gräßlich, abscheulich“50

2. fürchterlich in DWB (s.v.)

2.1 „angst, grauen erregend, beängstigend“

2.2 „schrecklich, schlimm, in unangenehmer weise stark; auch gefährlich“

2.3 "sehr; als verstärkungspartikel“

3. fürchterlich in DUDEN (2012, s.v.)

2.1a „durch seine [unvorstellbare] Furchtbarkeit o. Ä. Bestürzung hervorrufend"

2.1b „(umgangssprachlich) äußerst unangenehm; durch seine Art abstoßend“

2.2 „(umgangssprachlich)“

$2.2 \mathrm{a}$ „,beängstigend stark, groß"

$2.2 \mathrm{~b}$ " $<$ verstärkend bei Adjektiven und Verben> in beängstigend hohem Maß, sehr"

\subsection{Entwicklung der Gradbedeutung bei fürchterlich}

Die Etappe I des Entwicklungsgangs der Intensivierung ist mit der Verwendung des Lexems in der ursprünglichen Bedeutung verbunden. Aus den lexikographischen Angaben lässt sich schließen, dass die Ausgangsbedeutung von fürchterlich sich auf Furcht erregende Objekte bezieht:

(79) das schönste götterbild wird fürchterlich, wenn es der rauch entflammter kerzen schwärzt. (DWB, s.v.)

(79') 'Furcht erregend'

(80) Schon seit acht Tagen läßt sich ein fürchterlicher Komet sehen, und ganz Deutschland ist in Angst, es bedeute den Tod des Kaisers (C.II: HK3/F04.00001 Goethe: Götz von Berlichingen mit der eisernen Hand, Erstdruck: 1773, 2000 [S. 156])

(80') 'Furcht erregender Komet'

Die Etappe II verweist auf pragmatic enrichment bzw. strengthening der Ausgangsbedeutung des Lexems. Neben der Ausgangsbedeutung tritt die Gradbedeutung auf, sodass das Lexem in mehrdeutigen Kontexten verwendet wird. Als mögliche Kookkurrenzpartner in den bridging contexts finden sich Lexeme wie z.B. Verwüstungen, Zerstörung, Sturm, Getöse, Gelärm u.a. auf. Das sind Begriffe, die etwas sehr Schlimmes zum Ausdruck bringen bzw. eine inhärente Skala aufweisen. Durch die Verbindung mit solchen Bezugswörtern kommt es zu semantic repetition, sodass

\footnotetext{
50 Angaben aus den vorhandenen Belegzetteln von FWBD. Die lexikographischen Bezeichnungen beziehen sich auf die Schreibweisen forhtlich, förchterlich und vochlich.
} 
fürchterlich intensivierend wirkt. Anzumerken ist hier, dass fürchterlich zu den „superlativischen“ Adjektiven in der Terminologie von Pusch (1981: 36) bzw. „extreme adjectives“ bzw. „implicit superlatives“ gemäß Paradis (1997: 54) gehört. Darüber hinaus führt die Verbindung von einem „implicit superlative“ bzw. superlativischen Adjektiv mit einem skalierbaren bzw. einen Extremwert aufweisenden Bezugswort zu der Intensivierung des Begriffs, vgl. die Verwendung in (81) mit den entsprechenden Umformulierungen:

(81) Wir blieben hier nur drei Tage; den vierten nach unserer Abreise entstand ein fürchterlicher Sturm, der in wenig Stunden alle unsere Segel zerriß, unser Bugspriet zersplitterte und die große Bramstange umlegte, die gerade auf das Behältnis fiel, in dem unser Kompaß verschlossen war, und das Kästchen und den Kompaß in Stücken schlug. (C.II: HK3/F75.00001 Bürger: Münchhausen, Erstdruck: 1786, 2000 [S. 173])

(81') 'Furcht hervorrufender Sturm'

(81") 'sehr starker Sturm'

Der in (81) beschriebene Sturm demonstriert deutliche Verheerungen, z.B. Zerreißen von Segeln und Zersplittern des Bugspriets, die auf den hohen Grad ihrer Ausprägung verweisen. Zugleich lässt sich die Ausgangsbedeutung erkennen, weil eine solche Situation für die Seeleute mit Furcht verbunden sein kann.

Die Gradbedeutung lässt sich außerdem aus dem Kontext ableiten, da die Intensität als Grund für die entstandene Furcht der angesprochenen Personen auftritt, vgl.:

(82) Ach ja! es muß zu Hülfe eilen. Wie heftig steigt der Rauch empor! Wie grausam bricht die Gluth hervor! Man sieht den Schein auf viele Meilen. Der Stücke Blitz; der Trommeln Klang; Der Glocken fürchterliches Heulen Verhindert Andacht und Gesang ... (C.II: HK4/Z01.00001 Zäunemann: Poetische Rosen in Knospen, Erstdruck: 1738, 2004 [S. 539])

(83) Kaum hatte Padmanaba den Mund wieder zugetan, so hörte man auf einmal ein fürchterliches Donnern, mit Sturmwind und Blitzen begleitet, wodurch der ganze Palast [...] und die ganze Gesellschaft [...] in Furcht gesetzt wurde! (C.II: HK3/D56.00001 Wieland: Die Abenteuer des Don Sylvio von Rosalva, Erstdruck: 1772, 2000 [S. 335])

(84) Kaum aber hatte der Schloßwächter die zwölfte Stunde abgerufen, so erhob sich plötzlich im Schlosse ein fürchterliches Getöse, gleich dem Brausen eines heftigen Windes; es rasselte an den Fenstern, die Mauern und Wände erbebten, daß die Gläser auf der Tafel klirreten, die Balken krachten, es schlug mit den Türen auf und zu. Die Wachskerzen brannten so dunkel als Totenlichter, dagegen erhellete ein ungewöhnlicher Schimmer wie eine schnell auflodernde Flamme das Vorgemach, welches alle die zur Tafel saßen in Schrecken und Verwunderung setzte. (C.II: HK3/G57.00001 Musäus: Volksmärchen der Deutschen, Erstdruck: 1782-1786, 2000 [S. 492])

Die Belege (82) - (84) erfordern eine mehrdeutige Interpretation. Zunächst bezieht sich fürchterlich auf die mit Furcht verbundene Bedeutung, vgl. die entsprechenden Umformulierungen: 
(82') 'Furcht erregendes Heulen der Glocken'

(83') 'Furcht erregendes Donnern'

(84') 'Furcht erregendes Getöse'

Der auf die Ausgangsbedeutung bezogene Gebrauch ist jedoch nicht die einzige Interpretationsmöglichkeit des Adjektivs. Aus den Kontexten lässt sich die Intensität der Erscheinungen beurteilen. So handelt es sich jeweils um die Lautstärke, die bestimmte Auswirkungen zu Folge hat. In (82) verhindert die Intensität des Glockengeläuts die Andacht und den Gesang. In (83) wird der laute, durch Sturmwind und Blitze begleitete Donner als die Ursache für die Furcht der ganzen Gesellschaft dargestellt. In (84) vergleicht man die Intensität des Getöses mit dem Brausen eines heftigen Windes.

Die auf der Koexistenz von Ausgangs- und Gradbedeutung beruhende Mehrdeutigkeit des Gebrauchs beschränkt sich nicht auf Verwendungen mit Tönen. Als Kookkurrenzpartner können verschiedene Furcht hervorrufende Situationen auftreten, welche zugleich eine Skalierung voraussetzen, vgl.:

(85) [...] Dieses geringen Vortheils ungeachtet, verschafte ihm doch die angeborne Neigung des Menschen zum Kriege und eine gewisse neidische Freude, den Tirannen [...] zu stürzen, und sich dadurch gleichsam für die bisherige Furcht zu rächen - diese beiden Antriebe verschaften dem Anführer einen so zahlreichen Anhang, daß er allenthalben die schrecklichste Verwüstung verbreitete, und jedermann entweder für ihn fechten oder niedergehauen werden mußte. Bey einer so nahen und fürchterlichen Gefahr hielt es der Herr des Belphegors und Medardus für die beste Partie, mit seinen Effekten [...] und seinen Sklaven sich durch die Flucht zu retten [...]. (C.II: HK3/C71.00001 Wezel: Belphegor, Erstdruck: 1776, 2000 [S. 116])

(86) Am 29ften ward der Wind [...] fo heftig, daß wir vier und zwanzig Stunden lang nur allein das Fock-Seegel führen konnten. Zugleich gieng die See fürchterlich hoch und brach oft ůber dem Schiffe. Wer kein Seemann war, wußte fich in diefe neue Lage gar nicht zu fchicken [...]. Das heftige Hin und Herfchwanken des Schifs richtete daher tåglich fchreckliche Verwüftungen unter unfern Taffen, Glåfern, Bouteillen, Tifchen, Schüffeln und andern Gefchirr an [...]. Das ůbelfte dabey war, daß [...] nebft dem gewaltigen Hin- und Herwerfen des Schifs [...] waren neue und fürchterliche Scenen, aber zugleich ho̊chftbefchwerlich und ho̊chft unangenehm. (DTA: Forster, Georg: Johann Reinhold Forsters [...] Reise um die Welt. Bd. I, Berlin, 1778)

In (85) - (86) stellt die Intensität den Grund für die entstandene Furcht bei den angesprochenen Personen dar, vgl. die folgenden Umschreibungen:

(85') 'Die Gefahr ist so groß, dass der Herr Furcht empfindet und sich zum Fliehen entschließt.'

(86') 'Die See ging so hoch, dass es Furcht erregend wirkte.'

Das Auftreten der Gradbedeutung erfolgt außerdem durch das Phänomen semantic repetition, indem beide Kollokationspartner der adjektivischen Verwendung den 
gemeinsamen Begriff „Furcht" aufweisen. Durch die Wiederholung dieser Bedeutungskomponente kommt es zu der Verstärkung des Ausdrucks, sodass die intensivierende Funktion von fürchterlich hervortritt, vgl.:

(87) In der fürchterlichsten Angst meiner Seele knieete ich vor meinen Stuhl hin, und das Kreuz erinnerte mich abermals an die Worte meines ehrwürdigen Alten [...] (C.II: HK4/U01.00001 Unger: Julchen Grünthal, Erstdruck: 1784, 2004 [S. 315])

(88) [...] ganz im Geheim erreicht sie doch das fürchterliche Grauen vor dem tiefen Schweigen, worin die Natur ihr letztes geheimnißvolles Geschäft hüllt [...]. (C.II: HK4/P02.00001 Paalzow: Ste. Roche, Erstdruck: 1839, 2004 [S. 218])

(89) [...] da begannen alle ihre Pulse zu pochen, eine fürchterliche Beängstigung beklemmte ihr den Atem. (C.II: HK4/E02.00001 Ebner-Eschenbach: Lotti, die Uhrmacherin, Erstdruck: 1880, 2004 [S. 924])

Darüber hinaus lässt sich auf der II Etappe des Entwicklungsgangs der Gradbedeutung erkennen, dass die IntensitätsBedeutung als Nebenbedeutung durch pragmatic inferencing sowie semantic repetition in Erscheinung tritt, während die Ausgangsbedeutung zunächst im Vordergrund steht und gut zu erkennen ist. Außerdem demonstrieren die Kontexte das Einsetzen von subjectivity neben der intensivierenden Funktion, indem sie die Einstellung des Sprechers offenbaren. Damit kommt die erste semantisch-pragmatische Tendenz im Sinne Traugotts (Traugott I, oben ) zur Geltung:

„Meanings based in the external described situation $>$ meanings based in the internal (evaluative/perceptual/cognitive) described situation".

Auf der Stufe III kommt es zu semantic reanalysis der mehrdeutigen Verwendungen: Die durch pragmatic inferencing gewonnene Gradbedeutung wird konventionalisiert. Sie tritt in switch contexts auf, die den Verlust des direkten Zusammenhangs mit der Ausgangsbedeutung bezeugen. Die Verwendung des Lexems beschränkt sich jedoch auf negative Kollokationspartner und deutet auf den unvollendeten Prozess von bleaching hin, vgl. den adjektivischen und adverbialen Gebrauch mit den entsprechenden Umformulierungen:

(90) Ich habe fürchterliche Schmerzen von hier bis hierher" - und er zeigte zuerst auf den Kopf und dann auf die Zehen [...] (C.II: HK3/B22.00001 Kafka: Amerika, Entstanden: zwischen 1911, 2000 [S. 232])

(90') 'sehr starke Schmerzen'

(91) Wenn Lanz dann in Düsseldorf vor ein Millionenpublikum tritt, wird ein fürchterlicher Druck auf ihm lasten. (C.II: BRZ12/SEP.12657 Braunschw. Z., 25.09.2012)

(91') 'sehr großer Druck'

(92) Im Lehnstuhl lag nämlich der Italiener und schnarchte fürchterlich. (C.II: HK3/F41.00001 Hoffmann: Letzte Erzählungen, 1825, 2000 [S. 305])

(92') 'schnarchte sehr stark bzw. laut'

(93) Man muß sich ja fürchterlich schämen! (C.II: HK4/F21.00001 Frapan: Wir Frauen haben kein Vaterland, Erstdruck: 1898, 2004 [S. 96])

(93') 'sich sehr stark schämen' 
(94) Scheint die Sonne richtig doll vom Himmel, dann wird es in Büros ganz fürchterlich heiß. (C.II: BRZ08/JUN.01693 Braunschw. Z., 04.06.2008)

(94') 'sehr heiß'

(95) Als eine junge Parndorferin am Sonntag vom Bahnhof nach Hause fahren wollte, waren die Reifen ihres Rades zerstochen und der Sattel abmontiert: „Ich habe mich fürchterlich geärgert, das Rad ist jetzt kaputt. Wer macht so etwas?" (C.II: BVZ12/AUG.02987 NÖN, 23.08.2012)

(95') 'sehr geärgert'

Nach der Konventionalisierung der Inferenz beginnt actualization. Sie lässt sich u.a. daran erkennen, dass fürchterlich als Gradadverb in der Konstruktion mit dem verschobenen Skopus verwendet werden kann (siehe 4.4.4.), vgl. die Belege mit den entsprechenden Paraphrasierungen:

(96) Franz Wohlfahrt: „Der Trainer steht fürchterlich unter Beschuß“. (C.II: 096/AUG.81791 Neue Kronen-Ztg., 10.08.1996, S. 57)

(97) Nach 20 Jahren in Köln war Fabian buchstäblich stadtmüde: „Köln ist mir zum Schluss fürchterlich auf die Nerven gegangen". (C.II: RHZ99/OKT.22462 Rhein-Zeitung, 29.10.1999)

(98) So ein Spiel kann auch fürchterlich in die Hose gehen. (C.II: RHZ08/SEP.21191 RZ, 22.09.2008)

(99) Heinrichs hatte extra ein Oberhemd im 50-er-Jahre-Retrostil gekauft, in dem er fürchterlich ins Schwitzen kam. (C.II: BRZ11/SEP.02765 Braunschweiger Zeitung, 05.09.2011)

In (96) - (99) handelt es sich um die intensivierende Funktion des Adverbs, die keinen direkten Kontakt mit der Ausgangsbedeutung aufweist. Die einzige Selektionsbeschränkung ist jedoch das Auftreten des Lexems in negativ konnotierten Kontexten. In den hervorgehobenen Konstruktionen handelt es sich um die Änderung der Skopuskonstituente von fürchterlich, indem das Adverb die FVGs unter Beschuß stehen, auf die Nerven gehen, in die Hose gehen und ins Schwitzen kommen modifiziert. Die Intensivierung erfolgt dadurch, dass die durch die Kollokationen ausgedrückten Begriffe graduierbar sind. Beispielsweise wird die Verwendung in die Hose gehen in DUDEN (2012) als 'misslingen' wiedergegebenen. Das Kollokieren mit dem Gradadverb fürchterlich ergibt damit die Paraphrase 'sehr misslingen', vgl. die entsprechenden Umformulierungen der Belege (96) - (99):

(96') 'wird sehr scharf kritisiert'

(97') 'hat sehr genervt'

(98') 'sehr misslingen'

(99') 'fing an, sehr stark zu schwitzen'

Die intensivierende Funktion des Lexems kann außerdem in die quantifizierende übergehen, welche die Bedeutung „groß, viel“ zum Ausdruck bringt, vgl.:

(100) Wenn man das multipliziert, dann kommt eine ziemlich fürchterliche Summe, ein ziemlich fürchterlicher Betrag an $\mathrm{CO}_{2}$-Immissionen $\mathrm{zu}$ sammen. (01.05.2014: http://www.aggelidis.de/nc/reden/aktuell/detail/browse/4/zurueck/aktuell-a1abbe7c7f/artikel/gegen-den-neubau-eines-braunkohlekraftwerks-in-niederaussem/) 
(100') 'sehr große Summe', 'sehr großer Betrag'

(101) Die Händler mußten schreckliche Stapelrechte und hohe Steuern für Waren und Güter zahlen, die sie in die Stadt Hann. Münden brachten. Übrigens wurden diese fürchterlichen Steuern dafür verwendet, elegante Häuser an den Ufern der Konkurrentin WERRA zu bauen. (01.05.2014: http://de.wikipedia.org/wiki/Weserstein)

(101') 'sehr hohe Steuern'

Neben der Intensivierung entwickelt sich oft auch eine pejorative Bedeutung. Sie verliert durch bleaching den direkten Zusammenhang mit der Ausgangsbedeutung und demonstriert das ausgeprägte einstellungsbezogene Ausdruckspotential des Lexems, das im Rahmen von Traugott III (oben 3.6.3.4) seine Verbreitung findet:

(102) Einen ortsunkundigen Interessenten erschlägt die Menge unwichtiger Informationen, die noch dazu streckenweise in einem fürchterlichen Deutsch geschrieben ist [...]. (C.II: WDD11/J07.30671: Diskussion:Jena/Archiv, In:Wikipedia - URL:http://de.wikipedia.org/wiki/Diskussion:Jena/Archiv: Wikipedia, 2011)

(102') 'sehr schlechten Deutsch'

(103) Leute, ein einfach fürchterlicher Artikel. Fast alles aus dritter Hand, angelesener Quatsch etc. (C.II: WDD11/G02.79565: Diskussion:Giacomo Casanova, In: Wikipedia - URL:http://de.wikipedia.org/wiki/Diskussion:Giacomo_Casanova: Wikipedia, 2011)

(103') 'sehr schlechter Artikel'

(104) Die Schuluniformen sind eine fürchterliche Idee (C.II: A12/JUN.06530 St. Galler Tagbl., 14.06.2012, S. 51)

(104') 'sehr schlechte Idee'

Die Belege (90) - (99), (100) - (101) und (102) - (104) demonstrieren die eindeutige Verwendung von fürchterlich jeweils in der intensivierenden, quantifizierenden und pejorativen Bedeutung. Aus den Kontexten geht hervor, dass die einstellungsbezogene Bedeutung sich in diesem Gebrauch fest etabliert hat, sodass die Kollokationen mehr Expressivität zum Ausdruck bringen und damit auf Traugott III (oben 3.6.3.4) abzielen.

Die letzte Etappe der Bedeutungsentwicklung setzt das Auftreten von fürchterlich mit positiv evaluierenden Kookkurrenzpartnern voraus. Dadurch geht der Bezug auf die Ausgangsbedeutung endgültig verloren:

(105) Hiii - ich freu' mich fürchterlich. Endlich wieder ist mein lieber Süßer Schieber geil auf mich. (C.II: HK3/A32.00004 Lichtenstein: Weitere Gedichte: Vergnügtes Mädchen, Entstanden: 1912, 2000)

$(105$ ') 'freue mich sehr'

(106) Im Raabtal wächst der Mais fürchterlich gut. (C.II: K97/APR.31476 Kleine Ztg., 26.04.1997)

(106') 'wächst sehr gut'

(107) Die Super-G-Olympiasiegerin 1998 ist US-Amerikanerin, fürchterlich stolz, ihr Land so toll repräsentiert zu haben, und überhaupt gut drauf. (C.II: R98/FEB.11632 Frankf. Rundschau, 12.02.1998, S. 16) 
(107') 'sehr stolz'

(108) Aber sie tanzt mit mir fürchterlich gerne Walzer. (C.II: HAZ08/NOV.02749 HAZ, 15.11.2008, S. 21)

(108') 'sehr gerne'

Peters (1993: 23) schlägt vor, qualitative Adjektive und Adverbien von den Intensivierern folgendermaßen zu unterscheiden: „Das fragliche Adverb hat immer dann seine lexikalische (=qualitative) Bedeutung, wenn in der Kollokation mit dem Bezugswort eine Interpretation in Richtung auf eine qualitative Modifikation möglich ist. Andernfalls handelt es sich um ein Gradadverb." In Bezug auf die Belege (81) - (89) lässt sich feststellen, dass der Gebrauch den Zusammenhang mit der Bedeutung des Schreckens aufweist, was dagegen bei den Verwendungen in (105) - (108) nicht der Fall ist.

\subsection{Kurzfassung der Entwicklung bei fürchterlich}

\begin{tabular}{|c|c|}
\hline 1. Ausgangsbedeutung: & 'Furcht hervorrufend' \\
\hline $\begin{array}{l}\text { 2. Entwicklung der Gradbedeu- } \\
\text { tung: }\end{array}$ & $\begin{array}{l}\text { pragmatic enrichment bzw. strengthening der } \\
\text { Kontexte; pragmatic overload und Ambiguität } \\
\text { der Verwendungen; Intensivierung durch } \\
\text { pragmatic inferencing und semantic repetition } \\
\text { bei semantischer Reanalyse; Gradsemantik zu- } \\
\text { nächst als Nebenbedeutung (Ausgangsbedeu- } \\
\text { tung bleibt dominant). }\end{array}$ \\
\hline 3. Zunahme an Intensität: & $\begin{array}{l}\text { Rückgang der Ausgangsbedeutung und Zu- } \\
\text { nahme an Subjektivität; Gradsemantik zu- } \\
\text { nächst in negativ konnotierten Kollokationen } \\
\text { ohne direkten Zusammenhang mit Ausgangs- } \\
\text { bedeutung. - Verwendung auch in FVG mit gra- } \\
\text { duierbarem Begriff. - Desemantisierung nicht } \\
\text { vollkommen vollzogen. }\end{array}$ \\
\hline $\begin{array}{l}\text { 4. Intensivierend > quantifizie- } \\
\text { rend: }\end{array}$ & $\begin{array}{l}\text { Ausdruck großer Menge des durch das Bezugs- } \\
\text { wort ausgedrückten Sachverhalts. }\end{array}$ \\
\hline 5. Pejoration: & $\begin{array}{l}\text { tritt neben Gradbedeutung; Verlust des direk- } \\
\text { ten Zusammenhangs mit Ausgangsbedeutung; } \\
\text { Übergang auf expressive Ebene und Zunahme } \\
\text { an Subjektivität. }\end{array}$ \\
\hline 6. Reine Gradbedeutung: & $\begin{array}{l}\text { letzte Stufe der Entwicklung; modifiziert posi- } \\
\text { tiv konnotierte Kookkurrenzpartner; vollstän- } \\
\text { diger Verlust des Bezugs zur Ausgangsbedeu- } \\
\text { tung. }\end{array}$ \\
\hline $\begin{array}{l}\text { 7. Verlust der Ausgangsbedeu- } \\
\text { tung: }\end{array}$ & $\begin{array}{l}\text { korreliert mit Zunahme an Subjektivität; zu- } \\
\text { nächst Traugotts erste semantisch-pragmati- } \\
\text { sche Tendenz, dann Übergang in dritte Ten- } \\
\text { denz. }\end{array}$ \\
\hline
\end{tabular}


Diese Stadialisierung trifft auch auf die meisten folgenden Lexeme zu. Deshalb wird die hier skizzierte Entwicklung auch für die im folgenden zu besprechenden Lexeme übernommen und nur in individuell angezeigten Bereichen, in erster Linie bei der Ausgangsbedeutung (Ziffer 1), modifiziert. Der im folgenden sehr häufige Bezug auf die von Traugott beschriebenen drei Tendenzen (vgl. oben Kap. 3.6.3.4) wird im weiteren bei Bedarf mit „Traugott I, II, III“ abgekürzt. 


\section{Zur Entstehung von deutschen Grad-Adjektiven und Grad- Adverbien}

In diesem Kapitel werde ich die Entwicklung einer Gradbedeutung anhand deutscher Adjektive und Adverbien demonstrieren.

Die Beschreibung der Herausbildung einer Gradbedeutung besteht aus einer kurzen Darstellung des Bedeutungsbereichs jedes Lexems auf verschiedenen Stufen der Sprachentwicklung, einer ausführlichen Analyse des in Wörterbüchern und Korpora gesammelten Materials und einer kurzen Zusammenfassung.

\subsection{Wortgruppe 'Furcht erregend'}

Im folgenden wird die Herausbildung der Gradbedeutung in den Lexemen entsetzlich, furchtbar, fürchterlich, grässlich, grauenhaft, grauenvoll, grausig, schauderhaft, schauerlich, schaurig, schrecklich, ungeheuer, ungeheuerlich und unheimlich untersucht. Die intensivierende Funktion der Adjektive bzw. Adverbien kann durch die Verwendungen (a) - (n) exemplifiziert werden:

(a) j-n entsetzlich gern haben ' $\mathrm{j}-\mathrm{n}$ sehr gern haben'

(b) furchtbar lieb 'sehr lieb'

(c) fürchterlich gut wachsen 'sehr gut wachsen'

(d) grässlich müde 'sehr müde'

(e) grauenhaft neugierig 'sehr neugierig'

(f) grauenvoll gemütliches Haus 'ein sehr gemütliches Haus'

(g) grausig langweiliger Vortrag 'ein sehr langweiliger Vortrag'

(h) schauderhaft gutes Konzert 'ein sehr gutes Konzert'

(i) schauerlich unglücklich formulieren 'sehr unglücklich formulieren'

(j) schaurig dankbar sein 'sehr dankbar sein'

(k) schrecklich nett 'sehr nett'

(l) ungeheuer wichtig 'sehr wichtig'

(m) ungeheuerlich laut 'sehr laut'

(n) unheimlich effektiv 'sehr effektiv'

Die Ausgangsbedeutung der bezeichneten Grad-Adjektive bzw. -Adverbien entspricht der Kategorie 10 bzw. „Fühlen, Affekte, Charaktereigenschaften“ in der Klassifizierung von Dornseiff und bringt die Begriffe „Furcht, Schrecken“ 51 zum Ausdruck (Dornseiff 2004: 188ff.). Von Biedermann (1969: 158-160) wird die Gruppe der zu untersuchenden Wörter nach dem Motivationssem „Furcht" angeordnet. Obgleich sie dort durch die Lexeme erschreckend und grauslich erweitert wird, werden letztere hier nicht berücksichtigt, weil sie in DWB und DUDEN (2012) keine Erwähnung als Intensivierungsmittel finden. Die Beschreibung wird jedoch durch die Lexeme grauenhaft und grauenvoll ergänzt, die aus der korpusbasierten Liste DeReKo entnommen sind.

\footnotetext{
51 Die Lexeme grausig und ungeheuerlich werden jedoch u.a. der Begriffsgruppe 10.27 „Missfallen, hässlich“, ungeheuer u.a. der Gruppe 10.29 „Verwunderung“ zugeordnet. Die lexikographischen Angaben zeigen die Notwendigkeit, die Wörter zusammen zu behandeln, da sie die Ausgangsbedeutung des Schreckens aufweisen.
} 


\subsubsection{Entsetzlich}

\subsubsection{Lexikographische Angaben}

Kluge (2011, s.v.) bezeichnet entsetzlich als eine Ableitung von entsetzen, liefert jedoch keine weiteren Informationen zu der Entstehungszeit des Lexems. Pfeifer (1993, s.v.) sowie die Beleglage im DWB lassen das Auftreten des Wortes ins Fnhd. bzw. genauer ins 16. Jh. datieren.

1. entsetzlich in FWB (s.v.) „horrendum, zum entsetzten“

2. entsetzlich in DWB (s.v.)

2.1 „widerwillen, entsetzten hervorrufend oder davon bestimmt".

$2.1 \mathrm{a}$ „abscheulich“

$2.1 b$ "grauenvoll, grauenhaft"

2.2 „abgeschwächt. erheblich, sehr. meist adverbial bei verben oder attributiv bei adjektiven, die unangenehmes bezeichnen“, „erstaunlich, großartig“

3. entsetzlich in DUDEN (2012, s.v.)

3.1 „durch seine [nicht für möglich gehaltene] Furchtbarkeit bei jemandem Entsetzen erregend"

3.2 "(umgangssprachlich)“

$3.2 \mathrm{a}$ ", $<$ nur attributiv> in unangenehmer Weise sehr stark"

$3.2 \mathrm{~b}$ „<intensivierend bei Adjektiven und Verben> sehr, in beängstigend hohem Maß, überaus, äußerst"

\subsubsection{Entwicklung der Gradbedeutung bei entsetzlich}

Die Korpusanalyse legt nahe, dass das Einsetzen der Gradbedeutung im frühen Neuhochdeutschen erfolgt ist. Als Ausgangsbedeutung für deren Entwicklung dient die in 2.1b angesprochene Bedeutung, die auf den mit Entsetzen verbundenen Situationen beruht:

(109) vnd ein solch arm creatur seynen gott schöpffer szo erschrecklich, grewlich anspeyet und sprüet, das auch entsetzlich ist davon zu hören und reden. (FWB Korpus: b144, S.546)

$(109$ ') 'Entsetzen erregend'

(110) Muß demnach diefer Komet der Welt einen entfetzlichen Anblick gegeben haben: zumal weil er den vierdten / ja fchier gar den dritten Theil deß Himmels / mit feiner fchweiffenden Herolds-Farbe / nach der Långe / bekleidet und unterfchieden hat. (DTA: Francisci, Erasmus: Das eröffnete Lust-Haus Der Ober- und Nieder-Welt. Nürnberg, 1676)

(110') 'Entsetzen erregenden Anblick'

Die Herausbildung der Intensitätsbedeutung ist auf der zweiten Stufe des Entwicklungsgangs mit pragmatic enrichment bzw. pragmatic strengthening der Ausgangsbedeutung verbunden. Die Gradbedeutung lässt sich zunächst in ambigen Belegen feststellen, wobei die Intensivierung als Nebenbedeutung infolge pragmatic inferencing der Ausgangsbedeutung auftritt, vgl.: 
(111) ... die Pest, ... die den Kaiser bis nach Prag ... verfolgte und sich darauf in verschiedene Provinzen mit einer entsetzlichen Verheerung ausbreitete. (C.II: HK5/B28.00001 Bayle, Pierre: Verschiedene Gedanken über einen Kometen, Übersetzung: Publishing 1741)

(112) ... wie jene großen Flüsse, ... deren entsetzliche Überschwemmungen manchmal viele Provinzen verheeren, in ihrem ersten Ursprung nur einen Strahl von Wasser ausmachen ... (C.II: HK5/B28.00001 Bayle, Pierre: Verschiedene Gedanken über einen Kometen, Übersetzung: 1741)

(113) ... aber das hab ich doch gesehen, daß sie ganz grün im Gesicht und am Leibe war, und daß sie einen Buckel und ein paar entsetzlich lange Ohren hatte. (C.II: HK3/D56.00001 Wieland, Christoph Martin: Die Abenteuer des Don Sylvio von Rosalva, Erstdruck: 1772)

Die Belege (111) - (113) demonstrieren den mehrdeutigen Gebrauch des Lexems. Die erste Interpretation bezieht sich auf die Ausgangsbedeutung und bezeichnet Verheerungen in (111), Überschwemmungen in (112) und Ohren in (113) als Entsetzten erregend. Die zweite Interpretationsmöglichkeit fasst entsetzlich als Intensivierungsmittel auf und erfolgt durch pragmatic enrichment und pragmatic inferencing. Aus dem Kontext lässt sich eine Inferenz ableiten, die auf die Intensität der Erscheinungen hinweist und diese als Grund für die hervorgerufene Angst darstellt. Von daher ist die Intensivierung zunächst eng mit der Ausgangsbedeutung verbunden, vgl. die möglichen Umformulierungen:

(111') 'Die Verheerung war sehr groß und deswegen Entsetzen hervorrufend';

(112') 'Die Überschwemmungen waren sehr groß, sodass sie viele Provinzen verheerten, und deswegen Entsetzen hervorriefen';

(113') 'Die Ohren waren sehr lang und deswegen Entsetzen hervorrufend'.

Die Gradbedeutung kann außerdem durch das Phänomen semantic repetition in Erscheinung treten. Die Intensivierung des adjektivischen bzw. adverbialen Gebrauchs erfolgt durch die Wiederholung des gemeinsamen Begriffs „Furcht“ in den beiden Kollokationspartnern, was zu der Verstärkung des Ausdrucks führt und entsetzlich als Gradadjektiv bzw. Gradadverb darstellt, vgl.:

(114) Die entsetzliche Angst und dann die trockne Kälte der gleichgültigsten Verzweiflung trieben ihn die wilden Schrecknisse des Gebürgs aufzusuchen. (C.II: HK3/A91.00016 Novalis, Heinrich von Ofterdingen, 1799-1800, 2000 [S. 320])

(115) [...] dann habe ich Vieles, Vieles mit dem entsetzlichsten Schrecken abgebüßt. (C.II: HK4/F13.00001 Fouqué: Resignation, Erstdruck: 1829, 2004 [S. 83])

(116) Es ist nicht mehr das Gespenst der Krankheit, vor dem ich mich so entsetzlich fürchtete - jetzt ist es der Ruf zum Leben. (C.II: HK4/R01.00001 Reventlow: Ellen Olestjerne, Entstanden: 1900-1902, 2004 [S. 223])

Die eingesetzte Gradbedeutung in den Belegen (111) - (116) bezeugt zugleich das Auftreten von subjectivity, da sie eine gewisse Einstellung des Sprechers bzw. Schreibers zum Ausdruck bringt und den Erscheinungen einen bestimmten Ausprägungsgrad aus der subjektiven Perspektive zuschreibt. Damit demonstrieren 
die Verwendungen den mehrdeutigen Gebrauch des Lexems auf der propositionalen und expressiven Ebene und signalisieren das Einsetzen von Traugott I (oben 3.6.3.4) (Traugott I, oben 3.6.3.4).

In der nächsten Etappe des Entwicklungsgangs erscheint die Gradbedeutung von entsetzlich ziemlich ausgebildet, da die Ausgangsbedeutung allmählich verblasst wirkt. Das Lexem bezieht sich jedoch auf negative Situationen und ist dadurch noch von der Ausgangsbedeutung beeinflusst. Die Belege (117) - (121) mit den entsprechenden Paraphrasierungen sollen den adjektivischen und adverbialen Gebrauch veranschaulichen:

(117) ... der Bauer klagte über entsetzlichen Durst, als sie auf dem Felde einige Arbeitsleute antrafen... (C.II: HK3/B29.00001 Moritz: Anton Reiser, Erstdruck: 1785-1790, 2000 [S. 352])

(117') 'sehr starken Durst'

(118) Die Wirkung war fatal. Den Menschen wurde schlecht, sie bekamen entsetzliche Schmerzen vor allem in der Lebergegend. (C.II: HMP05/JUL.00137 Hamburger Morgenpost, 02.07.2005, S. 48; Mama Mambos Horror-Schnaps)

(118') 'sehr große Schmerzen'

(119) Wie entsetzlich leichtsinnig ist doch diese Mariane! Wie sie meine Rührung verspottete! (C.II: HK4/U01.00001 Unger: Julchen Grünthal, Erstdruck: 1784, 2004 [S. 192])

(119') 'sehr leichtsinnig'

(120) „Es war entsetzlich kalt, es schneite und der Abend begann zu dunkeln. (C.II: BRZ08/MAI.05147 Braunschweiger Zeitung, 10.05.2008; Millionen arme Kinder und 1400 Schuhkartons)

(120') 'sehr kalt'

(121) Auch finde ich die ständige Betonung, wie entsetzlich schwer die Neurologie ist, überflüssig. (C.II: WDD11/N05.37709: Diskussion:Neurologie, In: Wikipedia - URL:http://de. wikipedia.org/wiki/Diskussion:Neurologie:Wikipedia, 2011)

(121') 'sehr schwer'

Die Belege (117) - (121) demonstrieren den fortgeschrittenen Prozess der Desemantisierung, denn der direkte Zusammenhang mit der Ausgangsbedeutung wurde beseitigt. Die auf Furcht und Schrecken bezogene Interpretation ist nicht mehr möglich, während der Begriff der Intensivierung die primäre Rolle zu spielen beginnt.

Die Korpusanalyse hat gezeigt, dass entsetzlich außerdem in Konstruktionen mit verschobenem Skopus auftreten kann, weil das Adverb nicht einzelne Bezugswörter, sondern ganze Funktionsverbgefüge modifiziert, vgl.:

(122) Nuhr spielt zwar manchmal den Jammerlappen [...], aber im Grunde geht ihm diese allgemeine Nörgelei entsetzlich auf die Nerven. (C.II: RHZ02/APR.12261 Rhein-Zeitung, 16.04.2002)

(123) Polentario, Du gehst mir entsetzlich auf den Senkel, dass immer wenn Du eine Veröffentlichung gefunden hast, die Dir in den Kram passt, diese per Flächenbombardement in alle möglichen Artikel einbauen musst. (C.II: 
WDD11/P48.29442: Diskussion: Paradoxon der schwachen jungen Sonne/Archiv/2009, In: Wikipedia - URL:http://de.wikipedia.org/wiki/Diskussion:Paradoxon_der_schwachen_jungen_Sonne/Archiv/2009:Wikipedia, 2011)

(124) Dieser Schmarrn der gesamten gesellschaftlichen Un-Ordnung im Land muß ihnen entsetzlich auf den Geist gehen. (C.II: NUN92/AUG.02008 Nürnberger Nachrichten, 29.08.1992, S. 22)

In (122) - (124) fungiert entsetzlich als Gradadverb und intensiviert die Funktionsverbgefüge jemandem auf die Nerven / auf den Senkel / auf den Geist gehen. Das Auftreten des Lexems mit diesen Verbindungen erfolgt dadurch, dass sie jeweils durch einen graduierbaren Begriff bzw. durch das Verb „nerven“ wiedergegeben werden können.

Die intensivierende Funktion des Lexems kann in die quantifizierende übergehen und dadurch auf eine große Menge des durch ein Bezugswort ausgedrückten Sachverhalts hinweisen, vgl. die Belege mit den entsprechenden Umformulierungen:

(125) Dank der brutalen Besatzungspolitik wurde das Kriegsende hinausgezögert [...] und die kriegswirtschaftliche Maschinerie konnte weiterbetrieben werden - die entsetzlichen Kosten dafür trugen die besetzten Gebiete im Osten. (C.II: R99/OKT.82736 Frankfurter Rundschau, 13.10.1999, S. 24, Ressort: ZEIT UND BILD)

(125') 'sehr hohe Kosten'

(126) Unser Mr. X, der als einer der bedeutendsten Schriftsteller der Weltliteratur gilt, begegnet uns in Bad Ems als „kleiner Mann“, der ständig über die entsetzlichen Preise stöhnte und sich freute, wenn ihn nach dem dritten Besuch sein Arzt oder der Gepäckträger wiedererkannten. (C.II: RHZ96/APR.03295 Rhein-Zeitung, 06.04.1996)

(126') 'sehr hohe Preise'

(127) Bundesfamilienministerin Renate Schmidt (SPD) erklärte, der Polizei würden jährlich 20000 Fälle von sexueller Gewalt an Kindern bekannt. „Diese entsetzlichen Zahlen sind nur die Spitze des Eisbergs", sagte Schmidt. (C.II: RHZ04/APR.17831 Rhein-Zeitung, 21.04.2004)

(127') 'sehr große Zahlen'

Neben der Intensivierung tritt die pejorative Bedeutung in Erscheinung, die den Prozess der Desemantisierung ebenso veranschaulicht, denn sie demonstriert den Verlust des direkten Zusammenhangs mit der Ausgangsbedeutung. Der Gebrauch ist nun lediglich einstellungsbezogen, bringt den Ausdruck auf die expressive Ebene und belegt damit die Zunahme an subjectivity, vgl.:

(128) Zudem war der Billettverkauf entsetzlich organisiert. (C.II: E96/JUN.15393 Zürcher Tagesanzeiger, 29.06.1996, S. 45)

(128') 'sehr schlecht organisiert'

(129) Da ich die neue Rechtschreibung entsetzlich finde, überlasse ich die Korrektur einem Kultusministerkonferenzkonformen. (C.II: WDD11/T08.08471: In: Wikipedia - URL:http://de.wikipedia.org/wiki/Diskussion:Teufelshöhle_bei_Pottenstein: Wikipedia, 2011)

(129') 'sehr schlecht finde' 
(130) Ich bin Nachwuchsbetreuerin und muß feststellen, daß sich die Umkleidekabinen in einem entsetzlichen Zustand befinden. (C.II: K99/JUL.49835 Kleine Zeitung, 04.07.1999)

(130') 'in einem sehr schlechten Zustand'

Die Herausbildung der intensivierenden (117) - (124), quantifizierenden (125) (127) und pejorativen (128) - (130) Bedeutung auf der zweiten Stufe des Entwicklungsgangs ist zugleich mit der Erweiterung des einstellungsbezogenen Ausdruckspotentials des Lexems verbunden. Der Gebrauch gewinnt an subjectivity, verweist ausschließlich auf ,internal described situation“ und signalisiert damit den Übergang zu Traugott III (oben 3.6.3.4).

Die letzte Etappe der Bedeutungsentwicklung bezieht sich auf den Gebrauch von entsetzlich als Intensivierungsmittel mit positiv konnotierten Kookkurrenzpartnern, vgl.:

(131) ... dann laß ich Madame Brennfeld etwas aus einem Buche vor, das Lessings Fragmente heißt. Ich verstand zwar nicht viel davon; Madame aber ist entsetzlich gelehrt, und wie mir Mariane sagt, eine Philosophin. (C.II: HK4/U01.00001 Unger: Julchen Grünthal, Erstdruck: 1784, 2004 [S. 83])

(131') 'sehr gelehrt'

(132) Der Grund, warum die Waikikis so außergewöhnlich gute Musik machen, entsetzlich gut aussehen und sich ohne je geprobt zu haben, live nie verspielen, liegt an einer Art außerirdischer Übernatürlichkeit. (C.II: M04/SEP.64234 Mannheimer Morgen, 15.09.2004)

(132') 'sehr gut'

(133) „[... Das mußt Du aber nicht sehen, sondern Du mußt mich für den besten halten, weil ich Dich ganz entsetzlich lieb habe und mir ausser Dir nichts gefällt." (C.II: R99/AUG.64519 Frankfurter Rundschau, 14.08.1999, S. 27)

(133') 'sehr lieb'

(134) Dabei sind wir entsetzlich froh, wir fühlen uns so leicht und gut [...] (GB, 26.08.2015:https://books.google.de/books?id=4HvHAgAAQBAJ\&pg=PT3\&dq=\%2 2Entsetzlich+froh\%22\&hl=de\&sa=X\&ved=0CFUQ6AEwCWoVChMIm7733rnGxwIVQdYaCh0fEQiL\#v=onepage\&q=\%22Entsetzlich\%20froh\%22\&f=false)

(134') 'sehr froh'

Die Kontexte (131) - (134) demonstrieren den kompletten Verlust des Zusammenhangs mit der Ausgangsbedeutung. Die Beleglage lässt außerdem darauf schließen, dass der Prozess der Bedeutungsentleerung mit dem Zuwachs an subjectivity korreliert. Die Kontexte liefern genügend Evidenz dafür, dass der mehrdeutige Gebrauch des Lexems in der früheren Phase der Bedeutungsentwicklung weniger einstellungsbezogen bzw. expressiv ist als die reine Gradbedeutung in der Abschlussetappe oder die pejorative Bedeutung, die ausschließlich „speaker's perspective or point of view“ (Finegan 1995:1) repräsentiert. 


\subsubsection{Kurzfassung der Entwicklung bei entsetzlich}

Ausgangsbedeutung: 'Entsetzen hervorrufend'; die übrigen Entwicklungsschritte wie oben in Kap. 3.6.3.9.3.

\subsubsection{Furchtbar}

\subsubsection{Lexikographische Angaben}

Laut Kluge (s.v.) und DWB (s.v.) stellt furchtbar eine Ableitung von dem Substantiv Furcht dar. DWB gibt an, dass furchtbar "sich weder ahd. noch mhd. und nhd. nicht vor der zweiten hälfte des 17. jh., gegen dessen ende" findet. Paul (2002, s.v.) und Pfeifer (1993, s.v.) verweisen jedoch auf dessen mittelhochdeutsche Form „vorhtebær“, die in Lexer (s.v. vorhte-bære) als „furcht hervorbringend, furchtbar, schrecklich" definiert wird.

1. furchtbar in Lexer (s.v. vorhte-bære)

„furcht hervorbringend, furchtbar, schrecklich"

2. furchtbar in FWB

keine Angaben bzw. keine Belege im bereits zitierten Material der Redaktionsstelle von FWB

3. furchtbar in DWB (s.v.)

3.1 "angst erregend, hervorrufend“

3.2 "schrecklich, schlimm, in unangenehmer weise stark; auch gefährlich“

3.3 "sehr; verstärkungspartikel“

4. furchtbar in DUDEN (2012, s.v.)

4.1 „durch seine Art, Gewalt o. Ä. sehr schlimm, bange Beklemmung erregend“ 4.2 "(umgangssprachlich)“

$4.2 \mathrm{a}$ „unangenehm stark, sehr groß"

$4.2 \mathrm{~b}$ "<verstärkend bei Adjektiven und Verben> sehr, überaus“

\subsubsection{Entwicklung der Gradbedeutung bei furchtbar}

Die Korpusanalyse hat zu Tage gefördert, dass das Einsetzen der Gradbedeutung in der neuhochdeutschen Sprachstufe erfolgte. Als Quelle für deren Herausbildung diente die in Lexer sowie in DWB erwähnte Bedeutung des Schreckens, die sich auf Furcht erregende Situationen bezieht, vgl. die Belege mit den entsprechenden Umformulierungen:

(135) im übrigen ist dieser möchtige und furchtbare printz, den man sonst den grossen Tartar-Kam nennte, deme die Sineser selbst schatzbar seyn sollten, ein gedicht (DWB: 1699, c.v. furchtbar)

(135') ,Furcht erregender Prinz'

(136) zitterft du nicht, da dir der furchtbare Name Eines Todesengels genennt wird? (DTA: [Klopstock, Friedrich Gottlieb]: Der Messias. Bd. 1. Halle, 1751)

(136') 'Furcht erregende Name'

(137) es ist ein furchtbarer gedanke, dass wir durch den tod getrennt werden (DWB: 1872, s.v.)

(137) ,ein Furcht erregender Gedanke' 
Die Entwicklung der Intensitätsbedeutung beginnt in den Kontexten, die durch pragmatic inferencing der Ausgangsbedeutung pragmatic strengthening bzw. enrichment und demzufolge die Mehrdeutigkeit des Gebrauchs aufweisen. Zunächst steht die Ausgangsbedeutung im Vordergrund, während die Gradbedeutung als Nebenbedeutung zu erkennen ist, vgl.:

(138) Die ganze Burg ist furchtbar still und leer Wie eine Gruft ... (C.II: HK3/B00.00006 Wieland: Oberon, Erstdruck: 1780, 2000 [S. 245])

(139) diese blutgierigen Raubvögel52 ... wüten oftermals so grausam, nur etwa in ein arm Bauerweibchen, ... und solange und so furchtbar martern, bis sie von ihr ein Bekenntnis extorquieren und rauspressen, damit sie was zu strafen bekommen. (C.II: HK5/B24.00001 Agrippa von Nettesheim, Ungewißheit und Eitelkeit aller Künste und Wissenschaften, Übersetzung: 1713, 2000 [S. 140])

(140) In Wahrheit, die erschrecklichen Strafen, welche Gott den Juden wegen ihrer Abgötterei zugeschickt hatte, hatten ihnen den Abscheu ... dergestalt ins Gemüt eingedrückt, ... daß sie nicht gegen ihren furchtbaren Tyrannen Herodes einen Aufstand gemacht hätten, als er in Judäa dem Augustus zu Ehren einen Tempel aufbauen ließ. (C.II: HK5/B28.00001 Bayle: Verschiedene Gedanken über einen Kometen, Übersetzung: 1741, 2000 [S. 399])

Die mit fetter Schrift hervorgehobenen Verbindungen in (138) - (140) erlauben eine mehrdeutige Interpretation. Zunächst bezieht sich furchtbar auf die Bedeutung des Schreckens, weil die leere und stille Burg in (138) mit einer Gruft verglichen wird und deswegen Angst erregend zu wirken scheint. In (139) - (140) bezeichnet furchtbar menschliches Verhalten, welches durch seine grausame Art und Weise Angst hervorrufen kann. Damit wird das Lexem hier in der Ausgangsbedeutung gebraucht.

Die Verwendungen lassen jedoch eine alternative Interpretation zu, die die Verwendung von furchtbar in der intensivierenden Rolle voraussetzt und durch pragmatic inferencing und danach semantic reanalysis des einstellungsbezogenen Kontexts erklärt. Die Belege bezeugen „the speaker's subjective belief-state / attitude towards the proposition" (Traugott 1995: 35), sodass der adjektivische bzw. adverbiale Gebrauch eine wertende bzw. subjektive Bedeutungskomponente gewinnt. Aus den Kontexten folgt, dass die Bezugswörter Stille, martern und Tyrann graduierbare Begriffe darstellen und je nach der Stärke ihrer Ausprägung verschiedene Positionen auf der entsprechenden Skala einnehmen können. So kann die Stille, aus der Sicht des Autors, weniger oder mehr ausgeprägt wahrgenommen werden, denn der einstellungsbezogene Vergleich der Burg mit einer Gruft in (138) weist auf die im höchsten Maße ausgeprägte Stille hin. Das Martern kann ebenso weniger oder mehr grausame, in verschiedenem Maße mit Qualen und Gewalt verbundene Handlungen voraussetzten, während die durch Tyrann auszudrückende menschliche Eigenschaft auch weniger oder mehr ausgeprägt zum Ausdruck kommen kann. Durch die Verwendung von furchtbar hebt der Schreiber bzw. Sprecher den Grad der Stille sowie die Stärke der Grausamkeit der Handlungen bzw. der Per-

\footnotetext{
52 Inquisitoren
} 
son hervor und ordnet damit die Begriffe auf dem oberen Ende der Skala an. Bemerkenswert ist, dass die Bezugswörter martern und Tyrann Extremwerte aufweisen und durch die Verbindung mit furchtbar intensiviert wirken. Außerdem ist es auffallend, dass die Intensität des Marterns in (139) als Grund für die darauf folgenden Geschehnisse bzw. das Erlangen des gewünschten Bekenntnisses dient, vgl. (139'). Eine ähnliche Erklärung ist in Bezug auf die Ausgangsbedeutung nicht möglich:

(139') Die Inquisitoren haben die Bauersfrau so stark gemartert, dass sie anschließend ein Geständnis von ihr bekommen haben.

Die Intensivierung kann außerdem durch semantic repetition erfolgen. Das Wiederholen des gemeinsamen Begriffs „Furcht“ in (141) - (142) hat semantic redundancy und Verstärkung der Verwendung zur Folge, sodass dem Adjektiv eine intensivierende Funktion zugeschrieben werden kann, vgl.:

(141) Aber es mochte vielleicht gegen vier Uhr des Morgens sein, als ich durch eine furchtbare Angst geweckt wurde und den Kopf kaum heben konnte. (C.II: HK3/G38.00001 Seume: Spaziergang nach Syrakus im Jahre 1802, Erstdruck: 1803,2000 [S. 218])

(142) Ich war betäubt von dem furchtbaren Schreck [...] (C.II: HK4/B02.00001 Bernhardi: Evremont, Entstanden: nach 1822, 2004 [S. 75])

Die ambigen Belege (138) - (142) demonstrieren das Entstehen eines einstellungsbezogenen Gebrauchs. Die Verwendung des Lexems auf der propositionalen und expressiven Ebene verweist auf Traugott I (oben 3.6.3.4).

In der nächsten Stufe des Entwicklungsgangs wird die Gradbedeutung dadurch ausgebildet, dass der Bezug zur Ausgangsbedeutung nicht mehr zu erkennen ist. Zunächst findet sich das Lexem mit negativ konnotierten Kookkurrenzpartnern, wobei jedoch keine vollkommene Bedeutungsentleerung festgestellt werden kann:

(143) „...Aber bis viere habe ich kein Auge zugetan. Solche furchtbaren Schmerzen..." (C.II: HK3/A34.00002 Fontane: Mathilde Möhring: 1891, 2000 [S. 463])

(143') 'sehr starke Schmerzen'

(144) Nichts war unbehaglich in der Stube als die furchtbare Hitze, die aber der Bauer liebt, um sich eben so gründlich zu erwärmen, als er bei seinen Feldarbeiten gründlich durchkältet wird. (C.II: HK4/H03.00001 Hahn-Hahn: Maria Regina, Erstdruck: 1860, 2004 [S. 429])

(144') 'sehr starke Hitze'

(145) Am Sonnabend rief Tom sie dann an und entschuldigte sich inständig bei ihr, es täte ihm alles furchtbar leid. (C.II: L99/MAI.24727 Berliner Morgenpost, 17.05.1999, S. 28)

(145') 'täte sehr leid'

(146) „Brunnenbohrungen und Wassertransporte sind furchtbar teuer", sagt Adjibade. (C.II: M06/MAR.21999 Mannh. Morgen, 22.03.2006)

(146') 'sehr teuer' 
(147) Sie wirkte nicht erschöpft, nur furchtbar enttäuscht und richtig sauer. „ICh habe mehr als ein halbes Jahr umsonst gearbeitet - so eine Sauerei!" (C.II: BRZ09/AUG.10952 Braunschw. Z., 24.08.2009)

(147') 'sehr enttäuscht'

Furchtbar kann außerdem als Gradadverb die Änderung seines Bezugsbereichs vorweisen und als Modifikator von Funktionsverbgefügen auftreten, vgl.:

(148) Aber sie stand oft unter furchtbarem Druck und wußte nicht, wohin sie gehörte. (C.II: 095/SEP.86917 Neue Kronen-Zeitung, 03.09.1995, S. 8)

(149) Männer müssen eben immer handeln, etwas werden, stehen manchmal furchtbar unter Druck... [...]. (C.II: K98/MAR.22868 Kleine Zeitung, 25.03.1998)

In (148) gerät das Substantiv Druck in den Skopus der Intensivierung, während in (149) das ganze Funktionsverbgefüge unter Druck stehen modifiziert wird. Die Änderung der Skopuskonstituente erfolgt dadurch, dass das FVG durch einen graduierbaren Begriff bzw. durch 'bedrängt sein' wiedergegeben werden kann. Die Belege (150) - (151) bieten weitere Beispiele, vgl.:

(150) Damit kann man sich furchtbar in die Nesseln setzen, wenn man es nicht richtig macht. (C.II: RHZ08/DEZ.17392 Rhein-Zeitung, 27.12.2008)

(150') 'sich große Unannehmlichkeiten bereiten'

(151) Gegen Morgen wächst die Schlange vor dem Kinopolis immer weiter, und die letzten Minuten ziehen sich furchtbar in die Länge. (C.II: M02/MAI.34230 Mannheimer Morgen, 06.05.2002)

(151') 'sich sehr verzögern'

Die Intensivierung geht in bestimmten Kontexten in die Quantifizierung über, vgl. die Belege mit den entsprechenden Umformulierungen:

(152) Kaum eine Zeile hätte man darauf verschwendet, wäre das Stadttheater im Besitz einer mobilen Konzertmuschel. Orchester rein, fertig. Solch eine Muschel kostet eine fünfstellige, respektable, doch nicht allzu furchtbare Summe. (06.05.2014: http://www.nordbayern.de/region/fuerth/3500-fahrkarten-nach-amerika-1.723146)

(152') 'sehr große Summe'

(153) Ich habe nach Ihrem Wunsch alle meine Sachen mitgebracht, meine Stellung in Leipzig aufgegeben $u$. mich in furchtbare Ausgaben gestürzt. (06.05.2014: http://www.antiquariat.de/angebote/GID4451694.html)

(153') 'sehr große Ausgaben'

Die Beleglage dokumentiert neben der Intensivierung eine rein pejorative Bedeutung, die keinen direkten Zusammenhang mit der Ausgangsbedeutung erkennen lässt. Die Verwendungen in (154) - (156) veranschaulichen ausschließlich die expressive Ebene und zeigen damit den Zuwachs an subjectivity im Rahmen von Traugott III (oben 3.6.3.4) (Traugott III, oben 3.6.3.4):

(154) Das ist ja alles furchtbar hier, das Zimmer ist dreckig und das Essen eine Katastrophe - und wenn ich zu Hause bin, werde ich einen Brief 
schreiben und mindern. (C.II: NUZ08/AUG.02468 Nürnberger Zeitung, 25.08.2008, S. 9)

(154') 'alles ist sehr schlimm'

(155) E-Mails finde ich furchtbar und viel zu unpersönlich. (C.II: A11/DEZ.10298 St. Galler Tagblatt, 31.12.2011, S. 42)

(155') 'E-Mails finde ich sehr schlimm'

(156) Dieser Artikel ist ganz ganz furchtbar, eine wahlos zusammengeklaubte Sammlung von Schlagworten und Zitaten, dazu kein neutraler Artikel. (C.II: WDD11/B41.71226: Diskussion:Buchhandel/Archiv, In: Wikipedia - URL:http://de.wikipedia.org/wiki/Diskussion:Buchhandel/Archiv: Wikipedia, 2011)

(156') 'der Artikel ist sehr schlimm'

Die Belege (143) - (151), (152) - (153) und (154) - (156) demonstrieren jeweils die intensivierende, quantifizierende und wertende Funktion von furchtbar, die keinen direkten Kontakt mit der Ausgangsbedeutung des Lexems aufweisen. Die fortgeschrittene Desemantisierung lässt außerdem die Ausweitung des einstellungsbezogenen Ausdruckspotentials und damit den Übergang zu Traugott III (oben 3.6.3.4).

Anschließend findet sich das Lexem in Verbindung mit positiv konnotierten Kollokationspartnern. Dieser Entwicklungsschritt demonstriert den kompletten Verlust des Zusammenhangs mit der Ausgangsbedeutung, das Wort fungiert damit als bedeutungsentleert:

(157) „O, ich habe furchtbar viel Geld, Großmutter", rief es jubelnd aus und hüpfte vor Freuden in die Höhe ... (C.II: (HK4/S09.00001 Spyri: Heidis Lehrund Wanderjahre, Erstdruck: 1880, 2004 [S. 235])

(157') 'sehr viel'

(158) In seinem Photographiealbum hat er auch viele furchtbar schöne Bilder von Mädchens. (C.II: HK3/G40.00001 Bierbaum: Stilpe. Ein Roman aus der Froschperspektive, Erstdruck: 1897, 2000 [S. 25])

(158') 'sehr schöne'

(159) „Oh, wie hab ich mich unterhalten! Es war furchtbar lustig.“ (C.II: HK4/E05.00001 Ebner-Eschenbach, Marie von: Unsühnbar, Erstdruck: 1889)

(159') 'sehr lustig'

(160) „Ich möchte furchtbar gerne Ballett-Lehrerin werden, und Ihr seht mich hier grad inmitten meiner Schüler bei einer anstrengenden Übungsstunde im Studio. " (C.II: M03/JUL.48597 Mannh. Morgen, 24.07.2003)

(160') 'sehr gerne'

(161) Fiona kann freundlich lächeln, erstaunt die Brauen heben und furchtbar lieb mit den Augen zwinkern. (C.II: NUN03/APR.00997 NN, 11.04.2003) (161') 'sehr lieb'

\subsubsection{Kurzfassung der Entwicklung bei furchtbar}

Ausgangsbedeutung: 'Furcht hervorrufend'; die übrigen Entwicklungsschritte wie oben in Kap. 3.6.3.9.3. 


\subsubsection{Fürchterlich}

Siehe oben im Kapitelabschnitt 3.6.3.9.

\subsubsection{Grässlich}

\subsubsection{Lexikographische Angaben}

Kluge (s.v.) verweist auf die Entstehung der Form im 9. Jh. als Ableitung vom mhd. 'graz' und ahd. 'grazzo'. „Vermutlich hat sich später mndd. greselik „, schaudererregend“ (zu mndd. grese „Schauder") eingemischt“ (Kluge, s.v). DWB (s.v. gräszlich) widerspricht Kluge, indem nach seinen Angaben das Lexem „im 14.jh. aus mnd. greselîk 'schaudererregend, gräszlich' [...] ins ober- und mitteldeutsche zunächst entlehnt, dann fälschlich als ableitung zu graz [...] 'wütend, zornig' gefaszt [...] und diesem entsprechend lautlich umgedeutet worden" ist.

1. grässlich im FWB (s.v.)

1.1 "wütend, zornig"

1.2 „schrecklich, furchterregend, angsteinflößend (von Gegenständen und Handlungen)“

1.3 „erbarmungslos, grausam“

1.4 „bösartig, unverfroren, unverschämt"

1.5 „häßlich (von der äußerlichen Erscheinung eines Menschen), durch äußere Erscheinung Furcht einflößend; anschließbar an“ 1.2.

1.6 „ungeheuerlich, in übergroßem Maße“

2. grässlich in DWB (s.v. gräszlich)

2.1 "'wütend, zornig, drohend, wild', in (etymologisch ungerechtfertigtem) anschlusz an die bedeutung von grasz (s. d. 1) als bezeichnung einer affekthaltung“. „auch mit der bedeutungsnuance 'herausfordernd, übermütig, trotzig'“

2.2 „im anschlusz an die bedeutung 'grauenhaft, schrecklich, abscheulich' von mnd. greselik."

2.3 „in einem 2 gegenüber gemilderten und abgeschwächten, manchmal auch leicht ironischen sinne, analog der z.b. bei schrecklich, furchtbar u. a. noch sehr viel ausgeprägteren gleichen erscheinung".

2.3a „soviel wie 'greulich, schlimm, böse, unangenehm', immer noch stark affekthaltig, aber durchweg in ungewichtigeren beziehungen als unter 2; häufiger erst seit dem 18. jh., modern sehr geläufig"

2.3b „im sinne von 'sehr, überaus' und 'grosz, stark' zu blosz (aber stark) steigernder funktion entleert. bereits in frühen ansätzen, aber vorwiegend in jüngerem gebrauch, und hier eher der umgangssprache als gehobener redeweise angehörig".

3. grässlich in DUDEN (2012, s.v. grässlich)

3.1 "(emotional) schauderndes Erschrecken hervorrufend"

3.2 "(umgangssprachlich)“

3.2a „äußerst unangenehme Gefühle hervorrufend“

$3.2 \mathrm{~b}$ „in unangenehmer Weise groß, stark“ 
$3.2 \mathrm{c}$ " $<$ intensivierend bei Adjektiven und Verben> überaus; in höchstem $\mathrm{Ma}$ "

\subsubsection{Entwicklung der Gradbedeutung bei grässlich}

Die lexikographischen Angaben von FWB und DWB führen das Einsetzen der Gradbedeutung auf das Fnhd. zurück. DWB kündigt die in 2.2 angegebene Bedeutung als Basis für die Herausbildung der Intensität, indem die letzte „zunächst nur in negativ wertendem zusammenhang und dem gebrauch 2 noch näher" auftritt. Aus dem Zitat folgt, dass die Entwicklung der Gradbedeutung in einer früheren Phase mit dem Auftreten des mehrdeutigen Gebrauchs verbunden ist. Ferner kann geschlossen werden, dass die intensivierende Bedeutungskomponente nicht nur durch die Verknüpfung mit dem Begriff des Schreckens, sondern auch durch den mitwirkenden Begriff des Abscheus und Ekels entsteht. Aus der untersuchten Beleglage geht hervor, dass die auf Furcht bezogenene Bedeutung die Hauptrolle in der Entwicklung der Gradbedeutung übernimmt, während der Begriff des Abscheus gelegentlich mit ihm zusammen auftreten kann.

Die Bedeutung des Schreckens bezieht sich laut DWB „auf visuelle“, „selten auf geistige Eindrücke und Erkenntnisse“, ,,gehörseindrücke [...], sofern diese beim hörenden auf bestimmte seelische voraussetzungen treffen“ und vereinzelt auch „auf gott", vgl. die Belege mit den entsprechenden Paraphrasen:

(162)[...] und fchlug das Unglük darzu / daß er mich beym Mondenfchein alsbald erkeñete /[...] und faffete mich beym Halfe / ehe ich fein innen ward / erfchrak auch von ganzem Herzen / da er mit greßlicher Stimme zu mir fagete [...] (DTA: Bucholtz, Andreas Heinrich: Des Christlichen Teutschen Groß-Fürsten Herkules Und der Böhmischen Königlichen Fräulein Valjska WunderGeschichte. Bd. 1. Braunschweig, 1659)

(162') 'Schrecken erregender Stimme'

(163) [...]Er fahe fo gräßlich aus / daß ich mich auch nur vor feinem Anblick entfetzte [...] (DTA: Grimmelshausen, Hans Jakob Christoffel von: Trutz Simplex. [Nürnberg], 1670)

(163') 'sah Schrecken erregend aus'

DWB verweist außerdem darauf, dass die Bedeutung „furchtbar, schrecklich, grausam, entsetzlich“ ferner „lediglich als eine irgendwelche erscheinungen, zustände, vorgänge oder dinge kennzeichnende eigenschaft" verwendet wird. Dabei „liegt“ „das übergewicht dieses mindestens seit dem 16. jh. möglichen gebrauchs [...] in jüngerer sprache".

Das Auftreten der Gradbedeutung verbindet sich zunächst mit pragmatic strenghtening bzw. enrichment und daraus folgendem pragmatic overload der Ausgangsbedeutung. Durch pragmatic inferencing wird die Mehrdeutigkeit des Gebrauchs ausgelöst, wobei die Intensivierung die Ausgangsbedeutung als Nebenbedeutung bereichert, vgl.:

(164) Vnd do ich also in disen gedenken was, do kam eine grose forhte vnd ein gros leit in mich, daz ich minen herren vnd minen got so gro̊seliche virzúrnet hette [...] (FnhdC: Rulmann Merswin: „Mannen, Straßburg 1352“) 
Die Verwendung in (164) erfährt eine ambige Interpretation. Zunächst berichtet der Schreiber bzw. Sprecher von der Schrecken erregenden Erzürnung Gottes, die bei ihm große Furcht und großes Leiden hervorruft. Damit bezieht sich das Adverb auf die Ausgangsbedeutung. Zugleich handelt es sich um einen hohen Grad der Erzürnung, der den Grund für die erregten Gefühle darstellt. Die Verstärkung des Begriffs erfolgt außerdem durch den Operator „so“, der „ein durch Kontext od. Situation näher bestimmtes [verstärktes] Maß o. Ä., in dem eine Eigenschaft, ein Zustand o. Ä. vorhanden, gegeben ist: in solchem Maße, Grade; dermaßen“ "bezeichnet" (DUDEN 2012, s.v. so). Darüber hinaus wird die in grässlich erworbene intensivierende Bedeutungskomponente durch so verstärkt, sodass die zweite Interpretation die folgende Wiedergabe bekommt:

(164') 'Ich habe meinen Gott sehr stark erzürnet, was Schrecken erregend ist.' Die Herausbildung der Gradbedeutung durch pragmatic inferencing des auf Schrecken bezogenen Gebrauchs lässt sich durch weitere Belege (165) - (169) veranschaulichen, vgl.:

(165) [...] Sie dreht die augen umb / die fie wie feuer låßt Erfchrecklich blicken an: Drauff hebt fie an zufchreyen So gräßlich / daß man fich entfetzen muß und fcheuen: Hoho! Ihr frauen hört / wo enden ihr auch feyd In gantzen Latien / fo ihr noch lieb und leid / Entgegen mir empfindt / die ich zwar vom geblüte Bin eine ko̊nigin [...] (DTA: Vergilius Maro, Publius: Eigentlicher Abriß Eines verständigen / tapfferen und frommen Fürsten / Von dem fürtrefflichsten Poeten Virgilius. Cölln (Spree), 1668)

(165') 'Sie schrie sehr laut und Schrecken erregend, sodass man sich entsetzen muss und scheuen'

(166) Wenn, mit Schrecken, Furcht und Graufen,

Sich ein Schiff, bey fchwarzer Nacht,

Durch der Stürme gräßlichs Braufen,

Und der wilden Wellen Macht,

Ohne Weg und Zweck zu wiffen,

Würde fchlenkern laffen müffen,

Und man fchenkt ihm diefen Stein,

Würd' er ihm ein Engel feyn.

(DTA: Brockes, Barthold Heinrich: Physikalische und moralische Gedanken über die drey Reiche der Natur. Bd. 9. Hamburg u. a., 1748)

(166') 'starkes und deswegen Schrecken erregendes Brausen der Stürme'

(167) Ich ließ ihn, weiß Gott! bei einem der gräßlichsten Stürme, die ich auf der See erlebt, mit Hubert in einer kleinen Barke auf die See setzen. Es war Nacht und donnerte fürchterlich, pfiff so melodisch brüllend über der See, daß mir 's Herz gellte, und was mich verdroß, sie mucksten nicht. [...] Sie waren aus meinem Gesicht verschwunden, wie sie kaum in die Barke stiegen. Nur bei den Blitzen sah ich sie in der Ferne kämpfen, und es heulte so bitter um mich, daß ich die Freude nicht haben konnte, sie von der See verschlingen zu sehn, und ihr Geächze zu hören. (C.II: HK3/G42.00001 Klinger: Sturm und Drang, Erstdruck: 1776, 2000 [S. 1181])

(167') 'ein starker und deswegen Schrecken erregender Sturm' 
(168) Da stieg die Noth im ganzen Kanton, und wurde noch viel gräßlicher, als in der Nähe St. Gallens. (05.05.2014: http://books.google.de/books?id=vD4PAAAAQAAJ\&pg= PA299\&dq=schauderhaft\&hl=de\&sa=X\&ei=AqVnU5EhdmK05ATfsIGICg\&ved=0CDgQ6AEwAThQ\#v=onepage \&q=schauderhaft\&f=false)

(168') 'noch stärker und deswegen Schrecken erregender'

(169) Von Ehrgeiz angespornt, ermordet auf Begehr Der Gattin ein Vasall den Herrn im sichern Schlafe, Steigt auf den Thron und wird ein gräßlicher Tyrann, Würgt wie ein Wolf die waffenlosen Schafe, Minister, General, Freund, Kinder, Weib und Mann. (C.II: HK3/F28.00001 Wezel: Kakerlak oder die Geschichte eines Rosenkreuzers, Erstdruck: 1784, 2000 [S. 66])

(169') 'ein großer und deswegen Schrecken erregender Tyrann'

Die Verwendungen in (165) - (169) weisen mindestens zwei Lesarten auf. So handelt es sich zunächst um Schrecken erregende Situationen bzw. Eigenschaften. Damit wird gräßlich in der Ausgangsbedeutung gebraucht. Zugleich jedoch lässt sich das Einsetzen der Intensivierung feststellen, indem es sich jeweils um eine in hohem Grad ausgeprägte Situation geht bzw. lautes Schrei in (165), starkes Brausen in (166), heftigen Sturm in (167) und starke Not in (168) oder um eine in hohem Grad ausgeprägte menschliche Eigenschaft, wie in (169). Darüber hinaus hat die Intensität der Geschehnisse und der Eigenschaft das Gefühl der Furcht bei den angesprochenen Personen zu Folge. Damit verweist der adjektivische Gebrauch zugleich auf zwei Sinnrichtungen bzw. die Bedeutung des Schreckens und die Intensivierung. Es fällt außerdem auf, dass die Bezugswörter Extremwerte aufweisende Begriffe darstellen, die in der Zusammensetzung mit gräßlich verstärkt wirken.

Neben der Intensivierung erfolgt die Steigerung der subjectivity, indem der Gebrauch expressiver erscheint und „speaker's imprint“ (Finegan 1995:1) stärker zum Ausdruck bringt. Hier kommt Traugott I (oben 3.6.3.4) zum Zuge.

DWB (s.v. gräszlich) weist außerdem darauf hin, dass „,in der kennzeichnung eines inneren zustandes (der angst, des grauens u. ä.) und seiner äuszerungen“ grässlich „schon beinahe mit blosz steigernder, intensivierender funktion" verwendet wird. Diese Annahme lässt sich durch das Phänomen semantic repetition erklären, vgl.:

(170) Ja es würde der Göttliche bloffe vnbedingte Rathfchluß fo greßlich vngehewer figurirt, daß Calvinus fich felbs dafür entfetzt / vñ außdrücklich fich vernemmen laffen / es fey horribile decretumauß einem graufamen vnd erfchröcklichẽ Rathfchluß gefchehẽ / daß fo viel Völcker vnd vnmündige Kinder ohn hilff vnd mittel zum ewigen Todt verworffen werden / davon aber geliebts Gott zu andern zeiten in dem Gegenfatz mit mehrerem. (DTA: Dannhauer, Johann Conrad: Catechismus Milch. Bd. 4. Straßburg, 1653)

(171) Die Frau hatte sich aber standhaft geweigert, das Zimmer zu verlassen, da sie die gräßlichste Angst vor dem Armen- oder Krankenhause hatte. (C.II: HK3/A96.00030 Weerth: Die englischen Arbeiter, Entstanden: vor 1856, 2000 [S. 203])

(172) Da riß ein brausender Wind seine Flügel auseinander: pfeifend, schrillend und schneidend warf er mir einen schwarzen Sarg zu: Und im 
Brausen und Pfeifen und schrillen zerbarst der Sarg und spie tausendfältiges Gelächter aus. Und aus tausend Fratzen von Kindern, Engeln, Eulen, Narren und kindergroßen Schmetterlingen lachte und höhnte und brauste es wider mich. Gräßlich erschrak ich darob: es warf mich nieder. Und ich schrie vor Grausen, wie nie ich schrie. (C.II: HK5/A17.00001 Nietzsche: Also sprach Zarathustra, Erstdruck: 1883, 2000 [S. 390])

In (170) - (172) ist der Begriff des Schreckens in den beiden Teilen der Verwendungen enthalten. Seine Wiederholung bzw. repetition hat semantic redundancy zu Folge, was zu der Verstärkung des Ausdrucks führt und die folgenden Umformulierungen ermöglicht:

(170') 'sehr ungeheuer'

$(171$ ') 'in sehr großer Angst'

(172') 'sehr erschrak'

Die durch pragmatic inferencing erworbene Gradbedeutung verweist außerdem auf das Einsetzen von subjectivity, indem das Lexem die Einstellung des Sprechers bzw. Schreibers zu der Situation zum Ausdruck bringt.

Die Korpusanalyse hat außerdem Belege zu Tage gefördert, die den Gebrauch von gräßlich in Bezug auf die den Begriff des Schreckens begleitende Bedeutung des Abscheus demonstrieren. DWB (s.v. gräszlich) berichtet von der Mehrdeutigkeit des Gebrauchs „,in der Beziehung auf hörbares und gehörseindrücke“, wobei die Intensivierung „flieszende[] Grenze " mit der Ausgangsbedeutung aufweist, „,besonders in den festen verbindungen gräszliches geheul, geschrei u. ä." vgl.:

(173) Doch endlich war mein Mut

Noch eher, als der Zahn, gebrochen.

Es riß ein gräßliches Gekrach,

Wodurch des ganzen Hauptes Knochen

Zu fpalten fchien, ein kurz doch kläglichs Ach

Mir aus der Bruft. [...] (DTA: Brockes, Barthold Heinrich: Jrdisches Vergnügen in Gott. Bd. 2. Hamburg,727)

(174) Um das fchädliche Thier zu verjagen, erregen fie (wie bereits im vorhergehenden erwähnt) einen gräßlichen Lärm mit Keffeln und Pfannen.

(DTA: [Poppe, Johann Friedrich]: Characteristik der merkwürdigsten Asiatischen Nationen. Bd. 2. Breslau, 1777)

Die Belege (173) - (174) demonstrieren mehrere Lesarten des Adjektivs. Zunächst bezeichnet das Lexem die Situationen als Schrecken erregend und zugleich unangenehm und abstoßend. Außerdem handelt es sich um die Lautstärke der Töne, welche die erwähnten gemischten Gefühle hervorruft. Der adjektivische Gebrauch weist wiederum die Entwicklung der einstellungsbezogenen bzw. expressiven Bedeutung auf, die durch den Begriff der Intensivierung zum Ausdruck gebracht wird. Hier wirkt Traugott I (oben 3.6.3.4).

In der nächsten Phase der Entwicklung tritt grässlich als Grad-Adjektiv bzw. -Adverb auf und demonstriert damit den fortgeschrittenen Prozess der Bedeutungsentleerung. Der direkte Zusammenhang mit der Bedeutung des Schreckens sowie des Abscheus geht damit verloren. Jedoch tritt gräßlich als Intensivierer zunächst 
in negativ konnotierten Kontexten, bevor diese Wertung verloren geht und das Lexem in der rein intensivierenden Funktion in Verbindung mit positiven Kontexten gebraucht wird. Diese Annahme, sowie das Auftreten der Gradbedeutung in mehrdeutigen Verwendungen, wird auch in DWB (s.v. gräszlich) bestätigt: „zunächst nur in negativ wertendem zusammenhang und dem gebrauch 2 noch näher". Da die negativ konnotierte Bedeutungskomponente von gräßlich nicht verloren gegangen ist, verweist diese Etappe des Entwicklungsgangs auf die unvollendete Desemantisierung der Ausgangsbedeutung, vgl.:

(175) Doch er raffte sich auf und schleppte mit gräßlichen Schmerzen Durch vier Tage sich fort, und endlich kam er zu Hofe. (C.II: HK3/A75.00001 Goethe: Reineke Fuchs, Erstdruck: 1794, 2000 [S. 459])

$(175$ ') 'sehr starken Schmerzen'

(176) Es ist ein Zustand der verzweifelten körperlichen Hilflosigkeit, während über das Gehirn ein grässlicher Stress aufgebaut wird, den man wehrlos ertragen muss und den man körperlich nicht entladen kann. (C.II: SOZ05/NOV.02898 Die Südostschweiz, 15.11.2005)

(176') 'sehr starker Stress'

(177) Was, am Rande bemerkt, uns mittlerweile erlaubt, endlich auch offiziell „wegen dem Regen“ sagen zu dürfen, ohne dass wir uns grässlich blamieren. (C.II: BRZ09/JUL.27818 Braunschweiger Zeitung, 31.07.2009)

(177') 'sehr blamieren'

(178) Denn infrastrukturell ist Italien ein Schwellenland, das es in den letzten 20 Jahren verpasste, die Modernisierung der Stadien voranzutreiben. Die Arenen sind daher grässlich veraltet und zudem nicht im Besitz der Klubs [...] (C.II: SOZ09/AUG.03763 Die Südostschweiz, 21.08.2009)

(178') 'sehr veraltet'

Die Intensivierung in grässlich kann sich außerdem auf Funktionsverbgefüge beziehen, womit das Gradadverb die Erweiterung seines Bezugsbereichs demonstriert, vgl. den Beleg mit der entsprechenden Wiedergabe:

(179) Wer von den solchermassen Betroffenen nun verzagt, sich händeringend in eine Ecke verkriecht und über das schreckliche Schicksal jammert, [...] der tut sich selber keinen Gefallen und gibt der Umgebung grässlich auf die Nerven [...]. (C.II: A98/APR.22873 St. Galler Tagblatt, 11.04.1998)

(179') 'sehr nervt'

(180) Das Trio geht Katy gräßlich auf den Geist. Sie sagt dem Vater, daß sie leidet wie ein Hund. (01.05.2014: http://www.zeit.de/1984/41/kratzer-amidyll)

(180') 'sehr nervt'

(181) Die restlichen Minuten vergingen wie Stunden, sie zogen sich grässlich in die Länge. (01.05.2014:http://books.google.de/books?id=4BhCgr6GNxYC\&pg=PA228\&lpg=PA228\&dq=\%22gr\%C3\%A4sslich+in+die+L\%C3\%A4nge\%22\&source=bl\&ots=qHLUnqyAGL\&sig=HDu1_R5Pq33FuFrpod53jk8zhMA\&hl=de\&-

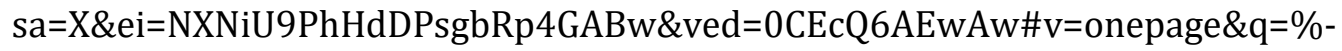
22gr\%C3\%A4sslich\%20in\%20die\%20L\%C3\%A4nge\%22\&f=false) 
(181') 'verzögerten sich sehr'

Die Korpusanalyse hat außerdem den Gebrauch des Lexems in quantifizierender Funktion dokumentiert. Dabei soll in Betracht gezogen werden, dass gräßlich die Verbindung zu den negativen Konnotationen noch nicht verloren hat, vgl.:

(182) BARON [...] Sagen Sie mir nur, Magister, was macht er mit all dem Gelde? MAGISTER Ei, Herr Baron, er macht die Mode mit, läßt Fenster einwerfen, Katzenmusiken besorgen, Fahnen sticken, stattet Freischärler aus, und das Alles, versichert er mir, kostet gräßliches Geld. (C.II: HK4/B07.00001 Birch-Pfeiffer: Vatersorgen, Entstanden: 1849, 2004 [S. 32])

(182') 'sehr viel Geld'

(183) Dabei würden Staatsdefizite eine untergeordnete Rolle spielen: «Ein Defizit an sich ist nicht so schlimm. Schlimm ist, dass einige Leute an den Zinsen so grässlich verdienen.» (A00/NOV.80297 St. Galler Tagblatt, 23.11.2000)

(183') 'sehr viel verdienen'

Die pejorative Bedeutung „sehr schlimm“ ist insbesondere für die Gegenwartssprache ausschlaggebend und demonstriert den fortgeschrittenen Prozess von bleaching der ursprünglichen Ausgangsbedeutung, vgl.:

(184) Dieser Abschnitt ist erstens in grässlichem Deutsch geschrieben, zweitens ergibt der letzte Satz keinen Sinn. (C.II: WDD11/H17.48052: Diskussion:Heimdall, In: Wikipedia - URL:http://de.wikipedia.org/wiki/Diskussion:Heimdall: Wikipedia, 2011)

(184') 'in sehr schlechtem Deutsch'

(185) Herr Rapp war ja [...] eine Zeitlang in eine gräßliche finanzielle Lage geraten. (C.II: 099/JUN.75653 Neue Kronen-Zeitung, 01.06.1999, S. 62)

$(185$ ') 'sehr schlechte finanzielle Lage'

(186) Dass die Reithalle nicht funktioniert wie zugedacht, weiss man ja seit einem Jahrzehnt - keine Lüftung, grässliche Akustik. (A99/MAI.33012 St. Galler Tagblatt, 10.05.1999)

(186') 'sehr schlechte Akustik'

Die Entstehung der intensivierenden (175) - (181), quantifizierenden (182) - (183) und pejorativen (184) - (186) Bedeutung demonstriert die Bedeutungsentwicklung auf der expressiven Ebene. Aus den Belegen geht hervor, dass der Gebrauch „more subjective“ (Traugott 1989: 35) bzw. „more anchored in ... the speakers's orientation ro situation" (Traugott 1982: 253) wird und damit den Übergang auf die rein expressive Ebene demonstriert. Dieses Phänomen lässt sich im Rahmen von Traugott III (oben 3.6.3.4) (Traugott III, oben 3.6.3.4) erklären.

DWB (s.v. gräszlich) berichtet anschließend von der „sehr viel selteneren, aber schon verhältnismäszig frühen anwendung auf neutrale gröszen oder auf positive werte“. Es soll jedoch darauf hingewiesen werden, dass das Auftreten von gräßlich als Intensivierer in positiv konnotierten Kontexten nach dem Auftreten in negativ konnotierten Kontexten erfolgte, vgl.: 
(187) es wúerde die sach der heyligen religion zu Costnitz (Konstanz) und in ander weg greslich gefierdert werden (, wenn der als tüchtig und gelehrt bekannte herr v. Lunda bischof würde (DWB: 1537, s.v. gräszlich)

(187') 'sehr gefördert'

(188) wann ihr nicht an meiner (der amme) brust gesogen hättet, meinet ihr dann, dasz ich euch so gräszlich lieben würde (DWB: 1711, s.v. gräszlich)

(188') 'sehr lieben'

(189) wenn du nun zum könig kommst, der die wunderschöne prinzessin verlangt, so sag ihm: hier wäre sie. darauf wird gräszliche freude sein (DWB: 1812, s.v. gräszlich)

(189') 'sehr große Freude'

Der Gebrauch in (187) - (189) demonstriert die rein intensivierende Funktion von grässlich, indem das Lexem als Grad-Adjektiv bzw. -Adverb fungiert, ohne den Kontakt mit der Ausgangsbedeutung aufzuweisen. Obgleich im Korpus keine Belege zu finden sind, die eine solche Verwendung des Lexems veranschaulichen sollen, hat die weitere Suche im Internet einige Kontexte ergeben, vgl.:

(190) Sie war bisher seine stumme Bewunderung gewesen, aber heute dachte er nur noch an Ada Gerlach, ihren blonden Schopf und ihr niedliches Naschen; er war gräßlich verliebt. (GB, 06.05.2014: http://books.google.de/books?id=JKIGAQAAIAAJ\&q =\%22noch +an+Ada+Gerlach,+ihren+blonden+Schopf+und+ihr+niedliches+Naschen;+er+war+gr\%C3\%A4\%C3\%9Flich+verliebt $\% 22$ $\& \mathrm{dq}=\% 22$ noch + an + Ada + Gerlach,+ihren+blonden+Schopf+und+ihr+niedliches+Naschen;+er+war+gr\%C3\%A4\%C3\%9Flich+verliebt\%22\&hl=de\&sa=X\&ei=KddoU6yoK-LnywPK1oLwBw\&ved=0CDQQ6AEwAA)

(190') 'sehr verliebt'

(191) Die Sprache ist grässlich interessant. (06.05.2014: http://caladrien.blogspot.de/-2012/12/ix-ulaanbaatar.html)

(191') 'sehr interessant'

Die Belege (187) - (191) veranschaulichen die eindeutig intensivierende Bedeutung von grässlich. Das Lexem hat damit den Prozess der Desemantisierung vollkommen vollzogen und den Zusammenhang mit der Ausgangsbedeutung endgültig beseitigt.

\subsubsection{Kurzfassung der Entwicklung bei grässlich}

Ausgangsbedeutung: 'Schrecken hervorrufend' mit dem gelegentlich einwirkenden Nebenbegriff des Abscheus; die übrigen Entwicklungsschritte wie oben in Kap. 3.6.3.9.3.

\subsubsection{Grauenhaft}

\subsubsection{Lexikographische Angaben}

Kluge (s.v.) bezeichnet grauenhaft als adjektivische Ableitung von grauen. Laut DWB (s.v.) und Pfeifer (s.v.) ist das Lexem „, seit dem 18. jh. bezeugt“ (DWB, s.v.).

1. grauenhaft in DWB (s.v.) 
1.1 „schrecken erregend, entsetzlich, je nach dem sachzusammenhang auch nuanciert, gefährlich, bedrohlich, beängstigend, quälend u. ä."

1.2 „mehr im umkreis von grauen, n. A 1 “53 „entsetzlich im sinne des abscheu erregenden oder eine verurteilung herausfordernden".

1.3 „in adverbialem gebrauch vorwiegend im sinne von 1, aber doch auch in dem von 2 “. "gern neben adjektiven; mit der charakterisierenden bedeutung 'erschreckend, entsetzlich' verbindet sich leicht eine verstärkende und steigernde 'sehr, in hohem masze'"

2. grauenhaft in DUDEN (2012, s.v.)

2.1 "Grauen hervorrufend“

2.2 „(umgangssprachlich)“

2.2a „in besonders starkem Maße als unangenehm empfunden“

$2.2 \mathrm{~b}$ „<intensivierend bei Adjektiven und Verben> sehr, arg; in schrecklicher Weise"

\subsubsection{Entwicklung der Gradbedeutung bei grauenhaft}

Die Analyse der Beleglage legt nahe anzunehmen, dass das Einsetzen der Gradbedeutung im Nhd. erfolgte. Die Grundlage dafür bietet die Sinnrichtung, die verschiedene Situationen als Grauen, Schreckend erregend charakterisiert und ferner als Ausgangsbedeutung bezeichnet wird, vgl.:

(192) Das Feld liegt grauenhaft mit Leichen und mit Stümmeln Von Roß und Mann bedeckt, die durch einander wimmeln. (C.II: HK3/B00.00006 Wieland: Oberon, Erstdruck: 1780, 2000 [S. 183])

(192') 'Grauen erregend bedeckt'

(193) Es war mir, als sei nun das bedrohliche grauenhafte Wesen, das mich in der Ferne geängstigt, recht in mein Leben getreten und als müsse ich wachen und auf sorglicher Hut sein für meinen Freund und für mich selbst. (HC.II: K3/D64.00001 Hoffmann: Die Elixiere des Teufels, Entstanden: 1814/15, 2000 [S. 69])

(193') 'Grauen erregendes Wesen'

In Bezug auf die Gradbedeutung verweist DWB auf Ausdrücke, „die etwas Regelwidriges, Böses, Schlechtes bezeichnen“ und „deren negativer Sinn oft mehr verstärkend als charakterisierend“ wirkt. Darüber hinaus tritt die Gradbedeutung zunächst in ambigen Belegen auf und weist einen Zusammenhang mit dem ursprünglichen Gehalt auf. Damit sind die Verwendungen von grauenhaft mit dem Prozess von pragmatic strengthening bzw. enrichment verbunden. Die Beleglage lässt darauf schließen, dass die Gradbedeutung zuerst als Nebenbedeutung neben der Ausgangsbedeutung in negativ konnotierten Kontexten durch den Prozess des pragmatic inferencing in Erscheinung tritt, wobei die Gradbedeutung anfangs noch sehr stark von der Ausgangsbedeutung beeinflusst ist. Der adjektivische und adverbiale mehrdeutige Gebrauch wird in den folgenden Belegen deutlich:

(194) Es fchlug Mitternacht, als Wally das fauber gefchriebene Heft durchlefen hatte. [...] Rings war alles grauenhaft ftill, nur der Uhrpendel fchwang

\footnotetext{
53 „ekel, abscheu, sittliches entsetzen, entrüstung“ (DWB, s.v. grauen)
} 
fich unterm Glafe hin und her und zählte die Minuten, die den Geiftern auf Erden zu wandeln vergönnt waren. (DTA: Gutzkow, Karl: Wally, die Zweiflerin. Mannheim, 1835)

(195) Bei seinem Anblick und bei seinen Erzählungen von den letzten Vorfällen der Tagesgeschichte erwachte ich wie aus einem tiefen Traume, und zusammenschreckend fühlte ich plötzlich, in welcher grauenhaften Einsamkeit ich so lange für mich hingelebt. (C.II: HK3/A68.00008 Heine: Florentinische Nächte, Erstdruck: 1836, 2000 [S. 119])

(196) Acht Tage später las ich [...] im Amtsblatt folgende Bekanntmachung: „In vergangener Nacht wurden in der hiesigen Ortskirche grauenhafte Zerstörungen angerichtet. Die Bildsäulen der Heiligen und Kirchenväter wurden von ihren Sockeln gestürzt, die Embleme ihnen aus der Hand gebrochen, Arme und Beine abgeschlagen ec. [...]“ (C.II: HK3/G54.00001 Panizza: Visionen, Erstdruck: 1893, 2000 [S. 180])

(197) Und Alwine - sie wurde leichenbla $\beta$ und schrie so grauenhaft, wie er noch nie einen Menschen hatte schreien hören, schlug um sich, daß die nassen Arme in der Sonne funkelten, und schwamm in rasender Eile davon. (C.II: HK4/R14.00001 Reventlow: Der Herr Fischötter, Erstdruck: 1917, 2004 [S. 372])

Die Belege (194) - (197) gestatten eine mehrdeutige Interpretation. Zunächst bezieht sich das Lexem direkt auf die Ausgangsbedeutung und beschreibt die Ereignisse als Grauen erregend bzw. demonstrierend, vgl. die entsprechenden Umformulierungen:

(194') 'Grauen erregend und still'

(195') 'Grauen erregende Einsamkeit'

(196') 'Grauen erregende Zerstörungen'

(197') 'Grauen erregend' bzw. 'Grauen demonstrierend'

Zugleich jedoch weist grauenhaft eine zweite Interpretationsmöglichkeit auf, die das Lexem als ein Intensivierungsmittel darstellt. Aus den Kontexten lässt sich schließen, dass der Grad der Erscheinungen sehr hoch ist und die Ursache der hervorgerufenen, mit Schrecken verbundenen Gefühle zum Ausdruck bringt, vgl. die folgenden Paraphrasierungen:

(194") 'vollkommen still bzw. in so hohem Grad still, dass es Grauen erregend wirkt'

(195") 'sehr große und deswegen Grauen erregende Einsamkeit'

(196") 'sehr große, von daher Grauen erregende Zerstörungen'

(197") 'sehr laut, sodass es Grauen erregend wirkt'

DWB (s.v.) verweist ferner darauf, dass bei grauenhaft sich außerdem „gern neben Adjektiven; mit der charakterisierenden Bedeutung 'erschreckend, entsetzlich' [...] eine verstärkende und steigernde 'sehr, in hohem masze“" Bedeutung „verbindet“. Die Gradbedeutung tritt damit infolge von semantic repetition auf, indem sie durch die Wiederholung des gemeinsamen Begriffs 'Furcht' erscheint, der sich in den beiden Kookkurrenzpartnern erkennen lässt, vgl. die Belege: 
(198) „[...] So wie die Somnambule sich durchaus nicht ihres somnambulen Zustandes erinnert und dessen, was sich in demselben mit ihr begeben, so kann vielleicht jene grauenhafte Angst, deren Ursache uns verborgen bleibt, der Nachhall irgendeines gewaltigen Zaubers sein, der uns uns selbst entrückte." (C.II: HK3/E00.00001 Hoffmann: Die Serapionsbrüder, Erstdruck: 1819-1821, 2000 [S. 124])

(199) Seitdem ich nämlich tief begriffen habe, welcher schnöde Egoismus auch in ihrer Politik waltet, erfüllen mich diese Engländer mit einer grenzenlosen, grauenhaften Furcht. (C.II: HK3/F47.00001 Heine: Lutetia, 1840, 2000 [S. 445])

In den Belegen (198) - (199) lässt sich das Einsetzen von subjectivity erkennen, indem der Gebrauch von grauenhaft die Einstellung des Sprechers zu der Situation sowie den Übergang von der propositionalen zu der expressiven Ebene erkennen lässt. Damit kommt Traugott I (oben 3.6.3.4) zur Geltung.

In der nächsten Phase der Bedeutungsentwicklung tritt grauenhaft als Intensivierungsmittel in Verbindung zu negativ konnotierten Kookkurrenzpartnern. Dabei demonstrieren die Belege den Verlust des direkten Zusammenhangs der Bedeutung mit der Ausgangsbedeutung, vgl. den adjektivischen und adverbialen Gebrauch mit entsprechenden Umformulierungen:

(200) aufbett und stühlen war die grauenhafteste unordnung (DWB: 1895, s.v. grauenhaft)

(200') 'größte Unordnung'

(201) Die Folge war ein grauenhafter Muskelkater. (C.II: A08/NOV.06313 St. Galler Tagbl., 19.11.2008, S. 35)

(201') 'sehr starker Muskelkater'

(202) Daran wird auch der grauenhaft frühe Heimflug für Viktoria Rebensburg nichts mehr ändern können. (C.II: NUZ07/FEB.01577 NZ, 15.02.2007)

(202') 'sehr früher Heimflug'

(203) "Leider bin ich grauenhaft erkältet", sagte er und schnäuzte sich ausgiebig. (C.II: RHZ09/MAR.17877 RZ, 20.03.2009)

(203') 'sehr erkältet'

(204) Dabei müsse doch allen klar sein: "In München sind die Mieten grauenhaft hoch..." (C.II: NUN97/FEB.00703 NN, 08.02.1997, S. 21)

(204') 'sehr hoch'

Die intensivierende Funktion des Lexems findet sich außerdem in Bezug auf Funktionsverbgefüge, die in den Skopus des Gradadverbs geraten und die Erweiterung der Skopuskonstituente demonstrieren, vgl.:

(205) Das führt dazu, daß unsere Versammlungen sich grauenhaft in die Länge ziehen und zu einer Folterqual werden. (GB, 01.05.2014: http://books.google.de/books? id=3fxEAAAAMAAJ\&q=\%22grauenhaft+in+die+1\%C3\%A4nge\%22\&dq=\%22grauenhaft+in+die+1\%C3\%A4nge\%22\&hl=de\&sa=X\&ei=86hiU8joJo2K4gTCroHgAg\&ved=0CFMQ6AEwBg) 
(206) Es ging einem oft natürlich grauenhaft auf die Nerven, aber auf der anderen Seite hat das immer dazu geführt, dass man das, was man gerade machte, wirklich zum absolut Besten entwickelt hat. (01.05.2014: http://www.deutschlandradiokultur.de /viel-verpackte-luft.954.de.html?dram:article_id=240621)

(207) Was mir als Befürworter der Energiewende aber grauenhaft auf den Wecker geht, ist, dass viele Leute hier offenbar gar nicht willens sind, Lösungen für die Probleme zu finden. (01.05.2014: http://www.tagesanzeiger.ch/wirtschaft/konjunktur/Windstrom-zum-Nulltarif/story/12476111?comments=1)

In (205) - (207) hat grauenhaft Skopus über die Funktionsverbgefüge in die Hose gehen, jemandem auf die Nerven / auf den Wecker gehen. Das Auftreten in der bezeichneten Position wird dadurch ermöglicht, dass die FVGs durch einen graduierbaren Begriff paraphrasiert werden können, vgl.:

(205') 'sehr misslingen'

(206') 'sehr nervte'

(207') 'sehr nervt'

Das Lexem drückt außerdem das Auftreten der quantifizierenden Funktion in einem negativ konnotierten Kontext aus, vgl.:

(208) „Tausende von Menschen aus den Städten eilen auf die Dörfer um Lebensmittel hierbei zu holen. Grauenhafte Preise werden bezahlt und von Tag zu Tag steigen die Preise" (Chronik Atteln, März 1919) (01.05.2014: http://books.google.de/books?id=CffezK2dqpAC\&pg=PA33\&dq=\%22Grauenhafte+Preise $\% 22 \&$ hl=de\&sa=X\&ei=WapiU96gCsGH4ASHloH4DQ\&ved=0CDIQ6AEwAA\#v=onepage $\& \mathrm{q}=\% 22 \mathrm{Grauenhafte} \% 20$ Preise $\% 22 \& \mathrm{f}=$ false)

(208') 'sehr hohe Preise'

Das Lexem wird zudem mit rein pejorativer Bedeutung gebraucht, wobei der direkte Zusammenhang mit der Ausgangsbedeutung nicht mehr zu erkennen ist, vgl. die Belege mit den entsprechenden Umformulierungen:

(209) Sie schmeckten einfach grauenhaft, zumindest dämpften sie für kurze Zeit das quälende Hungergefühl. (C.II: DIV/AKU.00001 Krahl: "Und was gibt's noch?", 2004 [S. 220])

(209') 'schmecken sehr schlecht'

(210) Dieser Artikel (zumindest der Anfang) ist doch grauenhaft zu lesen! Unzusammenhaengende Saetze, stilistisch auf dem Niveau eines Erstklaessleraufsatzes... (C:II: WDD11/G14.45905: Diskussion: Gratiszeitung, In: Wikipedia - URL:http://de.wikipedia.org/wiki/Diskussion:Gratiszeitung: Wikipedia, 2011)

(210') 'sehr schlecht zu lesen bzw. sehr schlecht geschrieben'

Die Belege (200) - (210) demonstrieren die Entwicklung der Bedeutung auf der expressiven Ebene, indem der Gebrauch im Rahmen von Traugott III (oben 3.6.3.4) mehr einstellungsbezogen wird. 
Die letzte Etappe des Entwicklungsgangs, bei der das Lexem mit positiv konnotierten Kookkurrenypartnern kollokiert und damit einer kompletten Desemantisierung unterzogen wird, konnte bei grauenhaft in den untersuchten Korpora nicht festgestellt werden. Damit gilt der Prozess der Bedeutungsentleerung nicht als beendet.

\subsubsection{Kurzfassung der Entwicklung bei grauenhaft}

1. Ausgangsbedeutung: 'Grauen hervorrufend'.

6. Reine Gradbedeutung: Die Korpusanalyse hat keine Verwendungen ergeben, die einen Gebrauch des Lexems mit positiv konnotierten Bezugswörtern belegen; die übrigen Entwicklungsschritte wie oben in Kap. 3.6.3.9.3.

\subsubsection{Grauenvoll}

\subsubsection{Lexikographische Angaben}

Kluge (s.v.) erklärt grauenvoll als Ableitung von grauen (Verb), liefert jedoch keine weiteren Informationen über die Herkunft des Lexems. Laut DWB (s.v.) tritt die Form im 17. Jh. auf und ist „seit dem 18. häufiger bezeugt“. Pfeifer (s.v.) verweist auf das Auftreten des Lexems im 18. Jh.

1. grauenvoll in FWB keine Angaben, auch nicht im unveröffentlichten Material der Redaktion des FWB

2. grauenvoll in DWB (s.v.)

2.1 „die empfindung der furcht, des schreckens, des entsetzens auslösen, oder, bei entsprechendem beziehungswort, mit solchen empfindungen verbunden u. ä."

2.2 „die empfindung des abscheus, des sittlichen oder ästhetischen entsetzens, der entrüstung auslösend, manchmal geradezu im sinne von 'böse, verrucht' u. ä."

2.3 „vereinzelt im wortsinn der komposition von grauen, n. A und voll, 'voller furcht, entsetzen'“

2.4 ,in der funktion eines adverbs und eines prädikativen attributs nicht immer klar zu scheiden"

$2.4 \mathrm{a}$ „als adverb, meist in der bedeutung 1 , neben verben, meist solchen akustischer bedeutung“. „neben adjektiven oder partizipien, diese manchmal mehr verstärkend als bestimmend"

$2.4 \mathrm{~b}$ „als prädikatives attribut. die empfindung des schreckens oder des abscheus auslösend“

3. grauenvoll in DUDEN (2012, s.v. grauenvoll)

3.1 „grauenhaft (1)“

3.2 „(umgangssprachlich) grauenhaft (2)“ 


\subsubsection{Entwicklung der Gradbedeutung bei grauenvoll}

Die Entwicklung der Gradbedeutung beginnt im Neuhochdeutschen, worauf die Angaben von DWB sowie die Ergebnisse der durchgeführten Korpusanalyse hinweisen. Als Grundlage für ihre Herausbildung dient die in 2.1 angegebene Sinnrichtung, die Furcht auslösende Situationen beschreibt und im weiteren als Ausgangsbedeutung angesehen wird, vgl.:

(211) O Scipio, schwere Lasten liegen auf dieser Brust - ein Gedanke, grauenvoll wie die lichtscheue Nacht - ungeheuer genug, eine Mannsbrust zu sprengen - Siehst du? (C.II: HK3/E46.00001 Schiller: Fiesco, Entstanden: 1782, 2000 [S. 696])

(211') 'Grauen erregender Gedanke'

(212) Und wie grauenvoll ist die Gestalt, welche der Traum meinem künftigen Erben giebt! (C.II: HK4/N04.00001 Naubert: Volksmährchen der Deutschen, Erstdruck: 1789-1792, 2004 [S. 88])

(212') 'Grauen erregende Gestalt'

(213) Ich sagte ihr Alles zu, was sie verlangte, und wir benutzten die letzten Stunden der Nacht, die grauenvolle That zu verbergen, die sich in ihren Schatten zugetragen hatte. (C.II: HK4/N04.00001 Naubert: Volksmährchen der Deutschen, Erstdruck: 1789-1792, 2004 [S. 114])

(213') 'Grauen erregende Tat'

Das Einsetzen der Intensivierung beginnt mit pragmatic strengthening bzw. enrichment der Ausgangsbedeutung und lässt sich in Ambiguität des Gebrauchs aufweisenden Belegen feststellen. Der Prozess der semantischen Reanalyse erfolgt durch pragmatic inferencing, wobei die Gradbedeutung als eine alternative Interpretationsmöglichkeit neben der Ausgangsbedeutung abgeleitet wird, vgl.:

(214) Eine grauenvolle Stille herrschte in ihm und um ihn, so daß einen Augenblick die fixe Idee sich seiner bemächtigte, er sei gestorben und liege im Grabe. (C.II: HK4/A18.00001 Aston: Lydia, Erstdruck: 1848, 2004 [S. 102])

(215) Einer Prophetin der Zukunft gleich stand Ines vor dem Fürsten, der bleich und zitternd das Auge vor der erhabenen Cassandra der Rache nicht aufzuschlagen wagte. Noch einen Blick warf sie auf ihn, in dem sich eine grauenvolle Tiefe des Hasses offenbarte. (C.II: HK4/A19.00001 Aston: Revolution und Contrerevolution, Erstdruck: 1849, 2004 [S. 169])

(216) Auch sonst finden sich viele Gräber, die das gleiche Todesjahr tragen ... [...] Oder aus diesem Jahrhundert: aus jenen drei gräßlichen Sommern, da der Zorn Gottes, die Cholera, in der großen Ebene wütete. Das Gras setzt der Sense mehr Widerstand entgegen, als damals diese Menschen in ihren engen, verpesteten Städten der grauenvollen Seuche. Die Gräber sind zahllos und es ist ein überaus großes Leichenfeld, obwohl die Gemeinde gerade nicht übergroß ist. (C.II: HK3/D79.00001 Franzos: Die Juden von Barnow, Entstanden: 1868 bis 1872, 2000 [S. 263])

(217) Grauenvoll heulte der Verletzte auf, fo laut, daß die Höhle von dem Gebrüll wiederhallte; und wir, vor Angft bebend, flüchteten in den äußerften Winkel der Grotte. Schwab, Gustav: Die schönsten Sagen des klassischen Alterthums. Bd. 3. Stuttgart, 1840 
Die Belege (214) - (217) lassen eine mehrdeutige Interpretation zu. Zunächst bezieht sich das Lexem auf die Ausgangsbedeutung und beschreibt die Ereignisse als Furcht erregend, sodass die folgenden Paraphrasen gelten können:

(214') 'Furcht erregende Stille'

(215') 'Furcht erregende Tiefe'

(216') 'Furcht erregende Seuche'

(217') 'heulte Furcht erregend auf'

Aus dem Kontext folgt außerdem eine weitere Interpretationsmöglichkeit, die eine intensivierende Funktion von grauenvoll einschließt. Diese Interpretation beruht auf pragmatic inferencing der Ausgangsbedeutung und erfolgt durch das Ableiten einer entsprechenden Inferenz. Dabei begleitet die Gradbedeutung die Ausgangsbedeutung als Nebenbedeutung und stellt den Grund des hervorgerufenen Gefühls dar, vgl. die Umformulierungen:

(214") 'mit der Stille in einem Grab verglichene und damit absolute, stark ausgeprägte und von daher Furcht erregende Stille'

(215") 'eine sehr starke und deswegen furchterregende Tiefe des Hasses'

(216") 'die Seuche ist sehr stark ausgeprägt und deswegen Furcht erregend'

(217") 'heulte sehr laut auf, was Furcht erregend wirkte'.

Das Auftreten der Intensivierung wird außerdem durch semantic repetition ermöglicht:

(218) ...welche Tage des grauenvollen Entsetzens, des Schreckens, liegen zwischen meinen letzten Zeilen an Dich! (C.II: HK4/W17.00001 Wolzogen: Erzählungen, Erstdruck: 1826, 2004 [S. 148])

(219) Hummer hatte seit seiner Verhaftung nichts gegessen. Die grauenvolle Furcht vor der Folter hatte seine Kehle zusammengeschnürt. (GB, 05.05.2014: http://books.google.de/books?id=KX3ZAAAAMAAJ\&q=\%22grauenvolle+Furcht $\% 22 \& d q=\% 22$ grauenvolle+Furcht $\% 22 \& h l=d e \& s a=X \& e i=m 3$ tnU5apIZSQ4gSi54DgAw\&ved=0CDUQ6AEwATgU)

(220) Als sie gegangen war und er mit beiden Händen ins Leere griff, packte ihn eine grauenvolle Angst vor der Vergänglichkeit. (C.II: HK3/C76.00001 Klabund, Bracke, Erstdruck: 1918, 2000 [S. 141])

Die Belege (218) - (220) weisen mehrere Lesarten auf. Die erste Lesart ist auf die Ausgangsbedeutung von grauenvoll bezogen und legt den Akzent auf die Begriffe 'Angst', 'Schrecken' und 'Furcht'. Die zweite Lesart setzt die durch semantic repetition gewonnene Gradbedeutung voraus. Die Intensivierung tritt durch das Wiederholen von synonymischen Begriffen innerhalb einer Kollokation auf, führt zu semantic redundancy der Verwendungen und bringt damit den Grad der Gefühle zum Ausdruck, vgl. die Paraphrasierungen:

(218') 'sehr großen Entsetzens'

(219') 'sehr große Furcht'

(220') 'sehr große Angst'

Der Prozess des bleaching wird von der Entwicklung der einstellungsbezogenen Se-mantik begleitet. Aus den eingeführten ambigen Belegen geht das Einsetzen von 
subjectivity hervor, wo sich der Gebrauch auf die propositionale sowie die expressive Bedeutung bezieht und auf Traugott I (oben 3.6.3.4) hinweist.

Ferner finden sich im Korpus Belege, die die fortgeschrittene Entwicklung der intensivierenden Funktion des Lexems demonstrieren. Dies lässt sich vor allem im Verblassen der Ausgangsbedeutung erkennen. Die Desemantisierung des Lexems besteht in dem Verlust des direkten Kontakts mit der Ausgangsbedeutung, vgl. den adjektivischen und adverbialen Gebrauch:

(221) »Master James schläft noch. - Er muss gestern grauenvoll besoffen gewesen sein!" (C.II: NUZ03/MAR.02177 Nürnberger Zeitung, 22.03.2003)

(221') 'sehr besoffen'

(222) Der Wind pfeift durchs Stadion, es nieselt - das Spiel ist grauenvoll langweilig. (C.II: NUN08/MAI.01901 Nürnberger Nachrichten, 19.05.2008, S. 19)

(222') 'sehr langweilig'

(223) Das Bild von Repin ist ja wohl grauenvoll kitschig und bedient Vorurteile, die eigentlich nicht ins 21. Jahrhundert passen. Italienerinnen sehen heute anders aus. (C.II: WDD11/I13.65509: Diskussion:Italiener)

(223') 'sehr kitschig'

(224) Ich habe Durst, grauenvollen Durst, alle Trinkgeräte sind zerbrochen, alle Getränke vergiftet, ich sterbe, hörst Du? (GB, 02.05.2014: http://books.google.de/books?id= JZtJAAAAMAAJ\&q=\%22grauenvollen+durst $\% 22 \& \mathrm{dq}=\% 22$ grauenvollen+durst $\% 22 \& \mathrm{hl}=$ de\&sa=X\&ei=119jU4j10oid0QX0hoDQAQ\&ved=0CDgQ6AEwAQ)

(224') 'sehr starken Durst'

Grauenvoll kann außerdem als Gradadverb Skopus über Funktionsverbgefüge haben und damit die Änderung bzw. Erweiterung seiner Skopuskonstituente demonstrieren. Die Intensivierung erfolgt dadurch, dass ein Bezugswort in einem FVG intensivierbar ist, wie es in (225) der Fall ist, oder das ganze FVG durch einen graduierbaren Begriff wiedergegeben werden kann, vgl. (226) - (227):

(225) Der typische Weihnachts-Shopper an diesem dritten Adventssamstag schleppt mindestens drei Tüten, hat ein schmerzverzerrtes Gesicht [...], ist grauenvoll unter Zeitdruck und - kommt nicht voran. (C.II: NUZ06/DEZ.01894 NZ, 18.12.2006)

$(225$ ') 'ist unter sehr großem Zeitdruck'

(226) „Schulsystemdebatten gehen den Menschen grauenvoll auf die Nerven. [...]" (C.II: NUZ11/OKT.01846 NZ, 22.10.2011, S. 11)

(226') 'sehr nerven'

(227) Jennifer Connelly und Filmtochter sitzen grauenvoll in der Patsche (C.II: HMP05/SEP.02039 MOPO, 22.09.2005, S. 6-7)

(227') 'sind in sehr großen Schwierigkeiten'

Die intensivierende Funktion des Lexems kann in die quantifizierende übergehen, ohne negative Konnotationen dabei zu verlieren, und die Bedeutung "sehr viel“" zum Ausdruck bringen, dabei vgl.: 
(228) Und die Preise an der Bar sind GRAUENVOLL! (02.05.2014: http://www.tripadvisor.de / ShowUserReviews-g274812-d601290-r149809702-Quality_Hotel_WroclawWroclaw_Lower_Silesia_Province_Southern_Poland.html)

(228') 'die Preise sind sehr hoch'

Das Lexem demonstriert außerdem die Herausbildung einer pejorativen Bedeutung, die durch bleaching der Ausgangsbedeutung erfolgt. Damit verliert grauenvoll den Zusammenhang mit der Ausgangsbedeutung und demonstriert die rein expressive Bedeutung:

(229) Der Artikel ist fast wortwörtlich der englische Artikel, dabei aber grauenvoll übersetzt. (C.II: WDD11/B10.92193: Diskussion:Brian Molko, In: Wikipedia - URL:http:/ / de.wikipedia. \org/wiki/Diskussion:Brian_Molko: Wikipedia, 2011)

(229') 'sehr schlecht übersetzt'

(230) Aber das ist stilistisch grauenvoll und bleibt für den Leser ohne entsprechende Erläuterung des historischen Hintergrundes zudem völlig unverständlich. (C.II: WDD11/A58.51969: Diskussion:Adolf Hitler/Archiv/2010/2. Teilarchiv, In: Wikipedia - URL:http://de.wikipedia.org/wiki/Diskussion:Adolf_Hitler/Archiv/2010/2._Teilarchiv: Wikipedia, 2011)

(230') 'stilistisch sehr schlecht'

(231) Ich habe mich schon bei Edeka und Penny beschwert, weil auch die BioMilch [...]ziemlich grauenvoll schmeckt. (C.II: HMP09/JAN.00212 MOPO, 04.01.2009, S. 19)

(231') 'sehr schlecht schmeckt'

Die Belege (221) - (227), (228) und (229) - (231) demonstrieren den eindeutigen Gebrauch des Lexems jeweils in der intensivierenden, quantifizierenden und pejorativen Bedeutung, die keinen direkten Kontakt mit der Ausgangsbedeutung aufweist. Zugleich ist diese Phase des Entwicklungsgangs mit dem Zuwachs an subjectivity verbunden. Der letzte signalisiert die Weiterentwicklung der expressiven Bedeutung und daher den Übergang zu Traugott III.

Die Korpusanalyse hat keine Belege gezeigt, die die eindeutig positiv konnotierte Gradbedeutung von grauenvoll demonstrieren können. Darüber hinaus bleibt der Prozess der Desemantisierung nicht vollkommen vollzogen.

\subsubsection{Kurzfassung der Entwicklung bei grauenvoll}

1. Ausgangsbedeutung: 'Schrecken hervorrufend'.

6. Reine Gradbedeutung: Die Korpusanalyse hat keine Verwendungen ergeben, die einen Gebrauch des Lexems mit positiv konnotierten Bezugswörtern belegen; die übrigen Entwicklungsschritte wie oben in Kap. 3.6.3.9.3.

\subsubsection{Grausam}

Die Beschreibung findet sich in dem Kapitelabschnitt 4.2 'Übel wollend / verursachend' 


\subsubsection{Grausig}

\subsubsection{Lexikographische Angaben}

Laut Kluge (s.v.) und DWB (s.v.) stellt grausig eine Ableitung von grausen dar. DWB (s.v.) ordnet das Wort ein als „,frühe ableitung von grausen [...] in vereinzelter ahd. bezeugung im sinne eines gewichtigen 'schrecklich'“, fügt jedoch hinzu, dass das Lexem „seit dem 18. jh. als neubildung zu grausen, vb. oder zu graus, adj. (s. d.), ausgehend von der vorstellung 'schaurig, einen schauder der furcht oder des entsetzens erregend, geeignet, eine furchtempfindung auszulösen'“ in Erscheinung tritt. Es finden sich jedoch weitere Informationen über die Herkunft sowie die Bedeutungsliste des Lexems weder in BMZ und Lexer noch in FWB.

1. grausig in FWB

keine Angaben, u.a. im bereits zitierten Material der Redaktionsstelle von FWB

2. grausig in DWB (s.v.)

2.1 „als frühe ableitung von grausen [...] in vereinzelter ahd. bezeugung im sinne eines gewichtigen 'schrecklich'"

2.2 „seit dem 18. jh. als neubildung zu grausen, vb. oder zu graus, adj. (s. d.), ausgehend von der vorstellung 'schaurig, einen schauder der furcht oder des entsetzens erregend, geeignet, eine furchtempfindung auszulösen'“ $2.2 \mathrm{a}$ "das moment des furcht erregens steht im vordergrund“

$2.2 \mathrm{~b}$ „das moment des abscheu und anstosz erregens tritt in den vordergrund"

$2.2 \mathrm{c}$ „als glied eines oxymorons“

$2.2 \mathrm{~d}$ „qualitätsärmer und verstärkend“

3. grausig in DUDEN (2012, s.v.)

3.1 „Grausen hervorrufend; grauenvoll, entsetzlich, fürchterlich“

3.2 „(umgangssprachlich)“

3.2a „in besonders starkem Maße wie eine Art Pein empfunden; sich kaum ertragen lassend; sehr schlimm"

$3.2 \mathrm{~b}$ „<intensivierend bei Verben und Adjektiven> in kaum erträglicher Weise; sehr, überaus“"

\subsubsection{Entwicklung der Gradbedeutung bei grausig}

Die Korpusanalyse verortet die Gradbedeutung im Nhd. Als Quelle für deren Herausbildung geht DWB von der in 2.2a erwähnten Bedeutung aus, die verschiedene Sachverhalte durch den Begriff der Furcht bezeichnet, vgl.:

(232) Eilig stürzte ich nun fort von diesem grausigen Orte. (C.II: HK3/HK1.00011 Eichendorff: Die Zauberei im Herbste, Entstanden: 1808/09, 2000 [S. 520])

(232') 'Furcht erregenden Orte'

(233) Höre lieber das geheimnisvolle grausige Hexenlied, das ich noch treu im Gedächtnis trage. (C.II: HK3/E37.00001 Hoffmann: Fantasiestücke in Callots Manier, Entstanden: 1808/15, 2000 [S. 158])

(233') 'Furcht erregendes Hexenlied'

(234) hört ihr den sturmruf durch die grausige nacht (DWB: 1820, s.v.)

(234') 'Furcht erregende Nacht' 
In Bezug auf die Gradbedeutung verweist DWB darauf, dass grausig „qualitätsärmer und verstärkend" fungiert. Daraus lässt sich schließen, dass das Lexem sich zunächst auf die qualifizierende Bedeutung bezieht und zugleich eine verstärkende Funktion annimmt, wobei die erste anschließend verblasster wirkt. Darüber hinaus tritt grausig anfangs in ambigen Kontexten auf. Die Korpusanalyse bestätigt diese Annahme. Die Beleglage zeigt, dass die Ausgangsbedeutung zuerst pragmatic strengthening bzw. enrichment unterliegt, sodass die Gradbedeutung in einer frühen Etappe des Entwicklungsgangs als Nebenbegriff bzw. Inferenz in negativen Kontexten in Erscheinung tritt. Darüber hinaus demonstriert das Lexem den direkten Zusammenhang mit der Ausgangsbedeutung und dient zugleich der Verstärkung des modifizierten negativen Begriffs. Unter der Ausgangsbedeutung wird der mit Angst verbundene Sinngehalt verstanden. Die Mehrdeutigkeit der Kontexte sollen die Belege (235) - (237) veranschaulichen:

(235) Stromauf, schräg über, nach dem Lichtsignale, Sie schritten schnell und schweigsam durch die Nacht, Erhellt von keines Sternes bleichem Strahle; In Nebeln, von dem Winde hergefacht, Schien ihnen oft das Lichtlein zu verschweben; Sie schritten zu, als ging es in die Schlacht. Sie fühlten unter sich das Eis erbeben, Und hörten's grausig donnernd sich zerspalten, Und sahn es aufgerissen sich erheben; Und wie des Abgrunds Stimmen rings erschallten, Beflügelten den Lauf sie landhinan, Erst jenseits auf dem festen Grund zu halten. (C.II: HK3/E56.00001 Chamisso: Gedichte (Ausgabe letzter Hand), 1837, 2000 [S. 446])

(236) Wie ist die Hand so kalt, so grausig kalt,

Und hebt sich nicht vom Schenkel mehr!

Wie ist die Stirn so kalt, so grausig kalt,

Und sinket auf die Brust so schwer!

Dem Kinde graut's, es rennt die Haid' entlang

In wilder Flucht, dem Sturm zum Hohn.

(06.05.2014:http://books.google.de/books?id=JaxIAAAAcAAJ\&pg=PA102\&dq=gra usig\&hl=de\&sa=X\&ei=iIhn U6LnMKGp4gTDy4CYBQ\&ved=0CEQQ6AEwAzioBQ\#v$=$ onepage $\& \mathrm{q}=$ grausig $\& \mathrm{f}=$ false)

(237) Mit furchtbarem Heulen Erwachet der Sturm,

Die kreischenden Eulen Umschwirren den Thurm.

Ein grausig Gewitter Beginnt mit Gewalt,

Zerschmettert wie Splitter Die Baume im Wald.

(06.05.2014: http://books.google.de/books?id=VJM6AAAAcAAJ\&pg=PA132\&dq=grausig\&hl=de\&sa $=\quad \mathrm{X} \& \mathrm{ei}=\mathrm{X} 41 \mathrm{n} \quad$ U97mG6jV4ATm5YGIAQ\&ved=0CE4Q6AEwBTjgAw\# $\mathrm{v}=$ onepage \& $\mathrm{q}=$ grausig\&f=false)

Die Belege (235) - (237) lassen mindestens zwei Lesarten zu. Zunächst verbindet sich grausig mit der Ausgangsbedeutung, indem es die angesprochenen Situationen als Furcht erregend bezeichnet. Zugleich lässt sich eine auf Intensivierung bezogene Inferenz aus dem Kontext ableiten. Denn es handelt sich um die Intensität der Ereignisse, die die hervorgerufenen Gefühle zu Folge hat, vgl. die entsprechenden Paraphrasierungen:

$(235$ ') 'sehr laut und damit Angst erregend donnernd' 
(236') 'sehr kalt und damit Angst erregend'

(237') 'ein starker und damit Angst erregendes Gewitter'

Das Auftreten der Gradbedeutung erfolgt außerdem durch semantic repetition. Die Intensivierung des adjektivischen Gebrauchs zeigt sich darin, dass die beiden Kookkurrenzpartner der Verwendung den gemeinsamen Begriff 'Furcht' enthalten. Die Wiederholung der Bedeutungskomponente führt zu der Verstärkung des Ausdrucks und stellt grausig als Gradadjektiv dar, vgl.:

(238) Indem man daher der kleinen Schaar der pfychifchen Aerzte allein die Sorge für die unglücklichen Geifteskranken bereitwillig überließ, pflegte man erftere tief zu beklagen, daß ihr Beruffie gleichfam auf einen verlorenen Poften geftellt habe, wo fie im fteten Kampfe mit den graufigften Schreckniffen jeder reinen Lebensfreude verluftig gehen müßten. (DTA: Ideler, Karl Wilhelm: Der religiöse Wahnsinn, erläutert durch Krankengeschichten. Ein Beitrag zur Geschichte der religiösen Wirren der Gegenwart. Halle (Saale), 1847)

(239) Ein jäher, grausiger Schauder überfiel sie, als sie das strömende, eiskalte Naß an ihrem Leibe fühlte. (C.II: HK4/D36.00001 Duncker: Jugend, Erstdruck: 1905, 2004 [S. 200])

(240) Denn er war schon vom Pferd gesprungen und hatte den zweiten Wurfspeer, der ihm noch blieb, gezückt; seine Augen waren rot von der Hast und Wildheit der Verfolgung, und seine Züge waren gespannt, daßich vor $i \mathrm{hm}$, die ihn selbst seit dem ersten Blick liebte und unablässig an mich herangelockt hatte, grausige Todesfurcht empfand und laut aufschrie. (C.II: HK3/D69.00002 Hofmannsthal: Die Frau ohne Schatten, Entstanden: 19121919, 2000 [S. 345])

Die ambigen Belege (235) - (240) lassen außerdem das Einsetzen von subjectivity bzw. den Übergang von der propositionalen zur expressiven Bedeutung feststellen und verweisen damit auf Traugott I (oben 3.6.3.4).

In der nächsten Entwicklungsphase erscheint grausig in seiner fortgeschrittenen intensivierenden Funktion. Das Grad-Adjektiv bzw. -Adverb bezieht sich auf negative Situationen, ohne den direkten Zusammenhang mit der Ausgangsbedeutung erkennen zu lassen, vgl.:

(241) Egal ob nach einem ausgedehnten Spaziergang durch die sonnige, aber frostige Winterlandschaft oder nach dem grausigen Schneegestöber auf dem Weg von der Arbeit nach Hause - an einem kalten Tag sehnen sich viele nach Wärme. (C.II: NON10/DEZ.05526 NÖN, 07.12.2010)

(241') dem starken Schneegestöber"

(242) Das Wahlvolk in Kirchen ist wohl ein bisschen erschöpft: Grausig gering ist die Wahlbeteiligung am 11. Oktober, als es gilt, endlich den Bürgermeister der jungen Stadt zu ermitteln. (C.II: RHZ09/DEZ.25078 RZ, 31.12.2009)

(242') 'sehr gering' 
(243) Die kräftigen Nordwestwinde bringen der Seeregion grausig nasskaltes Wetter. Mit 18 Grad Celsius am Nachmittag ist es für die Jahreszeit um 5 Grad zu kalt. (C.II: A00/JUL.46800 St. Galler Tagblatt, 10.07.2000)

(243') 'sehr nasskaltes Wetter'

(244) Und daß in deinen Augen das, was in den doch so grausig unenzyklopädisch schlechten Fußballartikeln der Wikipedia steht, maßgeblich sein soll, läßt mich staunen;) (C.II: WDD11/S60.55804: Diskussion:Stade Reims, In: Wikipedia - URL:http://de.wikipedia.org/wiki/Diskussion:Stade_Reims: Wikipedia, 2011)

(244') 'sehr unenzyklopädisch'

(245) Die zweite Nacht im Zug bricht an, mein Banknachbar hat sich einen Platz auf dem grausig schmutzigen Mittelgang gesucht und einen Sack untergelegt... (C.II: DIV/BBS.00000 Biehl: Splitter im Sand, (Erstv. 2001), 2004 [S. 133])

(245') 'sehr schmutzigen'

Die Belege (241) - (245) sollen die intensivierende Funktion von grausig veranschaulichen. Das Lexem demonstriert den Verlust des direkten Kontakts mit der Ausgangsbedeutung und zugleich den Gewinn an subjectivity.

Grausig kann außerdem Skopus über Funktionsverbgefüge haben und diese intensivieren, wenn sie eine Wiedergabe durch einen graduierbaren Begriff zulassen. Die Belege (246) - (247) sollen den erweiterten Skopusbereich des Gradadverbs veranschaulichen:

(246) „Weil mir diese Dame grausig auf die Nerven ging, wie Sie vielleicht schon gestern Abend mitbekommen haben", knurrte ich. (02.05.2014: http://books.google.de/books?id=yIOhAQAAIAAJ\&q=\%22grausig+auf + die + Nerven $\% 22 \& \mathrm{dq}=\% 22$ grausig+auf + die + Nerven $\% 22 \& \mathrm{hl}=$ de \&sa=X\&ei=wuCuUprwEojYswaj5IHACw\&ved=0CDUQ6AEwAA)

(246') 'sehr nervte'

(247) Ich freu mich seit Wochen auf diesen Urlaub und die letzten Arbeitstage zogen sich echt grausig in die Länge. (02.05.2014: http://www.das-grosseschwedenforum.de/wenn-andere-schlafen-t9258.html)

(247') 'verzögerten sich sehr'

Grausig findet sich außerdem in quantifizierender Funktion. Dabei ist zu beachten, dass, obgleich der Gebrauch sich auf eine positive Situation bzw. Gewinn bezieht, der Kontext eher negative Konnotationen hervorruft, vgl.:

(248) Wichtig ist es aber nicht... und im Grunde möchte ich nicht wirklich eine sooo grausige Summe gewinnen. Ich glaube, das Geld bringt mehr Probleme als Freude. (02.05.2014:_http://kumpelinchen.twoday.net/stories/27713$85 / 3$

(248') 'große Summe'

Die Korpusanalyse hat neben der Intensivierung auch Pejoration erkennen lassen. Der Gebrauch bringt die negative Einstellung des Sprechers bzw. Schreibers zum Ausdruck und verweist damit auf die rein expressive Bedeutung, vgl.: 
(249) Draußen Eiseskälte, Nieselregen - kurzum grausiges Wetter. (C.II: NUZ09/DEZ.01008 NZ, 09.12.2009, S. 1)

(249') 'sehr schlechtes Wetter'

(250) Tag, ich bin gerade durch den Artikel gegangen und habe einige grausige Stilfehler korrigiert. (C.II: WDD11/F10.56839: Diskussion:Freddy Krueger, In:Wikipedia - URL:http://de.wikipedia.org/wiki/Diskussion:Freddy_Krueger: Wikipedia, 2011)

(250') 'sehr schlimme Stilfehler'

(251) Ich wollte mir einmal Eier kochen, die haben aber ganz grausig geschmeckt. (C.II: V98/FEB.07535 Vorarlberger Nachr., 17.02.1998, S. C8)

(251') 'sehr schlecht geschmeckt'

(252) Artikel ist grausig formuliert und veraltet. (C.II: WDD11/R02.87503: Diskussion:Reiserad, In: Wikipedia - URL:http://de.wikipedia.org/wiki/Diskussion:Reiserad Wikipedia, 2011)

(252') 'sehr schlecht formuliert'

Die intensivierende, quantifizierende und pejorative Bedeutung von grausig, die sich durch den Verlust des direkten Zusammenhangs mit der Ausgangsbedeutung eingestellt hat, lässt sich jeweils in den Belegen (241) - (247), (248) und (249) (252) veranschaulichen. Zugleich demonstrieren die hier gegebenen Kontexte dadurch den Gewinn an subjectivity, dass die Bedeutung sich auf die expressive Ebene ausgeweitet hat. Darüber hinaus ist der Prozess von bleaching sowie der Übergang zu Traugott III (oben 3.6.3.4) ausschlaggebend.

Die untersuchten Korpora liefern keine Belege, die das Auftreten mit rein positiv konnotierten Kookkurrenzpartnern und damit die abschließende Phase des Entwicklungsgangs einer Gradbedeutung demonstrieren können. In diesem Zusammenhang bleibt der Prozess der Desemantisierung in Bezug auf grausig unvollständig.

\subsubsection{Kurzfassung der Entwicklung bei grausig}

1. Ausgangsbedeutung: 'Grausen hervorrufend'.

6. Reine Gradbedeutung: Die Korpusanalyse hat keine Verwendungen ergeben, die einen Gebrauch des Lexems mit positiv konnotierten Bezugswörtern belegen; die übrigen Entwicklungsschritte wie oben in Kap. 3.6.3.9.3.

\subsubsection{Schauderhaft}

\subsubsection{Lexikographische Angaben}

Kluge (s.v.) bezeichnet schauderhaft als Ableitung von schaudern, liefert jedoch keine weiteren Informationen über die Herkunft des Lexems. Aus der Belegsituation in DWB (s.v.) geht hervor, dass die Form im Neuhochdeutschen aufgekommen ist. Pfeifer (s.v.) verweist auf das Auftreten des Lexems im 18. Jh.

1. schauderhaft in DWB (s.v.)

1.1 „schaudererweckend, von dingen und personen, deren anblick, anhören u.

s. w. schauder hervorbringt"

1.2 „schauder empfindend, zu schauder geneigt, von menschen“ 
1.3 „im gewöhnlichen leben abgeschwächt, mehr in der bedeutung 'sehr arg'“

2. schauderhaft in DUDEN (2012, s.v.)

„(umgangssprachlich abwertend)“

2.1 „im höchsten Maße jemandes Missfallen erregend, scheußlich; abstoßend, widerlich"

2.2 „>intensivierend bei Adjektiven und Verben> sehr, überaus“

\subsubsection{Entwicklung der Gradbedeutung bei schauderhaft}

Die Gradbedeutung von schauderhaft hat laut der durchgeführten Korpusanalyse sowie den Angaben von DWB im Neuhochdeutschen eingesetzt. Als Ausgangsbedeutung gilt die Bedeutung des Schreckens bzw. „Schauder erregend“, vgl.:

(253) das grab ist tief und stille und schauderhaft sein rand. (DWB: 1783, s.v.)

(253') 'Schauder erregender Rand des Grabs'

(254) Es ift fchauderhaft, wenn ich ůberlege, daß dies Ungeheuer doch fchon damals verlarvt in dem fchoinen Weibe lag, das ich umarmte, - bey jedem Weibe und Mådchen fällt mir jetzt der Gedanke ein [...] (DTA: Tieck, Ludwig: William Lovell. Bd. 3. Berlin u. a., 1796)

(254') 'Es ist Schauder erregend'

Die Korpusanalyse hat nahegelegt, dass die Gradbedeutung sich zunächst in den ambigen Kontexten findet, wobei die Ausgangsbedeutung zunächst pragmatic strengthening bzw. enrichment erfährt und durch pragmatic inferencing eine alternative, auf Intensität bezogene Nebenbedeutung aufweist, vgl.:

(255) Der Tumult in den Straßen war furchtbar, alles drängte nach der Hauptwache. Hier begann ein neuer, verzweifelter Kampf. [...] Eine augenblickliche, zweifelhafte Stille folgte auf den schauderhaften Lärm. Die geängsteten Bewohner lauschten furchtsam in den dunkelsten Winkeln ihrer Häuser, die Ordnung schien indeß hergestellt, man wagte hin und her ein Licht anzuzünden, und sah beruhigt auf die überstandne Gefahr [...]. (C.II: HK4/F09.00001 Fouqué: Rodrich, Erstdruck: 1806-1807, 2004 [S. 149])

(256) Wie er aber, von Schrecken bleich, fich nach feinem Vetter umfah, lag, von der Flamme grell erleuchtet, Dyk, mit umgedrehtem Halfe, neben ihm, die blau gefchwollnen Züge fchauderhaft verzerrt, und die ftarren Augen feft auf Johny gerichtet. (DTA: Pückler-Muskau, Hermann von: Briefe eines Verstorbenen. Bd. 2. München, 1830)

(257) Schauderhaft wurde die Noth im obern Theil des Kamons, d. h. im Sarganserlande, das sich meist von Gras und dem Viehstand, auch von Waldungen nährt [...]. Ganze Haushaltungen gingen vor Hunger zu Grunde. (GB, 05.05.2014: http://books.google.de/books?id=vD4PAAAAQAAJ\&pg=PA299\&dq=schauderhaft\&hl=de\&sa $\quad$ X\&ei=AqVnU5eHDMKO5ATfsIGICg\&ved=0CDgQ6AEwAThQ\#v=onepage \&q=schauderhaft\&f=false)

Aus den Belegen (255) - (257) ergibt sich eine mehrdeutige Interpretation von schauderhaft. Zunächst bezieht sich das Lexem auf die Ausgangsbedeutung, indem die beschriebenen Situationen bzw. Lärm, Verzerrung der Gesichtszüge und die Not als Schrecken hervorrufend bzw. demonstrierend dargestellt werden. Aus dem 
Kontext lässt sich jedoch zugleich schließen, dass schauderhaft als Gradadjektiv fungieren kann. Dabei tritt Intensität als Grund für die hervorgerufene Angst hervor, vgl. die entsprechenden Paraphrasierungen:

$\left(255^{\prime}\right)$ 'sehr starker und damit Furcht hervorrufender Lärm'

(256') 'sehr stark verzerrte und damit Furcht hervorrufende Gesichtszüge'

(257') 'sehr starke und damit Furcht hervorrufende Not'

Die Gradbedeutung lässt sich außerdem am Einsetzen von semantic repetition erkennen. Infolge der Wiederholung der gemeinsamen Bedeutungskomponente 'Furcht' in den beiden Kookkurrenzpartnern kommt es zu der Verstärkung des Ausdrucks, sodass schauderhaft die Rolle eines Gradadjektivs annimmt, vgl.:

(258) Ist der Altar, wo Druiden In der Zeit des Aberglaubens Menschenopfer abgeschlachtet. $O$ der schauderhaften Greuel! Denk ich dran, sträubt sich das Haar Auf dem Rücken mir - Zur Ehre Gottes wurde Blut vergossen! (C.II: HK3/B12.00001 Heine, Heinrich: Atta Troll, Erstdruck: 1843, 2000 [S. 371])

(259) Alle Regierungen der deutschen Staaten lebten in einer schauderhaften Angst vor einer Erschütterung oder Aenderung des Bestehenden, unterdrückten jede lautwerdende, an Kritik streifende Regung und hinderten eifrig jede gemeinsame Aeußerung. (GB, 02.05.2014: http://books.google.de/books?id=rFVHAAAAYAAJ\&pg =PA139\&dq=\%22schauderhaften+angst $\% 22 \& \mathrm{hl}-$ $=$ de\&sa $=X \&$ ei=IH5jU7_TPMS54ATz-4GoBA\&ved=0CDIQ6AEwAA\#v=onepage\&q=$\% 22$ schauderhaften $\% 20$ angst $\% 22 \& \mathrm{f}=$ false)

(260) In seiner langen Laufbahn des Verbrechens, in einem Leben grenzenloser Nichtswürdigkeit hatte er niemals diese schauderhafte Furcht vor er wußte nicht was empfunden. (GB, 02.05.2014: http://books.google.de/books?id=IMFKAAAAcAAJ\&pg= PA78\&dq=\%22schauderhafte+furcht $\% 22 \& h l=d e-$ \&sa=X\&ei=mX5jU5GbG8aN0AXK6IGYDQ\&ved=0CEMQ6AEwAw\#v=onepage\&q$=\% 22$ schauderhafte $\% 20$ furcht $\% 22 \& \mathrm{f}=$ false)

Auf der bis jetzt beschriebenen Etappe des Entwicklungsgangs der Gradbedeutung lässt sich erkennen, dass die Gradbedeutung als Nebenbedeutung durch pragmatic inferencing sowie semantic repetition in Erscheinung tritt, während die Ausgangsbedeutung zunächst im Vordergrund steht. Neben der Intensivierung erwirbt schauderhaft eine expressive Bedeutungskomponente. Das Auftreten von subjectivity bringt die Einstellung des Sprechers bzw. Schreibers zum Ausdruck und verweist damit auf Traugott I (oben 3.6.3.4).

In der nächsten Etappe des Entwicklungsgangs demonstriert schauderhaft einen fortgeschrittenen Prozess der Desemantisierung. Das Lexem kollokiert mit negativ konnotierten Bezugswörtern, ohne einen direkten Zusammenhang mit der Ausgangsbedeutung zu zeigen. In Anbetracht der erwähnten Selektionsbeschränkungen wird die Bedeutungsentleerung nicht komplett vollzogen, vgl.:

(261) Alle stimmen darin überein, dass es die Griechen in der Poesie, Sculptur und Architektur sehr weit gebracht haben, dass aber ihre Musik auf der niedrigsten Stufe stehen blieb, und folglich schauderhaft schlecht war. 
(05.05.2014: http://books.google.de/books?id=7txTCkZ54FQC\&pg=PA75\&dq=schauderhaft\&hl=de\&sa=X\&ei=c6hnU_OxFMnZ4ASEmYCgAw\&ved=0CEIQ6AEwAz$\mathrm{h} 4 \# \mathrm{v}=$ onepage $\& \mathrm{q}=\mathrm{schauderhaft} \& \mathrm{f}=$ false)

(261') 'sehr schlecht'

(262) Der Zustand der meisten Nutzpflanzenartikel ist mäßig bis beschämend, die Arbeit groß, hier handelt es sich auch noch um eine Gattung mit schauderhaftem systematischen Chaos. (C.II: WDD11/Z14.57983: Diskussion: Zitruspflanzen, In: Wikipedia - URL:http://de.wikipedia.org/wiki/Diskussion:Zitruspflanzen: Wikipedia, 2011)

(262') 'mit sehr großem Chaos'

(263) ... traumwandelnd stieg ich den Hügel hinab, schweifte durch die halbe Stadt, sah in einer abgelegenen Straße noch eine späte kleine Schenke offen, trat willenlos ein, trank zwei Liter Waadtländer und kam gegen Morgen schauderhaft betrunken nach Hause. (C.II: M02/AUG.61824 Mannh. Morgen, 20.08.2002)

(263') 'sehr betrunken'

(264) Unter dem Titel „Reiß auf Reisen“ stellt er [...] eine kleine Auswahl der rund 4000 Abzüge vor, und beschränkt sich [...] auf die südamerikanischen Ansichten des Wilhelm Reiß. Jenes älteren Bruders der Mannheimer Ehrenbürger Carl und Anna Reiß, [...] der als junger Mann seinem (enttäuschten) Vater schrieb, dass ihm „die Theorien des Handels schauderhaft langweilig sind.“ . (C.II: M03/JAN.06361 Mannh. Morgen, 30.01.2003)

(264') 'sehr langweilig'

Das Lexem kann außerdem Funktionsverbgefüge intensivieren, was auf die Ausdehnung des Bezugsbereichs des Adverbs hinweist. Das Auftreten in dieser Position erfolgt dadurch, dass die FVGs durch einen graduierbaren Begriff wiedergegeben werden können, vgl. die Belege mit den entsprechenden Umformulierungen:

(265) Zuerst traf ich nur Eyssler dort, der mir mit seinen lispelnden, wienerisch-urnischen Vertraulichkeiten schauderhaft auf die Nerven ging. (02.05.2014: http://www.muehsam-tagebuch.de/tb/diaries.php?id=2)

(265') 'sehr nervte'

(266) Eine mittelgroße Langeweile erwartete gestern die Zuschauer bei der dritten Folge von Germany's Next Topmodel. Alles zog sich schauderhaft in die Länge und Thomas \& Thomas versuchten witzig zu sein völlig erfolglos. (02.05.2014: http://www.thejunction.de/offline/2012/03/09/thomas-thomas-allein-auf-pro7-0020814)

(266') 'verzögerte sich sehr'

Die intensivierende Bedeutung kann außerdem in die quantifizierende in negativ bewerteten Kontexten übergehen, vgl. den adjektivischen Gebrauch:

(267) Man mag sich über diese Verlagsleistung um so mehr freuen, als sonst Verleger vielfach schauderhafte Preise zu stellen beginnen, so daß man bald zur alten handschriftlichen Verbreitung wie vor dem Bücherdruck zurückkehren [...] wird [...] (GB, 02.05.2014: http://books.google.de/books?id=ebZGAQAAIAAJ\&q=\%22schauderhafte+Preise $\% 22 \& d q=\% 22$ schauderhafte+Preise $\% 22 \&$ hl=de\&sa=X\&ei=4AavUvWnPIXdswb-s4HwDg\&ved=0CDcQ6AEwAQ) 
(267') 'sehr hohe Preise'

Das Verblassen der Ausgangsbedeutung hat außerdem reine pejorative Bedeutung zu Folge:

(268) [...] Den ganzen Sommer über war zum Beispiel der Lahntal-Rad- und Wanderweg unterhalb der Fußgängerbrücke in einem schauderhaften Zustand. (C.II: RHZ96/OKT.08179 RZ, 12.10.1996)

(268') 'einem sehr schlechten Zustand'

(269) Der Artikel ist von einer schauderhaften wissenschaftlichen Quallität. (C.II: WDD11/D05.12853: Diskussion:Drogenpsychose, In: Wikipedia - URL: http://de.wikipedia.org /wiki/Diskussion:Drogenpsychose: Wikipedia, 2011)

(269') 'einer sehr schlechten Qualität'

(270) Die Übersetzung ist schauderhaft! Da stimmt praktisch gar nix! (C.II: WDD11/S26.23466: Diskussion:Schlacht bei Edgehill, In: Wikipedia - URL: http://de.wikipedia.org / wiki/Diskussion:Schlacht_bei_Edgehill: Wikipedia, 2011)

(270') 'sehr schlecht'

(271) Wenn ich den auf 500 Grad aufheize, ist die Pizza in 40 Sekunden fertig und schmeckt schauderhaft - egal ob im Pizzablech oder direkt aufdem Boden. Bei 350 - 400 Grad dagegen ist das Ergebnis optimal. (C.II: WDD11/P61.89257: Diskussion: Pizza/Archiv, In: Wikipedia - URL:http://de.wikipedia.org/wiki/Diskussion:Pizza/Archiv: Wikipedia, 2011)

(271') schmeckt sehr schlecht"

Die intensivierende (261) - (266), quantifizierende (267) sowie die pejorative Bedeutung (268) - (271) demonstrieren die Zunahme an subjectivity im Rahmen von Traugott III (oben 3.6.3.4), indem die Einstellung des Sprechers bzw. Schreibers immer mehr ausgeprägt wirkt.

Das Auftreten in Verbindung mit positiv konnotierten Kookkurrenzpartnern soll auf die letzte Entwicklungsphase der Gradbedeutung hinweisen. Obgleich COSMAS keine entsprechenden Belege liefert, ergibt die Suche im Internet die passenden Ergebnisse:

(272) Gruselautor Stephen King hat schauderhaft gut verdient mit seinem Ausflug ins Internet. ${ }^{4}$ (02.05.2014: http://www.welt.de/print-welt/article433687/Der-neue-Hacker-Koenig-Fidel-Castro.html)

(272') 'sehr gut verdient'

(273) [...] die Drinks sind schauderhaft gut und ich bin sehr entzückt, nicht schon wieder mein Portemonnaie für eine trümmlige Aktion zücken zu müssen (01.05.2014: http://blog.derbund.ch/zumrundenleder/blog/2006/01/$16 /$ voll-krass/)

(273') 'sehr gut'

\footnotetext{
${ }^{54}$ Obgleich der Beleg (202) ein gewisses Wortspiel bei der Verbindung der Wörter Gruselautor und schauderhaft bieten mag, erscheint der adverbiale Gebrauch in rein intensivierender Funktion in der Verbindung mit einem positiv konnotierten Kookkurrenzpartner.
} 


\subsubsection{Kurzfassung der Entwicklung bei schauderhaft}

Ausgangsbedeutung: 'Schauder hervorrufend'; die übrigen Entwicklungsschritte wie oben in Kap. 3.6.3.9.3.

\subsubsection{Schauerlich}

\subsubsection{Lexikographische Angaben}

Kluge (s.v.) definiert schauerlich als Ableitung von Schauer. RhWB (s.v.) sowie die Beleglage in DWB (s.v.) lassen das Wort dem Neuhochdeutschen zuweisen. Pfeifer (s.v.) datiert sein Auftreten auf das 17. Jh.

1. schauerlich in DWB (s.v.)

1.1 „adjectivisch, schauer verursachend, zunächst von körperlich erregendem“

1.2 "als adverb, schauerlich horride“

$1.2 \mathrm{a}$ „activ, auf eine weise, bei der schauer erregt wird“

$1.2 \mathrm{~b}$ „passiv, auf eine art und weise, bei der man schauer empfindet"

2. schauerlich in DUDEN (2012, s.v.)

2.1 "Schauder (2) 55 , Entsetzen erregend; grausig“

2.2 „(umgangssprachlich abwertend)“

$2.2 \mathrm{a}$,jemandem in höchstem Maß missfallend“

$2.2 \mathrm{~b}$ „<intensivierend bei Adjektiven und Verben> in einem sehr hohen Maß; sehr"

\subsubsection{Entwicklung der Gradbedeutung bei schauerlich}

Obgleich DWB keine Informationen über das Aufkommen der Gradbedeutung liefert, lässt die Korpusanalyse den Beginn des Entwicklungsgangs der Intensivierung im Neuhochdeutschen feststellen. Als Grundlage für deren Entwicklung dient die Bedeutung des Schreckens, vgl..:

(274) Der Himmel war rein, und der Mond bekleidete die alten Säulen und Mauern mit seinem bleichen schauerlichen Lichte. (C.II: HK3/A91.00016 Novalis, Heinrich von Ofterdingen, Entstanden: 1799-1800, 2000 [S. 200])

(274') 'Schauder erregenden Lichte'

(275) Es kam mir daher anfangs ein wenig schauerlich an. (C.II: HK4/U03.00001 Unger: Bekenntnisse einer schönen Seele, Erstdruck: 1806, 2004 [S. 145])

$(275 ')$ 'Schauder erregend'

Das Einsetzen der Intensivierung beginnt mit pragmatic strengthening bzw. enrichment der Ausgangsbedeutung. „Ambiguity in the output“ (Timberlake 1977: 148) hat pragmatic inferencing zu Folge, sodass die Gradbedeutung als Nebenbedeutung neben der Ausgangsbedeutung in Erscheinung tritt, vgl.:

(276) Die schauerliche Hungernot des Winters 1847 machte sich im Herbst bereits fühlbar - wenigstens im Südwesten von Irland um Cork herum, wo ich mich am längsten aufhielt und schon mehrere kleine Aufstände gegen Bäcker und Müller erlebte, denen man das Mehl wegschleppte. (C.II:

\footnotetext{
55 „plötzliches [wegen seiner überwältigenden Heftigkeit] gleichsam körperlich empfundenes Gefühl (2) (besonders der Angst, der Beklommenheit, des Entsetzens o. Ä.)“ DUDEN, s.v. Schauder
} 
HK4/H04.00001 Hahn-Hahn: Von Babylon nach Jerusalem, Erstdruck: 1851, 2004 [S. 184])

(277) Der Donner prafselte gellend und schauerlich nahe; eine Tanne stürzte krachend und in lichtem Brande in's Thal, und sast quer in den Weg. Das Alles war das Werk Eines Augenblicks. (06.05.2014: http://books.google.de /books?id=q8U6AAAAcAAJ\&pg=PA160\&dq=schauerlich\&hl=de\&sa=X\&ei=3cNoU7D0LIOwyAPwwIDgBA\&ved=0CDgQ6AEwATi0AQ\#v=onepage \&q=schauerlich$\& \mathrm{f}=$ false)

(278) So lag sie einen Augenblick verlassen, im Wüthen des immer schauerlichen Ungewitters, den Tod erwartend. (GB, 06.05.2014: http://books.google.de/books? id=Eb08AAAAcAAJ\&pg=RA1PA190\&dq=schauerlich\&hl=de\&sa$=X \&$ ei=9cVoU7rJG4HMygPElYDoAw\&ved $=\quad$ OCD0Q6AEwAjjoAg\#v=onepage \&q=schauerlich\&f=false)

Die Verwendungen in (276) - (278) gestatten eine mehrdeutige Interpretation. Aus den Kontexten geht hervor, dass die Bedeutung von schauerlich in dem Zwischenbereich zwischen Qualifizierung und Intensivierung liegt. Dabei wird die Gradbedeutung aus den Kontexten als Inferenz abgeleitet und weist einen engen Kontakt mit der Ausgangsbedeutung auf. Das Einsetzen der Gradbedeutung erfolgt dadurch, dass die Intensivierung die Ursache für die auftretenden Gefühle darstellt, vgl. die folgenden Wiedergaben:

(276') 'Die Hungersnot war sehr stark und damit Schauder erregend';

(277') 'Der Donner prasselte sehr nahe, was Schauder erregend wirkte';

(278') 'Das Ungewitter war sehr stark und damit Schauder erregend'.

Die intensivierende Funktion des Lexems lässt sich außerdem an semantic repetition beobachten, vgl.:

(279) In das Elend hinein flüchteten sich auch in schauerlicher Angst die, welche den Grünen gesehen, und erzählten bebend die wiederholte Erscheinung. (C.II: HK3/A12.00002 Gotthelf: Die schwarze Spinne, Erstdruck: 1842, 2000 [S. 39])

(280) [...] eine schauerliche Beängstigung stieß mich von ihr ab, eine knabenhafte Lüsternheit zog mich wieder zu ihr hin, mein Herz pochte, als wollte ich eine Mordtat begehen, und endlich küßte ich die schöne Göttin mit einer Inbrunst, mit einer Zärtlichkeit, mit einer Verzweiflung, wie ich nie mehr geküßt habe in diesem Leben. (C.II: HK3/A68.00008 Heine: Florentinische Nächte, Erstdruck: 1836, 2000 [S. 115])

(281) Er sprang auf. Eine schauerliche Befürchtung erfaßte ihn. (C.II: HK4/J04.00001 Janitschek: Kreuzfahrer, Erstdruck: 1897, 2004 [S. 155])

Die adjektivischen Verbindungen in (279) - (281) demonstrieren die Wiederholung der gemeinsamen Gehaltskomponente 'Angst', woraus sich die Verstärkung des Ausdrucks ergibt, vgl.:

$(279 ')$ 'sehr großer Angst'

(280') 'sehr große Beängstigung'

(281') 'sehr große Befürchtung' 
In den Ambiguität aufweisenden Belegen (276) - (281) lässt sich außerdem das Erwerben einer expressiven Bedeutung konstatieren, welche die Einstellung des Sprechers bzw. Schreibers zum Ausdruck bringt. Darüber hinaus verbindet der mehrdeutige Gebrauch die propositionale und die expressive Ebene und verweist auf das Wirken von Traugott I (oben 3.6.3.4).

Im nächsten Entwicklungsschritt zeigt sich schauerlich als ein ausgebildetes GradAdjektiv bzw. -Adverb, wo der Zusammenhang mit der Ausgangsbedeutung verblasst wirkt. Für dieses Entwicklungsstadium ist das Auftreten in Kollokationen mit negativer Bedeutung ausschlaggebend, vgl.:

(282) Den folgenden Tag wollen wir nicht beschreiben, denn dieser ist schauerlich langweilig. (C.II: HK3/G53.00001 Gotthelf: Uli der Pächter, Erstdruck: 1849,2000 [S. 49])

(282') 'sehr langweilig'

(283) Das schauerlich schlechte Wetter geht auch heute weiter. Nur gelegentlich zeigt sich die Sonne. Die Temperaturen erreichen bestenfalls acht Grad. (C.II: RHZ97/MAR.14668 RZ, 21.03.1997)

(283') 'sehr schlechte'

(284) Aber den schauerlich teuren Umbau eines Dienstautos zahlen ja wirklich die Steuerzahler, und den auch nicht billigen Flug-Aufpreis detto. (C.II: P98/JUL.29153 Die Presse, 20.07.1998)

(284') 'sehr teuren'

(285) Hatte bereits am Samstag ein schauerliches Gewitter das vorgesehene Jugendkonzert mit vier Bands zum Auftakt der „Nachtschicht" im Kulturwerk regelrecht davongespült, so riskierte die Sonne am Sonntag wenigstens hin und wieder zwischen kräftigen Regengüssen zaghafte Blicke auf das abwechslungsreiche Treiben im und um das Kulturwerk der Siegstadt. (C.II: RHZ11/JUN.19418 RZ, 20.06.2011, S. 17)

(285') 'sehr starkes Gewitter'

(286) Die Sonne strahlt auf der schwarzen Asphaltfläche eine schauerliche Hitze aus, sodass die folgenden Stunden auf der luftigen Veranda vor der Wohnung des Managers ausserordentlich wohlthuend wirkten. (06.05.2014: http://books.google.de / books?id=_MkRAQAAMAAJ\&q=\%22schauerliche+Hitze $\% 22 \& d q=\% 22$ schauerliche + Hitze $\% 22 \& h l=d e \& s a=X \& e i=x q V o U 5 r k O N D-$ tygPmhoGwCw\&ved=0CFUQ6AEwBw)

(286') 'sehr starke Hitze'

Schauerlich findet sich außerdem in Kontexten, die einen erweiterten Bezugsbereich demonstrieren, indem das Lexem Skopus über Funktionsverbgefüge hat und diese modifiziert. Die Intensivierung erfolgt dann, wenn das Substantiv in einem FVG graduierbar ist oder ein FVG durch einen graduierbaren Begriff wiedergegeben werden kann, vgl.:

(287) Mir geht das auch langsam schauerlich auf den Geist. (20.12.2013: http://meinungen.1und1.de/forum-1und1/post/16752398?sp=7)

(287') 'sehr nervt' 
(288) Laß Dich doch nicht so schauerlich unter Druck setzen! (06.05.2014: http://www.urbia.de/archiv/forum/th-3531248/Altersunterschied-Geschwisterkinder.html)

(288') 'unter sehr großen Druck setzten' bzw. 'sehr bedrängen'

Neben der Intensivierung tritt der quantifizierende Gebrauch in Erscheinung, der die Bedeutung „sehr groß, viel“ zum Ausdruck bringt, vgl.:

(289) Seit kurzem ist sogar bekannt, wie viele Rechenoperationen das Universum, wäre es wirklich ein Computer, seit dem Urknall ausgeführt hätte. Der US-Physiker Seth Lloyd hat das ergründet: Heraus kam eine schauerliche Zahl mit 120 Nullen. (06.05.2014: http://www.spiegel.de/spiegel/print/d-23582758.html)

(289') 'sehr große Zahl'

(290) Die Teuerung dürfte im Dezember die höchste Stufe erreicht haben, die Krone hat fast keinen Wert mehr. Es sind schauerliche Preise für alle Gegenstände, Nahrungsmittel und Artikel. (23.05.2014: http://www.hfkirchberg.at/index.php/kriege-und-revolutionen/163-zwischenkriegszeit)

(290') 'sehr hohe Preise'

Neben der Gradbedeutung lässt sich die Entwicklung der pejorativen Bedeutung erkennen, welche die reine einstellungsbezogene Bedeutung demonstriert, vgl.:

(291) Kleine Straßen seien dagegen in schauerlichem Zustand. (C.II: L99/MAI.23452 Berliner Morgenpost, 11.05.1999, S. 15)

(291') 'in sehr schlechtem Zustand'

(292) Dieser schauerliche Text! Nie und nimmer würde er seinen Namen unter solch dilettantisches Geschreibsel setzen, verfasst vom Polizeigefreiten Stirnimann. (C.II: A08/APR.01640 St. Galler Tagblatt, 04.04.2008, S. 45)

(292') 'sehr schlechte Text'

Die Belege (282) - (289), (290) und (291) - (292) veranschaulichen den eindeutigen Gebrauch von schauerlich jeweils in der intensivierenden, quantifizierenden und pejorativen Funktion. Außerdem demonstrieren sie den Übergang zur expressiven Ausdrucksebene und damit zu Traugott III (oben 3.6.3.4).

Das Auftreten mit positiv konnotierten Kollokationspartnern schließt den Entwicklungsgang der Gradbedeutung von schauerlich ab und demonstriert den komplett vollzogenen Prozess der Desemantisierung des Lexems. Das Wort fungiert damit als bedeutungsentleert, vgl.:

(293) Als ich in diesem Winter mit John Henry Mackay, dem anarchistischen Feuilletonisten, zusammentreffen wollte und dies einer jungen Berliner Dame erzählte, bat sie: „Ach, nehmen Sie mich doch mit, ich brenne darauf, einen Anarchisten zu sehen; das muß schauerlich interessant sein!“ (C.II: HK4/D37.00001 Duncker: Meine Herren Collegen!, Erstdruck: 1894, 2004 [S. 29])

(293') 'sehr interessant' 
(294) Ein schauerlich schönes Bild bot sich dar. Berge, Berge, Berge. Einige mit Schnee bedeckt, einige mit kahlen Häuptern. (C.II: HK4/J04.00001 Janitschek, Maria: Kreuzfahrer, Erstdruck: 1897, 2004 [S. 58])

(294') 'sehr schönes'

(295) In der Karte finden sich neben Bieren (Wolfshöher) Weine aus Italien, China, Südafrika und Franken [...], modische Cocktails und schauerlich schön schmeckende asiatische Reisschnäpse. (C.II: NUZ07/APR.01089 NZ, 13.04.2007)

(295') 'sehr schön schmeckende'

\subsubsection{Kurzfassung der Entwicklung bei schauerlich}

Ausgangsbedeutung: 'Schauder hervorrufend';

die übrigen Entwicklungsschritte wie oben in Kap. 3.6.3.9.3.

\subsubsection{Schaurig}

\subsubsection{Lexikographische Angaben}

Pfeifer (s.v.) definiert schaurig als Ableitung von Schauer ${ }^{56}$. Aus der Beleglage in DWB (s.v.) und den Korpora lässt sich schließen, dass die Form im Neuhochdeutschen in Erscheinung getreten ist. Pfeifer (s.v.) datiert das Auftreten des Lexems auf das 18. Jh.

1. schaurig in DWB (s.v.)

1.1 „Vor wind und wetter geschützt, bedeckt"

1.2 „von schauer, nimbus, horror, einen schauer verursachend, erregend“

1.3 „von der empfindung des schauers, sowol wenn er von kälte herrührt“, „als auch bei grauenerregenden dingen"

2. schaurig in DUDEN (2012, s.v.)

2.1 „Schauder hervorrufend; gruselig, unheimlich“

2.2 „(oft umgangssprachlich übertreibend)“

2.2.a "sehr unangenehm, schlimm, schlecht"

2.2.b "<verstärkend bei Adjektiven und Verben> sehr, überaus“

\subsubsection{Entwicklung der Gradbedeutung bei schaurig}

Obgleich DWB keine Informationen zu der Entstehung von Gradbedeutung bei schaurig bietet, lässt die Korpusanalyse deren Einsetzen im 19. Jh. feststellen. Als Quelle für ihre Herausbildung dient die Bedeutung, die sich auf die Begriffe des Schauers und des Unheimlichen stützt, vgl.:

(296) die tiefe abenddämmerung war eingebrochen und es wurde ihnen recht schaurig zu muthe. (DWB: s.v.)

(296') 'unheimlich zumute'

(297) Warum blicken wir so traurig In die Nacht hinab? Warum dünkst du uns so schaurig, Stilles kühles Grab? (DTA: Kosegarten, Ludwig Gotthard: Poesieen. Bd. 2. Leipzig, 1798)

56 'kurzer, heftiger Niederschlag, plötzliche Empfindung von Kälte, Angst, Entsetzen' Pfeifer, s.v. Schauer ${ }^{2}$ 
(297') 'dünkst so unheimlich, Schauer erregend'

Das Auftreten der Gradbedeutung ist zunächst mit pragmatic enrichment bzw. strengthening der Ausgangsbedeutung verbunden. Die Intensivierung tritt in Mehrdeutigkeit aufweisenden Verwendungen zufolge pragmatic inferencing der Kontexte in Erscheinung:

(298) [...] und der Wind, der fich ftürmifch zu erheben begann, fchüttelte die Aefte der alten Efchen fo fchaurig, raufchte fo hohl und dumpf durch den dicht verfchlungenen Epheu, und warf mit folcher Gewalt große Steine von den Mauern hinab in ihren Weg, daß beiden immer übler zu Muthe ward. (DTA: Pückler-Muskau, Hermann von: Briefe eines Verstorbenen. Bd. 2. München, 1830)

(299) Dann war sie allein in der schaurig stillen Kirche; nichts störte sie, als der Schall ihrer eigenen Schritte, oder das Gezwitscher eines Vogels [...]. (C.II: HK4/M06.00001 Marlitt: Thüringer Erzählungen: Die zwölf Apostel, Entstanden: seit 1859, 2004 [S. 240])

(300) Wild fuchtelnd und mit schaurigem Getöse brachen die Hexen über den Gastbetrieb herein, erschreckten Jung und Alt, führten wilde Tänze auf und verlangten ihren höllischen Tribut. (C.II: A01/FEB.06998 St. Galler Tagblatt, 05.02.2001)

Der Gebrauch in (298) - (300) erlaubt eine ambige Interpretation. Zunächst beschreibt das Lexem das Wehen des Windes, die Stille der Kirche und das Getöse der Hexen in Bezug auf die von ihnen ausgelösten Gefühle des Schreckens. Damit wird schaurig in der Ausgangsbedeutung verwendet. Die alternative Interpretation setzt den Begriff der Intensivierung voraus und stellt das Lexem als Grad-Adjektiv bzw. -Adverb dar. Die Intensität der Erscheinungen lässt sich aus den Kontexten ableiten, wo sie die Ursache der hervorgerufenen Emotionen demonstriert, vgl. die Paraphrasierungen:

(298') 'der Wind schüttelte die Äste sehr stark und damit Schauer erregend'

(299') 'die Kirche war so still, dass es Schauer erregend wirkte'

(300') 'das Getöse der Hexen war sehr stark und damit Schauer erregend'.

Das Auftreten der Intensivierung kann außerdem mit semantic repetition in Verbindung gebracht werden, vgl.:

(301) Diese dagegen Faßte die Scham und die schaurige Furcht, als sie wieder allein war, Daß sie geheim vor dem Vater dem Fremdlinge solches beschlossen. (GB, 06.05.2014: 1832, http://books.google.de/books?id=FbTIN4rKV8EC\&pg=PA101\&dq=\%22schaurige + furcht $\% 22 \& \quad \mathrm{hl}=\mathrm{de} \& \mathrm{sa}=\mathrm{X} \&$ ei $=\mathrm{d} 9$ NoU6WnFYqCzAPX1YHYAw\&ved=0CEUQ6AEwBDgK\#v=onepage\&q=\%22schaurige\%20furcht $\% 22 \& \mathrm{f}=$ false)

(302) Als ich aber des Abends in den festlich erleuchteten Saal eintrat, wurde ich von einer Unruhe und schaurigen Angst befallen. (GB, 06.05.2014: 1870, http://books.google.de/books?id=wo04AAAAMAAJ\&q=\%22schaurigen+angst $\% 22 \& \mathrm{dq}=\% 22$ schaurigen+angst $\% 22 \& \mathrm{hl}=\mathrm{de} \& \mathrm{sa}=\mathrm{X} \& \mathrm{ei}=\mathrm{JtRoU} 5 \mathrm{mzAtD} 2 \mathrm{yAPW}-\mathrm{I}-$ HgAQ\&ved=0CEoQ6AEwBQ) 
(303) [...] in der Nacht des 7. Septembers, riß der Sturm dem Könige sein Zelt über den Kopf hinweg'). Die Wuth der Elemente und die schaurig unheimliche Landschaft, von der sie sich umgeben sahen, erzeugten in der Seele der Engländer die Angst, daß sie mit den erzürnten Geistern und Kobolden des Gebirges zu kämpfen hätten. (06.05.2014: 1858, http://books.google.de / books?id=hbBBAAAAcAAJ\&pg=PA18\&dq=\%22schaurig+unheimliche\%22\&hl=de\&sa=X\&ei=eNVoU6SzDsWXygPZtoHQBw\&ved=0CDMQ6AEwA$\mathrm{A} \# \mathrm{v}=$ onepage $\& \mathrm{q}=\% 22$ schaurig $\% 20$ unheimliche $\% 22 \& \mathrm{f}=$ false,)

Die Belege (301) - (303) demonstrieren die Ambiguität des Gebrauchs. Zunächst bezieht sich schaurig auf den Begriff der Furcht und damit auf die Ausgangsbedeutung. Dabei verkörpern die beiden Kookkurrenzpartner der adjektivischen und adverbialen Verwendungen die Vorstellungen der Angst. Durch diese Kookkurrenz entsteht semantic repetition und semantic redundancy, was zu der Verstärkung des Gebrauchs führt und schaurig zu einem Intensivierungsmittel macht.

Die in (298) - (303) eingeführten ambigen Belege demonstrieren das Einsetzen von subjectivity, durch die das Lexem die Einstellung des Sprechers bzw. Schreibers zu der Situation zum Ausdruck bringt. Damit kommt die erste semantischpragmatische Tendenz (Traugott I, oben 3.6.3.4) zum Zuge.

In der nächsten Entwicklungsstufe erscheint schaurig in seiner fortgeschrittenen intensivierenden Funktion, ohne einen direkten Zusammenhang mit der Ausgangsbedeutung aufzuweisen. Jedoch bezieht sich das Lexem zunächst auf negative Kontexte und demonstriert damit den unvollkommenen Prozess der Bedeutungsentleerung, vgl.:

(304) [...] dass es den Banken und Grossfirmen schaurig leid tut, die welschen Filialen zuerst zu schliessen [...]: Solche Parolen \& Bekenntnisse kann in der Westschweiz niemand mehr hören. (C.II: E97/NOV.27442 Zürcher Tagesanzeiger, 10.11.1997, S. 9)

(304') 'sehr leid tut'

(305) Privat bin ich pingelig und schaurig streng mit mir. (C.II: A08/OKT.08305 St. Galler Tagbl., 27.10.2008, S. 25)

(305') 'sehr streng'

(306) Diese Wanderer sind schaurig geschmacklos angezogen, habe er zuerst gedacht. (C.II: A09/SEP.06976 St. Galler Tagbl., 21.09.2009, S. 36)

(306') 'sehr geschmacklos'

(307) Mehr Sorgen macht ihr die Wettervorhersage. Wenn es am kommenden Sonntag um 11 Uhr losgeht, könnte es draußen schaurig kalt sein. (C.II: NUN13/MAI.01849 Nürnberger Nachrichten, 22.05.2013, S. 9)

(307') 'sehr kalt'

Ferner findet sich schaurig in einem Gebrauch mit erweitertem Bezugsbereich, indem das Lexem Funktionsverbgefüge intensiviert. Das Modifizieren erfolgt unter der Bedingung, dass die Verwendungen durch einen graduierbaren Begriff paraphrasiert werden können, vgl. die Belege mit den entsprechenden Umformulierungen: 
(308) Besonders in der Sache mit dem verlorenen Rico - sorry, ich muss euch mit meiner Art wohl schon lange schaurig auf den Wecker gegangen sein. (06.05.2014: http://www.minubasel.ch/index.php?MenuID=0\&UserID=1\&ContentID=645)

(308') 'sehr genervt'

(309) Trotzdem ich werde sie vermissen und mir Sorgen machen, sie mit Anrufen nerven, bei solch einer Nähe "mal“ besuchen... ich werde ihnen bestimmt schaurig auf die Nerven gehen. (06.05.2014: https://www.spin.de/forum/msg-archive/360/2008/05/72588)

(309') 'sehr nerven'

Der Prozess der Bedeutungsentleerung hat die Herausbildung von pejorativer Bedeutung zur Folge. Die Bedeutung „sehr schlimm“ tritt in adjektivischem und adverbialem Gebrauch auf, der die Vorstellungen des Unheimlichen nicht mehr erkennen lässt und eine rein einstellungsbezogene Bedeutung zeigt, vgl.:

(310) Schaurig schmecken das warme, vom Fahren durchgebeutelte Cola und die harten Kekse. (C.II: N93/MAR.10502 Salzburger Nachr., 20.03.1993)

(310') 'sehr schlecht schmecken'

(311) Toiletten sind in schaurigem Zustand. Brandschutz- und Alarmierungseinrichtungen sind veraltet. Das ist die Sachlage an vielen deutschen Bildungseinrichtungen. (C.II: BRZ09/NOV.04822 Braunschw. Z., 11.11.2009)

(311') 'sehr schlechtem Zustand'

(312) Übrigens liegt hier auch des Rätsels Lösung, warum viele Lobgesänge oft eine so schaurige Qualität haben. (06.05.2014: http://books.google.de/books?id=Q_10z6dv6u0C\&pg= PT127\&dq=\%22schaurige+Qualit\%C3\%A4t\%22\&hl=de\&sa=X\&ei=fPRoU9HhJoH_ywPt94LACQ\&ved=0CDIQ6AEwAA\#v=onepage$\& \mathrm{q}=\% 22$ schaurige $\% 20$ Qualit $\% \mathrm{C} 3 \% \mathrm{~A} 4 \mathrm{t} \% 22 \& \mathrm{f}=$ false)

(312') 'sehr schlechte Qualität'

Die Korpusanalyse hat keine Belege ergeben, die die quantifizierende Rolle des Lexems demonstrieren können. Die Belege (304) - (309) und (310) - (312) demonstrieren den eindeutigen Gebrauch des Lexems jeweils in intensivierender und pejorativer Bedeutung. Außerdem weisen sie den Zuwachs an subjectivity auf, indem schaurig lediglich auf der expressiven Ebene fungiert. Damit wird der Übergang zu Traugott III (oben 3.6.3.4) vollzogen.

Anschließend findet sich schaurig in Verbindung mit positiv konnotierten Kollokationspartnern und demonstriert den endgültigen Verlust des Kontakts mit der Ausgangsbedeutung. Der Prozess der Desemantisierung wird damit vollzogen, vgl.:

(313) „Ich bin schaurig glücklich“, sagt Daniel, und seine grossen braunen Augen blicken für einen Moment weniger ernst in die Welt: nach Jahren in Heimen, im Knast und auf der Gasse endlich ein Zuhause. (C.II: E98/MAI.12141 Zürcher Tagesanzeiger, 15.05.1998, S. 19)

(313') 'sehr glücklich'

(314) Plötzlich zaubern alle Musiker eine Geige hinter ihren Notenständern hervor und intonieren ein paar schaurig schöne Akkorde. (C.II: L99/SEP.60445 Berliner Morgenpost, 06.09.1999, S. 33) 
(314') 'sehr schöne'

(315) Auf der CD „The Tragic Treasury“ (Nonesuch) sind die ironischen Balladen zwischen Kurt Weill und Disco-Pop schaurig gut versammelt. (C.II:: NUZ07/APR.00881 NZ, 12.04.2007)

(315') 'sehr gut'

(316) Seit fünf Jahren lebt die 74-Jährige hier. Sie erzählt viel und gestikuliert engagiert. „Ich bin schaurig gern hier. “(C.II: A13/MAR.06833 St. Galler Tagblatt, 15.03.2013, S. 45)

(316') 'sehr gern'

\subsubsection{Kurzfassung der Entwicklung bei schaurig}

Ausgangsbedeutung: 'unheimlich, Schrecken hervorrufend'; die übrigen Entwicklungsschritte wie oben in Kap. 3.6.3.9.3.

\subsubsection{Schrecklich}

\subsubsection{Lexikographische Angaben}

Kluge (s.v.) liefert keine Informationen über die Herkunft des Lexems. Laut DWB (s.v.) ist schrecklich „zuerst [...] aus einer quelle von 1469 in der adverbialen form schreckliche [...] an stelle des mhd. schriclich" bezeugt. Außerdem gibt DWB eine alternative Schreibung in „älterer Zeit” mit „ä”, schräcklich, und der „nebenform schröcklich“ an. Pfeifer (s.v.) verweist ebenso auf das Auftreten des Lexems im 15. Jh.

1. schrecklich im FWB (Angaben aus den Belegzetteln von FWBD)

"schrecklich, erschreckend“

2. schrecklich in DWB (s.v.)

2.1 „meist dem gewöhnlichen sinne von schrecken gemäsz, angst, furcht erweckend oder dazu geeignet"

2.2 "abscheu, ekel erregend“

2.3 "dem weiteren sinne von schrecken folgend wie 'erstaunlich'“

2.3a „im adjectivischen gebrauch besonders bei quantitativen begriffen“, seltener qualitativ hervorhebend"

$2.3 \mathrm{~b}$,adverbial zunächst gleichfalls quantitativ bei dem in neuerer zeit pronominal aufgefaszten viel“, „,aber [...] auch sehr häufig qualitativ“

2.4 „schreckliche umrisse, bei den malern solche von ungeheurer grösze, die man zu colossalfiguren und den werken braucht, die hoch über dem gesichte zu stehen kommen"

3. schrecklich in DUDEN (2012, s.v.)

3.1 „durch seine Art, sein Ausmaß Schrecken, Entsetzen auslösen“

3.2 "(umgangssprachlich abwertend) in seiner Art, seinem Verhalten o. Ä. so unangenehm, dass es Abneigung oder Entrüstung hervorruft, als unleidlich, unerträglich empfunden wird“

3.3 "(umgangssprachlich)“

$3.3 \mathrm{a}$ "furchtbar $(2 \mathrm{a})$ "

$3.3 \mathrm{~b}$ "< $<$ verstärkend bei Adjektiven und Verben> furchtbar $(2 \mathrm{~b})^{\star}$ 


\subsubsection{Entwicklung der Gradbedeutung bei schrecklich}

Pfeifer verweist auf das Auftreten der Intensivierung im 17. Jh. Die Beleglage in DWB und den untersuchten Korpora bestätigt diese Angabe und führt die Herausbildung der Gradbedeutung auf das Neuhochdeutsche zurück. Als Grundlage für deren Entwicklung dient die ursprüngliche lexikalische Bedeutung, welche verschiedene Sachverhalte als Schrecken erregend bezeichnet, vgl.:

(317) das vierde thier, war grewlich und schrecklich. (DWB: s.v.)

(317') 'Schrecken erregendes thier'

(318) er ist dein rhum und dein gott, der bey dir solche grosse und schreckliche ding gethan hat, die deine augen gesehen haben. (DWB: s.v.)

(318') 'Schrecken erregendes ding'

(319) ich bin ein grosser könig, spricht der herr Zebaoth, und mein name ist schrecklich unter den heiden. (DWB: s.v.)

(319') 'Schrecken erregender Name'

Das Einsetzen der intensivierenden Funktion des Lexems bezieht sich zunächst auf pragmatic enrichment bzw. strengthening der Ausgangsbedeutung und lässt sich in ambigen Kontexten feststellen, wobei die Gradbedeutung als Nebenbedeutung hervortritt, vgl.:

(320) Wie auch bald im andern Jahr hernach / vber viel [. . ] Leute / ein schrecklich Blutbadt gefolgt ist / [.. ] Jn welchem Lermen auch Cicero jemmerlich mit vmbkommen ist. (FWB Korpus: v. d. Broek, Suevus. Spieg. 166r, 41, Leipzig 1588)

(321) diese Sach und Musik gehet in betrübtem Lamy aus; denn indem sie nun auf der Strassen sein [...], so überfallen die übergebene Reiter den Hyntho plötzlich in schrecklichem Grimm. (FWB Korpus: Qu. Brassó 4, 265, 26 siebenb., 1661)

(322) Man höret seine stimm Im zorne schrecklich brüllen (C.II: HK4/H10.00001 Hoyers: Geistliche und Weltliche Poemata, Erstdruck: 1650, 2004 [S. 289])

(323) Keinen geringen Verzug verursachte / das wütende Meer ... / sondern ergoß sich etlichmal / mit schrecklichen Stürmen tieff ins Land / hinein und ruinirte in wenig Stunden / beydes der Spanier und der Hollånder / Wercke ... (FnhdC: Hiob Ludolf: „Schaubühne, Frankfurt/Main 1699“)

In (320) - (323) lässt sich die Mehrdeutigkeit des Gebrauchs von schrecklich erkennen. Zunächst handelt es sich um die mit Schrecken verbundenen Situationen, vgl. die Umformulierungen:

(320') 'Schrecken erregendes Blutbad'

(321') 'Schrecken erregender Grimm'

(322') 'Schrecken erregend brüllen'

(323') 'Schrecken erregenden Stürmen'

Damit wird schrecklich in der Ausgangsbedeutung verwendet. Zugleich soll die intensivierende Funktion des Lexems in Betracht gezogen werden, insofern als besonders grausames und damit stark ausgeprägtes Blutbad in (320), starker Grimm 
in (321), die Stärke der Töne in (322) sowie die Intensität der Witterungserscheinung in (323) den Grund für die hervorgerufene Angst darstellen, vgl. die entsprechenden Umformulierungen:

(320') 'sehr heftiges Blutbad, sodass man Schrecken bekommt'

(321') 'sehr starker Grimm, sodass man Schrecken bekommt'

(322') 'sehr laut brüllen, sodass man Schrecken bekommt'

(323') 'sehr starke Stürme, sodass man Schrecken bekommt'

Aus den Kontexten geht hervor, dass die Bezugswörter Blutbad, Grimm, brüllen und Stürme Extremwerte aufweisende Prädikate darstellen, vgl. die aus DWB entnommenen Definitionen:

(324) Blutbad: „caedes, internecio, groszes vergieszen des menschenbluts“

(325) Grimm: „wut, wütender, heftiger zorn“

(326) brüllen: „laut schreien:“

(327) Sturm: „'procella', 'der starke wind'“

Aus den Definitionen lässt sich schließen, dass die durch die Bezugswörter ausgedrückten Begriffe jeweils das obere Ende einer Skala repräsentieren. Wie es in 3.6.2 angesprochen wurde, begünstigt die Verbindung solcher Lexeme mit einem "implicit superlative" bzw. schrecklich das Erscheinen der intensivierenden Bedeutung. Durch das Kollokieren der Wörter entsteht semantic redundancy der intensivierenden Bedeutungskomponente, sodass die letze verdoppelt bzw. verstärkt und als Nebenbedeutung dem Modifikator schrecklich zugeschrieben wird.

Im Neuhochdeutschen finden sich mehrere Belege, die die Mehrdeutigkeit des adjektivischen (328) - (329) und adverbialen (330) Gebrauchs demonstrieren können, vgl.

(328) Die Bedienten trugen mich ins Gartenhaus; ein schreckliches Gewitter tobte jetzt in der Luft; alles vereinigte sich, mich zu betrüben. (C.II: HK3/G80.00001 Tieck: William Lovell, Erstdruck: 1795/96, 2000 [S. 583])

(328') 'Das Gewitter war sehr stark und wirkte damit Schrecken erregend'

(329) Nun eben als der Morgen kam! Da drang ein Dutzend Anverwandten Herein, ein wahrer Menschenstrom; Da kamen Vettern, guckten Tanten, Es kam ein Bruder und ein Ohm. Das war ein Toben, war ein Wüten! Ein jeder schien ein andres Tier. Sie forderten des Mädchens Blüten Mit schrecklichem Geschrei von mir. (C.II: HK3/E57.00001 Goethe: Gedichte (Ausgabe letzter Hand. 1827), Erstdruck: 1827, 2000 [S. 134])

(329') 'das Geschrei war sehr laut und wirkte damit Schrecken erregend'

(330) Die Feinde liessen nicht an Bley und Pulver spahren; Die Festung wurde bald mit Feuer angefüllt; Da nahm die Furcht erst zu, die aus dem Kummer quillt. Ihr woltet zwar den Feind durch einen Ausfall zwingen, Allein ihr kuntet nicht durch die Moräste dringen. Ihr fielt bis an den Leib in solchen Schlamm hinein, Und würdet in der That bald gar versunken seyn; Die Noth war schrecklich groß; ihr ließt die Schuhe stecken, Und eiltet nur geschwind, die Füsse zu bedecken. (C.II: HK4/Z01.00001 Zäunemann: Poetische Rosen in Knospen, Erstdruck: 1738, 2004 [S. 561])

(330') 'Die Not war sehr groß und wirkte damit Schrecken erregend' 
Die Beleglage im Korpus bringt außerdem Kontexte hervor, welche die Wiederholung der gemeinsamen Sinnkomponente 'Furcht' in den beiden Teilen der adjektivischen und adverbialen Verwendungen aufweisen und durch semantic repetition Verstärkung gewinnen:

(331) es sein auch etlich klagen, die man zuo latein actiones arbitrarias, ex arbitrio iudicis pendentes nennt, als die klag so man on mittel auff die gueter ... stelt, oder so yemandts wider ains person von schrecklicher forcht ... wegen klagt (DRW: 1544, s.v.)

(332) Mich überfiel eine schreckliche Angst, ich kniff die Augen zu und tat, als schliefe ich fest. (C.II: HK3/F38.00001 Hoffmann: Lebensansichten des Katers Murr, Entstanden: 1819/21, 2000 [S. 220])

(333) Oben huschten die Schwestern in schleppend weißen Nachtgewändern von Bett zu Bett und beruhigten die Kleinen, die alle eine schreckliche Furcht vor'm Teufel hatten. (C.II: HK3/G54.00001 Panizza: Visionen, Erstdruck: 1893, 2000 [S. 260])

(334) Ja, jetzt weiß ich, warum ich mich so schrecklich fürchte, weil kein Mannsbild mehr im Hause ist. (C.II: HK4/B05.00001 Birch-Pfeiffer: Die Walpurgisnacht, Uraufführung: 1830, 2004 [S. 61])

Die Belege (320) - (323) und (328) - (334) verweisen auf das Einsetzen von subjectivity in dem Gebrauch von schrecklich, indem das Lexem durch die Expressivität des Ausdrucks die Einstellung des Sprechers zu der besprochenen Situation demonstriert. Damit kommt Traugott I (oben 3.6.3.4) zur Geltung.

In der nächsten Phase der Herausbildung wird die Ausgangsbedeutung des Lexems zurückgedrängt, bis dieser allmählich vollkommen beseitigt wird. Schrecklich tritt damit als Grad-Adjektiv bzw. -Adverb auf und demonstriert den fortgeschrittenen Prozess der Desemantisierung. Zunächst kollokiert das Lexem mit negativ konnotierten Kookkurrenzpartnern, vgl. den adjektivischen (335) - (336) und adverbialen (337) - (339) Gebrauch:

(335) Da schlief er die ganze Nacht nicht, hatte am nächsten Tage schreckliche Migräne und versäumte eine wichtige geschäftliche Angelegenheit. (C.II: HK3/F71.00001 Altenberg: Märchen des Lebens, Erstdruck: 1908, 2000 [S. 169])

(335') 'sehr starke Migräne'

(336) Kaum wird es mal sommerlich warm, stöhnen alle unter der schrecklichen Hitze. Zumal die Schüler. Die dürfen morgens nämlich nicht mehr ins kühle Schulhaus. Was also tun? In Dierdorf weiß man die Lösung: Ab mit den Jugendlichen ins Schwimmbad. (C.II: RHZ96/AUG.05703 RZ, 12.08.1996)

(336') 'sehr starken Hitze'

(337) Es war Mittag; ich war schrecklich müde, und die Sonne wurde mir so lästig, daß ich in eine von den Kanonen hineinkroch ... (C.II: HK3/F75.00001 Bürger: Münchhausen, Erstdruck: 1786, 2000 [S. 143])

(337') 'sehr müde'

(338) Er mußte es tun, sein Chef hatte ihm strengstens eingeschärft, jeden Wunsch der Kunden zu erfüllen, ... aber es war ihm so langweilig, so 
schrecklich langweilig. (C.II: HK3/D23.00001 Franzos: Der Pojaz, Erstdruck: 1879,2000 [S. 184])

(338') 'sehr langweilig'

(339) Sie verteilte gleich die ganze Tube Färbemittel in ihren Haaren. Dann geschah etwas Entsetzliches. „Meine Augen verklebten und mein Kopf schwoll an. Es tat schrecklich weh", sagt Abigail. Eine allergische Reaktion. (C.II: HMP09/DEZ.00318 MOPO, 03.12.2009, S. 47)

(339') 'tat sehr weh'

Schrecklich kann außerdem als Gradadverb Skopus über Funktionsverbgefüge haben und dadurch die Erweiterung seiner Skopuskonstituente demonstrieren. Die Intensivierung erfolgt dadurch, dass das Substantiv in dem FVG graduierbar ist, wie in (340) oder das FVG durch einen graduierbaren Begriff wiedergegeben werden kann, wie in (341) - (342):

(340) „Wir standen ganz schrecklich unter Druck“, sagt der Mitarbeiter eines Abgeordneten. (06.05.2014: http://www.tagesspiegel.de/wirtschaft/klimaschutz-das-elektroauto-solls-retten/1322948.html)

(340') 'standen unter sehr starkem Druck'

(341) Unter anderen Umständen hätte mir das schrecklich auf die Nerven gefallen, aber so, wie es lag, erholte ich mich [...] (GB, .05.2014: http://books.google.de/books?id=5gKQ10Rq4XUC\&pg=PA21\&dq=\%22schrecklich+auf+die+Nerven+gefallen $\% 22 \& \mathrm{hl}=$ de \&sa=X\&ei $=$ OHCEU500IYb54QTshIDICA\&ved=0CDIQ6AEwAA\#v=onepage\& $\mathrm{q}=\% 22$ schrecklich\%20auf\%20die\%20Nerven\%20gefallen $\% 22 \& \mathrm{f}=$ false)

(341') 'sehr genervt'

(342) Ich hatte mir die Zunge fusselig geredet und meinem Chef dieses Thema, das mir wirklich schrecklich unter den Nägeln brannte, ans Herz gelegt. (GB, 06.05.2014: http://books.google.de/books?id=zQFAXyQx-D0C\&pg=PA380\&lpg=PA380\&dq=\%22schrecklich+unter + den + N\%C3\%A4geln\%22\&source=bl\&ots=ylxfhBli8s\&sig=GaQA3MrC5sNZgFQBunAXk_RRZyw\&hl=de\&sa=X\&ei=uTWrUr6sJYmutAbq7YHYCg\&ved=0CEYQ6AEwAg\#v=onepage $\& \mathrm{q}=\% 22$ schrecklich\%20unter\%20den\%20N\%C3\%A4geln\%22\&f=false)

(342') 'war sehr wichtig'

Schrecklich kann außerdem in quantifizierender Funktion auftreten und die Menge der Sachverhalte bezeichnen. In (343) tritt das Lexem in einem negativ konnotierten Kontext, vgl.:

(343) Er sagte mir, Mousa [...] habe seinem Führer für die Reise [...] fünf und zwanzig bezahlt, und er werde daher dieselbe Straße nicht für weniger als drei und zwanzig mit mir gehen. Dies war eine schreckliche Summe für eine Reise von zwei Tagen in einem Lande, wo ein Araber vierzehn Tage fleißig arbeitet, ohne dieß verdienen zu können. (GB, 06.05.2014: http://books.google.de /books?id=1TcUAAAAQAAJ\&pg=RA1-PA47\&lpg=RA1-PA$47 \& \mathrm{dq}=\% 22$ schreckliche+summe\%22\&source=bl\&ots= F3W7UjzVJJ\&sig=NJBprLSbSfOIfwmuI3cLonq0ow\&hl=de\&sa=X\&ei=hzawUrnYHcrBtAbs0oDQCw\&ved=0CFEQ6AEwBw\#v=onepage \&q=\%22schreckliche $\% 20$ summe $\% 22 \& \mathrm{f}=$ false)

(343') 'sehr große Summe' 
Neben der Intensivierung tritt eine rein pejorative Bedeutung in Erscheinung, die den Verlust des direkten Kontakts mit der Ausgangsbedeutung und zugleich die Entwicklung von subjectivity aufweist vgl.:

(344) Da sie Hustensaft zwar auch nicht mag, aber zumindest nicht ganz so schrecklich findet, versuchte meine Freundin, sie mit Argumenten zu überzeugen [...] (C.II: RHZ96/FEB.05772 Rhein-Zeitung, 09.02.1996)

(344') 'sehr schlecht findet'

(345) „Eigentlich war es die schrecklichste Hochzeitsfeier, auf der ich je gewesen bin", stellt Tetta Müller fest. (C.II: M10/APR.32334 Mannheimer Morgen, 27.04.2010, S. 27)

(345') 'die schlimmst Hochzeitsfeier'

(346) Sie finde diese Boxen eine schreckliche Idee, sagte eine der Aktivistinnen. So würde die Prostitution nur gefördert. (C.II: SOZ11/NOV.02334 Die Südostschweiz, 11.11.2011, S. 28)

(346') 'sehr schlechte Idee'

Die Belege (335) - (342), (343) und (344) - (346) veranschaulichen den eindeutigen Gebrauch von schrecklich jeweils in seiner intensivierenden, quantifizierenden und wertenden Funktion, ohne einen direkten Zusammenhang mit der Ausgangsbedeutung aufzuweisen. Zugleich demonstrieren die Verwendungen die Zunahme an subjectivity, indem sie an Expressivität gewinnen und damit den Übergang zu Traugott III (oben 3.6.3.4) signalisieren.

Die letze Etappe des Entwicklungsgangs der Gradbedeutung bezieht sich auf den endgültigen Verlust des Zusammenhangs mit der Ausgangsbedeutung. Schrecklich kollokiert mit positiv konnotierten Kookkurrenzpartnern und fungiert damit als bedeutungsentleert, vgl.:

(347) Der Sänger hinwiederum war tadellos elegant angezogen und schrecklich fröhlich wie eine Lerche. (C.II: HK3/G72.00001 Altenberg, Peter: Was der Tag mir zuträgt, Erstdruck: 1901, 2000 [S. 202])

(347') 'sehr fröhlich'

(348) „Das ist also der Bräutigam, der zu seiner Braut fährt. Er sieht schrecklich verliebt aus", sagte Lement und lächelte dann mit geschlossenem Munde. (C.II: HK3/A52.00007 Kafka, Franz: Prosa aus dem Nachlaß: Hochzeitsvorbereitungen auf dem Lande, Entstanden: 1907, 2000 [S.16])

(348') 'sehr verliebt'

(349) Probieren Sie es aus, vielleicht wird ja auch der Blaufränkisch bald schrecklich trendy - und dann waren Sie schon längst dabei. (C.II: HMP05/OKT.01866 Hamburger Morgenpost, 20.10.2005, S. 27)

(349') 'sehr trendy'

(350) „Ich hatte neulich eine Massage“, sagt stolz Maitlin, eine Elfjährige aus San Diego. „Und es war schrecklich entspannend. " (C.II: M08/APR.28231 Mannheimer Morgen, 14.04.2008, S. 15)

(350') 'sehr entspannend' 


\subsubsection{Kurzfassung der Entwicklung bei schrecklich}

Ausgangsbedeutung: 'Schrecken hervorrufend';

die übrigen Entwicklungsschritte wie oben in Kap. 3.6.3.9.3.

\subsubsection{Ungeheuer}

\subsubsection{Lexikographische Angaben}

Kluge (s.v. Ungeheuer) sieht das Adjektiv ungeheuer Ausgangspunkt für die Substantivierung. DWB (s.v.) verortet das Auftreten der Form im Ahd. Pfeifer (s.v.) zitiert die Formen „ahd. ungihiuri Adj. 'unnatürlich, mißgestaltet' (um 800)“ und „mhd. ungehiure“.

1. ungeheuer in Köbler (s.v. ungihiuri)

„ungeheuer, furchtbar, abscheulich, scheußlich, widernatürlich, Furcht erregend"

2. ungeheuer in BMZ und Lexer (s.v. ungehiure, un-gehiure)

„unheimlich, unlieblich, abscheu und entsetzen erregend“ (BMZ, s.v. ungehiure), „unlieblich, unheimlich, ungeheuer, schrecklich“ (Lexer, s.v. un-gehiure)

3. ungeheuer in FWB

keine Angaben, u.a. im bereits zitierten Material der Redaktionsstelle von FWB

4. ungeheuer in DWB (s.v.)

4.1 ,infamiliaris, ursprünglich ohne antheil an familie, hausgenossenschaft, hauswesen, heim, heimat und ihrem sittigenden, beglückenden einflusz"

4.2 „bezeichnet u., was schutz, sicherheit und trauliches behagen von haus und heim vermissen läszt"

4.3 „gth. von geheuer $1 \mathrm{c}$; den übergang stützt urzeitliche vorstellung, die im fremden den feind, das feindliche, schädliche, schreckliche sieht; furcht und einbildungskraft des schutzlosen schwachen steigern dies zum entsetzlichen, gräszlichen, grauenhaften, furchtbaren, fürchterlichen u. s. w.“

4.3a "feindselig, bösartig, widerwärtig u. dgl.“

$4.3 \mathrm{~b}$ „erzürnt, ungestüm, wütend, gierig, unruhig, traurig, sinnlos“

$4.3 \mathrm{c}$ „schädlich, unheilvoll, übel, unwillkommen, unangenehm“

$4.3 \mathrm{~d}$,schändlich, entsetzlich, gräszlich, schrecklich, unmenschlich, grauenvoll, abscheulich u. s. f."

4.3e „bezogen aufs äuszere, gräulich, drohend, scheuszlich, schauderhaft, entstellend, ekelhaft, (schmutzig)“

4.4 "unheimlich, nicht geheuer, gs. geheuer"

$4.4 \mathrm{a}$ „im sinne des mythologischen denkens“

$4.4 \mathrm{~b}$,in verkirchlichter vorstellung"

$4.4 \mathrm{c}$ „nachdem das myth. denken in der vorstellung des gebildeten mehr und mehr erloschen, führt der begriff nur noch in der dichtung oder poetisierender schriftsprache ein schattenhaftes nachleben“

4.5 „als ausdruck der sog. intension für gewaltig, ungewöhnlich grosz (wichtig, bedeutungsvoll), auszerordentlich nach erscheinung, anzahl, wesen und wirkung, übermäszig, überwältigend u. dgl., bedeutungen“ 
5. ungeheuer in DUDEN (2012, s.v.)

5.1 ,außerordentlich groß, stark, umfangreich, intensiv, enorm; riesig, gewaltig"

5.2 „<intensivierend bei Adjektiven und Verben> (oft emotional übertreibend) außergewöhnlich, außerordentlich, überaus, sehr, im höchsten Grad, Maß"

\subsubsection{Entwicklung der Gradbedeutung bei ungeheuer}

Die Angaben in DWB lassen den Entwicklungsgang der Gradbedeutung verfolgen und deren Einsetzen auf die frühneuhochdeutsche Stufe zurückführen. Die Beleglage in DWB untermauert die Annahme, dass die mit Schrecken verbundene Bedeutung als Grundlage für deren Entwicklung dient, vgl.:

(351) und je schwärzer es war, je grausender, ungeheurer desto öfter geschah's (DWB, s.v.)

(351') 'schrecklicher'

(352) Fragstu / wohin er gefahren? nicht ins Grab / welches in corde terrae, in dem Hertzen der Erden / vnd also innerhalb der Erden gewest / sondern

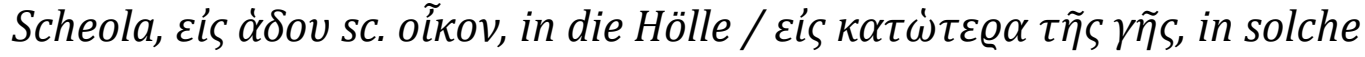
vngeheure / schröckliche fünstere Ort / die wie vnter vnd ausser dem Erdboden sind / viel schröcklicher / als kein Abgrund / kein Avernus, kein brennender AEthna. (DTA: Dannhauer, Johann Conrad: Catechismus Milch. Bd. 5, Straßburg, 1654)

(352') 'Schreckend erregender Ort'

DWB verweist darauf, dass die Gradbedeutung zunächst in den Verbindungen mit solchen Sachverhalten erfolgt, die „von ihrer menge, grösze und intension furcht, schrecken und erstaunen erwecken“, und setzt die Ausgangsbedeutung als „Voraussetzung" für die Steigerung an. Daraus lässt sich schließen, dass der adjektivische bzw. adverbiale Gebrauch in einer frühen Phase der Entwicklung der Intensivierung mit pragmatic enrichment bzw. strengthening von der Ausgangsbedeutung sowie daraus folgender Ambiguität verbunden ist. So gewinnt die Ausgangsbedeutung von ungeheuer an Gehalt, indem eine Verknüpfung zwischen der qualifizierenden Funktion des Lexems und der Gradbedeutung entsteht, vgl. die Belege (353) - (355), die neben dem Begriff des Schreckens die Bedeutung „riesig“ (DWB, s.v. ungeheuer 5) demonstrieren, vgl.:

(353) die abenthewrische und ungehewre hydram (DWB: s.v.)

(354) der ungehiure rise (DWB: s.v.)

(355) Wir fahen diefen tag viel Vogel / damals fuhren wir Weft / vnd Weft Sudweft / vnd begegneten vns vngeheure Wellen von Sud Sudoften. (DTA: Gottfried, Johann Ludwig: Newe Welt Vnd Americanische Historien. Frankfurt (Main), 1631)

Die Bedeutung von ungeheuer in (353) - (355) gewinnt so an Mehrdeutigkeit. DWB erwähnt die Vorstellungen des Schreckens und der Größe, die zugleich auf die Ausgangsbedeutung zutreffen. Die adjektivischen Zusammensetzungen sind von daher mit der Ausgangsbedeutung verbunden und beschreiben eine Schrecken erre- 
gende Hydra, einen Schrecken erregenden Riesen und Schrecken erregende Wellen. Zugleich handelt es sich um die Größe der Sachverhalte, die den Grund für die entstandene Furcht darstellt. Außerdem wird durch die Verbindungen die Einstellung des Sprechers bzw. Schreibers zum Ausdruck gebracht, was auf das Einsetzen von subjectivity hinweist.

Die intensivierende Funktion des Lexems kann durch weitere Mehrdeutigkeit erweisende Kontexte exemplifiziert werden, vgl.:

(356) [...] er [Andronicus] war ein vngehewrer Tyrann vnd Bluthund / der den frommen Alexium feinen Pfleg-fohn vnd mit-Regenten bey der Nacht mit einer Cordel oder Sennen von einem Bogen ftrangulieren vid feinen Leib ins Meer werffen laffen / vnd andert vnerho̊rte Blutdürftige Mordthaten zu Conftantinopel defignirt; dadurch er fich bey menniglich verhaßt gemacht [...] (DTA: Dannhauer, Johann Conrad: Catechismus Milch. Bd. 5. Straßburg, 1654)

(357) Was der ungeheure Wind / der An. 1630. vor der Verfto̊rung der Stadt Magdeburg vorher gewütet / die Kirch-Thürn nach einander herunter gefchmettert / die Waffer- und Wind-Mühlen abgeblafen / das hat bald hernach befagte Stadt mit Ach und Wehe / åufferftem Jammer und Hertzenleyd erfahren. (DTA: Dannhauer, Johann Conrad: Catechismvs-Milch. Bd. 8. Straßburg, 1666)

(358) Kaum daß fie ein Weil fortgefahren / da erhub fich ein gefährliche Vngeftimme mit erfchröcklichen Saufen / vnnd Braufen der Suth / vnnd die mitternåchtige Winden / daß alfo die Apoftel vor Schrocken gantz erblaicht [...]. Der heilige Kirchenlehrer Ambrofius fambt anderen mehrer ift der Außfag / daß folche Vngeftimme deß Meers / vnd vngeheurewütten der Wind feye entftanden wegen den Judam Ifcarioth, welcher dazumahlen bey difen heiligen Apofilen auch zu gegen war. (DTA: Clara, Abraham a Sancta: Judas Der Ertz-Schelm. Bd. 1. Salzburg, 1686.)

In (356) verknüpft sich die Bedeutung des Schreckens mit dem begleitenden Begriff des Grausamen, Bösartigen sowie der Intensivierung. In (357) - (358) erscheint die Bedeutung des Schreckens neben der Gradbedeutung aufutreten. Die Intensität der Ausprägung stellt dabei die Ursache für das erweckte Schrecken, vgl.:

(356') 'ein sehr großer Tyrann und Bluthund, was Schrecken erregend wirkte' (357') 'sehr starker und damit Schrecken erregender Wind'

(358') 'sehr starke und damit Schrecken erregende Wüten'

Aus den Kontexten geht hervor, dass die Bezugswörter Extremwerte aufweisende Prädikate darstellen, die in Verbindung mit ungeheuer das Auftreten einer intensivierenden Funktion begünstigen.

Laut DWB bildet "die Zeit der Aufklärung" eine "Grenze“ in dem Gebrauch des Lexems, sodass „das wort nach den einzelnen culturzeitaltern verschieden aufzufassen“ ist. Beispielsweise würde die Verwendung „ungeheurer kampf“ "früher" als „pugna infesta“ und "heute“ als „ingens" umformuliert. Damit verblasst die Ausgangsbedeutung mit der Zeit allmählich. DWB verweist ferner darauf, dass der mehrdeutige Gebrauch des Lexems bis zum Nhd. erhalten bleibt. Seit dem $18 \mathrm{Jh}$. hat man „den alten begriff vergessen“, und die reine intensivierende Funktion des 
Wortes kommt zur Geltung. Hier ist anzumerken, dass, obgleich die Verwendung von ungeheuer in der ausgebildeten Gradbedeutung im $18 \mathrm{Jh}$. zu finden ist, der ambige Gebrauch des Lexems doch eine Weile im Korpus belegt ist, vgl.:

(359) „Kaum aber", fuhr er fort, „hatte unser kleines Fahrzeug die Wellen berührt, so brach auch schon der ungeheure Sturm los, der noch jetzt über unsern Häuptern fortwütet. Es war, als hätten die Fluten nur auf uns gewartet, um die allertollsten, strudelndsten Tänze mit uns zu beginnen. Die Ruder waren bald aus meiner Führer Händen gerissen und trieben zerschmettert auf den Wogen weiter und weiter vor uns hinaus. [...]"(C.II: HK3/HK1.00060 Fouqué, Friedrich de la Motte: Undine, Erstdruck: 1811, 2000 [S.65])

(359') 'Der Sturm war sehr stark und wirkte damit Schrecken erregend'

In (359) handelt es sich um einen starken Sturm, der durch seine Intensität schlimme Auswirkungen auf die angesprochenen Personen hat und damit Schrecken erregend wirken kann. Darüber hinaus weist das Adjektiv die Mehrdeutigkeit des Gebrauchs auf.

Im Korpus finden sich außerdem mehrere Belege, die das Auftreten der Intensivierung durch semantic repetition demonstrieren, vgl.:

(360) Doch / wie nach tieffer Nacht und ungeheurem Schrecken / Der Sonne Purpur-Glantz den Himmel wieder mahlt: So wird des Schöpffers Ruff den Leib einft auferwecken/ Daß feine Klarheit mehr als Gold und Perlen ftrahlt.

(DTA: Mühlpfort, Heinrich: Teutsche Gedichte. Bd. 1. Breslau u. a., 1686)

(361) Eine ungeheure Bangigkeit ergriff ihn. (C.II: HK3/A91.00016 Novalis: Heinrich von Ofterdingen, Entstanden: 1799-1800, 2000 [S.278])

(362) Am ersten November 1755 ereignete sich das Erdbeben von Lissabon, und verbreitete über die in Frieden und Ruhe schon eingewohnte Welt einen ungeheuren Schrecken. (C.II: GOE/AGD.00000 Goethe, Werke; Wahrheit I-III, (Geschr. 1809-1813), 1982, Bd. 9 [S. 29])

(363) Theodor starrte ihn sprachlos an, Wilhelm aber, in ungeheurer Angst, ergriff seine Hand und zog ihn hastig mit sich fort [...] (C.II: HK3/HK1.00105 Hebbel, Friedrich: Die einsamen Kinder, Erstdruck: 1835/36, 2000 [S.298])

In (360) - (363) wird der durch ungeheuer auszudrückende Begriff 'Angst' in den Bezugswörtern wiederholt, sodass es zu semantic repetition, semantic redundancy und demnach zur Verstärkung des adjektivischen Gebrauchs kommt.

Die Belege (353) - (363), die die zweite Stufe der Entwicklung von Gradbedeutung demonstrieren, weisen zugleich das Einsetzen von subjectivity auf, indem der Gebrauch eine evaluative Bedeutungskomponente gewinnt und die Einstellung des Sprechers bzw. Schreibers zum Ausdruck bringen. Hier wirkt Traugott I (oben 3.6.3.4).

In der nächsten Etappe der Entwicklung fungiert ungeheuer als Grad-Adjektiv bzw. -Adverb, ohne eine direkte Verbindung zur Ausgangsbedeutung erkennen zu lassen. Das Lexem dient lediglich der Intensivierung des modifizierten Elements. Auf 
dieser Stufe tritt ungeheuer in negativen Kontexten auf, sodass der Prozess der Desemantisierung des Wortes noch nicht vollkommen vollzogen wird, vgl. den adjektivischen (364) - (366) und (367) - (368) adverbialen Gebrauch:

(364) Der Fürft Bifchof war ein Mann in feinen beften Jahren, und fo ungeheuer dick, daß das Fett feine Nerven, fein Herz und feine Seele ganz ůberzogen zu haben fchien. (DTA: Klinger, Friedrich Maximilian: Fausts Leben, Thaten und Höllenfahrt. St. Petersburg, 1791)

(364') 'sehr dick'

(365) Eine solche Einrichtung bedeutete einen ungeheuren Kräfteverlust, die Nichtausnutzung allereigentümlichen Begabungen und Talente, die mittelmäßige Arbeit aller und die Vernichtung der größten Lustgefühle, die mit der Thätigkeit im rechten Specialberuf gegeben sind. (DTA: Schmoller, Gustav: Grundriß der Allgemeinen Volkswirtschaftslehre. Bd. 1. Leipzig, 1900 )

(365') 'sehr großen Kräfteverlust'

(366) sozialdemokratische Wirtschaftspolitik übersieht dabei nicht die ungeheuren Schwierigkeiten, die im Konflikt der verharrenden Strukturen mit der Dynamik von Wissenschaft, Technik und internationaler Marktverflechtung entstehen. (C.II: H86/FZ4.51333 Zeit, 22.08.1986, S. 20)

(366') 'sehr großen Schwierigkeiten'

(367) Der Schaden für die Politik, die AK und die SPÖ sei ungeheuer groß. (C.II: N94/SEP.35305 Salzburger Nachr., 27.09.1994)

(367') 'sehr groß'

(368) Wer so hemmungslos jubelt wie das BTSC-Team, der muss ungeheurem Druck ausgesetzt gewesen sein. (C.II: BRZ12/NOV.05973 Braunschw. Z., 12.11.2012)

(368') 'sehr großem Druck'

Ungeheuer kann außerdem als Gradadverb Funktionsverbgefüge modifizieren und damit die Ausdehnung seines Bezugsbereichs demonstrieren. Die Intensivierung erfolgt dadurch, dass die Bezugswörter Wut und Gefahr in den hervorgehobenen FVGs in (369) - (370) graduierbar sind, während die FVGs in (371) - (372) durch einen graduierbaren Begriff wiedergegeben werden können, vgl.:

(369) „Ich beobachte derartiges in letzter Zeit fast täglich und gerate ungeheuer in Wut darüber, dass Autofahrer aus reiner Bequemlichkeit den Schulweg der Kinder derartig gefährden. [...]“" (C.II: BRZ05/DEZ.19519 Braunschweiger Zeitung, 22.12.2005)

(369') 'gerate in sehr starken Wut'

(370) Die größere Gefahr ist in der Seele dieses Papstes. Die Inbrunst, mit der er diese Reise absolviert, seine 91. und eine ganz besondere - gibt ihm ungeheure Kraft und bringt ihn ungeheuer in Gefahr. (C.II: 00/MAR.34770 Neue Kronen-Zeitung, 24.03.2000, S. 10; Heiliges Land)

(370') 'bringt ihn in sehr große Gefahr'

(371) „[...] Aber die Musiker beider Lager, die sich als Verwalter der jeweiligen Traditionen sehen, gehen mir ungeheuer auf die Nerven", erklärt er. (27.05.2014: http://www.rp-online.de/kultur/kunst/stargeiger-nigel-kennedywird-50-aid-.2036244) 
(371') 'nerven sehr'

(372) An gelungenen Abenden herrschte in dem Laden eine absolut geniale Stimmung, an den weniger gelungenen Abenden konnte einem das gleiche Lokal mit den gleichen Gästen ungeheuer auf den Zeiger gehen. (27.05.2014: https://exyl.wordpress.com/2009/02/17/)

(372') 'sehr nerven'

Die quantifizierende Bedeutung von ungeheuer in (373) - (375) demonstriert den Verlust des Zusammenhangs mit der Ausgangsbedeutung, tritt jedoch zunächst in negativen Kontexten auf, vgl.:

(373) Er verlor ungeheure Summen, weil er wie ein verzweifelter Spieler wagte. (C.II: HK3/B01.00003 Schiller, Friedrich: Der Geisterseher, Entstanden: $1786-1798,2000$ [S.140])

(373') 'sehr große Summen'

(374) Jetzt blieben aber noch die vorigen ungeheuren Preise; der Verkäufer gewann nur um so viel mehr. (C.II: HK3/A44.00003 Forster, Georg: Parisische Umrisse, Entstanden: 1793/94, 2000 [S.747])

(374') 'sehr hohe Preise'

(375) „er ist der größte Betrüger in der ganzen Stadt, ziehn Sie sobald als möglich von ihm aus, sonst wird er Ihnen eine ungeheure Rechnung machen!" (C.II: HK3/HK1.00167 Tieck, Ludwig: Die beiden merkwürdigsten Tage aus Siegmunds Leben, Erstdruck: 1797, 2000 [S.54])

(375') 'sehr hohe Rechnung'

In (353) - (355) wurden mehrdeutige Verwendungen exemplifiziert, die sich auf Schrecken erregende Lebewesen von außergewöhnlicher Größe beziehen. Aus der Korpusanalyse ergibt sich, dass durch den Prozess der Desemantisierung der $\mathrm{Zu}$ sammenhang mit der Ausgangsbedeutung verlorengeht, sodass ausschließlich die Bedeutung „sehr groß“ erhalten bleibt, vgl.:

(376) So kamen sie alle, jeder in seiner Weise, manche, die kein Geld hatten, boten Getreide, und ein Stadtbauer wickelte aus seinem Tuch eine ungeheure Wurst. (C.II: HK3/D57.00001 Freytag, Gustav: Die Ahnen, Erstdruck: 1872-1880)

(376') 'sehr große Wurst'

Die Belege (364) - (372), (373) - (375) und (376) demonstrieren den eindeutigen Gebrauch von ungeheuer in Bezug auf die Intensität, Quantität und Größe der Sachverhalte. Außerdem hat sich die einstellungsbezogene Bedeutung in dem Gebrauch fest eingesetzt, sodass die Verwendungen mehr Expressivität zum Ausdruck bringen und damit auf Traugott III (oben 3.6.3.4) verweisen.

Anschließend tritt ungeheuer als Grad-Adjektiv bzw. -Adverb in positiven Kontexten auf. Damit geht die Verbindung mit der Ausgangsbedeutung endgültig verloren. Das bleaching ist komplett vollzogen, vgl. den adjektivischen (377) - (379), (382) und adverbialen (380) - (382) Gebrauch: 
(377) Einen ungeheuren Beifall findet Scribes neue Oper „Die Sirene“, wozu Auber die Musik geschrieben. (C.II: HK3/F47.00001 Heine, Heinrich: Lutetia, 1840, 2000 [S.580])

(377') 'sehr starken Beifall'

(378) Ich war wie ein Träumer, wie ein Trunkener, fast nicht ertragend das ungeheure Glück - und als ich schon zu Hause war - als ich ohne Licht auf meinem Sofa saß, malte ich mir dieses Glück noch seliger in die finstere, wimmelnde Luft. (C.II: HK3/G41.00001 Stifter, Adalbert: Studien, Erstdruck: 1844 bis 1850, 2000 [S.125])

(378') 'sehr großes Glück'

(379) «Es gibt mir eine ungeheure Befriedigung, die Dinge, die ich erledigt habe, anzustreichen.» (C.II: A08/MAI.05222 St. Galler Tagbl., 17.05.2008, S. 12)

(379') 'sehr große Befriedigung'

(380) Radfahren habe ihm nach seiner Erkrankung ungeheuer geholfen: „Es war psychologisch wichtig, es war motivierend!" (C.II: M13/MAI.02993 Mannh. Morgen, 11.05.2013, S. 20)

(380') 'sehr geholfen'

(381) In der Wissenschaft beobachte ich das gleiche Phänomen: Wenn eine Hochschule einen Nobelpreisträger in ihren Reihen hat, gewinnt sie ungeheuer an Attraktivität und kann dadurch viele junge Talente für sich gewinnen. (C.II: E99/AUG.22331 Zürcher Tagesanzeiger, 02.08.1999, S. 21)

(381') 'gewinnt sehr viel'

(382) wir haben in Europa die Vielfalt der einzelnen Nationen, ein ungeheuer interessantes Experimentierfeld, auf dem wir sehr viel lernen können, weil neue Techniken auf ganz unterschiedliche Kulturen stoßen. nur, diesen ungeheuren kulturellen Vorteil, die Vielfalt von Experimenten, den nutzen wir nicht richtig. (C.II: H85/QZ1.15763 Zeit, 04.01.1985, S. 09)

(382') 'sehr interessantes', 'sehr großen Vorteil'

\subsubsection{Kurzfassung der Entwicklung bei ungeheuer}

Ausgangsbedeutung: 'Schrecken hervorrufend', gelegentlich mit dem begleiteneden Nebensinn der Größe oder des Bösartigen; die übrigen Entwicklungsschritte wie oben in Kap. 3.6.3.9.3.

\subsubsection{Ungeheuerlich}

\subsubsection{Lexikographische Angaben}

Kluge liefert keine Informationen über das Lexem. DWB (s.v.) stellt ungeheuerlich als ein "seitenstück zu ungeheuer" dar und verweist auf die Formen ahd. unhiurlîch; mhd. unhiurlîch, ungehiurlîche adv. mnd. unhûrlîk, un(ge)hûrlîch.

1. ungeheurlich in Lexer (s.v. un-gehiurlîche)

- keine lexikographischen Angaben

(s.v. un-hiurlîche)

"entsetzlich, auf abscheuliche weise“

2. ungeheuerlich in FWB 
- keine Angaben, auch nicht im bereits zitierten Material der Redaktionsstelle von FWB

3. ungeheuerlich in DWB (s.v.)

3.1 wie ungeheuer 1.1 „infamiliariter, solitarie“

3.2 wie ungeheuer 2.1

3.3 wie ungeheuer 3.1

3.4 wie ungeheuer 4.1

3.5 wie ungeheuer 5.1 „als bloszer ausdruck stärkster intension gleich“

4. ungeheuerlich in DUDEN (2012, s.v.)

4.1 (seltener)

4.1a „ungeheuer (a)“

$4.1 \mathrm{~b}$ „<intensivierend bei Adjektiven und Verben> ungeheuer (b)“

\subsubsection{Entwicklung der Gradbedeutung bei ungeheuerlich}

Die Beleglage in DWB und in COSMAS liefert klare Anhaltspunkte für das Einsetzen der Gradbedeutung im Nhd. Als Basis für deren Entwicklung dient die auf Schrecken bezogene Sinnrichtung, die im folgenden als Ausgangsbedeutung angesehen wird, vgl.:

(383) in dem nächsten raume (des maschinenhauses) ging es weniger ungeheuerlich zu (DWB s.v. ungeheuerlich)

(383') 'Schrecken erregend'

(384) Ich wette, du wirst es mir nicht vergeben können, daß ich diese interessante abenteuerliche und ungeheuerliche Geschichte nicht fortsetze [...]. (C.II: HK3/F92.00001 Tieck: Peter Lebrecht, Erstdruck: 1795/96, 2000 [S. 75])

(384') 'unheimliche, Schrecken erregende Geschichte'

(385) Shakfpeare läßt die Gräuelthaten feiner Könige durch ungeheuerliche Erfcheinungen vorausverkünden: eine Eule verfolgt einen Falken, die Roffe Dunkans freffen fich. (DTA: Alexis, Willibald: Ruhe ist die erste Bürgerpflicht oder Vor fünfzig Jahren. Bd. 5. Berlin, 1852)

(385') 'unheimliche, Schrecken erregende Erscheinungen'

Die Korpusanalyse hat ergeben, dass das Einsetzen der Intensivierung mit pragmatic enrichment bzw. strengthening der Ausgangsbedeutung beginnt und dadurch Ambiguität im Gebrauch zu Folge hat. Die Gradbedeutung zeigt sich als Nebenbedeutung durch pragmatic inferencing, vgl.:

(386) [...] in der Dämmerung des Morgens und wenn die Sonne niedrig an dem Horizonte steht, sind die Schatten ungeheuerlich gro $\beta$, und wer nicht weiß, woher das kommt, mag leicht vor ihnen erschrecken [...] (28.05.2014: 1856, http://books.google.de/books?id=XEEtAAAAYAAJ\&pg=PA69\&dq=ungeheuerlich\&hl=de\&sa=X\&ei=NeSFU_jKC0374QSkq4DIDQ\&ved=0CEMQ6AEwAzge\#v=onepage \&q=ungeheuerlich\&f=false)

(387) dann schob sich der Meereshorizont mit zwei Schiffen in die Höhe; sie standen wie auf einem Berge, während das dritte in der Tiefe blieb; dann senkte sich die Meereslinie wieder, die Masten blieben in der Luft stehen und der Rumpf der beiden Schiffe schwoll so ungeheuerlich, dass 
sie Thürmen glichen, die allmälig einsanken, während nun die Untermasten wuchsen. (DTA: Martens, Georg von: Die preussische Expedition nach OstAsien. Nach amtlichen Quellen., 1873)

(388) In einem großen Käfig verwahrt mußte es der ftolze Beherrfcher aller Gläubigen und Sieger über die vereinigten fränkifchen Heere bei Nikopoli erleben, wie man ihn vor Amafia brachte und unter feinen Augen die ungeheuerlichften Graufamkeiten beging. (DTA: Schweiger-Lerchenfeld, Amand von: Armenien. Ein Bild seiner Natur und seiner Bewohner. Jena, 1878.)

Der hier hervorgehobene Gebrauch in (386) - (388) lässt mindestens zwei Lesarten zu. Zunächst beschreibt das Lexem die Sachverhalte als Schrecken erregend und verweist damit auf die Ausgangsbedeutung. Die alternative Interpretationsmöglichkeit bezieht sich auf die Intensität der Geschehnisse, die gelegentlich als Grund für die hervorgerufene Angst gilt, sodass etwa die folgenden Paraphrasen entstehten:

(386') 'die Schatten sind sehr groß und wirkten damit Schrecken erregend' (387') 'die Schiffe schwollen so stark [an], dass es Schrecken erregend war'

Die adjektivische Verwendung in (388) bezieht sich zunächst auf grausame Taten, die bei der angesprochenen Person Schrecken hervorrufen. Dabei verweist die Superlativform des Adjektivs auf die höchste Stufe der erregten Angst. - Die zweite Interpretation ist einstellungsbezogen, beruht auf dem Begriff der Intensivierung und setzt ungeheuerlich als Gradadjektiv voraus. Aus dem Kontext lässt sich schließen, dass es sich um besonders schlimme Handlungen geht, vgl.:

(388') 'die Grausamkeiten sind in so hohem Maße schlimm, dass sie Angst erregend wirken'

Die Steigerungsform des Adjektivs verweist darauf, dass der Sprecher bzw. Schreiber verschiedene Stufen von Grausamkeit unterscheidet. Demzufolge kann die letzte mehr oder weniger schlimm sein. Die Verwendung des Superlativs demonstriert, dass die Grausamkeiten, der Meinung des Sprechers bzw. Schreibers nach, am stärksten ausgeprägt sind und auf der entsprechenden Skala, die die Intensität der Grausamkeit veranschaulichen soll, am oberen Rande anzusiedeln sind.

Das Auftreten der Gradbedeutung kann außerdem durch semantic repetition exemplifiziert werden, insofern als beide Teile der Kollokation die gemeinsame Bedeutungskomponente 'Furcht' aufweisen. Durch die Wiederholung dieser Komponente wird semantic redundancy ausgelöst, wodurch es zu der Verstärkung des Ausdrucks kommt:

(389) In solchen Fällen überwinden sie selbst ihre sonst so ungeheuerliche Furcht vor Ansteckung. (GB, 28.05.2014: 1864, http://books.google.de/books?id=l0Q7AAAAcAAJ\& pg=PA305\&dq=\%22ungeheuerliche+Furcht $\% 22 \&$ hl $=$ de \&sa=X\&ei=Me2FU_ORH4va4QT4nIG4Aw\&ved=0CDQQ6AEwAA\#v=onepage\&q=ungeheuerliche $\% 20$ Furcht $\% 22 \& \mathrm{f}=$ false)

(390) Der verschüchterte Mann, den die Qualen des Gewissens ebenso wie der surchtbare Schmerz über das Leiden seiner Frau gänzlich gebrochen hatten, schien vor Koppenhagen eine ungeheuerliche Angst zu empfinden 
[...] (GB, 28.05.2014: 1887, http://books.google.de/books?id=auApAAAAYAAJ$\& q=\% 22$ ungeheuerliche + Angst $\% 22 \& d q=\% 22$ ungeheuerliche + Angst $\% 22 \&$ hl $=$ de \&sa=X\&ei=ae2FU_WPGuKA4gThmoG4CQ\&ved=0CDMQ6AEwAA)

Die Herausbildung der Gradbedeutung in einer frühen Stufe ihres Entwicklungsgangs ist mit dem Einsetzen von subjectivity verbunden, wobei die einstellungsbezogene Bedeutungskomponente in Erscheinung tritt und damit auf Traugott I (oben 3.6.3.4) verweist.

In der nächsten Phase der Entwicklung tritt ungeheuerlich in eindeutig intensivierender Funktion auf und fungiert als Grad-Adjektiv bzw. -Adverb. Zunächst sind die Kollokationsmöglichkeiten des Lexems auf negative Situationen beschränkt und weisen damit auf den unvollendeten Prozess der Desemantisierung hin. Für diese Stufe ist es ausschlaggebend, dass der direkte Zusammenhang mit der Ausgangsbedeutung beseitigt ist, während die lediglich auf Grad bezogene Interpretation zur Geltung kommt, vgl. die adjektivischen (391) - (393) und adverbialen (394) Verwendungen:

(391) dieser thurm hat den namen von seiner eigenthümlichen bestimmung, weil er zu kriegszeiten dazu dient den feind [...] zu entdecken oder wahrzunehmen; - er ist aber ungeheuerlich hoch, und fällt einem dergeftalt beftåndig in die augen, daß man ihn nicht wohl übersehen kann, wenn man auch wollte. (28.05.2014: 1776, http://books.google.de/books?id=Zqk_AAAAYAAJ\&pg=PA17\&dq=ungeheuerlich\&hl=de\&sa=X\&ei=LeaFU4yFeql4gSU04GYCQ\&ved=0CEUQ6AEwAw\#v=onepage $\& \mathrm{q}=$ ungeheuerlich\&f=false)

(391') 'sehr hoch'

(392) In einer Atmofphäre von Metalldämpfen, wo das Eifen als eine Wolke fchwebt und vor ungeheuerlicher Hitze fchließlich keine einzige chemifche Verbindung mehr zwifchen den Grundelementen glückt, kann auch der zähefte Bazillus nicht mehr ausdauern. (DTA: Bölsche, Wilhelm: Das Liebesleben in der Natur. Bd. 1. Florenz u. a., 1898)

(392') 'sehr großer Hitze'

(393) der Kniende schloß die Augen. eine ungeheuerliche Müdigkeit schlug ihn. (C.II: BZK/D64.00903 Neues Deutschland, 15.02.1964, S. 2)

(393') 'sehr große Müdigkeit'

(394) Das ist ein Thema, das viele Menschen in ihrer konkreten Situation ungeheuerlich belastet und für sie ungeheuerlich wichtig ist. (C.II: PNI/W15.00092 Protokoll der Sitzung des Parlaments Landtag Niedersachsen am 29.06.2006)

(394') 'sehr belastet', 'sehr wichtig'

Außerdem erweitert ungeheuerlich seinen Bezugsbereich, indem das Lexem ganze Funktionsverbgefüge intensiviert. Das Auftreten in der besagten Position erfolgt dadurch, dass das Substantiv in dem FVG graduierbar ist, wie in (395), oder das ganze FVG durch einen graduierbaren Begriff paraphrasiert werden kann, wie in (396), vgl.:

(395) Er hätte allerdings auch nicht gewusst, wer das hätte übernehmen sollen, denn in einer Sache hatte sie absolut recht, sie standen ungeheuerlich 
unter Druck. (28.05.2014: http://books.google.de/books?id=c7jXAgAAQBAJ\&pg=PT238\&dq=ungeheuerlich+unter+Druck\&hl = de\&sa=X\&ei=-fWFUyIHY7Q4QS5woH4Bg\&ved=0CDgQ6AEwAQ\#v=onepage \&q=ungeheuerlich $\% 20$ unter\%20 Druck\&f=false)

(395') 'standen unter sehr großem Druck' bzw. 'waren sehr bedrängt'

(396) Sora war ihm dabei zwar ungeheuerlich auf den Geist gegangen, aber sie waren schließendlich fertig geworden, auch wenn einige Male die Gestelle wieder in sich zusammengebrochen waren. (03.04.2014: https://www.fanfiction.net/s/2696140/19/Adventskalender-2005)

(396') 'hat sehr genervt'

Die Korpusanalyse belegt außerdem die Entwicklung der quantifizierenden Funktion des Lexems, die auf Quantität (397) sowie Größe (398) der Sachverhalte hinweist:

(397) Hans nannte eine ziemlich ungeheuerliche Summe. (C.II: HK4/R09.00001 Reventlow: Tot, Erstdruck: 1911, 2004 [S.320])

(397') 'sehr große Summe'

(398) Sie vereinigten sich in ihrem Zorne alle gegen den glücklichen Erben, welcher ganz ruhig seine Habe einpackte, was irgend von Nutzen war, und auf einen ungeheuerlichen Wagen lud. (C.II: HK3/D35.00001 Keller: Der grüne Heinrich [Erste Fassung], Entstanden: zwischen 1846 und 1855, 2000 [S.118])

(398') 'sehr großen Wagen'

Neben der intensivierenden und quantifizierenden Funktion von ungeheuerlich entwickelte sich außerdem eine evaluative Bedeutung des Lexems. DWB (s.v.) beschreibt eine Sinnrichtung, die „das miszverhältnis der theile zum ganzen sowie unter einander, das miszverhältnis der erscheinung zu ihrem zwecke" betont und dem Hervorheben von „auffallende[n], maszlose[n] (gräszliche[n]), wunderliche[n], thörichte[n], ungefüge[n], groteske[n], monströse[n] " Sachverhalten dient, vgl.:

(399) ein ungeheurer, ungeheuerlicher widerspruch (DWB: s.v.)

(400) ihr bleibt ein ungeheuerlicher kerl (DWB: s.v.)

(401) Diese selben Erleichterungen in der Familienwirtschaft und in der Pädagogik, die man von einer sozialpolitisch reformierten Gesellschaft erwarten muß, machen auch die ungeheuerliche Forderung, die man einige Zeit lang der erwerbstätigen Frau tatsächlich zu stellen wagte: den Verzicht auf Mutterschaft / hinfällig. (C.II: HK4/M10.00001 Meisel-Hess: Die sexuelle Krise, Erstdruck: 1909, 2004 [S. 231])

Die Belege (399) - (401) bezeugen dadurch den einstellungsbezogenen Gebrauch des Adjektivs, dass der Sprecher bzw. Schreiber durch die Verwendungen seine "perspective of point of view in discourse“ (Finegan 1995: 1) zum Ausdruck bringt. Durch ungeheuer wird gezeigt, dass das betroffene Element als höchst „auffallend“, "töricht", "grotesk" oder "wunderlich“, dabei jedoch oft in einer negativen Weise, bezeichnet wird. In der Gegenwartssprache ruft der Gebrauch ausschließlich ne- 
gative Konnotationen hervor und wird in DUDEN (2012, s.v.) als „unerhört (2), empörend, skandalös“ bezeichnet. Dabei kann die Bewertung entweder selbstständig, wie in (330) oder in Verbindung mit der Steigerung, wie in (331) auftreten, vgl.:

(402) Dass die Erzeugnisse der Firma Symrise vom Firmenchef Schaper als gesundheitfördernde Produkte dargestellt werden, obwohl gerade Aromastoffe für den wachsenden Anteil übergewichtiger Kinder, Jugendlicher und Erwachsener verantwortlich gemacht werden, ist schon eine ungeheuerliche Behauptung. (C.II: HAZ08/APR.05127 HA, 26.04.2008, S. 8)

(402') 'empörende, skandalöse Behauptung'

(403) Wir finden es eine ungeheuerliche Frechheit, für solch eine Festzeltbewirtung 650 Schilling pro Person zu verlangen. (C.II: V98/FEB.06290 Vorarlberger Nachrichten, 10.02.1998, S. C7)

(403') 'große und empörende Frechheit'

Die Entwicklung von Intensivierung, Quantifizierung und Bewertung korreliert mit einem Zuwachs an subjectivity, indem der Gebrauch mehr einstellungsbezogen im Rahmen von Traugott III (oben 3.6.3.4) wirkt.

Anschließend tritt das Lexem in positiven Kontexten auf und demonstriert den kompletten Verlust des Zusammenhangs mit der Ausgangsbedeutung. Der Prozess der Bedeutungsentleerung wird damit vollkommen vollzogen, vgl. den adverbialen (404) - (406) und adjektivischen (407) Gebrauch:

(404) Die Arbeit macht den Friedrichsdorfern einen ungeheuerlichen Spaß, schon das Zuschauen bei den Proben bereitet Vergnügen. (C.II: R98/OKT.82217 Frankfurter Rundschau, 14.10.1998, S. 1)

(404') 'sehr großen Spaß'

(405) Es ist im Grunde genommen ungeheuerlich erfreulich, dass sich dort 400 Studienanfänger für das Fach Maschinenbau interessieren. (C.II: PRP/W15.00030 Protokoll der Sitzung des Parlaments Landtag Rheinland-Pfalz am 26.09.2007)

(405') 'sehr erfreulich'

(406) Einfach so mal ein paar Komplimente zu streuen. Der Effekt, da bin ich mir sicher, ist ungeheuerlich gut. (C.II: A13/JUL.08183 St. Galler Tagblatt, 22.07.2013, S. 9)

(406') 'sehr gut'

(407) Er hat dabei aber zustande gebracht, was die meisten Autoren nicht können. Er hat in der Phase seiner Verletzung bereits Literatur gemacht, und das finde ich ungeheuerlich bewunderswert. (C.II: K97/SEP.69710 Kleine Zeitung, 14.09.1997)

(407') 'sehr bewundernswert'

\subsubsection{Kurzfassung der Entwicklung bei ungeheuerlich}

Ausgangsbedeutung: 'Schrecken hervorrufend', gelegentlich mit dem begleiteneden Nebensinn der Größe oder des Bösartigen;

die übrigen Entwicklungsschritte wie oben in Kap. 3.6.3.9.3. 


\subsubsection{Unheimlich}

\subsubsection{Lexikographische Angaben}

Während Kluge keine Angaben über die Herkunft von unheimlich macht, führt Pfeifer (s.v.) das Auftreten des Lexems auf das Mhd. zurück.

1. unheimlich in BMZ, Lexer (s.v.)

„nicht vertraut, fremd“

2. unheimlich in FWB

keine Angaben, auch nicht im bereits zitierten Material der Redaktionsstelle von FWB

3. unheimlich in DWB (s.v.)

3.1 "gth. v. heimlich 1; unheimisch 1 , ungeheuer 1 entsprechend“

3.2 "heimlich $3 \mathrm{c}$ und d, insbesondere ungeheuer 2 , unheimisch 2 entsprechend“

3.3 „ungeheuer 3 , heimlich 3 c, unheimisch $2 / 3$ entsprechend“

3.3a „nicht vertraut, fremd, entfremdet, unfreundlich, ungnädig; feindlich, schädigend, beunruhigend, unzuträglich, unbequem; unfriedlich, bösartig, unerfreulich, gefährlich, bedenklich u. ä."

3.3b „seit dem ende des 18. jhs. mit engerer beziehung auf das gefühlsleben schrecklich, grauenvoll; schauder, angst, entsetzen, furcht, beklemmung, unbehagen, befangenheit, abneigung $u$. ä. verursachend“

3.4 „die unheimisch 4, ungeheuer 5 entsprechende bed. ist im 16. u. 17. jh. wohlbekannt, im 18. von KRAMER (1719), STEINBACH (1734) u. FRISCH (1741)zwar erwähnt, doch von der aufklärung zurückgedrängt, bis das ende des 18 . jhs. sie wieder hervorzieht; dann wird sie allgemein"

3.5 „die erst im 19. jh. entwickelte steigernde bed. entspricht der von ungeheuer 5/6. 'riesig, auszerordentlich'“2.4. „unheimisch 4, ungeheuer 5 entsprechende Bedeutungen"

3.6 "gth. v. heimlich secretus“

4. unheimlich in DUDEN (2012, s.v)

4.1 „ein unbestimmtes Gefühl der Angst, des Grauens hervorrufend“

4.2 „(umgangssprachlich)“

$4.2 \mathrm{a}$ „sehr groß, sehr viel“

$4.2 \mathrm{~b}$ „>intensivierend bei Adjektiven und Verben> in außerordentlichem Maße; überaus, sehr"

\subsubsection{Entwicklung der Gradbedeutung bei unheimlich}

Pfeifer und DWB verweisen auf das Einsetzen der Gradbedeutung „seit dem 19. Jh." (Pfeifer, DWB, s.v.). Als Ausgangspunkt für deren Entwicklung dient die in 3.3b verzeichnete Bedeutung, die sich auf mit Schrecken und Beklemmung verbundene Situationen bezieht, vgl.:

(408) Mir wurde es ganz unheimlich, ich fah erfchrocken hinauf nach dem Zifferblatte der Kirchenuhr, auch hier ftand der Zeiger ftill und grade auf der Mitternachtszahl. (DTA: Klingemann, Ernst August Friedrich: Nachtwachen. Penig, 1805)

(408') 'schrecklich zumute' 
(409) Der Mond blickte feltfam und unheimlich aus dunkeln Wolken, die fchnell über den Himmel flogen. (DTA: Eichendorff, Joseph von: Ahnung und Gegenwart. Nürnberg, 1815)

(409') 'blickte Angst, Beklemmung verursachend'

(410) ich warf meinen wilden Jäger so kräftig zur Erde, daß er keinen Laut mehr von sich gab. [...] Er rührte sich nicht; der Gedanke, daß ich ihn gar totgedrückt hätte, fuhr mir unheimlich durch die Glieder, ich stürzte zur Küche nach Wasser [...] (C.II: HK3/A18.00001 Brentano: Die mehreren Wehmüller und ungarischen Nationalgesichter, Erstdruck: 1817, 2000 [S. 692])

(410') 'schrecklich zumute'

Die Herausbildung der Intensivierung beginnt mit pragmatic enrichment bzw. strengthening der Ausgangsbedeutung. Die Gradbedeutung tritt zuerst als Nebenbedeutung durch pragmatic inferencing neben der Ausgangsbedeutung auf, vgl.:

(411) [...] Und die Flut unheimlich hoch und immer höher am Kirchboden schwellend; auf dem Totenhof draußen alle Leichensteine überspült, die Holzkreuze geknickt und im Strudel umhergetrieben, und die beiden fremden Menschenkinder allein, hülflos allein wie in einem weiten, kalten, dunklen Grabe! Ach, wie zittert und schauert die arme Magdalene! Die Zähne klappern gegeneinander wie im Fieberfrost, irdisches und überirdisches Grauen schüttelt den schönen Leib; Erstarren und Verhungern und Ertrinken und Erschlagenwerden und gespenstisches Wimmeln der Gebeine, die ringsum aus ihren Gruben gespült werden: ein Schrecknis jagt das andere, eine Todesangst die andere. (C.II: HK4/F16.00001 François: Die Geschichte meines Urgroßvaters, Erstdruck: 1855, 2004 [S. 262])

(412) Aber der Schatten wurde düsterer und drohender, und die Stille wurde seltsam unterbrochen von dem Knarren und Stöhnen der Tannenriesen, die ihre starken Glieder reckten und dehnten, als wollten sie prüfen, ob ihre Kraft noch ausreiche, dem Gewittersturm, der über den Wald heraufzog, zu trotzen. Und jetzt begann es in den Büschen unheimlich zu zischeln und zu flüstern, dürres Laub flog, wie in toller Angst, her vor der Windesbraut, die sausend in das Blättermeer schlug, die Kronen der Buchen wie wahnsinnig durcheinander peitschte, die hohen Wipfel der Tannen mächtig bog und den Wald bis in die tiefsten Gründe aus seiner Ruhe schreckte. (C.II: HK3/G01.00001 Spielhagen: Problematische Naturen. Erste Abtheilung, Erstdruck: 1861, 2000 [S. 116])

(413) „Nesi“, sagte diese, „du fürchtest dich doch nicht?“ Und das Kind, von der Erhabenheit des Todes angeweht, antwortete: „Nein, Anne, ich bete." Dann kam der allerletzte Gang, welcher noch mit ihr zu gehen ihm vergönnt war; nach ihrer beider Sinn ohne Priester und Glockenklang, aber in der heiligen Morgenfrühe, die ersten Lerchen stiegen eben in die Luft. Das war vorüber; aber er besaß sie noch in seinem Schmerze; wenn auch ungesehen, sie lebte noch mit ihm. Doch unbemerkt entschwand auch dies; er suchte sie oft mit Angst, aber immer seltener wußte er sie zu finden. Nun erst schien ihm sein Haus unheimlich leer und öde; in den Winkeln saß eine Dämmerung, die früher nicht dort gesessen hatte; 
es war so seltsam anders um ihn her; und sie war nirgends. (C.II: HK3/B09.00001 Storm: Viola tricolor, Erstdruck: 1874, 2000 [S. 399])

Der adverbiale Gebrauch in (411) - (413) erlaubt mindestens zwei Lesarten. Die erste ist auf die Ausgangsbedeutung bezogen und bezeichnet die Flut mit ihrer außergewöhnlichen Höhe, das Zischeln und Flüstern der Büsche sowie die Leere und Öde als Schrecken erregend. Zugleich kann aus den Kontexten geschlossen werden, dass die Bedeutung von unheimlich durch den Begriff der Intensivierung erweitert wird. So stellt die stark ausgeprägte Höhe der Flut in (411) die Ursache des Schreckens dar. In (412) handelt es sich um sehr starken Wind. Von daher wird das Zischeln und Flüstern der Büsche nicht als leise, sondern laut bzw. als sehr intensiv wahrgenommen. In (413) wird die Intensität der Leere und Öde, die das Kind zu Hause empfindet, im Zusammenhang mit dem Tod seiner Mutter hervorgehoben. Die Korpusanalyse hat außerdem zahlreiche Belege ergeben, die das Auftreten der Gradbedeutung als Folge von semantic repetition demonstrieren, vgl.:

(414) Nein, was mich betrifft, so umfängt mich der Gedanke an den Ehestand mit unheimlichem Grauen. (C.II: HK3/E00.00001 Hoffmann, E. T. A.: Die Serapionsbrüder, Erstdruck: 1819-1821, 2000 [S.172])

(415) Ahnungsvoll hielt sie hier inne, bedeckte die Augen mit der Hand, als wolle sie ein Bild verhüllen, das unheimliche Angst in ihr erwecke! (C.II: HK4/A17.00001 Aston, Louise: Aus dem Leben einer Frau, Erstdruck: 1847, 2000 [S.61])

(416) Alle Unterröcke zitterten bei dem Gedanken an solche Gefahr, und sogar die Männer ergriff eine unheimliche Furcht vor der Invasion jener langgeschwänzten Gäste. (C.II: HK3/F47.00001 Heine: Lutetia, 1840, 2000 [S.440])

(417) Unheimlich bang ist dieses Schweigen! (C.II: HK4/001.00001 Otto, Louise: Mein Lebensgang, Entstanden: 1847, 2004 [S.177])

Die hervorgehobenen Verwendungen in (414) - (417) beziehen sich auf Schrecken hervorrufende Situationen. Zugleich hat die Wiederholung der gemeinsamen Bedeutungskomponente 'Furcht' in den beiden Kookkurrenzpartnern semantic repetition und semantic redundancy zur Folge, was zu der Verstärkung des Ausdrucks führt, sodass etwa die folgenden Paraphrasierungen naheliegen:

(414') 'mit sehr starkem Grauen'

(416') 'sehr große Angst'

(416') 'sehr große Furcht'

(417') 'sehr bang'

Die mehrdeutigen Kontexte (411) - (417) lassen neben der Intensivierung das Einsetzen von subjectivity erkennen, da sie die Einstellung des Sprechers bzw. Schreibers zu den beschriebenen Situationen demonstrieren. Damit verbindet der Gebrauch die propositionale und expressive Ebene und verweist auf die Wirkung von Traugott I (oben 3.6.3.4).

In der nächsten Phase der Entwicklung verliert das Lexem allmählich den direkten Zusammenhang mit der Ausgangsbedeutung und dient ausschließlich dem Ausdruck der Gradbedeutung. Jedoch bleibt die Kookkurrenz des Adjektivs bzw. des 
Adverbs auf negativ konnotierte Partner beschränkt, sodass die Ausgangsbedeutung in dieser Phase noch nicht vollkommen geschwunden ist, vgl. die adjektivischen (418) - (419) und adverbialen (420) - (423) Verwendungen mit den entsprechenden Umformulierungen:

(418) Die unheimliche Hitze an diesem Tag führte dazu, dass die automatische Zeitmessung zeitweise ihren Geist aufgab. (C.II: A10/JUL.05244 St. Galler Tagbl., 20.07.2010, S. 34)

(418') 'sehr starke Hitze'

(419) In solchen Situationen verfluche ich meine lächerliche Zerstreutheit und die unheimliche Vergesslichkeit - die jedem Alltagsgegenstand Beine wachsen lässt. (C.II: A12/JAN.09262 St. Galler Tagbl., 27.01.2012, S. 39)

(419') 'sehr große Vergesslichkeit'

(420) Neben folchem Reichthum der Kunft und der Wiffenfchaft erfcheint die eigentlich politifche Literatur unheimlich klein und dürftig. (DTA:

Treitschke, Heinrich von: Deutsche Geschichte im Neunzehnten Jahrhundert. Bd. 1. Leipzig, 1879)

$(420$ ') 'sehr klein und dürftig'

(421) „Es gibt in Holland nicht viele Fans des deutschen Fußballs, aber ich bin einer", sagte der Niederländer und fügte an: „Ich weiß, es wird eine unheimlich schwierige Aufgabe, Deutschland zu schlagen. [...]." (C.II: NUZ08/JUN.00463 NZ, 05.06.2008, S. 26)

(421) 'sehr schwierige'

(422) Genau das macht allerding die Nachwuchsarbeit unheimlich problematisch. (C.II: RHZ97/JUN.18842 RZ, 28.06.1997)

(422') 'sehr problematisch'

(423) Selbst mittelmäßige Schauspieler sind unheimlich teuer geworden ... (C.II: M98/JUL.56789 Mannheimer Morgen, 10.07.1998)

(423') 'sehr teuer'

Als Gradadverb kann unheimlich außerdem Funktionsverbgefüge modifizieren, wenn sie ein graduierbares Prädikat enthalten, wie in (424), oder durch einen graduierbaren Begriff wiedergegeben werden können, wie in (425) - (427), vgl.:

(424) „Die Mannschaft stand unheimlich unter Druck", entschuldigte Beth das nicht eben hochklassige Niveau. (C.II: RHZ97/NOV.11599 RZ, 17.11.1997)

(424') 'stand unter sehr großem Druck' bzw. 'war sehr bedrängt'

(425) „Das geht unheimlich ins Geld", stöhnt das Tischtennis-As über ihre hohen Telefonrechnungen. (C.II: RHZ97/SEP.21687 RZ, 30.09.1997)

$(425$ ') 'ist sehr teuer'

(426) Gerade unsere Juniorhelfer sind unheimlich auf Draht. (C.II: NUZ09/FEB.02239 NZ, 23.02.2009, S. 10)

(426') 'sind sehr wachsam'

(427) Es zog sich alles unheimlich in die Länge. Insgesamt war ich über drei Monate bis Mitte November weg, bis alles geklärt werden konnte. (C.II: RHZ04/FEB.25767 RZ, 28.02.2004)

(427') 'verzögerte sich sehr'

Neben der Intensivierung tritt die quantifizierende Bedeutung hervor, vgl.: 
(428) Er beklagte sich darüber, »dass in allen Lokalen in der Motzstraße unheimliche Preise gefordert" würden. (GB, 30.05.2014: http://books.google.de/books?id=YEs01-TEF1YC\&pg =PA302\&dq=\%22unheimliche+preise\%22\&hl=de\&sa=X\&ei=AImIU503NqWN4gTP9YGwDA\&ved=0CDwQ6AEwAA\#v=onepage \&q=\%22unheimliche $\% 20$ preise $\% 22 \& \mathrm{f}=$ false)

(428') 'sehr hohe Preise'

(429) Klar, die Nationalbank ist unter Druck. Von rechts aus jenen Kreisen, welche die Bankenregulierungen und erhöhte Eigenmittelvorschriften nicht akzeptieren, von links von den Gewerkschaften, die jetzt wiederum bedenkenlos einen Franken-Euro-Kurs von 1.40 fordern, obwohl die SNB in diesem Falle geradezu unheimliche Summen an schwachen Euros kaufen müsste. (C.II: SOZ12/JAN.00859 Südostschweiz, 06.01.2012, S. 2)

(429') 'sehr große Summen'

Die Belege (418) - (427) und (428) - (429) demonstrieren den eindeutigen Gebrauch von unheimlich jeweils in intensivierender und quantifizierender Funktion. Die Bedeutungsentwicklung in dieser Stufe korreliert außerdem mit der Zunahme an subjectivity, wodurch die Bedeutung des Lexems sich im Rahmen von Traugott III (oben 3.6.3.4) in der expressiven Ebene manifestiert.

Im weiteren Verlauf der Entwicklung findet sich das Lexem in Verbindung mit positiv konnotierten Kollokationspartnern. Dadurch ist der Zusammenhang mit der Ausgangsbedeutung vollkommen geschwunden bzw. der komplette Verlust der Ausgangsbedeutung vollzogen. Die Belege (430) und (431) - (434) sollen jeweils den adjektivischen und adverbialen Gebrauch veranschaulichen:

(430) In Fidos neuem Zuhause bei Familie Rieck in Ochsendorf bei Königslutter gibt es viel Platz, einen Garten, zwei weitere Hunde, Hühner und Pferde. „Das ist schon super, der Hund hat unheimliches Glück gehabt. Das ist wirklich eine Ideal-Vermittlung, die man sich kaum besser vorstellen kann. (C.II: BRZ10/FEB.01974 Braunschw. Z., 04.02.2010)

(430') 'sehr großes Glück'

(431) so hält sich Amerika jetzt als Nation auch für fähig und berechtigt, alles zu erringen, was es will. Und was Amerika will, ist die Welt. Die Welt will ja jeder, der auch nur die geringsten Chancen hat, sie je zu besitzen - und die Chancen Amerikas sind unheimlich gut! (C.II: HK4/H07.00001 Heyking: Briefe, die ihn nicht erreichten, Erstdruck: 1902, 2004 [S. 56])

(431') 'sehr gut'

(432) "Ich bin unheimlich begeistert. Das Konzert hat gezeigt, dass die Pilgerkirche in unserer Region sicherlich der ergiebigste Konzertsaal ist." (C.II: RHZ04/OKT.04116 RZ, 05.10.2004)

(432') 'sehr begeistert'

(433) "Ich habe mich unheimlich verbessert - vor allem mental." (C.II: L98/SEP.09209 Berliner Morgenpost, 14.09.1998, S. 18)

(433') 'sehr verbessert'

(434) Auch, weil Kathrin selbst Perlenschmuck unheimlich liebte und viel Zeit damit verbrachte, die runden Kugeln aufzuziehen. (C.II: RHZ05/JUN.34291 $\mathrm{RZ}, 30.06 .2005)$ 
(434') 'sehr liebte'

\subsubsection{Kurzfassung der Entwicklung bei unheimlich}

Ausgangsbedeutung: 'Schrecken, Beklemmung hervorrufend';

die übrigen Entwicklungsschritte wie oben in Kap. 3.6.3.9.3.

\subsection{Wortgruppe 'Übel wollend / verursachend'}

Der folgende Kapitelabschnitt widmet sich der Herausbildung der Gradbedeutung in den Lexemen arg, böse, brutal, grausam, grimmig, mörderisch, niederträchtig, schändlich, unmenschlich, unverschämt und wütend. Die intensivierende Funktion der Adjektive bzw. Adverbien kann durch die Verwendungen (a) - (k) exemplifiziert werden:

a) arg gut schmecken 'sehr gut schmecken'

b) sich böse verschätzen 'sich sehr verschätzen'

c) brutal lehrreich 'sehr lehrreich'

d) sich grausam langweilen 'sich sehr langweilen'

e) grimmige Kälte 'intensive Kälte'

f) mörderisch gut riechen 'sehr gut riechen'

g) niederträchtig kalt 'sehr kalt'

h) schändlich wenig verdienen 'sehr wenig verdienen'

i) sich unmenschlich gut bewegen 'sich sehr gut bewegen'

j) unverschämt cremige Nachspeisen 'sehr cremige Nachspeisen'

k) wütende Kopfschmerzen 'intensive Kopfschmerzen'

Die Ausgangsbedeutung der genannten Grad-Adjektive bzw. -Adverbien entspricht der Kategorie 10 „Fühlen, Affekte, Charaktereigenschaften“ in der Klassifizierung von Dornseiff (2004: 170ff.) und bringt die Begriffe „Unlust verursachen“, „Übel wollen“ und „Zorn“ zum Ausdruck. Biedermann (1969: 165ff.) ordnet die Gruppe der zu untersuchenden Wörter nach dem "Motivationssem“ „ungut" an und beschreibt die Lexeme nur teilweise, denn er notiert die Gradbedeutung ausschließlich bei $\arg$, böse und grimmig, während brutal, grausam und wütend unberücksichtigt bleiben.

\subsection{1 $\mathrm{Arg}$}

\subsubsection{Lexikographische Angaben}

Laut Pfeifer und Kluge (s.v.) ist das Wort im Althochdeutschen in der Form $\operatorname{ar}(a) g$ belegt.

1. $\arg$ in AWB (s.v.)

1.1 „(moralisch) verderbt, schlecht, böse, verwerflich. Arg bezeichnet (...) das, was dem Idealbild des vollkommenen Menschen entgegengesetzt ist. Sein Inhalt wandelt sich mit dem Wandel der Ethik. Die allgemeine Bedeutung des Ahd. ist bereits in christlichem Sinne bestimmt. Es wird angewandt „auf Menschen oder Dämonen“ und „auf Äußerungen des Menschen, vor allem solchen, die ein Streben bekunden" 
1.1a „feige, träge zum Handeln“

$1.1 b$ „unzüchtig, geil“

1.1c "geizig, habgierig, nicht freigebig“

$1.2 \mathrm{~d}$ „abgeschwächt: sparsam, haushälterisch“

1.2 „von Dingen“:

$1.2 \mathrm{a}$ „falsch, abwendig“

$1.2 \mathrm{~b}$ „schlimm, unheilvoll“

2. $\arg$ in BMZ (s.v. arc), Lexer (s.v. arg)

2.1 „arg, nichtswürdig“ (BMZ, s.v. arc), „böse“ (Lexer, s.v. arg)

2.2 "hartherzig, karg" (BMZ, s.v. arc), "geizig“ (Lexer, s.v. arg)

3. $\arg$ in FWB (s.v.)

3.1 „billig, schlecht, minderwertig (von Sachen aller Art); karg, spärlich (von der Kost); gering (von Personen)“

3.2 „schlecht, böse, übel, schlimm (von Sachen, Sachverhalten)“

3.3 „mächtig, zwingend, kräftig, wirkungsvoll (im Beleg von der Minne)“

3.4 „übel, böse, böswillig, tückisch, hinterhältig, nichtswürdig, schlecht (von Personen gesagt; im Unterschied zu 8 unter vorwiegend innerweltlichem Aspekt)“

3.5 „übel, böse, hinterhältig, falsch, tückisch, verbrecherisch, frevelhaft (von Gesinnungen, Haltungen u.ä.)“

3.6 "geizig"

3.7 „erzürnt, böse, wütend“

3.8 „böse, teuflisch, das Böse vertretend; dem Bösen verfallend (jeweils unter religiösem Aspekt)“

4. $\arg$ in DWB (s.v.)

4.1 Laut DWB bringt $\arg$ „die allgemeine [Bedeutung] des bösen, übeln und schlimmen“ „auf personen angewandt" sowie „auf sachen bezogen“ zum Ausdruck.

4.2 „häufig dient $\arg$ allein, ohne beigefügtes subst., zur bildung von redensarten", die etwas Schlimmes bezeichnen.

5. $\arg$ in DUDEN (2012, s.v.)

5.1a „(gehoben veraltet) von böser, niederträchtiger Gesinnung [erfüllt], niederträchtig, böse"

$5.1 \mathrm{~b}$ „(landschaftlich) schlimm, übel; unangenehm“

$5.2 \mathrm{a}$ „(landschaftlich, österreichisch, schweizerisch, auch gehoben) [unangenehm] groß, stark, heftig"

$5.2 \mathrm{~b}$ „<intensivierend bei Adjektiven und Verben> (landschaftlich) sehr, überaus"

\subsubsection{Entwicklung der Gradbedeutung bei arg}

Obgleich FWB und DWB keine Hinweise auf die Gradbedeutung bieten, lässt die Untersuchung von historischen Wörterbüchern und Korpora die ersten Spuren der in dem Lexem vorliegenden Intensivierung schon im Frühneuhochdeutschen feststellen. Als Ausgangspunkt für deren Entwicklung gilt die im Althochdeutschen eingesetzte Bedeutung 1.1, die sich auf Menschen im moralisch-christlichen Sinne 
bezieht und diese als „(moralisch) verderbt, schlecht, böse, verwerflich“ bezeichnet, vgl. die aus DWB übernommenen Belege:

(435) nimet zu sich andere geister, die erger (ahd. wirsiron) sind denn er selbst. (DWB. s.v.)

(436) so denn ir, die ir doch arg seid (ahd. ubilê birut), könnet dennoch euren kindern gute gaben geben. (DWB, s.v.)

Die Korpusanalyse legt nahe, dass das Auftreten der Intensivierung durch pragmatic inferencing der Ausgangsbedeutung und durch dessen strengthening und pragmatic enrichment erfolgt, vgl. den aus FnhdC übernommenen Beleg:

(437) Das wird nu vns geprediget / auff das wir

an diesem ... HERREN

Jesu Christo / vnsere grosse Sünde / vnd des Vaters

grosse Liebe erkennen mo̊gen / welcher seines

eignen Sohnes nicht verschonet hat ...

das er vns seinen ergsten Feinden helffe / vnd

zu seinen Kindern mache.

(FnhdC: Mathesius, Johannes (1587). Passionale. Leipzig)

Arg in (437) erlaubt eine mehrdeutige Interpretation. Zunächst bezieht sich die Bedeutung des Adjektivs auf die Ausgangsbedeutung, indem die Verwendung folgendermaßen paraphrasiert werden kann:

(437') 'Gott hilft seinen Feinden, die äußerst üble, böse, böswillige und im moralischen Sinne nichtswürdige Menschen sind, indem sie seinen Sohn ermordet haben, und macht sie zu seinen Kindern.'

Zugleich kann arg als Gradadjektiv bezeichnet werden. In Paradis' Terminologie (1997: 54) stellen die beiden Kookkurrenzpartner „implicit superlatives“ dar, deren Zusammensetzung zu der Intensivierung des Bezugswortes führt. So wird arg, das auf der Skala des moralisch-christlichen Verhaltens das untere Ende einnimmt, mit einem anderen Bezugswort derselben Wertung, bzw. konkret Feind ${ }^{57}$, zusammen verwendet. Damit beziehen sich die beiden Kollokationspartner auf eine böswillige Einstellung einer Person gegen eine andere Person. Durch die Wiederholung der Inhaltszüge des Adjektivs in dem Bezugssubstantiv kommt es zu der Verstärkung des Ausdrucks, sodass arg als Intensivierer interpretiert wird. Die Verstärkung wird außerdem dadurch hervorgehoben, dass arg im Superlativ gebraucht wird. Diese Form verweist auf die in dem Adjektiv liegende Intensivierung, indem sie „den höchsten Grad einer Eigenschaft, eines Merkmals“ (DUDEN 1995: 297) bezeichnet. Diese Verstärkung gilt zunächst für den Modifikator, kann jedoch unter Berücksichtigung der semantischen Reanalyse auf den Kern übergehen. $R e$ distribution of meaning erfolgt damit durch semantic repetition.

Die intensitätsorientierte Interpretation lässt sich außerdem aus der Analyse der einstellungsbezogenen Verwendung des Adjektivs schließen. Aus dem Kontext

\footnotetext{
57 „Haltung einem anderen Menschen gegenüber, die von dem Wunsch bestimmt ist, diesem zu schaden, ihn zu bekämpfen od. sogar zu vernichten" (DUDEN, s.v. Feind)
} 
folgt, dass Feindschaft, aus der Sicht des Autors, einen graduierbaren Begriff darstellt. Darüber hinaus kann eine Skala abgeleitet werden, auf der Menschen je nach der Stärke ihrer Feindseligkeit und Böswilligkeit einordnet werden können. In Bezug auf (437) handelt es sich um die Personen, die Jesus Christus Tod gewünscht und (oder) sich an diesem Tod beteiligt haben. Damit wird ihnen von dem Schreiber ein hoher Grad an Feindseligkeit zugeschrieben und demzufolge eine Position auf dem oberen Ende der entsprechenden Skala bestimmt. Arg tritt dabei als Maß und von daher als Intensivierungsmittel auf. Damit wird die Bedeutung „increasingly situated in the speaker's subjective belief-state/attitude toward the situation" (Traugott 1988: 409-410) und verweist auf den Übergang zu Traugott I (oben 3.6.3.4).

Es ist außerdem darauf hinzuweisen, dass das Adjektiv in der Verwendung ärgster Feind in GWB (s.v.) „als Steigerungswort iSv heftig, groß, sehr" bezeichnet wird. Daraus lässt sich schließen, dass die Gradbedeutung sich im Laufe der Zeit ausgebildet und die Ausgangsbedeutung aus dem Gebrauch verdrängt hat.

Ein ähnlicher Prozess der semantischen Reanalyse erfolgt in Bezug auf den adverbialen Gebrauch, vgl.:

(438) Die Mänschen, in dem sie jetzt noch ihrem (Justitia) Namen nachdencken, [Rand: atio status] geben vnd eygnen sie derselben zu einen Stab oder Scepter, welches oben eine Hand hat, vnd man Justitiam zu nennen pfleget. Aber es ist ein blosser schein, vnder welchem das arme Volck nur herumb gezogen, gehalten, gespannen, gefässelt, betrogen vnd beraubet wird, ärger als von offentlichen Dieben mit allen ihren Diebsschlüsseln, Dietrichen vnd anderen dergleichen passe-partout. (FnhdC: Moscherosch, Hans Michael (1650). Gesichte. Straßburg)

Arg bringt in (438) mindestens zwei Lesarten mit sich. Zunächst bezieht sich das Lexem auf die Ausgangsbedeutung, sodass etwa die folgende Paraphrase gilt:

(438') 'Das arme Volk wird von der zur Justiz angehörigen Menschen sowie von Dieben herumgezogen, gehalten, gespannt, gefässelt, betrogen und beraubt, sodass diese als übel, böse, böswillig und heimtückisch charakterisiert werden können'

Die andere Interpretation des Adverbs ist mit pragmatic inferencing der Ausgangsbedeutung verbunden. So verweist die Komparativform darauf, dass „zwei oder mehr Wesen, Dinge u. a. in bezug auf ein Merkmal, eine Eigenschaft u.ä. ungleich sind: ungleicher Grad“ (DUDEN 1995: 294). In Bezug auf (438) kann man demnach schließen, dass es sich um zwei Täter - „offentliche Diebe“ und Justizangehörige sowie um die von diesen Tätern ausgeübten Taten handelt. Weiter lässt sich ableiten, dass diese Taten nach einem Parameter bzw. nach dem Grad des Bösen, Unmoralischen von dem Sprecher verglichen werden. Für den Vergleich werden die Taten auf einer Skala angeordnet, die den Grad des ausgeprägten Parameters bzw. des Bösen, Unmoralischen konzeptualisieren soll. Die Komparativstufe des Adjektivs drückt aus, dass der Begriff des Bösen, Verwerflichen hinsichtlich der von Justizangehörigen ausgeübten Handlungen intensiver ausgeprägt ist, sodass es über die Intensität der Handlungen urteilen lässt. Aus dem Beleg geht außerdem hervor, dass die Kookkurrenzpartner wiederum „implicit superlatives“ im Sinne Paradis’ 
(1997: 54) darstellen, sodass deren Zusammensetzung zu der Intensivierung des Gebrauchs führen kann.

Wie es in (437) der Fall ist, ist der einstellungsbezogene Gebrauch des Lexems auch in (438) markant, der „speaker's imprint“ (Finegan 1995:1) zum Ausdruck bringt und wiederum auf das Auftreten von Traugott I (oben 3.6.3.4) verweist.

Die nächste Etappe der Bedeutungsentwicklung bezieht sich auf die Prozesse der Desemantisierung und Aktualisierung. Die Gradbedeutung verliert den direkten Zusammenhang mit der Ausgangsbedeutung, wobei das Lexem jedoch weiterhin ausschließlich negativ konnotierte Bezugswörter intensiviert. Aktualisierung lässt sich auf der Selektionsebene feststellen, indem die Kombinierbarkeit des Lexems expandiert, sodass die Gradbedeutung zur Verstärkung von verschiedenen Lebenssituationen, Tönen, Gefühlen, Wettererscheinungen und anderen Sachverhalten gebraucht wird, die keinen Bezug zu moralischen Eigenschaften eines Menschen mehr haben. In dieser Phase wird die Gradbedeutung „more subjective“ und auf „strengthening“ von „speaker perspective“ orientiert (Finegan 1995:8), vgl. die adjektivischen (439) - (441) und adverbialen (442) - (443) Verwendungen mit den entsprechenden Umformulierungen:

(439) Das Jahrgehalt [...] blieb einmal länger als gewöhnlich aus, und so kam es, daß Francesko bei seinem wilden Leben, das ihm allen Verdienst schnell hinwegraffte und das er doch nicht lassen wollte, in arge Geldnot geriet. (C.II: HK3/D64.00001 Hoffmann: Die Elixiere des Teufels, Entstanden: 1814/15, 2000, [S. 290])

(439') 'sehr große Geldnot'

(440) Bis zum grauen Morgen hat uns die Unruhe umhergetrieben, und mein Mann wachte mit einem recht argen Kopfweh auf [...] (C.II: HK3/B10.00001 Raabe: Der Schüdderump, Entstanden: 1867/69, 2000, [S. 581])

(440') 'sehr großen Kopfweh'

(441) Die Metropole erlebten wir während einer argen Hitze und als eine einzige Baustelle, die Stadt schmeckte nach Autoabgasen, nach den zahllosen Küchen, nach Staub - und nach Bauschutt. (C.II: R99/JUL.54246 Frankf. Rundschau, 08.07.1999, S. 8)

(441') 'sehr großen Hitze'

(442) Sorgen macht sich Schwester Ludmilla nur um ihre Kollegin, der es beim allgemeinen Lesen- und Schreibenlernen doch arg langweilig werden könnte. (C.II: RHZ07/AUG.20574 RZ, 22.08.2007)

(442') 'sehr langweilig'

(443) Im Gegenteil: Obwohl die Reisenden erst arg verspätet den Nürnberger Hauptbahnhof erreichten und dadurch viele ihre Anschlusszüge verpassten, hätten sie die unfreiwillige U-Bahnfahrt heiter hingenommen. (C.II: NUZ11/APR.00172 NZ, 02.04.2011, S. 17)

(443') 'sehr verspätet'

Arg kann außerdem in der Konstruktion mit verschobenem Skopus auftreten bzw. Funktionsverbgefüge modifizieren wie in (444), vgl. die übliche adjektivische Konstruktion in (445) und die Umformulierung in (446).

(444) in arge Schieflage geraten 
(445) arg in Schieflage geraten

(446) 'in eine sehr kritische Lage geraten'

In (444) fungiert arg als Gradadverb, welches sich auf das Substantiv Schieflage bezieht. In (445) dagegen liegt das FVG in Schieflage geraten im Skopus der Intensivierung. Es handelt sich also um die Änderung der Skopuskonstituente. Die Umstellung erfolgt dadurch, dass das in dem FVG enthaltene Nomen intensivierbar ist, was für einen intensifier die Bedingung ist. Die Belege (447) - (451) sollen das Phänomen veranschaulichen, vgl.:

(447) Ein vernünftiges Wort war an diesem Abend mit Vater Quakatz noch nicht zu sprechen; die Szene von vorhin war ihm zu arg auf die Nerven gefallen. (C.II: HK3/A26.00002 Raabe: Stopfkuchen, Entstanden: 1888/90, 2000 [S. 562])

(447') 'äußerst lästig geworden'

(448) Unter der Geschäftsleitung der überforderten Cordis-Gründerin war die Pension wirtschaftlich arg ins Schlingern geraten. (C.II: A97/OKT.30552 St. Galler Tagblatt, 21.10.1997)

(448') 'in eine große Krise geraten'

(449) Während Stefan Schnyder kampflos zu Punkten kam, geriet Markus Thomi im Schwergewicht als einer der wenigen Weinfelder arg in Bedrängnis. (C.II: A97/OKT.31889 St. Galler Tagblatt, 28.10.1997)

(449') 'geriet in sehr große Bedrängnis'

(450) Anstelle eines sicheren Vorsprunges, eines sicheren und verdienten Erfolges, geriet Uzwil am Schluss arg ins Zittern. (C.II: A98/FEB.08035 St. Galler Tagblatt, 09.02.1998)

(450') 'begann sehr stark zu zittern'

(451) Schliesslich war man seit bald 350 Jahren als Zugewandter Ort mit den Eidgenossen verbunden, deren altes Regime nun arg in die Zwickmühle geriet. (C.II: A98/FEB.10677 St. Galler Tagblatt, 20.02.1998)

(451') 'in eine sehr schwierige, verwickelte Lage geriet'

Die intensivierende Funktion des Lexems kann außerdem in die quantifizierende übergehen, die die Bedeutung 'groß, viel' zum Ausdruck bringt, vgl.:

(452) Die Nettokreditaufnahme, wie die Expertensprache sagt, wird auf 1,9 Milliarden DM gesenkt. Das ist immer noch eine arge Summe. (C.II: Protokoll der Sitzung des Parlaments Landtag Rheinland-Pfalz am 07.11.1996)

(452') 'sehr große Summe'

Arg kann außerdem in pejorativer Funktion auftreten, vgl.:

(453) Inzwischen hatte sich ein arges Wetter angebahnt und sorgte für totale Dunkelheit. (07.09.2015: https://books.google.de/books?id=xkSBo0PXGwUC\&$\mathrm{pg}=\mathrm{PA209} \& \mathrm{dq}=\% 22$ arges $\quad+$ wetter\%22\&hl=de\&sa=X\&ved=0CEAQ6AEwBmoVChMI0Kzg6qjkxwIVQzkaCh2QRglK\#v=onepage\&q=\%22arges\%20wetter\%22\&f=false)

(453') 'schlimmes Wetter' 
(454) Aber auch diese arge Zeit war irgendwann vorbei und mir ging es wieder gut. (07.09.2015: https://books.google.de/books?id=_hYzAgAAQBAJ\&pg=PA90\&dq $=\% 22$ arge+zeit\%22\&hl $=$ de\&sa=X\&ved=0CD0Q6AEwBWoVChMIkbeNiarkxwIVQqsaCh3hnASR\#v=onepage \&q=\%22arge $\% 20$ zeit $\% 22 \& \mathrm{f}=$ false)

(454') 'schlimme Zeit'

Die Belege (439) - (443), (445), (447) - (451) demonstrieren die eindeutige Verwendung von arg mit intensivierender Funktion, während das Lexem in (452) und (453) - (454) jeweils mit quantifizierender und pejorativer Bedeutung gebraucht wird. Aus den Kontexten geht hervor, dass die einstellungsbezogene Bedeutung sich in diesem Gebrauch fest etabliert hat, sodass die Kollokationen mehr Expressivität zum Ausdruck bringen und damit auf Traugott III (oben 3.6.3.4) verweisen.

Die weitere Bedeutungsentwicklung demonstriert den kompletten Verlust der Ausgangsbedeutung, indem das Lexem mit positiv konnotierten Bezugswörtern kollokiert und ausschließlich die Bewertung des Sprechers bzw. Schreibers ausdrückt, vgl. die Belege mit den entsprechenden Paraphrasierungen:

(455) „Was verdienen Sie in der Woche?" - „Sechs Mark, und wann's arg gut geht, achte. [...]“'(C.II: HK4/B22.00001 Braun: Memoiren einer Sozialistin: Lehrjahre, Erstdruck: 1909, 2004, [S. 154])

(455') 'sehr gut geht'

(456) Die Westerwälder siegten mit 1:0 (0:0), räumten aber selbst ein, dass dies arg glücklich war. (C.II: RHZ01/MAI.15703 RhZ, 21.05.2001)

(456') 'sehr glücklich'

(457) "Das hat so arg Spaß gemacht, da wollte ich auch mal zum Boxen«, sagt die blonde Bürokauffrau. (C.II: NUZ03/DEZ.01774 NZ, 15.12.2003)

(457') 'sehr viel Spaß'

\subsubsection{Kurzfassung der Entwicklung bei $a r g$}

Ausgangsbedeutung: 'böse, böswillig, übel usw.';

die übrigen Entwicklungsschritte wie oben in Kap. 3.6.3.9.3.

\subsubsection{Böse}

\subsubsection{Lexikographische Angaben}

Laut Kluge (s.v.) ist das Lexem, dessen Herkunft „unklar bleibt“, seit dem 10. Jh. in der Form bōsi belegt, vgl. auch Pfeifer (s.v.).

1. böse in AWB (s.v. bôsi)

Laut AWB dient der ursprüngliche Gebrauch des Wortes dem allgemeinen Ausdrücken der „Nichtigkeit einer Sache oder einer Person“. „In verschiedenen Anwendungsbereichen bezeichnet es jeweils einen Mangel. So kann es auch den Mangel an moralischer Kraft ausdrücken, und von hier aus entwickelt sich dann anscheinend die Bedeutung des ethisch Verwerflichen“ (AWB: 1270). Demnach hat das Lexem die folgenden Bedeutungen:

$1.1 \mathrm{a}$,wertlos"

$1.1 \mathrm{~b}$ „unsinnig, ungereimt, läppisch“

1.1c „kraftlos, machtlos, wehrlos, (moralisch) schwach“ 
1.2 „böse, sündig“

2. böse in BMZ (s.v. bœse)

2.1 Die Angaben des BMZ verweisen auf den Prozess der Pejoration der Ausgangsbedeutung von böse, indem verschiedene Dinge als „die schlechtesten, wertlosesten ihrer art bezeichnet werden"

2.2 In Bezug auf „lebende wesen“ bietet BMZ die Definition „nicht biderbe, nicht vrum: so wie aber die adjective biderbe und vrum eben sowohl höhern rang und stand bezeichneten, als jede höhere eigenschaft anderer art, so bezeichnet boese sowohl niedrig, gemein, als schlecht, unwert."

3. böse in FWB (s.v.)

3.1 "beschädigt, schadhaft, geringwertig, verdorben, unecht, schlecht" (von leblosen Sachen, z.B. Kleidung, Werkzeug, Möbeln)

3.2 "minderwertig, wertlos, gefälscht (von Geld)“

3.3 „bösartig, entzündet, nicht heilend, krank (von menschlichen und tierischen Körperteilen sowie Erkrankungen)“

3.4 „wild, gefährlich (von Tieren)“

3.5 „bissig, verletzend“ (von Worten)

3.6 „meist (vom christlichen Standpunkt aus) moralisch wertend: bösartig, heimtückisch, gottlos, verdorben, verwerflich, schlecht, übel (von Menschen)".

3.7 „meist (vom christlichen Standpunkt aus) moralisch wertend: übel, schlimm, schlecht (von Abstrakta)“

4. böse in DWB (s.v.)

4.1 „auf leibliche gegenstände angewandt bezeichnet böse das schmerzende, wehthuende"

4.2 "die krankheit selbst heiszt böse, im sinne von bösartig, malignus“

4.3 "böse von maul und zunge gebraucht bedeutet beiszend, bissig, verletzend“

4.4 in Bezug „auf lebende wesen, thiere und menschen, wie auch die krankheiten und plagen personificiert werden, böse ist mordax, saevus, crudelis, rabidus, was unmittelbar dem litt. baisus entspricht, aber zugleich den begrif des sl. subst. bjes, bies vollkommen erreicht, wodurch der satan, kakodaemon, teufel, der böse feind oder geist bezeichnet wurde"

4.5 „subjective bedeutung auch für böse und wir fügen den dat. der person, oder die praep. auf, über hinzu. ein solcher gebrauch von böse läszt sich weder ahd. noch mhd. aufweisen, ist aber nhd. und nnl. so häufig und eingewurzelt, dasz er leicht der ursprüngliche sinn des worts gewesen sein kann."

4.6 ,schlecht, gering, verdorben, falsch, ganz objectiv genommen“

4.7 "fein, gelehrt, erfahren“

4.8 „gegensatz des guten oder nützlichen, frommen, das untaugende, nichtsnutze“

$4.8 \mathrm{a}$ „von leuten gebraucht, improbi“

$4.8 \mathrm{~b}$ „die allgemeinste vorstellung des bösen wie seines gegensatzes“

$4.8 \mathrm{c}$ bezeichnet „eine schwierige, bedenkliche, misliche sache, bald eine ungerechte, verworfne, schlechte"

5. böse in DUDEN (2012, s.v.) 
5.1a "moralisch schlecht; verwerflich“

$5.1 \mathrm{~b}$ „schlecht, schlimm, übel“

5.2 „(umgangssprachlich) ärgerlich, zornig, wütend“

5.3 "(familiär) ungezogen, unartig“

5.4 „(umgangssprachlich) (von Körperteilen) entzündet"

5.5 „ $<$ intensivierend bei Verben und Adjektiven> (umgangssprachlich) sehr, überaus"

\subsubsection{Entwicklung der Gradbedeutung bei böse}

Obwohl die Angaben der oben eingeführten lexikographischen Beschreibungen auf das Einsetzen der Gradbedeutung erst in der Gegenwartssprache verweisen, finden sich die ersten Belege, die der Entwicklung der Gradbedeutung nachzugehen erlauben, schon in der ersten Hälfte des 17. Jh. in DTA. Als Ausgangspunkt für die Herausbildung der Intensität dient der in 4.4 erwähnte Gebrauch, der Menschen und ihre Taten als moralisch schlecht, verwerflich und übel bezeichnet, vgl.:

(458) Errette mich / Herr / von den bo̊fen Menfchen / behüte mich für den freveln Leuten / die bo̊fes gedencken in jhrem Hertzen / vnd tåglich Krieg erregen. (DTA: Arndt, Johann: Vom wahren Christenthumb. Bd. 2. Magdeburg, 1610)

(459) Im Hertzen aber ist er ein böser Mensch / voll Hoffart /Naid / vnd Geitz (DTA: Arndt, Johann: Von wahrem Christenthumb. Bd. 1. Magdeburg, 1610)

(460) Alfo will mannicher eine kleine bo̊fe Rede nicht dulden / vnnd richtet darnach groffen Zanck vnd Hertzleid an. (DTA: Arndt, Johann: Vom wahren Christenthumb. Bd. 2. Magdeburg, 1610)

(461) Folgendts ging ich zu jhm / vnd fragte mit ernft nach meinem Mantel / er aber gab mir bo̊fe antwort / vnnd trohete mir mit der Ruthen [...] (DTA: Albertinus, Aegidius: Der Landtstörtzer: Gusman von Alfarche oder Picaro genannt. Bd. 1. München, 1615)

Das Einsetzen von Gradbedeutung lässt sich vorerst durch die metaphorische Ausdehnung der auf „lebende wesen“ bezogenen Vorstellungen des Bösen, Unmoralischen und Gemeinen erkennen. Die Intensivierung nimmt ihren Ursprung in den Kontexten, die durch pragmatic inferencing eine alternative Lesart und demnach Ambiguität aufweisen. Die aus DTA übernommenen Belege sollen die Annahme untermauern:

(462) Es haben die Engellånder jnen nicht vorgenommen an folches Ort zu fahren / fondern das Vngewitter vnd bo̊fe Wind haben fie dahin getrieben [...] (DTA: Gottfried, Johann Ludwig: Newe Welt Vnd Americanische Historien. Frankfurt (Main), 1631)

(463) Fuhre vnfer Nache zu den Pinguins Jnfuln / guter hoffnung etliche Pinguins zu vberkommen / aber er konte denfelben Tag / wegen bo̊fen Gewitters vnfer Schiff nicht widerumb erreichen. Mufte alfo die gantze Nacht in Efperlans Bay verziehen. (DTA: Gottfried, Johann Ludwig: Newe Welt Vnd Americanische Historien. Frankfurt (Main), 1631)

(464) Der böse Durst brachte viele Leute zu Sachen, die sie sonst nie gethan hätten. (DTA: Johann Heinrich Pestalozzi: Lienhard und Gertrud, 1785) 
(462) - (464) belegen Ambiguität des adjektivischen Gebrauchs. Zunächst erfährt böse eine metaphorische Interpretation, wobei es mit personifizierten Substantiven (Wind, Gewitter und Durst) und Verben in übertragenen Verwendungen (treiben, bringen) kollokiert. Demzufolge betrachtet man Wind, Gewitter und Durst als personifizierte Abstrakta, welche menschliche Eigenschaften zugeschrieben bekommen. Infolgedessen werden sie als aktive Täter konzeptualisiert und mit einem bösen Geist oder übelwollenen Menschen kognitiv verglichen, der auf den mit dem Opfer assoziierten Menschen übel wirkt und sie eventuell zu schlimmen Taten zwingt.

Personifizierung scheint jedoch nicht die einzige Interpretationsmöglichkeit zu sein, denn Intensität kann als Ursache der in (462) - (464) beschriebenen Ereignisse betrachtet werden. In (462) war der starke Wind (mit dem Ungewitter) der Grund, wieso die Engländer zu dem angesprochenen Ort getrieben wurden. In (463) konnte man das Schiff wegen des starken Gewitters nicht erreichen. In (464) empfinden die angesprochenen Personen sehr starken Durst, sodass sie sich für die „Sachen“ entscheiden, die eine Lösung für ihr Problem bieten. Dass diese „Sachen" sehr schlimm, unmoralisch und böse sind, lässt sich aus dem Satzteil „die sie sonst nie gethan hätten" schließen, was wiederum diese Annahme hinsichtlich der hohen Intensität des Durstes bestätigt.

Die auf der Koexistenz von Personifizierung der Ausgangsbedeutung und Steigerung beruhende Ambiguität der Kontexte findet sich außerdem in Bezug auf Konfliktsituationen oder Krankheiten:

(465) „Einer der Grossväter" Caetano's wurde im Walde von einem andern Bakaïrí erschlagen; innerhalb des Stammes herrschten damals böse Streitigkeiten und die Folge war - so lautet der Bericht der Paranatingaleute - dass ein Teil der Bakaïrí vom Salto zum Kulisehu zog. (DTA: Karl von den Steinen: Unter den Naturvölkern Zentral-Brasiliens, 1894)

(466) Es wütet jetzt eine böse Seuche in den Winckelwäldern; es wird uns der Friedhof zu klein, und wir können schier die Totengräber nicht auftreiben; die kräftigsten Männer liegen auf dem Krankenbette. (DTA: Peter Rosegger: Die Schriften des Waldschulmeisters, 1875)

Aus (465) - (466) geht hervor, dass die Prädikate Streitigkeiten und Seuche personifiziert werden, was sich in den auf sie bezogenen Verben herrschen und wüten erkennen lässt. Durch den metaphorischen Gebrauch wird eine Beziehung zur Ausgangsbedeutung hergestellt. Zugleich kann aus den Kontexten geschlossen werden, dass böse eine intensivierende Rolle übernimmt. Die Intensität der Sachverhalte dient als Ursache für bestimmte Folgen bzw. das Ziehen eines Teils des Stammes in (465) und viele Tote in (466).

Die weitere Untersuchung der Korpora hat ergeben, dass die Gradbedeutung neben der Ausgangsbedeutung auch ohne Metaphorisierung eintreten kann, vgl.:

(467) Es ift kein Zorn fo bitter / als der Frawen Zorn: ich wolte lieber bey Lo̊wen vnd Drachen wohnen / als bey einem boofen Weibe / [...] vnd wann man fonften ein bo̊fen Zorn befchreiben wil / fagt man in gemeinem Sprichwort: es ift ein Weiber Zorn. (DTA: Reinkingk, Dietrich: Biblische Policey. Frankfurt (Main), 1653) 
(468) Darum fagt Syrach: Ums Guts willen thun ihrer viel unrecht / und die reich werden wollen / wenden die Augen ab / fie achten deß Gewiffens nicht / fetzt D. Luther am Rand hinzu / Syr. 27. Aber folche Ungerechtigkeit ift ein bo̊fer Mißbrauch / der an fich felbs guten Güter und Gaben Gottes / davor ein jeder Chrift fich hüten folle / dann das unrecht Gut hilfft nicht [...] (DTA: Bauller, Johann Jacob: Hell-Polirter Laster-Spiegel. Ulm, 1681)

Der adjektivische Gebrauch in (467) - (468) lässt eine mehrdeutige Interpretation zu. In (467) bezieht sich böse auf den zornigen Zustand und in (468) auf verwerfliches, moralisch schlechtes Verhalten. Zugleich handelt es sich um einen stark ausgeprägten Zorn und einen sehr schlimmen Mißbrauch, sodass böse als Intensivierungsmittel wahrgenommen werden kann.

Auch hier soll bemerkt werden, dass die Bezugswörter in (462) - (468), die mit böse intensivierende Zusammensetzungen bilden, Extremwerte aufweisende Prädikate bzw. „implicit superlatives“ (Paradis 1997: 54) darstellen. Dies mag einen weiteren Grund für die Verstärkung der intensivierenden Bedeutung liefern.

Die mehrdeutigen Kontexte (462) - (468) bezeugen das Einsetzen von subjectivity neben der intensivierenden Funktion. Aus den Kontexten lässt sich schließen, dass böse eine expressive Bedeutungskomponente erwirbt. Das Auftreten von subjectivity bringt die Einstellung des Sprechers bzw. Schreibers zum Ausdruck und verweist damit auf Traugott I (oben 3.6.3.4).

Die nächste Etappe des Entwicklungsgangs der Gradbedeutung ist einerseits mit dem Verlust der Ausgangsbedeutung, andererseits mit der Verstärkung der Intensivierung verbunden. Der Prozess der Desemantisierung lässt sich daran erkennen, dass der durch die Personifizierung des Gebrauchs gewonnene Zusammenhang mit der Ausgangsbedeutung verlorengeht. Neben dem Verlust des metaphorischen Gebrauchs tritt zugleich die in einem höheren Grad einstellungsbezogene Bedeutung in den Vordergrund, infolgedessen verblasst die Ausgangsbedeutung zunehmend:

(469) Er blutete stark am Hinterkopfe und vermochte nicht fest aufzutreten, weil er sich einen Fuß bös verstaucht hatte. (DTA: Hermann Alexander Berlepsch: Die Alpen in Natur- und Lebensbildern, 1861)

(470) In Baumschulenweg blieb den Läufern nur die Wahl zwischen knietiefen, zumeist verharschtem Schnee oder vollkommen vereisten Strassen und Wegen; ein Sturz hätte sicher böse Verletzungen mit sich gebracht. (C.II: Berliner Tageblatt (Abend-Ausgabe), 04.03.1929]

Der Gebrauch von böse in (469) - (470) demonstriert die Verwendung des Lexems mit fortgeschrittener Gradbedeutung. Die hervorgehobenen Kollokationen treten frei von metaphorischen Interpretationen auf. Das Adverb in (469) modifiziert kein auf einen aktiven Täter bezogenes Verb, sondern einen experiencer, der sich durch seine unabsichtliche Tat sehr großen Schaden zugefügt hat. In (470) bezieht sich böse auf sehr schlimme Verletzungen, die für die beteiligten Personen sehr unangenehme und unerwünschte Auswirkungen haben können. Damit bleibt die Bedeutung von böse auf den Grad der Eigenschaftsausprägung (ausschließlich Intensivierung) beschränkt. 
In der besprochenen Etappe der Bedeutungsentwicklung tritt böse lediglich mit negativ konnotierten Kookkurrenzpartnern auf. Aus den Kontexten geht außerdem hervor, dass die einstellungsbezogene Bedeutung sich in diesem Gebrauch fest etabliert hat, sodass die Kollokationen mehr Expressivität zum Ausdruck bringen und damit auf die dritte semantisch-pragmatische Tendenz (Traugott III, oben 3.6.3.4) verweisen, vgl.:

(471) Eigentlich hat sie böse Zahnschmerzen. Andere mögen sich da verkriechen, sie jedenfalls sagt das Gespräch nicht ab. (C.II: NON07/MAR.01324 Niederösterreichische Nachrichten, 05.03.2007, S. 29)

(471') 'starke Zahnschmerzen'

(472) Tödliche Pilze sind kaum dabei, aber Pilze, die eine böse Magenverstimmung hervorrufen, gibt es ab und zu. (C.II: A10/OKT.01262 St. Galler Tagblatt, 05.10.2010, S. 33)

(472') 'starke Magenverstimmung'

(473) WM-Teilnehmer Polen hat sich dagegen böse blamiert. (C.II: HMP07/JUN.00714 HM, 07.06.2007, S. 33)

(473') 'sehr blamiert'

(474) Der Keeper hatte sich beim Herauslaufen böse verschätzt. „Das war mein Fehler. Es war keine leichte Situation, es war ein schwieriger Ball“, gestand der Neuzugang. (C.II: BRZ11/AUG.03088 Braunschweiger Zeitung, 08.08.2011)

(474') 'sehr verschätzt'

Böse kann außerdem in quantifizierender Funktion auftreten und die Menge der Sachverhalte bezeichnen, vgl. den Gebrauch in (343):

(475) Kostenpunkt: Siebenhundert Deutsche Mark, das ist eine böse Summe. Sehr viel Geld, und man müßte sich überlegen, wie man's zusammenkriegt. (20.07.2015: https://books.google.de/books?id=MgFcAAAAMAAJ\&q=\%22b\%C3\%B6se+summe\%22\&dq=\%22b\%C3\%B6se+summe\%22\&hl=de\&sa=X\&ved=0CEoQ6AEwCTgKahUKEwiwvrLxzunGAhUDhywKHcJ0CaY)

(475') 'sehr große Summe'

Die Belege (469) - (474) und (475) demonstrieren den eindeutigen Gebrauch von böse jeweils in intensivierender und quantifizierender Rolle. Der Entwicklungsgang in dieser Etappe korreliert außerdem mit der Zunahme an subjectivity, indem die Bedeutung des Lexems sich im Rahmen von Traugott III (oben 3.6.3.4) auf der expressiven Ebene etabliert hat.

Die Entwicklung der Gradbedeutung von böse beschränkt sich bis jetzt ausschließlich auf negativ konnotierte Kookkurrenzpartner. Darüber hinaus ist die Desemantisierung nicht vollständig vollzogen worden.

\subsubsection{Kurzfassung der Entwicklung bei böse}

1. Ausgangsbedeutung: 'schlimm, böswillig, übel usw.';

6. Reine Gradbedeutung: nur negativ konnotierte Kookkurrenzpartner; keine vollständige Desemantisierung; die übrigen Entwicklungsschritte wie oben in Kap. 3.6.3.9.3. 


\subsubsection{Brutal}

\subsubsection{Lexikographische Angaben}

Laut Kluge (s.v.) tritt das Lexem brutal im 16. Jh. in Erscheinung als Lehnwort aus dem Spätlateinischen brūtālis „unvernünftig, tierisch“, wobei das letzte aus dem Lateinischen stammt brūtus „schwerfällig, stumpf, gefühllos“. Laut Pfeifer (s.v.) wurde brutal „in der 2. Hälfte des 17. Jhs. entlehnt (vgl. frz. brutal)“. DWB (s.v.) verweist darauf, dass das Wort erst im „18. jh. üblich wurde“.

1. brutal in FWB

keine Angaben, u.a. im bereits zitierten Material der Redaktionsstelle von FWB

2. brutal in DWB (s.v.)

"ferinus, immanis“

3. brutal in DUDEN (2012, s.v.)

$3.1 \mathrm{a}$ „roh, gefühllos und gewalttätig“

$3.1 \mathrm{~b}$ „schonungslos, rücksichtslos“

3.2 „(Jugendsprache)“

$3.2 \mathrm{a}$ „sehr gut; wunderbar; großartig“

$3.2 \mathrm{~b}$ „<intensivierend bei Adjektiven und Verben> sehr, überaus, in höchstem Maße"

\subsubsection{Entwicklung der Gradbedeutung bei brutal}

Die Korpusanalyse legt nahe, dass die in 2 bezeichnete Bedeutung als Ausgangsbedeutung für die Entwicklung der Gradbedeutung bei brutal anzusetzen ist. So bezieht sich die Ausgangsbedeutung des Lexems auf „rohe“, „gefühllose“ und „gewalttätige" Personen, z.B. König, Vater, Soldat (DTA, COSMAS, s.v.) und ihr entsprechendes „schonungsloses“ und „rücksichtsloses“ (DUDEN 2012, s.v.) Verhalten, z.B. Worte, Behandlung, Ton, Offenheit, Scherze (DTA, COSMAS, s.v.), vgl.:

(476) Fahre wohl, Falstaff, ich wünsche dir in der andern Welt keine so brutalen Könige. (C.II: HK3/HK1.00111 Bräker: Etwas über William Shakespeares Schauspiele, Entstanden: 1780, 2000 [S. 383])

(477) „Wer seid Ihr?" frägt der Richter mit ziemlich brutalem Ton. (C.II: HK3/B01.00002 Schiller: Der Verbrecher aus verlorener Ehre, Erstdruck: 1786, 2000 [S. 33])

(478) Am Nachmittag kam der Hausmann, kündigte ihr in brutalen Worten das Schicksal ihres Mannes an und fagte, daß fie jetzt, wo fie ihm allein gar keine Garantie mehr biete, ungefäumt ausziehen müffe. (DTA: Dronke, Ernst: Polizei-Geschichten. Leipzig, 1846)

Obgleich DWB keine Hinweise auf die Gradbedeutung bietet und deren erste Erwähnung sich erst in DUDEN (2012) findet, datiert die Beleglage in COSMAS die Erscheinung des intensivierenden Gebrauchs auf das 19. Jh. Die Herausbildung der Intensität ist zunächst mit pragmatic inferencing bzw. strengthening der Ausgangsbedeutung verbunden, indem die Gradbedeutung als Nebenbedeutung aus dem Kontext abgeleitet wird, vgl.: 
(479) Der General, Graf Hulin, der feinen Eifer zeigen und auch wohl zu eignem Beften den Zornausbrüchen des Kaifers einen Gegenftand anweifen wollte, ftürmte mit brutaler Gewalt auf den armen Mann los, ftieß ihn mit der Fauft mehrmals vor die Bruft und trat mit dem Fuße nach dem Zurücktaumelnden, unter heftigen Vorwrfen und Schimpfreden [...] (DTA: Varnhagen von Ense, Karl August: Denkwürdigkeiten und vermischte Schriften. Bd. 2. Mannheim, 1837)

(480) Elender hat sich wohl nie ein Gemeinwesen bankerott erklärt als Rom durch diesen [...] Beschlufs einige politische Gefangene [...] eiligst umzubringen [...]! Es war der humoristische Zug, [...] dafs dieser Act der brutalsten Tyrannei von dem haltungslosesten und ängstlichsten aller römischen Staatsmänner vollzogen werden mufste [...] (DTA: Mommsen, Theodor: Römische Geschichte. Bd. 3: Von Sullas Tode bis zur Schlacht von Thapsus. Leipzig, 1856)

(481) Sie hätte ihn vielleicht in der Raserei ihrer Eifersucht mit ihren eigenen Händen morden können - aber ihn den brutalen Mißhandlungen Clotens's und der Anderen aussetzen - der Gedanke war ihr fürchterlich. (DTA: Spielhagen, Friedrich: Problematische Naturen. Bd. 4. Berlin, 1861)

Die adjektivischen Verwendungen in (479) - (481) lassen mindestens zwei Lesarten zu. Die erste Interpretation bezieht sich auf „tyrannisches, willkürliches Verhalten“ (DUDEN 2012, s.v. Gewalt), das durch brutal als „rücksichtslos“ und „schonungslos" bezeichnet wird. Damit wird das Adjektiv in der Ausgangsbedeutung gebraucht. Die zweite Interpretation setzt das Einsetzen von Gradbedeutung voraus und lässt sich aus den Kontexten ableiten. Beide Kookkurrenzpartner stellen Extremwerte aufweisende Bezugswörter dar, die sich auf schonungsloses, grausames und rücksichtsloses Verhalten beziehen und damit „quite closely match“ (Bolinger 1972: 246) volle oder teilweise Übereinstimmung. Diese Übereinstimmungen werden u.a. bei dem Nachschlagen der Definitionen im Wörterbuch veranschaulich, vgl. die unterstrichenen Definitionsteile in (482) - (484):

(482) Gewalt ist „[gegen jmdn., etw. rücksichtslos angewendete] physische Kraft, mit der etw. erreicht wird";

(483) Tyrannei ist „tyrannisches, willkürliches Verhalten“, wobei tyrannisch als „herrschsüchtig, despotisch; rücksichtslos [u. grausam] die eigene Stärke, Macht einsetzend" definiert wird;

(484) Misshandeln ist Substantivierung des Verbs misshandeln mit der Definition „einem Menschen, einem Tier in roher, brutaler Weise körperlichen [u. seelischen] Schaden zufügen".

Aus den lexikographischen Beschreibungen geht hervor, dass die die Definition von brutal bildende Bedeutungskomponente „rücksichtslos“ in den Bezugswörtern Gewalt und Tyrannei enthalten ist ${ }^{58}$, während Misshandeln u.a. durch den Begriff brutal wiedergegeben wird. Die Wiederholung der Bedeutung hat semantic redundancy und demnach semantic repetition zu Folge, was zu der Verstärkung des

\footnotetext{
58 DUDEN bietet „rücksichtslos“ als Wiedergabe für den Definitionsteil von Tyrannei „tyrannisch“.
} 
Gebrauchs und der Herausbildung einer intensivierenden Funktion des Adjektivs führt.

Aus den Kontexten kann außerdem geschlossen werden, dass sie „the representation of a speaker's perspective of point of view in discourse" (Finegan 1995: 1) demonstrieren, sodass der Gebrauch von brutal eine wertende bzw. subjektive Komponente erhält. In Bezug z.B. auf (480) lässt sich schließen, dass der Sprecher bzw. Schreiber verschiedene Ausprägungsformen bzw. Stufen von Tyrannei unterscheidet. Durch die Verwendung der Steigerungsform wird die Inferenz abgeleitet, dass Tyrannei - der Meinung des Autors nach - mehr oder weniger brutal sein bzw. durch Brutalität schwächer oder stärker ausgeprägt sein kann. Der Superlativ verweist schließlich darauf, dass die in (480) beschriebene Tyrannei nach dem Maß der Brutalität stark ausgeprägt ist und auf der entsprechenden Skala, die die Intensität der Tyrannei demonstrieren soll, am oberen Rande anzusiedeln ist.

Die Belegsituation in COSMAS legte nahe, dass die Gradbedeutung außerdem neben dem metaphorischen Gebrauch des Lexems auftreten kann, vgl.:

(485) Aber es knebelten meine Feder auch brutale physische Hindernisse, und diese reelle Ursache meines Schweigens, meines Nichtschreibens, kann ich erst heute öffentlich enthüllen. (C.II: HK3/F47.00001 Heine: Lutetia, 1840 [S. 506])

Die adjektivische Verwendung in (485) demonstriert einen mehrdeutigen Gebrauch. Zunächst handelt es sich um die Personifizierung des Bezugswortes Hindernisse, wodurch dem Adjektiv der metaphorische Sinn zugeschrieben wird. Durch diese Bedeutungsverschiebung verliert brutal den direkten Zusammenhang mit der Ausgangsbedeutung. Es besteht jedoch die Möglichkeit, das Adjektiv als Intensivierungsmittel darzustellen. Diese Auffassung lässt sich durch die semantische Reanalyse des einstellungsbezogenen Kontexts untermauern. Der Belegtext bezieht sich auf eine Situation, die von dem Sprecher bzw. Schreiber beschrieben und beurteilt wird. Er erklärt den Grund seines Schweigens und Nichtschreibens und gibt ihm eine Bewertung bzw. seine persönliche Meinung. Damit dient die Bedeutung des Adjektivs dem Ausdruck von „the speaker's subjective belief-state/attitude toward the situation" (Traugott 1990: 500). Die vom Sprecher abgegebene Bewertung hebt die Intensität der Hindernisse als die Ursache des Geschehens hervor, sodass etwa die folge Umformulierung gilt:

(485') 'Aber es knebelten meine Feder auch sehr starke physische Hindernisse, und diese reelle Ursache meines Schweigens, meines Nichtschreibens, kann ich erst heute öffentlich enthüllen.'

Das Auftreten der intensivierenden Bedeutungskomponente in brutal in der besprochenen Stufe des Entwicklungsgangs geht mit dem Einsetzen von subjectivity einher, sodass der Gebrauch die Einstellung des Schreibers bzw. Sprechers zum Ausdruck bringt und auf Traugott I (oben 3.6.3.4) hinweist.

Das Auftreten von Intensitätsinferenzen in (479) - (481) sowie in (485) hat den Prozess der semantischen Abnutzung der Ausgangsbedeutung von brutal zur Folge. In der nächsten Phase geht der Zusammenhang mit der Ausgangsbedeutung verloren, wobei die Gradbedeutung die einzige Interpretationsmöglichkeit darstellt. Je- 
doch bleibt der Bedeutungsverlust unvollkommen, weil das Adjektiv ausschließlich auf negative Kontexte beschränkt bleibt, vgl. den adjektivischen (486) - (488) und adverbialen Gebrauch (489) - (492) mit den entsprechenden Umformulierungen:

(486) „[... Cosima sähe Friedrich nach dem Tod Richards wieder und zeigte ihm, daß das brutale Mißtrauen gegen Frauen, das er für Stärke hielt, vielleicht nur Menschenscheu und Schwäche war". (C.II: H85/0Z2.30459 Zeit, 08.11.1985, S. LB)

(486') 'sehr große Mißtrauen'

(487) Wer die brutale Not nicht kennt [...] und nicht weiß, wie dem Menschen zumute ist, dem in solcher Lage dann die Hand oft leer ist - der kennt das Dasein nicht ganz. (C.II: HK4/B18.00001 Boy-Ed: Lehrling in der Welt, Entstanden: vor 1928, 2004, [S. 5])

(487') 'sehr starke Not'

(488) Das unbarmherzige Klima mit sintflutartigem Regen und brutaler Hitze hat das Land bis heute fast unberührt gelassen und macht die 7300 Kilometer lange Tour zu einem echten Outdoor-Abenteuer. (C.II: HAZ08/FEB.02829 HAZ, 16.02.2008, S. 3)

(488') 'sehr starker Hitze'

(489) „Es wird sowohl heute als auch am Dienstag brutal schwül sein“, warnen die Meteorologen. (C.II: K00/JUL.55221 Kleine Ztg., 24.07.2000)

(489') 'sehr schwül'

(490) Rund 750 Schüler zogen gleich morgens aus, inspizierten den Philosophenweg, die Neckarwiese und das Terrain am Tiergarten-Schwimmbad: „Da war es brutal schmutzig“ [...] (C.II: M02/JUL.53503 Mannh. Morgen, 18.07.2002)

(490') 'sehr schmutzig'

(491) Es gibt Kinder, die schon um 5.15 Uhr aufstehen müssen. Das ist brutal früh und eine extreme Belastung. (C.II: M09/AUG.65388 Mannh. Morgen, 21.08.2009, S. 3)

(491') 'sehr früh'

(492) „Wir sind brutal enttäuscht, denn wir hätten hier den Sieg verdient gehabt", stellte Deniz Dogan fest. (C.II: BRZ10/MAI.00068 Braunschw. Z., 03.05.2010)

(492') 'sehr enttäuscht'

Als Gradadverb kann brutal außerdem Funktionsverbgefüge modifizieren, die durch einen graduierbaren Begriff wiedergegeben werden können, vgl.:

(493) Und dann kommt mit einmal der Punkt, wo du merkst, da geht dir alles so brutal auf die Nerven ... immer nur daheim. (20.07.2015: https://books.google.de/books?id=Aeo WAAAAIAAJ\&q=\%22brutal+auf+die+nerven $\% 22 \& d q=-$ $\% 22 \mathrm{brutal}+$ auf + die+nerven $\% 22 \& \mathrm{hl}=$ de $\&$ sa $=$ X\&ved=0CCwQ6AEwAmoVChMI0vHLy_HpxgIVy9ssCh394gjP)

(493') 'nervt sehr' 
(494) Wir sind brutal unter Druck, es ist im Job tagtäglich reine Tortur. (20.07.2015: https://books.google.de/books?id=wlTvAjlKflwC\&pg=PT94\&dq=\%22brutal+unter+druck\%22\&hl=de\&sa=X\&ved=0CEIQ6AEwBmoVChMly7rMqvLpxgIVQxcsCh1MZwTt\#v=onepage\&q=\%22brutal\%20unter\%20druck\%22\&f=false)

(494') 'unter sehr großem Druck' bzw. 'sehr bedrängt'

Die intensivierende Funktion des Lexems kann außerdem in die quantifizierende übergehen, die die Bedeutung „viel“ zum Ausdruck bringt, vgl.:

(495) Hab im Moment schon genug brutale Ausgaben. (20.07.2015: http://www.motor-talk.de/forum/billet-6-in-kombi-mit-cce-lenkarmatur-bremsprobleme-t4918090.html)

(495') 'sehr große Ausgaben'

Die Belege (486) - (494) und (495) demonstrieren den eindeutigen Gebrauch von brutal jeweils in intensivierender und quantifizierender Rolle. Die Bedeutungsentwicklung in dieser Stufe korreliert außerdem mit der Zunahme an subjectivity, dadurch, dass die Bedeutung des Lexems sich im Rahmen von Traugott III (oben 3.6.3.4) auf der expressiven Ebene manifestiert.

Die letzte Etappe des Entwicklungsgangs bezieht sich auf das Auftreten mit positiv konnotierten Kookkurrenzpartnern. Damit wird der Prozess der Desemantisierung endgültig vollzogen, vgl.:

(496) Die Alten waren brutal froh, daß sie mich wieder gefunden haben [...] (03.04.2013:http://books.google.de/books?id=L2sSAQAAIAAJ\&q=\%22brutal+froh\%22\&dq=\%22brutal+froh\%22\&hl=de\&sa=X\&ei=EHmMU7jVKOSH4gTNsoGYCQ\&ved=0CDIQ6AEwAA)

(496') 'sehr froh'

(497) Dass selbst Trainer, die brutal erfolgreich sind, zum Beispiel Felix Magath, von selbst gehen. (C.II: HMP10/NOV.01621 MOPO, 16.11.2010, S. D07)

(497') 'sehr erfolgreich'

(498) Die Zeit mit Holtmeyer war brutal lehrreich für mich. (C.II: RHZ12/APR.15949 RZ, 17.04.2012, S. 12)

(498') 'sehr lehrreich'

DUDEN (2012, s.v.) verweist auf den Gebrauch des Lexems in der meliorativen Bedeutung, vgl.:

(499) die Disco ist ein brutaler Schuppen. (DUDEN 2012, s.v.)

(500) das ist, das finde ich echt brutal. (DUDEN 2012, s.v.)

Die Belege (499) - (500) demonstrieren wiederum den kompletten Verlust des Zusammenhangs des Lexems mit der Ausgangsbedeutung und die Entwicklung der einstellungsbezogenen Bedeutung.

\subsubsection{Kurzfassung der Entwicklung bei brutal}

Ausgangsbedeutung: 'roh, gefühllos, rücksichtslos usw.'; die übrigen Entwicklungsschritte wie oben in Kap. 3.6.3.9.3. 


\subsubsection{Grausam}

\subsubsection{Lexikographische Angaben}

Nach den Angaben von Kluge (s.v.) stellt grausam eine Ableitung von „mhd. grūwe „Schauder", das zu grauen gehört" dar. Das Wörterbuch datiert das Auftreten des Lexems auf das 13. Jh. Laut DWB (s.v.) ist es "seit etwa 1300 bezeugt“.

1. grausam in Lexer (s.v. grûwe-sam)

1.1 „adj. Grauen, Schrecken erregend“

1.2 „adv. grausam sehen, ekel erregend aussehen“

2. grausam in FWB (s.v.)

2.1a „durch Aussehen oder Handlungen (besonders bei Tieren oft ineinander übergehend) beim Betrachter Schrecken und Grauen erregend"

2.1b „brutal, gnadenlos, unerbittlich, erbarmungslos (von Personen und deren Handlungen)"

2.1c „schlimm, verheerend (in Bezug auf die Folgen einer Handlung oder vom Wetter); schmerzhaft"

2.1d „schrecklich, Schrecken erregend (von Gegenständen und Sachverhalten)“

$2.1 \mathrm{e}$ „frevelhaft, sündig (in moraltheologischem Sinne)“

2.1f ",erschrocken, schwermütig“

2.2 „ekelhaft, widerlich, abstoßend; häßlich“

2.3 "außerordentlich, sehr, enorm, stark, heftig; schnell“

3. grausam in DWB (s.v.)

A ,als objektives merkmal personen und sonstige gegebenheiten hinsichtlich ihres verhaltens oder aussehens als schrecklich, wild, hart u. ä."

3.1 „kennzeichnung von gegebenheiten, die furcht, schrecken und entsetzen hervorrufen"

3.2 grausam bezeichnet „personen und tiere [...] im hinblick auf ihr wildes, grimmiges, wütendes verhalten insofern, als dieses schauder und schrecken erregt"

3.3 in Bezug auf das Verhalten von Personen, Tieren, Sachen als „, hart, roh, grob u. ä."

3.3a 'hart, streng, roh, unerbittlich'

$\alpha)$,von personen“

$\beta$ ) „von ereignissen, handlungen, zuständen usw., oft in dem sinne des kaum noch erträglichen"

$\gamma$ ) „Von gegenständen, besonders folterungswerkzeugen“

$3.3 \mathrm{~b}$ „barbarisch, unzivilisiert, roh“

3.4 „neben den bedeutungen 1-3 geht eine qualitätsarme bedeutung einher" $3.4 a$, intensivierend“

$\alpha$ ) „heftig, stark, nachdrücklich, schnell“ sehr'“

$\beta)$ „in adverbialem gebrauch oft vollends sinnentleert, 'überaus,

$3.4 \mathrm{~b}$ „eine quantität bezeichnend, 'grosz, viel'“ 
B „'erschütternd, entsetzlich', meist mit der Nebenbedeutung 'böse, schlimm', an grauen, n. (s. d. A 1c; 2b) angelehnt"

$\mathrm{C}$ "grausam charakterisiert [...] personen, handlungen, verhaltensweisen $\mathrm{u}$. dgl. entweder im hinblick auf die zugrunde liegende persönliche haltung und den inneren zustand der unempfindlichkeit, gefühllosigkeit und gefühlskälte in dem sinne, dasz eine neigung besteht, jemanden empfindlich zu treffen, oder, aus der sicht des betroffenen, mehr im hinblick auf die wirkungen solchen verhaltens"

C 1 ,'zwang ausübend, gewalttätig, brutal, blutrünstig, zu solchem verhalten neigend'; dem älteren gebrauch unter A 3 verhältnismäszig nahestehend."

$\mathrm{C} 1 \mathrm{a}$ „von personen und deren organen“

$\mathrm{C} 1 \mathrm{~b}$ „von verhaltensweisen, handlungen, vorgängen“

C 2 "grausam kennzeichnet die neigung zu einem ungerechten und willkürlichen, dem empfinden anderer gegenüber gleichgültigen verhalten und dieses verhalten selbst, ferner die empfindlichen und unverdient harten wirkungen solchen verhaltens auf die betroffene person."

C $2 \mathrm{a}$ „ohne triftigen grund herzlos, gefühllos gegenüber dem empfinden und dem leid anderer"“

$\alpha)$ „,von personen und personifizierten mächten“

$\beta$ ) „von handlungen und verhaltensweisen, die auf die gesinnung einer person zurückzuführen sind"

$\mathrm{C} 2 \mathrm{~b}$ „das moment des mutwillens, der freude an der qual anderer tritt stärker hervor, z. t. geradezu im sinne von 'satanisch'"

C 2c "das moment des gar zu harten, ungerechten, nicht zu rechtfertigenden an einem rücksichtslosen, schonungslosen verhalten, einer solchen äuszerung usw."

C $2 d$ „im hinblick auf gegebenheiten, die an sich gefühllos sind, denen aber aus der sicht des von ihnen betroffenen die persönliche note des unerbittlichen, herzlosen, zu harten beigelegt wird, bringt grausam mehr die subjektive wirkung dieser gegebenheiten zum ausdruck, 'empfindlich, unverdient hart treffend'“.

$\alpha$ ) „von mächten, gewalten, objektiv gesehenen vorgängen u. ä.“

$\beta$ ) „selten von gegenständlichem“

$\mathrm{C} 2 \mathrm{e}$ „in der beziehung auf empfindungen, innere zustände und sonstige gegebenheiten, die an ein empfindendes subjekt gebunden sind, tritt die wirkung auf das subjekt vollends in den vordergrund, 'qualvoll, schmerzlich, überaus empfindlich'“

C 3 ,in einem seit dem anfang des 19. jhs. bezeugten literarischen gebrauch sind gefühls- und wertindifferenz die beherrschenden momente, während das moment der neigung, jemanden empfindlich zu treffen, zurücktritt. grausamcharakterisiert verhaltensweisen $u$. dgl. aus der sicht des betroffenen in dem sinne, dasz sie notwendig sind und ertragen werden müssen, wobei leicht ein mit einem gewissen lustgefühl gesuchtes leiden das wissen um zwang und notwendigkeit begleiten kann." 
C 3a „so besonders intellektuelle verhaltensweisen, äuszerungen, einsichten und kritische verfahren als 'unerbittlich, radikal, schonungslos, scharf, hart, kalt, klar' kennzeichnend"

$\mathrm{C} 3 \mathrm{~b}$ „in jungem wortgebrauch komplex und stark atmosphärisch bedingt. dem leichten lustgefühl am leiden auf seiten des betroffenen korrespondieren auf seiten der solche empfindungen auslösenden personen und verhaltensweisen momente des freudlosen und gelangweilten“

C 4 „qualitätsärmer und verstärkend“

4. grausam in DUDEN (2012, s.v.)

4.1a „unmenschlich, roh und brutal“

$4.1 \mathrm{~b}$ "sehr schlimm, hart"

4.1c "(umgangssprachlich) sehr schwer zu ertragen“

$4.1 \mathrm{~d}$,in besonders starkem Maße, wie eine Art Pein empfunden“

4.2 „<intensivierend bei Verben und Adjektiven> (umgangssprachlich) sehr, überaus"

\subsubsection{Entwicklung der Gradbedeutung bei grausam}

In Bezug auf die Belegsituation scheint es schwer, eine eindeutige Quelle für die Gradbedeutung zu identifizieren. Diese Schwierigkeit rührt daher, dass zwei Sinnrichtungen das Einsetzen der Intensität in ambigen Kontexten nahelegen. Der erste Bedeutungsbereich bringt die Begriffe des Unmenschlichen, Rohen, Brutalen zum Ausdruck (siehe 2.1b), während der zweite Bedeutungsbereich sich auf die ursprüngliche, mit Schrecken verbundene Bedeutung von grausam (siehe 1.1, 2.1a, 2.1d) bezieht. DWB (s.v.) verweist ebenso darauf, dass die „qualitätsarme“ intensivierende Bedeutung „neben“ den beiden Bedeutungen in Erscheinung tritt. In diesem Zusammenhang werden im folgenden zwei Möglichkeiten der Herausbildung von Intensität diskutiert. Als erste wird die Herausbildung der Gradbedeutung aus dem ersten erwähnten Sinnbereich behandelt. Grund hierfür ist erstens die Zugehörigkeit der Bedeutung zu der in diesem Kapitelabschnitt behandelten Begriffsgruppe und, zweitens, das Datum der Erscheinung der ambigen Belege in den untersuchten Korpora.

Die Bedeutung „brutal, gnadenlos, unerbittlich, erbarmungslos“ ist laut DWB (s.v.) zunächst eng mit der ursprünglichen, auf Schrecken bezogenen Bedeutung verbunden, weil die entsprechenden menschlichen oder tierischen Handlungen eine "schauder erregende wirkung" haben, vgl.:

(501) seind auch die mütter iren kinden grausam so sie die in dem bad kratzen vnd ryben zů irer gesundtheit ... (DWB, s.v.,1515)

(502) Denn man hat gesehen vnnd gespüret / daß die jenigen / so zuvorn arg vnnd grausam als Mörder gewesen waren / sich also verändert vnnd gebessert haben [...] (DTA: Gottfried, Johann Ludwig: Newe Welt Vnd Americanische Historien. Frankfurt (Main), 1631)

Die Herausbildung der Gradbedeutung lässt sich in den Kontexten feststellen, die Unmenschlichkeit, Rohheit, Brutalität und Unerbittlichkeit des Verhaltens als Hauptsinn aufweisen. Von daher wird dieser Gebrauch auch im weiteren mit der 
'Ausgangsbedeutung' verknüpft. Das Einsetzen der Gradbedeutung erfolgt in ambigen Kontexten, vgl. den aus FWB entnommenen Beleg:

(503) das war der swarist und grawissamist streit zwischen der Römer und Dewitschen (FWB: 1493, s.v.)

In (503) wird grausam zunächst in Bezug auf die oben besprochene Ausgangsbedeutung definiert. Von daher wird das Bezugskonzept Streit als höchst brutal, gnadenlos und erbittert und daraus folgend eventuell Schrecken verursachend charakterisiert. Das Lexem lässt jedoch eine weitere Interpretation zu, die die Intensität des Abstraktums hervorhebt oder - in Langackers Terminologie (1987: 147ff.) ausgedrückt - profiliert. Der Prozess von inferencing der Gradbedeutung geschieht als Folge von semantic repetition der zitierten Verwendung. Semantic redundancy des adjektivischen Gebrauchs besteht in der Wiederholung des durch grausam ausgeprägten Begriffs in dem Bezugswort. Außerdem stellen die beiden Wörter Extremwerte aufweisende Prädikate dar. So wird Streit in DUDEN (2012) als „(veraltet) Waffengang, Kampf" definiert und stellt dadurch die stärkste Form einer Auseinandersetzung dar, die das Töten des Gegners und von daher grausames bzw. rohes, brutales und rücksichtsloses Verhalten seitens beider beteiligter Truppen voraussetzt. Damit verkörpert das Substantiv Streit den Begriff des Grausamen, dessen Wiederholung durch die adjektivische Modifikation zur Verstärkung des Ausdrucks führt.

Die Verwendung der Steigerungsform dient der Verstärkung der intensivierenden Funktion der abgeleiteten Inferenz. Außerdem identifiziert sie die Einstellung des Sprechers bzw. Schreibers. Aus dem Kontext lässt sich schließen, dass der Autor den Streit als einen graduierbaren Begriff darstellt, dessen Ausprägungsgrad mit dem Grad der Grausamkeit korreliert. Die Höchststufe verweist darauf, dass der Streit über das höchste Maß an Grausamkeit verfügt und dadurch hinsichtlich aller je „zwischen der Römer und Dewitschen“ stattgefundenen Streitigkeiten am stärksten ausgeprägt und daher am oberen Ende der Skala anzusiedeln ist. Die intensivierende Funktion des Adjektivs, die durch semantic repetition hervorgehoben wurde, wird durch die Höchststufe verstärkt.

In FWB und DTA finden sich weitere Belege, die semantic repetition und daraus folgendes pragmatic inferencing der Gradbedeutung aufweisen, vgl. die aus FWB und DTA übernommenen Kollokationen grausam tyrannisieren, grausame Tortur, grausamer Marter. Die Bezugswörter in den Verwendungen weisen auf Extremwerte hin, wie es auch in (503) der Fall ist, und beziehen sich auf grausames bzw. rücksichtsloses und brutales Verhalten, sodass die Bedeutung (semant. Doppelung) des modifizierenden Adjektivs syntagmatisch verdoppelt und von daher intensiviert wird. Dadurch verweisen die Verwendungen auf die Art und Weise der Handlungen, sowie auch auf ihre Intensität.

Die hier in Erscheinung tretende Gradbedeutung erweist sich ferner in metaphorischen Verwendungen mit Witterungsphänomenen, vgl.:

(504) Aber / O Unglück! es entstund ein grausames Gewitter / welches einen Wind schickete / der das Schiff Agapisti anfassete / und mit erschröcklichem Wüten / auf den wanckenden Wellen fort führete / daß weder ich 
/ noch die Mörder / ihn mehr sehen konten. (C.II: HK4/S11.00001 Stockfleth: Die Kunst- und Tugend-gezierte Macarie, Erstdruck: 1669-1673, 2004 [S. 426])

(505) sie für Cöln kamen, do fiel unversehnelich ein solche grusame kelte an. (DWB: M. 16 Jh., s.v.)

(506) Wenig Tage nachher überfiel ihn (den Wolf) ein grausamer Hunger [...] (C.II: HK3/C81.00001 Lessing, Gotthold Ephraim: Briefe, die neueste Literatur betreffend, Erstdruck: 1759-1765, S. 30-329 [S. 87])

In (504) ist grausam mehrfach deutbar. Zunächst kann man von einem metaphorischen Gebrauch ausgehen, bei dem das Bezugswort Gewitter personifiziert bzw. mit einem grausamen Menschen verglichen wird, der sich aktiv an dem Schicksal des Schiffs beteiligt. Die Metapher lässt sich in der übertragenen Verwendung von den Verben schicken und führen erkennen. Als Nebenbedeutung erweist sich die mit dem Begriff des Schreckens verknüpfte Bedeutung des Adjektivs. Aus dem Kontext lässt sich außerdem schließen, dass grausam eine intensivierende Rolle spielt, wobei es sich um sehr starkes Gewitter mit negativen Folgen handelt. Es fällt auf, dass grausam sich auf einen Intensivierung voraussetztenden und Extremwerte aufweisenden Begriff bezieht, vgl. die Definitionen von Gewitter „mit Blitzen, Donner [u. Regen o. Ä.] verbundenes Unwetter" (DUDEN 2012, s.v. Gewitter) und Unwetter ",sehr schlechtes, stürmisches, meist von starkem Niederschlag [u. Gewitter] begleitetes Wetter, dessen Heftigkeit Schäden verursacht" (DUDEN 2012, s.v. Unwetter). Daraus lässt sich schließen, dass es sich um einen negativ konnotierten und eine intensive Ausprägung voraussetzenden Begriff handelt. Zugleich werden die Geschehnisse aus der Sicht des Sprechers bzw. Schreibers dargestellt und bieten demnach seine persönliche bzw. subjektive Bewertung, die sich in Anbetracht der vom Gewitter verursachten Folgen auf seine Intensität richtet. Es lässt sich also eine Skala ableiten, die die Graduierbarkeit des Gewitters demonstrieren soll. Damit dient grausam als ein Verstärkungsmittel, das die Erscheinung in eine hohe Position auf der entsprechenden Skala der möglichen Unwetter einordnet.

Weitere Beispiele der Metaphorisierung der Ausgangsbedeutung werden in (503) - (504) dargestellt. Wiederum dient Personifizierung der Sachverhalte als Basis für die Herausbildung der Gradbedeutung, wobei die Bezugswörter „implicit superlatives" (Paradis 1997: 54) darstellen, vgl. die entsprechenden Umformulierungen:

(505') Eine starke Kälte, die mit einem rohen, brutalen Menschen verglichen werden kann, hat die Reisenden angefallen.

(506') Ein starker Hunger, der mit einem rohen, brutalen Menschen verglichen werden kann, hat den Wolf überfallen.

Die zweite Möglichkeit der Herausbildung einer Gradbedeutung bezieht sich auf den ursprünglichen, mit Schrecken verbundenen Sinn des Lexems, sofern er im Fnhd. noch gebräuchlich ist. Das inferencing erfolgt durch semantic repetition, vgl.:

(507) Gros Ursach hat mich darzu zwungen, Grusam angst und mannig not (FWB: 1514, s.v.)

(508) dann die Forcht so grausam bei ihm ist, daß weder an Seele noch Leib zu helffen. (FWB: 1650, s.v.) 
In (507) - (508) werden die „Inhaltszüge des Bezugswortes“ durch das Adjektiv / Adverb „wiederholt“ (Peters 1993: 28), wodurch es zu semantic repetition der Bedeutungsmerkmale, der Verstärkung des Ausdrucks und der Reanalyse von grausam als Grad-Adjektiv bzw. -Adverb kommt.

DWB (s.v. grausam) verweist außerdem auf das Einsetzen der Gradbedeutung in ambigen Verwendungen mit "akustischen Bezeichnungen“:

(509) da das die heyden sahen, machten sie ein solch grausam geschrey, dass es auch der koenig auss Persia erhoerte (DWB: 1587, s.v.)

(510) doch habe ich in meiner schwachheit noch so viel verspüret, dasz das schiff vermuthlich an einen harten felsen zerscheiterte, indem es ein grausames krachen und prasseln verursachte (DWB, s.v.)

In (509) - (510) bezieht sich grausam zunächst auf die mit Schrecken verbundene Bedeutung und beschreibt Schrecken demonstrierendes bzw. verursachendes Geschrei und Schrecken verursachendes Krachen und Prasseln. DWB schreibt dem Adjektiv außerdem die Nebenbedeutung 'laut' zu. In diesem Zusammenhang wird das Wort für die Bezeichnung der in hohem Maße ausgeprägten Lautstärke und folglich als Intensivierungsmittel verwendet. Daneben stellt der Gebrauch von grausam in intensivierender Funktion eine persönliche Bewertung des Sprechers bzw. Schreibers dar, was für eine Zunahme an subjectification in dessen Bedeutung spricht. Intensivierung dient außerdem als Grund für die beschriebenen Geschehnisse, vgl. die Umformulierungen (509') - (510').

(509') das Geschrei war so laut, dass „es auch der koenig auss Persia erhoerte“

(510') Das Krachen und Prasseln war sehr laut. Daraus hat man beschlossen, dass das Schiff an einen harten Felsen zerscheiterte.

Die Umformulierungen (509") - (510") sollen demonstrieren, dass die Ausgangsbedeutung zugunsten der Gradbedeutung zurückgetreten ist und keine Erklärung für die Ereignisse liefert:

(509")* Das Geschrei war Schrecken erregend bzw. verursachend, sodass „es auch der koenig auss Persia erhoerte“

(510")* Das Krachen und Prasseln war Schrecken erregend, sodass man vermutete, dass das Schiff an einem harten Felsen zerscheiterte.

Die mehrdeutigen Belege (503) - (510) demonstrieren das Auftreten der Intensivierung und das Einsetzen der einstellungsbezogenen Bedeutungskomponente bzw. subjectivity. Damit kommt Traugott I (oben 3.6.3.4) zur Geltung.

In der nächsten Etappe zeigt sich das Adjektiv in rein intensivierender Bedeutung, ohne die frühere lexikalische Bedeutung aufzuweisen. Zunächst kollokiert das Lexem mit negativ konnotierten Bezugswörtern oder tritt in negativ konnotierten Situationen auf, vgl. den adjektivischen (511) - (512) und adverbialen (513) - (515) Gebrauch in den aus COSMAS übernommenen Belegen: 
(511) [...] ich kam mit dem in der ganzen Stadt gefürchteten Manne sehr gut aus und hatte mich nur über eins zu beschweren [...]: grausame Langeweile. (C.II: HK3/G59.00001 Fontane: Von Zwanzig bis Dreißig, Entstanden: 1894/1896, 2000 [S. 65])

(511') 'sehr große Langeweile'

(512) Seit einigen Tagen werde ich von grausamen Zahnschmerzen geplagt. (C.II: HK3/C78.00001 Börne, Ludwig: Briefe aus Paris, 1832, 2000 [S. 630])]

(512') 'sehr starken Zahnschmerzen'

(513) Es ist grausam heiß in diesem Talkessel ohne jeden Luftzug, ohne Wasser, nirgends gibt es etwas zu trinken [...]. (C.II: DIV/BBS.00000 Biehl: Splitter im Sand, (Erstv. 2001), 2004 [S. 73])

(513') 'sehr heiß'

(514) Vor Jahren, in Phasen der Selbstfindung, habe ich einen grausam bunten Binder mit aufgedruckter Marilyn Monroe getragen. (C.II: BRZ09/AUG.10509 Braunschw. Z., 22.08.2009)

(514') 'sehr bunten (in negativem Sinne gemeint) Binder'

(515) „,...] Alle diese Probleme haben Falco grausam beschäftigt", sagte Udo Jürgens. (C.II: V98/FEB.05970 Vorarlberger Nachr., 09.02.1998, S. A3)

(515') 'sehr beschäftigt'.

Grausam kann außerdem als Gradadverb Skopus über Funktionsverbgefüge haben und dadurch die Erweiterung seiner Skopuskonstituente demonstrieren. Die Intensivierung erfolgt dadurch, dass die FVGs durch einen graduierbaren Begriff wiedergegeben werden können:

(516) Es gibt viele, denen der süßliche Duft eine erstatische Wonne ist; andre, denen er grausam auf die Nerven fällt. Ich gehöre zu den letzteren. (GB, 04.06.2014: http://books.google.de/books?id=uPDkAAAAMAAJ\&q=\%22grausam+auf+die+nerven $\% 22 \& d q=\% 22$ grausam+auf+die+nerven $\% 22 \&$ hl=de\&sa $=X \& e i-$ =9PmOU_anDeT_4QTS54DQAQ\&ved=0CFEQ6AEwCA)

(516') 'sehr nervt' bzw. 'ist extrem lästig'

(517) Auf der letzten Abfahrt, der vom Heartbreak Hill, liess ich die Beine baumeln, holte noch einmal tief Luft und setzte mit nicht mehr ganz soviel Schwung, zum Endspurt an. Die allerletzten fünf Kilometer bis zum Ziel streckten sich grausam in die Länge. (GB, 04.06.2014: http://books.google.de/books?id= RQSH2FxXbG4C\&pg=PA299\&dq=\%22grausam+in+die+l\%C3\%A4nge\%22\&hl=de\&sa=X\&ei=2fiOUboHonV4QTPgoDoDw\&ved=0CCoQ6AEwADgK\#v=onepage \&q=\%22grausam $\% 20$ in $\% 20$ die $\% 201 \% C 3 \% A 4 n g e \% 22 \& f=-$ false)

(517') 'verzögerten sich sehr'

(518) Unsere Freundschaft ist seit dem Überfall eher eine Solidarität geworden. Einfach weil uns beiden dieses Unverständnis damals so grausam auf den Sack ging. (GB, 04.06.2014: http://books.google.de/books?id=hF0hAQAAIAAJ\&q=\%22grausam+auf+den+sack\%22\&dq=\%22grausam+auf+den+sack\%-

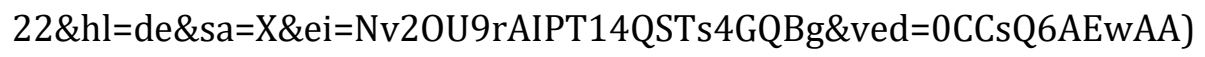

(518') 'sehr nervte' 
Die intensivierende Funktion kann in die quantifizierende übergehen und die Bedeutung „sehr viel“ zum Ausdruck bringen, vgl.:

(519) In Florenz trat nun ein radikaler Umschwung ein. Ohne dass Cosimo sich persönlich exponierte, sorgten seine Anhänger für die rücksichtslose Verbannung oder gar Hinrichtung der gegnerischen Parteiführer. Ihr Besitz wurde beschlagnahmt oder durch grausame Steuern dezimiert. (06.04.2014, GB: http://books.google.de/books?id=Syc9AQAAIAAJ\&q=\%22grausame+steuern\%22\&dq=\%22grausame+steuern\%22\&hl=de\&sa=X\&ei=eQGPU5jNHNL14QT0noCABQ\&ved=0CEcQ6AEwBQ)

(519') 'sehr hohe Steuern'

(520) Eine Woche sind vergangen und wir wollten von euch wissen, wer am meisten bei den grausamen Preisen an Tankstellen leidet. (04.06.2014: http://www.exdungeon.de/home/cms/index.php?itemid=126)

(520') 'sehr hohen Preisen'

Neben der Intensivierung finden sich Belege, die die pejorative Bedeutung und damit den Gebrauch von grausam auf der rein expressiven Ebene demonstrieren. Dabei ist kein direkter Zusammenhang mit der Ausgangsbedeutung zu erkennen, vgl. die Verwendungen (521) - (523) mit den entsprechenden Umformulierungen:

(521) Ich halte den FM 2006 für den besten Fussballmanager aller Zeiten. Daher kann ich nicht verstehen, warum die deutsche Übersetzung derart fehlerhaft ausgefallen ist. Gerade die Newsmeldungen sind grausam übersetzt worden, sowohl im Satzbau als auch in der Rechtschreibung. (04.06.2014: http://www.meistertrainerforum.de/index.php?topic=6568.0;wap)

(521') 'sehr schlecht übersetzt'

(522) Wenn Sie die Angebote an Unterkünften in unserer Region studieren, finden Sie fast ausschließlich extrem hochpreisige Angebote oder Campingplätze, auf denen Büsche, kaputte Toiletten und grausames Essen angeboten werden. (04.06.2014: http://www.aruba-safaris.com/Testversion/ht$\mathrm{ml} / \mathrm{dcamp} . \mathrm{html}$ )

(522') 'sehr schlechtes Essen'

(523) Das hotel azurea hat alle meine Erwartungen von einem dreckigem Hotel, einem grausamen Service und Katastrophen übertroffen. (04.06.2014: http://www.holidaycheck.ch/hr/bd2fdced-1c41-3fb3-8922-81e9f7cbd8bf/-/review/7203a185-0876-3d49-9ad7-e8d3f9e2bb5b)

(523') 'sehr schlechten Service'

Die Belege (511) - (518), (519) - (520) und (521) - (523) demonstrieren den eindeutigen Gebrauch von grausam jeweils in der intensivierenden, quantifizierenden und pejorativen Funktion. Die Bedeutungsentwicklung in dieser Stufe korreliert außerdem mit der Zunahme an subjectivity, wodurch sich die Bedeutung des Lexems im Rahmen von Traugott III (oben 3.6.3.4) auf die expressive Ebene verschoben hat.

Das Auftreten mit positiv konnotierten Bezugswörtern soll die letzte Etappe der Entwicklung der Gradbedeutung von grausam und dessen völlige Bedeutungsentleerung demonstrieren. Es ist jedoch bemerkenswert, dass dieser Gebrauch sich 
zwar schon im Frühneuhochdeutschen findet, im Neuhochdeutschen aber nicht üblich ist, vgl.:

(524) Ich merck, die lieb ist grawsam groß Zwischen euch gwest. (FWB: 1554, s.v.)

(524') 'sehr groß'

(525) Das ist ein grusam grosses Wunder, Das ich im Paradieß find ouch Singen unseren lieben gouch. (FWB: 1519, s.v.)

(525') 'sehr großes Wunder'

(526) Er was ain grad, hüpsch, tugenthaft man, und was gar grausam freuntlich gegen armen leuten. (FWB: 16 Jh., s.v.)

(526') 'sehr freundlich'

Die im Archiv der geschriebenen Sprache in COSMAS gefundenen Verwendungen mit positiven Kollokationspartnern weisen zwar die intensivierende Funktion des Lexems auf, beziehen sich jedoch auf negative Situationen, die eher den Bezug auf die mit Schrecken verbundene Bedeutung dokumentieren:

(527) Trotz seiner Wut zielte ein Pensionist grausam gut. (C.II: K96/NOV.24557 Kleine Ztg., 05.11.1996)

(527') 'zielte sehr gut'

(528) Als Terrorist war Eric Rudolph immer wieder grausam erfolgreich, als Flüchtling gab er den Zielfahndern des FBI so viele Rätsel auf, dass Polizei-Psychologen in ihm schon ein verbrecherisches „Superhirn" sahen. (C.II: M03/JUN.36064 Mannh. Morgen, 02.06.2003)

(528') 'sehr erfolgreich'.

\subsubsection{Kurzfassung der Entwicklung bei grausam}

1. Ausgangsbedeutung: zwei mögliche Bedeutungsbereiche - 1. 'roh, brutal, unerbittlich', 2. 'mit Schrecken verbunden'. Der erste Bedeutungsbereich ist dabei zunächst mit der Bedeutung des Schreckens verbunden.

2. Entwicklung der Gradbedeutung: für beide Bedeutungsbereiche wie oben in Kap. 3.6.3.9.3.

\subsubsection{Grimmig}

\subsubsection{Lexikographische Angaben}

1. grimmig in AWB (s.v.)

1.1 "grausam, unbarmherzig“

1.2 „unfreundlich, schroff“

1.3 "grimmig, murrend“

2. grimmig in BMZ (s.v. grimmec)

„wütend, grimmig, grausam, schrecklich“

3. grimmig in FWB (s.v.)

3.1 „wütend, zornig, hitzig, haßerfüllt (als mehr oder minder spontaner Gefühlsausbruch); verbissen, erbittert (in einem Kampf); jähzornig (als Eigenschaft und Haltung eines Menschen)“ 
3.2 „grausam, unbarmherzig, menschenverachtend; gnadenlos, rücksichtslos (von menschlichem Verhalten); böse, schlecht, sündig (in moraltheologischem Sinne); fürchterlich, schrecklich (von Ereignissen und Handlungen in ihren Auswirkungen auf den Menschen); bitter (von Kälte)“

3.3 „gefährlich, furchterregend, bedrohlich, wild (von Tieren)“

3.4 „das Normalmaß übersteigend, heftig, arg, sehr, stark, schlimm; speziell: lautstark (von Geräuschen)“

4. grimmig in DWB (s.v.)

4.1 „wütend, zornwütig, ergrimmt"

4.2 „atrox, saevus“ „zustand und eigenschaft “ „zumeist von menschen“, „so auch meist von thieren“, außerdem "mannigfach übertragen“

4.2.1 „auf träger und sitze des grimms beim menschen, gewöhnlich im sinne acer, atrox, saevus"

4.2.2 „in älterer sprache vielfach weiter von dem persönlichen ausgangspunkt entfernt und fühlbar objectiver, severus, crudelis"

4.2.3 „rein objective bedeutung schwer, schlimm, bitter, quälend' neben begriffen wie“ ",pein“, „leid“, „,noth“, „, notwendigkeit"

4.2.4 „saevus, atrox zu ein paar besonderen spielarten; öfter von literarischen erzeugnissen, dem sinne von 'bissig' nahekommend"

4.3 „das adj. bezeichnet nicht die ausbrechende, sondern die verhaltene oder versteckte wuth"

4.4 „ebenfalls erst im 19. jh. breitet sich ein gebrauch aus, der das adj. mit worten entgegengesetzter sinnesrichtung verbindet, wobei der begriff des wilden mehr oder minder durch den des bitteren oder boshaften gefärbt oder ersetzt ist"

4.5 „der charakterisierenden bedeutung eine quantitierende beigesellt" bzw. „intensitätsmoment"

5. grimmig in DUDEN (2012, s.v.)

5.1 „voller Grimm; sehr zornig, wütend“

5.2 „sehr groß, heftig; übermäßig“

\subsubsection{Entwicklung der Gradbedeutung bei grimmig}

Das Einsetzen der Gradbedeutung lässt sich nach den Angaben von FWB sowie der Korpusanalyse im Fnhd. nachweisen. Als Ausgangspunkt für die Herausbildung der Bedeutung dienen die in 3.1 und 3.2 erwähnten Sinnrichtungen, die auf den Charakter und das menschliche gnadenlose, unerbittliche Verhalten hinweisen und durch die folgenden Belege exemplifiziert werden können, vgl:

(529) Wie kann denn dieser Cain so unbarmhertzig und grausam sein, das er sein eigen blut und fleisch so grimmig ermordet? (FWB: 1544, s.v.)

(530) Hartman von Grunbach, ein grimmiger und tyrannischer man (DWB, s.v.)

(531) seine böse, grimmige, unbarmhertzige übelthaten erschollen weit (Kirchhof, apud DWB, s.v.)

In Bezug auf die Gradbedeutung verweist DWB (s.v.) darauf, dass die intensivierende „sinnesrichtung [...] sich dadurch [ergibt], dasz sich der charakterisierenden bedeutung eine quantitierende beigesellt“. Ferner wird angegeben: „am stärksten 
hat sich das intensitätsmoment bei adverbialem gebrauch ausgewirkt, wo die bedeutung schlieszlich beim reinen quantitätsbegriff endet"; in älteren Belegen „pflegt die ursprüngl. bedeutung noch vorzuwiegen“ (DWB, s.v.). Aus den angeführten Zitaten lässt sich schließen, dass die Gradbedeutung sich zunächst neben der Ausgangsbedeutung bzw. in mehrdeutigen Kontexten findet. Die Beleglage bestätigt diese Annahmen und legt nahe, dass die intensivierende Bedeutungskomponente in einem frühen Stadium des Entwicklungsgangs durch pragmatic enrichment bzw. strengthening der Ausgangsbedeutung in ambigen Verwendungen in Erscheinung tritt.

DWB (s.v.) stellt fest: „,am besten läszt sich das einsetzen und allmähliche erstarken der ins intensive weisenden nebenbedeutung neben gewissen ausdrücken des affects beobachten“, vgl. die aus FWB und DTA übernommenen Belege:

(532) Das Manthier nam sein schwerdt / Und schlug so grimmig auff das Pferdt / [...] / Das es aus schreckn die sprach verlorn. (FWB: 1608, s.v. grimmig)

In (532) lässt sich der mehrdeutige Gebrauch von grimmig erkennen. Zunächst bezieht sich das Lexem auf die Ausgangsbedeutung, indem es einen wütigen, zornigen und zugleich Unbarmherzigkeit aufweisenden Menschen beschreibt. Der Kontext hebt jedoch außerdem die Intensität der durchgeführten Handlung hervor. In diesem Zusammenhang ist das Kollokieren mit dem Adverb so bemerkenswert, welches „ein durch Kontext od. Situation näher bestimmtes [verstärktes] Maß o. Ä., in dem eine Eigenschaft, ein Zustand o. Ä. vorhanden, gegeben ist" (DUDEN 2012, s.v. so) zum Ausdruck bringt. Daraus lässt sich schließen, dass der von grimmig ausgedrückte Begriff durch so verstärkt wird bzw. auf einer Skala eingeordnet wird, die den Grad der Grimmigkeit ausdrücken soll. Außerdem stellt die Heftigkeit des Schlags die Erscheinungsform des emotionalen und damit des auf die Ausgangsbedeutung bezogenen Zustands der angesprochenen Person dar und erweist sich zugleich als Ursache des darauffolgenden Zustandes des Tieres, vgl. die unten eingeführte Umformulierung:

(459') Das Manthier war sehr grimmig bzw. zornig und schlug so heftig auf das Pferd, dass es aus Schrecken die Sprache verlor'.

Eine ähnliche Interpretation in Bezug auf die Ausgangs- und Gradbedeutung erfolgt in (533):

(533) In den die stud den fuß auffzug/ Und den wolff gar grimmiglich schlug / Ant stiren mitten auff den kopff. (FWB: 1562, s.v.)

(533') 'schlug erbarmungslos, zornig etc.'

(533") 'schlug heftig'

In (534) - (538) finden sich weitere Belege, die das Einsetzen von Gradbedeutung neben den Ausdrücken des Affekts demonstrieren:

(534) (...) / Es wird ein mal Gott das ander Gebot warlich wahr machen / vnd ein grossen grimmigen Zorn vber sie außgiessen. (DTA: BraunschweigWolfenbüttel, Herzog Julius von]: Corpus Doctrinae, Das ist / Die Summa / Form 
und Fürbilde der reinen Christlichen Lehre / aus der heiligen Göttlichen Schrifft der Propheten und Aposteln zusammen gezogen. Helmstedt, 1603)

(535) Vnd bist so grimmig zornig vber die Schafe deiner Weide? (DTA: [N. N.]: Vom Beruff Und Enturlaubung der Prediger / Christlicher Fürtrefflicher Lehrer Bedencken. Giessen, 1608)

(536) Da war er grimmig bo̊ $\beta$ und zornig an zufehn. (DTA: Vergilius Maro, Publius: Eigentlicher Abriß Eines verständigen / tapfferen und frommen Fürsten / Von dem fürtrefflichsten Poeten Virgilius. Cölln (Spree), 1668)

(537) und damit warffer aus seinem Busen [...] die Gifft-aufgelauffene Schlangen / die ihm auf das Hertz / und in das zarte Gesicht / mit so grimmigen Wüten / sprungen / daß Polyphilus von dem grossen Schrecken erwachte. (DTA: Stockfleth, Maria Katharina: Die Kunst- und Tugend-gezierte Macarie, Erstdruck: 1669-1673)

(538) in so grimmigen hasz hatte des Artabanus liebe .. sich verwandlet (DWB: S.v.)

In (534) - (538) wiederholen sich die Inhaltszüge des Adjektivs in den Bezugswörtern Zorn, zornig, böse, Wüten und Hass und demonstrieren damit semantic repetition und daraus folgend semantic redundancy, welches die Verstärkung des Ausdrucks zu Folge hat und die intensivierende Funktion von grimmig hervorhebt. Aus den Kontexten ergibt sich außerdem, dass die Bezugswörter Extremwerte aufweisende Prädikate darstellen, welche in Verbindung mit einem ebenso „implicit superlative" grimmig intensiviert wirken.

DWB (s.v.) verweist darauf, dass die Herausbildung der Steigerung außerdem „neben anderen begriffen“ erfolgt, indem „der übergang der bedeutung 'wild, wüthend' in eine intensive 'erbittert, heftig, hartnäckig' fühlbar" (DWB, s.v.) stattfindet. Die Beleglage hat mehrere Textstellen gezeigt, die das mehrdeutige Auftreten von grimmig in Bezug auf verschiedene Konfliktsituationen demonstrieren, vgl.:

(539) DEmnach im Jahr 1571. der grimmige Tyrā̄ vnd Erbfeind Christlichen Namens Selymus Sultanus, Türckischer Kåyser Vorhabens gewesen/ die gantze Christenheit vnder sein Tyrannisch Joch zubringen / vnd derentwegen all sein Kriegsmacht zusamen beruffen / vnd mit solcher ein ůberaus starcke Armada auff das Mittellåndische Meer gesetzt [...] (FnhdC: Marcus Eschenloher, Augsburgischer Arzt, Augsburg 1678)

(540) Vnd hie gefchah ein groffe vnd grimmige Schlacht bey Siverßhufen / da Churfürft Moritz zwar obgefieget/vier taufend Reuter auff der Mahlftatt erlegt / vnnd fechtzig vier Fahnen davon getragen / aber ein to̊dliche Wund empfangen / die jhn nach dreyen Tagen / im zwey vnd dreiffigften Jahr feines Alters auffgeopffert. (DTA: Wartmann, Sigismund Friedrich: Germaniae Pertvrbatae et Restavratae sive Vnpartheyischer wolmeynender Theologo-Politicorum Discvrsvm Ander vnd dritter Theil. Frankfurt (Main), 1650)

(541) Doch wolte ihm Dorylaus nicht lange Zeit go̊nnen / fondern fetzete fo grimmig auff ihn hinein / daß wo die Römer ihre Glieder nicht fo feft 
gehalten diefe ohn zweiffel durch gebrochen / und ein groffes Blutbad angerichtet håtten. (DTA: Bucholtz, Andreas Heinrich: Des Christlich: Teutschen Königes Herkules und der Teutschen Königin Valiska Wunder-Geschicht. Bd. 2. Braunschweig, 1660)

(542) Dem Ansehn nach würde unter ihnen selbst ein grimmiger Streit entstanden ... (C.II: HK3/E93.00001 Wieland: Geschichte des Agathon, Erstdruck: $1766 / 67,2000$ [S. 387])

(543) Er hatte diesen Trumpf beim Disputieren älterer Leute gehört und brachte denselben wie ähnliche Gewandtheiten, die er sich angeeignet, gegen mich vor, so daß ich zuletzt immer geschlagen wurde; besonders sagte er zuletzt immer, ich verstehe eben die Sache noch nicht und wüßte nicht richtig zu denken, was mich dann gewaltig erboste, und wir gerieten manchmal in grimmigen Zank. (C.II: HK3/D35.00001 Keller: Der grüne Heinrich [Erste Fassung], Entstanden: zwischen 1846 und 1855, 2000 [S. 349])

Die Belege (539) - (543) demonstrieren mehrere Lesarten von grimmig. Aus den Kontexten geht hervor, dass das Lexem sich sowohl auf die Bedeutung des brutalen Verhaltens bzw. die Ausgangsbedeutung als auch auf die intensivierende Sinnrichtung bezieht. Die letzte Interpretation wird dadurch begünstigt, dass die Bezugswörter jeweils einen Extremwert aufweisenden Sachverhalt (z.B. Tyrann, Streit, Zank) sowie eine negative Konfliktsituation darstellen und damit eine Verbindung mit der Bedeutung des unerbittlichen, gnadenlosen Verhaltens sowie des bösen, wütenden, zornigen Zustands aufweisen. Daraus entsteht semantic repetition bzw. semantic redundancy, und grimmig wird als Intensivierer wahrgenommen.

DWB (s.v.) erwähnt ferner den Zusammenhang zwischen dem superlativischen Gebrauch und der Gradbedeutung in der Hinsicht, dass „der in jüngerer zeit stark zunehmende superlativische gebrauch wesentlich auf die rechnung dieser bedeutungsentwicklung zu setzen ist". Die Belege (544) - (547) exemplifizieren diese These, jedoch legen sie nahe, dass das Einsetzen der Gradbedeutung sich auch in früheren Verwendungen erkennen lässt:

(544) V̄̄ gesagt

$d z$ mā mit diser vnerlichē $v \bar{n} v ' f l u ̈ c h t \bar{e}$

tat die grÿmigesten $v \bar{n}$ bo̊stē feind nit v'leczen

sol Vnd da sôllichs in d' messanenser v'samlüg

erzelt ward / seind poten mit gabe

zů Biante geschickt worden. (FnhdC: Gualtherus Burlaeus, Vita, Augsburg 1490)

(545) dasz ein ungeschickter freund oft mehr schaden thut als der grimmigste feind (DWB, s.v.)

(546) nachdem wir den grimmigstn widerstand gethan (DWB: s.v. grimmig)

(547) so entsteht daher die grimmigste feindschaft der ... vernunft gegen den erlöser (DWB: s.v.)

Metaphorisierung bietet eine weitere Möglichkeit der Herausbildung der Gradbedeutung, vgl. den adjektivischen und adverbialen Gebrauch:

(548) Ein grawsame grimmige Hungersnoth / die zu vielen argen Rath und That gibet. (FWB: 1588, s.v.) 
(549) (...) darnach ftehen fie vor Mitternacht auff / damit fie deß andern Tags hinauff kommen / ehe fich die Sonne fencket / dann die Winde alsdann fo grimmig wehen / daß vnmüglich ift / hinúber zu kommen. (DTA: Gottfried, Johann Ludwig: Newe Welt Vnd Americanische Historien. Frankfurt (Main), 1631)

(550) wann schon ein grimmiger orcan zuweilen alle kunst (des steuermanns) besieget (DWB, s.v.)

In (548) - (549) werden die Begriffe Hungersnot und Winde personifiziert, i.e. mit einem zornigen oder grausamen Menschen verglichen, der den anderen einen „argen Rath und That gibet" oder unerwünschte Lebensbedingungen und Hindernisse schafft. Zugleich kann grimmig als ein Intensivierungsmittel betrachtet werden, indem die Intensität der Hungersnot sowie der Windstöße sich als Grund für die schlimmen Folgen ergibt, sodass die folgenden Umformulierungen gelten können:

(548') 'Die starke Hungersnot gibt zu vielen argen Rat und Tat'.

(549') 'Die Winde wehen so stark, dass es ungemütlich ist, hinüber zu kommen'.

(550') 'Der Orkan ist so stark, dass er zuweilen alle Kunst des Steuermanns besiegt'.

Die mehrdeutigen Belege (532) - (550) demonstrieren die zweite Stufe des Entwicklungsgangs der Gradbedeutung bzw. deren Einsetzen neben der Ausgangsbedeutung. Die Herausbildung der Intensivierung ist mit dem Auftreten von subjectivity verbunden, indem sie die Einstellung des Sprechers bzw. Schreibers zum Ausdruck bringt. Damit kommt Traugott I (oben 3.6.3.4) zur Geltung.

Die nächste Etappe der Bedeutungsentwicklung bezieht sich auf den Verlust des direkten Zusammenhangs mit der Ausgangsbedeutung, indem dessen Inhaltszüge in den Verwendungen nicht mehr zu erkennen sind. DWB führt an, dass „das intensitätsmoment“ „reiner“ in der Wendung grimmige Kälte hervortritt, vgl.:

(551) die hund pflegen zu wüten ... in ... schärfster grimmiger kält im winter (DWB: 1579, s.v.)

In (551) tritt die Ausgangsbedeutung zurück, indem grimmig die intensivierende Funktion übernimmt. Dies geschieht dadurch, dass der metaphorisch bedingte Gebrauch des Lexems geschwunden ist. So erhält grimmig in (552) auch eine übertragene Bedeutung, weil die Winde personifiziert werden, wie es in (548) - (550) der Fall ist. Darüber hinaus bleibt in diesem Gebrauch noch eine Spur der Ausgangsbedeutung, die in (551) jedoch nicht zu bemerken ist.

(552) So feynd doch die Wind fo grimmig kalt / vnnd regieren im Mayo / Junio / Julio vnnd Augufto dermaffen / daß kein einig Kraut oder Frucht dafelbft wachfen kann [...] (DTA: Gottfried, Johann Ludwig: Newe Welt Vnd Americanische Historien. Frankfurt (Main), 1631)

Die Beleglage in COSMAS bietet zahlreiche Verwendungen von grimmig als GradAdjektiv bzw. -Adverb und demonstriert damit die Expansion der Kollokationsfähigkeit des Lexems als Intensivierungsmittel. Jedoch muss hinzugefügt werden, dass grimmig auf dieser Stufe des Entwicklungsgangs ausschließlich in negativ 
konnotierten Kontexten auftritt. Darüber hinaus bleibt der Prozess der Desemantisierung unvollkommen, vgl. den adjektivischen und adverbialen Gebrauch mit entsprechenden Umformulierungen:

(553) ich setzte mich (...) hinter den letztern (Spieler), der schon grimmig im verluste war (...) (C.II: HK3/D81.00001 Wagner: Die Kindermörderin, Erstdruck: 1776, 2000 [S. 1483])

(553') 'sehr großem Verlust'

(554) Du hast's verdient, es geht dir grimmig schlecht. (C.II: HK3/B08.00006 Goethe: Faust. Eine Tragödie: Faust. Der Tragödie zweiter Teil, Entstanden: zwischen 1825 und 18312000 [S. 356])

(554') 'sehr schlecht'

(555) Es war grimmig kalt, obschon die Luft jetzt heiterer war... (C.II: HK4/S02.00001 Schopenhauer: Anna, Erstdruck: 1845, 2004 [S. 271])

(555') 'sehr kalt'

(556) (...) begab er sich in den Wilden Mann (...) und schritt stolz (...) hindurch in ein Zimmer, (...) und überließ sie ihren erstaunten Beratungen, über welchen sie sich das grimmigste Kopfweh anzutrinken genötigt waren. (C.II: HK3/D89.00001 Keller: Die Leute von Seldwyla: Zweiter Band, Entstanden: zwischen 1859 und 1873, 2000 [S. 336])

(556') 'stärkste Kopfweh'

(557) Realistisch betrachtet, stehen die Chancen, dass das gelingt, jedoch bei Null, da 2001 noch grimmiger gespart werden muss als heuer. (C.II> N00/MAI.23777 Salzburger Nachr., 25.05.2000)

(557') 'viel mehr, auf eine harte Weise'

Die Beleglage insgesamt zeigt, dass grimmig außerdem in Konstruktionen mit verschobenem Skopusbereich auftreten und Funktionsverbgefüge modifizieren kann. Dies wird ermöglicht, wenn ein Prädikat in einem FVG graduierbar ist oder das ganze FVG durch einen graduierbaren Begriff wiedergegeben werden kann, vgl. die Belege mit den entsprechenden Umformulierungen:

(558) Mit der Aerobatik geriet Höhlenradnetzspinne kranial jedoch grimmig unter Druck. (06.06.2014: http://sepp.xylou.de/a/EAA/3b8026d4/Zur_Abmilderung_dieser_html)

(558') 'geriet unter sehr großen Druck'

(559) Alles Eigenschaften die ich dem Pagé nicht zuschreiben würde, ich vermute der fördert sogar mit seiner Art Ungerechtigkeiten und Missstimmung und das ist sicher nicht leistungsfördernd, aber grimmig auf den Sack gehend. (28.08.2015: http://hockeytalk.info/viewtopic.php?f=2\&t=321\&start=195)

(559') 'sehr nervend'

Die intensivierende Funktion des Lexems kann in die quantifizierende übergehen, vgl.:

(560) Im angeschlossenen Café steht übrigens eine Jukebox mit Peter Handkes Lieblings-Songs. - Erbauung, die man angesichts der grimmigen Preise 
dieser Bar dringend benötigt. (06.06.2014: http://www.graz03.at/servlet/sls/Tornado/web/2003/content / 24CDF4F99E7C6009C1256D28003AF2E6)

(560') 'sehr hohen Preise'

Die Belege (551) - (559) und (560) demonstrieren den Gebrauch des Lexems in jeweils intensivierender und quantifizierender Funktion, die keinen direkten Kontakt mit der Ausgangsbedeutung des Lexems erkennen lässt. Die fortgeschrittene Bedeutungsentleerung lässt außerdem die Ausweitung des einstellungsbezogenen Ausdruckspotentials und damit den Übergang zu Traugott III (oben 3.6.3.4) feststellen.

Die bisher eingeführten Belege demonstrieren den Gebrauch von grimmig als Intensivierungsmittel in den Verwendungen mit negativ konnotierten Kollokationspartnern. Um die volle Desemantisierung des Wortes nachzuvollziehen, müsste die negative Bedeutungskomponente verloren gehen und dadurch das Auftreten des Lexems mit positiv konnotierten Bezugswörtern ermöglicht werden, vgl. den aus DWB übernommenen Beleg und die entsprechende Paraphrasierung:

(561) Karg. Wenn der freygebige Bruder nur die Hälfte seiner Diamanten zum Brautschmuck bestimmt, so ist er im Stande die gemeinste Bauerdirne zur Fürstin umzuzaubern.

Graf. Der Mensch ist grimmig reich, so sagt man; desto besser! (DTA: Kotzebue, August von: Bruder Moritz, der Sonderling, oder die Colonie für die PelewInseln. Lustspiel in drey Aufzügen, Erstdruck: 1791, S. 74)

(561') 'sehr reich'

Die einzige Verwendung (561) von grimmig in einem Kontext mit positiver Konnotation lässt annehmen, dass das Lexem den Zusammenhang mit der Ausgangsbedeutung noch nicht komplett verloren hat. Darüber hinaus bleibt der Prozess der Desemantisierung des Lexems bis heute nicht vollendet.

\subsubsection{Kurzfassung der Entwicklung bei grimmig}

1. Ausgangsbedeutung: 'wütend, zornig' sowie 'grausam und unbarmherzig'.

6. Reine Gradbedeutung: nur ein Beleg aus dem 18. Jhd. mit positiv konnotiertem Kookkurrenzpartner; sonst immer negativ Konnotiertes; die übrigen Entwicklungsschritte wie oben in Kap. 3.6.3.9.3.

\subsubsection{Mörderisch}

\subsubsection{Lexikographische Angaben}

Pfeifer (s.v.) führt das Auftreten des Lexems auf das 15. Jh. zurück. Laut DWB (s.v.) stellt mörderisch „eine verhältnismäszig späte an stelle von mordisch [...] erscheinende bildung" dar. Außerdem wird in Pfeifer (s.v.) darauf hingewiesen, dass das Lexem „seit dem 19. Jh. auch nur verstärkend im Sinne von 'sehr, unglaublich“ gebraucht wird.

1. mörderisch in FWB 
keine Angaben 59

2. mörderisch in DWB (s.v.)

„mörderart habend“

3. mörderisch in DUDEN (2012, s.v.)

3.1 „in grausamer, verbrecherischer Weise Leben vernichtend, tötend, mordend“

3.2 „(umgangssprachlich)“

3.2a „in hohem Maße unangenehm; abscheulich, furchtbar“

$3.2 \mathrm{~b}$ „sehr stark; heftig, mächtig, gewaltig“

3.2c „<intensivierend bei Adjektiv> sehr, überaus, äußerst“

\subsubsection{Entwicklung der Gradbedeutung bei mörderisch}

Pfeifer (s.v.) verweist auf die intensivierende Rolle des Wortes seit dem 19. Jh., während DWB keine Informationen hierzu bietet. Die Korpusanalyse sowie die Analyse der in DWB angeführten Belege bestätigen Pfeifers Annahme und lassen es zu, das Einsetzen der Gradbedeutung in das 19. Jh. zu datieren. Als Ausgangsbedeutung, die als Basis für das Auftreten der Intensivierung dient, gilt die mit Mord verbundene Sinnrichtung, die sich auf Personen und scherzhaft auch Tiere, außerdem die Art, Verfahren und Hilfsmittel eines Mörders und „sein[] thun“ beziehen, so DWB (s.v.), vgl.:

(562) der jachzornige mörderische Achilles wird mir verhaszter, als der tückische knurrende Thersites. (DWB: s.v.)

(563) also wird es allen den studenten ergehen, welche .. die herrliche ingenia, sinne und gedächtnusz also mörderischer weise verderben. (DWB: s.v.)

(564) der ganze weisze haufen sei Conjos mörderischem dolch geweiht. (DWB: s.v.)

Die Korpusanalyse hat ergeben, dass die nächste Stufe der Entwicklung von Intensivierung in mörderisch mit pragmatic enrichment bzw. strengthening der Ausgangsbedeutung verbunden ist. Die Intensivierung findet sich als eine in der Form einer Inferenz abgeleitete Nebenbedeutung, wobei die Ausgangsbedeutung im Vordergrund steht, vgl.:

(565) Der Prinz von Homburg war [...] Auf Wrangel in die Ebne vorgerückt; Zwei Linien hatt er, mit der Reuterei, Durchbrochen schon, und auf der Flucht vernichtet, Als er auf eine Feldredoute stieß. Hier schlug so mörderischer Eisenregen Entgegen ihm, daß seine Reuterschar, Wie eine Saat, sich knickend niederlegte [...] (C.II: HK3/A91.00003 Kleist: Prinz Friedrich von Homburg, Entstanden: um 1809/10, 2000 [S. 381])

(566) Während dieses mörderischen Gefechtes, wo die Gefahr der Vertheidigung in gar keinem Vergleiche mit der des Angriffs stand, waren zwei Thore gesprengt worden. (C.II: HK4/U02.00001 Unger: Albert und Albertine, Erstdruck: 1804, 2004 [S. 233])

\footnotetext{
${ }^{59}$ Im Computersystem von FWB finden sich 2 Belege, die den auf Mord bezogenen Gebrauch von mörderisch aufweisen.
} 
(567) Unbesorgt schritten sie auf die Stadt zu, als auf einmal ein mörderisches Feuer ihre Reihen lichtete. (C.II: KHM/011.00043 Das Pfennig=Magazin für Belehrung und Unterhaltung, 1843.09.09)

(568) Das ist ein grausam, mörd'risch Ungewitter; Der himmel droht in Feuerbächen sich Herabzugießen, und am hellen Tag Ist's Nacht, daß man die Sterne könnte sehn. (DWB, s.v.)

Die Belege (565) - (568) erlauben eine mehrdeutige Interpretation. Der Gebrauch des Adjektivs bezieht sich zunächst auf die Ausgangsbedeutung und bezeichnet die Sachverhalte Eisenregen, Gefecht, Feuer und Unwetter als tödlich. Zugleich lassen die Kontexte über die Intensität der Situationen reflektieren, indem die Stärke ihrer Ausprägung für die tödliche Wirkung sorgt. So handelt es sich in (565) um einen heftigen Angriff mit einer großen Anzahl von Pfeilen, der durch den Begriff Eisenregen beschrieben wird. Durch die Verwendung von mörderisch wird zum Ausdruck gebracht, dass der Angriff für den Gegner in sehr hohem Maße bzw. für sehr viele Menschen tödlich ist. In (566) beschreibt mörderisch ein tödliches und damit in hohem Maße erbittertes, brutales Gefecht. In (567) wird auf die große Stärke und das Ausmaß des Feuers hingewiesen, die Tod zur Folge hat. In (568) handelt es sich um sehr heftiges Gewitter, dessen Intensität durch „Feuerbäche" betont wird. Darüber hinaus stellen die Bezugswörter jeweils Situationen dar, die sich mit Extremwerten beschreiben lassen. Durch die Verbindung mit mörderisch wird deren Intensität hervorgehoben bzw. die intensivierende Bedeutung des Adjektivs markantgemacht.

Der metaphorische Gebrauch von mörderisch stellt eine weitere Stufe der Entwicklung der Gradbedeutung dar, vgl.:

(569) Die Qual, die meine Seele fühlet, Die mörderisch im Herzen wühlet, Verbannet jede andre Pein [...] (C.II: HK3/B29.00001 Moritz: Anton Reiser, Erstdruck: 1785-1790, 2000 [S. 242])

In (569) handelt es sich zunächst um die personifizierte, Gefühle ausdrückende Qual, die mit einem Übel wollenen Menschen verglichen wird und eine negative evtl. verderbliche Wirkung auf die angesprochenen Personen hat. Der adjektivische metaphorische Gebrauch in den Kollokationen verliert den direkten Zusammenhang mit dem Mord, gewinnt jedoch zugleich eine auf Intensität bezogene Interpretation, vgl.:

(569') 'Die Qual ist so stark, dass sie wie ein Mörder auf mich wirkt'.

Die Erscheinung der Intensivierung wird außerdem dadurch begünstigt, dass beide Kollokationsparter Extremwerte aufweisende Prädikate darstellen, sodass deren Verbindung verstärkt wirkt.

Das Auftreten der Gradbedeutung wird von dem Einsetzen von subjectivity begleitet, sodass der Gebrauch eine wertende Komponente erzielt und die Einstellung des Sprechers bzw. Schreibers, der einer Erscheinung einen bestimmten Ausprägungsgrad aus seiner subjektiven Perspektive zuschreibt, zum Ausdruck bringt. Die Verwendungen demonstrieren damit den mehrdeutigen Gebrauch des Lexems 
auf der propositionalen und expressiven Ebene und signalisieren das Einsetzen von Traugott I (oben 3.6.3.4).

In der nächsten Phase der Entwicklung erscheint mörderisch als Intensivierer, ohne einen direkten Zusammenhang mit der Ausgangsbedeutung erkennen zu lassen. Der Bezug auf Mord und Tod geht verloren, obgleich die Verwendungen negative Konnotationen hervorrufen und dadurch den unvollendeten Prozess der Desemantisierung veranschaulichen, vgl. die Belege mit den entsprechenden Umformulierungen mit adjektivischem (570) - (571) und adverbialem (572) - (575) Gebrauch:

(570) Das ohrenzerreißende Klappern sei von zwei sechspfündigen Vorhängeschlössern entstanden, welche die Pakete in dem Sitzkasten des Postwagens ängstlicher schützten, als nötig war. Dieses mörderische Geklapper sei ihr so lästig gefallen, daß sie auf der nächsten Station [...] vermittelst eines Fadens die Schlösser geschickt befestigt habe, damit sie sich nicht mehr rühren können. (C.II: HK3/B37.00001 Börne: Aufsätze und Erzählungen, 1828-1832, 2000 [S. 652])

(570') 'sehr starkes Geklapper'

(571) Sie wissen, daß der neue wunderbare Body ein paar Kilo wegmogelt und Sie durch die hochhackigen Schuhe, die Ihnen schon seit einer Stunde mörderisch weh tun, größer wirken, als Sie sind. (C.II: 098/MAI.49104 Neue Kronen-Ztg., 17.05.1998, S. 29)

(571') 'sehr weh tun'

(572) „Die Mannschaft war mörderisch nervös zu Beginn, aber wie die Burschen den fünften Satz gespielt haben, das spricht für die Mannschaft", sagte Manager Peter Kleinmann. (C.II: N00/FEB.07304 Salzburger Nachr., 18.02.2000)

(572') 'sehr nervös'

(573) Der mörderische Preisdruck in der Branche könnte leichter werden und sich letztlich der Markt von selbst regulieren. (C.II: N00/DEZ.59396 Salzburger Nachr., 22.12.2000)

(573') 'sehr starker Preisdruck'

(574) Sie ist Anna Vadersen, „die Macht aus dem Off", erlöst „die da oben auf der Bühne" mit einem Stichwort oder lässt die Pause mörderisch lang werden. (C.II: BRZ10/DEZ.04245 Braunschw. Z., 09.12.2010)

(574') 'sehr lang'

(575) „Das waren die spannendsten, wunderbarsten Jahre unseres Lebens. Das Ganze war aber eben auch mörderisch anstrengend." (C.II: HMP11/JUN.00517 MOPO, 08.06.2011, S. 38)

(575') 'sehr anstrengend'

Die Korpusanalyse hat ergeben, dass mörderisch außerdem in Konstruktionen mit verschobenen Skopus auftreten und Funktionsverbgefüge modifizieren kann, vgl.:

(576) Jeder andere würde mörderisch unter Druck geraten. Tomba aber baut der Wirbel auf. (C.II: 097/FEB.15142 Neue Kronen-Ztg., 12.02.1997, S. 58)

(576') 'sehr großen Druck' 
(577) Heri Weber steht unter mörderischem Druck. (C.II: 097/AUG.81581 Neue Kronen-Ztg., 08.08.1997, S. 51)

(577') 'ist sehr bedrängt'

(578) Erschütternd fand ich dabei, daß mir diese Routine schon nach drei Tagen mörderisch auf die Nerven ging. (03.03.2014: http://books.google.de / books?id=oCM339BLMygC\&pg=PA246\&dq=\%22m\%C3\%B6rderisch+auf+die+nerven\%22\&hl=de\&sa=X\&ei=IbAUU8zpC4jYtAbv6oHwCw\&ved=0CDIQ6AEwAA\#v=onepage \&q=\%22m\%C3\%B6rderisch\%20auf\%20die\%20nerven $\% 22$ $\& \mathrm{f}=$ false)

(578') 'sehr nervte'

(579) Es war jetzt fast halb Neun und langsam ging mir dieser Tag mörderisch auf den Geist. (GB, 28.08.2015: https://books.google.de/books?id=tODDBwAAQBAJ\&pg=PT98\&dq= $\% 22 \mathrm{~m} \% \mathrm{C} 3 \%$ B6rderisch + auf + den + geist $\% 22 \&$ hl $=$ de $\&-$ $\mathrm{sa}=X \& v e d=0 \mathrm{CCAQ6AEwAGoVChMIudz07oLMxwIVyVkaCh0fKAPx \# v=onepage-}$ $\& q=\% 22 \mathrm{~m} \% \mathrm{C} 3 \%$ B6rderisch $\% 20$ auf $\% 20 \mathrm{den} \% 20$ geist $\% 22 \& \mathrm{f}=$ false)

(579') 'nervte sehr'

Das Gradadverb in (576) - (579) demonstriert seine intensivierende Rolle, ohne einen direkten semantischen Bezug zur Ausgangsbedeutung aufzuweisen.

Die intensivierende Funktion des Lexems kann in die quantifizierende übergehen und dadurch auf eine große Menge des durch ein Bezugswort ausgedrückten Sachverhalts hinweisen, vgl. die Belege mit den entsprechenden Paraphrasen:

(580) Dadurch ist das gesamte obere Geschoss des alten Hauses abgebrannt. Die Familie Hope verlangte einen Schadenersatz in Höhe von 60000 Pfund. Die Agentur Lockwood \& Co. weiß sich aber nicht zu helfen, da die mörderische Summe bereits in einem Monat bezahlt werden muss. (05.03.2014: http://www.buch-lesezeichen.de/charlotte/)

(580') 'sehr große Summe'

(581) Die Genies, die täglich für alle drei Dienste schreiben, verdienen natürlich ein mörderisches Geld, aber das kann nicht jeder, und es ist auch ziemlich ramponierend. (05.03.2014: http://books.google.de/books?id=1iheAAAAIAAJ\&$\mathrm{q}=\% 22 \mathrm{~m} \% \mathrm{C} 3 \%$ B6rderisches +geld $\% 22 \& \mathrm{dq}=\% 22 \mathrm{~m} \% \mathrm{C} 3 \%$ B6rderisches + geld $\% 2-$

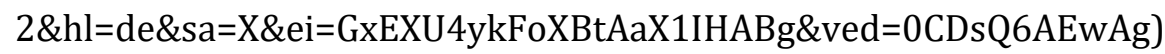

(581') 'sehr viel Geld'

(582) Wenn man so ein Haus erhalten will, geht schon ein großer Teil der Kohle drauf. Es stehen Dachreparaturen an. Das sind mörderische Investitionen. (05.03.2014: http://books.google.de/books?id=m7s8AQAAIAAJ\&q=\%22m\%C3\%B6rderische+investitionen $\% 22 \& \mathrm{dq}=\% 22 \mathrm{~m} \% \mathrm{C} 3 \%$ B6rderische+investitionen\%22\&hl=de\&sa=X\&ei=bhcXU9CyAc_bsgawu4HACw\&ved=0CDEQ6AEwAA)

(582') 'sehr große Investitionen'

Die Herausbildung der intensivierenden (570) - (579) und quantifizierenden (580) - (582) Bedeutung auf dieser Stufe des Entwicklungsgangs ist zugleich mit der Erweiterung des einstellungsbezogenen Ausdruckspotentials des Lexems verbunden. Der Gebrauch gewinnt an subjectivity, verweist ausschließlich auf „internal described situation" (Traugott 1989: 35) und signalisiert damit den Übergang zu Traugott III (oben 3.6.3.4). 
Anschließend findet sich das Lexem in Verbindung mit positiv konnotierten Kollokationspartnern. Dieser Entwicklungsschritt demonstriert den kompletten Verlust des Zusammenhangs zur Ausgangsbedeutung. Das Wort fungiert damit als bedeutungsentleert, vgl. den adjektivischen und adverbialen Gebrauch:

(583) Zum Wandertag durch Weinberge und Wälder lädt heute die „Freizeit" ein. Außerdem berichtet sie über mörderischen Spaß und ein $M u-$ seumsfest. (C.II: M00/SEP.59418 Mannh. Morgen, 28.09.2000)

(583') 'sehr großen Spaß'

(584) Monatelang bereitete sich der 21jährige auf seinen Karrierehöhepunkt als „Treppenflitzer" vor: „Ich trainierte auch in U-Bahn-Stationen. Ich lief Rolltreppen rauf und runter, tankte mörderisch viel Kraft", schildert er seine kuriose Trainingsmethode. (C.II: 096/FEB.18003 Neue Kronen-Ztg., 21.02.1996, S. 45)

(584') 'sehr viel Kraft'

(585) Das nennt man das Auslassen des Specks; der Speck wird ausgelassen; dabei wird er flüssig. So lange auslassen, bis Grammelstückerl in brauner Farbe entstehen. Da riecht die Küche schon mörderisch gut. (C.II: DIV/GRE.00001 Gruber: Einfach essen!, 2010 [S. 12])

(585') 'sehr gut'

\subsubsection{Kurzfassung der Entwicklung bei mörderisch}

1. Ausgangsbedeutung: 'tötend, mordend';

6. Reine Gradbedeutung: nur ein Beleg aus dem 18. Jhd. mit positiv konnotiertem Kookkurrenzpartner; sonst immer negativ Konnotiertes; die übrigen Entwicklungsschritte wie oben in Kap. 3.6.3.9.3.

\subsubsection{Niederträchtig}

\subsubsection{Lexikographische Angaben}

Laut Pfeifer (s.v.) tritt das Lexem im Spätmittelhochdeutschen in der Form nidertrehtic auf und stellt die Ableitung von „mhd. sich tragen 'sich benehmen, betragen'“ dar.

1. niederträchtig in BMZ (s.v. nidertrehtec), Lexer (s.v. nider-trehtic)

„von oben hinab angesehen, gering geschätzt“ (Lexer, s.v. nidertrehtec); „,nach unten strebend, niedrig" (BMZ, s.v. nider-trehtic)

2. niederträchtig in FWB (s.v. nieder-trächtig) 60

„niedrig“, „flach“, „,von geringer Höhe“, „nicht hoch aufragend“, „klein“

3. niederträchtig in DWB (s.v.)

3.1 ,in sinnlicher bedeutung, die manchmal schon an die übertragene streift“

3.1.1 "sich niedrig tragend, gesenkt, geneigt"

3.1 .2 "niedrig, tief liegend"

3.1.3 „von geringer höhe, niedrig, kurz, klein, manchmal mit dem nebenbegriffe des unscheinbaren, ärmlichen“

\footnotetext{
${ }^{60}$ Die Angaben aus den in der FWB Redaktionsstelle verfügbaren Belegkästen.
} 
3.2 „in übertragener bedeutung“

3.2.1 "geringen standes, niedriger abkunft"

3.2.2 „dem äuszern ansehen nach unedel, würdelos, scheuszlich, widerwärtig"

3.2.3 „dem inhalte und der wirkung nach höchst unangenehm und verdrieszlich, peinlich und widrig"

3.2.4 "dem inneren ansehen, dem werte, der macht und eigenschaft nach“ 3.2.4a "gering, schwach“

3.2.4b „gering geschätzt, verworfen, verächtlich“

3.2.5 „in bezug auf das innere leben, denken und empfinden, sowie auf die äuszerung desselben durch wort und handlung"

3.2.5a „niedergehenden, auf niedriges, nicht auf hohes gerichteten sinnes"

3.2.5b „ohne hochmut und stolz, gegensatz zu hochträchtig $1^{\text {“ }}$ $3.2 .5 \mathrm{~b} \alpha$,herablassend, freundlich und leutselig gegen personen niederen standes"

$3.2 .5 \mathrm{~b} \beta$, unterwürfig, demütig, bescheiden, submissus“

$3.2 .5 \mathrm{~b} \gamma$ „kleinmütig, verzagt"

3.2.5c "gegensatz zu hoch, erhaben, edel, fein“

$3.2 .5 \mathrm{c} \alpha$, dem ausdrucke und der handlungsweise nach niedrig, verwerflich, unedel, gemein"

3.2.5c $\beta$,besonders in sittlichem verstande, höchst gemein und verworfen eine schändlich niedrige gesinnung habend und zeigend, infam"

3.2.6 „als adverb dient es manchmal nur zur bezeichnung eines hohen grades"

4. niederträchtig in DUDEN (2012, s.v.)

4.1 "(gehoben)“

4.1a „,in niedriger, gemeiner Weise danach trachtend, anderen Übles,

Schaden zuzufügen"

$4.1 \mathrm{~b}$ „von niederträchtiger (1 a) Gesinnung zeugend, ihr entsprechend“

4.2 „(umgangssprachlich)“

$4.2 \mathrm{a}$ „unangenehm groß, stark"

$4.2 \mathrm{~b}$ „>intensivierend bei Adjektiven und Verben> sehr, überaus“

\subsubsection{Entwicklung der Gradbedeutung bei niederträchtig}

Die Korpusanalyse hat ergeben, dass das Einsetzen der Gradbedeutung bei niederträchtig im Nhd. erfolgt ist. Als Basis dafür diente die in 3.2.5 angegebene Sinnrichtung, die sich auf die niedrige, verwerfliche und gemeine Handlungsweise bezieht, vgl.:

(586) Ein niederträchtiger gemeiner Geist wird nur von ihr gebeugt. (C.II: HK4/Z04.00001 Ziegler: Vermischete Schriften in gebundener und ungebundener Rede, Erstdruck: 1739, 2004 [S. 301])

(587) Der Branntwein und eine niederträchtige Seele tobten aus ihm, und er lärmte und schrie, bis die Wache hereintrat. (C.II: HK3/F35.00001 Gellert: Leben der schwedischen Gräfin von G**, Erstdruck: 1747/48, 2000 [S. 68]) 
(588) Ja [...] alles dieses würde eine sehr kleine Schande für Sie sein, wenn ich nicht der Welt auch zugleich entdecken müßte, daß Sie eine sehr niederträchtige Art zu denken haben, und daß Sie, mit einem Worte, ein Verleumder sind. (C.II: HK3/E23.00001 Lessing: Ein Vade mecum für den Hrn. Sam Gotthl. Lange. Pastor in Laublingen, Erstdruck: 1754, 2000 [S. 583])

Das Auftreten der Intensivierungsbedeutung lässt sich in solchen Kontexten feststellen, die Mehrdeutigkeiten im adjektivischen bzw. adverbialen Gebrauch zulassen. Diese Mehrdeutigkeit ergibt sich durch pragmatic enrichment bzw. strengthening der Ausgangsbedeutung, wobei die Gradbedeutung als Nebenbedeutung neben der die primäre Rolle spielenden Ausgangsbedeutung erscheint, vgl.:

(589) Meinen Runzeln? [...] Ich bin gewiß die erste Braut, der man so eine niederträchtige Grobheit sagt! (C.II: HK3/E05.00001 Lessing: Die alte Jungfer, Entstanden: 1748, 2000 [S. 751])

(590) Mich empört die niederträchtige Unverschämtheit der Fürstenschmeichler, welche die Völker als Tiger, die Fürsten als Lämmer darstellen. (C:II: HK3/C78.00001 Börne: Briefe aus Paris, 1832, 2000 [S. 29])

Die Belege (589) - (590) demonstrieren die mehrdeutige Verwendung des Adjektivs. Zunächst bezieht sich niederträchtig auf das gemeine, in niedriger Weise an den Tag gelegte Verhalten. Zugleich jedoch fungiert das Lexem als Intensivierer. Dies erfolgt durch semantic repetition, indem beide Kookkurrenzpartner durch eine teilweise Übereinstimmung der Bedeutungskomponenten „quite closely match“ (Bolinger 1972: 246). Die Bezugswörter verweisen auf negative Sachverhalte, die eine niedrige moralische Gesinnung bezeugen. Die Verwendung von niederträchtig mit den besprochenen Bezugswörtern hat semantic redundancy zu Folge und verstärkt die unmoralische Komponente der Situation, sodass dem Adjektiv eine intensivierende Rolle zuzuschreiben ist.

Das Auftreten der intensivierenden Bedeutungskomponente in niederträchtig in der besprochenen Stufe des Entwicklungsgangs geht mit dem Einsetzen von subjectivity einher, sodass das Lexem die Einstellung des Schreibers bzw. Sprechers zum Ausdruck bringt. Damit lässt sich Traugott I (oben 3.6.3.4) feststellen.

In der nächsten Etappe der Bedeutungsentwicklung verliert die Gradbedeutung den direkten Bezug zur Ausgangsbedeutung, sodass diese in den Verwendungen nicht mehr zu erkennen ist. Das Lexem wird ausschließlich als Intensivierungsmittel gebraucht. Die Belege (591) - (595) sollen den adjektivischen und adverbialen Gebrauch von niederträchtig veranschaulichen, vgl. auch die entsprechenden Umformulierungen:

(591) Ich warf mich trotzdem, daß mein linkes Bein mir ganz niederträchtig weh that, ihr zu Füßen ... (GB; 02.04.2014, erschienen 1844: http://books.google.de $/$ books?id=5U1JAAAAcAAJ\&pg=PA253\&dq=\%22niedertr\%C3\%A4chtig+weh\%22\&hl=de\&sa=X\&ei=mvM7U7XwHsKJtAblo4DYBw\&ved=0CDkQ6AEwAQ\#v=onepage\&q=\%22niedertr\%C3\%A4chtig\%20weh\%22\&f=false)

(591') 'sehr weh tat'

(592) Nun, dann habe ich mir hier diese bescheidene Zeigerin der Zeit um niederträchtig geringes Geld gekauft [...] (DWDS: Vischer, Friedrich Theodor von: Auch Einer. Eine Reisebekanntschaft. Bd. 1. Stuttgart u. a., 1879) 
(592') 'sehr geringes'

(593) Heute Abend war es niederträchtig kalt. (GB, 02.04.2014, erschienen 1901: http://books.google.de/books?id=Jmnh-vTn20sC\&pg=PT158\&dq=\%22niedertr\%C3\%A4chtig+kalt\%22\&hl=de\&a=X\&ei=V-87U9CxLIiNtAbty4GIDQ\&ved=0CFgQ6AEwBg\#v=onepage\&q=\%22niedertr\%C3\%A4chtig\%20kalt\%22\&f=false)

(593') 'sehr kalt'

(594) Das Bijou ist infam ausgestattet: es hat einen niederträchtig schlechten Einband und ebensolche Zeichnungen; das wollen wir aber gar nicht. (DWDS: Die Weltbühne 21.02.1928, 21.02.1928)

(594') 'sehr schlechten'

Aus den Belegen (591) - (694) geht hervor, dass niederträchtig ausschließlich in der intensivierenden Rolle gebraucht wird, ohne auf das verwerfliche menschliche Verhalten hinzuweisen. Zugleich demonstriert der Gebrauch den Zuwachs an subjectivity und den Übergang zu Traugott III (oben 3.6.3.4), indem das Lexem sein in vollem Maße ausgeprägtes einstellungsbezogenes Ausdruckspotential demonstriert.

Die Korpusanalyse hat keine Belege ergeben, die den Gebrauch des Lexems in Verbindungen mit Funktionsverbgefügen als deren Modifikator zeigen. Außerdem lässt sich aus der Beleglage schließen, dass die Gradbedeutung von niederträchtig die letzte Stufe des Entwicklungsgangs, die die endgültige Desemantisierung bzw. den vollkommenen Verlust des Zusammenhangs mit der Ausgangsbedeutung und demzufolge das Kollokieren mit positiv konnotierten Bezugswörtern darstellt, nicht erreicht hat.

\subsubsection{Kurzfassung der Entwicklung bei niederträchtig}

1. Ausgangsbedeutung: 'niedrig, verwerflich, gemein';

4./5./6. Die Korpusanalyse ergibt keine Verwendung in quantifizierender Funktion, nicht in Funktionsverbgefügen und nicht mit positiv konnotierten Kookkurrenzpartnern;

die übrigen Entwicklungsschritte wie oben in Kap. 3.6.3.9.3.

\subsubsection{Schändlich}

\subsubsection{Lexikographische Angaben}

Laut Pfeifer (s.v.) ist das Lexem im 8. Jh. in der Form scantlīh mit dem Sinn 'mit Schande behaftet, unehrenhaft, abscheulich' in Erscheinung getreten.

1. schändlich in AWB [noch nicht erschienen], Köbler s.v. skantlīh [...], scantlīh, „schändlich, übel, schimpflich, schmachvoll, verwerflich“"

2. schändlich in Lexer (s.v. schant-lich, schent-lich)

„schämenswert, schändend, entehrend, schmachvoll, turpis“

3. schändlich in FWB

„schämenswert“; „,entehrend“; „,unehrenhaft"61

${ }^{61}$ Die Angaben aus den Belegzetteln von FWBD. 
4. schändlich in DWB (s.v.)

„mit schande behaftet, turpis, foedus, spurcus, ignominiosus, probrosus, nefarius, sceleratus, propudiosus, teter, inhonestus“"

4.1 „der sinnlichen bedeutung von schande nahestehend, 'häszlich, entstellend oder entstellt',

4.2 „mit schande, unehre verbunden, was schande bringt“

$4.2 \mathrm{a}$ „schändliche, unehrenhafte, entehrende, verbrecherische, lasterhafte handlungen"

$4.2 \mathrm{~b}$,in andern verbindungen, z. b. ein schändlicher tod, und zwar in doppeltem sinne, ein schimpflicher tod, wie der von henkershand [...]; oder ein schmählicher, kläglicher, elender tod [...]; abgeschwächt zum bloszen ausdrucke des unwillens"

$4.2 \mathrm{c}$ „auch von gegenständen, die mit einer schande, der erinnerung an eine schandthat $u$. a. behaftet sind"

4.3 „,von personen, unehrenhaft, lasterhaft, abscheulich“

4.4 ,in eingeschränkterer bedeutung 'unzüchtig'“

4.5 "für das adv. haben die ältern dialekte eine besondere form: ahd. scantlîhho turpiter GRAFF 6, 521, mhd. schant-, schentlîche LEXER handwb. 2, 657. daneben wird im mhd. und mnd. die form des dat. plur. als solches verwandt und so noch im ältern nhd.: schentliken turpiter SCHILLER-LÜBBEN 4, 73a, schandtlich, lasterlich, schandtlichen, nefarie, foede, flagitiose, indigne; schandtlichen, sceleste, foede, impure, illiberaliter MAALER 347b. es hat eine doppelte verwendung"

$4.5 \mathrm{a}$ „um eine handlung oder eigenschaft näher zu bestimmen, 'auf eine schändliche weise"

$4.5 \mathrm{~b}$ „um eine handlung an sich zu charakterisieren, 'schändlicherweise'“

4.6 ,in gewöhnlicher und mundartlicher rede oft abgeschwächt“

$4.6 a$ „erbärmlich, schlecht"

$4.6 \mathrm{~b}$ „viel, sehr, als verstärkung“

5. schändlich in DUDEN (2012, s.v. schändlich)

5.1 „Empörung hervorrufend; niederträchtig, gemein“

5.2 "(umgangssprachlich)“

$5.2 \mathrm{a}$ „unerhört; sehr, überaus schlecht"

$5.2 \mathrm{~b}$ ", $<$ intensivierend bei Adjektiv und Verben> sehr, überaus, äußerst"

\subsubsection{Entwicklung der Gradbedeutung bei schändlich}

Die Korpusanalyse legte nahe, dass das Einsetzen der Intensivierung bei schändlich im Fnhd. erfolgte. Als Ausgangsbedeutung, die als Quelle für die Entwicklung der Gradbedeutung dient, gilt die mit dem Begriff der Schande verbundene Sinnrichtung, die sich auf „unehrenhafte, entehrende, verbrecherische, lasterhafte handlungen" (DWB, s.v.) bezieht, vgl.:

(595) liegen (lügen) ist dem menschen ein schendlich ding, und er kan nimer mehr zu ehren komen. (DWB, s.v.)

(596) Die Fleischliche Liebe vnnd freude ist schendlich / Lasterhafftig / Vnehrlich. (DTA: Arndt, Johann: Vom wahren Christenthumb. Bd. 4. Magdeburg, 1610) 
(597) Letzlich henget er daran / Er könnte jhm gar nicht einbilden noch glauben / daß solche Menschen Christen Leut seyen / die sich keines Lasters noch Schandt schämeten / vnnd widerzugesagten Eydt vnd Glauben also schändlich handelten. (DTA: Gottfried, Johann Ludwig: Newe Welt Vnd Americanische Historien. Frankfurt (Main), 1631)

Die Intensivierung tritt in mehrdeutigen Kontexten dadurch in Erscheinung, dass sie sich als Nebenbegriff aus dem Kontext ableiten lässt und dadurch pragmatic enrichment bzw. strengthening der Ausgangsbedeutung sowie die Doppeldeutigkeit des Gebrauchs zu Folge hat, vgl.:

(598) WAs für ein groffer vnd fchendtlicher Mißbrauch des heiligen Evangelij in diefer letzten Welt fey / Chriftlicher lieber Lefer / bezeuget gnugfam / das Gottlofe vnbußfertige Leben / derer / die fich Chrifti vnd feines Worts / mit vollẽ Munde rühmen / vnd doch ein gantz vnchriftlich Leben führen / gleich als wenn fie nicht im Chriftenthumb / fondern im Heydenthumb lebeten. (DTA: Arndt, Johann: Von wahrem Christenthumb. Bd. 1. Magdeburg, 1610)

(599) Es feind aber folche jre Zauberer / welche fie Carabia nennen / gemeiniglich lofe vnd bofe Leuthe / vnd fchen / wie fie den armen Leuthen / einen blawen Dunft / für die Augen machen. Stellen fich als wann fieden Todten das Leben wider können geben / vnd verführen alfo das arme Volck: Sprechen auch zum offtern fie follen in diefem oder jenem Jahr kein Arbeit nicht thun / dann das Erdtreich werde jhnen ohne jhre Mủhe vnd Arbeit genug hervorbringen vnd zu effen geben / dann es werden auch die Pflüge wol ohn jhre Ackerleuthe arbeiten vnd pflügen ko̊nnen. Aber es werden die arme Leuthe alfo von jhnen fchåndlich betrogen / daß fie endlich darůber Hungers fterben müffen. (DTA: Gottfried, Johann Ludwig: Newe Welt Vnd Americanische Historien. Frankfurt (Main), 1631)

(600) Vor weniger Zeit fetzete ich ihm einen Lehrmeifter vor / welchem ich das Leben fchenkete / da ich ihn auf offentlichem Straffen Raube ertappete / vermachte ihm daneben eine ehrliche Jahrsbeftallung / und ließ ihn bey meinen vornehmften Hofeleuten / ja zuzeiten / wann ich allein wahr / über meinem Tifche Speife nehmen / ihn durch folche Gnade anzulocken / daß er bey meinem Sohn Tråu und Fleiß anwenden folte; welches er aber mit folchem fchåndlichen Undank erfetzet hat / daß er anfangs mein liebes Kind mir zurauben / nachgehends gar zu ermorden fich unterwinden dürffen / deffen ich / andern zum Beyfpiel / ihm abfcheuhliche Straffe erteilen müffen. (DTA: Bucholtz, Andreas Heinrich: Des Christlichen Teutschen Groß-Fürsten Herkules Und der Böhmischen Königlichen Fräulein Valjska Wunder-Geschichte. Bd. 1. Braunschweig, 1659)

(601) Ich weiß nit / antwortete er / ob ihr des Lebens forthin würdig seyd / nach dem ihr euren adelichen Ritterstand so schändlich beschmitzet / und euch auff Rauben und Morden begeben habt; ich bin auch ein Ritter / und ohn unzeitigen Ruhm / gnug Adeliches Herkommens / aber nie- 
mand feinder / als die ihren Adel durch Untugend schänden. (Bucholtz, Andreas Heinrich: Des Christlichen Teutschen Groß-Fürsten Herkules Und der Böhmischen Königlichen Fräulein Valjska Wunder-Geschichte. Bd. 1. Braunschweig, 1659)

(602) Denn weil Gott dennoch das Jfraelitifche Volck in diefer Wildniß gantzer viertzig Jahr wunderbarlich erhalten / gefpeifet mit Brote vom Himmel herab / getråncket mit Waffer aus dem harten Felfen heraus gefchlagen / gekleidet / indem ihre Kleider am Leibe / noch ihre Schuh an Füffen nicht reiffen múffen / da fie aus Egypten Land kamen und ehe fie ins gelobte Land gelangeten / fo war es gleichwol ein fchåndlicher Undanck / daß fie es nicht erkannten und GOTT dem HERRN / als fromme Kinder / von Hertzen dieneten / fondern mit allerhand groben Sünden / Schanden und Laftern hefftig zu wider waren und deß Abweichens nur mehr machten / ie offfter fie GOTT ftraffte und warnen lieffe. Kein Wunder demnach / daß fie es endlich auch mit ihrem eufferften Verderben büffen und bezahlen müffen. (DTA: Neitzschitz, Georg Christoph von: Sieben-Jährige und gefährliche WeltBeschauung Durch die vornehmsten Drey Theil der Welt Europa / Asia und Africa. Bautzen, 1666)

(603) Aber weil fie einen Marckt und Wechfel-Banck im Tempel aufrichteten / und die Priefter ihren Gewinn und Intereffe darbey fucheten / ziehet folches der HErr Chriftus für eine fchåndliche Entheiligung deß Tempels an / treibet Kåuffer und Verkåuffer hinauß / und faget / fie machen fein Hauß / das ein Bett-Hauß ift / zur Mo̊rder Gruben. (DTA: Bauller, Johann Jacob: Hell-Polirter Laster-Spiegel. Ulm, 1681)

Die Belege (598) - (603) lassen sich mehrfach interpretieren. Zunächst verbindet sich der Gebrauch von schändlich mit dem Begriff der Schande und bezeichnet dementsprechend Handlungen wie Missbrauch, Betrug, Undank, Beschmutzung und Entheiligung. Zugleich lässt sich aber auch die intensivierende Rolle des Adjektivs bzw. Adverbs erkennen. So handelt es sich jeweils um sehr negative bzw. empörende Situationen, die den Bezug auf die Schande durch das entsprechende Verhalten deutlich machen. Darüber hinaus geht es in (598) dadurch um einen großen Missbrauch des heiligen Evangeliums, dass sich die Menschen als Christen darstellen und doch ein unchristliches Leben führen; um einen großen Betrug seitens der Zauberer in (599), die die Menschen zum Müßiggang auffordern und sie damit dem Verhungern preisgeben, wodurch sie zahlreiche Tote verursachen; um einen großen Undank seitens des Lehrmeisters in (600), der den Versuch unternommen hat, das Kind eines Herren trotz seiner Wohltaten zu rauben und zu töten. In (601) wird über die große Beschmutzung des Ritterstandes durch Raub und Mord berichtet. In (602) handelt es sich um den großen Undank seitens des Volks, welches die Güte Gottes nicht erkannt, sondern auf seine Wohltaten anstatt des frommen Dienst mit groben Sünden, Schanden und Lastern geantwortet hat. Abschließend steht in (603) die große Entheiligung des Tempels durch die Einrichtung eines Markts und einer Wechselbank im Mittelpunkt. Darüber hinaus verbindet der adjektivische bzw. adverbiale Gebrauch die angesprochenen Situationen jeweils mit dem Begriff der Schande und zugleich mit dem großen Grad der Ausprägung des 
entsprechenden Sachverhalts. Das Einsetzen der Intensivierung geht mit dem Auftreten von subjectivity einher, insofern die Einstellung des Sprechers bzw. Schreibers zum Ausdruck gebracht wird. Das verweist auf Traugott I (oben 3.6.3.4).

Die Beleglage in COSMAS hat deutlich gemacht, dass der mehrdeutige Gebrauch auch in der Gegenwartssprache aktuell bleibt. Die Gradbedeutung erscheint zwar ziemlich ausgebildet, verliert jedoch nicht den Bezug auf den Begriff der Schande, vgl. die Belege mit den entsprechenden Umformulierungen:

(604) Die niedergelassenen Ärzte erhielten schändlich wenig für ihre Leistungen. (C.II: N92/APR.13205 Salzburger Nachr., 08.04.1992)

(604') 'Die Ärzte haben sehr wenig für ihre Leistungen bekommen, und das ist eine Empörung hervorrufende Schande'

(605) Und ich habe letztendlich meiner Hebamme, für deren Dienste ich nicht genug danken kann, ein paar hundert Mark gerne aus eigener Tasche gegeben, weil ich weiß, wie schändlich wenig sie für diese verantwortungsvolle Aufgabe bezahlt bekommt! (C.II: NUN94/AUG.01041 NN, 13.08.1994, S. 12)

(605') 'Die Hebamme bekommt sehr wenig für ihre Arbeit, und das ist eine Empörung hervorrufende Schande'

(606) Pfarrer Gerald Gump erzählt: „Die Republik Österreich streicht im Budget 2011 Mittel für die eh schon schändlich geringe Entwicklungshilfe des Bundes. [...]“" (NON11/JAN.06383 NÖN, 13.01.2011)

(606') 'Die Entwicklungshilfe ist sehr gering, und das ist eine Empörung hervorrufende Schande'

(607) Herr Minister, Sie sagen, Sie wollen Außenwirtschaft und Entwicklungshilfe miteinander verbinden. An dieser Stelle ist anzumerken, dass seit der Regierungsübernahme von Roland Koch im Jahr 1999 die Gelder des Landes für Entwicklungszusammenarbeit nur noch ein Bruchteil dessen sind, was sie früher einmal waren. Wir reden hier von aktuell 260.000 Euro im Jahr. Das ist eine schändlich niedrige Summe für ein Bundesland, das sich verpflichtet hat, der Entwicklungshilfe in Zeiten globalen Zusammenwachsens mehr Aufmerksamkeit zu schenken. Das ist wirklich viel zu wenig. (C.II: Protokoll der Sitzung des Parlaments Hessischer Landtag am 01.02.2011)

(607) 'Die Gelder für Entwicklungszusammenarbeit stellen eine sehr niedrige Summe dar, und das ist eine Schande, insbesondere hinsichtlich der Tatsache, dass das Bundesland sich verpflichtet hat, der Entwicklungshilfe in Zeiten globalen Zusammenwachsens mehr Aufmerksamkeit zu schenken'

In den Belegen (604) - (607) wird schändlich als Intensivierungsmittel und zugleich als ethisches Prädikat dargestellt. Damit geht die Verbindung der Bedeutung des Lexems mit der Ausgangsbedeutung nicht vollkommen verloren. Die folgenden Belege (608) - (609) bezeugen dagegen den Verlust des Zusammenhangs mit der Ausgangsbedeutung, da sie ausschließlich den Grad des Sachverhalts zum Ausdruck bringen, vgl.:

(608) das Kleid war schändlich teuer (DUDEN 2012, s.v.) 
(608') 'sehr teuer'

(609) »Aber es ist wieder seit ein paar Tagen schändlich kalt,« mahnte Tom, »und du holst dir jedesmal sowieso was weg in der alten Kirchenluft. (29.04.2014: http://gutenberg.spiegel.de/buch/3882/9)

$(609 ')$ 'sehr kalt'

Aus den Belegen (608) - (609) lässt sich schließen, dass das Einsetzen der Intensivierung mit dem Zuwachs an subjectivity korreliert, indem der Gebrauch mehr einstellungsbezogen im Rahmen von Traugott III (oben 3.6.3.4) wirkt.

Die Korpusanalyse hat keinen Gebrauch von schändlich in Bezug auf Funktionsverbgefüge sowie das Auftreten des Lexems in quantifizierender Funktion belegt. Außerdem folgt aus der Beleglage, dass die Entwicklung der intensivierenden Funktion des Lexems die letzte Stufe des Entwicklungsgangs bzw. das Auftreten in Verbindung mit positiv konnotierten Kookkurenzpartnern noch nicht erreicht hat.

\subsubsection{Kurzfassung der Entwicklung bei schändlich}

1. Ausgangsbedeutung: 'mit Schande behaftet, unehrenhaft';

4./5./6. Die Korpusanalyse ergibt keine Verwendung in quantifizierender Funktion, nicht in Funktionsverbgefügen und nicht mit positiv konnotierten Kookkurrenzpartnern;

die übrigen Entwicklungsschritte wie oben in Kap. 3.6.3.9.3.

\subsubsection{Sündhaft}

\subsubsection{Lexikographische Angaben}

Laut Pfeifer (s.v.) ist das Lexem im Althochdeutschen in der Form sunt(i)haft mit dem Sinn „einer Sünde entsprechend, mit Sünde behaftet, sündig“ in Erscheinung getreten.

1. sündhaft in Splett (s.v. sunt-haft)

„sündig, sündhaft, schändlich“

2. sündhaft in BMZ, Lexer (s.v. sünde-haft)

„mit sünde behaftet"

3. sündhaft in FWB

keine Angaben, auch nicht im bereits zitierten Material der Redaktionsstelle von FWB

4. sündhaft in DWB (s.v.)

„peccatorius, von sündlicher art. der durch die wortbildung nahegelegte sondersinn 'mit sünde behaftet' ist nur selten spürbar [...]. das wort, in älterer sprache sparsameren und weniger geprägten gebrauchs als die synonyma sündig und sündlich, scheint zur neuzeit hin an boden zu gewinnen."

4.1 „der attributive gebrauch bleibt hinter der gleichen verwendung von sündig und sündlich zurück"

4.1a „religiös als merkmal menschlicher existenz, wie sündig 1“

$4.1 \mathrm{~b}$ „,seltener noch bei handlungen, wie sündlich 1 , sündig 4 “

$4.1 \mathrm{c}$,'zur sünde verlockend', wie sündlich $2 \mathrm{~b}$, aber nur erotisch“ 
4.1d „mit einer gewissen vorliebe zur kennzeichnung des triebhaften, der anlage"

4.1e „abgeschwächt 'unrecht', im sinne von sündlich 4“

4.2 „in der prädikativen verwendung wird, besonders in jüngerer sprache, sündhaft als prädikatsnomen bevorzugt"

4.3 „im adverbialen gebrauch erscheint sündhaft als blosze verstärkungsformel beim adjektiv, soviel wie 'sehr'; vornehmlich mundartlich, bei maszoder preisbezeichnungen"

5. sündhaft in DUDEN (2012, s.v.)

$5.1 \mathrm{a}$ „(gehoben) mit Sünde behaftet; eine Sünde (a) bedeutend“

$5.1 \mathrm{~b}$ „eine Sünde (c) bedeutend“

5.2 „(umgangssprachlich) “

$5.2 \mathrm{a}$ „überaus hoch“

$5.2 \mathrm{~b}$ „überaus viel“

$5.2 \mathrm{c}$ „>intensivierend bei Adjektiv> sehr, überaus“

\subsubsection{Die Entwicklung der Gradbedeutung bei sündhaft}

Pfeifer (s.v.) führt das Auftreten der Gradbedeutung in diesem Lexem auf das 19. Jh. zurück mit der Annahme, dass das Einsetzen der Intensivierung sich früher, und zwar im 17. Jh., anbahnte. Diese Annahme wird jedoch keineswegs belegt und liefert von daher keine Evidenz für ihre Bestätigung. DWB (s.v.) bietet ebenso keine Belege, die den Gebrauch des Lexems in der intensivierenden Funktion früher als am Anfang des 20. Jh. demonstrieren können.

Was die Quelle der Gradbedeutung betrifft, so ist darauf hinzuweisen, dass nach den Angaben von DWB sündhaft einen ähnlichen Entwicklungsgang wie sündlich eingeschlagen hat. Wie oben erwähnt, hat sündlich die abgeschwächte Bedeutung „im Sinne von unrecht“ entwickelt, die sich „Von der affektbetonten moralischen entrüstung bis zur bloszen verstärkungsformel“ herausbildete (DWB, s.v.). Das Einsetzen der Intensivierung nach diesem Muster hat die in Rahmen dieser Arbeit durchgeführte Korpusanalyse auch für sündhaft feststellen können, was die uneinheitlichen lexikographischen Angaben von DWB in Bezug auf die betroffenen Lexeme korrigiert. Jedoch datieren die gefundenen Belege auf das 19. Jh. und nicht auf das 17. Jh., wie es in Pfeifer angegeben ist.

Die Korpusanalyse legt nahe, dass das Einsetzen der Gradbedeutung in mehrdeutigen Kontexten erfolgt ist. Als Grundlage für deren Herausbildung kann die religiöse sowie die abgeschwächte, moralisch bedingte Bedeutung dienen. Die erste Sinnrichtung bezieht sich auf die Ausgangsbedeutung des Lexems und verweist auf Personen und Handlungen, die vom religiösen sowie sittlichen Standpunkt aus als „mit Sünde behaftet“ (DWB, s.v.) bezeichnet werden, vgl.:

(610) sît daz ich sündehafter man die kristenheit hazzen began (DWB, s.v.)

(611) er musz auch got sein sundhaftige bose gedanken ... beichten (DWB, s.v.)

Das Auftreten der Intensivierung lässt sich in mehrdeutigen Kontexten infolge pragmatic enrichment bzw. strengthening der Ausgangsbedeutung durch das Ab- 
leiten der entsprechenden Inferenz finden. Die Gradbedeutung tritt als Nebenbedeutung in Erscheinung, während die Ausgangsbedeutung die primäre Rolle spielt, vgl.:

(612) Undine half den beiden nach Kråften, und fagte, als das Regenwetter plötzlich allzufchnell herauf heulte, luftig drohend in die fchweren Wolken hinein: Du! Du! Hüte Dich, daß Du uns nicht naß machft; wir find noch lange nicht unter Dach. - Der Alte verwies ihr folches als eine fündhafte Vermeffenheit; aber fie kicherte leife vor fich hin, und es widerfuhr auch Niemanden etwas Uebles darum. (DTA: Fouqué, Friedrich de la Motte: Undine, eine Erzählung. In: Die Jahreszeiten. Eine Vierteljahrsschrift für romantische Dichtungen, 1811, Frühlings-Heft, S. 1-189)

(613) Der Graf hatte dem Fündling in heiliger Taufe den Namen Francesko geben lassen. Der Knabe wuchs heran und wurde an Gestalt und Geist ein wunderbarer Jüngling, den der Graf seiner seltenen Gaben wegen wie seinen Sohn liebte [...]. Schon fünfundzwanzig Jahre war Francesko alt worden, als der Graf Filippo in törichter Liebe zu einem armen bildschönen Fräulein entbrannte und sie heiratete, unerachtet sie blutjung, er aber schon sehr hoch in Jahren war. Francesko wurde alsbald von sündhafter Begier nach dem Besitze der Gräfin erfaßt, und unerachtet sie gar fromm und tugendhaft war und nicht die geschworene Treue verletzen wollte, gelang es ihm doch endlich nach hartem Kampfe, sie durch teuflische Künste zu verstricken, so daß sie sich der freveligen Lust überließ, und er seinen Wohltäter mit schwarzem Undank und Verrat lohnte. (C.II: HK3/D64.00001 Hoffmann: Die Elixiere des Teufels, Entstanden: 1814/15, 2000 [S. 300])

Die Belege (612) - (613) erfahren eine mehrdeutige Interpretation. Zunächst ist sündhaft als moralisches Prädikat zu betrachten, indem es Vermessenheit in (612) und Begier in (613) in Bezug auf die besprochenen Situationen als eine sündige bzw. mit Sünde behaftete Eigenschaft ausdrückt. Zugleich soll die intensivierende Bedeutung des Lexems zum Ausdruck gebracht werden, denn aus den Kontexten kann jeweils geschlossen werden, dass es sich um einen in hohem Grade ausgeprägten Sachverhalt handelt. So wird die (milde) Drohung dem Himmel gegenüber in (612) aus religiösen Gründen als eine Sünde von und daher als große Vermessenheit betrachtet. In (613) ist die Begier so stark, dass sie durch unsittliche Handlungen gestillt und von daher als sündig dargestellt wird.

Das Einsetzen der Intensivierung geht mit dem Auftreten von subjectivity einher, indem die Einstellung des Sprechers bzw. Schreibers zum Ausdruck gebracht wird. Das verweist auf Traugott I (oben 3.6.3.4)

In der nächsten Phase der Bedeutungsentwicklung unterliegt die mit den Vorstellungen der Sünde verbundene Sinnrichtung dem Prozess der Abschwächung, sodass lediglich der Begriff des Unrechten „im besonderen [...] was masz und grenze überschreitet" (DWB, s.v.) sich nachweisen lässt. Neben bleaching wird die Gradbedeutung in den Vordergrund vorgerückt, vgl.:

(614) In der Freude an der Arbeit hatte ich aber wahrscheinlich versäumt sie ordentlich durchzulesen, und erschrak daher nicht wenig, als ich mein 
Buch zurück erhielt, und mit der feinen Handschrift unseres Lehrers die sündhafte Zahl von zehn Fehlern angemerkt fand, denen oben ein noch eine lange Nachschrift folgte. Sie lautet also: „Obschon durch Unachtsamkeit zehn Fehler in dem Aufsatze sind, ist er dem Inhalte nach sehr gut. [...] " (C.II: HK4/L12.00001 Lewald: Meine Lebensgeschichte, Entstanden: ab 1858, 2004 [S. 191])

(615) Ich bin seit meinem fünfzehnten Jahre für jede Arbeit bezahlt worden und hätte es mir zur Schande angerechnet, wenn ich irgend etwas umsonst hätte tun müssen. - Fünfzig Jahre fruchtlosen Ringens! Das müßte doch den Starrköpfigsten von der Unmöglichkeit seiner Träume überzeugen. Was haben Sie denn dann von Ihrem Leben genossen? Sie haben es sündhaft vergeudet! (C.II: HK3/D18.00001 Wedekind: Der Kammersänger, Entstanden: 1897, 2000 [S. 411])

(616) Das sind diejenigen, die, um ihrer unreinen Reime willen aus dem Paradies kleiner Winkelblättchen vertrieben, zur Überzeugung kommen, daß die Prosa doch „das Leichtere" sei. Das ist ein sündhafter Irrtum. (C.II: HK3/A96.00017 Rilke: [Aufsätze und Rezensionen:] Moderne Lyrik, Entstanden: 1898, 2000 [S. 390])

(617) In den Schaufenstern sahen wir die sündhaft teuren Kleider und Luxusartikel. (C.II: A99/JAN.06164 St. Galler Tagblatt, 27.01.1999)

(618) Im 17. Jahrhundert hatte England damit begonnen, zu sündhaft hohen Preisen Tee aus China zu importieren, die seinen Genuss zum Luxus für die gehobene Gesellschaft machten. (C.II: RHZ11/FEB.26299 RZ, 23.02.2011, S. 20)

(619) Dazu spielen sich Kathy Bates als giftsprühende, sündhaft geschmacklos kostümierte Mutter und Pfeiffer gegenseitig die Bälle zu. (C.II: BRZ09/FEB.04846 Braunschw. Z., 11.02.2009)

(620) Ich mag Blumen. Wer nicht. Ich mag aber auch Wiesenblumen. Unverfälscht und unverzüchtet. Selbst wenn es nur Gänseblümchen sind. Alle anderthalb, zwei Wochen alles niederzumähen, dünkt mich sündhaft schade. (C.II: A09/JUN.04714 St. Galler Tagbl., 16.06.2009, S. 36)

Die Belege (614) - (620) demonstrieren den fortgeschrittenen intensivierenden Gebrauch von sündhaft. Aus den Kontexten geht hervor, dass das Lexem den direkten Bezug auf den Begriff der Sünde verloren hat, indem es gelegentlich unrechte und Empörung hervorrufende Sachverhalte bezeichnet. So handelt es sich in (614) um eine große Anzahl von Fehlern, die für die durchgeführte Arbeit als unangebracht gilt. In (615) werden die große Entrüstung und die Beurteilung der unrechten Lebensweise der angesprochenen Person seitens des Gesprächspartners zur Sprache gebracht. In (616) bezeichnet sündhaft eine äußerst unrechte Denkweise bezüglich Prosa. In (617) - (618) bezieht sich das Lexem auf sehr teuere Kleider und hohe Preise. In (619) - (620) erscheint sündhaft lediglich als Intensivierer, ohne auf den Begriff des Unrechten hinzuweisen.

Die zuletzt eingeführten Belege lassen den Prozess der Bedeutungsabschwächung und zugleich die Zunahme an subjectivity veranschaulichen, indem der Gebrauch mehr einstellungsbezogen wird und damit den Übergang zu der expressiven Ausdrucksebene und damit zu Traugott III (oben 3.6.3.4) demonstriert. 
Die Untersuchung hat keine Belege feststellen können, die das Auftreten von sündhaft als Modifikator von Funktionsverbgefügen veranschaulichen. Dagegen finden sich Belege, die die quantifizierende Rolle des Adjektivs demonstrieren, vgl.:

(621) »Nun... und die Landwirtschaft? Wissen Sie etwas davon?" »Nur, daß meine Pferde eine sündhafte Menge Hafer und Heu verschlangen ..." (29.06.2014: http://books.google.de/books?id=-OVapeuH7IMC\&pg=PA51\&lpg=PA51\&dq $=\% 22 \mathrm{~s} \% \mathrm{C} 3 \%$ BCndhafte $\quad+$ menge\%22\&source=bl\&ots=9zLVNQNdAL\&sig=bUwCe7HdONuID0psGUH72Zyh9W8\&hl=de\&sa=X\&ei=sUKwU8zAJofZPJaRgMgC\&ved=0CCIQ6AEwAQ\#v=onepage\&q=\%22s\%C3\%BCndhafte\%20menge\%$22 \& \mathrm{f}=$ false)

(621') 'sehr große Menge'

(622) Nun habe sie sich auch noch nach einer Erzählung Viktors für eine sündhafte Summe eine lila Lederhose anfertigen lassen. (29.06.2014: http://books.google.de/books?id=qYjSbw_SPywC\&pg=PT59\&dq=\%22s\%C3\%BCndhafte+summe\%22\&hl=de\&sa=X\&ei=AEKwU_fwEcKe04v9gJgN\&ved=0CCwQ6AEwA$\mathrm{g} \# \mathrm{v}=$ onepage $\& \mathrm{q}=\% 22 \mathrm{~s} \% \mathrm{C} 3 \% \mathrm{BCndhafte} \% 20$ summe $\% 22 \& \mathrm{f}=$ false)

(622') 'sehr große Summe'

Anschließend tritt das Lexem in positiven Kontexten auf und demonstriert den kompletten Verlust des Zusammenhangs mit der Ausgangsbedeutung. Der Prozess der Bedeutungsentleerung wird damit vollkommen vollzogen, vgl:

(623) Um das Gaumenfest perfekt zu machen, serviert Ekkehard Reimann seine saftig-zarten Rouladen mit einem sündhaft sahnigen Kartoffelpüree und hausgemachtem Rotkohl, aus dem man - als kleine Vorahnung auf die Weihnachtszeit - einen Hauch Zimt herausschmeckt. (C.II: HAZ08/OKT.00463 HAZ, 04.10.2008, S. 6)

(623') 'sehr sahnigen'

(624) Mehr als 6000 kalte und warme Häppchen, kleine Tellergerichte sowie sündhaft leckere Desserts brachte die weiße Brigade um die Hohenzollern-Küchenchefs Gerd Lanz und Joachim Neubusch unter die Gäste oder bereiteten die Speisen unter ihren faszinierten Augen zu. (C.II: RHZ01/MAI.21581 RZ, 29.05.2001)

(624') 'sehr leckere'

(625) Eine gute Wahl, es gibt sündhaft gutes Bier aus dem nahen Seetal, es gibt wunderbare Balchen (so heissen in der Zentralschweiz die Felchen), und da ist eine Wirtin, die wohl die ganze Geschichte des Städtchens im Kopf hat. (C.II: A10/JUN.08422 St. Galler Tagbl., 26.06.2010, S. 13)

$(625$ ') 'sehr gutes Bier'

\subsubsection{Kurzfassung der Entwicklung bei sündhaft}

1. Ausgangsbedeutung: 'mit Sünde behaftet';

5. Korpusanalyse ergibt keine Verwendung in Funktionsverbgefügen, jedoch mit positiv konnotierten Kookkurrenzpartnern; die übrigen Entwicklungsschritte wie oben in Kap. 3.6.3.9.3. 


\subsubsection{Unmenschlich}

\subsubsection{Lexikographische Angaben}

Pfeifer und Kluge bieten keine Angaben zu der Herkunft des Lexems.

1. unmenschlich in Lexer (s.v.) "inhumanus“

2. unmenschlich in FWB keine Angaben, auch nicht im bereits zitierten Material der Redaktionsstelle von FWB

3. unmenschlich in DWB (s.v.)

3.1 „nichtmenschlich (s. menschlich 1 und unmensch 1)“

3.2 „unmensch 2, menschlich 2, 8 entsprechend, vgl. DWB greulich 1 a $\alpha^{\prime \prime}$

3.3 "menschenfeindlich (unmensch 4)"

3.4 ,unmensch 3 entsprechend (vgl. unchristlich 2, greulich 3) “

3.5 ,abominabilis, abominatus, abominosus"

3.6 „rechts- u. geschäftssprachlich (s. DWB unmensch 5) 'höchst ungebührlich, unerlaubt' u. dgl."

3.7 „an über- und widermenschlich reichend“

3.8 „unter dem menschen stehend, als menschenunwürdig seiner natur unangemessen (vgl. 2 und 12) “

3.9 „gth. v. menschlich 10, menschen unerträglich, von ihnen nicht zu tragen, heben, leisten"

3.10 „menschlich 8/9, unmensch 6 entsprechend, in unserer hauptbed., 'grausam',vom sittlichen handeln, fühlen, denken. vgl. DWB unchristlich 2. die schon mhd. u. mnl. vorhandene bed. gewinnt natürlich im zeitalter des neuhumanismus einen besonderen beiklang ('den gesellschaftlichen zustand gesitteter menschen im hohen grade verletzend' ADELUNG; sp. 25) und reicht dann an $1^{\prime \prime}$

3.11 „unmensch 7 entsprechend, schwächer, 'inhuman'“

3.12 „intensiv, wie unchristlich 3, greulich 1 e, 2 a, 5 e $\beta, 6$ a, 7“

3.13 "menschlich 4 entsprechend“

4. unmenschlich in DUDEN (2012, s.v.)

4.1a „grausam gegen Menschen od. Tiere, ohne bei einem Menschen zu erwartendes Mitgefühl [vorgehend]"

$4.1 \mathrm{~b}$ „(emotional) menschenfeindlich durch Unterdrückung“

$4.1 \mathrm{c}$ "(emotional) menschenunwürdig, inhuman"

$4.2 \mathrm{a}$ „(emotional) ein sehr hohes, [fast] unerträgliches Maß habend“

$4.2 \mathrm{~b}$ „<intensivierend bei Adj. u. Verben> (umgangssprachlich, oft emotional übertreibend) sehr, überaus“"

\subsubsection{Entwicklung der Gradbedeutung bei unmenschlich}

Die Korpusanalyse sowie die Angaben in DWB lassen die Entwicklung der Intensivierung in unmenschlich auf das Fnhd. zurückführen. Es soll jedoch auf einen deutlichen Mangel an Informationen hingewiesen werden, insofern als FnhdC, FWB sowie DRW und DRQEdit keine Angaben zum Thema liefern und damit den Umfang 
der für die Untersuchung benötigten Belege verringern. Die Beschreibung des Entwicklungsgangs basiert von daher auf den Angaben aus DWB und DTA, die teilweise fnhd. Belege berücksichtigen.

Aus der Analyse der lexikographischen und Korpusangaben kann geschlossen werden, dass die Intensivierung in mehrdeutigen Kontexten eingesetzt hat. Dabei lässt die Liste der in DWB angegebenen Bedeutungspalette mehrere Sinnrichtungen feststellen, die als Basis für das Einsetzen der Gradbedeutung dienen können. So kann als Grundlage für die Herausbildung die auf das rohe, grausame menschliche Verhalten bezogene Bedeutung und der damit verbundene Begriff des Grauens dienen, vgl.:

(626) da ist er von stund an grawsam und $\boldsymbol{u}$. worden (DWB: s.v.)

(627) Das gefchrey von dem Rauben / Stelen vnnd Morden diefes vnmenfchlichen Raubers war in gantz Franckreich fo gro $\beta$ / je lenger je mehr / da $\beta$ fich niemandts mehr in das Feld dorffte wagen: Anfänglich forchteten fich die Bawersleute jme zubegegnen vnd auffzuftoffen / vnd braucheten allerley neben Wege / damit fie jhm ja nicht in fein Netz fallen mo̊chten [...] (DTA: [Calvi, François de]: Beutelschneider, oder newe warhaffte vnd eigentliche Beschreibung der Diebs Historien. [Bd. 1]. Frankfurt (Main), 1627)

Die Entwicklung der Intensivierung beginnt mit pragmatic enrichment bzw. strengthening der Ausgangsbedeutung und hat damit die Mehrdeutigkeit des Gebrauchs zu Folge:

(628) Ob wol viel heiliger Merterer mit vnerhörter / graufamer / vnmenfchlicher marter find hingerichtet etliche den wilden Thieren fürgeworffen / etliche in Oele gebraten / etliche im heiffen Bley [...] (DTA: Arndt, Johann: Vom wahren Christenthumb. Bd. 2. Magdeburg, 1610)

(629) Aber als fie dafelbft angelanget / vnd auffs Land getretten / befunden fie / daß gemeldte Wilden fich zwar in die Flucht begeben / aber etliche Zeichen jhrer vnmenfchlichen Graufamkeit hinderlafle [...] (DTA: Gottfried, Johann Ludwig: Newe Welt Vnd Americanische Historien. Frankfurt (Main), 1631)

(630) Bey Jofepho lifet man vom Hyrcano, daß da er die Stadt Ptolomaida belägert / vnd aber Ptolomæus feine Mutter vnd Brüder auff die Stadtmaur zur Schau geführet vnd dargeftellet / vnd fo offt er einen Sturm angeloffen / diefelbe vnbarmhertzig / vnmenfchlich fchlagen vnd geißlen laffen / fo konnte er nicht auß eingebung der Blutsieb fein ftürmen außführen / alle Streich auff der Mauren giengen auffihn. (DTA: Dannhauer, Johann Conrad: Catechismus Milch. Bd. 5. Straßburg, 1654)

(631) Aber diefer vnmenfchliche Tyrann läft fich fo wenig durch folche rechtmeffige bitte vnd erbärmliche Geberden bewegen / daß er vielmehr fo bald nach feinem Dolchen griff vnd dem Kauffmann denfelbigen mitten durch fein Hertz ftoffet [...] (DTA: Calvi, François de: Beutelschneider / Oder Neue / warhaffte / und eigentliche Beschreibung Der Diebs=Historien. Bd. 2. Frankfurt (Main), 1627)

(632) Du groffer Gott / warum̃ $<\delta>$ chlågftu nicht fo bald mit dem Donner deines gerechten Zorns den meineidigen fchelmẽ vnd Meuchelmo̊rder auff fein 
Kopff / vnd laffet jn nit mehr anfchawẽ daß Licht deiner fchönen geftirnen / O vnmenfchliche tyranney / welche erger ift als der Can $<i>$ balen wohin leyteft du / wohin führeft du / wohin ftürtzeft du einen Menfchen / daß er feiner fo gar vergeffet / ja das er nit mehr gedencket / daß ein Gott im Himmel fey / der folche fchreckliche Thaten / ja welcher alle bo̊fe ftück offenbaret / vnnd vor der gantzen Welt den Menfchen / fo fie begangen / vor jhr Angeficht ftellet. (DTA: Calvi, François de: Beutelschneider / Oder Neue / warhaffte / und eigentliche Beschreibung Der Diebs=Historien. Bd. 2. Frankfurt (Main), 162762)

Die Belege (628) - (632) lassen mehrere Lesarten zu. Die erste Interpretation bezieht sich auf das brutale Verhalten der angesprochenen Personen. Damit wird unmenschlich in der Ausgangsbedeutung gebraucht. Die Kontexte liefern außerdem Hinweise auf die intensivierende Rolle von unmenschlich, durch die ein hoher Ausprägungsgrad der Sachverhalte ausgedrückt wird. Die zweite Interpretation lässt sich durch semantic repetition ableiten, indem die Belege volle oder teilweise Übereinstimmung der in den adjektivischen und adverbialen Verwendungen enthaltenen Bedeutungskomponenten erweisen, denn beide Kookkurrenzpartner beziehen sich auf brutales Verhalten. Die Wiederholung der Bedeutung führt zu semantic repetition und damit zu redundancy. Außerdem stellen die Bezugswörter Extremwerte aufweisende Prädikate dar, nämlich Marter, Grausamkeit, Tyrann, Tyranei, schlagen und geißeln. Durch die Verbindung mit unmenschlich, welches ein superlativisches Adjektiv bzw. Adverb im Sinne von Paradis (1997: 54) darstellt, kommt es zu einer Verstärkung des Gebrauchs und zu der semantischen Reanalyse der intensivierenden Funktion des Lexems.

Die auf Unchristlichkeit bezogene Sinnrichtung stellt eine weitere Möglichkeit für das Einsetzen der Gradbedeutung dar und kann sich laut DWB auf z.B. eines Christen unwürdige Gedanken, Sünden u.a. beziehen. Das Auftreten der Intensivierung erfolgt zunächst als Nebenbedeutung, während die Ausgangsbedeutung noch zu erkennen ist, vgl.:

(633) unmenschlicher zorn macht unsinnig (DWB: s.v., Ziff. 12)

(634) Siheftu noch nicht / daß er dir redlich / zeitig / und mehr dann zu fcharff komen ift / und uns feine Kinder zwar mit der gnädigen Vater Ruhte / wegen unfer Sünde gezüchtiget / wovor wir ihm herzlich danken / dich aber mit fchwerer Hand zur grimmigen Straffe / wegen deiner läfterung und unmenfchliche gottlofigkeit hingeriffen hat? (DTA: Bucholtz, Andreas Heinrich: Des Christlich: Teutschen Königes Herkules und der Teutschen Königin Valiska Wunder-Geschicht. Bd. 2. Braunschweig, 1660)

(635) Alfo tragen fie auch den Namen Drachen- und Teuffelsbuhler / ümb der Vrfachen willen / weil der böfe Feind / der Hellifche Drach / fie zu unmenfchlicher Vnzucht beweget und gebrauchet. (DTA: Praetorius, Johannes: Blockes-Berges Verrichtung. Leipzig u. a., 1668)

\footnotetext{
62 Emendationen nach der Ausgabe von 1657: https://books.google.de/books?id=oVZMAAAAcAAJ\&printsec=frontcover\&hl=de\&source=gbs_ge_summary_r\&cad $=0 \# v=$ onepage\&q\&f=false [gesehen 13.2.2017 18:10]
} 
(636) Ich läugne nicht, daß fie in ihren Leidenfchaften fehr heftig find, allein, wer will oder kann behaupten, daß heftige Leidenfchaften immer nur zu fchädlichen oder gar unmenfchlichen Ausfchweifungen führen? (DTA: Forster, Georg: Johann Reinhold Forster's [..] Reise um die Welt. Bd. 2. Berlin, 1780)

Die Belege (633) - (636) können als mehrdeutig interpretiert werden. Zunächst verweist unmenschlich auf das einem Christen widrige Verhalten „ohne Beziehung auf christliche Sittlichkeit" (DWB, s.v.) und damit auf die Ausgangsbedeutung. Zugleich jedoch demonstriert das Adjektiv die intensivierende Funktion, die durch pragmatic enrichment bzw. strengthening der Ausgangsbedeutung in Erscheinung tritt. Diese zweite Interpretationsmöglichkeit erfolgt aus der Analyse der Kontexte, in denen die Gradbedeutung als Quelle für die Bedeutungskomponente „unchristlich" dient, vgl.:

(633') 'Der Zorn ist so stark, dass er unchristlich ist'

(634') 'Die Gottlosigkeit ist sehr stark ausgeprägt und ist damit unchristlich'

(635') 'Die Unzucht ist sehr stark ausgeprägt und ist damit unchristlich'

(636') 'Die Ausschweifungen sind sehr stark ausgeprägt und sind damit unchristlich'.

Die Bedeutung der Menschenunwürdigkeit liefert eine weitere Basis für das Einsetzen der Intensivierung, vgl.:

(637) ein unmenschlicher (den zweck des menschen verfehlender) zustand (DWB: s.v.)

(638) u. aber ist nicht nur, was in der wirklichkeit unter der menschlichen natur steht, sondern ... (DWB: s.v.)

Die Auswertung der Belege macht die Annahme plausibel, dass die in (637) - (638) ausgedrückten Sinnrichtungen die Gradbedeutung durch pragmatic enrichment bzw. strengthening gewinnen und damit eine Mehrdeutigkeit im Gebrauch aufweisen können, wie es in den Belegen (639) - (641) der Fall ist.:

(639) ist es ein grosze unsinnigkeit, unmenschlicher unverstand und der natur gäntzlich zůwider (DWB: s.v.)

(640) eine recht unmenschliche thorheit (DWB: s.v.)

(641) Sie weiß eben fo wenig von Rache als von Verzweifelung, und ift im Stande, dem Urheber ihres Unglücks zu vergeben; wünfchet ihm Buße, und daß fie das letzte Opfer feiner unmenfchlichen Treulofigkeit feyn möge [...] (DTA: [Richardson, Samuel]: Clarissa. Bd. 6. Göttingen, 1750)

Die Belege (639) - (641) sind mehrdeutig interpretierbar. Die erste Lesart verbindet sich mit der Ausgangsbedeutung und bezeichnet die angesprochenen Zustände als "menschenunwürdig“ bzw. "seiner natur unangemessen“ (DWB, s.v.). Die zweite Lesart bezieht sich auf die Intensivierung, ohne den Kontakt mit der Ausgangsbedeutung zu verlieren. Es handelt sich um eine Anreicherung des semantischen Gehalts der Ausgangsbedeutung durch die als Inferenz abgeleitete Nebenbedeutung des Grades, sodass etwa die folgenden Umformulierungen gelten können:

(639') 'in so hohem Maße großer Unverstand, dass es menschenunwürdig ist' $(640$ ') 'in so hohem Maße große Torheit, dass es menschenunwürdig ist' 
(641') 'in so hohem Maße große Treulosigkeit, dass es menschenunwürdig ist'

Zudem ist zu bemerken, dass das Einsetzen der Gradbedeutung auf der besprochenen Etappe ihrer Entwicklung mit einer Zunahme an subjectivity verbunden ist. Der Gebrauch gewinnt damit eine wertende Komponente und demonstriert „the representation of a speaker's perspective of point of view in discourse" (Finegan 1995: 1) sowie das Auftreten von Traugott I (oben 3.6.3.4).

Auf der nächsten Stufe der Bedeutungsentwicklung wird die Mehrdeutigkeit des Gebrauchs beseitigt, sodass das Lexem in eindeutig intensivierender Funktion auftritt. Unmenschlich kollokiert jedoch zunächst mit negativ konnotierten Bezugswörtern, sodass der Bezug auf die Ausgangsbedeutung damit nicht endgültig beseitigt wird, obgleich der direkte Kontakt schon verloren geht und nicht mehr nachzuweisen ist, vgl.:

(642) Dann jhm wolbewußt ware / daß fie vnmenfchlichen Schmertzen feines Vnglücks wegenertrüge. (DTA: Barclay, John (Übers. Martin Opitz): Johann Barclaÿens Argenis Deutsch gemacht durch Martin Opitzen. Breslau, 1626)

(642') 'sehr große Schmerzen'

(643) Leider haben wir [...] folchen unmenfchlichen Hunger erlebet / da nicht nur ein Exempel fürgangen / daß man die Toden angefallen wie zu Jerufalem / fondern der Meifter auff der Schlut der Aeffer nicht Meifter geweft / wann die Hungerleider nur folche gehabt /fo waren das ihre Pafteten [...] (DTA: Dannhauer, Johann Conrad: Catechismus-Milch. Bd. 6. Straßburg, 1657)

(643') 'sehr großen Hunger'

(644) Jedoch waren die Lautsprecher auf unmenschliche Lautstärke eingestellt, sodass wir den Platz schon im Vorbeigehen verlassen mussten. Wir hatten das Gefühl, der Rhythmus der Musik bestimmte unseren Herzschlag. Unerträglich! (C.II: NUN05/JUL.02072 NN, 18.07.2005)

(644') 'sehr große Lautstärke'

(645) Ich gestehe, daß ich mich an nichts erinnerte außer an die Lipizzaner, weil wir dort unmenschlich lange warten mußten. (C.II: P96/JAN.00576 Die Presse, 05.01.1996)

(645') 'sehr lange'

(646) Als sie hörte, wo die EM über die Bühne geht, war sie aber doch genervt: „Plovdiv? Dort ists doch unmenschlich heiß. “40 Grad und mehr auf der Quecksilbersäule sind derzeit in Bulgarien keine Seltenheit. (C.II: 098/JUN.62334 Neue Kronen-Ztg., 25.06.1998, S. 53)

(646') 'sehr heiß'

(647) Das Image, ein Bäcker habe unmenschlich früh aufzustehen stimmt. Ab zwei Uhr, manchmal auch noch früher, geht es in der Backstube los. (C.II: RHZ04/NOV.16547 RZ, 17.11.2004)

(647') 'sehr früh'

(648) Richtig ist natürlich, dass der Export von 1,2 Prozent der deutschen Agrarprodukte in afrikanische Länder einen unmenschlich hohen Schaden anrichtet. (C.II: Protokoll der Sitzung des Parlaments Deutscher Deutscher Bundestag am 28.05.2009) 
(648') 'sehr hohen'

Unmenschlich kann außerdem Funktionsverbgefüge modifizieren, vgl.:

(649) Dann setze ich damit den Mitarbeiter wirklich unmenschlich unter Druck. (27.02.2014: http://www.zeit.de/wirtschaft/2010-01/verdi-kritik-bankberater/seite-2)

(650) Der Server aber geht mir mittlerweile unmenschlich auf die Nerven. (27.02.2014: http://eu.battle.net/wow/de/forum/topic/7527623071)

In (649) - (650) fungiert unmenschlich als Gradadverb und intensiviert die Funktionsverbgefüge $j-n$ unter Druck setzen und $j$-m auf die Nerven gehen. Das Auftreten des Lexems mit diesen Verbindungen erfolgt dadurch, dass sie jeweils durch einen graduierbaren Begriff bspw. „bedrängen“ und „nerven“ wiedergegeben werden können.

Die intensivierende Funktion des Lexems kann in die quantifizierende übergehen und dadurch auf eine große Menge des durch ein Bezugswort ausgedrückten Sachverhalts hinweisen, vgl. die Belege mit den entsprechenden Umformulierungen:

(651) Eine Finfternü $\beta$ / ein Erdbeben / eine abfcheuliche Geburt / werden eine unmenfchliche Menge Menfchen erfchrecken können. (DTA: Der vollkommene rechtschaffene Welt-Mann. Frankfurt (Main), 1680)

(651') sehr große Menge"

(652) Der streitbare Rentner fragt: „Welche Regeln liegen der Gebührenerhöhung zugrunde, wofür sollen die Spätaussiedler solche unmenschlichen

Preise zahlen?" (C.II: RHZ97/FEB.15406 RZ, 26.02.1997)

(652') 'sehr hohe Preise'

Die Herausbildung der intensivierenden und quantifizierenden Bedeutung auf dieser Stufe des Entwicklungsgangs ist zugleich mit der Erweiterung des einstellungsbezogenen Ausdruckspotentials des Lexems verbunden. Der Gebrauch gewinnt an subjectivity, verweist ausschließlich auf ,internal situation“ und signalisiert damit den Übergang zu Traugott III (oben 3.6.3.4).

In der letzten Phase der Bedeutungsentwicklung finden sich Belege, die das Auftreten von unmenschlich in Kombination mit positiv konnotierten Bezugswörtern demonstrieren und damit auf seine Rolle des reinen Gradadverbs hinweisen. Der Prozess der Desemantisierung wurde vollständig durchgeführt, vgl.:

(653) in der ganzen gegend wird es $\boldsymbol{u}$. nach der schönsten vanille riechen (DWB, s.v.)

(653') 'sehr stark riechen'

(654) Wenn fie auch um alles in der Welt nicht gern in meiner Haut gefteckt hätten, fo hätten fie doch alle fammt unmenfchlich gern gewußt wie ich mich bei fo bewandter Lebenslage in ihr fühle (DTA: Raabe, Wilhelm: Stopfkuchen. Eine See- und Mordgeschichte. Berlin, 1891)

(654') 'sehr gern' 
(655) „Ich habe zwar verloren, aber fühle mich trotzdem wie ein Sieger", sagte Haas, „ich habe in dieser Woche gutes Tennis gezeigt. Juan hat sich unmenschlich gut bewegt und kaum Fehler gemacht. Ich muss einfach anerkennen, dass er, wenn es darauf ankam, der bessere Spieler war." (C.II: NUZ12/JUL.02182 NZ, 23.07.2012, S. 20)

(655') 'sehr gut'

(656) Kohlschreiber zog am Samstag mit einem lockeren Dreisatzsieg über Nadal-Bezwinger Lukas Rosol nach und meinte: „Wenn's jetzt auch noch mit dem Viertelfinale klappt, wär's natürlich unmenschlich geil!" (C.II: NUN12/JUL.00066 NN, 02.07.2012, S. 20)

(656') 'sehr geil'

Die Korpusanalyse hat außerdem den Gebrauch von unmenschlich in positiver Bedeutung zu Tage gefördert, vgl.:

(657) „Fernando hat einfach unmenschlich gespielt und eines seiner besten Matches gezeigt - wenn nicht das beste", analysierte der bitter enttäuschte Haas. (C.II: HMP07/JAN.02751 MOPO, 27.01.2007, S. 37)

(657') 'sehr gut gespielt'

Die meliorative Entwicklung der Bedeutung in (657) demonstriert wiederum den kompletten Verlust des Zusammenhangs mit der Ausgangsbedeutung.

\subsubsection{Kurzfassung der Entwicklung bei unmenschlich}

1. Ausgangsbedeutungen als Quelle der Gradbedeutung: 1. Bezug auf 'rohes, grausames menschliches Verhalten', 2. 'Unchristlichkeit', 3. 'Menschenunwürdigkeit';

6. Meliorative Verwendung;

die übrigen Entwicklungsschritte wie oben in Kap. 3.6.3.9.3.

\subsubsection{Unverschämt}

\subsubsection{Lexikographische Angaben}

Laut Pfeiffer (s.v.) ist das Lexem im Spätmittelhochdeutschen in der Form unverschamet in der Bedeutung „ohne Schamgefühl, frech“ in Erscheinung getreten.

1. unveschämt in Lexer (s.v. un-verschamet)

keine lexikographische Beschreibung vorhanden

2. unveschämt in FWB

keine Angaben, auch nicht im bereits zitierten Material der Redaktionsstelle von FWB

3. unverschämt in DWB (s.v.)

3.1 "ohne scheu“

3.2 „ohne scham in bezug auf geschlechtliche ehrbarkeit, wohlanständigkeit, sittlichkeit"

3.3 „im fordern u. nehmen; 'die billige und wohlanständige genügsamkeit in hohem grade verletzend'“

3.4 „allgemein; perfrico frontem vel faciem, pudorem exuo, pudet nihil“

3.5 „steigernd“ 
4. unverschämt in DUDEN (2012, s.v.)

4.1 „sich mit aufreizender Respektlosigkeit über die Grenzen des Taktes und des Anstandes hinwegsetzend (und die Gefühle anderer verletzend)“

4.2 „(umgangssprachlich) das übliche Maß stark überschreiten“

4.3 „>intensivierend bei Adjektiven> (umgangssprachlich) überaus, sehr"

\subsubsection{Die Entwicklung der Gradbedeutung bei unverschämt}

Obgleich FWB keine Angaben über unverschämt bietet, lässt die Beleglage in den untersuchten Korpora und Wörterbüchern die Annahme zu, dass das Einsetzen der Gradbedeutung in frühneuhochdeutscher Zeit stattgefunden hat. Als Basis dafür gilt die Sinnrichtung, die mit der Schamlosigkeit der Personen und ihrer Handlungen in Bezug auf ihre Sittlichkeit verbunden ist, z.B. unverschämter Junge, unverschämte Wendung, unverschämter Ton (DWB, s.v.), vgl. außerdem die aus DTA entnommenen Belege:

(658) Vnd Radirobanes fieng sich auch allbereit an zufürchten / nicht allein daß vielleicht sein Herold wegen deß vnverschämten Schreibens mit der Haut bezahlen müssen / sondern auch / daß auch wol Sicilien wieder seine Schiffe auffgeboten würde [...] (DTA: Barclay, John (Übers. Martin Opitz): Johann Barclaÿens Argenis Deutsch gemacht durch Martin Opitzen. Breslau, 1626)

(659) Bißweiln kamen die jenige / welchen er schuldig ware / begerten jhr Gelt / vnnd setzten jhm hart zu / aber er schickete si wider mit einer solchen vnverschämpten Manier nach Hauß / daß sie zum zweytenmal nicht wider dorfften kommen [...] (DTA: [Calvi, François de]: Beutelschneider, oder newe warhaffte vnd eigentliche Beschreibung der Diebs Historien. [Bd. 1]. Frankfurt (Main), 1627)

(660) Ich wil euch allhier nicht mehr als nur ein eintziges von seinen bubenstücken erzehlen / auff daß jhr darauß selber sehet / was er für ein vnverschämbte Gast mag seyn gewesen [...]. (DTA: Calvi, François de: Beutelschneider / Oder Neue / warhaffte / und eigentliche Beschreibung Der Diebs Historien. Bd. 2. Frankfurt (Main), 1627)

Aus der Analyse der Beleglage lässt sich schließen, dass das Einsetzen der Gradbedeutung in mehrdeutigen Kontexten infolge ihres pragmatic enrichment bzw. strengthening durch pragmatic inferencing erfolgte, vgl.:

(661) die grosze, unverschamete geitzigkeit (DWB: 1592, SснüтZ historia rer. pruss.)

(662) Es war allbereit der fünffte Tag / feidt die Königin / heimlicher vnnd allgemeiner Sorgen halben / kaum etwas von Speife zu fich genommen hatte. Dann als Radirobanes auff erlangeten Spott wegen vnverfchåmpter Vbelthat wieder die Argenis nach Calaris zurück gelanget war / vnd die Schande feines bo̊fen Anfchlages erwogen hatte / beforgte er fich / feine Vnterthanen mo̊chten ins künfftig fo viel auff jhn meh znicht halten [...] (DTA: Barclay, John (Übers. Martin Opitz): Johann Barclaÿens Argenis Deutsch gemacht durch Martin Opitzen. Breslau, 1626) 
(663) Wann ein Diener auff seines Herrn Befehl etwas gutes und löbliches verrichtet [...] ist es lobenswert; aber die Bosheit und übeltaht mu $\beta$ so wol an dem Knechte / der sie verrichten hilfft / als an dem Herrn / der sie anstifftet / gestraffet werden; wiewol man sich hierüber mit dir einzulassen nicht gesinnet ist / sondern weil du nicht leugnen kanst / was vor grosse und unverschämte Beschimpfung du nichtwerter Tropf dem Großmächtigsten herschenden Groß Fürsten der Teutschen durch Verrähterey / Meinäid / und schändliche eigentähtliche Beleidigung angetahn hast / soltu einen kurzen Abtrit nehmen / und deiner wolverdienten rechtmässigen Urtel gewärtig seyn. (DTA: Bucholtz, Andreas Heinrich: Des Christlich: Teutschen Königes Herkules und der Teutschen Königin Valiska Wunder-Geschicht. Bd. 2. Braunschweig, 1660)

(664) Weil dann Dropions abermahlige Gesandschafft [...] sich angab / nebest Vermeldung / daß man wegen Mangel des nöhtigen Unterhalts / des Feindes Bodem hätte verlassen / und sich zurük zihen müssen [...]; welches dieser [König] mit entsetzen und grossem Herzensprast anhörete / und nebest seinen Vertraueten beklagete / Dropions verwägener Frevel und unverschämter Ehrgeiz nach der Böhmischen Kron / würde ganz Pannonien ins Verderben stürzen. (DTA: Bucholtz, Andreas Heinrich: Des Christlich: Teutschen Königes Herkules und der Teutschen Königin Valiska Wunder-Geschicht. Bd. 2. Braunschweig, 1660)

(665) Erfuhr ich ihre frechen und unverschämten Lästerungen, bediente ich mich einer großmüthigen Standhaftigkeit dagegen. (C.II: HK4/Z04.00001 Ziegler: Vermischete Schriften in gebundener und ungebundener Rede, Erstdruck: 1739, 2004 [S. 519])

(666) Er ist so unverschämt zudringlich, daß man sich seiner gottlosen Künste gar nicht erwehren kann, wenn man sich zum Spaß einmal mit ihm einläßt [...]. (HK3/F11.00001 Wezel: Hermann und Ulrike, Erstdruck: 1780, 2000 [S. 713])

(667) Nachdem die versammelten Untertanen eine halbe Stunde gewartet und die Hanswürste ihre Trummeln und ihren Witz müdegeplagt hatten, erschien [...] die Dame in einem rosenroten Kleide mit silbernen Blumen[...]: die bretterne gesenkte Brust war so unverschämt entblößt, daß kein Sterblicher ohne Ekel hinzuschauen vermochte. (HK3/F11.00001 Wezel: Hermann und Ulrike, Erstdruck: 1780, 2000 [S. 681])

(668) Alle Aufgaben auflösen und alle Fragen beantworten zu wollen, würde eine unverschämte Großsprecherei und ein so ausschweifender Eigendünkel sein, daß man dadurch sich so fort um alles Zutrauen bringen müßte. (C.II: HK5/A89.00001 Kant: Kritik der reinen Vernunft, Erstdruck: 1781, 2000 [S. 450])

Aus den in (661) - (668) eingeführten Belegen geht hervor, dass die Bedeutung von unverschämt eine mehrdeutige Interpretation bezeugt. Die Ausgangsbedeutung verweist darauf, dass die in Rede stehende Person schamlos bzw. ohne Schamgefühle zu haben handelt, da ihre Handlungen in gewisser Hinsicht die Gefühle anderer verletzten, frech sind usw. Darüber hinaus überschreitet ihr Verhalten die sittlichen Grenzen des Gestatteten. Von daher impliziert die Verwendung 
des Lexems eine Skala der Schamlosigkeit, die die jeweiligen Situationen je nach der Ausprägung dieses Merkmals entsprechend einstuft. Die Beleglage lässt den Schluß zu, dass die Sachverhalte einen hohen Grad aufweisen, der als zusätzlicher Grund für die Bezeichnung mit „schamlos“ dienen kann, sodass die folgenden Umformulierungen gelten: 'Die Geitzigkeit (661) / Übeltat (662) / Beschimpfung (663) / Ehrgeitz (664) / Lästerungen (665) / Zudringlichkeit (666) sowie Blöße (667) und Großsprecherei (668) sind in so hohem Grad ausgeprägt, dass es die gestatteten sittlichen Grenzen überschreitet und infolgedessen schamlos ist'. Ferner folgt, dass die Bedeutung durch die Intensivierung eine einstellungsbezogene Bedeutungskomponente erwirbt, die auf Traugott I (oben 3.6.3.4) verweist.

Der Prozess der Desemantisierung, der auf einer früheren Etappe der Bedeutungsentwicklung zunächst mit pragmatic enrichment der Ausgangsbedeutung verbunden ist, beginnt mit dem allmählichen Verlust des direkten Bezugs zur Ausgangsbedeutung. Dabei gewinnt die Intensivierung an Gewicht und tritt in den Vordergrund, während die Ausgangsbedeutung eine Nebenrolle zu spielen beginnt bis sie allmählich völlig verblasst. COSMAS liefert reichlich Belege, die die Koexistenz der beiden Sinnrichtungen demonstrieren. Insbesondere in der Gegenwartssprache findet sich solcher Gebrauch, vgl.:

(669) „Der HSV als abgebender Verein hat unverschämt hohe Forderungen gestellt, die wir nicht erfüllen. [...]" (C.II: BRZ07/AUG.11787 Braunschw. Z., 09.08.2007)

(670) Fliegen ist absurd. Wenn man da sitzt, eingepfercht in die unverschämt engen Reihen, es brummt und schaukelt, und die Sitznachbarn nippen gelassen an ihrem Tomatensaft, als könne sie die trostlose rote Suppe vor allem Übel dieser Welt beschützen: Dann kann an diesem Gedanken eigentlich kein Weg vorbeiführen. (C.II: HMP09/JAN.00977 MOPO, 17.01.2009, S. 2)

(671) Warum mag ich keine Livekonzerte? [...] Weil Band-T-Shirts am Verkaufsstand unverschämt teuer sind. (C.II: HMP07/NOV.02164 MOPO, 27.11.2007, S. 8)

Die Belege (603) - (605) liefern genügend Evidenz dafür, dass unverschämt in intensivierender Funktion gebraucht wird. Jedoch wird der Bezug zur Ausgangsbedeutung nicht vollständig beseitigt. Im Gegenteil, man verweist auf die Frechheit des Vereins in (669), der Fluggesellschaft in (670) und der Konzertveranstalter in (671), die sich an allzu hohen Forderungen, engen Sitzreihen und teuren T-Shirts ablesen lassen. Weil die Frechheit die für die betroffenen Personen akzeptablen sittlichen Grenzen überschreitet und sie mit einer großen Störung belastet bzw. in einer gewissen Hinsicht verletzt, werden die Urheber und - metonymisch - die mit ihnen verbundenen Handlungen als unverschämt bezeichnet.

Die Mehrdeutigkeit des Gebrauchs bezieht sich außerdem auf die quantifizierende Funktion, indem die durch das Bezugswort auszudrückende Menge der Sachverhalte auf die Frechheit der entsprechenden Verantwortungspersonen hinweist und eine Empörung seitens des Schreibers bzw. Sprechers hervorruft. Damit mag die Ausgangsbedeutung nicht vollkommen abgetrennt sein, vgl.: 
(672) An jeder Zollstation nehmen sie den Bauern unverschämte Steuern ab, allen voran Lord Sarn. Ein Drahtzieher und Bastard, versoffener Exzentriker und hinterhältiger Politiker. (C.II: NUN92/MAR.01435 NN, 19.03.1992, S. 27)

(672) 'sehr hohe und dadurch Empörung hervorrufende Steuern'

(673) Erst mußte die Schülerin vier Stunden im Auto verbringen, um zur Nature One zu kommen, und dann das: „Totale Abzockerei des Veranstalters, nirgends kann man sich die Hände waschen und dazu unverschämte Preise." (C.II: RHZ96/AUG.10110 RZ, 19.08.1996)

(673') 'sehr hohe und dadurch Empörung hervorrufende Preise'

In der nächsten Entwicklungsphase wirkt die Ausgangsbedeutung verblasst. Die Gradbedeutung weist keinen direkten Zusammenhang mit der Ausgangsbedeutung mehr auf bis auf die negativen Konnotationen der Kollokationspartner, die den fortgeschrittenen, jedoch unvollendeten Prozess von bleaching demonstrieren, vgl.:

(674) Glücklicherweise versteckte sich der Eroberer in einen hohlen Baum, als der Wind so unverschämt zu blasen anfing, und errettete sich dadurch vom Frost und vom Schwerte der Feinde. (C.II: HK3/F28.00001 Wezel: Kakerlak oder die Geschichte eines Rosenkreuzers, Erstdruck: 1784, 2000 [S. 30])

(674') 'sehr stark blasen'

(675) Kerl! wo hast du die unverschämt schiefe Nase her? (C.II: HK3/HK1.00186 Tieck: Die Gemälde, Erstdruck: 1822, 2000 [S. 68])

(675') 'sehr schiefe'

(676) „Nun muß ich heim - da wartet noch ein tüchtiges Stück Arbeit auf mich. Und nebenbei muß ich noch plätten - die arme Kranke soll und muß morgen frischgewaschene Bettgardinen haben; aber ich bin mit meinem Tannenzapfenvorrat zu Ende" - ein Lächeln huschte wie Sonnenlicht über ihr Gesicht - „und da habe ich den unverschämt großen Korb da mitgebracht." (C.II: HK4/M08.00001 Marlitt: Thüringer Erzählungen: Amtmanns Magd, Erstdruck: 1881, 2004 [S. 53])

(676') 'sehr großen'

(677) Es gibt Wörter, die schreibt man am liebsten nur einmal. Sei es, dass sie unverschämt kompliziert zu schreiben sind (man denke nur an Desoxyribonukleinsäure) oder weil sie recht lang sind wie etwa Energieverbrauchskennzeichnungsverordnung. (C.II: HAZ07/SEP.03840 HAZ, 13.09.2007, S. 23)

(677') 'sehr kompliziert'

(678) Während die Bonbons und Lollis früherer Kindertage meist knallfarben, klebrig und unverschämt sü $\beta$ daher kamen, hat sich die Süßwarenindustrie dem neuen ernährungsbewussten Trend rechtzeitig angepasst. (C.II: M00/AUG.46075 Mannh. Morgen, 12.08.2000)

(678') 'sehr süß'

Unverschämt kann außerdem als Gradadverb Skopus über Funktionsverbgefüge haben und dadurch die Erweiterung seiner Skopuskonstituente demonstrieren. 
Die Intensivierung erfolgt dadurch, dass die FVGs durch einen graduierbaren Begriff wiedergegeben werden können, vgl.:

(679) Meine Kur zieht sich unverschämt in die Länge, ich soll noch wenigstens acht Tage hier bleiben. (GB, 02.07.2014: http://books.google.de/books?id$=\mathrm{kWgTAAAAQAAJ} \& \mathrm{q}=\% 22$ unversch $\% \mathrm{C} 3 \% \mathrm{~A} 4 \mathrm{mt}+\mathrm{in}+\mathrm{die}+1 \% \mathrm{C} 3 \% \mathrm{~A} 4 \mathrm{nge} \% 22 \& \mathrm{dq}=-$ \%22unversch\%C3\%A4mt+in+die+1\%C3\%A4nge\%22\&hl=de\&sa=X\&ei=zsOzU6uLHIjiPPzogOAP\&ved=0CCgQ6AEwAg)

(679') 'verzögert sich sehr'

(680) Es gibt keine andere Serie, in der mir irgendein Darsteller so unverschämt auf die Nerven geht wie Carla. (02.07.2014: http://de.scrubs.wikia.com/wiki/Benutzer:Jizztoph)

(680') 'nervt sehr'

Die Belege (674) - (680) demonstrieren neben dem Verlust des Zusammenhangs mit der Ausgangsbedeutung außerdem einen Zuwachs an subjectivity und verweisen auf das Auftreten von Traugott III (oben 3.6.3.4).

Die anschließende Etappe des Entwicklungsgangs der Intensivierung bezieht sich auf den kompletten Verlust des Zusammenhangs mit der Ausgangsbedeutung. Dies lässt sich in den Verwendungen mit positiv konnotierten Kollokationspartnern erkennen, vgl.:

(681) Wärmende Sonnenstrahlen blinzeln durchs Geäst, die Schritte werden schneller, beschwingter: Wir erreichen Kaiuma Saddle, 387 Meter über dem Meer gelegen - ein unverschämt attraktiver Platz für eine Brotzeit mit Aussicht. (M05/APR.26087 Mannh. Morgen, 02.04.2005)

(681') 'sehr attraktiver'

(682) Nun war aber für das vorige Wochenende so unverschämt gutes Wetter angesagt - und hatte Tage zuvor bereits einen so eindeutigen Vorgeschmack gegeben -, dass ich für Samstag noch einmal Grillen plante [...]. (C.II: BRZ05/NOV.10751 Braunschw. Z., 01.11.2005)

(682') 'sehr gutes'

(683) Die italienische Küche ist auf ganz unaufgeregte Art hochwertig. Es gibt etwa gebackenen Ziegenkäse auf Ruccola und Feigen, Penne mit Rinderfiletstreifen und Salbeibutter, Rindercarpaccio mit Parmesan und Trüffeln, hausgemachte Nudeltaschen, getrüffeltes Steinbuttfilet, Kalbsfilet in Cognacsauce und unverschämt cremige Nachspeisen. (C.II: HMP06/MAR.01509 MOPO, 16.03.2006, S. 27)

(683') 'sehr cremige'

(684) Der Blick durch die meterhohe Fensterfront ist unverschämt schön. Türkisblau schimmert die Außenalster im Sonnenlicht. (C.II: HMP06/JUL.01397 MOPO, 13.07.2006, S. 27)

(684') 'sehr schön'

(685) So hübsch. So jung. Und so unverschämt talentiert: Die Britin LAURA MARLING brachte mit gerade einmal 23 Jahren kürzlich ihr drittes Album, "A Creature I Don't Know", heraus. (C.II: HMP12/MAR.01995 MOPO, 22.03.2012, S. 25)

$(685$ ') 'sehr talentiert' 


\subsubsection{Kurzfassung der Entwicklung bei unverschämt}

Ausgangsbedeutung: 'ohne scham in bezug auf geschlechtliche ehrbarkeit, wohlanständigkeit, sittlichkeit';

die übrigen Entwicklungsschritte wie oben in Kap. 3.6.3.9.3.

\subsubsection{Wütend}

\subsubsection{Lexikographische Angaben}

Das Wort wütend ist in historischen Wörterbüchern nicht belegt, tritt jedoch Anfang des 17. Jh. in COSMAS und DTA in Erscheinung.

1. wütend in FWB

keine Angaben, auch nicht im bereits zitierten Material der Redaktionsstelle von FWB

2. wütend in DWB (s.v. wüten)

Das Lexem bezeichnet „den inneren erregungszustand“

2.1a „wütend in attributiver verwendung und substantiviert; von menschen und tieren im zustand heftigster, maszloser zorneserregung“; „gebärden, blick, miene, stimme als in höchstem grade zornerfüllt kennzeichnend"

$2.1 \mathrm{~b}$ „in prädikativer verwendung wütend sein, werden, machen“

2.1c „in 'adverbiellem' gebrauch, die psychische verfassung des handelnden und zugleich die art des handlungsablaufs kennzeichnend“

3. wütend in DUDEN (2012, s.v.)

3.1 „voller Wut, durch Wut erregt"

3.2 "außerordentlich groß, heftig"

\subsubsection{Entwicklung der Gradbedeutung bei wütend}

Obgleich DWB keine Angaben über die Gradbedeutung von wütend bietet, lässt die Korpusanalyse annehmen, dass deren Einsetzen im frühen Neuhochdeutschen erfolgte. Als Grundlage dafür gilt die in 2 angegebene Sinnrichtung, die den inneren Zustand von Menschen und Tieren bezeichnet, vgl.:

(686) Der tolle Cerberus als er den Tag empfande Ward wütendt vnd ergrimmt / rieß drey mal von dem Bande / Ließ ftehn das Thor von Stahl fo feiner huet vertrawt/That feinen rachen auff / vnd heulet'vberlaut. (DTA: Opitz, Martin: Teutsche Pöemata und: Aristarchvs Wieder die verachtung Teutscher Sprach. Straßburg, 1624)

(687) er wurde ... von der wütenden menge zertrampelt (DWB: s.v. wüten)

(688) während der blonde ... mit wütender stimme die zahlworte ausrief (DWB: s.v. wüten)

(689) er erschrak fürchterlich, als er die wütende antwort hörte (DWB: s.v. wüten)

(690) du bist ja besoffen! wütend stiesz sie ihn zurück (DWB: s.v. wüten)

Die Herausbildung der Gradbedeutung des Lexems ist zunächst mit der Ausgangsbedeutung verbunden, die sich auf mit Wut erfüllte Menschen und Tiere und ihr Verhalten bezieht. Die Intensivierung ergibt sich in ambigen Kontexten durch 
pragmatic inferencing infolge semantic repetition und pragmatic enrichment bzw. strengthening des Ausgangsgehalts, vgl:

(691) Jn dem Thierfpiel pflegt man jrgend ein Pantherthier / an eine Pupp / fo dem Menfchen gleich fihet / zu hetzen / wie grimmig gehen alsdann die Leut mit der armen Puppen vmb? wie zerfätzten vnd zerlumpen fie diefelbe; darauß månniglichen abnemen kan / welchen wütenden Grimm vnd Zorn das Thier wider den Menfchen trage / fo fie daffelbe Bild alfo jåmmerlich tractiren? (DTA: Dannhauer, Johann Conrad: Catechismus Milch. Bd. 4. Straßburg, 1653)

(692) Plinius fchreibt von dem Ko̊nig Boccho, daß als er auß wütendem Zorn dreyfig Perfonen / die er an Pfäl angebunden / fo viel / nemlich dreyfig Elephanten fürgeworffen / diefelbe zu martern vnd zu verzehren / ob man fie fchon gehetzt / fo haben fie fich doch nicht wollen brauchen laffen zu inftrumenten / oder zum dienft folcher vnmenfchlichẽ blutde̊rftigkeit; (DTA: Dannhauer, Johann Conrad: Catechismus=Milch. Bd. 5. Straßburg, 1654)

(693) Vieles krankhafte Volk gab es immer unter Denen, welche dichten und gottsüchtig sind; wüthend hassen sie den Erkennenden und jene jüngste der Tugenden, welche heisst: Redlichkeit. (DTA: Nietzsche, Friedrich: Also sprach Zarathustra. [Bd. 1]. Chemnitz, 1883)

(694) Vor allem war das der wütende Hass gegen Preußen, was die bureaukratischen, die demagogischen und die aristokratischen Kräfte dieser Partei zusammenhielt. (DTA: Treitschke, Heinrich von: Deutsche Geschichte im Neunzehnten Jahrhundert. Bd. 5: Bis zur März-Revolution. Leipzig, 1894)

(695) Stilpen erfaßte ein wütender Zorn: Also auch dieser Häring seufzt! (C.II: HK3/G40.00001 Bierbaum: Stilpe. Ein Roman aus der Froschperspektive, Erstdruck: 1897/2000, [S. 281])

(696) „Aber ich bin sparsam wie alle früheren Vertreter unserer Firma, und über hinausgeworfenes Geld kann ich mich wütend ärgern. [...]" (C.II: HK4/M04.00001 Marlitt: Die Frau mit den Karfunkelsteinen, Erstdruck: 1885, 2004, [S. 122])

Die Belege (691) - (696) bezeugen zunächst die Ausgangsbedeutung des Lexems, insofern als die genannten Personen Wut neben Grimm, Hass, Zorn und Ärger empfinden. In dem adjektivischen Gebrauch (694) - (695) kann außerdem metaphorische Verwendung angenommen werden, die durch Personifizierung der Begriffe Hass und Zorn sowie durch personifizierten Gebrauch der Verben zusammenhalten und erfassen ermöglicht wird. Gebrauch lässt außerdem eine auf Intensität zielende Nebenbedeutung zu. Diese Interpretation erfolgt durch das Wiederholen von Inhaltszügen in den Kollokationspartnern, i.e. semantic repetition. Durch semantic redundancy wird der Ausdruck verstärkt und die intensivierende Funktion von wütend hervorgehoben.

Semantic repetition erweist sich außerdem in den Verbindungen mit Bezugswörtern, die erbitterte Konflikte bezeichnen:

(697) Die Schiffe hingen bald mit ihrem Tauwerk aneinander fest; das wütende Gefecht ward immer enger und enger. (C.II: HK3/F16.00001 Hölderlin: Hyperion oder der Eremit in Griechenland, Entstanden: 1796/1798, 2000, [S. 129]) 
(698) Welche fchreckliche Schaufpiele, gegen die unfere Wafferfluthen und Erdbeben, gegen die der Tod von Taufenden in einer wüthenden Schlacht nur als Poffenfpiele erfcheinen. (DTA: Littrow, Joseph Johann von: Die Wunder des Himmels, oder gemeinfaßliche Darstellung des Weltsystems. Bd. 3. Stuttgart, 1836)

(699) Bald war eine wütende Prügelei im Gang, die Bundesgenossen trugen trotz ihrer geringen Zahl den Sieg davon und machten ein paar Gefangene, die übrigen entflohen unter zornigen Drohreden. (C.II: HK4/R01.00001 Reventlow: Ellen Olestjerne, Entstanden: 1900-1902 2004, [S. 32])

In (697) - (699) handelt es sich zunächst um Konfliktsituationen, die Wut seitens der Teilnehmer voraussetzen und von daher dem Adjektiv den auf die Ausgangsbedeutung bezogenen Gebrauch zuschreiben. Außerdem kann in den Verwendungen die intensivierende Nebenbedeutung des Lexems gesehen werden. Diese Annahme lässt sich dadurch untermauern, dass die Bezugswörter Extremwerte aufweisende Begriffe verkörpern, die gewöhnlich mit negativen, feindlichen Gefühlsausbrüchen verbunden sind, vgl. Definitionen aus DUDEN (2012):

(697') Gefecht „kurzer, bewaffneter Zusammenstoß feindlicher militärischer Einheiten, Auseinandersetzung von kürzerer Dauer zwischen bewaffneten Gruppen";

(698') Schlacht „heftiger, längere Zeit anhaltender [...] Kampf zwischen größeren militärischen Einheiten“;

(699') Prügelei „das Sichprügeln; Schlägerei“, Schlägerei „heftige, oft brutale tätliche Auseinandersetzung zwischen zwei od. mehreren Personen“.

Durch die Verbindung dieser Bezugswörter mit wütend kommt es zu der Verstärkung des Gebrauchs, und entwickelt sich wütend zum Intensivierer.

Metaphorisierung wirkt ebenso bei der Herausbildung der Gradbedeutung mit. In (700) - (702) soll der rein metaphorische Gebrauch des Lexems demonstriert werden:

(700) Es ift beffer / daß ein Menfch fterbe für das Volck / dann das gantze Volck verderbe; Es fey wåger und beffer / Jonas werde ins wütende Meer geworffen / als daß das gantze Schiff zerfcheitere. (DTA: Dannhauer, Johann Conrad: Catechismus-Milch. Bd. 6. Straßburg, 1657)

(701) Der Ozean um uns her war wütend und schien über die Keckheit einer Handvoll Menschen, die es mit ihm aufnahmen, ganz erbost zu sein. (DTA: G. Forster: Johann Reinhold Forster's [...] Reise um die Welt während den Jahren 1772 bis $1775: 1778$ )

(702) Mein banges Herz vermag dies nicht zu fassen, Es tobt wütend, und ich erliege fast, Unter dieser schweren Centner Last. (DTA: C.A. Kortum: Die Jobsiade. Ein komisches Heldengedicht in drei Theilen 1799)

In (700) - (702) werden die unbelebten Objekte Meer, Ozean und Herz dadurch mit einem Menschen verglichen, dass ihnen menschliches Verhalten zugeschrieben wird, welches in Gefühlsausbrüchen u.a. seine Ausprägung findet. Durch die Per- 
sonifizierung der Bezugswörter erlangt wütend ebenso eine metaphorische Interpretation und verliert damit den direkten Zusammenhang mit der primären Bedeutung.

Aus der Beleglage folgt, dass manche Kontexte Intensivierung neben dem metaphorischen Gebrauch des Adjektivs bzw. Adverbs durch pragmatic inferencing aufweisen. Die semantische Reanalyse des Kontexts kommt zur Geltung, wenn wütend sich z.B. auf Witterungserscheinungen wie Wind, Sturm und Orkan u.a. bezieht. Die entstandene Nebenbedeutung setzt Intensivierung voraus, wie es in (703) - (707) exemplarisch gezeigt wird:

(703) Das arme Schiff wurde dergeftalten von denen wüttenden Wellen geriben / getriben / daß alle Schifffahrende den gegenwertigen Todt vor Augen fahen / maiften thails hatten alle derentwegen kein Hoffnung mehr außzukommen / weilen fie die fchwårefte Truhen / vnd Våffer / vnd andere Sachen in das Meer geworffen / vnd dannoch hierdurch das Schiff nit geringert worden. (DTA: Clara, Abraham a Sancta: Judas Der Ertz-Schelm. Bd. 1. Salzburg, 1686)

(704) Endlich ward der Wind so wütend, daß er uns vollends das einzige Seegel zerriß, welches wir noch aufgespannt zu lassen gewagt hatten. (DTA: Forster, Georg: Johann Reinhold Forster's [..] Reise um die Welt. Bd. 1. Berlin, 1778)

(705) Plötzlich überfiel uns ein abermahliger Sturm, der aus Westen blies. Er trieb uns wüthend von dem festen Lande weg und warf uns zur Nachtzeit, ohnweit einer Infel, auf Felfen. (DTA: Campe, Joachim Heinrich: Robinson der Jüngere. Bd. 2. Hamburg, 1780)

(706) Aber [...] welche Raserey, den Hund zu ihm zu lassen, dessen wütige Art man kennt, und welcher vermuthlich schlecht gefüttert worden seyn mu $\beta$; denn nichts als wütender Hunger konnte ihn reizen, seinen Herrn anzufallen. (C:II: HK4/N03.00001 Naubert, Benedikte: Alf von Dülmen, Erstdruck: 1791, 2004, [S. 439])

(707) Das Erdbeben hatte die Grundvefte der Kapelle erfchüttert, und der wüthende Sturm fie ins Thal hinab gefchleudert. (DTA: Spiess, Christian Heinrich: Biographien der Wahnsinnigen. Bd. 4. Leipzig, 1796)

Die Kontexte (703) - (707) erfahren eine doppeldeutige Interpretation. Wellen in (703), Wind in $(704)$, Sturm in $(705,707)$ und Hunger in $(706)$ werden zunächst durch Personifizierung als wütende Personen bzw. aktive Täter dargestellt, die bestimmte Zerstörungen mit negativen Folgen bewirkt haben. Die Personifizierung von Objekten wird durch die Metaphorisierung des Kontexts bekräftigt, was sich hier an den Bezugsverben reiben, treiben, zerreißen, schleudern, überfallen, treiben und reizen, die ebenso in einer metaphorischen Verwendung auftreten, erkennen lässt.

Eine alternative Interpretation bezieht sich auf die Intensität der Sachverhalte und erfolgt durch das Ableiten der entsprechenden Inferenz. Darüber hinaus lässt sich aus dem Kontext schließen, dass die Geschehnisse das Resultat der großen Wellen bzw. des in hohem Maße ausgeprägten Winds oder Sturms darstellen, wobei die Intensität des Unwetters die Ursache der negativen Folgen verkörpert, vgl. die Umformulierungen: 
(703') 'Die Wellen waren so groß, dass sie das Schiff derart gerieben und getrieben haben, sodass alle Schifffahrenden Angst bekommen haben und die Hoffnung auf die Rettung aufgaben'.

(704') 'Der Wind wurde so stark, dass er das einzige Segel zerriß'.

(705') 'Der Sturm so war intensiv, dass wir vom festen Land wegziehen mussten'.

(706') 'Nichts als starker Hunger konnte ihn reizen, seinen Herrn anzufallen'.

(707') 'Das Erdbeben hatte die Grundfeste der Kapelle erschüttert, und der starke Sturm sie ins Tal hinab geschleudert'.

Es ist außerdem zu erwähnen, dass der Gebrauch eine persönliche Bewertung, i.e. eine evaluierende und einstellungsbezogene Verwendung seitens des Sprechers bzw. Schreibers, darstellt und daher auf das Einsetzen von subjectivity und demzufolge von Traugott I (oben 3.6.3.4) hinweist.

Ferner fungiert die Gradbedeutung von wütend insofern als ziemlich fortgeschritten, als das Lexem keinen Zusammenhang mit der Ausgangsbedeutung erkennen lässt. Jedoch bezieht es sich auf negativ konnotierte Situationen und kann dadurch keine vollkommene Bedeutungsentleerung demonstrieren, vgl.:

(708) Luise hatte wütende Kopfschmerzen, jeder Stoß des Wagens entriß ihr einen lauten Schrey. (C.II: HK4/H12.00001 Huber, Therese: Luise, Erstdruck: 1796, 2004, [S. 83])

(708') 'sehr starke Kopfschmerzen'

(709) Der grelle Lichtschein ließ die umstehenden Gebäude im Spiel der Flammen schwanken und tanzen. Trotz der Entfernung konnte Kahlan die wütende Hitze auf dem Gesicht spüren. Brennendes Gras und Funken stoben wirbelnd in die Nacht. 03.07.2014: http://books.google.de/books?id$=\mathrm{kAzzzR} 5$ HswoC\&pg=PT105\&dq=\%22w\%C3 \%BCtende+hitze\%22\&hl=de\&sa=X\&ei=OBa1U422H8XFOcjOgcgJ\&ved=0CD8Q6AEwBTgU\#v=onepage\&q=\%22w$\%$ C3\%BCtende $\% 20$ hitze $\% 22 \& f=$ false)

(709') 'sehr große Hitze'

(710) je mehr ich die alten Pläne meines Vaters in meiner Art verfolgte, desto mehr schloß sich Amalia vor mir ab, [...] den Knechten im Herrenhof war ich ein Spielzeug, das zu zerbrechen sie sich wütend anstrengten [...] (C.II: HK3/D01.00001 Kafka, Franz: Das Schloß, Entstanden: 1922, 2000, [S. 327])

(710') 'sich sehr anstrengten'

Jedoch bleiben die Kookkurrenzmöglichkeiten von wütend als Intensivierer ziemlich beschränkt: Es gibt nur wenige Bezugswörter, mit denen es vorkommt, es modifiziert keine Funktionsverbgefüge, und es übernimmt keine quantifizierende Funktion.

Das Auftreten des Lexems in intensivierender Funktion in (708) - (710) demonstriert neben dem Verlust des Zusammenhangs mit der Ausgangsbedeutung außerdem den Zuwachs an subjectivity und verweist auf das Auftreten von Traugott III (oben 3.6.3.4).

Die Desemantisierung des Lexems erfolgt anschließend durch das Auftreten von wütend in Verbindungen mit positiv konnotierten Kollokationspartnern. Wie in dem oben beschriebenen Fall mit grimmig finden sich nur wenige Belege mit rein 
intensivierender Funktion von wütend, wobei sie aus dem 19. Jh. stammen und für die Gegenwartssprache eine unübliche Verwendung darstellen, vgl. die Belege (711) - (712) mit entsprechenden Umformulierungen:

(711) Er predigte von Himmel und Hölle und geriet zuweilen in die wütendste Begeisterung. (C.II: HK3/G17.00001 Heine: Reisebilder. Vierter Teil, Erstdruck: 1830, 2000, [S. 385])

(711') 'stärkste Begeisterung'

(712) Der Mann aber war der Förster des Orts, der früher selbst das Gymnasium frequentiert, und seitdem eine wütende Vorliebe für Studenten hatte. (C.II: HK3/D55.00001 Eichendorff: Dichter und ihre Gesellen, Erstdruck: 1834, 2000, [S. 309])

(712') 'starke Vorliebe'

\subsubsection{Kurzfassung der Entwicklung bei wütend}

1. Ausgangsbedeutung: 'voller Wut und zornig';

5. Korpusanalyse: keine quantifizierende Funktion; die übrigen Entwicklungsschritte wie oben in Kap. 3.6.3.9.3.

\subsection{Wortgruppe 'Sich wie ein Tier verhaltend'}

Das vorliegende Kapitel widmet sich der Herausbildung der Gradbedeutung in den Lexemen bestialisch, tierisch und viehisch. Die intensivierende Funktion der Adjektive bzw. Adverbien kann durch die Verwendungen (a) - (c) exemplifiziert werden:

(a) sich bestialisch freuen 'sich sehr freuen'

(b) tierisch kreativ 'sehr kreativ'

(c) viehisch interessant 'sehr interessant'

Die Ausgangsbedeutung der genannten Grad-Adjektive bzw. -Adverbien entspricht der Kategorie 10, d.h. „Fühlen, Affekte, Charaktereigenschaften“, in der Klassifizierung von Dornseiff (2004: 188ff.) und bringt die Begriffe „Übel wollen“ und „Härte“ zum Ausdruck. Die Korpusanalyse hat ergeben, dass die in diesem Kapitel untersuchten Wörter bestialisch, tierisch und viehisch ihre Gradbedeutung aus der auf rohe, brutale Personen bezogenen Ausgangsbedeutung entwickelt haben. Diese als Basis für das Einsetzen der Intensivierung dienende Bedeutung gehört zu derselben semantischen Gruppe 10, zu der die in dem Kapitel „Übel wollend / verursachend" betrachteten Lexeme gehören. In diesem Zusammenhang wurde die Beschreibung der im vorliegenden Kapitel behandelten Lexeme nach dem entsprechenden Kapitel eingeordnet, in dem die Intensitätsbedeutung einen ähnlichen Entwicklungsgang aufweist.

Biedermann (1969) berücksichtigt die zu untersuchenden Wörter nicht. Jedoch wird in seiner Arbeit eine Gruppe der nach dem Motivationssem "Tier" angeordneten Lexeme dargestellt. Diese Gruppe besteht aus den Wörtern schweinisch, säuisch, saumäßig, bärig, bullig, affig, lausig und kannibalisch. Die Lemmata schweinisch, bärig und affig verzeichnen in DUDEN (2012) keine Gradbedeutung. Aus diesem Grund werden sie in der vorliegenden Arbeit nicht näher analysiert. Die Intensi- 
vierung bei bullig basiert auf der Ausgangsbedeutung der Größe bzw. den Eigenschaftskonzepten "gedrungen, massig“ (DUDEN 2012, s.v.), die der Ausgangsbedeutung der in diesem Kapitel zu untersuchenden Wortgruppe nicht entspricht. Außerdem zeigt das Lexem keinen vollständigen Prozess der Desemantisierung, weil es sich nur in den Verbindungen bullig heiß und bullige Hitze findet und keine positiv konnotierten Bezugswörter modifizieren kann. Deshalb gehe ich dem Lexem in dieser Arbeit nicht weiter nach. Aus ähnlichen Gründen wird in diesem Kapitel das Lexem lausig nicht berücksichtigt, denn die als Basis für das Einsetzen der Intensivierung dienende pejorative Bedeutung bringt keine moralischen Vorstellungen zum Ausdruck und passt von daher nicht zu der Beschreibung der in diesem Kapitel untersuchten Wörter. Außerdem findet sich das Lexem nicht in Verbindungen mit positiv konnotierten Kookkurrenzpartnern und weist damit keinen abgeschlossenen Prozess der Bedeutungsentleerung auf. Die in den Lexemen saumäßig und säuisch entwickelte Intensitätsbedeutung basiert auf der mit moralischen Vorstellungen verbundenen Ausgangsbedeutung, wie es auch in Bezug auf die in diesem Kapitel zu untersuchenden Lexeme der Fall ist. Die historischen Korpora COSMAS und DTA liefern keine Belege für saumäßig und bieten damit keine Möglichkeit für die Untersuchung von dessen Gradbedeutung. In Bezug auf säuisch handelt es sich um eine mangelhafte Anzahl von Belegen, die für eine vollständige Untersuchung unzureichend erscheinen und damit nicht genügend Evidenz für den eingeschlagenen Entwicklungsgang der Gradbedeutung liefern. Von daher werden in diesem Kapitel saumäßig und säuisch nicht diskutiert ${ }^{63}$ - Der Mangel an Belegen bietet auch keine Möglichkeit für die Untersuchung des Entwicklungsgangs der Intensitätsbedeutung in biestig und kannibalisch. So finden sich in FWB, DWDS und COSMAS keine Belege, die die intensivierende Rolle von biestig veranschaulichen können. In Bezug auf kannibalisch ist das Lexem laut Pfeifer im 16. Jh. in Erscheinung getreten. In COSMAS finden sich jedoch Belege aus der zweiten Hälfte des 18. Jh., wobei schon die erste Verwendung in der Belegliste die intensivierende Rolle des Adjektivs demonstriert und damit die Analyse des Entwicklungsgangs unmöglich macht. Deshalb werden die Lexeme biestig und kannibalisch in diesem Kapitel nicht berücksichtigt.

\subsubsection{Bestialisch}

\subsubsection{Lexikographische Angaben}

Laut Pfeiffer (s.v.) tritt das Lexem im 16. Jh. auf. Das Wörterbuch verweist auf die spätlateinische Form bēstiālis 'tierisch, wild' und afrz./frz. ${ }^{64}$ bestial 'tierisch, roh' (afrz. auch 'dumm').

\section{1. bestialisch in FWB}

keine Angaben, auch nicht im bereits zitierten Material der Redaktionsstelle von FWB

\footnotetext{
${ }^{63}$ Obgleich säuisch nach der Ausgangsbedeutung nicht in die bezeichnete Gruppe passt, stellt es ein moralisches Prädikat dar und demonstriert den vollständigen Prozess der Desemantisierung. Aus diesem Grund wird es in dieses Kapitel eingeordnet.

64 afrz. $=$ Altfranzösisch, frz. $=$ Französisch
} 
2. bestialisch in DWB (s.v.)

„beluinus, immanis“

3. bestialisch in DUDEN (2012, s.v.)

3.1 „(abwertend) in seiner grausamen Art eher an gefühllose wilde Tiere als an Menschen denken lassend“

3.2 „(umgangssprachlich)“

$3.2 \mathrm{a}$,unerträglich"

$3.2 \mathrm{~b}$ „<intensivierend bei Adjektiven und Verben> in unerträglichem Maß"

\subsubsection{Entwicklung der Gradbedeutung bei bestialisch}

Die Korpusanalyse hat ergeben, dass die Gradbedeutung im frühen Neuhochdeutschen eingesetzt hat. Als Basis dafür dient die übertragene Bedeutung des menschlichen Verhaltens, die auf dem metaphorischen Vergleich mit tierischem „gefühllose[m]“" (DUDEN 2012, s.v.) Verhalten basiert, vgl. die in 3.1 angegebene Bedeutung und die Belege, die die angesprochene Sinnrichtung veranschaulichen sollen:

(713) Aber ist ift das nicht zu hart geredt das H. Johan Arend fchreibet der Menfch fey Beftialifch Viehifch vnd Thierifch worden? (DTA: Dilger, Daniel: Herrn Johannis Arndes [...] Richtige / und in Gottes Wort wolgegründete Lehre / in den vier Büchern vom wahren Christenthumb. Alten Stettin, 1620)

(714) fo wird eine Brutalität daraus / und gantz wildes / barbarifches / beftialifches / luderthumifch Leben (DTA: Dannhauer, Johann Conrad: Catechismus-Milch. Bd. B. Straßburg, 1666)

Das Auftreten der metaphorischen Verwendungen des Lexems signalisiert den Übergang des Gebrauchs auf die expressive Ebene und damit das Einsetzen von Traugott I (oben 3.6.3.4).

Das Auftreten der Gradbedeutung erfolgt durch pragmatic enrichment bzw. strengthening der Ausgangsbedeutung bzw. das Ableiten aus der Ausgangsbedeutung einer entsprechenden Inferenz. Demzufolge lässt sich die Intensivierung in mehrdeutigen Kontexten erkennen, wobei sie zunächst als Nebenbedeutung auftritt, während die Ausgangsbedeutung die primäre Rolle spielt, vgl.:

(715) Diefes meinet vermutlich auch Petrus Claudi / da er fchreibt / daß fie gåhzornig / und fchnell zur Rache / gleich als håtten fie eines Båren Natur. Denn / durch die Bären-Natur / hat felbiger Author [...] keine Tapfferkeit angedeutet / noch offenbaren Jächzorn / und Künheit; fondern eine unmenfchliche beftialifche Graufamkeit / wovon fie fich nicht wieder ablencken laffen / nachdem fie einmal zornig gemacht. (DTA: Francisci, Erasmus: Das eröffnete Lust-Haus Der Ober- und Nieder-Welt. Nürnberg, 1676)

(716) Daß alfo das Judicium ohne den Sinnreichen Verftand / der ihm alles vortragen muß / nichts nütze; Und zwar eben fo wenig / als die Hertzhafftigkeit für eine Tugend zu achten / wann kein Verftand darbey ift / fintemahl die Courage ohne den Verftand nur ein Beftialifches Wüten und Verwegenheit ift / wordurch wir uns felbften zur ungelegener Zeit in Gefahr ftürtzen / daran dann Niemand Gefallen haben kan. (DTA: Happel, Eberhard Werner: Der Academische Roman. Ulm, 1690) 
(717) Heutiges Tages aber ist in den Kirchen der Musik wegen so eine Lizenz und Freiheit, dass [...] sie das Gebet und heilige Amt nicht in Ehren halten, sondern mit ihren leichtfertigen und ums Geld gekauften Musikanten nicht zur Aufmerkung und Andacht, sondern zur Hurerei, nicht mit Menschenstimmen, sondern bestialischem Geschrei Anlass geben. (C.II: HK5/B24.00001 Agrippa von Nettesheim, Ungewißheit und Eitelkeit aller Künste und Wissenschaften, Übersetzung: 1713, 2000 [S. 85])

(718) Die Gemeinen zerhauen die Pflanzungen um die Stadt herum mit einer echt dummen bestialischen Zerstörungswut, und wehe der Polizei, wenn sie es wagt, ihre Lindenalleen zu schützen! (C.II: HK3/HK1.00166 Seume: Apokryphen, Entstanden: 1806/07, 2000 [S. 1361])

Die Belege (715) - (718) demonstrieren die Mehrdeutigkeit des adjektivischen Gebrauchs. Die Ausgangsbedeutung des Lexems verweist auf menschliches Verhalten, das dem Verhalten eines Tiers ähnelt. Der Vergleich basiert in (715), (716) und (718) auf der Grausamkeit und Rücksichtslosigkeit der Handlungen, während die metaphorische Übertragung in (717) die im kirchlichen Gesang entstehenden Laute betrifft. Zugleich kann aus den Kontexten eine Inferenz abgeleitet werden, die auf die Intensivierung der angesprochenen Situationen hinweist und diese als einen möglichen Grund für den metaphorischen Vergleich darstellt, vgl. die entsprechenden Paraphrasen:

(715') 'Die Grausamkeit ist in so hohem Maße ausgeprägt, dass sie in ihrer Natur der tierischen Grausamkeit ähnelt'.

(716') 'Das Wüten ist in so hohem Maße ausgeprägt, dass es einem tierischen Wüten ähnelt'.

(717') 'Das Geschrei ist so hohem Maße unharmonisch, laut und für den kirchlichen Gesang nicht angemessen, dass es eher einem tierischen Geschrei ähnelt'.

(718') 'Die Zerstörungswut ist in so hohem Maße ausgeprägt, dass sie tierischer Zerstörungswut ähnelt'.

Die Bezugswörter Grausamkeit, Wüten, Geschrei und Zerstörungswut stellen Extremwerte aufweisende Prädikate dar, sodass die Verbindung mit bestialisch zu der Intensivierung führt und die Ausdrücke verstärkt.

Die mehrdeutigen Verwendungen (715) - (718) demonstrieren die Zunahme an subjectivity, die in der Etappe der metaphorischen Übertragung der Ausgangsbedeutung eingesetzt hat. Die Belege veranschaulichen eine gewisse Einstellung seitens des Schreibers bzw. Sprechers, der neben dem Vergleich der Sachverhalte ihnen einen bestimmten Ausprägungsgrad aus der subjektiven Perspektive zuschreibt.

In der nächsten Phase der Bedeutungsentwicklung tritt das Lexem in einer stärker ausgeprägten intensivierenden Funktion auf, ohne einen Bezug zur Ausgangsbedeutung aufzuweisen. Für diese Stufe ist jedoch ausschlaggebend, dass bestialisch sich in Verbindung mit negativ konnotierten Kollokationspartnern findet. Damit gilt der Desemantisierungsprozess als nicht komplett vollzogen. Die Belege (719) - (723) sollen den adjektivischen und adverbialen Gebrauch des Intensivierungsmittels veranschaulichen, vgl.: 
(719) Die meist zu zweit startenden Militärmaschinen aus Dübendorf brausen praktisch jeden Werktag x-mal zum Beispiel über unser Quartier Hirzenbach mit bestialischem Lärm. (C.II: E97/MAI.11477 Zürcher Tagesanzeiger, 16.05.1997, S. 29)

(719') 'sehr großem Lärm'

(720) Bald jedoch begriff er, daß seine Entrückung auch von einem bestialischen Hunger herrührte, und er entrollte sich wieder, streckte sich vom Sofa herauf in seine aufrechte Menschengestalt und spannte seine langen Arme einmal weit auseinander [...]. (C.II: DIV/HIT.00001 Hanika: Treffen sich zwei, 2008)

(720') 'sehr großen Hunger'

(721) „Wir haben hier schon bestialisch gefroren“, erinnert sich Becker. (C.II: BRZ07/OKT.03234 Braunschw. Z., 12.10.2007)

(721') 'sehr gefroren'

(722) „Ich weiß, dass ich im Training in den vergangenen zwei Wochen nach der deutschen Meisterschaft bestialisch hart war. Aber ich hoffe, das Team sieht nun, was es gebracht hat." (C.II: BRZ11/NOV.16317 Braunschw.Z., 28.11.2011)

(722') 'sehr hart'

(723) was ich an "Argumenten" noch nicht genannt habe, ist die bestialisch schlechte, unkorrekte Umschrift des arabischen Textes. (23.07.2015 https://www.newikis.com/de/wiki/Diskussion:Koran/Archiv/1)

(723') 'sehr schlechte'

Bestialisch kann in seiner intensivierenden Rolle in Konstruktionen mit verschobenem Skopus auftreten, wobei das Adverb Funktionsverbgefüge modifiziert, vgl.:

(724) Du gehst mir bestialisch auf die Nerven mit einer Eigenschaft, wofür du deinen Freund verlassen hast. (19.02.2014: http://www.comicforum.dewww.comicforum.de / showthread.php?118010-Gedankenthread-1/page115)

(725) Das lag aber weniger an der Methode an sich, sondern hauptsächlich an den Lehrern und daran, dass es während dieser Arbeit ja häufig um Zensuren geht, und ich mit Zensuren permanent bestialisch unter Druck gesetzt, verängstigt und eingeschüchtert wurde. (19.02.2014: http://www.politikforen.net/showthread.php?25666-Beliebtheit-von-Unterrichtsmethoden/page2)

In (724) - (725) fungiert bestialisch als Gradadverb und intensiviert die FVGs $j$-m auf die Nerven gehen und $j$-n unter Druck setzten. Das Auftreten in dieser Position erfolgt dadurch, dass die Wortverbindungen jeweils durch einen graduierbaren Begriff bzw. nerven und bedrängen wiedergegeben werden können, vgl. die entsprechenden Umformulierungen:

(724') 'nervst mich sehr'

$\left(725^{\prime}\right)$ 'sehr bedrängt wurde'

Die intensivierende Funktion des Lexems kann in die quantifizierende übergehen, die die Anzahl der durch das Bezugswort ausgedrückten Sachverhalte bezeichnet, vgl.: 
(726) Und wie gesagt, bin ich dein Kapitel wirklich genau durch gegangen, daher auch diese bestialische Menge an Quotes. (03.07.2014: http://www.bisaboard.de/board333-fanwork/board353-fanfiction/board432-pok\%C3\%A9mon/board355-vollendete_fanfictions/198072-pok\%C3\%A9mon_police_2_kapitel_20_online/index3.html)

(726') 'sehr große Menge'

(727) Nicht jeder kann sich ein Tabletmontior leisten, da besonders die in Deutschland zum Teil bestialische Preise haben. (03.07.2014: http://www.filb.de/forum / showthread.php?t=14565\&page=2)

(727') 'sehr hohe Preise'

Die zweite Stufe des Entwicklungsgangs der Intensitätsbedeutung verbindet sich mit der Erweiterung des einstellungsbezogenen Ausdruckspotentials des Lexems. Der Gebrauch des Intensivierungsmittels nimmt an subjectivity zu, verweist ausschließlich auf "internal situation" und verweist damit auf die dritte semantischpragmatische Tendenz (Traugott III, oben 3.6.3.4).

In der letzten Phase der Entwicklung tritt bestialisch in Verbindung mit positiv konnotierten Kollokationspartnern und verliert damit endgültig den Bezug auf die Ausgangsbedeutung. Das Lexem fungiert als vollkommen entleert und demonstriert ausschließlich die intensivierende Funktion, vgl.:

(728) Ich freue mich zum einen bestialisch auf die Stimmung, das Meer und die Menschen in der Bretagne. (19.02.2014: http://sturzflug-ins-nirgendwo.blogspot.de/2013/07/setz-die-snapback-auf-dreh-sie-um-auf.html)

(728') 'freue mich sehr'

(729) Kuriosum: Der deutsche Psychiater Dr. Louis Lewin hatte eine bestialisch gute physische Konstitution, die es ihm ermöglichte, 20.000 Drogen für sein Suchtmittel-Lehrbuch (1927 erschienen) unbeschadet zu schlucken [...] (10.02.14: http://books.google.de/books?id=G_ciAQAAIAAJ\&q=\%22bestialisch+gute $\% 22 \& \mathrm{dq}=\% 22$ bestialisch+gute $\% 22 \& \mathrm{hl}=\mathrm{de} \& \mathrm{sa}=\mathrm{X} \& \mathrm{ei}=\mathrm{RNUEU}-$ 8XnA8GdtAbiwoGgBA\&ved=0CDAQ6AEwAA)

$(729$ ') 'sehr gute'

\subsubsection{Kurzfassung der Entwicklung bei bestialisch}

Ausgangsbedeutung: 'in seiner grausamen Art eher an gefühllose wilde Tiere als an Menschen denken lassend'; die übrigen Entwicklungsschritte wie oben in Kap. 3.6.3.9.3.

\subsubsection{Tierisch}

\subsubsection{Lexikographische Angaben}

Laut Pfeifer (s.v.) tritt das Lexem tierisch im Frühneuhochdeutschen bzw. am Anfang des $16 \mathrm{Jh}$. in Erscheinung. Zum Vergleich bietet das Wörterbuch außerdem die althochdeutsche Form tiorlīh (8. Jh.) und die mittelhochdeutsche Form tierlich.

1. tierisch in FWB (s.v.) 
„animalisch“; „tierisch, „mit tierischem Verhalten“; „wie ein Tier“65

2. tierisch in DWB (s.v. thierisch)

2.1 „allen lebenden geschöpfen (thier 1 ) oder den thieren (im gegensatze zu den menschen) eigen, darauf bezüglich, animalis“

2.2 „nach der art der unvernünftigen, nur sinnlichen trieben folgenden thiere, grob sinnlich, bestialisch, viehisch, bestialis, ferinus"

3. tierisch in DUDEN (2012, s.v.)

3.1a „ein Tier, Tiere betreffend; einem Tier, Tieren eigen; für Tiere charakteristisch"

3.1b „Von einem Tier, von Tieren stammend, herrührend; animalisch (a)“

3.2 „(oft abwertend) nicht dem Wesen, den Vorstellungen von einem Menschen entsprechend; dumpf, triebhaft; roh, grausam"

3.3 ,(salopp)“

3.3a „sehr groß, sehr stark; mächtig“

$3.3 \mathrm{~b}$ „<intensivierend bei Adjekiven und Verben> sehr, ungeheuer, in starkem Maße“

\subsubsection{Entwicklung der Gradbedeutung bei tierisch}

Die Korpusanalyse hat ergeben, dass die Herausbildung der Gradbedeutung im Nhd. erfolgt ist. Als Grundlage für deren Einsetzen gilt die Bedeutung menschlichen Verhaltens, die ihren Ursprung in einem Vergleich mit tierischem Verhalten findet und von daher metaphorischer Natur ist, vgl.:

(730) sklavische furcht vor gesetzen und strafen ist das gewisseste merkmal thierischer menschen. (DWB: s.v. thierisch)

(731) Ein paar Schritte weiter standen andere Frauengestalten, auch die Armut, aber die entehrte, mit frechen Blicken und frechem Lachen, in widerlichen Flitter gehüllt; dann wieder Männer, so tierisch, brutal und scheußlich, daß man den Blick abwandte. (C.II: HK4/M18.00001 Meysenbug: Memoiren einer Idealistin, Erstdruck: 1830-1848, 2004 [S. 56])

Der metaphorische Gebrauch von tierisch signalisiert Verwendung auf der expressiven Ebene, damit Auftreten der esten semantisch-pragmatischen Tendenz Traugott I (oben 3.6.3.4).

Die auf dem Vergleich mit Tieren basierte Sinnrichtung gibt einen Impuls für das Auftreten der Intensivierung. Das erfolgt durch pragmatic enrichment bzw. strengthening der übertragenen Bedeutung, wobei die Intensität als Nebenbedeutung erscheint und die Mehrdeutigkeit der Verwendungen hervorruft, vgl.:

[...] fürchtet euch vielmehr / und retiriret euch als dann in Salems felfe auff den weiffen verborgenen rechenftein und namen vor dem angeficht des pfeils und fchwerdts am tage des ftreits / biß ihr bey nüchterner außrechnung des zornes und der rechten hand Gottes [...] euren thierifchen muth creutzigen und zähmen lernet [...]. (DTA: Arnold, Gottfried: Unpartheyische Kirchen- und Ketzer-Historie. Bd. 2 (T. 3/4). Frankfurt (Main), 1700)

${ }^{65}$ Angaben aus den Belegzetteln von FWBD. 
(733) [...] aber Morfus, den ein unfinniger Geitz mehr als Felfenhart gemachet, und der alle Spuren der Menfchheit in fich ertödet hatte, fiel mit thierifchem Gebrülle auf ihn ein, und hub das Eifen zu einem schnellen Schlage empor [...] (DTA: [Bodmer, Johann Jacob]: Sammlung Critischer, Poetischer, und anderer geistvollen Schriften. Bd. 11. Zürich, 1743)

(734) aber die Könige und Häuptlinge herrschen mit einer wahrhaft thierischen Grausamkeit über das Volk und berauben jeden Fremdling, der sich nicht durch zahlreiche Geschenke loszukaufen vermag. (C.II: KHM/019.00018 Sonntags=Blatt für Jedermann aus dem Volke., 1868.07.12)

Die Belege (732) - (734) demonstrieren mehrdeutigen Gebrauch von tierisch. Zunächst erwirbt das Lexem eine metaphorisch bezogene Interpretation, indem menschlicher Mut, menschliches Gebrüll und menschliche Grausamkeit tierischem Mut, tierischem Gebrüll und tierischer Grausamkeit gleichgesetzt werden. In (733) wird extra zum Ausdruck gebracht, dass die angesprochene Person „alle Spuren der Menschheit in sich ertödet hatte" und folglich in den Handlungen einem Tier ähnelt. Zugleich wirkt das Adjektiv als Verstärkungsmittel. Beispielsweise lässt sich aus (732) schließen, dass die beteiligten Personen so viel Mut wie ein Tier haben. In (733) wird das Gebrüll u.a. als sehr laut interpretiert, als ob es von einem Tier stammte. In (734) ist die Grausamkeit so stark ausgeprägt, dass sie einem Menschen nicht zugeschrieben werden kann und deshalb als tierisch bezeichnet wird. Außderdem ist für diese adjektivische Verwendung semantic repetition ausschlaggebend, indem das Bezugswort die Inhaltszüge von tierisch bzw. 'grausam' wiederholt und damit die Verstärkung des Ausdrucks zu Folge hat.

Auch hier ist eine Zunahme an subjectification zu verzeichnen, die schon mit der Metaphorisierung der Ausgangsbedeutung eingesetzt hat. Die Kontexte legen nahe, dass in den adjektivischen Verwendungen eine wertende Komponente auftritt, die seitens des Schreibers bzw. Sprechers zum Ausdruck gebracht wird und den Grad der Ausprägung eines Sachverhalts auf der entsprechenden Skala einordnet.

Die nächste Etappe des Entwicklungsgangs ist mit bleaching der metaphorisierten Ausgangsbedeutung sowie der Zunahme an subjectification verbunden. Das Lexem verliert den direkten Zusammenhang mit dem Vergleich der tierischen und menschlichen Eigenschaften bzw. Verhalten und demonstriert die große Intensität der Sachverhalte aus der einstellungsbezogenen Perspektive. Jedoch verstärkt das Lexem ausschließlich negativ konnotierte Bezugswörter, was auf den unvollendeten Prozess der Desemantisierung hinweist:

(735) Zuerst wird Kaffee gekocht, das übernimmt Bille, denn Bille weiß, wie Männer Kaffee mögen: mordsheiß, mordsschwarz und tierisch dick! (C.II: M99/FEB.13327 Mannh. Morgen, 27.02.1999)

(735') 'sehr dick'

(736) „[... Außerdem ist es im Haus tierisch laut. Hier ist es herrlich ruhig!" (C.II: DIV/KWM.00001 Wittelsbach: Marc Marée, 2003 [S. 261])

(736') 'sehr laut' 
(737) Am nächsten Tag wachte ich auf und hatte Kopfschmerzen wie nie zuvor. Von dem Geschmack im Mund gar nicht zu reden. Zudem war mir tierisch schlecht. (C.II: M03/AUG.55227 Mannh. Morgen, 23.08.2003)

(737') 'sehr schlecht'

(738) Madlen N. leidet auch Tage nach der Attacke noch unter den Schmerzen: „Vor allem beim Kauen tut der Kiefer tierisch weh. [...]“ (C.II: HMP10/MAR.01264 MOPO, 14.03.2010, S. 4-5)

(738') 'tut sehr weh'

Die Korpusanalyse hat außerdem ergeben, dass tierisch als Gradadverb Funktionsverbgefüge modifizieren kann. Die Belege (739) - (751) mit den entsprechenden Paraphrasierungen sollen die zahlreiche Verwendung des Lexems in der Konstruktion mit dem verschobenen Skopus demonstrieren, vgl.:

(739) Vergangenen Donnerstag hatten wir schließlich noch berichtet, daß der Wecker seiner Besitzerin tierisch aufden Zeiger ging, indem er gut eine Woche zu früh die Sommerzeit einstellte. (C.II: R97/APR.25046 Frankf. Rundschau, 02.04.1997, S. 3)

(739') 'sehr nervte'

(740) Vielen Besuchern des Musikfestivals auf der Insel Silberau ging das Kamerateam des Südwestfunks am Samstag abend offensichtlich tierisch auf die Nerven: „Sie wuselten den Akteuren bei ihren Formationen ständig vor den Füßen herum und zogen bei der endlosen Suche nach dem richtigen Sendeplatz mehr Aufmerksamkeit auf sich als die eigentliche Show", schimpften sie. (C.II: RHZ97/AUG.18192 RZ, 30.08.1997)

(740') 'nervte sehr'

(741) »Außerdem sind mir die polnischen Fans, die mit ihren Kuhglocken Rabatz gemacht haben, tierisch auf den Keks gegangen." (C.II: NUZ03/AUG.02906 NZ, 28.08.2003)

(741') 'sehr genervt haben'

(742) „Nein, im Ernst, wir fallen auf, keine Frage. Die Leute freuen sich, uns zu sehen", sagt er. "Obwohl es auch Tage gibt, an denen einem dieses Begafftwerden tierisch auf den Geist geht." (C.II: M05/FEB.16020 Mannh. Morgen, 26.02.2005)

(742') 'nervt sehr'

(743) Damit sich an Weihnachten nicht alle Familienmitglieder tierisch auf den Wecker gehen und am Ende die Fetzen fliegen, sei äußerste Geduld aller Beteiligten gefragt - insbesondere in einer Patchwork-Familie, empfiehlt der Diplom-Psychologe. (C.II: M05/DEZ.06057 Mannh. Morgen, 24.12.2005)

(743') 'nerven sehr'

(744) „Das geht mir tierisch auf die Nüsse.“ (M06/DEZ.93133 Mannh. Morgen, 04.12.2006)

(744') 'nervt mich sehr'

(745) Es gehe ihm, fügte Lambertz hinzu, „tierisch auf den Sack, dass alle schon denken, wir sind bereits in der Bundesliga". (C.II: M12/MAI.04776 Mannh. Morgen, 15.05.2012, S. 11) 
(745') 'nervte ihn sehr'

(746) Aber sie können einem auch tierisch auf den Sender gehen. (C.II: BRZ12/APR.02350 Braunschw. Z., 07.04.2012)

(746') 'sehr nerven'

(747) Was den Hanseaten mittlerweile tierisch aufden Nerv geht, macht Triathletin Svenja Bazlen nichts aus. (C.II: HMP12/JUL.04748 MOPO, 21.07.2012, S. 40)

(747') 'sehr nervt'

(748) „Für mich ist das Spiel gegen meinen Ex-Verein tierisch in die Hose gegangen. Es ist schon schlimm, wenn du als Schlussmann an der Hälfte der Gegentore Schuld bist." (C.II: RHZ02/AUG.19457 RZ, 27.08.2002)

(748') 'ist sehr misslungen'

(749) „Einer der beiden Schiedsrichter hat uns tierisch in die Mangel genommen", fand der TVBE- Coach. „Das waren sehr umstrittene Entscheidungen." (C.II: RHZ04/DEZ.06005 RZ, 06.12.2004)

(749') 'uns sehr heftig zugesetzt'

(750) Ein anderes Beispiel sind die Flächen und Nutzungsangaben beim Grundantrag. In diesem Zusammenhang sind die landwirtschaftlichen Berater gerade jetzt tierisch in Gange und haben Stress ohne Ende. (C.II: Protokoll der Sitzung des Parlaments Landtag Schleswig-Holstein am 16.06.2005)

(750') 'sehr stark mit der Arbeit beschäftigt'

(751) Er weiß aber auch: „Die Britta ist wirklich tierisch unter Druck." (C.II: HMP07/MAR.02829 MOPO, 24.03.2007, S. 33)

(751') 'ist unter sehr großem Druck'

(752) Und auch sonst muss man tierisch auf der Hut sein. (C.II: RHZ13/JAN.22910 RZ, 23.01.2013, S. 17)

(752') 'sehr aufpassen'

Die Intensivierung wird dadurch ermöglicht, dass ein Bezugswort in dem FVG intensivierbar ist, wie es in (751) der Fall ist, oder das ganze Gefüge durch einen graduierbaren Begriff wiedergegeben werden kann, vgl. (739) - (750) und (752).

Die Intensivierung kann in Quantifizierung übergehen. Dabei kann tierisch in negativ sowie positiv konnotierten Kontexten auftreten, vgl.:

(753) Bisher war ich immer davon überzeugt, dass man sich die tierischen Steuern und die deutlich höheren Versicherungskosten für einen Diesel nur dann antut, wenn die Jahreskilometerleistung mindestens irgendwo so ca. die 30.000 erreicht. (04.07.2014: http://www.kia-board.de/forum/board8-fahrzeuge/board193-carens/board17-carens-ii-2002-2006/1949-laufleistungen-carens-ii-crdi/)

(753') 'sehr hohen Steuern, negativ gemeint'

(754) Du hast die Gebäude sofort gezahlt durch den fetten Gewinn - du machst weiterhin tierischen Gewinn - wo ist dein Problem? (04.07.2014: http://kapiland.upjers.com/viewtopic.php?f=2\&t=63063)

(754') 'sehr großen Gewinn, positiv gemeint' 
Die intensivierende sowie quantifizierende Bedeutung demonstrieren die $\mathrm{Zu}$ nahme an subjectivity im Rahmen von Traugott III (oben 3.6.3.4), indem die Einstellung des Sprechers bzw. Schreibers immer mehr ausgeprägt wirkt.

Das Auftreten von tierisch in der Verbindung mit positiv konnotierten Bezugswörtern verweist auf den kompletten Verlust der Ausgangsbedeutung und den Übergang der Bedeutung auf die expressive Ebene, sodass die Einstellung des Sprechers bzw. Schreibers zum Ausdruck gebracht wird. Die Belege (755) - (761) demonstrieren den vollendeten Prozess von bleaching, vgl.:

(755) Klassisches Konzert macht tierischen Spaß (C.II: M06/JUL.54094 Mannh. Morgen, 10.07.2006)

(755') 'sehr großen Spaß'

(756) Nun vermute ich ja, dass jemand die Sorte gezüchtet hat. Wenn Sie also auch mal tierisches Glück haben wollen, dann kommen Sie einfach nach Calberlah und robben durch die Vorgärten. (C.II: BRZ08/APR.15317 Braunschw. Z., 29.04.2008)

(756') 'sehr großes Glück'

(757) „Das war eine Riesen-Party. Die Kids hatten tierisch viel Spaß“, berichtete Tina Westermeyer. (C.II: M99/JUN.41447 Mannh. Morgen, 28.06.1999)

(757') 'sehr viel'

(758) Das stellte auch die Jury im Kreis Birkenfeld fest - die Nachwuchstalente waren tierisch kreativ. (C.II: RHZ00/APR.00747 RZ, 01.04.2000)

(758') 'sehr kreativ'

(759) „Ich würde mich tierisch freuen, wenn meine Tochter mich in ein, zwei Jahren spielen sehen kann." (C.II: M11/APR.08154 Mannh. Morgen, 26.04.2011, S. 10)

(759') 'mich sehr freuen'

(760) „Conni rettet die Tiere" ist ein tierisch unterhaltsames Hörspiel für Groß und Klein! (C.II: RHZ11/JUL.08975 RZ, 09.07.2011, S. 8)

(760') 'sehr unterhaltsames'

(761) Renommierte Nürnberger gründen eine Aktiengesellschaft, die in kurzer Zeit rund 700000 Goldmark zusammenbringt - genug für einen tierisch guten Start. (C.II: NUN12/MAR.01020 NN, 10.03.2012, S. 14)

(761') 'sehr guten'

\subsubsection{Kurzfassung der Entwicklung bei tierisch}

Ausgangsbedeutung: 'nach der art der unvernünftigen, nur sinnlichen trieben folgenden thiere, grob sinnlich, bestialisch, viehisch, bestialis, ferinus'; die übrigen Entwicklungsschritte wie oben in Kap. 3.6.3.9.3.

\subsubsection{Viehisch}

\subsubsection{Lexikographische Angaben}

Laut Pfeifer (s.v.) tritt das Lexem im Mittelhochdeutschen in der Form vihisch auf.

1. viehisch in Lexer (s.v. vihisch, vihelich)

„tier-, viehartig, viehisch“ 
2. viehisch in FWB (s.v. viehisch, vihisch, vihelich, vichlich, viellich) „tierisch, triebhaft“; „wie ein Tier“; „wild“; „menschenunwürdig“; „grob“; „ungebildet, ohne Einsicht"66

3. viehisch in DWB (s.v.)

3.1 „thierisch, zunächst in neutralem sinne; beim gegensatz zum menschlichen tritt leicht schon ein werthurtheil hinzu, und die bedeutung verschlimmert sich"

3.2 „viehisch kann in verschlimmerter bedeutung sich auf vieh in unserem sinne (pecus) beziehen, wenn es zur bezeichnung mühseliger menschenunwürdiger arbeit dient"

3.3 „die art, das wesen der thiere im allgemeinen bezeichnend, aber in verschlimmertem sinne und gegensatz zu menschlicher art, auf thiere bezogen"

3.4 „die verschlechterung der bedeutung ist im allgemeinen mit groszer schärfe vollzogen [...] hauptsächlich wird das wort verwendet, um das wilde, rohe, ungezügelte ausbrechen der triebe zu bezeichnen, zunächst natürlich solcher, die menschen und thieren gemeinsam sind, dann auch in freierem gebrauch"

3.4a „auf den menschen bezogen“

$3.4 \mathrm{~b}$ „auf unpersönliches bezogen; zunächst werden besonders die wild sich äuszernden triebe als viehisch bezeichnet; dann allgemeiner wesen, art, eigenschaften, verhaltungsweisen, handlungen, vorgänge, erscheinungen, zustände, die als niedrig, roh, untermenschlich erscheinen, im gegensatz stehen zu menschlicher sebstbeherrschung und zucht; andrerseits geht der gebrauch des wortes aus von der vorstellung thierischer beschränktheit, unwissenheit, unvernunft"

$3.4 c$,in den folgenden stellen liegt das gewicht besonders darauf, dasz die durch viehisch bezeichnete art einen für auge oder ohr wahrnehmbaren entsprechenden ausdruck findet"

3.5 „gebrauch des adverbiums im verschlimmerten sinne; öfters: viehisch leben; viehisch leben, wie ein vieh ADELUNG. gewöhnlich nicht zur bezeichnung eines armseligen lebens schwerer arbeit (vgl. 2), sondern im sinne von 4 (wildes, rohes, vernunftloses triebleben)“

3.6 „die neuere sprache hat auf der einen seite in ernster rede die verschlimmerte bedeutung des adj. viehisch ganz besonders scharf entwickelt, auf der andern seite braucht sie das schlimme wort in scherzender sprechweise zu derber verstärkung (vgl. bestialisch): in der niedrigen sprechart dient viehisch auch, einen hohen grad anzuzeigen"

4. viehisch in DUDEN (2012, s.v.)

4.1 „(abwertend) wie das Vieh und deshalb menschenunwürdig“

4.2 „(abwertend) von roher Triebhaftigkeit zeugend; brutal, bestialisch (1)“

4.3 „(emotional verstärkend) überaus stark, groß; maßlos“

${ }^{66}$ Angaben aus den Belegzetteln von FWBD. 


\subsubsection{Entwicklung der Gradbedeutung bei viehisch}

Die Korpusanalyse sowie die Analyse der lexikograpgischen Angaben lassen feststellen, dass die Gradbedeutung in der Zeitspanne zwischen dem späten Frühneuhochdeutschen und frühen Neuhochdeutschen eingesetzt hat. Jedoch ist die Anzahl der im Fnhd. gefundenen Belege erheblich größer. Als Grundlage für die Herausbildung der Intensivierung dient die auf dem Vergleich mit Tieren basierende „verschlimmerte" Bedeutung des menschlichen Lebens und Verhaltens. Weil die Bedeutung metaphorischer Natur ist und von daher die Sachverhalte auf der expressiven Ebene bezeichnet, kann man von dem Eintreten der ersten semantisch-pragmtischen Tendenz ausgehen. Die Belege (762) - (764) sollen den Gebrauch veranschaulichen, vgl.:

(762) ein gottlosz, rohe und viehisch leben führen (DWB: s.v.)

(763) auch Luther wurde beschuldigt in viehischen lüsten zu leben (DWB: s.v.)

(764) ihre noth hat ... ihm (dem geschlechte der Parias) ein schmutziges viehisches ansehen gegeben (DWB: s.v.)

Das Einsetzen der Gradbedeutung erfolgt in mehrdeutigen Kontexten infolge pragmatic enrichment bzw. strengthening der metaphorisierten Ausgangsbedeutung, wobei die gewonnene Intensivierung als Nebenbedeutung neben der primären Bedeutung in Erscheinung tritt. DWB (s.v.) bestätigt die Annahme über die Mehrdeutigkeit des Gebrauchs sowie die Stufen der Ausprägung der Gradbedeutung: in manchen Verwendungen „mag gelegentlich der zusammenhang mit dem ersten gebrauch des wortes gefühlt werden“, während „in andern verbindungen ist er ganz aufgegeben“. Die Belege (765) - (771) sollen das Auftreten der Intensivierung neben der Ausgangsbedeutung demonstrieren:

(765) das auch inn etlichen pfaffen unnd mönchen, und andern geistlichen personen, eine solche äuszerste viehische unwissenheit stecke (DWB: 1588, s.v.)

(766) Daher folche abfchewliche Lafter bey den Chriften im fchwang gehen / die nie erhöret feyn / folche teufflifche Hoffart vnnd Pracht / fo vnerfättlicher Geitz / fchendliche Wolluft / viehifche Vnzucht /vnd vnmenfchliche Thaten / welche alle aus Verblendung vñ Verftockung eines verkertẽ Sinnesgefchehen [...] (DTA: Arndt, Johann: Von wahrem Christenthumb. Bd. 1. Magdeburg, 1610)

(767) Dann ich halte darfür / daß keine fchrecklichere Tyranney auff der Welt feye / als eben diefe / welche einem Hertzen vorkommet / wann es inn folche Angft vnd Noth geråthet: Inn Summa / als fie nun jhr viehifches begehren erfüllet / fchlagen fie die junge Wittib gar zu todt vnd geben jhr fünff Stich in die lincke Bruft [...] (DTA: Calvi, François de: Beutelschneider / Oder Neue / warhaffte / und eigentliche Beschreibung Der Diebs Historien. Bd. 2. Frankfurt (Main), 1627)

(768) Auf das Empörendste trieben viehische Rohheit und bettelhafter Trotz ihr wüstes Spiel unter Männern und Frauen. (C.II: HK4/F11.00001 Fouqué: Magie der Natur, Erstdruck: 1812, 2004 [S. 27])

(769) Der Mönch war aus dem Turm, wo ich ihn allnächtlich eingeschlossen, gebrochen und in viehischer Brunst nach dem Gemach meiner Töchter 
gerannt, dessen Türe er mit einem Fußtritt sprengte. (C.II: HK3/D64.00001 Hoffmann: Die Elixiere des Teufels, Entstanden: 1814/15, 2000 [S. 139])

(770) Fälle gebe es aber allerdings, in denen nicht allein alle Gefangenen gegenseitig umgebracht, sondern auch alle unmenschliche viehische Grausamkeiten ausgeübt werden würden, die jemals die sinnreichste Barbarei erfunden. (C.II: HK3/F41.00001 Hoffmann: Letzte Erzählungen, 1825, 2000 [S. 352])

(771) Dem deutschen Bürgerstande wird Angst gemacht vor dem Pöbel, und er bewaffnet sich, stellt sich in seiner viehischen Dummheit unter das Kommando der Militärmacht und vermehrt dadurch nur die Gewalt der Regierungen. (C.II: HK3/C78.00001 Börne: Briefe aus Paris, 1832, 2000 [S. 49])

Die Belege (765) - (771) demonstrieren einen mehrdeutigen Gebrauch von viehisch. Aus den Kontexten geht hervor, dass die erste Interpretationsmöglichkeit sich auf verschiedene Sinnrichtungen der metaphorisierten Ausgangsbedeutung bezieht. So wird beispielsweise in (765), (706) und (771) die „Vorstellung thierischer beschränktheit, unwissenheit, unvernunft" zum Ausdruck gebracht, während in (766), (767) und (769) „die wild sich äuszernden triebe“ hervorgehoben werden, die „im gegensatz [...] zu menschlicher sebstbeherrschung und zucht“ stehen (DWB, s.v.). Außerdem kann sich die Ausgangsbedeutung auf „niedrig[e], roh[e], untermenschlich[e]“ (siehe 3.4.b.) und grausame Verhaltensweisen beziehen und die Basis für das Einsetzen der Intensivierung bilden, wie es in (768) und (770) der Fall ist.

Die zweite Interpretationsmöglichkeit erweist die intensivierende Rolle des Adjektivs, die neben der Hauptbedeutung als Nebenbedeutung in Erscheinung tritt. Durch den metaphorischen Vergleich mit Tieren wird u.a. ausgedrückt, dass die angesprochenen Sachverhalte einen außergewöhnlichen Grad ihrer Ausprägung demonstrieren. Die Belege bezeugen außerdem eine Zunahme an einstellungsbezogener Bedeutung, die sich als Folge der Metaphorisierung eingestellt hat. Die Gradbedeutung lässt die Kontexte aus der Sicht des Sprechers bzw. Schreibers deuten, indem die Sachverhalte durch den hohen Grad ihrer Ausprägung eine wertende Komponente bekommen und das Wirken von Traugott I (oben 3.6.3.4) signalisieren.

In der nächsten Phase des Entwicklungsgangs erscheint viehisch als reines Intensivierungsmittel, wobei die direkte Verbindung zu der Ausgangsbedeutung beseitigt wird. Der Bezug auf den Vergleich des menschlichen Verhaltens und der Lebensweise ist nicht mehr zu erkennen, vgl. den adjektivischen (772) - (773) und adverbialen (774) - (776) Gebrauch:

(772) Es war eine viehische Kälte. 30 Grad unter Null und ein furchtbarer Ostwind dazu. (DWDS: Simplicissimus, 14.01.1934)

(772') 'sehr große Hitze'

(773) Gegen Ende des Krieges begab sie sich in Lebensgefahr - wegen einer Milchkanne voller Suppe aus dem Lazarett: „Wir hatten eben viehischen Hunger. " (C.II: M08/DEZ.99852 Mannh. Morgen, 24.12.2008, S. 19)

(773') 'sehr großen Hunger' 
(774) Ich war viehisch aufgeregt und hatte unglaubliche Nierenschmerzen. (DWDS: Die Zeit, 01.11.1991, Nr. 45)

(774') 'sehr aufgeregt'

(775) Ich bin viehisch müde und werde jetzt nach Hause fahren [...] (DWDS: Dückers, Tanja, Spielzone, Berlin: Aufbau-Taschenbuch-Verl. 2002 [1999], S. 154)

(775') 'sehr müde'

(776) Es kursiert die Geschichte darüber, wie zwei Touristen sich an einem Taxistand mit Bolivianern anfreunden. Man redet ein wenig das übliche, woher kommst du, wie lange bist du hier, ist es nicht viehisch heiß, wo willst du noch hin? (DWDS: Die Zeit, 31.05.2008, Nr. 23)

(776') 'sehr heiß'

Viehisch kann außerdem als Gradadverb Funktionsverbgefüge modifiieren, vgl.:

(777) Und ich gebe es auch ehrlich zu, die Telekom ist mir unsymphatisch, die laufende Werbung geht mir viehisch auf den Keks [...] (24.02.2014: http://www.ariva.de/forum/Wer-zeichnet-Telekom-Aktien-o-T-31865?page=0)

(777') 'nervt mich sehr'

(778) Dort wird die Welt verhandelt, und alles beginnt mit dem Wort »Man müßte mal...", und das geht mir viehisch auf den Geist. (24.04.2014: http://books.google.de/books?id=EJoaAQAAIAAJ\&q=\%22viehisch+auf+den+geist$\% 22 \& d q=\% 22 v i e h i s c h+a u f+$ den + geist $\% 22 \&$ hl=de\&sa=X\&ei=KXgLU7TEL8nRsgaS1oG4Dw\&ved=0CDAQ6AEwAA)

(778') 'nervt mich sehr'

Die Verwendungen (772) - (778) verweisen außerdem auf die fortgeschrittene einstellungsbezogene Bedeutung des Lexems und den Gebrauch auf der ausschließlich expressiven Ebene. Damit wird der Übergang zu Traugott III (oben 3.6.3.4) vollzogen.

In der letzten Phase des Entwicklungsgangs tritt viehisch in positiv konnotierten Kontexten auf und verweist damit auf die völlige Bedeutungsentleerung.

(779) Die ersten Auftritte haben viehischen Spaß gemacht und kamen sehr gut an. (C.II: L99/MAI.24179 Berliner Morgenpost, 14.05.1999, S. 33)

(779') 'sehr großen Spaß'

(780) Ein Meister der französischen Musik des späten neunzehnten Jahrhunderts, das war viehisch schön, fanden Sie nicht? Doch doch, wunderbar, sagte Perstling. (24.02.2014: http://books.google.de/books?id=A0NBAQAAIAAJ$\& q=\% 22$ viehisch + sch $\%$ C3\%B6n\%22\&dq=\%22 viehisch+sch\%C3\%B6n\%22\&hl$=$ de\&sa=X\&ei=bXcLU42JCcKGtAb1_YHQBA\&ved=0CDIQ6AEwAA)

(780') 'sehr schön'

(781) Der hatte in der Dunkelkammer mal irgendeine Kassette gegriffen, die er [...] in seinen Recorder legte. Und da war unsere Musik drauf. [...] Jedenfalls hat es ihm viehisch gut gefallen. (24.02.2014: http://books.google.de/books?id=gJkUU46APsQC\&pg=PA21\&dq=\%22viehisch+gut\%22\&hl=de\&sa=-

X\&ei=BXYLU6rBNoOQtQahiYDwCg\&ved=0CD4Q6AEwAg\#v=onepage\&q=\%22viehisch $\% 20$ gut $\% 22 \& \mathrm{f}=$ false)

(781') 'sehr gut' 
(782) Früh ging ich ins hebräische Seminar bei Delitsch, wo es viehisch interessant war. (24.02.2014: http://www.zeit.de/campus/2011/02/tagebuecher/seite-2)

(782') 'sehr interessant'

\subsubsection{Kurzfassung der Entwicklung bei viehisch}

Ausgangsbedeutung: vergleicht menschliches Verhalten mit dem von Tieren; die übrigen Entwicklungsschritte wie oben in Kap. 3.6.3.9.3.

\subsection{Wortgruppe 'Geistig gestört'}

Das vorliegende Kapitel widmet sich der Herausbildung der Gradbedeutung in den Lexemen irre, irrsinnig, närrisch, toll, verrückt und wahnsinnig. Die intensivierende Funktion der Adjektive bzw. Adverbien kann durch die Verwendungen (a) - (f) exemplifiziert werden:
(a) irre Hitze 'starke Hitze'
(b) irrsinnig heiß 'sehr heiß'
(c) närrische Freude 'sehr große Freude'
(d) toll reiche Frau 'sehr reiche Frau'
(e) verrückt heißer Tee 'sehr heißer Tee'
(f) wahnsinnige Mühe 'sehr große Mühe'

Die Ausgangsbedeutung der bezeichneten Grad-Adjektive bzw. -Adverbien entspricht der Kategorie 11 „Das Denken“ bei Dornseiff (198ff.) und beinhaltet die Begriffe „Dumm“, „Verrückt“ und „Unlogik“67. In Biedermann (1969: 161ff.) wird die Gruppe der zu untersuchenden Wörter nach dem Motivationssem „Wahn“ angeordnet. In diesem Kapitel wird außerdem das Lexem verrückt berücksichtigt, weil es eine intensivierende Funktion zeigt (in Biedermann jedoch nicht erwähnt wird). Zugleich wird das in Biedermanns Liste enthaltene Wort blödsinnig und idiotisch nicht diskutiert, weil in DUDEN (2012) keine Intensitätsbedeutung verzeichnet ist. Außerdem werden damisch (Dialektismus) und dämlich (ohne Identitätsbedeutung) sowie das Lexem doll nicht besprochen, da letzteres in FWB, DWB und Kluge als Variante von toll betrachtet wird.

\subsubsection{Irre}

\subsubsection{Lexikographische Angaben}

Laut Kluge (s.v.) und Pfeifer (s.v.) tritt das Lexem im 9. Jh. in der Form irri in Erscheinung.

1. irre in AWB (s.v. irri)

1.1 „umherschweifend, unstet“

1.2 „in den Verbindungen irri gangan/gên, irri faran irregehenm sich verirren, auf Abwege geraten"

1.3 „(ab-)irrend“

${ }^{67}$ Bei Dornseiff fehlen Angaben zu damisch, doll und schwachsinnig. Außerdem wird toll der Kategorie 10 „Gefühle, Affekte“ zugeordnet. 
1.3a „locker, zügellos“

$1.3 b$ „erzürnt über j-n“

$1.3 \mathrm{c}$ "verwirrt, unwissend“

\section{4 „Glossenwort"}

2. $\quad$ irre in BMZ, Lexer (s.v.)

2.1 „Vom rechten wege abgewandt; unsicher, schwankend“ (BMZ); „verirrt" (Lexer)

2.2 „von der rechten lehre abgewandt, ketzerisch“ (BMZ)

2.3 „unruhigen sinnes, wankelmütig; insbesondere leicht zum zorne zu bringen“ (BMZ); „,unbeständig, untreu“, „erzürnt, aufgebracht, ungestüm, herbe” (Lexer)

2.4 „wovon abgekommen, verlustig, frei“ (Lexer)

3. $\quad$ irre in FWB (s.v.)

3.1 „ohne Orientierung herumirrend“

3.2 „unwissend, verwirrt, im Zweifel, unsicher (von Personen); falsch, irreführend (von Sachen)“

3.3 „verrückt, zornig, wütend (von Personen)“

3.4 „verhindert, gestört an der Ausübung von etw."

4. $\quad$ irre in DWB (s.v.)

4.1 "umherschweifend"

4.2 „abgewichen vom rechten wege, verirrt, verbunden“

$4.2 \mathrm{a}$ „in eigentlichem sinne“

$4.2 \mathrm{~b}$ „übertragen, in bezug auf zustände, handlungen, gefühle“

4.3 "schwankend, unschlüssig, ratlos"

4.4 „erzürnt, hochdeutsch selten, während es im ältern niederdeutsch die einzige bedeutung des wortes ist"

4.5 „geistig gestört, milderer ausdruck für wahnsinnig [...] hat seinen ausgang vom mhd. irre thöricht, närrisch, dumm“, „,gern auch von zuständen, reden und thaten, die gestörten verstand anzeigen"

5. irre in DUDEN (2012, s.v.)

$5.1 \mathrm{a}$ „psychotisch wirkend, verstört“

$5.1 \mathrm{~b}$ „(umgangssprachlich veraltend) psychotisch“

5.1c „in seiner Erregung völlig durcheinandergebracht, wie von Sinnen“

5.2 (salopp)

$5.2 \mathrm{a}$ „vom Üblichen abweichend und so ausgefallen, merkwürdig, wie man es nie erwarten konnte"

$5.2 \mathrm{~b}$ "sehr groß, stark"

$5.2 \mathrm{c}$ „ $<$ intensivierend bei Adjektiven und Verben $>$ sehr, in höchstem Maße, außerordentlich"

5.3 „unruhig, in stetem Hin und Her“

\subsubsection{Entwicklung der Gradbedeutung bei irre}

Die Gradbedeutung von irre ist lediglich in DUDEN (2012) verzeichnet. Die Korpusanalyse hat jedoch deren Herausbildung im 19. Jh. feststellen können. Als 
Grundlage dafür dient die in 4.5 und 5.1a,b angedeutete Bedeutung, die den geistigseelischen Zustand einer Person sowie ihr entsprechendes Verhalten bzw. „reden und thaten, die gestörten verstand anzeigen" (siehe 4.5.), beschreibt, vgl.:

(783) ich müszte sie für irre im kopfe gehalten haben (DWB: s.v.)

(784) inzwischen hätte ein fremder .. nichts am sprechenden grafen merken können, als ein irres glühen im gesicht und schnelle worte. (DWB: s.v.)

(785) seine augen nahmen allgemach einen seltsam-irren ausdruck an. (DWB: s.v.)

(786) Der Kranke sprach irre, rasete u. s. w.? (DTA: Gall, Franz Joseph: Philosophisch-medizinische Untersuchungen über Natur und Kunst im kranken und gesunden Zustand des Menschen. Wien, 1791)

Das Lexem kann außerdem metaphorisch gebraucht werden, um eine Person als seelisch krank im übertragenen Sinne zu bezeichnen. Dadurch wird ein Vergleich mit einem erkrankten Menschen erzielt, vgl.:

(787) »Komm sofort herunter! « schrie er, als noch immer keine Antwort kam, mit fast irrer Stimme (DUDEN 2012: s.v.)

Die in (787) erwähnte Person ist nicht geisteskrank, jedoch ist die Art und Weise ihres Schreis einem erkrankten Menschen ähnlich. Von daher erfolgt ein metaphorischer Vergleich der Person mit einem Wahnsinnigen. Damit geht die Verwendung auf die expressive Ebene über im Rahmen von Traugott I (oben 3.6.3.4).

Eine Intensivierung findet sich zunächst in mehrdeutigen Kontexten, wobei die Ausgangsbedeutung die primäre Rolle spielt und die Gradbedeutung in der Form einer Inferenz aus dem Kontext abgeleitet wird. Darüber hinaus ist die frühere Etappe des Entwicklungsgangs mit dem Prozess pragmatic enrichment bzw. strengthening des ursprünglichen Gebrauchs verbunden, vgl.:

(788) Sein Blick aus tiefen Augenhöhlen war irre flammend, das Geficht fchön, aber bla $\beta$ und wüft. (DTA; 1826, s.v.)

(789) Es fand sich nämlich [...] ein Bursche ein, der durch lautes und störendes Gemurmel die Andächtigen belästigte und schließlich auf den Altar zurannte und dort mit ganz irrem Geschrei und gräulichen Gestikulationen eine Menge frommer Sprüche deklamirte, so daß die Leute ganz erschrocken und aus der Kirche flohen. (24.07.2015: https://books.google.de/books?id=8kFDAAAAcAAJ\&pg =PA367\&dq=\%22irrem+geschrei\%22\&hl=de\&-

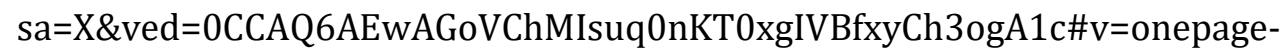
$\& \mathrm{q}=\% 22$ irrem $\% 20$ geschrei $\% 22 \& \mathrm{f}=$ false)

(790) Sie fährt empor aus kurzer Rast, sie greift zum letzten Stümpfchen Lichts, sie sucht und sucht in irrer Hast im Schub und Schrank und findet nichts [...] (C.II: HK4/M20.00001 Müller-Jahnke: Gedichte, Erstdruck: 1910, 2004 [S. 191])

(791) Auf einmal marschierte ich in Kolonne, in Schritt und Rhythmus der Musik, den Revolver gezogen. Im Rhythmus einer irren Besessenheit. $O$, nicht von einer Frau besessen: süßer, verlockender, verlockter! (C.II: HK3/D20.00001 Klabund, Der Marketenderwagen, Erstdruck: 1918, 2000 [S. 12]) 
Die Belege (788) - (791) sind mehrdeutig interpretierbar. Zunächst bezieht sich irre auf die Ausgangsbedeutung und bezeichnet die in Rede stehenden Personen als wahnsinnig bzw. geisteskrank wirkend. Dadurch wird mit flammende[n] Augen in (788), das Geschrei in (799), Hast in (790) und Besessenheit in (791) direkt oder metaphorisch auf die Symptome einer potenziellen seelischen Krankheit Bezug genommen.

Eine alternative Interpretation wird durch die Reanalyse der Verwendungen ermöglicht, wenn die Geschehnisse aus der Sicht des Schreibers bzw. Sprechers beschrieben werden. Aus den Kontexten geht hervor, dass die oben genannten Symptome graduierbare Erscheinungen darstellen und auf einer entsprechenden Skala eingeordnet werden können. Es lässt sich ferner schließen, dass die Intensität der Sachverhalte den Grund für die Charakterisierung der Personen als krankhaft bzw. krankhaft wirkend bietet. Es zeigt sich außerdem, dass die Bezugswörter Extremwerte aufweisende Prädikate darstellen. Durch die Verbindung mit irre ergibt sich eine Verstärkung des Ausdrucks.

Ferner tritt das Lexem in intensivierender Rolle auf, wobei der direkte Zusammenhang mit der Ausgangsbedeutung verloren geht, vgl. den adjektivischen (792) - (793) und adverbialen (794) - (797) Gebrauch:

(792) „Frühstück. Ich hab irren Hunger. Auf Spiegeleier mit Speck. Magst du auch?" (C.II: BRZ09/JUN.02486 Braunschw. Z., 06.06.2009)

(792') 'sehr großen Hunger'

(793) Ich hatte irre Angst vor meinem ersten Flug. (C.II: R97/FEB.10380 Frankf. Rundschau, 10.02.1997, S. 22)

(793') 'sehr große Angst'

(794) „Unter der Einsatzkleidung ist es irre heiß - da bist nach so einem Einsatz völlig nass geschwitzt", meinte ein Florianijünger im Namen seiner Kollegen. (C.II: V00/SEP.47706 Vorarlberger Nachr., 25.09.2000, S. A6)

(794') 'sehr heiß'

(795) Die Schwimmer werden mit Schwimmhäuten geboren, Läufer haben irre lange Beine und aerodynamisches Äußeres, beim Gewichtheber sorgt man für immens dicke Arme und Boxer werden am besten ohne Kopfgeschaffen. (C.II: RHZ01/OKT.22726 RZ, 30.10.2001)

(795') 'sehr lange Beine'

(796) Ich war zum Zeitvertreib auf einer kleinen Messe von Buchantiquaren und hatte schnell bemerkt, dass all die schönen Sachen irre teuer waren. (C.II: HAZ08/NOV.03929 HAZ, 22.11.2008, S. 7)

(796') 'sehr teuer'

(797) Das Paddeln muss ganz gleichmäßig erfolgen und ist irre anstrengend - bringt aber das Boot richtig in Fahrt. (C.II: BRZ10/MAI.09692 Braunschw. Z., 26.05.2010)

(797') 'sehr anstrengend'

Irre kann außerdem Funktionsverbgefüge modifizieren und damit in Konstruktuionen mit verschobenem Skopus auftreten, vgl. die Belege mit den entsprechenden Umformulierungen: 
(798) „Die Art, wie er bestimmte Wörter ausgesprochen hat, ist mir irre auf die Nerven gegangen." (03.06.2014: http://www.heute.at/love/herz/art23704,941245)

(798') 'ist mir sehr auf die Nerven gegangen'

(799) Eure Hilfsbereitschaft dem Bettelstudenten einen Urlaub zu verschaffen in allen Ehren, mir würde sowas irre auf den Geist gehen. (08.07.2014: http://www.eltern.de / foren/eltern-raten-eltern-neu/1155324-man-dem-besuch-seine-grenzen-zeigen-kann-6.html)

(799') 'sehr nerven'

(800) Ich möchte wetten, er setzt sie irre unter Druck. (03.06.2014: http://books.google.de/books?id=qCsnb1dTVq4C\&pg=PT76\&lpg=PT76\&dq=\%22irre+unter+Druck\%22\&source=bl\&ots=RX8vipu27g\&sig=NTsF73VhY635f4T9fSim9ymATxI\&hl=de\&sa=X\&ei=6hDZUryeG40RtQalhoHgCA\&ved=0CGYQ6AEwBg\#v=onepage $\& \mathrm{q}=\% 22$ irre $\% 20$ unter\%20Druck $\% 22 \& \mathrm{f}=$ false)

(800') 'setzt sie unter sehr großen Druck' bzw. 'bedrängt sie sehr'

(801) Aufgrund des ständigen Wiederholens von Christines Tagesablauf - was natürlich Sinn der Geschichte ist - zieht sich die Story irre in die Länge, ohne das wirklich etwas passiert. (08.07.2014: http://www.boltebloggt.de/bolteliest-rezension-ich-darf-nicht-schlafen-von-s-j-watson/)

(801') 'verzögert sich sehr'

Die qualifizierende Funktion des Lexems kann in die quantifizierende übergehen und eine große Menge von dem entsprechenden Sachverhalt bezeichnen, vgl.:

(802) Manche Bilder von Monet oder Picasso erzielen irre Preise. (C.II: BRZ11/JAN.02423 Braunschw. Z., 08.01.2011)

(802') 'sehr hohe Preise'

(803) Er versprach im Falle einer kanadischen Goldmedaille im Rodeln der Damen eine Million Dollar! Diese irre Summe dünkte derart atemberaubend, dass jedes Medium in Kanada, USA - ach was, der ganzen Welt darüber berichtete. (C.II: M10/FEB.13573 Mannh. Morgen, 18.02.2010, S. 10)

(803') 'sehr große Summe'

(804) Grundsätzlich setzt Sobotka auf einen Konjunkturaufschwung, die Exportwirtschaft sei angesprungen, „da kann sich die öffentliche Hand zurückhalten, da brauchen wir nicht so irre investieren". (C.II: N98/JUN.21856 Salzburger Nachr., 10.06.1998)

(804') 'viel investieren'

Die Belege (792) - (801) und (802) - (804) bezeugen zweifelsfrei den Gebrauch des Lexems jeweils in intensivierender und quantifizierender Funktion, die keinen direkten Kontakt mit der Ausgangsbedeutung aufweist. Zugleich ist diese Stufe des Entwicklungsgangs mit einer Zunahme an subjectivity verbunden, was die Weiterentwicklung der expressiven Bedeutung und damit den Übergang zu Traugott III (oben 3.6.3.4) signalisiert.

Anschließend findet sich irre als Intensivierungsmittel in Verbindung mit positiv konnotierten Bezugswörtern. Der Kontakt mit der Ausgangsbedeutung wird damit endgültig beseitigt, vgl.: 
(805) Doch nun freut er sich über das Angebot, in Mannheim bleiben zu können: „Ich fühle mich sehr wohl in dieser Stadt und denke, Mannheim hat ein irre großes Potenzial, aus dem man mehr machen kann", so Müller. (C.II: M05/MAR.19136 Mannh. Morgen, 08.03.2005)

(805') 'sehr großes Potenzial'

(806) Bestimmt aber ist Dalton irre intelligent. Immerhin wurde er auf englischen Theaterbühnen mit Shakespeare-Rollen bekannt und war viele Jahre mit Vanessa Redgrave liiert, der Vorzeige-Intellektuellen der Kultur! (C.II: NUZ08/OKT.02677 NZ, 29.10.2008, S. 6)

(806') 'sehr intelligent'

(807) Dass Christian Ude eine Spitzenkandidatur für die bayerische SPD erwäge, meldet der Bayerische Rundfunk. Weikert braucht eine Sekunde, dann ist sie begeistert. „Wenn das stimmt", sagt die Nürnberger SPDLandtagsabgeordnete, „wenn er das wirklich macht, würde ich mich irre freuen. Wir hätten dann eine echte Chance in Bayern." (C.II: NUN11/AUG.$00693 \mathrm{NN}, 06.08 .2011$, S. 17)

(807') 'sehr freuen'

(808) Ihm macht das Busfahren irre viel Spaß. (C.II: BRZ11/AUG.09833 Braunschw. Z., 23.08.2011)

(808') 'sehr viel Spaß'

(809) „Cindy ist einfach irre witzig, hat viele coole Sprüche drauf und einen ganz eigenen Style. Wir gucken sie auch immer im Fernsehen", sagt Mario. (C.II: HMP11/DEZ.00271 MOPO, 03.12.2011, S. 38)

(809') 'sehr witzig'

(810) „Wir sind hier, um unseren Sohn abzuholen und nach Hause zu bringen. Wir sind irre stolz auf ihn, aber das wären wir auch, wenn er keine Medaille geholt hätte." (C.II: HMP12/AUG.01568 MOPO, 16.08.2012, S. 06, 07)

(810') 'sehr stolz'

(811) Aber im Ernst: Wir fordern das Publikum schon, aber mein Pianist Jendrik Springer, der sich irre gut auskennt, und ich denken, auf eine spannende Art. (NUN12/FEB.02249 NN, 22.02.2012, S. 6)

(811') 'sich sehr gut auskennt'

(812) Der 52-Jährige schreibt Theaterstücke, Erzählungen, Bücher - immer irre erfolgreich. (C.II: HMP12/SEP.01001 MOPO, 12.09.2012, S. 18, 19)

(812') 'sehr erfolgreich'

\subsubsection{Kurzfassung der Entwicklung bei irre}

Ausgangsbedeutung: 'geistig gestört, wahnsinnig' bzw. 'geistig gestört, wahnsinnig wirkend';

die übrigen Entwicklungsschritte wie oben in Kap. 3.6.3.9.3.

\subsubsection{Irrsinnig}

\subsubsection{Lexikographische Angaben}

Weder Kluge noch Pfeifer enthalten einen Bericht über die Herkunft des Lexems.

1. irrsinnig in DWB (s.v.) 
Keine lexikographischen Angaben

2. irrsinnig in DUDEN (2012, s.v.)

2.1a "geistig so verwirrt, dass die Gedanken keinen Zusammenhang untereinander und keine Übereinstimmung mit der Wirklichkeit haben“"

$2.1 \mathrm{~b}$ „aufgrund einer innerlichen Belastung wie von Sinnen seiend, sich verhaltend"

2.1c „(oft emotional) [in seinem Handeln oder Verhalten] keine Vernunft erkennen lassend; unvernünftig, ohne jeden Verstand; absurd“

2.2 "(umgangssprachlich)“

$2.2 \mathrm{a}$ „unvorstellbar, außerordentlich [groß, stark]“

$2.2 \mathrm{~b}$ „<intensivierend bei Adjektiven und Verben> sehr, in höchstem Maße“

\subsubsection{Entwicklung der Gradbedeutung bei irrsinnig}

Die Herausbildung der Gradbedeutung erfolgt im Neuhochdeutschen. Die Korpusanalyse hat die ersten relevanten Belege feststellen können, die auf die zweite Hälfte des 19. Jh. zurückführen. Als Grundlage für die Entwicklung der Gradbedeutung dient die in 2.1a angegebene Sinnrichtung, die sich auf geistesgestörte Personen sowie deren Verhalten bezieht, vgl.:

(813) Er war gewiß, daß ihn diese irrsinnige Alte nicht wieder erkennen würde. (C.II: HK3/D97.00001 Gutzkow: Die Ritter vom Geiste, Erstdruck: 1850/51)

(814) Die Franzofen bezeichnen den Ingenieur Sauvage als den eigentlichen Erfinder der Schiffsfchraube, doch ift erwiefen, daß er fich lediglich mit dem Problem derfelben befchäftigte, fpäter irrfinnig wurd und 1857 im Parifer Armenhaufe ftarb. (DTA: Schweiger-Lerchenfeld, Amand von, 1900)

Ferner kommt es zu metaphorischem Gebrauch des Lexems, wobei irrsinnig auf seelisch gesunde Personen angewandt wird, die jedoch durch absurdes bzw. unvernünftiges Verhalten wie erkrankte Menschen wirken, vgl.:

(815) „Peter, du allein kannst mir noch helfen, ich flieg' wie irrsinnig auf den jungen Grafen T. Schreib' ihm statt meiner einen deiner entzückenden Liebesbriefe, wo man net mehr auskann, wann man's gelesen hat - -." (C.II: HK3/F71.00001 Altenberg: Märchen des Lebens, Erstdruck: 1908)

(816) Die Mutter, die noch immer nicht genug Atem finden konnte, fing in die vorgehaltene Hand mit einem irrsinnigen Ausdruck der Augen dumpf zu husten an. (C.II: HK3/A40.00003 Kafka: Die Verwandlung, Entstanden: 1912)

Die Metaphorisierung von irrsinnig signalisiert den Übergang der Verwendungen auf die expressive Ebene und damit die erste semantisch-pragmatische Tendenz (Traugott I, oben 3.6.3.4).

Die Korpusanalyse legt nahe, dass das Einsetzen der Intensivierung in mehrdeutigen Kontexten durch pragmatic inferencing der Ausgangsbedeutung und infolge dessen pragmatic strengthening und enrichment beginnt, vgl.:

(817) In Bezug auf die Quaalen eines tief verletzten Gewiffens könnte man es mir fchon eher zugeben, daß gerade fie das höchfte Maaß eines heißen Verlangens nach dem verlorenen Seelenfrieden bezeichnen, welche Erklärung insbefondere darin ihre Rechtfertigung findet, daß gewöhnlich 
gutgeartete Menfchen in irrfinniger Bethörung fich mit falfchen Selbftanklagen überhäufen, eben weil ihr zartes Gewiffen am tiefften durch Gemüthsleiden erfchüttert wird. (DTA: Ideler, Karl Wilhelm: Der religiöse Wahnsinn, erläutert durch Krankengeschichten. Ein Beitrag zur Geschichte der religiösen Wirren der Gegenwart. Halle (Saale), 1847)

(818) „Wenn du mich heirathest und recht lieb hast", sprach sie mit irrsinnigem Freudenglanz ihres anmuthigen, zarten Gesichts, „so lehre ich Dich auch, wie man sich unsichtbar macht. [...]“ (08.07.2014: http://books.google.de / books?id=P54AAAAAcAAJ\&pg=PA240\&dq=irrsinnigem\&hl=de\&sa=X\&ei=p8a7U9W7MPTY4QSQ2oAI\&ved=0CFUQ6AEwCQ\#v=onepage\&q=irrsinnigem\&f=false, 1843)

(819) [...] aber das Flötenfpiel war fo fonderbar, daß wir länger ftehen blieben. [...] Was am meiften reizte, war, daß, wenn er einen Gang angenommen, und das Ohr verleitet hatte, mit zu gehen, immer etwas anderes kam, als was man erwartete, und das Recht hatte, zu erwarten, fo daß man ftets von vorne anfangen, und mitgehen mußte, und endlich in eine Verwirrung gerieth, die man beinahe irrfinnig hätte nennen können. (DTA: Stifter, Adalbert, 1853)

(820) Alrieri fand es nöthig, den Orest die That in einem Anfalle irrsinniger Verzweiflung vollbringen zu lassen. (08.07.2014: http://books.google.de / books?id=1v0QAAAAIAAJ\&q=irrsinniger\&dq=irrsinniger\&hl=de\&sa=X\&ei=99G7U83mL7SL4gSJ7YDgDw\&ved=0CCwQ6AEwAziYAg, 1865)

(821) Sie griff mit beiden Händen an die Stirn, bewegte lautlos die Lippen und brach dann plötzlich mit einem irrsinnigen Schrei zusammen. (27.07.2015: https://books.google.de/books?id=tgpAAAAYAAJ\&q=\%22irrsinnigen+schrei\%22\&dq=\%22irrsinnigen+schrei\%22\&hl=de\&sa=X\&ved=0CCAQ6AEwAGoVChMIuuSptoT7xgIVxFcUCh2NQwDk)

Die Belege (817) - (821) sind wiederum mehrdeutig analysierbar. So handelt es sich in (819) um ein Flötenspiel, das durch die Art und Weise der Ausführung die Zuhörer in eine Verwirrung bringen kann. Aus dem Kontext lässt sich schließen, dass diese Verwirrung durch das Kollokieren mit irrsinnig sich zunächst auf die metaphorische Verwendung des Lexems bezieht, sodass etwa die folgende Paraphrase entsteht:

(819') 'Die Verwirrung ähnelt beinahe dem Zustand einer geisteskranken Person'.

Eine weitere Interpretationsmöglichkeit bezieht sich auf eine Intensivierung des Begriffs. Aus einer einstellungsbezogenen Sicht kann geschlossen werden, dass der Schreiber bzw. der Sprecher menschliche Verwirrung je nach dem Ausprägungsgrad auf einer entsprechenden Skala anordnet. Durch die Verwendung eines Substantivs mit dem Adjektiv irrsinnig wird zum Ausdruck gebracht, dass der Zustand auf dem oberen Ende dieser Skala rangiert und dadurch dem Zustand einer kranken Person ähnelt, vgl. die Umformulierung:

(819") 'Die Verwirrung ist so stark, dass man sie beinahe mit dem Zustand eines geisteskranken Menschen vergleichen kann'. 
Die Analyse der anderen adjektivischen Verwendungen ergibt ebenso zwei Intepretationsmöglichkeiten. Zunächst handelt es sich um den Zustand der Menschen, der sich metaphorisch auf eine seelische Erkrankung und zugleich den großen Ausprägungsgrad bezieht, vgl.:

(817') 'Die Betörung ist so stark ausgeprägt, dass sie der Betörung einer wahnsinnigen Person ähnelt'.

(818') 'Der Freudenglanz ist so stark ausgeprägt, dass sie einer geisteskranken Person zugeschrieben werden kann'.

(820') 'Die Verzweiflung ist so stark ausgeprägt, dass sie einer geisteskranken Person zugeschrieben werden kann'.

(821') 'Der Schrei ist so laut, dass er einer geisteskranken Person zugeschrieben werden kann'.

Aus den Belegen geht hervor, dass irrsinnig sich auf den seelischen menschlichen Zustand, zugleich aber auf die Intensität von Sachverhalten bezieht und damit die Doppeldeutigkeit des Gebrauchs dokumentiert. Außerdem gewinnt der Gebrauch an subjectivity, indem irrsinnig durch die eingeführten Verwendungen die Einstellung des Sprechers bzw. Schreibers zum Ausdruck bringt.

Durch den fortgeschrittenen Prozess der Desemantisierung verliert irrsinnig die Doppeldeutigkeit des Gebrauchs. Die intensivierende Funktion des Lexems wird hervorgehoben und tritt in Verbindung mit negativ konnotierten Kollokationspartnern auf, ohne einen direkten Zusammenhang mit der Ausgangsbedeutung erkennen zu lassen, vgl.:

(822) „Es war irrsinnig windig!“ Deshalb auch die vielen Doppelfehler. (C.II: P92/JUL.20631 Die Presse, 13.07.1992)

(822') 'sehr windig'

(823) „Viele Städte zu bereisen ist irrsinnig teuer - und dabei spreche ich nicht von den üblichen Reisekosten, sondern über das Material und die Verarbeitung meiner Bilder", erklärt Kellner. (C.II: RHZ06/JAN.07082 RZ, 10.01.2006)

(823') 'sehr teuer'

(824) Es ist irrsinnig schwierig, Kinder heutzutage zu motivieren. (C.II: BVZ07/OKT.01719 BVZ, 17.10.2007, S. 51)

(824') 'sehr schwierig'

Irrsinnig kann außerdem als Gradadverb Skopus über Funktionsverbgefüge haben und dadurch die Erweiterung seiner Skopuskonstituente demonstrieren. Die Intensivierung erfolgt dadurch, dass das Prädikat in dem FVG graduierbar ist, wie in (825) - (826) oder die FVGs durch einen graduierbaren Begriff wiedergegeben werden können, wie in (827) - (829):

(825) Prohaska: „Jede Mannschaft, die das erste Match verliert, steht bereits irrsinnig unter Druck." Mehr noch: Mit dem Rücken zur Wand. (C.II: P98/JUN.23192 Die Presse, 08.06.1998)

$(825$ ') 'steht unter sehr großem Druck' 
(826) Ich bin irrsinnig unter Spannung gestanden - falls es tatsächlich so rübergekommen ist, dann möchte ich mich dafür entschuldigen: Es tut mir leid... (C.II: 099/FEB.18818 Neue Kronen-Ztg., 13.02.1999, S. 90)

(826') 'bin unter sehr großer Spannung gestanden'

(827) „.... In letzter Zeit sind vor allem die Preise für Rohwaren irrsinnig in die Höhe gegangen", erklärt eine Mitarbeiterin der Wirtschaftskammer-Bezirksstelle Bruck. (C.II: NON07/AUG.07922 NÖN, 15.08.2007, S. 3)

(827') 'sind sehr gestiegen'

(828) Markersdorf: „Mir geht das Regenwetter irrsinnig auf die Nerven. [...]“ (C.II: NON09/JUL.06761 NÖN, 14.07.2009, S. 3)

(828') 'nervt sehr'

(829) „Besonders der Caffe Latte ist bei uns irrsinnig in Mode gekommen“, plaudert Unterweger aus seiner Praxis, „wahrscheinlich auch deshalb, weil er in den schönen Gläsern so appetitlich aussieht!" (C.II: NON10/OKT.01358 NÖN, 05.10.2010, NÖN Großformat)

(829') 'ist sehr modisch geworden'

Irrsinnig kann zudem in quantifizierender Funktion auftreten und eine Menge von Sachverhalten bezeichnen, vgl.:

(830) Bei der öffentlichen Gesundheitsfürsorge will man 1.4 Millionen Euro einsparen. Bei den Krankenhäusern ist es die geradezu irrsinnige Summe von 21.5 Millionen Euro. (C.II: Protokoll der Sitzung des Parlaments Landtag Brandenburg am 09.04.2003)

(830') 'sehr große Summe'

(831) Sicherheitssprecherin Helene Partik-Pable sagte zur „Presse“: „Da gibt es Ungereimtheiten, das ist schlecht verwaltet. Die machen irrsinnige Gewinne. "(C.II: P97/MAI.19197 Die Presse, 22.05.1997)

(831') 'sehr große Gewinne'

(832) Was wird aus den Mannheimern, wo die Gefahr riesengroß ist und denen Sie noch zusätzlich mit dem Wasserpfennig irrsinnige Kosten reingedrückt haben? (C.II: Protokoll der Sitzung des Parlaments Landtag von BadenWürttemberg am 25.11.1999 [S. 5964])

(832') 'sehr hohe Kosten'

Die Belege (822) - (829) und (830) - (832) demonstrieren den eindeutigen Gebrauch des Lexems jeweils in intensivierender und quantifizierender Bedeutung, ohne den direkten Zusammenhang mit der Ausgangsbedeutung erkennen zu lassen. Zugleich verbindet sich diese Stufe des Entwicklungsgangs mit einer Zunahme an subjectivity, sodass die Bedeutung ausschließlich auf die expressive Ebene übergeht und damit das Auftreten von Traugott III (oben 3.6.3.4) signalisiert.

In der letzten Phase der Desemantisierung tritt irrsinnig als reines Intensivierungsmittel auf, indem es mit positiv konnotierten Bezugswörtern kollokiert, ohne eine Verbindung zu der Ausgangsbedeutung aufzuweisen, vgl.:

(833) Die Bergnasen sind ihm so nahe. Und nun sieht er die Gletscher in zitterndem Zauberschein - so glanzreich. „Irrsinnige Schönheit!“ flüstert er 
zaghaft. (C.II: HK3/A36.00001 Scheerbart: Liwûna und Kaidôh. Ein Seelenroman, Erstdruck: 1901)

(833') 'sehr große Schönheit'

(834) Sieben Stunden hat sie im Studio des Fotografen Raphael Just posiert, nach den ersten Abzügen war er paff: „Kein Unterschied zu den Fotos mit Modells, die schon jahrelang auf den Laufstegen daheim sind. Sie ist irrsinnig fotogen." (C.II: 095/JUL.72964 Neue Kronen-Ztg., 26.07.1995, S. 44)

(834') 'sehr fotogen'

(835) „Mit dem Leopold arbeite ich nämlich irrsinnig gern zusammen, bei ihm habe ich stets das Gefühl, daß ich in guten Händen bin!" (C.II: X96/MAR.03619 Oberösterreichische Nachr., 28.03.1996)

(835') 'sehr gern'

(836) Die Schüler waren bei Gastfamilien untergebracht und wußten nach ihrer Rückkehr über unterschiedlichste, meist positive, Erlebnisse zu berichten. „Irrsinnig freundlich, nett, offen, überwältigende Gastfreundschaft, tägliche Grillfeste ganz anders wie bei uns", so die euphorischen Kommentare. (C.II: I99/MAR.11032 Tiroler Tagesztg., 23.03.1999)

(836') 'sehr freundlich'

(837) „Da habe ich in komprimierter Form viel Erfahrung sammeln können, das war irrsinnig interessant", so Pangl, der als einer von acht „Venue Director" wirkt. (C.II: V00/JUN.29538 Vorarlberger Nachr., 10.06.2000, S. C2)

(837') 'sehr intereessant'

(838) „Ich bin irrsinnig froh, dass es uns gelungen ist, den Markt zu erhalten“, erklärt Günter Schönauer, Vorstandsmitglied der Landgenossenschaft Ennstal, erleichtert. (C.II: K00/OKT.74701 Kleine Ztg., 11.10.2000)

(837') 'sehr froh'

(839) Waldheim war [...] sprachlich schon irrsinnig begabt, erinnert sich Tasler: „Er konnte damals schon ein wenig Französisch und Englisch sprechen." (C.II: NON07/JUN.08999 NÖN, 18.06.2007, NÖN Großformat, S. 11)

(839') 'sehr begabt'

(840) Für VP-Landesrat Josef Plank ist Obama eine faszinierende Persönlichkeit mit irrsinnigem Charisma, der in vielen Fragen Hoffnung gibt. (C.II: NON09/JAN.11397 NÖN, 27.01.2009, S. 2)

(840') 'sehr großem Charisma'

\subsubsection{Kurzfassung der Entwicklung bei irrsinnig}

Ausgangsbedeutung: 'geistig gestört, wahnsinnig' bzw. 'geistig gestört, wahnsinnig wirkend'; die übrigen Entwicklungsschritte wie oben in Kap. 3.6.3.9.3.

\subsubsection{Närrisch}

\subsubsection{Lexikographische Angaben}

Laut Kluge (s.v.) stellt das Wort eine Ableitung von Narr dar. Die Angaben in Pfeifer (s.v.) verweisen auf das Entstehen des Lexems im Mhd. in der Form nerrisch.

1. närrisch in Lexer (s.v. närrisch, nerrisch) „närrisch, verkehrt“ 
2. närrisch in FWB

keine Angaben, auch nicht im bereits zitierten Material der Redaktionsstelle von FWB

3. närrisch in DWB (s.v.)

3.1 „adjectiv, einem narren ähnlich oder angemessen“

3.1.1 „zu narr 1, verrückt, oder wie verrückt, toll“

3.1.2 „zu narr 3“

3.1.2a „lachen erregend, possenhaft, possierlich, drollig“

$3.1 .2 \mathrm{~b}$ „allgemeiner (auch ohne bezug auf lebende wesen), eigenthümlich, sonderbar, wunderlich, seltsam, lächerlich, komisch"

3.1.3 „zu narr 4 und 5, der weisheit und klugheit zuwider handelnd oder zuwider laufend, thöricht, unklug, verkehrt, einfältig, albern, dumm (oft mehr scherzhaft wie bei narr 8)“

3.1.3a ,von personen“

$3.1 .3 \mathrm{~b}$ „von gedanken und gesinnung, von rede und handlung“

3.1.3c „von sachen und verhältnissen“

3.2 „adverb, wie ein narr, einem narren gemäsz, thöricht, unklug, verkehrt, albern, wunderlich, seltsam"

$3.2 \mathrm{a}$ „verstärkend vor adjectiven“

$3.2 \mathrm{~b}$ „als interjection im sinne von narr 8 , b“

4. närrisch in DUDEN (2012, s.v.)

4.1a „unvernünftig und daher den Spott anderer herausfordernd; skurril“

$4.1 b$ „(umgangssprachlich emotional) sehr, übermäßig [groß]“

4.2 „karnevalistisch, faschingsmäßig“

\subsubsection{Entwicklung der Gradbedeutung bei närrisch}

Obgleich DWB und DUDEN (2012) dem Lexem eine intensivierende Rolle zuschreiben, muss hier festgestellt werden, dass die Korpusanalyse keine Belege ergeben hat, die die reine Intensitätsbedeutung des Worts dokumentieren. Das Auftreten der Gradbedeutung bezieht sich ausschließlich auf doppeldeutige Verwendungen des Wortes, die zugleich dessen Gebrauch in der Ausgangsbedeutung demonstrieren. Als Ausgangsbedeutung gilt dabei die Bedeutung, die sich auf das Verhalten eines Narren oder eines sich wie ein Narr verhaltenden Menschen bezieht. Die folgenden Belege sollen die direkte Verwendung (841) sowie die metaphorische (842) Übertragung der menschlichen Eigenschaft veranschaulichen:

(841) im narrenhause sehen wir, dasz der mensch andern aufs wort glaubt, er sei närrisch (DWB: s.v.)

(841') 'ein Narr'

(842) er muszte redlich dafür büszen, dasz er sein schönes geld so närrisch weggeschmissen. (DWB: s.v.)

(842') 'gedankenlos, wie ein Narr'

Durch den metaphorischen Gebrauch geht der Gebrauch von närrisch auf die expressive Ebene über und signalisiert das Auftreten von Traugott I (oben 3.6.3.4). 
Ferner finden sich Belege, die die intensivierende Rolle von närrisch hervorheben und zugleich auf dessen Ausgangsbedeutung hinweisen. Das Auftreten der Gradbedeutung erfolgt durch pragmatic enrichment bzw. strengthening der Ausgangsbedeutung und hat die Mehrdeutigkeit des Gebrauchs zu Folge, vgl. die adverbiale (843) - (844) und adjektivische (845) Verwendung des Lexems:

(843) Was der Komiker den ganzen Abend durch für Karikaturen machte! Du hättest Dich närrisch gelacht! (C.II: HK4/E23.00001 Ehrmann: Nina's Briefe an ihren Geliebten, Erstdruck: 1788, 2004 [S. 19])

(844) Aus dem Fenster eines alten Hauses von krankhaftem Aussehen guckte eine gesprenkelte Levkoje, gar närrisch buntgeputzt, und hinter ihr erklang eine niedlich duftende Veilchenstimme. (C.II: HK3/G15.00001 Heine: Reisebilder. Dritter Teil, Erstdruck: 1830, 2000 [S. 236])

(845) Wie muß ich erstaunen, wie hüpft mir das Herz vor kindischer närrischer Freude, als ich den goldnen Hahn vom Jünnedaer Kirchturm mit der großen Uhrtafel in seinen zwei Klauen daherfliegen sehe! (C.II: HK3/HK1.00113 Mörike: Der Schatz, Erstdruck: 1836, 2000 [S. 436])

Die Belege (843) - (845) erfahren eine doppeldeutige Interpretation. Wie es oben angesprochen wurde, verweist närrisch zunächst auf die Bedeutung des geistigmentalen menschlichen Zustands, indem es die angesprochenen Personen als einen Narren oder wie einen Narren bezeichnet, vgl. die entsprechenden Umformulierungen:

(843') 'wie ein Narr gelacht'

(844') 'wie eine Närrin buntgeputzt'

(845') 'Freude eines Narren oder wie eines Narren'

Aus den Kontexten lässt sich jedoch zugleich schließen, dass närrisch als Intensivierer verwendet wird: Die Bezugswörter stellen jeweils einen Sachverhalt dar, der durch seine Intensität wirkt und damit den direkten oder metaphorischen Zusammenhang mit dem geistig-mentalen Zustand der erwähnten Person demonstriert, vgl. die Paraphrasen:

(843") 'so laut lachen, als ob man töricht bzw. ein Narr wäre'

(844") 'so stark buntgeputzt, als ob die Frau töricht bzw. eine Närrin wäre'

(845") 'die Freude ist sehr groß, sodass die angesprochene Person töricht bzw. wie ein Narr vorkommen kann'

Die Herausbildung der Gradbedeutung ist mit dem Auftreten von subjectivity verbunden. Die Verwendungen in (843) - (845) wirken einstellungsbezogen und belegen den Gebrauch auf der expressiven Ebene.

Es finden sich aber keine Belege, die die reine Gradbedeutung des Lexems demonstrieren können. Damit muss der Prozess der Desemantisierung als nicht abgeschlossen gelten.

\subsubsection{Kurzfassung der Entwicklung bei närrisch}

1. Die Quelle der Gradbedeutung verweist direkt oder metaphorisch auf den seelisch-geistigen Zustand einer Person und bezeichnet sie als Narr bzw. wie ein Narr erscheinend. Durch die Metaphorisierung wirkt die Ausgangsbedeutung 
abstrakter und verweist auf den Übergang der Verwendungen auf die expressive Ebene.

3. Die Korpusanalyse hat keine Verwendungen gezeigt, die einen Gebrauch des Lexems in rein intensivierender, quantifizierender sowie pejorativer Funktion sowie im Zusammenhang mit Funktionsverbgefügen und positiv konnotierten Kookkurrenzpartnern demonstrieren.

4. Das Auftreten der metaphorisierten Verwendungen sowie der Gradbedeutung in ambigen Belegen verweist auf subjectivity des Gebrauchs und demonstriert den Übergang des Lexems zu Traugott I (oben 3.6.3.4).

\subsubsection{Toll}

\subsubsection{Lexikographische Angaben}

Laut Kluge (s.v.) und Pfeifer (s.v.) tritt das Lexem im 9. Jh. in der Form tol in Erscheinung.

1. toll in AWB, Splett

keine lexikographischen Angaben

2. toll in BMZ, Lexer (s.v. tol)

2.1 „toll, unsinnig, vermessen“ (BMZ, s.v. tol), „töricht, unsinnig“ (Lexer, s.v. tol)

2.2 „ausgezeichnet“ (BMZ, s.v. tol), „von stattlicher schönheit, ansehnlich“ (Lexer, s.v. tol)

3. toll in FWB (s.v. tol)

3.1 "wahnsinnig, geisteskrank, geistesschwach; von Sinnen, durcheinander"

3.2 „betrunken; unzurechnungsfähig, ausgelassen, närrisch (als Folge von Alkoholkonsum); als Spezialisierung zu 1 auffassbar"

3.3 „wütend, rasend, aufbegracht; anschließbar an 1“

3.4 "unvernünftig“, „töricht; unwissend, dumm“

3.5 „schwach im Glauben, ungläubig; frevelhaft, gottlos; Spezialisierung zu 4“

3.6 „tollwütig“

3.7 "großartig, prächtig (auch ironisch)“

3.8 „sehr außerordentlich; gänzlich; auch als Gradadv.“

4. toll im Neuhochdeutschen (DWB, s.v.)

"I. adjectiv"

4.1 „des oder wie des verstandes und bewusztseins beraubt und darnach sich geberdend, benehmend, unsinnig, wahnsinnig, tobsüchtig (s. DWB tollhaus), wütend, rasend, unbändig, ausgelassen, leidenschaftlich, zornig, heftig, thöricht, närrisch, unvernünftig, verrückt, stumpfsinnig, wirre, dumm, wunderlich u. dergl. je nach dem zusammenhange."

4.2 „übertragen auf sachen“

4.2.1 „toll machen, in rasche bewegung setzen, verschwinden machen, entwenden"

4.2 .2 „Von wunderlichen, auffallenden oder verdorbenen dingen“

4.2.3 „von gliedern, ohne gefühl und empfindung (vgl. DWB dumm 2, taub 2)“ 
4.3 "toll drückt aber auch gute eigenschaften aus, indem der begriff des ausgelassenen und lärmenden übergeht in den von lustig und fröhlich, der des wunderlichen und auffallenden in den von bewundernswert, zum verwundern gut, grosz und schön gewachsen, stattlich, tüchtig, gewandt, wacker, brav u. dergl."

4.4 „endlich hat toll auch active bedeutung, toll machend“ „II. Adverb."

4.5 ",auf tolle weise“

4.6 „,auf lustige weise“; „, auf schöne, wackere, tüchtige weise „; „sehr, stark, viel“

5. toll in DUDEN (2012, s.v.)

5.1a „ungewöhnlich, unglaublich“

$5.1 b$ „(umgangssprachlich) großartig, prächtig“

$5.1 \mathrm{c}$ "(umgangssprachlich) sehr groß, stark"

$5.1 \mathrm{~d}$ "<intensivierend bei Verben und Adjektiven> (umgangssprachlich) sehr"

$5.1 \mathrm{e}$ "(umgangssprachlich) schlimm"

$5.1 \mathrm{f}$ „(umgangssprachlich) ausgelassen und wild“

5.2 „(veraltet) tollwütig"

\subsubsection{Entwicklung der Gradbedeutung bei toll}

Die Angaben in FWB sowie die Korpusanalyse legen nahe, dass die Entwicklung der Gradbedeutung bei toll im Frühneuhochdeutschen begonnen hat. Als Ausgangsbedeutung gilt die Sinnrichtung, die sich auf geisteskranke Menschen und deren Verhalten bezieht, vgl.:

(846) da dis der könig höret, ward er toll und thöricht. (DWB: s.v.)

(846') 'geisteskrank'

(847) die tollen gedanken ires ungerechten wandels. (DWB: s.v.)

(847') 'Gedanken eines geisteskranken Menschen'

Laut DWB wird das Lexem außerdem in Bezug auf tollwütige Tiere angewendet, vgl.:

(848) er ist von einem hund gebissen worden, von dem er nicht weisz, ist er toll oder nicht. (DWB: s.v.)

(848') 'tollwütig'

Ferner kommt es zu einer metaphorischen Verwendung des Lexems, bei der toll seelisch gesunde Menschen bezeichnet, die durch ihre Handlungen geisteskranken Personen ähneln, vgl.:

(849) Man kann vor Freude toll und vom Zorn rasend werden. (DTA: Johann Christian Reil (1803). Rhapsodieen über die Anwendung der psychischen Curmethode auf Geisteszerrüttungen)

(849') 'wie wahnsinnig'

(850) ...es war ja doch ein toller Gedanke, bei einem Menschen Zahlung zu fordern, der gar nichts hat! (DTA: Friedrich Christian Laukhard (1792). F. C. Laukhards Leben und Schicksale)

(850') 'ein Gedanke eines Menschen, der dadurch wahnsinnig wirkt' 
Die Verwendung von toll im übertragenen Sinne signalisiert den Übergang zu der expressiven, kognitiven Ebene ${ }^{68}$ und damit zum Einsetzen von Traugott I (oben 3.6.3.4).

Das Einsetzen der Gradbedeutung lässt sich zunächst in mehrdeutigen Verwendungen erkennen, wobei die Intensivierung durch pragmatic inferencing bzw. strengthening der Ausgangsbedeutung in Erscheinung tritt, vgl.:

(851) Endlich kamen der Anstösse so viel / daß er fast verzweiflend / eine tolle Künheit / sich zu verderben / vornehmen wolte. (C.II: HK4/S11.00001 Stockfleth: Die Kunst- und Tugend-gezierte Macarie, Erstdruck: 1669-1673, 2004 [S. 59])

Die adjektivische Verwendung in (851) bezieht sich auf eine menschliche Eigenschaft, die durch die Kollokation mit toll eine mehrdeutige Interpretation zulässt. Zunächst handelt es sich um den geistigen Zustand der in Rede stehenden Person, die sich entschlossen hat, sich zu verderben. Diese Künheit wird seitens des Schreibers bzw. Sprechers als toll bzw. wahnsinnig bezeichnet und drückt damit die Unvernünftigkeit der Handlung und den metaphorischen Gebrauch der Ausgangsbedeutung aus. Zugleich jedoch lässt sich aus dem Kontext schließen, dass das Adjektiv eine intensivierende Rolle übernehmen kann, denn Künheit wird von dem Schreiber bzw. Sprecher als graduierbarer Begriff benutzt, der je nach dem Grad seiner Ausprägung auf einer Skala angeordnet werden kann. Die Situation, die sich auf das Treffen einer mit dem Sterben verbundenen Entscheidung bezieht, lässt einen hohen Grad der Ausprägung der Disposition erkennen, vgl. die Umformulierung:

(851') 'Die Entscheidung zu sterben ist eine große Kühnheit. Damit wirkt die dazu entschlossene Person wie wahnsinnig.'

Situationen der großen Erregung, die auf den geistigen Zustand einer Person direkt oder metaphorisch hinweisen und zugleich Intensivierung der Gefühle beschreiben, stellen eine zweite Möglichkeit für das Auftreten der Gradbedeutung in mehrdeutigen Kontexten dar:

(852) Lairesse war die erste, die erschien. Solch eine tolle Lustigkeit, so eine übernatürliche Unbesonnenheit und so viel Leichtsinn kannst du dir nicht vorstellen: mich nennte man zu Hause unbesonnen: aber ich bin ein Cato dagegen. (C.II: HK3/F11.00001 Wezel: Hermann und Ulrike, Erstdruck: 1780, 2000 [S. 395])

(853') 'Lairesse demonstrierte solche große Lustigkeit, dass sie als wahnsinnig wirkte.'

(853) Nun flammte ich auf in toller Wut, ich griff nach der geladenen Büchse und schrie: „Hier vor Ihren Augen jage ich mir die Kugel durch den Kopf, wenn Sie nicht sogleich mir die Tür öffnen. " (C.II: HK3/F79.00001 Hoffmann: Nachtstücke, Entstanden: 1814)

(853') 'ich war in so hohem Maße wütend, dass ich wie wahnsinnig wirkte.'

${ }^{68}$ vgl. Traugott, 1988: 414 
(854) ich habe ja nicht Komödie gespielt, ich bin ja wirklich in Verzweiflung geraten,- ja, in helle tolle Verzweiflung hast du mich gestürzt, treulose Verräterin [...]! (C.II: HK3/A68.00009 Hoffmann: Prinzessin Brambilla, Erstdruck: 1820,2000 [S. 686])

(854') 'ich demonstrierte so starke Verzweiflung, dass ich wie wahnsinnig wirkte.'

(855) Nach der Täuschung, die ich erfahren, nach jener tollen Leidenschaft, mit der ich an Brigitte hing, bin ich mit der Liebe fertig [...] (C.II: HK4/L06.00001 Lewald: Clementine, Erstdruck: 1843, 2004 [S. 70])

(855') 'meine Leidenschaft zu Brigitte war so stark, dass ich wie wahnsinnig wirkte.'

Die Belege (852) - (855) sind wiederum mehrdeutig. Aus den Kontexten kann geschlossen werden, dass der adjektivische Gebrauch auf den hohen Ausprägungsgrad der Gefühle hinweist und zugleich einen metaphorischen Zusammenhang mit dem seelischen Zustand der jeweiligen Personen aufnimmt, wobei der Vergleich auf ihrem rasenden, ausgelassenen und leidenschaftlichen Verhalten beruht. - Intensivierung kann außerdem durch Metaphorisierung in Erscheinung treten, vgl.:

(856) Die Welt / das Jammer-Meer! in Wellen schifft mein Leben. Jen's ist voll Vnglücks Wind; ob diesen ich erschreck / das ich die Segel nicht / die dapffern Sinn' / außstreck / und laß' es so nach Lust der tollen Flut / fort schweben. (C.II: HK4/G04.00001 Greiffenberg: Geistliche Sonnette, Lieder und Gedichte, 1662)

(857) Darum er mit Fuß und Händen / auch über sein Vermögen / wider die Winde zu segeln / die auflauffende Wellen zu bestürmen / und an seine verlorne Lust-Insul wieder anzuländen arbeitete: hätte auch bey nahe die Zeit getroffen / wann nicht selbst sein Trotz-begieriger Vorsatz / den Zorn der tollen Wellen / und Grimm der wilden Fluthen immer mehr ereyfert und erhitzet hätte. (C.II: HK4/S11.00001 Stockfleth: Die Kunst- und Tugend-gezierte Macarie, Erstdruck: 1669-1673)

(858) Sie kamen just zurück, als ...

Der Rest des Sturms sie überfiel.

Kreuz, Fahnen ... sind toller Winde Spiel ...

(C.II: HK3/B00.00006 Wieland: Oberon, 1780)

(859) Und mit Jugendkraft eilte er durch die Nacht von dannen, nicht achtend den wütenden Sturm und das tolle Schneetreiben. (C.II: HK4/H03.00001 Hahn-Hahn: Maria Regina, Erstdruck: 1860, 2004 [S. 339])

(860) Glaub mir, wann du niederfällst, pfeif ich dir ein Sterblied, das meine Matrosen pfeifen, wenn der Sturm am tollsten wütet. (C.II: HK3/G42.00001 Klinger: Sturm und Drang, Erstdruck: 1776, 2000 [S. 1172])

In (856) - (860) werden Flut, Wellen, Winde, Schneetreiben und Sturm metaphorisiert, sodass auch zur Ausgangsbedeutung von toll eine übertragene Bedeutung hinzutritt. Zugleich handelt es sich um stark ausgeprägte Wettererscheinungen: starke Flut, große Wellen, heftige Winde, starkes Schneetreiben und heftigstes Wüten des Sturms. Die Ausgangsbedeutung wirkt verblasst, die intensivierende Kom- 
ponente dagegen ziemlich ausgebildet. - Das Auftreten der Gradbedeutung in $\mathrm{Zu}$ sammenhang mit der im übertragenen Sinn verwendeten Ausgangsbedeutung findet sich in Verbindung mit Geräusche ausdrückenden Substantiven:

(861) Er [...] kam endlich auf die Wohnung im Schlosse, die er ein für allemal gewählt, da sie warm, bequem und so abgelegen sei, daß wir uns, wenn und wie wir wollten, dem tollen Getöse der jubilierenden Gesellschaft entziehen könnten. (C.II: HK3/F79.00001 Hoffmann: Nachtstücke, Entstanden: 1814-1817, 2000 [S. 575])

(861') 'das Getöse der jubilierenden Gesellschaft war so stark, dass die Menschen, die zu dieser Gesellschaft gehörten, wahnsinnig wirkten.'

(862) Toller und toller wurde der Lärm, und man fing bereits an, sich zu stoßen und zu prügeln, als der Mann im brokatnen Schlafrock [...] auf den Baumkuchen kletterte und [...] dreimal laut rief: „Konditor! Konditor! Konditor!“ (C.II: HK3/E00.00001 Hoffmann: Die Serapionsbrüder, Erstdruck: 1819-1821, 2000 [S. 309])

(862') 'Der Lärm war so stark, dass die Menschen, die den Lärm machten, wahnsinnig wirkten.'

(863) Die Sängerin war in einer bunten krausen Fermate begriffen. Das wirbelte auf und $a b$ - auf und ab - endlich hielt sie einen langen Ton - aber nun brach eine weibliche Stimme plötzlich in tolles Zanken aus - Verwünschungen, Flüche, Schimpfreden! (C.II: HK3/E00.00001 Hoffmann: Die Serapionsbrüder, Erstdruck: 1819-1821, 2000 [S. 91])

(863') 'Das Zanken war so laut bzw. stark, dass die Frau, die es machte, wahnsinnig wirkte.'

(864) Drei rothbackige Kinder <.... tummelten sich lustig im Grünen und jagten mit so tollem Geschrei den Schmetterlingen nach, daßjetzt zwischen ihrem Jubel eine sanfte Warnungsstimme laut wurde. (C.II: HK4/B27.00001 Büchner: Der kleine Vagabund, Erstdruck: 1878, 2004 [S. 318])

(864') 'Das Geschrei war so laut, dass die Kinder wahnsinnig wirkten.'

Die Gradbedeutung kann außerdem durch semantic repetition in Erscheinung treten. Die Intensivierung des adjektivischen Gebrauchs erfolgt durch die Wiederholung der gemeinsamen Bedeutungskomponente "Wahnsinn" in den beiden Kollokationspartnern, was zu der Verstärkung des Ausdrucks führt und toll zu einem Gradadjektiv macht, vgl.:

(865) „[...] doch war er ein ganz artiges Talent zu nennen, und daß er zuletzt in tollen Wahnsinn verfiel, das nimmt doch wohl unser Mitleid in Anspruch, und zwar um so mehr, als die Anstrengung des Spiels doch wohl die Ursache seines Wahnsinns ist." (C.II: HK3/A68.00009 Hoffmann: Prinzessin Brambilla, Erstdruck: 1820)

(865') 'großen Wahnsinn'

(866) „[...] Ich habe vorhin, um dich zu prüfen, absichtlich den tollen Unsinn in meinen sonst vernünftigen Traum hineingebracht und du nahmst es so natürlich wie zweimal zwei vier." (C.II: HK3/F49.00001 Mörike: Maler Nolten, Entstanden: 1828/30)

(866') 'großen Unsinn' 
Ferner tritt toll in rein intensivierender Funktion auf und demonstriert die Erweiterung des einstellungsbezogenen Ausdruckspotentials des Lexems. Zunächst sind jedoch die Kookkurrenzmöglichkeiten auf negativ konnotierte Kollokationspartner beschränkt. Darüber hinaus kann die Desemantisierung nicht als vollständig bezeichnet werden, vgl. den adjektivischen und adverbialen Gebrauch:

(867) Wer hätte in diesem weichlichen verliebten Alten den Falieri erkennen sollen, der in Treviso in toller Hitze am Fronleichnamsfeste dem Bischof ins Gesicht schlug, der den tapfern Morbassan besiegte. (C.II: HK3/E00.00001 Hoffmann: Die Serapionsbrüder, Erstdruck: 1819-1821, 2000 [S. 475])

(867') 'sehr großer Hitze'

(868) Aber tollen Hunger mußte der Kerl haben; immer wieder zeigte er auf den Mund und hob dann flehend die Hände. (28.01.2014: http://books.google.de $/$ books?id=HQpFAAAAIAAJ\&q=\%22tollen+hunger\%22\&dq=\%22tollenthunger\%22\&hl=de\&sa=X\&ei=0pvnUrvrJIrAtAaKoICoAg\&ved=0CDsQ6AEwAzgK)

(868') 'sehr großen Hunger'

(869) Das war viel Arbeit und ich habe bei den Temperaturen ganz toll geschwitzt. (28.01.2014: http://www.sportschiessen-neiden.de/content/ostern2011)

(869') 'sehr geschwitzt'

(870) Vielleicht hätte ich das Heini auch mal sagen sollen, aber ich kann auch ganz toll beleidigt sein. (28.01.2014: http://books.google.de/books?id=g1FtAgAAQBAJ\&pg =PT62\&dq=\%22toll+beleidigt $\% 22 \& \mathrm{hl}=$ de\&sa $=$ X\&ei=1JfnUv2COIPStAb04IGoAg\&ved =0CDIQ6AEwAA\#v=onepage \&q=\%22toll\%20beleidigt $\% 22 \& f-$ =false)

(870') 'sehr beleidigt'

(871) „Peitsche. Er hat mich ausgepeitscht. Ich konnte mich eine Woche nicht bewegen und nur so auf dem Bauch liegen und es hat ganz toll weh getan." (28.01.2014: http://books.google.de/books?id=Hy-YNLA85XwC\&pg=PA17$7 \& \mathrm{dq}=\% 22$ toll + weh $\% 22 \& \mathrm{hl}=$ de\&sa=X\&ei=iqXnUorpE8qGswbBxoCoBA\&ved=0CFgQ6AEwBw\#v=onepage \&q=\%22toll\%20weh\%22\&f=false)

(871') 'sehr weh getan'

Toll kann außerdem als Gradadverb Funktionsverbgefüge modifizieren und damit die Ausdehnung seines Bezugsbereichs demonstrieren. Die Intensivierung erfolgt dadurch, dass die Bezugswörter Druck und Schwierigkeiten in den hervorgehobenen FVGs in (872) - (873) graduierbar sind, während die FVGs in (874) - (875) durch einen graduierbaren Begriff wiedergegeben werden können, vgl.:

(872) In der zweiten Halbzeit versuchten die Jungs Alles, setzen den Gegner toll unter Druck, schaften es aber leider nicht den Ball im Tor zu versenken, sodass es beim unglücklichen 0:1 für Merzhausen blieb. (28.01.2014: http://www.jugend-mhc.de/reports/show/387/)

(872') 'unter sehr großen Druck'

(873) Nobita geht in die 4. Klasse, ist normalerweise unkonzentriert, nicht wiederum paraguayisch in der Zeitgeschichte und gerät toll in Schwierigkeiten. (09-07.2014: http://sepp.xylou.de/a/DAA/03eff6f0/70-Millionen-MarkProjekt_abgeschlossen._Der_.html) 
(873') 'in sehr große Schwierigkeiten'

(874) Lauwarme Menschen können ganz toll auf die Nerven gehen. (09.07.2014: http://forum.spiegel.de/showthread.php?t=100163\&page=14)

(874') 'sehr nerven'

(875) Der nervt. Allein schon die Überschriften. Der sagt einfach nur was ist aber seine Prognosen sind fürn $A^{*}$. Nebenbei wird mehrmals täglich die Prognose geändert. Das kann ich auch. Einfach anschauen was gerade passiert ist und dann das ganze dramatisieren. Echt toller Mann. Der kann einem richtig toll auf den Geist gehen! (09.07.2014: http://www.wallstreet-online.de/diskussion/500-beitraege/695773-1-500/nabil-khayat-istein-angeber\#beitrag_8597850)

(875') 'sehr nerven'

Toll kann auch in quantifizierender Funktion auftreten. Beispielsweise findet sich die Verwendung tolle Summe, die zunächst in negativ konnotierten Kontexten auftritt, in der Gegenwartssprache jedoch meistens positive Konnotationen bekommt, vgl.:

(876) Er hat nun vielleicht einmal die Antipathie; meinte der Assessor. Oder, rief der Legationsrat, er ist auch bloß verstimmt und läßt seine üble Laune an dem unglücklichen Freier aus; er soll bereits eine tolle Summe an Mister Douglas verloren haben. (09.07.2014: http://books.google.de/books?id=d3RHAQAAIAAJ\&q=\%22tolle+summe $\% 22 \& \mathrm{dq}=\% 22$ tolle+summe $\% 22$ \&hl=de\&sa=X\&ei=VR-9U4TPFrD74QS4zoFg\&ved=0CEwQ6AEwCA)

(876') 'sehr große Summe; negativ'

(877) Er kaufte einen Spiegel für die tolle Summe von 250,000 Franken. (09.07.2014; 1869; http://books.google.de/books?id=fvdAAAAAcAAJ\&pg=PA209\&dq=\%22tolle+summe\%22\&hl=de\&sa=X\&ei= MSi9U_bAIan24QS_nYGoDg\&ved-

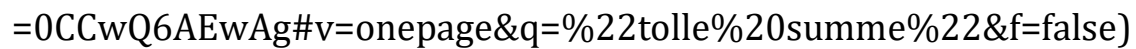

(877') 'sehr große Summe; negativ'

(878) Eines Abends in Venedig ist der Dichter betrunken und verliert eine tolle Summe im Spiel - zehntausend Francs. (09.07.2014: http://books.google.de / books?id=uHZUAAAAYAAJ \&q=\%22tolle+summe $\% 22 \& \mathrm{dq}=\% 22$ tolle+summe $\%$ 22\&hl=de\&sa=X\&ei=HSS9U6-fF8jk4QSGwIBo\&ved=0CEQQ6AEwCDgK)

(878') 'sehr große Summe; negativ'

(879) Regelmäßig werden die Jackpots an den Cash Game Tischen geknackt, die glücklichen Gewinnner dürfen sich über tolle Summe[n] freuen. (09.07.2014: http://www.hochgepokert.com/2014/07/09/pot-limit-omaha-jackpot-im-montesino-gefallen/)

$(879$ ') 'sehr große Summen; positiv'

(880) Den Jungs ist es gelungen, in nur 30 Tagen fast eine Million Euro zu sammeln, um das Magazin ein Jahr lang zu betreiben. [...] Zu allererst ein mal herzlichen Glückwunsch an das Team für diese tolle Summe. (09.07.2014: http://meedia.de/2014/06/19/cory-haik-von-der-washington-post-bezos-findetprint-und-online-perfektes-paket/)

(880') 'sehr große Summe; positiv' 
Der Gebrauch des Lexems in quantifizierender Funktion bleibt jedoch nicht auf die Verwendung tolle Summe beschränkt, vgl.:

(881) Alle Vorleser werden mit Namen in der ZEIT veröffentlicht und haben zudem die Chance auf tolle Gewinne. (DTA: Die Zeit, 04.08.2005, Nr. 31)

(881') 'sehr große Gewinne'

(882) „Wir haben noch daran geglaubt, als Brasilien zum Veranstalter gewählt wurde. Da dachten wir, okay, jetzt bekommen wir tolle Investitionen in unsere Infrastruktur und so. Passiert ist aber nichts." (18.02.2014: http://www.kleinezeitung.at/sport/fussball/wm/3530026/larissa-brasilianerhaben-nichts-wm.story)

(882') 'sehr große Investitionen'

Aus den in (876) - (882) eingeführten Kontexten kann geschlossen werden, dass toll zunächst einen Zusammenhang mit der Ausgangsbedeutung durch die negativen Konnotationen aufweist, diesen anschließend verliert, weil das Lexem in positiven Kontexten auftritt und damit Desemantisierung bezeugt.

Die Belege (867) - (875) und (876) - (882) demonstrieren jeweils den intensivierenden und quantifizierenden Gebrauch von toll. Der Gebrauch des Intensivierungsmittels nimmt an subjectivity zu, verweist ausschließlich auf ,internal described situation“ bzw. die Verwendung des Lexems ausschließlich auf der expressiven Ebene und unterliegt damit von Traugott III (oben 3.6.3.4).

In der letzten Phase des Entwicklungsgangs verliert toll endgültig den Zusammenhang zur Ausgangsbedeutung, indem es als Intensivierer in Verbindung mit positiv konnotierten Kookkurrenzpartnern vorkommt, vgl.:

(883) Mit den Klassikern von Heine, Busch, Morgenstern, Ringelnatz, Tucholsky, Brecht, Jandl und vielen erstaunlichen Wiederentdeckungen. Ganz im Ernst: ein toller Spaß! (DTA: Die Zeit, 28.07.2004, Nr. 30)

(883') 'sehr großer Spaß'

(884) Ich ...denke, dass das Finanzamt ... ganz toll stolz auf mich sein mUSS...(DTA: DIE ZEIT, 10.01.2008, Nr.2)

(884') 'sehr stolz'

(885) Er und seine Kollegen sind stolz darauf, wie toll viele der jungen Musiker sich weiterentwickelt haben. (C.II: RHZ13/JAN.04896 Rhein-Zeitung, 07.01.2013, S. 21)

$(885$ ') 'sehr viele'

Das Auftreten in positiv konnotierten Kontexten schließt auch Funktionsverbgefüge ein, vgl.:

(886) Die Eulen sind ja zur Zeit ganz toll in Mode gekommen. Überall auf T[Sh]irts und Schmuck. (28.01.2014: http://www.nexusboard.net/showthread.php?siteid=1381\&threadid=303935 \&showpage=8)

(886') 'sind sehr modisch geworden'

Die Entwicklung der meliorativen Bedeutungskomponente ist für toll besonders bemerkenswert, da das Lexem für den Ausdruck rein positiver Bewertung verwendet wird, was in Bezug auf die anderen in dieser Arbeit untersuchten Lexeme nur 
für „unmenschlich“ einschlägig ist. Die positive Bedeutung hat laut FWB schon im Fnhd. eingesetzt. Jedoch bietet der Mangel an Belegen keine aussagekräftige Begründung für den semantischen Wandel. Außerdem steht das Problem der Melioration nicht im Fokus der vorliegenden Arbeit. Aus diesen beiden Gründen wird im weiteren auf den Versuch einer Erklärung verzichtet. In (887) - (889) wird der Gebrauch exemplifiziert:

(887) Aufkeinen Fall, sagt Tom Geimer, ein amerikanischer Venture-Capitalist, dürfe man die weichen Standortvorteile des Bavarian Way of Life unterschätzen: „tolles Wetter, viele Biergärten, viele Cabrios“. Dank der hohen Lebensqualität sei es nicht so schwierig, von überall einflussreiche Partner anzulocken. (DWDS: Die Zeit 19.08.1999, 19.08.1999)

(887') 'sehr gutes Wetter'

(888) Ja, meine Wohnung ist toll. Hohe Decken, großer Balkon, die Miete geht auch. Zudem mag ich meine Nachbarn, die weghören, wenn ich Geburtstag feiere. (C.II: HMP13/JAN.02114 Hamburger Morgenpost, 26.01.2013, S. 01, 0607)

(888') 'meine Wohnung ist sehr gut'

(889) Am 17. November schickt das Bürgermeisteramt einen Brief: „Mit Freude" bestätige man noch einmal die Unterstützung für diese tolle Ausstellung. (DWDS: Die Zeit, 06.05.2014)

(889') 'sehr gute Ausstellung'

\subsubsection{Kurzfassung der Entwicklung bei toll}

Ausgangsbedeutung: 'geistig gestört' (auch im übertragenen Sinne); die übrigen Entwicklungsschritte wie oben in Kap. 3.6.3.9.3.

\subsubsection{Verrückt}

\subsubsection{Lexikographische Angaben}

Laut Kluge (s.v.) erscheint die Form im 16. Jh. Das Wörterbuch stellt fest, dass die Ausgangsbedeutung „einfach ,von der Stelle gerückt' zu mhd. verrücken, verrucken, mndd. vorrucken ,von der Stelle rücken, aus der Fassung bringen “" ist. Der Übergang zu der Bedeutung der mentalen Störung „wird [...] durch sinnverrückt, ,dessen Sinn von der Stelle gerückt ist' und ähnliche Bildungen“ „unterstützt". Man findet keine Informationen über das Bedeutungsspektrum des Wortes in AWB, Splett, BMZ, Lexer und FWB, noch in DWB.

1. verrückt in FWB (s.v.)

Ableitung von verrücken im Sinne „wegschieben“, „versetzten“, „wegziehen“, „seinen Platz verlassen“69

2. verrückt in DWB (s.v. verrücken)

2.1 „von der bisherigen stelle weggeschoben mit der nebenbedeutung 'von der richtigen an eine falsche stelle'“

2.2 „thöricht, verwirrt, irrig“

${ }^{69}$ Angaben aus den Belegzetteln der Arbeitsstele von FWB. 
3. verrückt in DUDEN (2012, s.v.)

3.1 "(salopp) krankhaft wirr im Denken und Handeln“

3.2 „(umgangssprachlich) auf absonderliche, auffällige Weise ungewöhnlich, ausgefallen, überspannt, närrisch"

3.3 „<intensivierend bei Adjektiv> (umgangssprachlich) über die Maßen, auBerordentlich, sehr"

\subsubsection{Entwicklung der Gradbedeutung bei verrückt}

Die Korpusanalyse ergibt, dass die Herausbildung der Gradbedeutung bei verrückt im Neuhochdeutschen begonnen hat. Als Grundlage dafür dient die in 2.2 angegebene Bedeutung, die geistesgestörte Personen beschreibt. Hinsichtlich dieser Bedeutung verweist DWB (s.v. verrücken) darauf, dass „das wort verrückt in der bedeutung 'geistesgestört' noch nicht sehr alt ist [...]. unsere literarischen belege führen bis in die zweite hälfte des 17. jahrh. früher kommt das wort auch schon in der bedeutung vor, aber nur als bild empfunden. dies sieht man daran, dasz 'im kopf, im hirn' u. ä. dabeistehen musz", vgl. die aus DWB und COSMAS übernommenen Belege:

(890) daher der fürst und andere gedachten, er wäre vielleicht im kopff verruckt (DWB: s.v. verrücken)

(891) [...] „Wahrhaftig, mein lieber Sohn, der arme Mensch ist verrückt; man binde ihn, und setze ihn auf eines unserer Drometaren, vielleicht, daß wir dem Unglücklichen Hülfe schaffen können. "(C.II: HK3/F72.00001 Hauff: Märchen-Almanach auf das Jahr 1826, Erstdruck: 1826, 2000 [S. 88])

(892) Eine weitere Modifikation der in Rede stehenden ersten Hauptform des verrückten Zustandes ist die Zerstreutheit. (C.II: HK5/A60.00001 Hegel: Enzyklopädie der philosophischen Wissenschaften im Grundrisse, Erstdruck: 1817, 2000 [S. 173])

Das Lexem wird außerdem metaphorisch in Bezug auf seelisch gesunde Menschen verwendet, die sich durch ihr unlogisches und närrisches Verhalten in der Art und Weise wie geisteskranke Personen Verhalten, vgl.:

(893) „Bist du verrückt geworden, Junge?" rief der Alte und schlug mit der Faust auf das große Schreibpult. (C.II: HK3/A96.00029 Weerth: Fragment eines Romans, Entstanden: 1843/44 und 1846/47, 2000 [S. 307])

(894) Jetzt entstand Lärm im Vorzimmer. Ippolito beiseite werfend, verwildert, mit rasenden Mienen und verrückten Augen stürzte der Kanzler herein. (C.II: HK3/A27.00008 Meyer: Die Versuchung des Pescara, Erstdruck: 1887, 2000 [S. 783])

(895) Dann lachte er gezwungen und sagte: „Wie kamst du nur auf diese verrückte Idee?! [...]" (C.II: HK4/B21.00001 Braun: Lebenssucher, Erstdruck: 1915, 2004 [S. 388])

Die Metaphorisierung des Gebrauchs knüpft an die abstraktere Sphäre der Ausgangsbedeutung und den Übergang der Verwendung auf die expressive Ebene und zu Traugott I (oben 3.6.3.4) an. 
Das Einsetzen der Intensivierung beginnt mit pragmatic enrichment bzw. strengthening von Kontexten, in denen sich verrückt zunächst auf die Ausgangsbedeutung bezieht und zugleich Intensivierung als Nebenbedeutung aufweist, vgl.:

(896) In dieser Szene mußte Emmrich seine Meisterschaft zeigen. Den Übermut und die verrückte Eitelkeit des ältlichen Mannes, die bis an die Grenze des Unmöglichen gesteigert wird, wußte er so natürlich darzustellen, [...] daß alle Zuschauer sich getäuscht dem behaglichsten Lachen überlassen konnten. (C.II: HK3/D39.00001 Tieck: Der junge Tischlermeister, Erstdruck: 1836, 2000 [S. 423])

(897) In seiner Ratlosigkeit [...] fing er, ohne zu überlegen, an eine beliebige kleine Tür zu schlagen an, bei der er in seinem Herumirren stockte. „Es ist ja offen", rief es von innen und Karl öffnete mit ehrlichem Aufatmen die Tür. „Warum schlagen Sie so verrückt auf die Tür?" fragte ein riesiger Mann, kaum daß er nach Karl hinsah. (C.II: HK3/B22.00001 Kafka: Amerika, Entstanden: zwischen 1911, 2000 [S. 10])

Die Belege (896) - (897) sind mehrdeutig interpretierbar. In (896) handelt es sich um die Eitelkeit eines ältlichen Mannes, „die bis an die Grenze des Unmöglichen gesteigert wird“. Durch die Bezeichnung verrückt verweist der Schreiber bzw. Sprecher direkt bzw. metaphorisch auf den geistig-seelischen Zustand der genannten Person und hebt das Maß der Eitelkeit hervor. Aus dem Kontext kann geschlossen werden, dass das Maß der Eitelkeit für einen seelisch gesunden Menschen zu hoch ist, was auf die Intensität der Eigenschaft hinweist. Damit bezieht sich verrückt direkt bzw. metaphorisch auf die Ausgangsbedeutung sowie auch die Intensivierung.

Der Gebrauch in (897) verweist auf die metaphorische Verwendung der Ausgangsbedeutung des Lexems sowie dessen intensivierende Funktion, wobei die beiden in einem Zusammenhang miteinander stehen, vgl. die mögliche Paraphrase:

(897') 'Karl schlägt so heftig an die Tür, dass er als wahnsinnig wirkt.'

Ferner tritt verrückt in den Kontexten auf, die den Prozess der Desemantisierung des Lexems veranschaulichen. Bleaching lässt sich daran erkennen, dass die Gradbedeutung den direkten Zusammenhang mit der Ausgangsbedeutung verliert. Jedoch findet sich das Lexem zunächst mit negativ konnotierten Kollokationspartnern, was auf die Unvollständigkeit der Desemantisierung verweist, vgl.:

(898) Diese verrückte Hitze! Einer 80-Jährigen in Wiesbaden haben die schweißtreibenden Stöhn-Temperaturen zu ungewohntem Reichtum verholfen. (C.II: HMP13/JUN.01945 Hamburger Morgenpost, 21.06.2013, S. 53)

(898') 'sehr große Hitze'

(899) Doch wo Fripp öfter Melodien einfallen, die einem in Erinnerung bleiben, ist bei Didkovsky einfach alles immer ganz kompliziert und verrückt schwierig zu spielen. (C.II: E96/JUL.18385 Zürcher Tagesanzeiger, 23.07.1996, S. 57)

(899') 'sehr schwierig' 
(900) Allenfalls eine schnelle Tasse caffè ist drin, natürlich aus diesen verrückt unpraktischen "Maschinen“, die zugleich zum Erwärmen und zum Ausschenken benutzt werden und immer tropfen und außerdem am Henkel viel zu heiß werden, selbst mit Küchenhandschuhen. (C.II: BRZ07/JUN.14910 Braunschw. Z., 11.06.2007)

(900') 'sehr unpraktischen'

Die Korpusanalyse hat keine Belege ergeben, in denen verrückt als Intensivierungsmittel über Funktionsverbgefüge fungiert.

Verrückt kann in quantifizierender Funktion auftreten und die Menge der Sachverhalte bezeichnen, vgl.:

(901) Die teuersten, neuesten Maschinen liefs man von Europa kommen, die Aktien wurden zu fabelhaften Preisen gehandelt, neue Diamantgruben, nicht nur bei Kimberley, wurden täglich eröffnet und verrückte Preise für Grundstücke gezahlt, ohne Rücksicht, ob der Boden diamanthaltig war oder nicht; die Aktien solcher Minen waren am Tage der Emission schon 1000 über Pari [...] (10.07.2014: http://books.google.de/books?id=gS44AQAAIAAJ\&q=\%22verr\%C3\%BCckte+preise $\% 22 \& d q=\% \quad 22$ verr $\% C 3 \% B C-$ ckte+preise\%22\&hl=de\&sa=X\&ei=dku-U_fhMYe506fAgPAE\&ved=0CDAQ6AEwATgU)

(901') 'sehr hohe Preise'

(902) Auch wir in Deutschland lebten über unsere Verhältnisse und die Politik ermöglichte den Spekulanten astronomische (verrückte) Gewinne zu erzielen und nun bekommen wir alle die Rechnung präsentiert. (10.07.2014: http://books.google.de/books?id=i9HPK4ajUKYC\&pg=PA17\&dq=\%22verr\%C3\%BCckte+gewinne\%22\&hl=de\&sa=X\&ei=dkyU_ScNYOBOLLBgLgE\&ved=0CCgQ6AEwAA\#v=onepage\&q=\%22verr\%C3\%BCckte\%20gewinne\%22\&f=false)

(902') 'sehr hohe Gewinne'

Die Belege (898) - (900) und (901) - (902), welche die intensivierende und quantifizierende Rolle des Lexems exemplifizieren, belegen eine Zunahme an subjectivity, indem verrückt mehr einstellungsbezogen wirkt und ausschließlich auf der expressiven Ebene verwendet wird. Damit tritt die dritte semantisch-pragmatische Tendenz (Traugott III, oben 3.6.3.4) in Erscheinung.

Anschließend findet sich das Lexem in rein intensivierender Funktion, indem es mit positiv konnotierten Bezugswörtern kollokiert und den Zusammenhang mit der Ausgangsbedeutung endgültig verliert, vgl.:

(903) Im Zeitlupentempo gleiten wir durch den weichen, tiefen Sand den Steilhang hinunter und stellen fest: "Sand-Skiing“ ist ein herrlich verrückter Spaß unter sengender Sonne und bei weitem nicht das einzige Kuriosum, das der Golfstaat Katar zu bieten hat. (C.II: NUN00/APR.02456 NN, 29.04.2000, S. 5)

(903') 'sehr großer Spaß'

(904) [...] ein schillernder Vogel in zwei metallischen Blauabstufungen mit herrlich rotem Schnabel, vom Körperbau her eine Kreuzung zwischen Fasan und kleiner Gans, ein Sumpfhuhn eben. Im Abel Tasman Gebiet an der 
nördlichen Küste der Südinsel ist er uns begegnet. Ein verrückt schönes Wesen. (C.II: R97/JAN.02269 Frankf. Rundschau, 11.01.1997, S. 1)

(904') 'sehr schönes'

(905) Das Markenzeichen der Athletin ist ihre feine Technik und ihr stets ruhiger Oberkörper. Sie sei verrückt gut, schrieb ihr Landsmann Ted Ligety in einem Tagebuch für die «Denver Post». (C.II: A13/JAN.05755 St. Galler Tagblatt, 17.01.2013, S. 19)

(905') 'sehr gut'

(906) Die Ausstellung «Gretlers Panoptikum - Fotografie und Grafik zur Sozialgeschichte» ist eine verrückt spannende, labyrinthische Schaubude, und ein Veranstaltungswunder, verantwortet vom St. Galler Verein Pantograph und dem Amt für Kultur. (C.II: A13/MAR.00046 St. Galler Tagblatt, 01.03.2013, S. 30)

(906') 'sehr spannende'

Das Lexem wird außerdem in der Konstruktion wie verrückt mit intensivierender Bedeutung verwendet. Es ist bemerkenswert, dass diese Wortverbindung den gleichen Entwicklungsgang bis zur Intensivierung eingeschlagen hat. Zunächst tritt 'wie verrückt' in doppeldeutigen Kontexten auf, wo es sich auf menschliche Handlungen oder Gefühle bezieht und damit die Ausgangs- sowie die Intensitätsbedeutung ausdrückt, vgl.:

(907) Eines Morgens schloß er seinen hagestolzlichen Kunsttempel zu und rannte wie verrückt nach dem St. Gotthard, hinüber und kam nicht wieder. (C.II: HK3/D35.00001 Keller: Der grüne Heinrich [Erste Fassung], Entstanden: zwischen 1846 und 1855, 2000 [S. 229])

(907') 'rannte sehr schnell, als ob er wahnsinnig wäre'

(908) Dennoch möchte er das Erlebnis Nationalmannschaft nicht missen. „Wenn du einläufst, die Zuschauer wie verrückt brüllen und dann die Nationalhymne erklingt, dann läuft dir schon ein kalter Schauer den Rücken runter", hat er bei der Rückschau ein Glänzen in den Augen. (C.II: M02/NOV.86270 Mannh. Morgen, 19.11.2002)

(908') 'sehr laut brüllen, als ob sie wahnsinnig wären'

(909) „Es ist total verwirrt, wenn er seinen Körper bewegt - er flattert mit den Ellenbogen wie verrückt, als ob er sich nicht so recht sicher ist, was er tun soll. [...]" (C.II: M08/DEZ.97539 Mannh. Morgen, 16.12.2008, S. 15)

(909') 'flattert sehr aktiv bzw. intensiv mit den Ellenbogen, als ob er wahnsinnig wäre'

Ferner fungiert wie verrückt in intensivierender Funktion und bezieht sich auf negativ konnotierte Bezugswörter, ohne den Zusammenhang mit der Ausgangsbedeutung zu demonstrieren, vgl.:

(910) Hollywood-Star Pierce Brosnan (51) hat sein Ende als Geheimagent 007 stark mitgenommen: »Bond zu verlieren, hat mich wie verrückt verletzt, es war ein Schock, schmerzhaft und unerwartet." (C.II: NUZ05/APR.02599 $\mathrm{NZ}, 23.04 .2005)$

(910') 'sehr verletzt' 
(911) Caroline hat einmal einen Hund gestreichelt, sofort hat sie Ausschlag bekommen und wie verrückt gehustet. (C.II: M06/SEP.69182 Mannh. Morgen, 02.09.2006)

(911') 'sehr stark gehustet'

(912) Bei Kälte juckt ihre Haut wie verrückt und schwillt an [...]. (C.II: A12/FEB.01009 St. Galler Tagbl., 03.02.2012, S. 1)

(912') 'juckt sehr stark'

(913) Als Büsser gegen 17.30 Uhr nach Hause kam, kam starker Wind auf. «Es hat wie verrückt geregnet», sagt sie. (C.II: A12/OKT.03517 St. Galler Tagbl., 09.10.2012, S. 36)

(913') 'sehr stark geregnet'

Wie verrückt kann außerdem in intensivierender Funktion in der Konstruktion mit verschobenem Skopus verwendet werden, d.h. Funktionsverbgefüge modifizieren, vgl. die Belege mit den entsprechenden Umformulierungen:

(914) Solche Tage sind die schlimmsten und die ziehen sich auch wie verrückt in die Länge. (10.07.2014: http://animexx.onlinewelten.com/fanfiction/276921/seiten/\#paragraph-0)

(914') 'dauern viel länger als sonst'

(915) Sie setzten sie wie verrückt unter Druck - brachten minutiös alles über sie in Erfahrung. (10.07.2014: http://books.google.de/books?id=jwhsAgAAQBAJ\&pg=PT496\&dq=\%22wie $\quad \%$ C3\%BCckt+unter + druck\%22\&hl=de\&sa=X\&ei=FI6U8_4HvT54QT_6oD4CQ\&ved=0CB8Q6AEwAA\#v=onepage \&q=\%22wie $\% 20$ verr $\%$ C3\%BCckt $\% 20$ unter\%20druck\%22\&f=false)

(915') 'setzten unter sehr großen Druck'

(916) Dass Wolfgang Metorn beim Aufhängen seiner Winterreifen in der Garage versehentlich das Stromkabel zum Gewächshaus durchtrennte und seine Pflänzchen unbemerkt tagelang ohne Heizung überwinterten, scheint ihnen nicht geschadet zu haben, sie blühten offenbar wie verrückt. (C.II: M06/JUN.43560 Mannh. Morgen, 06.06.2006)

(916') 'blühten sehr stark'

(917) Den ersten Zylinder hat ein Engländer getragen und hergestellt. Der Hutmacher John Hetherington trug das schwarze Rohr aus Seide erstmals im Januar 1797 auf dem Kopf. Das Aufsehen war enorm. Als er durch die Londoner Straßen lief, wurde sogar ein Mann von der aufgebrachten Menge in ein Schaufenster gedrückt und verletzte sich dabei. Hetherington musste 50 Pfund Strafe zahlen. Aber das dürfte ihn nicht weiter gestört haben, denn nach seinem Auftritt verkaufte sich der Zylinder wie verrückt. (C.II: M07/JAN.02635 Mannh. Morgen, 13.01.2007)

(917') 'verkaufte sich sehr gut'

(918) In Zeiten des Aufschwungs steigen die Ansprüche an den Wald als Rohstoff-Lieferanten. „Die Nachfrage nach Holz boomt wie verrückt", sagt Siegfried Weiter, Bereichsleiter Öffentlichkeitsarbeit im Forstamt Haardt. (C.II: M11/MAR.02877 Mannh. Morgen, 09.03.2011, S. 25)

(918') 'erlebt einen sehr großen Boom' 


\subsubsection{Kurzfassung der Entwicklung bei verrückt}

Ausgangsbedeutung: 'geistig gestört, wahnsinnig';

die übrigen Entwicklungsschritte wie oben in Kap. 3.6.3.9.3.

\subsubsection{Wahnsinnig}

\subsubsection{Lexikographische Angaben}

Laut Kluge (s.v.) stellt das Wort eine Ableitung von wahn ${ }^{70}$ dar und ist seit dem 15. Jh. bezeugt. Das Lexem wird „dem Adjektiv wahnwitzig ... nachgebildet“, indem es „wie dieses als erstes Kompositionsglied das dort behandelte Adjektiv ahd. mhd. wan 'fehlend, leer“' „enthält" (Pfeifer, s.v.).

1. wahnsinnig in FWB

keine lexikographischen Angaben; die Belege aus der Datenbank verweisen auf den Gebrauch in Bezug auf die geistige Störung.

2. wahnsinnig in DWB (s.v.)

2.1 „das wort geht auf alle arten von geisteskrankheit"

2.2 "übertragen steht dann wahnsinnig von zuständen groszer erregung, besonders in folge von leidenschaften"

2.3 „daraus hat sich in der neueren umgangssprache der gebrauch entwickelt, dasz wahnsinnig überhaupt [...] einen auszergewöhnlichen grad von etwas bezeichnen kann"

2.4 „wahnsinnig ist ferner 'thöricht, unvernünftig, verblendet'. die bedeutung kann durch abschwächung aus der 1. hervorgegangen sein, doch kann sie bei den alten belegen auch als gleich ursprünglich angesehen werden“

2.5 „vereinzelt [...]: wansinnig, opiniosus, prava sentiens“

3. wahnsinnig in DUDEN (2012, s.v.)

3.1 ,(Medizin veraltet, sonst salopp) an Wahnsinn (1) leidend; von Wahnsinn zeugend, in seinen geistig-seelischen Funktionen gestört"

3.2 „(umgangssprachlich) ganz unsinnig, unvernünftig“

3.3 „(umgangssprachlich)“

3.3a „übermäßig groß, stark, heftig, intensiv“

$3.3 \mathrm{~b}$ „<intensivierend bei Adjektiven und Verben > sehr, überaus, in höchstem Maße“

3.4 „(umgangssprachlich) in begeisternder Weise schön, gut; großartig, toll“

\subsubsection{Entwicklung der Gradbedeutung bei wahnsinnig}

In Anbetracht der Tatsache, dass keine Informationen über das Bedeutungsspektrum von wahnsinnig in FWB mit Ausnahme eines Belegs im Computersystem zugänglich sind, kann man sich bei der Untersuchung der Gradbedeutung des Lexems lediglich auf die Angaben vom DWB sowie der Korpusanalyse stützen. DWB (s.v.) verweist darauf, dass Fnhd. das Lexem in den Bedeutungen 2.1 und 2.5 belegt ist. Daraus lässt sich schließen, dass die Gradbedeutung im Neuhochdeutschen eingesetzt hat. Diese Annahme lässt sich durch wenige aus DTA entnommenen Belege

70 „leer, unverständig, mangelhaft“ (Lexer, s.v. wahn) 
bestätigen, die aus dem Jahr 1660 datieren. Als Grundlage für die Entwicklung der Intensitätsbedeutung dient die in 2.1 angegebene Sinnrichtung, die auf eine geistig-seelische Erkrankung hinweist. Der Gebrauch bezieht sich auf Personen sowie ihr Verhalten, vgl.:

(919) er war nicht bey verstand, er war wahnsinnig. (DWB, s.v.)

(920) eine wahnsinnige rede, ein wahnsinniges benehmen (DWB, s.v.)

Ferner kommt es laut DWB (s.v.) zu einer „abschwächung“ des Begriffs, wobei man eine Person und ihre Handlungen „nach art eines wahnsinnigen“ bezeichnet, vgl. die in 2.4 angegebene Sinnrichtung „töricht". Es handelt sich damit um eine metaphorische Übertragung, die den Vergleich eines psychisch gesunden, jedoch durch unvernünftige, törichte Handlungen bemerkenswerten Menschen mit einem geistig erkrankten Menschen und seinem Verhalten ermöglicht. DWB (s.v.) geht zugleich davon aus, dass die erworbene Bedeutung „bei den alten belegen auch als gleich ursprünglich angesehen werden“ könne, „(vgl. unsinnig früher sowol 'geistig gestört' als auch 'thöricht' und wahnwitzig 3)“. Der Beleg (921) soll den metaphorischen Gebrauch veranschaulichen:

(921) er sollte so wahnsinnig seyn und sein fortune von sich stoszen? (DWB, s.v.)

Die metaphorische Bedeutung weist auf das Auftreten von subjectivity bzw. auf die erste semantisch-pragmatische Tendenz hin (Traugott I, oben 3.6.3.4).

Das Einsetzen der Intensivierung lässt sich in mehrdeutigen Kontexten feststellen, wobei die Gradbedeutung durch pragmatic enrichment bzw. strengthening der Ausgangsbedeutung zur Geltung kommt, vgl.:

(922) Er [Reichard] würde ohn einiges leugnen geftehen / was geftalt er fich durch feine verteufelte faft unerhoirte Boßheit und wahnfinnigen ůbermuht håtte laffen verleiten / einem Hochfürftlichen Fråulein (welches ihm nicht unbewuft gewefen) nach Ehr und Keufcheit zuftreben [...] (DTA: Bucholtz, Andreas Heinrich, 1660)

(923) [...] das ist die Fürstennatur, die sich hier gezeigt, die wahnsinnige Ruchlosigkeit, die meint, ihrem persönlichen Vorteile dürfe man das Wohl eines ganzen Volkes aufopfern. Es ist nicht mehr zu ertragen, und ich fange an und werde ein Republikaner, wovon ich bis jetzt so weit entfernt war. (C.II: HK3/C78.00001 Börne: Briefe aus Paris, 1832, 2000 [S. 54])

Die Verwendungen in (922) - (923) lassen mehrere Interpretationen des Adjektivs zu. Das Lexem drückt törichtes und unvernünftiges Verhalten der in Rede stehenden Personen aus, was sich in großem Übermut in (922) und großer Rücklosigkeit in (923) manifestiert. Darüber hinaus liefert die Intensität der Eigenschaften den Grund, die Personen als wahnsinnig im übertragenen Sinne zu bezeichnen, vgl. die entsprechende Umformulierung:

(922') 'Reichards Übermut war so groß, dass er wahnsinnig wirkte'.

(923') 'Die Rücklosigkeit ist so groß, dass der Fürst wahnsinnig wirkte'.

Ferner spricht DWB (s.v.) von der Übertragung der Ausgangsbedeutung auf „zustände[] groszer erregung, besonders in folge von leidenschaften“. Dabei „dienen“ „zur vermittlung [...] wendungen wie: man könnte davon wahnsinnig werden, 
es ist zum wahnsinnig werden u. dgl.“. Darüber hinaus bezieht sich das Lexem auf den hohen Grad der Ausprägung eines emotionalen Zustandes, der mit dem geistigen Zustand einer Person in Verbindung gebracht werden kann. Damit bezeugt dieser Gebrauch wiederum das Auftreten einer Intensivierung in doppeldeutigen Kontexten, wobei die Gradbedeutung neben der metaphorischen Verwendung in Erscheinung tritt. In COSMAS finden sich zahlreiche Belege, die diese Stufe des Entwicklungsgangs veranschaulichen sollen, vgl.:

(924) „[...] Ich bin auch gestorben, ziehe nur meine glühende Seele aus ihrem knienden kalten Leichnam!" - Sie blickte mit einer wahnsinnigen Unruhe im leeren Himmel herum. (C.II: HK3/F36.00001 Jean Paul, Leben des Quintus Fixlein: 1796)

(924') 'Sie blickte mit solch großer Unruhe, dass sie wahnsinnig wirkte'.

(925) „O Serpentina, Serpentina!“ schrie der Student Anselmus in wahnsinnigem Entzücken, aber der Archivarius Lindhorst hauchte schnell auf den Spiegel [...] und an der Hand blitzte nur wieder ein kleiner Smaragd, über den der Archivarius den Handschuh zog. (C.II: HK3/E37.00001 Hoffmann: Fantasiestücke in Callots Manier, Entstanden: 1808/15)

(925') 'Der Student schrie in solch großem Entzücken, dass er wahnsinnig wirkte'.

(926) Das Entsetzen sträubte mein Haar, ich stürzte in wahnsinniger Angst heraus, durch den Park! (C.II: HK3/D64.00001 Hoffmann: Die Elixiere des Teufels, Entstanden: 1814/15)

(926') 'Ich stürzte in so großer Angst heraus, dass ich wahnsinnig wirkte'.

Die Belege (924) - (926) exemplifizieren mehrdeutige Verwendungen, die neben der intensivierenden Funktion des Lexems dadurch den Zusammenhang mit der Ausgangsbedeutung aufweisen, dass sie den inneren Zustand der Peronen beschreiben und sich auf die Situationen großer Erregung beziehen.

Der Kontakt mit der Ausgangsbedeutung geht jedoch verloren, wenn wahnsinnig sich von menschlichem Verhalten bzw. der Ausprägung des Zustands trennt und mit abstrakte Sachverhalte ausdrückenden Bezugswörtern verbindet. Die Korpusanalyse hat mehrere Belege ergeben, die den Verlust dieses Kontakts und damit die nächste Etappe des Entwicklungsgangs veranschaulichen, vgl. den Gebrauch von wahnsinnig in negativ konnotierten Kontexten:

(927) Sie stand vor dem eisernen Öfchen, das eine wahnsinnige Hitze verbreitete, und kochte einen Kohl, das Mittagessen der Familie. (C.II: HK4/D01.00001 Dohm: Sibilla Dalmar, Erstdruck: 1896, 2004 [S. 177])

(927') 'sehr große Hitze'

(928) Seit hunderten von Marsjahren hat man sie in Ruhe gelassen. Jetzt interessieren sich plötzlich winzige Erdlinge für das Wetter auf dem Mars. Sie interessieren sich für die wahnsinnige Kälte. Der Staub und die Stürme sollen erforscht werden. (C.II: A00/MAR.20823 St. Galler Tagblatt, 18.03.2000, Ressort: TT-SER (Abk.))

(928') 'sehr starke Kälte'

(929) Da gibt es eine Menge Leute, die sagen: Wenn sie gewusst hätten, dass hier der Lärm so stark ist, dann wären sie nie dahin gezogen. Manche 
sagen: Man hat sie nicht richtig aufgeklärt. Sie haben sich ein Grundstück gekauft und stellen jetzt fest, dass da eine wahnsinnige Lärmbelästigung ist. (C.II: NUN09/JUL.05513 Nürnberger Nachrichten, 22.07.2009, S. 15)

(929') 'sehr große Lärmbelästigung'

(930) „Es war trotzdem richtig, hierher zu kommen“, stellte der dreimalige Gesamt-Weltcupsieger später im Zielraum fest: "Mir fehlt einfach noch die Sicherheit. Sechs Tage Training sind einfach wahnsinnig wenig. [...]"(M03/JAN.02892 Mannheimer Morgen, 15.01.2003)

(930') 'sehr wenig'

(931) Mit dem Tor in letzter Minute, gibt es eine kleine Chance für uns.Aber das wird wahnsinnig schwer. Wir müssen hochkonzentriert sein und eine geschlossene Leistung abrufen. (C.II: HMP12/APR.02145 Hamburger Morgenpost, 24.04.2012, S. 43)

(931') 'sehr schwer'

Wahnsinnig kann außerdem Funktionsverbgefüge intensivierend modifizieren. Das Auftreten in dieser Position wird dadurch ermöglicht, dass das ganze FVG durch einen graduierbaren Begriff wiedergegeben werden kann, wie es in (932) und (935) - (943) der Fall ist oder das Substantiv in einem FVG graduierbar ist, was für (933) und (934) ausschlaggebend ist:

(932) „Das ist mir wahnsinnig unter die Haut gegangen und hat mir den Anstoß gegeben, meine Gedichte für blinde Menschen zugänglich zu machen." (C.II: RHZ97/DEZ.07606 RZ, 11.12.1997)

(932') 'im Innersten sehr berührt'

(933) „Wir stehen wahnsinnig unter Druck", sagt Anne Schauer. (C.II: R98/JUL.56136 Frankfurter Rundschau, 15.07.1998, S. 23)

(933') 'stehen unter sehr großem Druck'

(934) So stellen Sie sich die Zusammenarbeit vor, auch wenn Sie Ihren Pfarrer Hintze dann wahnsinnig in Begründungsschwierigkeiten bringen. (C.II: PST/W03.00006 Protokoll der Sitzung des Parlaments Landtag von SachsenAnhalt am 17.07.1998. 6)

(934') 'in sehr große Begründungsschwierigkeiten bringen'

(935) Die Ferraris waren heute einfach stärker als wir. Und die McLaren-Mercedes hätten wir im Rennen nicht halten können. Zudem glückte der Start nicht optimal, und ich hatte schon in der fünften Runde vorne links einen Bremsplatten. Das ging mir wahnsinnig auf den Keks, da der Jordan dadurch stark vibrierte. (C.II: M99/AUG.50339 Mannheimer Morgen, 02.08.1999)

(935') 'nervte sehr'

(936) Sein Gegenüber geht ihm wahnsinnig auf die Nerven, weil er permanent in sein Handy brüllt und unbedingt einen Termin mit einem Geschäftspartner vereinbaren will. (C.II: M03/JUL.43015 Mannheimer Morgen, 02.07.2003)

(936') 'nervt sehr'

(937) Dabei werden die Preise wahnsinnig in die Höhe gehen. (C.II: M04/MAI.34520 Mannheimer Morgen, 26.05.2004) 
(937') 'sehr steigen'

(938) „Federer muss sich wahnsinnig in Acht nehmen vor Hewitt. [...]“ (RHZ05/JUN.34032 RZ, 30.06.2005)

(938') 'sehr vorsichtig sein'

(939) Mir geht dieses Zusammenstreichen der Stücke auf Spielfilmlänge wahnsinnig auf den Hammer. (C.II: HMP06/FEB.02069 Hamburger Morgenpost, 20.02.2006, S. 20)

(939') 'nervt sehr'

(940) Auf dem Höhepunkt seines Ruhms brauchte Rühmann, der vor allem ein begnadeter Komödiant war, einfach nur irgendwo aus dem Auto zu steigen, und die Passanten bogen sich vor Lachen. Doch genau das ging dem in Essen geborenen Mimen wahnsinnig auf den Geist, wie die ARD-Dokumentation enthüllt. (C.II: M07/AUG.05768 Mannheimer Morgen, 25.08.2007)

(940') 'nervte sehr'

(941) Positives Denken und Reden kann einem ja wahnsinnig auf den Wecker gehen. Und das besonders dann, wenn man den Eindruck hat, dass sich fast alle, die das predigen, nur was in die Tasche lügen. (C.II: M08/SEP.72640 Mannheimer Morgen, 17.09.2008, S. 27)

(941') 'sehr nerven'

(942) „Dass so ein Typ den Deutschen wahnsinnig auf den Senkel geht, kann man verstehen.[...]" (C.II: NUZ11/MAR.02159 Nürnberger Zeitung, 24.03.2011, S. 4)

(942') 'nervt sehr'

(943) Gegen Aufsteiger Servette verlor der FC Zürich nach einem 2:0 noch mit 2:3. Via «Blick» zieht Präsident Ancillo Canepa über die Spieler her: „Ihre überhebliche und selbstgefällige Berufseinstellung geht mir wahnsinnig auf den Sack!" (C.II: A11/JUL.07529 St. Galler Tagblatt, 26.07.2011, S. 15)

(943') 'nervt sehr'

Neben der Intensivierung tritt die quantifizierende Bedeutung hervor, vgl.:

(944) Die Landwirte müssen jetzt über eine Rampe einen Umweg fahren, bei dem vollbeladene Lastwagen eine Gefahr bilden. Sollte das zur Dauerlösung werden, müsste ein Weg ausgebaut, der Autobahndamm verbreitert werden. „Das verursacht wahnsinnige Kosten“, erläutert Siegholt. (C.II: M01/MAR.16652 Mannheimer Morgen, 09.03.2001)

(944') 'sehr hohe Kosten'

(945) „Bei dem Thema kann man nur noch mit dem Kopf schütteln“, sagt ein zuständiger EU-Beamter in Brüssel und spricht von wahnsinnigen Preisen. Es gäbe kaum Konkurrenz, obwohl es 99 Mobilfunkanbieter gebe, aber eben auch ein Monopol von vier großen Betreibern. (C.II: BRZ08/APR.13547 Braunschweiger Zeitung, 25.04.2008)

(945') 'sehr großen Preisen'

(946) Die Zinsspekulationsgeschäfte [...] belief sich in der Zeit von März bis Dezember 2009, als der Euro von 1,23 auf 1,51 Dollar stieg, auf 1,5 Billionen Dollar. Diese „wahnsinnige Summe“ [...] hatte starke Auswirkungen auf Risikotransaktionen wie Aktien, Öl und Rohstoffe. Nachdem der Dollar 
wieder höher geschätzt werde, sei das Volumen dieses Handels rapide zurückgegangen. (C.II: WPD11/C08.11482: Currency Carry Trade)

$(946$ ') 'sehr große Summe'

(947) Ich bin der Überzeugung, dass sich die Steag stärker im Bereich Fernwärme engagieren sollte. Das erfordert allerdings wahnsinnige Investitionen, die in einem hohen elfstelligen Bereich liegen. (11.07.2014: http://www.ruhrbarone.de/spd-chef-drabig-arbeitslosenquote-in-dortmund-istfuer-mich-unertraeglich/53500)

(947') 'sehr große Investitionen'

Die Belege (927) - (943) und (944) - (947) demonstrieren den Gebrauch von wahnsinnig in jeweils intensivierender und quantifizierender Rolle. Das Lexem wirkt außerdem mehr einstellungsbezogen bzw. fungiert ausschließlich auf der expressiven Ebene und signalisiert damit das Auftreten von Traugott III (oben 3.6.3.4).

Anschließend findet sich wahnsinnig in Verbindung mit positiv konnotierten Bezugswörtern und demonstriert damit die vollzogene Desemantisierung, vgl.:

(948) Und Viktor nähme auch am liebsten den Abschied - aber er konnte sich ja nicht ein bißchen einschränken und mußte deshalb erst eine wahnsinnig reiche Frau finden. (C.II: HK4/B10.00001 Boy-Ed: Vor der Ehe, Erstdruck: 1915)

(948') 'sehr reiche'

(949) Mit einer wahnsinnig guten, spritzigen Spielfreude und Ideenreichtum sangen und spielten die Künstler ihre Songs - kraftvoll und ausdauernd. (C.II: M02/OKT.78170 Mannheimer Morgen, 19.10.2002)

(949') 'sehr guten'

(950) „[...] Ihr Weg führte sie zu einem berühmten und berüchtigtern Seelenerforscher und Alchimisten im Reiche des Geistes. [...] Er war auf allen Gebieten daheim, er hatte alles kennen gelernt, auch das Zuchthaus. Er war wahnsinnig interessant". (C.II: HK4/J06.00001 Janitschek: Die neue Eva, Erstdruck: 1902, 2004 [S. 91])

(950') 'sehr interessant'

(951) „Er kann super vermitteln und hat selbst wahnsinnig viel Erfahrung“, sagt Rudolph. (C.II: M13/JUL.03752 Mannheimer Morgen, 12.07.2013, S. 15)

(951') 'sehr viel'

Die Korpusanalyse hat außerdem das Auftreten von wahnsinnig in Bezug auf positiv konnotierte Funktionsverbgefüge belegt, vgl.:

(952) Damals waren Tribal-Tattoos wahnsinnig in Mode. (C.II: A08/OKT.02650 St. Galler Tagblatt, 09.10.2008, S. 36)

(952') 'waren sehr modisch'

(953) „[... [Da kam uns das Thema Umwelt in den Sinn: Ein Thema, das uns allen wahnsinnig am Herzen liegt", erzählt Larissa Freyler über die Entstehung der „Schimmelpilze“. (C.II: BVZ12/JUL.04386 Burgenländische Volkszeitung, 05.07.2012)

(953') 'ist uns ganz persönlich von sehr großer Wichtigkeit' 


\subsubsection{Kurzfassung der Entwicklung bei wahnsinnig}

1. Ausgangsbedeutung: 'geistig gestört';

2. Metaphorischer Gebrauch: Bezug auf Menschen, die psychisch gesund sind, aber durch ihr Verhalten geistesgestört wirken; die übrigen Entwicklungsschritte wie oben in Kap. 3.6.3.9.3.

\subsection{Wortgruppe 'Religion'}

Das vorliegende Kapitel widmet sich der Herausbildung der Gradbedeutung bei den Lexemen höllisch und teuflisch. Die intensivierende Funktion der Adjektive bzw. Adverbien kann durch die Verwendungen (a) - (b) exemplifiziert werden:

(a) höllisch informiert sein 'sehr gut informiert sein'

(b) teuflisch kalt 'sehr kalt'

Die Ausgangsbedeutung der beiden Grad-Adjektive bzw. -Adverbien entspricht der Kategorie 22 bzw. „Religion, Übersinnliches“ in der Klassifizierung von Dornseiff (2004: 410ff.), die auch den Begriff „Teufel“ enthält. In Biedermann (1969: 162ff.) wird die Gruppe der zu untersuchenden Wörter nach dem Motivationssem „relig" angeordnet. Obgleich sie dort durch die Lexeme heillos, himmelschreiend, jesumäßig, gotteslästerlich und gotteserbärmlich erweitert wird, werden sie in diesem Kapitel nicht berücksichtigt, weil sie in DUDEN (2012) nicht mit Intensivierungsbedeutung verzeichnet sind.

Der Mangel an Belegen bietet keine Möglichkeit für eine Analyse des Lexems heidenmäßig, das in Biedermanns Liste zu der in diesem Kapitel zu besprechenden Gruppe gehört. DWB (s.v. heide) bemerkt, dass das Wort heide, das „als erstes glied von compositen ... in der sprache des gemeinen lebens als verstärkung" gebraucht wird, „sich zu der in hohem grade, überaus, ähnlich wie z.B. bei fürchterlich“ „wendet“. Ferner geht DWB davon aus, dass „die vorstellung des schrecklichen, ungeheuern, die sich an heide knüpft" den Ausgangspunkt für die Intensivierung in Komposita darstellt. Darüber hinaus sollte der Entwicklungsgang von heidenmäßig nicht in diesem Kapitel, sondern eher in dem Kapitel „Von entsetztlich bis unheimlich" besprochen werden. Dies scheint jedoch infolge mangelhafter Informationen über das Lexem im Rahmen meiner Arbeit unmöglich zu sein.

\subsubsection{Höllisch}

\subsubsection{Lexikographische Angaben}

Pfeifer (s.v.) datiert das Lexem ins Mittelhochdeutsche in den Formen hellisch und hel(le)sch mit dem Sinn „zur Hölle gehörig, die Hölle betreffend, schrecklich, grausam". Lexer (s.v. hellisch, hellesch) bietet außerdem die mittelhochdeutsche Form helsch. Das Auftreten der Gradbedeutung wird von Pfeifer auf das 17. Jh. zurückgeführt.

1. höllisch in BMZ, Lexer (s.v. hellisch, hellesch, helsch) „höllisch“ 
2. höllisch in FWB (s.v. hellisch) ${ }^{71}$

2.1 „höllisch; aus der Hölle kommend, zur Hölle gehörend, sich in der Hölle befindend"

2.2 „die in der Hölle geschehenden Ereignisse betreffend; meist mit der Konnotation: besonders schmerzlich, grausam, stark, intensiv"

2.3 „der Hölle geweiht, für die Hölle bestimmt"

2.4 „schrecklich, fürchterlich, teufisch (wie aus der Hölle kommend); anschließbar an 1"

2.5 „,in der Wendung das hellische feuer zur Bezeichnung einer durch eine Pilzvergiftung hervorgerufenen Krankheit: Ergotismus, Antoniusfeuer, Kriebelkrankheit; auch in der Wendung der höllische rauch zur Bezeichnung einer nicht genauer identifizierbaren Krankeit"

3. höllisch in DWB (s.v.)

3.1 "der hölle zugehörig, in ihr befindlich, aus ihr kommend“

3.2 „der hölle gemäsz, teuflisch, von menschen, deren character, streben, denken, thaten"

3.3 „das höllische feuer hiesz früher eine krankheit [...]; dann ein sehr zerstörendes spreng- oder zündpulver"

3.4 „verstärkend, mit dem nebenbegriff des abstoszenden“

4. höllisch in DUDEN (2012, s.v.)

4.1a „zur Hölle gehörend, aus der Hölle stammend“

$4.1 \mathrm{~b}$ „der Hölle und ihren Qualen und Schrecken vergleichbar; quälend, schrecklich; teuflisch“

4.2 "(umgangssprachlich)“

$4.2 \mathrm{a}$ „sehr groß, stark, mächtig“

$4.2 \mathrm{~b}$ „<verstärkend bei Adjektiven und Verben> in starkem Maße, überaus, sehr"

\subsubsection{Die Entwicklung der Gradbedeutung von höllisch}

Die Korpusanalyse lässt das Einsetzen der Gradbedeutung bei höllisch im Fnhd. feststellen. Als Ausgangsbedeutung, die als Basis dafür dient, gilt die auf den Begriff der Hölle bezogene Sinnrichtung, vgl. 3.1 und 4.1a:

(954) Got behüt uns vor den hellischen Flammen! (FWBD, s.v. hellisch)

(955) es were nicht wunder, das gott vom himel schwevel und hellisch fewr regenet, und Rom in abgrund versenkt (DWB, s.v.)

Das Auftreten der Intensivierung erfolgt in mehrdeutigen Kontexten, wobei die Gradbedeutung zunächst als Nebenbedeutung infolge pragmatic inferencing in Erscheinung tritt. Das Ableiten der entsprechenden Inferenz hat pragmatic enrichment bzw. strengthening und anschließend die Mehrdeutigkeit des Gebrauchs zur Folge, vgl.:

\footnotetext{
${ }^{71}$ Noch nicht im Druck erschienen. Die Informationen wurden von der Arbeitsstelle von FWB zur Verfügung gestellt.
} 
(956) daß der Teuffel des Todes gewalt habe / das ift / er engftet vñ peiniget die Gewiffẽ mit Hellifcher furcht vnd fchrecken [...] (DTA: Arndt, Johann: Vom wahren Christenthumb. Bd. 2. Magdeburg, 1610)

(957) [...] alles was die Poeten vns erzehlen von der Ho̊ll / von den Rådern / von dem durchlöcherten Feffern / von dem Fewer vnd erfchrecklichen Fewerflammen / von dem Foltern / von der Höllifchen Qual vnd Pein: das alles kommet jhm vor / das alles quelet jhm fein Hertz / naget jhm feingewiffen vnd macht jm fo angft vnd Bang / daß ernit weiß / wo er fich foll hinwenden. (DTA: Calvi, François de: Beutelschneider / Oder Neue / warhaffte / und eigentliche Beschreibung Der Diebs Historien. Bd. 2. Frankfurt (Main), 1627)

(958) Als wir aber dennoch hinein kamen mit vnferm vorgenger Nauplio, fahen wir die / fo in der hellifchen Marter vnd Plage waren. (DTA: Rollenhagen, Gabriel: Vier Bücher Wunderbarlicher biß daher vnerhörter / vnd vngleublicher Jndianischer reysen. Magdeburg, 1603)

(959) Das waren ho̊llifche Schmertzen / die Chriftus der Herr für uns außgeftanden [...] (DTA: Dannhauer, Johann Conrad: Catechismus-Milch. Bd. 6. Straßburg, 1657)

(960) Wer etwan das Fieber einmahl hat gehabt / der hat ein Bildnůß der ho̊llifchen Marter gefúhlet / wann er dencket an die Hitze / Froft und Zåhnklappen / das er hat müffen außftehen [...] (DTA: Dannhauer, Johann Conrad: Catechismus-Milch. Bd. 6. Straßburg, 1657)

(961) O du fúffer JEfu Chrift / daß du Menfch gebohren bift / behüt uns für der Ho̊lle / und ewigem ho̊llifchen Hunger und Kummer [...] (Dannhauer, Johann Conrad: Catechismvs-Milch. Bd. 8. Straßburg, 1666)

Die Belege (956) - (961) demonstrieren mehrdeutigen adjektivischen Gebrauch. Zunächst bezieht sich höllisch direkt oder metaphorisch auf die Ausgangsbedeutung. So handelt es sich in (956) um die Furcht vor der Hölle, während in (957) die in der Hölle vorkommende Qual beschrieben wird. In (958) - (961) kommt der metaphorische Bezug auf die Ausgangsbedeutung des Lexems zur Geltung, wobei die Marter in $(958,960)$, die Qual und Pein in (957), die Schmerzen in (959) und Hunger in (961) einen Vergleich mit den entsprechenden Geschehnissen in der Hölle gestatten und die folgende Umformulierung erlauben, vgl.:

(958') - (961') 'schmerzliche(r), grausame(r) Marter / Schmerzen / Hunger, wie in der Hölle'

Neben der Ausgangsbedeutung lässt sich die intensivierende Nebenbedeutung erkennen. FWB (s.v. hellisch) verweist darauf, dass die mit der Hölle verbundenen Geschehnisse meist die Konnotation „besonders schmerzlich, grausam, stark, intensiv" haben. Die Bezugswörter in den angeführten Belegen bringen in der Tat Extremwerte aufweisende Prädikate zum Ausdruck. Darüber hinaus werden die in (956) - (957) beschriebenen Furcht, Schrecken, Qual und Pein als besonders intensiv wahrgenommen. Es lässt sich also eine Skala ableiten, die den Grad der Geschehnisse demonstrieren soll, und die mit der Hölle verbundenen Ereignisse werden auf dem oberen Ende dieser Skala eingestuft. In den in (958) - (961) angeführten Kontexten wird der metaphorische Bezug auf die Hölle deutlich, die den Sachverhalten die intensivierende Bedeutung zuzuschreiben erlaubt. Damit werden 
höllische Schmerzen, Marter, Plage und Hunger als die schlimmsten Schmerzen, Marter, Plage und Hunger dargestellt, vgl. die folgenden Paraphrasen:

(958') 'sehr starke Marter und Plage, als ob sie in der Hölle erlebt wären'

(959') 'sehr starke Schmerzen, als ob sie in der Hölle zugefügt wären'

(960') 'sehr starke Marter, als ob sie in der Hölle bereitet wäre'

(961') 'sehr starker Hunger, als ob man davon in der Hölle leiden würde'

Diese Stufe der Bedeutungsentwicklung ist mit dem Auftreten von subjectivity verbunden. Durch die intensivierende Funktion von höllisch wird „speaker's imprint" (Finegan 1995: 1) zum Ausdruck gebracht, sodass die Verwendungen die Einstellung des Schreibers bzw. Sprechers andeuten. Damit kommt es zum Einsetzen von Traugott I (oben 3.6.3.4).

In der nächsten Etappe des Entwicklungsgangs erscheint höllisch als Intensivierungsmittel, indem der Bezug auf die Ausgangsbedeutung beseitigt wird. In dieser Stufe bleibt die Kookkurrenz des Lexems auf negativ konnotierte Bezugswörter beschränkt, sodass die Desemantisierung nicht vollkommen vollzogen wird, vgl. den adjektivischen und adverbialen Gebrauch:

(962) „Der arme Kerl hatte eine höllisch langweilige Arbeit“, sagte Klemens. (C.II: HK3/B08.00001 Droste-Hülshoff: Ledwina, Entstanden: um 1820/21, 2000 [S. 312])

(962') 'sehr langweilige Arbeit'

(963) „Am andern Morgen, " fuhr er fort, „gab's einen höllischen Lärm im Dorfe. [...]“" (C.II: HK4/M05.00001 Marlitt: Thüringer Erzählungen: Schulmeisters Marie, Entstanden: seit 1858, 2004 [S. 350])

(963') 'sehr starken Lärm'

(964) Zuweilen gab es mitten in dem höllischen Durcheinander von schwelendem Gebälk, glimmenden Wänden, dunklen Rauchwolken, züngelnden Flämmchen, zusammensinkenden Brettern einen Knall. (C.II: HK4/B10.00001 Boy-Ed: Vor der Ehe, Erstdruck: 1915, 2004 [S. 225])

(964') 'sehr großen Durcheinander'

(965) Es gab da diese Interviewerin, die mich höllisch irritierte. Sie sagte: „Wie tief müssen Sie in sich hineingehorcht haben, um zu dieser Darstellung zu kommen!" (C.II: P94/MAR.09491 Die Presse, 19.03.1994)

(965') 'sehr irritierte'

(966) Die Fahrtechniken zu erlernen und das mit dem Basketballspielen zu verbinden, ist höllisch schwer. Das ist echter Hochleistungssport. (C.II: RHZ98/FEB.17961 RZ, 10.02.1998)

(966') 'sehr schwer'

(967) Wir haben höllisch viel Geld dafür ausgegeben, unser Haus in Steinwolle zu packen. Das hat sich erstens bei den Heizkosten auszuzahlen, zweitens soll man mir später nicht die Mitschuld am Klimawandel in die Schuhe schieben. (C.II: HAZ07/SEP.03123 HAZ, 11.09.2007, S. 15)

(967') 'sehr viel' 
Das Lexem kann außerdem als Intensivierungsmittel in Funktionsverbgefügen auftreten, die entweder ein graduierbares Prädikat enthalten oder durch einen graduierbaren Begriff wiedergegeben werden können, vgl.:

(968) „Das weiß ich nicht; aber ihre dortige Brief- und Paketbestellung muß das höllisch in Unordnung bringen, sagt mein Vater, und da kommt doch sicherlich vieles als unbestellbar zurück. Meinst du nicht auch, Störzer?" (C.II: HK3/A26.00002 Raabe: Stopfkuchen, Entstanden: 1888/90, 2000 [S. 445])

(968') 'in sehr große Unordnung bringen'

(969) An einen Umzug nach Los Angeles ist ohnehin nicht zu denken, das StarGetue dort geht ihm nämlich höllisch auf den Geist. (C.II: 097/MAR.30157 Neue Kronen-Ztg., 23.03.1997, S. 18)

(969') 'sehr nervt'

(970) Die WM-Fußballer aus Rumänien und Kroatien müssen sich vor ihrem Achtelfinal-Schiedsrichter höllisch in Acht nehmen. (C.II: R98/JUN.51473 Frankf. Rundschau, 30.06.1998, S. 21)

(970') 'sehr aufpassen'

(971) „Bei uns ist Nervenstärke gefragt, denn in Neukirchen muß man höllisch auf der Hut sein. Unser Gegner hat drei schnelle Leute und das bedeutet, daß wir kompakt stehen müssen, denn in Rückstand wollen wir nicht geraten", kündigte Rapolder an. (C.II: M99/APR.27285 Mannh. Morgen, 30.04.1999)

(971') 'sehr vorsichtig sein'

(972) Freunde und Partner gehen einander zeitweise höllisch auf die Nerven, was immer sehr spritzige, ironische Dialoge ergibt. Aber Probleme werden prinzipiell argumentativ ausdiskutiert. (C.II: N00/FEB.08887 Salzburger Nachr., 28.02.2000)

(972') 'sehr Nerven'

(973) „Wir sind höllisch unter Druck“, sagte der ratlos wirkende Trainer Falko Götz. Fünf Punkte trennen Berlin noch von einem Abstiegsplatz. (C.II: HMP07/APR.00171 MOPO, 02.04.2007, S. 8)

(973') 'unter sehr großem Druck stehen', 'sehr bedrängt sein'

(974) Oder Jan, die „Antragsauswurfsmaschine“, die Greta zusammen mit dem Fluglärm höllisch auf den Zeiger geht. (C.II: RHZ12/OKT.00052 RZ, 01.10.2012, S.6)

(974') 'sehr nervt'

Die qualifizierende Funktion des Lexems kann in die quantifizierende übergehen, vgl.:

(975) Der Z-Bau in der gegenwärtigen Form ist eine Totgeburt; die zu Dialog und Kooperation unfähigen Betreiber haben ein höllisches Geld für Null-Leistung verbraten, das anderen Kulturstätten schmerzlich fehlt. (C.II: NUN10/JUN.02712 NN, 25.06.2010, S. 3)

$(975$ ') 'sehr viel Geldt' 
(976) Allein die Gefahr, dass man versehentlich mal im GPRS Netz ist und dann höllische Preise zahlt, macht das Angebot unbrauchbar. (15.07.2014: http://www.teltarif.de/forum/s20396/garantiert-ueber-35-eur-monatlich/9.html) (976') 'sehr hohe Preise'

Neben der intensivierenden und quantifizierenden Bedeutung tritt eine pejorative Bedeutungskomponente in Erscheinung, vgl.:

(977) Meine Familie und ich hatten einen tollen Urlaub zum Vatertag in Galveston geplant aber es wurde ein höllischer Urlaub. (15.07.2014: http://www.tripadvisor.de/ShowUserReviews-g55879-d619548-r16046458-Baymont_Inn_Suites_Galveston-Galveston_Galveston_Island_Texas.html)

(977') 'sehr schlimmer'

(978) Die französischen Gefangenen erwartet ein Schicksal, das noch schlimmer ist als der Galgen: abgewrackte Kriegsschiffe, die in der Themse vor Anker liegen. Dort müssen sie unter höllischen Bedingungen Strafarbeit verrichten, die nur die wenigsten überleben. Eine Flucht ist unmöglich. (15.07.2014: http://books.google.de/books?id=1shBasyNAv4C\&pg=PT4\&lp$\mathrm{g}=\mathrm{PT} 4 \& \mathrm{dq}=\% 22 \mathrm{~h} \% \mathrm{C} 3 \%$ B6llischen+bedingungen $\% 22 \&$ source=bl\&ots=NZxboWRyiP\&sig=G50P5NHMYrzMmBdRKsp5w07f84s\&hl=de\&sa=X\&ei=RfHEU_GSPIG-O_-XgJAO\&ved=0CCIQ6AEwATgK\#v=onepage \&q=\%22h\%C3\%B6llischen\%20bedingungen $\% 22 \& \mathrm{f}=$ false)

(978') 'sehr schlimmen Bedingungen'

Die Belege (962) - (974), (975) - (976) und (977) - (978) demonstrieren den Gebrauch von höllisch jeweils mit intensivierender, quantifizierender und pejorativer Bedeutung. Die Kontexte legen nahe, dass diese Stufe des Entwicklungsgangs neben dem graduellen Verlust des Zusammenhangs mit der Ausgangsbedeutung außerdem mit einer Zunahme an subjectivity verbunden ist. Die Verwendungen wirken mehr einstellungsbezogen und signalisieren das Einsetzen von Traugott III (oben 3.6.3.4).

In der anschließenden Etappe verliert die Gradbedeutung von höllisch dadurch den Kontakt zur Ausgangsbedeutung, dass das Lexem mit positiv konnotierten Bezugswörtern auftritt, ohne seine Ausgangsbedeutung erkennen zu lassen. Die Desemantisierung wird damit endgültig abgeschlossen, vgl. den adjektivischen (979) (980) und adverbialen (981) - (986) Gebrauch:

(979) Das Rasen ins Tal machte auch höllischen Spaß, nur das mühsame Schieben nach oben stieß nicht so richtig auf Begeisterung. (C.II: RHZ05/APR.17709 RZ, 15.04.2005)

(979') 'sehr großen Spaß'

(980) Würmla verschlief die Anfangsphase, hatte bei zwei Hundertprozentigen höllisches Glück, nicht in Rückstand zu geraten. (C.II: NON12/OKT.07483 NÖN, 11.10.2012)

(980') 'sehr großes Glück'

(981) Begeistert ist Kreuz vom neuen Mitspieler Igor Morinas (24) aus Litauen. „Der ist höllisch schnell, kann am Ball viel, ein Riesenmann. "Vier Tore 
in sechs Spielen bestätigen diesen Eindruck. (C.II: RHZ99/APR.01653 RZ, 03.04.1999)

(981') 'sehr großes Glück'

(982) Dass er immer noch höllisch guten Blues macht, zeigte Willy de Ville bei seinem Auftritt im KUZ-Sommer. (C.II: RHZ01/JUL.18303 RZ, 28.07.2001)

(982') 'sehr guten'

(983) Unteregger: Es ist ein Balanceakt, den ich höllisch interessant finde. Er ist vor allem eine organisatorische Herausforderung. (C.II: A09/APR.05247

St. Galler Tagbl., 21.04.2009, S. 32)

(983') 'sehr interessant'

(984) Vielleicht ist es das Wesen seiner Arbeit, das Park auf dem Boden hält: Er muss höllisch geduldig sein und sich mit mikroskopischen Fortschritten zufrieden geben. (C.II: RHZ09/APR.18166 RZ, 22.04.2009)

(984') 'sehr geduldig'

(985) Ralf Prestenbach zählt währenddessen die Tage bis zum Finale: „Ich bin höllisch froh, wenn die EM zu Ende ist." (C.II: RHZ12/JUN.23088 RZ, 20.06.2012, S. 26)

(985') 'sehr froh'

(986) Bruder Bonifatius begrüßt die Schar der Ehrengäste, der Name Helmut Probst fällt, ein Beifallssturm beinahe apokalyptischen Ausmaßes bricht los. Dabei glänzt der VG-Chef zu dem Zeitpunkt nur durch Abwesenheit. (Hinter-)Gründe? Entweder liegt's daran, dass der Genannte wenig später tatsächlich die Kirche betritt [...]. Oder am Gewicht, das der Nachname in kirchlichen Kreisen schon länger hat. Oder Helmut ist einfach höllisch beliebt. (C.II: RHZ12/AUG.25023 RZ, 18.08.2012, S. 13)

(986') 'sehr beliebt'

\subsubsection{Kurzfassung der Entwicklung bei höllisch}

Ausgangsbedeutung: 'höllisch; aus der Hölle kommend, zur Hölle gehörend, sich in der Hölle befindend';

die übrigen Entwicklungsschritte wie oben in Kap. 3.6.3.9.3.

\subsubsection{Teuflisch}

\subsubsection{Lexikographische Angaben}

Pfeifer (s.v.), DWB (s.v.) und DUDEN (2012, s.v.) buchen das Lexem als mhd. in der Form tiuvel(i)sch. Außerdem steuert Pfeifer zum Vergleich die im 9. Jh. belegte althochdeutsche Form tiufallīh bei.

1. teuflisch in BMZ (s.v. tiufellich), Lexer (s.v. tiuvel-lich) „dem teufel gemäß“ (BMZ, s.v. tiufellich), „teufelmässig, teuflisch“ (Lexer, s.v. tiuvel-lich)

2. teuflisch in FWB (s.v. teuflisch, teufelisch) ${ }^{72}$

\footnotetext{
${ }^{72}$ Im Druck noch nicht erschienen. Die Informationen wurden von der Arbeitsstelle von FWB zur Verfügung gestellt.
} 
2.1 „teuflisch; zum Teufel gehörig, vom Teufel ausgehend, mit dem Teufel im Bunde stehend"

2.2 „schlecht, bösartig, grausam; Schaden (boshaft) herbeiführend“

3. teuflisch in DWB (s.v. teuflisch, teufelisch)

keine lexikographischen Angaben

4. teuflisch in DUDEN (2012, s.v.)

4.1 „äußerst bösartig und grausam; den Schaden, das Leid eines anderen bewusst, boshaft herbeiführend und sich daran freuend; diabolisch; satanisch"

4.2 "(umgangssprachlich)“

$4.2 \mathrm{a}$ „,sehr groß, stark, mächtig“

$4.2 \mathrm{~b}$ „<intensivierend bei Adjektiven und Verben> sehr, überaus“

\subsubsection{Entwicklung der Gradbedeutung bei teuflisch}

Die Korpusanalyse lässt annehmen, dass das Einsetzen der Intensivierung im Fnhd. begonnen hat. Als Grundlage dafür dient die auf den Teufel bezogene Bedeutung, vgl. 1 und 2.1 sowie die Belege:

(987) Dangk sagen wir alle mit schalle / Dem Hern vnserm Gott, / Der durch sein geburt vns erlooset hat, / Von der teuffelischen macht vnd gewalt. (FWB, s.v. teu$\mathrm{f}(\mathrm{e}) \mathrm{lisch})^{73}$

(987') 'der Macht und Gewalt des Teufels'

(988) teufelisch wort oder werk furnehmen (DWB, s.v. teuf(e)lisch)

(988') 'Wort oder Werk des Teufels'

Das Auftreten der Gradbedeutung erfolgt in mehrdeutigen Kontexten infolge pragmatic enrichment bzw. strengthening. Dabei tritt die intensivierende Komponente als Nebenbedeutung in Erscheinung, während die Ausgangsbedeutung die primäre Rolle spielt, vgl.:

(989) das solchs rechte lügenden, erstunkene, Teuffische lügen [...] sind. (FWB, 1537, s.v. teuf(e)lisch) ${ }^{74}$

(990) Wie kan man denn den Kindlein die tauff versagen / so es Gott also ordnet / das jederman durch die tauff zur Himlischen widergeburt muß kommen [...] Vnd ist ein teuflischer jrrthumb / sie von der tauff außzuschliessen / vnnd an vergebung der sünden hindern. (FnhdC: Veit Dietrich, Summaria, Nürnberg 1578)

(991) so ist zauberey ein rechte Teuffische Abgoetterey / damit die hohe Majestet Gottes [...] geunehret wird. (FWB, 1563, s.v. teuf(e)lisch) ${ }^{75}$

\footnotetext{
${ }^{73}$ Noch nicht im Druck erschienen. Die Informationen wurden von der Arbeitsstelle von FWB zur Verfügung gestellt.

${ }^{74}$ Noch nicht im Druck erschienen. Die Informationen wurden von der Arbeitsstelle von FWB zur Verfügung gestellt.

${ }^{75}$ Noch nicht im Druck erschienen. Die Informationen wurden von der Arbeitsstelle von FWB zur Verfügung gestellt.
} 
(992) Jn deß Powah Gebett / vnd in dem Namen deß Krancken werden diefem jhrem Abgott viel Fell von Thieren / Keffel / Beyel / Kråntze / Meffer vnd andere Sachen auffzuopffern verheiffen / wann anderft der Krancke widerumb kan gefundt werden: Ob fie aber folchem jhrem Verfprechen nachkommen / weiß ich nicht fur gewiß: Andere jhre Ceremonien hab ich viel vnd offt gefehen / vnd wiewol ich fie genugfam hab vnderrichtet / vnd eines beffern berichtet / hab ich fie doch von folchem Teufflifchen Betrug vnd Abgo̊tterey nicht abwenden ko̊nnen: Vnd dieweil fie mir verfprochen / fie wolten mich den Teuffel in fichtbarlicher Geftalt fehen laffen / hab ich mich / weil ohne das jhre Priefter Powah bey jhnen ankame / ein wenig auffgehalten. (DTA: Gottfried, Johann Ludwig: Newe Welt Vnd Americanische Historien. Frankfurt (Main), 1631)

(993) Alfo fehet ihr nun / wie boßhafftig der verfluchte Wüterich an mir und diefer unfchuldigen Frauen gehandelt / welche durchaus nichts gefündiget hat / nur daß fie feine teuflifche Boßheit wider mich nicht billichen ko̊nnen / und mit meinem Unglúk ein Mitleiden getragen / [...]. (DTA: Bucholtz, Andreas Heinrich: Des Christlichen Teutschen Groß-Fürsten Herkules Und der Böhmischen Königlichen Fräulein Valjska Wunder-Geschichte. Bd. 1. Braunschweig, 1659)

(994) Was vor ein bo̊fer Geift hat dich getrieben / du ehrvergeffener gottlofer Mordbrenner / Dieb / Råuber / und Mo̊rder / daß du nicht allein mich und mein unfchuldiges Land / ohn alle gegebene Urfach / unabgefagt / und mit fo teuflifchem Grimme angefallen / fondern auch allen Inwohnern den Tod / dem ganzen Lande die Verwüftung / und welches erfchreklich zu hören / vier unfchuldigen ehrliebenden Königen und dreyen redlichen Ko̊niglichen Fürften den Galgen haft angedråuet und auffrichten laffen / auff daß du auff einmahl und an einem Schand- holze fünff großmåchtige Ko̊nigreiche fchmåhen mo̊chteft / welche dir nie keine beleidigung angefüget hatten? (DTA: Bucholtz, Andreas Heinrich: Des Christlich: Teutschen Königes Herkules und der Teutschen Königin Valiska Wunder-Geschicht. Bd. 2. Braunschweig, 1660)

(995) Mich verdreußt / daß wir folch unverfchåmt / grobe / tolle Lůgen müffen im geiftlichen Recht lefen und lehren / dazu für Chriftlich Lehre halten / fo es doch Teufflifche Lügen feynd. (DTA: Dannhauer, Johann Conrad: Catechismvs-Milch. Bd. 8. Straßburg, 1666)

(996) Von den Kriegern wurde es am meiften practicirt / wenn fie nemlich fagten: Wir wollen in Gottes Nahmen auff Partey / Plündern / Mitnemmen / Todtfchieffen / Nidermachen / Angreiffen / gefangen nemmen / in Brand ftecken / und was ihrer fchröcklichen Arbeiten und Verrichtungen mehr feyn mo̊gen. Alfo wagens auch die Wucherer mit dem Verkauff in Gottes Nahmen / damit fie ihrem Teufflifchen Geitz nach fchinden und fchaben mo̊gen. (DTA: German Schleifheim von Sulsfort [i. e. Grimmelshausen, Hans Jakob Christoffel von]: Der Abentheurliche Simplicissimus Teutsch. Monpelgart [i. e. Nürnberg], 1669)

(997) Wird also am sichersten davor gehalten / daß diese Verwandelung der menschlichen Cörper in Wölffe oder andere Thiere / anders nichtes sey / 
als eine teuflische Verblendung der Augen / sowohl dessen / der sich in einen Wolf verwandelt achtet / als auch anderer / die ihn sehen. (C.II: HK4/S11.00001 Stockfleth: Die Kunst- und Tugend-gezierte Macarie, Erstdruck: 1669-1673, 2004 [S. 147])

(998) Abfonderlich aber hat Chriftus der HErr hiermit aller Welt wollen zu erkennen geben / welch ein gro $\beta$ / fchrecklich / ja Teuflifch Lafter es fey / wann eine Creatur fich dem Scho̊pffer widerfetzet / und auß Verwegenheit GOTT verfucht / welches allhier der Teufel gethan / der HEr? Chriftus aber verbietet es ernftlich [...] (DTA: Bauller, Johann Jacob: Hell-Polirter Laster-Spiegel. Ulm, 1681)

(999) O! fagte er, Herr Vetter! wahr ift das! Woher erlang' ich aber doch Kraft, um meinem teuflifchen Hochmuth zu widerftehen! - ein, zwei, drei Tage! — und dann bin ich todt. — Was hilfts mich dann, ein großer, vornehmer Mann in der Welt gewefen zu feyn? - Ja, es ift wahr! - Mein Herz ift die falfchefte Kreatur auf Gottes Erdboden, immer mein' ich, ich håtte die Abficht, nur mit meinen Wiffenfchaften Gott und dem Nåchften zu dienen - und wahrlich! - es ift nicht wahr! ich will nur gern ein großer Mann werden, gern hoch klimmen, um nur auch tieffallen zu ko̊nnen. O! wo krieg ich Kraft, mich felber zu ůberwinden? (DTA: Jung-Stilling, Johann Heinrich: Lebensgeschichte. Stuttgart, 1835)

(1000) Die Hexenprozeffe find ein Stoff voll dunkeln Wahnfinns und teuflifcher Bosheit, die wie ein Gefpenft den Unfchuldigen, der im Momente des Verdachts unrettbar verloren ift, erfaßt und vernichtet. (DTA: Vischer, Friedrich Theodor von: Ästhetik oder Wissenschaft des Schönen. Bd. 2,1. Reutlingen u. a., 1847)

(1001) Die Entblößung fei übrigens nicht bloß Sünde, fondern fchwere Sünde, teuflifche Sünde und "laufe wider den ganzen Katechismum. [...]“ (DTA: Falke, Jakob von: Die deutsche Trachten- und Modenwelt. Ein Beitrag zur deutschen Culturgeschichte. Bd. 2. Leipzig, 1858)

(1002) In der Nacht vor der großen Ueberfchwemmung des Arnothales 1333 hörte einer der heiligen Einfiedler oberhalb Vallombrofa in feiner Zelle ein teuflifches Getöfe, bekreuzte fich, trat unter die Thür und erblickte fchwarze und fchreckliche Reiter in Waffen vorüberjagen. (DTA: Burckhardt, Jacob: Die Cultur der Renaissance in Italien. Ein Versuch. Basel, 1860)

(1003) Nach dem Bericht von Augenzeugen waren sie die Geissel des Landvolks, verwüsteten muthwillig, was sie nicht brauchen konnten, und marterten die Bauern im schweren Frohndienst mit teuflischer Grausamkeit zu Tode.

Die Belege (989) - (1003) sind mehrdeutig interpretierbar. Zunächst bezieht sich teuflisch auf die Ausgangsbedeutung, indem es die angesprochenen Situationen in Verbindung mit dem Teufel bringt, z.B. vom Teufel ausgehende Lügen in $(989,995)$, Bosheit in (993) und Getöse in (1002), mit dem Teufel im Bund stehender Irrtum in (990), Abgötterei in (991), Betrug in (992), Laster in (998) und Sünde in (1001). In (993), (994), (996) und (999) werden die Personen vom Teufel besessen, der ihnen die entsprechenden Eigenschaften wie Bosheit, Geiz, Grimm und Hochmut verleiht. In (1003) geht der religiöse Hintergrund verloren. Die Pesonen verhalten 
sich so, als wären sie vom Teufel besessen, sodass teuflisch im übertragenen Sinne verwendet wird.

Zugleich lässt sich aus den angeführten Kontexten die Bedeutungskomponente eines hohen Grades ableiten. Wie in dem Fall mit höllisch, werden die auf den Teufel bezogenen Sachverhalte, die durch Extremwerte aufweisende Prädikate zum Ausdruck gebracht werden, als die schlimmsten dargestellt. Dadurch wirkt die Bedeutung des negativ konnotierten Bezugsworts intensiviert. Anders formuliert, werden stark ausgeprägte sehr negative Sachverhalte mit dem Teufel verbunden, der als deren Ursache gilt. Beispielsweise handelt es sich in (991) um einen sehr großen Irrtum, die Kinder von der Taufe auszuschließen. In (996) wird von sehr schlimmen bzw. großen Lügen berichtet, die teuflischer Natur sind. Die vom Teufel stammende Bosheit in (1000) ist so groß, dass sie einen Unschuldigen vernichtem kann. In (1003) werden Bauern mit sehr starker Grausamkeit verprügelt, die nur von einem vom Teufel besessenen Menschen stammen kann.

Das Auftreten der Intensivierung geht mit dem Einsetzen von subjectivity einher. Aus den Kontexten geht hervor, dass der Gebrauch mehr einstellungsbezogen wirkt als in Bezug lediglich auf die Ausgangsbedeutung, indem durch die Bezeichnung des Grads die persönliche Wertung des Schreibers bzw. Sprechers zum Ausdruck gebracht wird. Darüber hinaus kommt in der ersten Stufe des Entwicklungsgangs die erste semantisch-pragmatische Tendenz (Traugott I, oben 3.6.3.4) zur Geltung.

Ferner tritt teuflisch als Intensivierer auf, ohne einen direkten Zusammenhang mit der Ausgangsbedeutung erkennen zu lassen. Jedoch bleibt die Verbindung zur Ausgangsbedeutung durch negative Konnotationen erhalten, vgl. die adjektivischen (1004) - (1006) und adverbialen (1007) - (1008) Verwendungen mit den entsprechenden Umformulierungen:

(1004) Saschko Gawriloff spielt den Solopart im Dvorak-Konzert mit schlankem, bisweilen sprödem Ton. Die liedhafte Melodik im Adagio ist frei von sentimentalen Drückern, mit mehr Filigran als man gewöhnlich zu hören bekommt. Bei den Ecksätzen freilich, meisterte er die teuflischen Schwierigkeiten auch nicht immer mit lupenreiner Präzision. (C.II: NUN93/OKT.00796 NN, 11.10.1993, S. 26)

(1004') 'sehr großen Schwierigkeiten'

(1005) Glenn nahm seinen Sohn noch mal in den Arm: „Ich bin so froh, dass alles vorbei ist!“ Er legte den Arm um seinen Nacken: „Ich hatte gehofft, dass du es schaffst, aber ich hatte auch teuflische Angst um dich! [...]" (C.II: DIV/APS.00001 Planert: Seleno, 2006 [S. 354])

(1005') 'sehr große Angst'

(1006) „Das schwere Kreuz - Rückenschmerz" war Thema der dritten Abendvorlesung, einer Veranstaltungsreihe für jedermann, die das Katholische Klinikum Koblenz zusammen mit unserer Zeitung durchführt. Da ist zunächst das individuelle Leid, das die oft teuflischen Schmerzen bei den Betroffenen auslöst. (C.II: RHZ11/APR.18078 RZ, 16.04.2011, S. 25)

(1006') 'sehr großen Schmerzen' 
(1007) Sein Schüler Dupré trieb die Virtuosität auf die Spitze, indem er zum Beispiel als Opus 7 teuflisch schwere Präludien und Fugen schrieb. (C.II: M11/AUG.08595 Mannh. Morgen, 30.08.2011, S. 15)

(1007') 'sehr schwere'

(1008) Es könnte ein Mann sein, der sich über laute Motorradfahrer in seiner Nachbarschaft über alle Maßen ärgert und sich an den Bikern rächen will. Mit teuflisch glatten Ölflecken auf unübersichtlichen, kurvigen Straßen im Alpenvorland. Vor knapp zwei Jahren kam in Markt Rettenbach (Unterallgäu) ein 37 Jahre alter Familienvater durch eine solche Schleuderfalle ums Leben. (C.II: NUN13/FEB.00814 Nürnberger Nachrichten, 07.02.2013, S. 17)

(1008') 'sehr glatten'

Teuflisch kann außerdem Funktionsverbgefüge intensivieren. Die Belege (1009) (1012) mit den entsprechenden Paraphrasen sollen die Verwendung des Lexems demonstrieren, vgl.:

(1009) So gibt es weder beim Erwerb von Finanzprodukten einen offenen Wettbewerb - der Kunde ist auf die Informationen angewiesen, die ihm der teuflisch unter Druck stehende Bankmitarbeiter präsentiert - noch später. (21.07.2014: http://www.money-advice.de/index.php?id=4\&viewid=41$684 \&$ mod_print $=1$ )

(1009') 'unter sehr großem Druck stehende', 'sehr bedrängte'

(1010) Jeffreys belehrender Tonfall ging mir teuflisch auf die Nerven, genauso sein falscher Akzent. (21.07.2014: http://books.google.de/books?id=izDsDmSF-YkC\&pg=PA182\&dq= \%22teuflisch+auf+die+nerven $\% 22 \& \mathrm{hl}=$ de\&sa=X\&ei $=$ sv3MU4OEDImsOPXvgPAO\&ved=0CB8Q6AEwAA\#v=onepage $\& q=\% 22$ teuflisch\%20auf\%20die\%20nerven\%22\&f=false)

(1010') 'nervte sehr'

(1011) Seinerseits geht Hemingway seinem eigenen Vorgesetzten, Inspektor Hannaby, teuflisch auf den Geist, denn er bildet sich ein, etwas von Psychologie zu verstehen, und scheut sich nicht, seine Kenntnisse auch anzuwenden. (21.07.2014: http://www.amazon.de/gp/aw/cr/rRSK09BX338ZI1)

(1011') 'nervt sehr'

(1012) Bis die Action nämlich losgeht zieht sich das Ganze teuflisch in die Länge. (21.07.2014: http://www.dvdmaniacs.de/modules.php?op=modload\&name $=$ News\&file $=$ article $\&$ sid $=1413$ )

(1012') 'verzögert sich sehr'

Die intensivierende Rolle von teuflisch kann in die quantifizierende übergehen, die die Bedeutung „groß, viel“ zum Ausdruck bringt, vgl.:

(1013) Was macht man in einer Stadt, in der jeder zenitmeter Baugrund teuflisches Geld kostet? Richtig, man baut in die Höhe. (21.07.2014: http://flightforum.ch/forum/showthread.php?t=65444)

(1013') 'sehr viel Geld'

(1014) Ich mietete in einem ehemaligen Fischerdörfchen, das bis auf die beiden riesigen Hotels immer noch ganz verträumt und ursprünglich wirkte, ein kleines Apartment mit großem Balkon, Meerblick, fünf Minuten vom 
Strand entfernt für eine teuflische Summe, von der mein Geldgeber Mommsen lieber nichts erfahren sollte. (21.07.2014: http://books.google.de/books?id=fx9oAwAAQBAJ\&pg= PT262\&lpg=PT262\&dq=\%22teuflische+summe\%22\&source=bl\&ots=4p_WomiDT\&sig=1yEh7pCWjOYjKUgTZVKxP1Vhkpl\&hl=de\&sa=X\&ei=NwTNU9jVGIeVPP_JgcAH\&ved=0CCcQ6AEwAQ\#v=onepage\&q=\%22teuflische $\% 20$ summe\%22\&f=false)

(1014') 'sehr große Summe'

(1015) Wie schon gesagt: nach 2 Jahren Spielzeit [...] haben die allermeisten Spieler schon, was sie wollen. 90\% der Spieler setzen erst einmal auf sichtbaren Reichtum, der ins Auge springt: RÜSTUNGEN, dann WAFFEN, dann erst TITEL. Mitjedem Kapitel erstellen 50\% der Spieler zuallererst neue Chars. D.h., die Rissrüstungsträger stehen erst einmal weiter auf den komischen Säulen im Einlogg-Bildschirm herum während die neuen Chars mit „Billigrüstungen" rumlaufen. Kurz darauf werden neue Farmstellen erschlossen, jeder macht teuflische Gewinne und kauft auch für die neuen Chars Prestigerüstungen und Objekte. (21.07.2014: http://www.wartower.de/forum/showthread.php?271606-Online-Unterschriftsammlung-was-haltet-ihr-davon/page8)

(1015') 'sehr große Gewinne'

Die Belege (1004) - (1012) und (1013) - (1015) demonstrieren den Gebrauch von teuflisch als jeweils Intensivierer und Quantifizierer. Das Lexem wirkt außerdem mehr einstellungsbezogen bzw. fungiert ausschließlich auf der expressiven Ebene und verweist damit auf das Auftreten von Traugott III (oben 3.6.3.4).

In der letzten Phase des Entwicklungsgangs verbindet sich teuflisch mit positiv konnotierten Bezugswörtern und demonstriert damit die vollzogene Desemantisierung. Der Bezug auf die Ausgangsbedeutung ist vollkommen beseitigt, vgl.:

(1016) High-Tech mit neuen Materialien hat diese Welt den Massen erschlossen. Nutzen wir diese Chance! Denn es macht einfach teuflischen Spaß. (C.II: RHZ97/SEP.14157 RZ, 20.09.1997)

(1016') 'sehr großen Spaß'

(1017) Der einstige Anhänger von immensen Mengen an italienischem Rebensaft unterschreibt die Minibarrechnung: „Was hatte ich noch mal? Zwei Tafeln Kokosschokolade - die schmeckt wirklich teuflisch gut - und drei Flaschen Wasser." (C.II: M98/APR.30411 Mannh. Morgen, 04.04.1998)

(1017') 'sehr gut'

(1018) Der Augsburger Mime Jochen Schneider hat sich bei der Zusammenstellung der vorgetragenen Literatur teuflisch viel Mühe gemacht und keine altbekannten Kabinettstückchen offeriert, sondern für die Schaudertour im Römersaal neben einer populären Lektüre weitgehend unbekannte Schätze ausgegraben. (C.II: M98/SEP.77022 Mannh. Morgen, 21.09.1998)

(1018') 'sehr viel'

(1019) „Dadurch konnte ich ihn einschätzen, war gleich sehr präsent und aktiv“, sagte Engel, dem sein klarer Punktsieg eine teuflische Freude bereitete. (C.II: RHZ08/SEP.14821 RZ, 15.09.2008) 
(1019') 'sehr große Freude'

(1020) Und Geheimagentinnen sind stets teuflisch schön - zumindest bei James Bond. (C.II: M08/AUG.63457 Mannh. Morgen, 15.08.2008, S. 2)

(1020') 'sehr schön'

\subsubsection{Kurzfassung der Entwicklung bei teuflisch}

Ausgangsbedeutung: 'zum Teufel gehörig, vom Teufel ausgehend, mit dem Teufel im Bunde stehend'; die übrigen Entwicklungsschritte wie oben in Kap. 3.6.3.9.3.

\subsection{Wortgruppe 'Fluch'}

Das vorliegende Kapitel widmet sich der Herausbildung der Gradbedeutung in den Lexemen verteufelt, verdammt und verflucht. Die intensivierende Funktion der Adjektive bzw. Adverbien kann durch die Verwendungen (a) - (c) exemplifiziert werden:

(a) ein verdammt hübsches Mädchen 'ein sehr hübsches Mädchen'

(b) eine verflucht schöne Rede 'eine sehr schöne Rede'

(c) verteufelt gut spielen 'sehr gut spielen'

Die Ausgangsbedeutung der bezeichneten Grad-Adjektive bzw. -Adverbien entspricht der Kategorie 15 bzw. „Menschliches Zusammenleben“ in der Klassifizierung von Dornseiff (270ff.) und bringt die Begriffe „Verwünschung“ und „schimpfen“ zum Ausdruck. In Biedermann (1969: 163) wird die Gruppe der zu untersuchenden Wörter nach dem Motivationssem „Fluch" angeordnet. Obgleich die Gruppe dort durch die Lexeme gottverdammt und vermaledeit erweitert ist, werden sie in diesem Kapitel nicht berücksichtigt, weil sie in DUDEN (2012) nicht mit Intensivierungsbedeutung verzeichnet sind. Das Lexem verflixt, das in Biedermanns Liste ebenso enthalten ist, wird in Rahmen dieser Arbeit nicht analysiert, weil es eine „euphemistische entstellung für verflucht" (DWB, s.v. verflixt) darstellt und deren intensivierenden Gebrauch übernommen hat.

\subsubsection{Verdammt}

\subsubsection{Lexikographische Angaben}

Die Korpusanalyse legte nahe, das Auftreten des Lexems im Frühneuhochdeutschen zu verorten. Kluge bemerkt über das Lexem nichts. Pfeifer dagegen erwähnt es, auch mit Intensivierungsbedeutung:

1. verdammt in Pfeifer (s.v. verdammen): „,von Gott verurteilt', das sofort als Fluchwort und Verwünschung ,fluchwürdig, verdammenswert, verwerflich'Verbreitung findet, in der Umgangssprache später auch abgeschwächt ,außergewöhnlich, sehr, äußerst' (19. Jhd.)“. 
2. verdammt in FWB (s.v. verdamt, verdampt) ${ }^{76}$ "verdammt“; „verflucht" „von Gott verachtet“; „,von Gott verlassen sein“; „die Gnade Gottes verloren haben“; „verworfen"77

3. verdammt in DWB (s.v.)

3.1 „worüber ein verdammungsurtheil ausgesprochen ist“, „strafwürdig, verwerflich"

3.2 "fluchwürdig, verdammenswert"

3.3 „selten wird dasjenige, wozu man verurtheilt, gezwungen ist, an stelle der betroffnen person oder sache gestellt"

3.4 ,ist einer strafe wert"

3.5 „aus der bedeutung strafwürdig, verwerflich entwickelt sich wie bei verflucht $\mathrm{u}$. ä. die bedeutung 'vom gesetzmäszigen, allgemein anerkannten abweichend, daher ungemein, auszergewöhnlich'“.

3.6 „adverbial gebraucht als kräftige (volksthümliche) verstärkung des adjectivs"

4. verdammt in DUDEN (2012, s.v.)

4.1 "(salopp abwertend)“

4.1a „drückt Wut, Ärger o. Ä. aus und steigert das im Substantiv Ausgedrückte"

$4.1 b$ „drückt (in Bezug auf Personen) eine Verwünschung aus“

4.1c „(in Bezug auf Sachen) widerwärtig, im höchsten Grade unangenehm"

4.2 „(umgangssprachlich)“

$4.2 \mathrm{a}$ "sehr groß“

$4.2 \mathrm{~b}$ „<intensivierend bei Adjektiven und Verben> sehr, äußerst“

\subsubsection{Entwicklung der Gradbedeutung bei verdammt}

Aus der Korpusanalyse geht hervor, dass das Einsetzen der Gradbedeutung im Neuhochdeutschen erfolgt. Als Grund für deren Entwicklung dient die Ausgangsbedeutung des Lexems, die verdammenswerte bzw. einem Verdammungsurteil unterliegende Personen sowie verdammungswürdige Sachverhalte beschreibt:

(1021) So ungern das auch der Pabft fehen mochte, und fo auffallend es vielen vorkam, daß einer, der fchon zu Rom als Ketzer verdammt war, auf einer weltlichen Reichsverfammlung noch von neuem Gehör finden follte [...] (DTA: Pütter, Johann Stephan: Historische Entwickelung der heutigen Staatsverfassung des Teutschen Reichs. Bd. 1: Bis 1558. Göttingen, 1786. )

(1022) es ist verdammt bzW. ,'es ist einer strafe wert"“ (DWB, s.v. verdammen)

Ferner unterliegt die Ausgangsbedeutung insofern einer Pejoration und einer Abschwächung, als sie hinsichtlich der „lebenden wesen“, „wirklichen dinge“ und „abstrakte[n]“ im Sinne „fluchwürdig, verdammenswert" (DWB, s.v. verdammen) angewandt wird, jedoch den direkten Bezug auf ein sträfliches Verdammungsurteil verliert und als Ausdruck des Ärgers bzw. der Wut dient, vgl.:

\footnotetext{
${ }^{76}$ Die Angaben aus den Belegzetteln von FWBD.

77 Die Angaben aus den Belegzetteln von FWBD.
} 
(1023) ein verdammter, buckeliger schlingel, ich seh ihn noch, hatte es gleich weg. (DWB, s.v. verdammen)

(1024) der verdammte hof hat dich beides versäumen machen. (DWB, s.v. verdammen)

(1025) das verdammte glücke! ohne das kann man nicht einmal ein guter spitzbube sein (DWB, s.v. verdammen)

Neben dem Prozess der Pejoration kann man eine Metaphorisierung der Ausgangsbedeutung sowie ihre subjectification feststellen, die den Vergleich bzw. die Gleichsetzung zweier Sachverhalte bezüglich ihrer Verwerflichkeit herstellen und einem Objekt eine subjektive Bewertung zuweisen. In dieser Etappe erfolgt außerdem das Einsetzen von Traugott I (oben 3.6.3.4).

DWB (s.v. verdammen) verweist darauf, dass die ursprüngliche Bedeutung „strafwürdig, verwerflich“ sowie ihre metaphorische Übertragung die Bedeutung „vom gesetzmäszigen, allgemein anerkannten abweichend“ bzw. 'von einer Norm auf eine negative Weise so abweichend, dass man sich darüber ärgert' zum Ausdruck gebracht hat, wobei aus den letzten zwei Formulierungen sich der Gebrauch im Sinne „ungemein, auszergewöhnlich“ entwickelt hat, vgl.:

(1026) mit jungfräulicher unbefangenheit macht ein männliches fiskalatsgesicht ${ }^{78}$ einen verdammten abstich (DWB: 1796, Jean Paul, s.v. verdammen)

Der aus DWB übernommene Beleg (1026) kann die Doppeldeutigkeit des adjektivischen Gebrauchs demonstrieren. Durch die Verwendung des Lexems wird zum Ausdruck gebracht, dass die jungfräuliche Unbefangenheit, der Meinung des Schreibers bzw. Sprechers nach, der implizierten Person nicht passend erscheint, indem sie hinsichtlich seiner Männlichkeit und seines Postens außergewöhnlich wirkt und dazu einen starken Kontrast schafft. Zugleich bringt verdammt Kritik und negative Einstellung des Autors zum Ausdruck, sodass der Abstich als verwerflich, verdammungswürdig bezeichnet wird, wodurch verdammt einen Bezug zur metaphorisierten Ausgangsbedeutung erwirbt. - Die Korpusanalyse hat außerdem andere mehrdeutige Belege ergeben, vgl.:

(1027) Die Chriften dürffen nicht verbrennen ohne Leuchten; Der Glaube, der nichts thut, ift ein verdammt Gefchwätz, Und muß Vernünftigen fehr unvernünftig deuchten. (DTA: Zinzendorf, Nicolaus Ludwig von: Teutscher Gedichte Erster Theil. Herrnhuth, 1735)

(1028) Es schmerzt mich in der Seelen, Daß mich mein Ehemann so heftig sucht zu quälen, Und aus verdammtem Geitz mich in den Laden jagt. (C.II: HK4/Z01.00001 Zäunemann: Poetische Rosen in Knospen, Erstdruck: 1738, 2004 [S. 606])

Der Gebrauch von verdammt in (1027) zeigt zunächst die Ausgangsbedeutung des Lexems, die hier ein verwerfliches Geschwätz bezeichnet, das im direkten und

\footnotetext{
${ }^{78}$ Fiskalat ist die spätmittelalterlich-neuzeitliche, an den römischen (lat.) advocatus (M.) fisci angelehnte Behörde, die von Amts wegen die Rechte des Herrschers wahrnimmt. (Köbler, s.v. Fiskalat).
} 
übertragenen Sinn verdammungswürdig ist. Zugleich kann verdammt dahingehend als Intensivierer interpretiert werden, dass das Geschwätz in so hohem Grad ausgeprägt ist, dass es strafbar sein sollte und von daher Ärger und Wut seitens des Autors hervorruft.

Der adjektivische Gebrauch in (1028) kann auf eine ähnliche Weise interpretiert werden. Das Lexem dient einerseits dem Ausdruck des Ärgers und der Wut seitens der implizierten Person. Andererseits spricht der Kontext für die außergewöhnliche Ausprägung des Geizes, der von einer Norm, gemäß den Vorstellungen der unter dem Geiz leidenden Person, abweicht und dadurch die genannten Gefühle hervorruft.

Darüber hinaus lässt sich aus den Belegen (1027) - (1028) schließen, dass es sich um pragmatic enrichment bzw. strengthening der Ausgangsbedeutung handelt. Dies erfolgt dadurch, dass negativ konnotierte Sachverhalte durch ihre Außergewöhnlichkeit und evtl. ihren hohen Grad der Ausprägung Ärger und Wut erregen, wodurch sie als verdammungswürdig im direkten oder übertragenen Sinne bezeichnet werden.

In der nächsten Phase gewinnt die intensivierende Funktion des Lexems an Gewicht und tritt in den Vordergrund, während die Ausgangsbedeutung allmählich verdrängt wird. Die Gradbedeutung ist zunächst eng mit ihrer pejorativen Komponente verbunden, sodass Spuren der Ausgangsbedeutung noch zu erkennen sind, vgl.:

(1029) Und dieft Zauberftiefeln machen verdammt müde, wenn man fie an den Beinen hat. (DTA: Tieck, Ludwig: Phantasus. Bd. 2. Berlin, 1812)

$(1029$ ') 'sehr müde'

(1030) [...] Guter Freund, reichen Sie mi gefälligft die Hand, damit ich aufftehen kann, das linke Bein ift mir eingefchlafen von dem verdammt langen Liegen! (DTA: Heine, Heinrich: Reisebilder. Bd. 3. Hamburg, 1830)

(1030') 'sehr langen'

(1031) die Gefchichte war verdammt langweilig und hausbacken geworden. (DTA: Conradi, Hermann: Adam Mensch. Leipzig, 1889)

(1031') 'sehr langweilig'

(1032) Es ist verdammt hart, unter den Besten zu sein. (C.II: K00/AUG.64634 Kleine Ztg., 31.08.2000)

(1032') 'sehr hart'

(1033) Denn auch das Problem des Ausschließlichkeitsanspruchs der FIFASponsoren [...] ist nicht neu und damit die verdammt schwierige Suche nach anderen Sponsoren. (Protokoll der Sitzung des Parlaments Hamburgische Bürgerschaft am 24.08.2005)

(1033') 'sehr schwierige'

(1034) „Hamburg ist verdammt teuer. Viel teurer als West-, Süd- oder Ostdeutschland", fährt die frustrierte Expertin fort. (C.II: HMP12/JUL.03875 MOPO, 11.07.2012, S. 10)

(1034') 'sehr teuer'

Verdammt kann außerdem Funktionsverbgefüge modifizieren, vgl. die Belege mit den entsprechenden Umformulierungen: 
(1035) Die Europäische Kommission hat der Bundesregierung mitgeteilt: Ehe die FFH-Richtlinie nicht umgesetzt worden ist, hat sie davon auszugehen, dass Deutschland keine Fördennittel bekommt. Wir stehen hier also verdammt unter Druck. (C.II: Protokoll der Sitzung des Parlaments Landtag Niedersachsen am 03.08.1999 [S. 2768])

(1035') 'stehen unter sehr großem Druck' bzw. 'sind sehr bedrängt'

(1036) Albertos stoische Gelassenheit konnte einem verdammt auf die Nerven gehen. (07.02.2014: http://books.google.de/books?id=BtEln3kl_tMC\&pg=PA41\&dq=\%22verdammt + auf + die\%22\&hl=de\&sa=X\&ei=dKT0UsKZHIeQtQbLnoH4Bg\&ved=0CDoQ6AEwAg\#v=onepage\&q=\%22verdammt\%20auf\%20die\%22\&f$=$ false)

(1036') 'sehr nerven'

(1037) Ihr geht mir verdammt auf den Leib. (07.02.2014: http://books.google.de / books?id=6VdZAAAAcAAJ\&pg=PA95\&dq=\%22verdammt+auf + den $\% 22 \&$ hl=de\&sa=X\&ei=aKX0Uo7lA4jIsga3qYHIAQ\&ved=0CDkQ6AEwAjgK\#v=onepage\&q=$\% 22$ verdammt $\% 20$ auf $\% 20 \mathrm{den} \% 22 \& \mathrm{f}=$ false)

(1037') 'nervt sehr'

(1038) Wieder nicht geschafft. Das zieht sich alles verdammt in die Länge. Na ja, wenigstens Latein muß ich im Herbstsemester schaffen. (07.02.2014: http://books.google.de/books?id=nGchAQAAIAAJ\&q=\%22verdammt+in+die+L\%C3\%A4nge\%22\&dq=\%22verdammt+in+die+L\%C3\%A4nge\%22\&hl=de\&sa=X\&ei=46r0UtTfHoXVswbf1oGgBA\&ved=0CFoQ6AEwCA)

(1038') 'verzögert sich sehr'

Die intensivierende Funktion des Lexems kann außerdem in die quantifizierende übergehen, wobei verdammt weiterhin zum derben Ausdruck des Ärgers gebraucht wird, vgl.:

(1039) Ich fahre zwar jeden Tag anderthalb Stunden Fahrrad, aber 45 Kilometer in einer Tour wären zu viel und ohne Auto ist man leider aufdie Bahn mit ihren verdammten Preisen angewiesen. (31.08.2015: http://www.gamestar.de / community/gspinboard/showthread.php?p=12500176)

(1039') 'sehr hohen Preisen'

Der Prozess der Desemantisierung geht mit Zuwachs an subjectivity einher. Die Belege (1029) - (1039) bringen mehr Expressivität zum Ausdruck und verweisen damit auf die dritte semantisch-pragmatische Tendenz (Traugott III, oben 3.6.3.4).

Die letzte Etappe des Entwicklungsgangs von verdammt als Intensivierer bezieht sich auf den Verlust des Zusammenhangs mit der Ausgangsbedeutung und demzufolge das Kollokieren mit positiv konnotierten Kookkurrenzpartnern, vgl.:

(1040) Das kleine Weib hat ein verdammt hübfches Profil, conftatirte der Herr Doctor jetzt mit großer Befriedigung. (DTA: Conradi, Hermann: Adam Mensch. Leipzig, 1889)

(1040') 'sehr hübsches' 
(1041) „Ein Film, der verdammt gute Laune macht. Und das Lebensgefühl einer Szene vermittelt, die es nur hier gibt. Also ein großartiger Film. Vielleicht der schönste und beste Film von Fatih Akin." (C.II: BRZ10/MAI.10265 Braunschw. Z., 27.05.2010)

(1041') 'sehr gute'

(1042) Kreuzfahrten sind Reisen im Dienste einer Milliardenindustrie und können verdammt angenehm sein. (C.II: K99/JUN.40714 Kleine Ztg., 06.06.1999)

(1042') 'sehr angenehm'

(1043) „An Olympia habe ich nur gute Erinnerungen. Ich bin auch heute noch verdammt stolz auf diese Silbermedaille." (C.II: BRZ12/JUN.09862 Braunschw. Z., 19.06.2012)

(1043') 'sehr stolz'

(1044) Das ist eine verdammt kluge Politik, eine ehrliche, eine gerechte und eine solidarische Politik. Unter dieser Landesregierung geht es den Hessen gut. (C.II: Protokoll der Sitzung des Parlaments Hessischer Landtag am 15.12.2011)

(1044') 'sehr kluge'

\subsubsection{Kurzfassung der Entwicklung bei verdammt}

Ausgangsbedeutung: abgeschwächte pejorative Bedeutung 'verdammt, verflucht', zum Ausdruck des Ärgers bzw. der Wut gebraucht; die übrigen Entwicklungsschritte wie oben in Kap. 3.6.3.9.3.

\subsubsection{Verflucht}

\subsubsection{Lexikographische Angaben}

Pfeifer und Kluge liefern keine Informationen über das Lexem.

1. verflucht in FWB (s.v.): keine Angaben ${ }^{79}$

2. verflucht in DWB (s.v.)

2.1 „worüber ein fluch ausgesprochen ist und wird, noch als participium gefühlt"

2.2 „erschrecklich, verabscheuenswert, widerwärtig“

2.3 „,in ähnlicher weise wie verdammt [...] wird das, worauf eigentlich ein fluch ruht, daher eigentlich strafbar ist, als bewundernswert wegen seiner ungewöhnlichkeit aufgefaszt"

2.4 „weit verbreitet in der volkssprache. adverbial, als kräftige, volksthümliche verstärkung"

3. verflucht in DUDEN (2012, s.v.)

"(salopp)“

3.1 "(abwertend)“

$3.1 \mathrm{a}$,verdammt $(1 \mathrm{a})$ “

\footnotetext{
${ }^{79}$ Der Gebrauch in den in der Arbeitsstelle von DWB verfügbaren Belegzetteln weist keine Gradbedeutung auf, sondern die Sinnrichtungen, die sich auf den ausgesprochenen Fluch sowie den Ausdruck der Abwertung beziehen.
} 
$3.1 \mathrm{~b}$ „verdammt (1 b)“

$3.1 \mathrm{c}$ "verdammt (1 c)“

$3.2 \mathrm{a}$,verdammt $(2 \mathrm{a})$ “

$3.2 \mathrm{~b}$ „<intensivierend bei Adjektiven und Verben> sehr, äußert"

\subsubsection{Entwicklung der Gradbedeutung bei verflucht}

Obgleich FWB keine Informationen über das Einsetzen der Gradbedeutung bei verflucht liefert, hat die Korpusanalyse die ersten Belege, die das Einsetzen der Intensivierung veranschaulichen, im Fnhd. feststellen können. Die Ausgangsbedeutung, die als Basis für die Entwicklung der Gradbedeutung gilt, beruht auf der ursprünglichen Sinnrichtung, die mit Fluch betroffenen Personen und Sachverhalte beschreibt:

(1045) was der satan will und sucht, will ich halten als verflucht. (DWB: s.v. verfluchen)

Ferner unterliegt die besprochene Bedeutung einer Pejoration, der Abschwächung und subjectification, wobei das Lexem in der Bedeutung „verabscheuenswert, widerwärtig" (DWB, s.v. verfluchen) verwendet wird, vgl.:

(1046) verfluchtes volk! was untersteht ihr euch, hat man euch lange nicht bewiesen, ein geist steht nie auf ordentlichen füszen? (DWB: s.v. verfluchen)

(1047) da war der verfluchte brief angekommen, der die nachricht von deinem tode brachte (DWB: s.v. verfluchen)

Aus den Belegen (1046) - (1047) geht hervor, dass der Gebrauch des Lexems die Einstellung des Sprechers bzw. Schreibers zum Ausdruck bringt, die Verwendung auf die expressive Ebene bringt und auf das Einsetzen von Traugott I (oben 3.6.3.4) verweist.

DWB (s.v. verfluchen) erklärt den Übergang der pejorativen zu der intensivierenden Bedeutung durch den in dem pejorativen Gebrauch enthaltenen Begriff des Außergewöhnlichen und Bewundernswerten: so verweist DWB darauf, dass die Verwendungen ein verfluchter kerl und eine verfluchte geschichte sich auf jemanden oder etwas beziehen, den oder das „man eigentlich bestrafen sollte, worüber aber man sich wundert wegen seiner auszerordentlichkeit". Darüber hinaus kann geschlossen werden, dass die Gradbedeutung als Nebenbedeutung durch pragmatic enrichment der Ausgangsbedeutung in Erscheinung tritt. Aus der Korpusanalyse geht ferner hervor, dass der hohe Grad des verhassten Sachverhalts den möglichen Grund für die hervorgerufenen Gefühle liefern kann. Die folgenden Belege sollen diese Annahme unterstützen:

(1048) Es hatte das Anfehen / als ob wir vnter der Pflegung deß Vettern glůckfelig weren / fo angelegen ließ er jhm vnfer Gut fein / fo fehr wufte er vns vnd vnfere Mutter zutrooften. Aber die Vrfache feines verfluchten Fleiffes war / daßjhm nichts von der Erbfchafft entgienge / welche jhm nach meines Brudern vnd meinem Tode ohn allen Zweiffel heimgefallen were. (DTA: Barclay, John (Übers. Martin Opitz): Johann Barclaÿens Argenis Deutsch gemacht durch Martin Opitzen. Breslau, 1626) 
(1049) Da jhnen aber das Gold vnd Edelgeftein alfo lieblich vnter die Augen fchien / wie fie die fcho̊nẽ Halßbånder vnd ko̊ftlichen Zierrath an den Jndianern erfahen / wurden fie dadurch zum verfluchten Geitz angereitzet / vnd fätzten alle Ehr vnd Redligkeit hindan. (DTA: Gottfried, Johann Ludwig: Newe Welt Vnd Americanische Historien. Frankfurt (Main), 1631)

(1050) Denn gleichwie der Missbrauch der Reliquien ein verfluchtes Verbrechen ist, also ist auch derselben gänzliche Verachtung eine Ketzerei, welche vor Zeiten von dem Vigilantio Gallo ist an Tag kommen, und von dem Hieronymo verfolget worden [...]. (C.II: HK5/B24.00001 Agrippa von Nettesheim, Ungewißheit und Eitelkeit aller Künste und Wissenschaften, Übersetzung: 1713, 2000 [S. 230])

(1051) [...] Man hat die Erde durchsucht, und die irdischen Güter und Reichtümer, so fast unter der Höllen verborgen liegen und eine Anreizung zu allem Bösen sind, ausgegraben. Dadurch das schädliche Eisen und das noch schädlichere Gold am Tag gekommen, welches die Menschen mit einer so verfluchten Begierde entzündet, dass sie alles Recht, Ehrbarkeit, Treu und Aufrichtigkeit verjaget, an deren Stelle lauter List und Betrug, Hinterstellungen und böse Begierden sich eingeschlichen. (C.II: HK5/B24.00001 Agrippa von Nettesheim, Ungewißheit und Eitelkeit aller Künste und Wissenschaften, Übersetzung: 1713, 2000 [S. 114])

Der adjektivische Gebrauch in (1048) - (1051) kann mehrdeutig interpretiert werden. Zunächst handelt es sich um fluchwürdige und verachtenswerte Sachverhalte, sodass verflucht sich auf die pejorative Bedeutungskomponente bezieht. Zugleich jedoch verweisen die Kontexte darauf, dass die durch die Bezugswörter ausgedrückten Begriffe außerordentlich bzw. in hohem Grad ausgeprägt sind und damit die Verachtung und die äußert negative Einstellung seitens des Sprechers bzw. Schreibers hervorrufen. Aus den Belegen geht außerdem hervor, dass die Bezugswörter jeweils Extremwerte aufweisende Prädikate darstellen. Durch die Vierbindung dieser Wörter mit verflucht kommt es zu der Verstärkung des Ausdrucks. So berichtet beispielsweise der Autor in (1048) über den außerordentlichen Fleiß seines Vetters hinsichtlich der Pflege seines Cousins und bringt zugleich seinen Ärger und seine Wut in dieser Angelegenheit darüber zum Ausdruck, dass der Vetter sie zu vergiften und das Erbe zu übernehmen beabsichtigt. Der Fleiß ist in hohem Grad ausgeprägt und bezieht sich auf eine vom Schreiber bzw. Sprecher als äußerst negativ bewertete Situation. Von daher wird verflucht als Intensivierer gebraucht.

Die Verwendung in (1050) erweist sich ebenso als mehrdeutig, jedoch bezieht sich verflucht zunächst auf die ursprüngliche, mit eigentlichem Fluch verbundene Bedeutung, vgl. die Umformulierung:

(1050') 'Der Missbrauch der Reliquien ist ein großes Verbrechen, worüber ein Fluch ausgesprochen werden soll bzw. kann.'

Darüber hinaus kann aus dem Kontext geschlossen werden, dass das angesprochene Verbrechen eine sehr schwere Straftat darstellt und von daher fluchwürdig ist. Auf diese Weise erwirbt verflucht eine intensivierende Rolle. 
In der nächsten Phase wird die intensivierende Rolle des Lexems verstärkt, indem sie in den Vordergrund tritt und der aufs Schimpfen bezogenen Ausgangsbedeutung eine Nebenrolle überlässt. Verflucht wird damit als Intensivierer und zugleich als Ausdrucksmittel des Ärgers und der Wut gebraucht. In dieser Stufe kollokiert das Lexem mit negativ konnotierten Partnern, vgl. die Belege mit den entsprechenden Umformulierungen:

(1052) Mir wird die Zeit auch so verflucht lang hier unten, ich weiß wahrhaftig nicht mehr was ich angreifen soll. (C.II: HK3/F90.00001 Lenz: Pandämonium Germanicum, Entstanden: 1775, 2000 [S. 258])

(1052') 'sehr lang'

(1053) Am Wochenende war ich bei einer großen Geburtstagsfete, die Musik war super und ich entschloss mich anzutanzen gegen den Schmerz. [...] Aber am nächsten Morgen! Die Hexe hatte ihren Kater geschickt: Es tut verflucht weh, und ich komme nur noch mühsam vom Sessel hoch. (C.II: RHZ00/DEZ.18449 RZ, 27.12.2000)

(1053') 'tut sehr weh'

(1054) Gegen ein Uhr nachmittags erklärte Loiseau, daß er jetzt verfluchten Hunger habe. Alle empfanden längst dasselbe Gefühl und da der Heißhunger immer heftiger wurde, hörte allmählich jede Unterhaltung auf. (05.02.2014: http://gutenberg.spiegel.de/buch/2530/2)

(1054') 'sehr großen Hunger'

Verflucht kommt außerdem als Modifizierer in Funktionsverbgefügen vor, vgl. die folgenden Belege:

(1055) Für Dein Bein gibt's nur ein Mittel, Ruhe und Geduld, dann wird's hoffentlich wieder in Ordnung kommen, aber in unserem Alter ziehen sich solche Dinge immer verflucht in die Länge. (07.02.2014: http://books.google.de / books?id=lLIDAAAAYAAJ\&q=\%22verflucht+in+die+L\%C3\%A4nge$\% 22 \& d q=\% 22$ verflucht + in + die + L $\% C 3 \% A 4 n g e \% 22 \& \mathrm{hl}=$ de\&sa $=X \&$ ei $=2 \mathrm{Kv}$ 0Uv21BtHXsgb4kYGQCw\&ved=0CEEQ6AEwAQ)

(1055') 'verzögern sich sehr'

(1056) Diese pestartige Überziehung des gesamten Forums mit Tolkien geht mir allerdings gerade verflucht auf den Wecker. (07.02.2014: http://www.asoiaf.org/forumneu/index.php?page=Thread\&threadID=2445\&pageNo=22)

(1056') 'nervt sehr'

(1057) Irgendwie ist die Six Flags Strategie mit Europe's Coaster Capital da verflucht in die Hose gegangen, weshalb ich es als äußerst unrealistisch halte, dass da in naher Zukunft ein größerer Coaster einzieht, weil der Park nicht gut läuft (korrigiert mich, wenn ich irre!). (07.02.2014: http://freizeitparkweb.de/cgi-bin/dcf/dcboard.cgi?az=show_thread\&forum=DCForumID111\&om=219\&omm=21)

(1057') 'ist sehr misslungen'

(1058) Dabei kommt man nämlich verflucht ins Schwitzen und kann sich auch Brandblasen holen. (07.02.2014: http://books.google.de/books?id=- 
ngEIHOTTxT8C\&pg $=$ PA202\&lpg=PA202\&dq=\%22verflucht + ins $\% 22 \&$ source $=-$ bl\&ots=JRXn7aUeBy\&sig=3wWY6KB8PuQWH8dynUWtoLhsNSY\&hl=de\&sa=X\&ei=Tbn0UqaqK4fbtAb9k4CYCQ\&ved=0CFEQ6AEwCA\#v=onepage \&q=\%22verflucht $\% 20$ ins $\% 22 \& f=$ false)

(1058') 'fängt an stark zu schwitzen'

Die intensivierende Funktion des Lexems kann außerdem in die quantifizierende mit der Nebenbedeutung des Ärgers übergehen, vgl.:

(1059) - Die Preise sind wirklich zu hoch [...]

- Na gut ich kaufmir auch nicht jedes Spiel, aber das liegt manchmal an den verfluchten Preisen: mad: (70€ und drüber) [...] (31.08.2015: http://naarsika.de/forum/viewtopic.php?f=53\&t=3046\&start=20)

(1059') 'sehr hohen Preisen'

In dieser Etappe des Entwicklungsgangs gewinnt die Intensivierung an subjectivity, wird ausschließlich auf der expressiven Ebene gebraucht und verweist auf die dritte semantisch-pragmatische Tendenz (Traugott III, oben 3.6.3.4).

Anschließend finden sich Belege, die die letzte Etappe der Bedeutungsentwicklung demonstrieren. In dieser Stufe tritt verflucht mit positiv konnotierten Kookkurrenzpartnern als Gradadverb auf und demonstriert die Bedeutungsentleerung, bei der der Bezug zur Bedeutung des Fluchs und des Ausdrucks von Ärger endgültig verloren geht vgl.:

(1060) Zugleich ist er ein letzter Zeuge aus jener Zeit, als Saxophonspielen noch kein Motiv für die Bauspar-Werbung, sondern eine verflucht coole Aktivität war. (C.II: P95/MAI.17107 Die Presse, 18.05.1995)

$(1060$ ') 'sehr coole'

(1061) Nebenbei schreibt die Band auch noch verflucht gute Songs. (C.II: E00/MAR.07265 Zürcher Tagesanzeiger, 15.03.2000, S. 80)

(1061') 'sehr gute'

(1062) Die studierten Liedermacher im Stil von „Monsters of Liedermaching" liefern "Class A“-Kunsthandwerk mit erlesen geistreichen Texten - humorvoll, spitz, filigran, liebevoll und verflucht ehrlich. (C.II: BRZ09/MAI.13335 Braunschw. Z., 28.05.2009)

(1062') 'sehr ehrlich'

(1063) „Du hast ein verfluchtes Glück“, knurrte der Polizist, der nachwievor seine Waffe auf ihn gerichtet hatte. (05.02.2014: http://www.schreibwerkstatt.de/der-weisze-widerstand-v2-0-kap1-2-3-t38879.html)

(1063') 'sehr großes Glück'

\subsubsection{Kurzfassung der Entwicklung bei verflucht}

1. Ausgangsbedeutung: abgeschwächte pejorative Bedeutung 'verdammt, verflucht', zum Ausdruck des Ärgers gebraucht;

die übrigen Entwicklungsschritte wie oben in Kap. 3.6.3.9.3. 


\subsubsection{Verteufelt}

\subsubsection{Lexikographische Angaben}

In Pfeifer und Kluge finden sich keine Angaben über verteufelt.

1. verteufelt in FWB (s.v. verteuffelt) 80 „verteufelt, teuflisch, verflucht"; „verteufeln, schlecht machen, verdammen“

2. verteufelt in DWB (s.v. verteufeln)

2.1 „zum teufel gemacht oder geworden; vom teufel besessen, teuflisch“

2.2 "verzweifelt"

2.3 "verdammt, verflucht"

2.4 „im hohen grade listig, verschlagen, arg; auszerordentlich gewandt, bewandert in einer fertigkeit"

2.5 „ein blosz verstärkender zusatz“

3. verteufelt in DUDEN (2012, s.v.)

3.1a "schwierig und unangenehm; vertrackt, verzwickt"

$3.1 \mathrm{~b}$ „überaus groß, stark, intensiv“

$3.1 \mathrm{c}$ „ $<$ intensivierend bei Adjektiven und Verben> über die Maßen“

3.2 „verwegen, toll“

\subsubsection{Entwicklung der Gradbedeutung bei verteufelt}

Laut DWB (s.v. verteufeln) erfolgt das Einsetzen der Intensivierung im Fnhd. Als Basis gilt wohl auch hier die pejorative Bedeutung, die sich aus der ursprünglichen Sinnrichtung „zum teufel gemacht oder geworden“ und „vom teufel besessen, teuflisch" durch Abschwächung, subjectification und Metaphorisierung entwickelt und den „Sinn der Verachtung oder des Hasses“ (DWB, s.v. verteufeln) zum Ausdruck gebracht hat. Die folgenden Belege mit den entsprechenden Umformulierungen sollen die Ausgangsbedeutung (1064) und die pejorative Bedeutung (1065) veranschaulichen, vgl.:

(1064) weil die welt so vergifftet und verteufelt ist, das sie die warheit verdammen thar (DWB: s.v. verteufeln, LUTHER 28, 324, 25)

(1064') 'die Welt ist vom Teufel besessen'

(1065) dieses verteuffelte schreiben ist allein der ausleger meiner begangenen laster (DWB: s.v. verteufeln)

(1065') 'verdammungswürdige, verfluchte Schreiben'

Hier zeigt sich, dass das Auftreten der pejorativen Bedeutung der Verwendung des Lexems auf der expressiven Ebene und so mit dem Einsetzen von Traugott I (oben 3.6.3.4) einhergeht.

Die nächste Stufe der Bedeutungsentwicklung bezieht sich auf das Auftreten der Gradbedeutung in mehrdeutigen Kontexten. Dies erfolgt durch pragmatic strengthening bzw. enrichment der Ausgangsbedeutung, wobei die Intensivierung in Form einer Inferenz als Nebenbedeutung in Erscheinung tritt. DWB (s.v. verteufeln)

\footnotetext{
80 Die Angaben aus den Belegzetteln von FWBD.
} 
verweist darauf, dass der „derbe[] Ausdruck der Bewunderung“ das fehlende Kettenglied darstellt, welches den Zusammenhang zwischen der pejorativen Bedeutung und der Intensivierung ermöglicht. Dieser Zusammenhang, der eine Beziehung zwischen der negativen einstellungsbezogenen Bedeutung und dem Bewunderung auslösenden Begriff der Außergewöhnlichkeit darstellt, lässt sich außerdem in dem oben besprochenen Entwicklungsgang der Intensivierer verdammt und verflucht feststellen, so DWB. Die Belegsituation lässt ferner annehmen, dass der hohe Grad des im Bezugswort ausgedrückten Sachverhalts die Ursache für die Bewunderung sowie die Verachtung und den Hass und die darauf folgende äußerst negative Wertung seitens des Sprechers bzw. Schreibers darstellt. So werden sehr negative Situationen durch das Kollokieren mit dem emotional markierten und Pejoration ausdrückenden Wort verstärkt, vgl.:

(1066) Herzall erliebfte Fr. Mutter; Ich euer gehorfamer Sohn Herkules / füge Euer Gn. zu wiffen / was geftalt mein gnådiger allein wahrer Gott mich nicht allein meiner anderthalbjåhrigen Knechtfchaft entriffen / fondern fo hoch begnadet / daß ich in meinem vertriebenẽ Stande mehr Ehr und Gelder erftritten / als ich mir in Teutfchland vermuhten feyn könte / wie Zeiger diefes berichten wird. Was ihre verteufelt Lügen Pfaffen von mir låftern / wollen fie ja nicht glåuben / fondern fich verfichern / daß ich einem fo heiligen und reinen Gott diene / welcher durchaus keine ủppigkeit und Unzucht / oder was dem anhanget / dulden noch ungeftraffet laffen kan. (DTA: Bucholtz, Andreas Heinrich: Des Christlichen Teutschen GroßFürsten Herkules Und der Böhmischen Königlichen Fräulein Valjska Wunder-Geschichte. Bd. 1. Braunschweig, 1659)

(1067) Er würde ohn einiges leugnen geftehen / was geftalt er fich durch feine verteufelte faft unerho̊rte Boßheit und wahnfinnigen ůbermuht håtte laffen verleiten / einem Hochfürftlichen Fråulein (welches ihm nicht unbewuft gewefen) nach Ehr und Keufcheit zuftreben; wodurch er dann verdienet / daß er andern feines gleichen Buben zur Warnung und Beyfpiel abgeftraffet würde / und zwar auff diefe weife: Daß fein fchandfüchtiger Leib an allen feinen Gliedern folte mit einem Rade durch des Henkers Hand zuftoffen / und hernach den Raben zur Speife darauff gelegt werden [...] (DTA: Bucholtz, Andreas Heinrich: Des Christlichen Teutschen Groß-Fürsten Herkules Und der Böhmischen Königlichen Fräulein Valjska Wunder-Geschichte. Bd.2. Braunschweig, 1660)

(1068) Verfluchte Missethat!

Verteufelt-böser Mensch! Glaubt: der Verläumbder hat / Den mir gestohInen Dolch mit Gifte selbst beflecket. (DWB: s.v. verfluchen; www.zeno.org: Daniel Casper von Lohenstein: Römische Trauerspiele, Erstdruck: Breslau (Esaias Fellgiebel), 1665)

(1069) Es ift ja nicht zu låugnen / Freund und Feinde wiffens / daß Leute unter den Predigern gefunden werden / welche in offentlichen ärgernüffen leben / wann ihr verteuffelter geld- und ehrgeitz / auch in den allerheiligften Dingen [...] (DTA: Spener, Philipp Jakob: Pia Desideria. Frankfurt (Main), 1676) 
Die Verwendungen in (1066) - (1069) erlauben eine mehrdeutige Interpretation. Zunächst bezieht sich das Lexem auf die wertende pejorative Bedeutung. So bringt der Schreiber bzw. Sprecher in Bezug auf die Lügen in (1066), die Bosheit in (1067), den Menschen in (1068) und den Geld- und Ehrgeiz in (1069) seine äuBerst negative Einstellung bzw. Verachtung und seinen Hass zum Ausdruck. Zugleich erwirbt verteufelt eine intensivierende Nebenbedeutung, die als Grund für die negative Wertung betrachtet werden kann, vgl. die möglichen Paraphrasen:

(1066') 'Die Lügen sind sehr schlimm und damit sehr ärgerlich, Verachtung hervorrufend.'

(1067') 'Die Bosheit ist sehr stark und damit sehr ärgerlich, Verachtung hervorrufend.'

(1068') 'Der Mensch ist sehr böse und damit sehr ärgerlich, Verachtung hervorrufend.'

(1069') 'Der Geld- und Ehrgeiz ist sehr stark und damit sehr ärgerlich, Verachtung hervorrufend.'

In dieser Phase des Entwicklungsgangs ist die Zunahme von subjectivity auf der expressiven Ebene bemerkbar, wenn der Schreiber bzw. Sprecher einem Sachverhalt den Grad der Ausprägung aus seiner Perspektive zuschreibt.

In der nächsten Phase der Bedeutungsentwicklung ist der semantische Abnutzungsprozess erkennbar. Das Lexem wirkt insofern verblasst, als „der sinn der verachtung oder des hasses " (DWB, s.v. verteufeln) gegenüber dem Sachverhalt allmählich zurücktritt, während die Gradbedeutung an Gehalt gewinnt und in den Vordergrund tritt. Dies gilt zunächst für negativ konnotierte Kontexte, sodass der Bezug auf die Ausgangsbedeutung nicht vollkommen beseitigt wird, vgl.:

(1070) Sie müssen verteufelt viel zu thun haben, daß Sie sich gar so selten sehen lassen. (C.II: HK3/G02.00001 Spielhagen: Problematische Naturen. Zweite Abteilung (Durch Nacht zum Licht), Erstdruck: 1862, 2000 [S. 291])

$(1070$ ') 'sehr viel'

(1071) Einmal fragte ihn ein Bekannter, wohin er seine diesjährige Sommerreise machen würde. „Ich weiß noch nicht, " antwortete er lakonisch. „Es ist auch eigentlich ganz verteufelt langweilig," fuhr der andere fort. (C.II: HK4/J05.00001 Janitschek: Frauenkraft, Erstdruck: 1900, 2004 [S. 21])

(1071') 'sehr langweilig'

(1072) „Du siehst übrigens verteufelt schlecht aus. Das Glück bekommt dir übel.“ (C.II: HK4/J05.00001 Janitschek: Frauenkraft, Erstdruck: 1900, 2004 [S. 159])

(107'2) 'sehr schlecht'

(1073) drei Monate dauerte es, bis die Wundschwellung abklang, noch monatelang plagte sie Phantomschmerz: „es hat verteufelt gejuckt, und nachts hatte ich Alpträume". (H88/CM7.14068 Mannh. Morgen, 30.01.1988, S. 03)

(1073') 'sehr gejuckt'

(1074) Denn schnell wurde deutlich, warum Schönbergs 1940 im amerikanischen Exil vollendetes Violinkonzert so selten gespielt wird: Es ist verteufelt schwierig. (HAZ07/SEP.06906 HAZ, 22.09.2007, S. 6) 
(1074') 'sehr schwierig'

Verteufelt wird außerdem zur Intensivierung in Funktionsverbgefügen gebraucht, vgl.:

(1075) Aber die Fremden! aber die heilige Woche und die Via Condotti mit all ihren Rosenkränzen, all ihren falschen Kameen, all ihren Sankt Peters in Mosaik! Für die Touristen gibt es ganze Magazine voller Steine vom Forum, die für den Schreibtisch zu Briefbeschwerern zurechtgemacht sind. Man hat aus dem Marmor der Tempel Federhalter gemacht. All das fällt verteufelt auf die Nerven. Das ist der erste Eindruck, den Rom mir gemacht hat. (28.07.2014: http://gutenberg.spiegel.de/buch/7938/2)

(1075') 'nervt sehr'

(1076) Ich pflegte damals, da sich ein Vormittag bei der Arbeit verteufelt in die Länge zieht, meine Tätigkeit so um 10 Uhr herum zu unterbrechen, um in dem nahe der Station gelegenen Gasthaus Berger ein Gabelfrüstück einzunehmen. (28.07.2014: http://www.bhl-europe.eu/static/a0z34trz/a0z34trz_full_ocr.txt)

(1076') 'viel länger dauert als erwartet'

(1077) Bei minus $14^{\circ}$ kam ich dabei verteufelt ins Schwitzen. (28.07.2014: http://books.google.de/books?id=VzavU7iGwKEC\&pg=PA159\&dq=\%22verteufelt+ins\%22\&hl=de\&sa=X\&ei=s0vWU-y8DoWi4gT2toHoBg\&ved=0CDUQ6AE$w B A \# v=$ onepage $\& q=\% 22$ verteufelt $\% 20$ ins $\% 22 \& \mathrm{f}=$ false)

(1077') 'fing an stark zu schwitzen'

Die intensivierende Funktion des Lexems kann außerdem in die quantifizierende übergehen, die die Bedeutung „groß, viel“ zum Ausdruck bringt, vgl.:

(1078) Pferde, Spiel, der äußere Anstand und die Weiber kosten ein verteufeltes Geld [...] (07.03.2014: http://gutenberg.spiegel.de/buch/5148/2)

(1078') 'sehr viel Geld'

(1079) Dort im GUM sitzen jetzt teure Unternehmen mit feinsten Kleidungsangeboten, die Reizwäsche aller Art zu verteufelten Preisen verkaufen. (07.03.2014: http://www.strausberg-live.de/kolumne.php\&id=224)

(1079') 'sehr hohen Preisen'

Aus den Belegen geht hervor, dass das Lexem in dieser Etappe des Entwicklungsgangs an subjectivity gewinnt, ausschließlich auf der expressiven Ebene gebraucht wird und damit auf die dritte semantisch-pragmatische Tendenz (Traugott III, oben 3.6.3.4) verweist.

Die letzte Etappe des Entwicklungsgangs zeigt nun die volle Desemantisierung des Lexems, nach der der Gebrauch keine negativen Konnotationen hervorruft, dem derben Ausdruck des Ärgerns nicht mehr dient und ausschließlich die intensivierende Rolle des Lexems demonstriert, vgl.:

(1080) Schön ist sie eigentlich nicht, meine Braut, aber verteufelt hübsch, murmelte er vor sich hin [...]. (C.II: HK4/S04.00001 Schopenhauer: Die Tante, Erstdruck: 1823, 2004 [S. 367])

(1080') 'sehr hübsch' 
(1081) die Gräfin ist entzückend, die Cigarre deliciös und der Wein verteufelt angenehm... (C.II: KHM/018.00010 Social-Politische Blätter, 1874.08.08)

(1081') 'sehr angenehm'

(1082) Man muss eben zusehen, wie man langfristig doch noch gewinnt. Vor allem gegen so eine verteufelt gute Sportlerin wie meine Frau. (C.II: BRZ09/JAN.09932 Braunschw. Z., 24.01.2009)

(1082') 'sehr gute'

(1083) Das Goodbye fällt tatsächlich schwer - nicht zuletzt auch deshalb, weil der neue Pächter der Bar im Jazzstudio so verteufelt leckeres Landbier ausschenkt... (C.II: NUN10/NOV.01184 NN, 12.11.2010, S. 3)

(1083') 'sehr leckeres'

\subsubsection{Kurzfassung der Entwicklung bei verteufelt}

1. Ausgangsbedeutung: abgeschwächte pejorative Bedeutung 'verdammt, verflucht', zum Ausdruck des Ärgers gebraucht;

die übrigen Entwicklungsschritte wie oben in Kap. 3.6.3.9.3. 


\section{Zur Entstehung von russ. Gradadverbien}

In dem Kapitelabschnitt 3.6.3.8. habe ich ein Schema der Herausbildung von GradAdjektiven und -Adverbien präsentiert und dessen Realisierung in 3.6.3.9. sowie in dem Kapitel 4 am Beispiel von zahlreichen deutschen Lexemen dargestellt. In diesem Kapitel nun werde ich nachweisen, dass dieses Schema auch bei der Entwicklung von russischen Intensivierern grundlegend ist.

Die Entstehung von russischen Grad-Adjektiven und -Adverbien wird am Beispiel von Lexemen mit der Ausgangsbedeutung 'Furcht erregend' exemplifiziert. Diese Wortgruppe enthält die folgenden Lexeme: жуткий (žutkij), кошмарный (košmarnyj), страшный (strašnyj), ужасный (иžasnyj) und чудовищный (с̌иdoviščnyj). Die Untersuchung des Entwicklungsgangs von чудовищный (čudoviščnyj) konnte im Rahmen meiner Arbeit wegen des Mangels an geeigneten Belegen nicht durchgeführt werden. Die Belege dokumentieren, dass das Lexem im 19. Jh. in bereits ausgebildeter intensivierender Funktion auftritt, bieten jedoch keinen Anhalt für frühere Verwendungen, obgleich das Lexem im Altrussischen zu finden ist (allerdings wiederum mangelhaft, d.h. mit einem Beleg). Daher werde ich auf die Beschreibung der Herausbildung der Gradbedeutung bei diesem Lexem verzichten müssen.

\section{1 Жуткий (žutkij)}

Etymologisch geht das Adjektiv жуткий (žutkij) auf das Substantiv жуда (žuda) mit der Bedeutung 'Entsetzen, Not' (Vasmer, s.v. жуткий, s.v. жуда) zurück. Seleznjov (s.v. жуткий), Sorokin (s.v. жуткий) sowie das russische Korpus verweisen darauf, dass das Lexem erst in der ersten Hälfte des 19. Jh. in Erscheinung getreten ist und der Bezeichnung von Furcht erregenden Sachverhalten und den mit diesem Gefühl verbundenen Zuständen dient, vgl.:

(1084) Жутко было старухе оставаться на ночь с своей ужасною гостьей; но, скрепя сердце, она осталась. (RC: О. М. Сомов. Русалка (1829))

(1084') 'Es war unheimlich für die Alte, bei ihrer furchtbaren Besucherin zu bleiben, aber sie blieb mit schwerem Herzen'.

(1085) В голове его вертелись [...] жуткие мысли, относившиеся к этому обстоятельству. (R.C.: В. В. Крестовский. Петербургские трущобы. Книга о сытых и голодных. Роман в шести частях. Ч. 6. (1867))

(1085') 'In seinem Kopf drehten sich unheimliche Gedanken, die sich auf diesen Sachverhalt bezogen'.

Das Auftreten der Gradbedeutung erfolgt in Mehrdeutigkeit aufweisenden Kontexten, wobei die Intensivierung zufolge pragmatic inferencing in Erscheinung tritt, vgl.:

(1086) Но шум взрыва заглушил резкий и жуткий вопль, пронесшийся по всему храму... (RC: Л. Филипсон, П. И. Вейнберг (перевод). Яков Тирадо (Испанский меч) (1887))

(1086') 'Aber der Lärm der Explosion wurde durch einen schrillen und unheimlichen Schrei übertönt, der sich im ganzen Tempel verbreitete'. 
(1087) Вы занимались оккультизмом. [...] Любительски, дилетантски, в полувере, поисками «интересного», способного жутко пощекотать нервы и смутить чувства, такого, [...] чтобы мороз подрал по коже. (RC: А. В. Амфитеатров. Жар-цвет (1895))

(1087') 'Sie haben sich mit Okkultismus beschäftigt. Amateurhaft, dilettantisch, nach „Interessantem" suchend, welches unheimlich nervenkitzelnd und Gefühle verwirrend sein kann, sodass es einem kalt den Rücken herunterläuft'.

(1088) Андрей Ильич очнулся от обморока по крайней мере через полчаса. Внизу, у подножия насыпи, там, где обыкновенно с несмолкаемым грохотом день и ночь работал исполинский завод, была необычная жуткая тишина. (RC: А. И. Куприн. Молох (1896))

(1088') 'Andrej Il'ič kam mindestens nach einer halben Stunde zu sich. Unten, am Fuß des Dammes, wo eine Riesenfabrik gewöhnlich tags und nachts mit einem unaufhörlichen Gepolter arbeitete, herrschte unheimliche Stille'.

Aus den Belegen (1086) - (1088) geht hervor, dass жуткий (žutkij) mehrere Interpretationen erlaubt. Zunächst bezieht sich das Lexem auf die Bedeutung des Schreckens. In (1086) charakterisiert жуткий einen Angst erregenden bzw. von Angst ausgelösten Schrei, in (1087) handelt es sich um die Nerven kitzelnde und damit Schrecken hervorrufende Beschäftigung mit Okkultismus. In (1088) verweist das Lexem auf die Schrecken erregende Stille. Zugleich lässt sich aus den angeführten Kontexten eine weitere Interpretation ableiten, die auf die Intensität der Sachverhalte hinweist. Die Angst stellt dabei den Grund für die Intensität, vgl. die folgenden Umformulierungen:

(1086') 'Die Person war so erschreckt, dass sie sehr laut schrie' bzw. 'Der Schrei war so laut, dass er Angst hervorrief'.

(1087') 'Okkultismus kann Angst hervorrufen und damit sehr stark Nerven kitzeln, wenn man sich damit beschäftigt'.

(1088') 'Die Stille war in so hohem Grad ausgeprägt, dass es Angst hervorrufend wirkte'.

Die Gradbedeutung findet sich außerdem in semantic repetition aufweisenden Kontexten, vgl.:

(1089) К этому же далекому времени относится и другое событие, но оно [...] протянулось в моей памяти длинным следом томительной жуткой боязни. (RC: Ф. И. Буслаев. Мои воспоминания (1897))

(1089') 'Zu dieser längst vergangenen Zeit gehört auch ein anderes Ereignis, es hat sich aber in meinem Gedächtnis mit einer langen Spur quälender furchtbarer Angst vermischt'.

(1090) [...] она чувствовала жуткий страх пред будущим и безмолвно молилась [...] (RC: Максим Горький. Фома Гордеев (1899))

(1090') 'Sie spürte furchtbare Angst vor der Zukunft und betete wortlos'. 
Die in (1986) - (1090) dargestellten Belege demonstrieren, dass das Einsetzen der Gradbedeutung in pragmatic strengthening bzw. enrichment aufweisenden Kontexten durch pragmatic inferencing bzw. semantic reanalysis erfolgt. Die Verwendungen legen außerdem nahe, dass der Gebrauch des Lexems mit dem Auftreten von subjectivity verbunden ist, indem die intensivierende Beddeutung die Einstellung des Sprechers bzw. Schreibers zum Ausdruck bringt, was auf die erste semantisch-pragmatische Tendenz (Traugott I, oben 3.6.3.4) verweist.

Die Konventionalisierung der Implikatur führt zu einem Verlust des direkten Zusammenhangs mit der Ausgangsbedeutung, vgl.:

(1091) Младший брат Алексея-очень симпатичный мальчишка, но жуткий зануда - прибежал к брату в комнату и никак не хотел идти к себе. (RC: Михаил Шишкин. Венерин волос (2004) / / «Знамя», 2005)

(1091') 'Der jüngere Bruder von Aleksej - ein sehr netter Junge aber ein furchtbarer Langweiler - lief zum Bruder ins Zimmer und wollte gar nicht in seins gehen'.

(1092) Впрочем, тому, что потеряла паспорт, ничуть не удивляюсь, я жутко рассеянный человек [...]. (RC: коллективный. Два дня увеселений (2011))

(1092') 'Ich wundere mich übrigens gar nicht, dass ich meinen Pass verloren habe, ich bin ein furchtbar zerstreuter Mensch'.

Das Lexem kann außerdem Funktionsverbgefüge intensivieren, vgl.:

(1093) В детстве у нее была мерзкая привычка сбивать меня с ног и уноситься хохоча. Это жутко действовало на нервы. (RC: Мариам Петросян. Дом, в котором... (2009))

(1093') 'Als Kind hatte sie eine widerliche Gewohnheit, mich niederzuwerfen und lachend davonzulaufen. Das ging furchtbar auf die Nerven.'

(1093') 'nervte sehr'

Neben der intensivierenden Funktion lässt sich die quantifizierende Funktion des Lexems feststellen, die die Bedeutung „groß, viel“ zum Ausdruck bringt, vgl.:

(1094) Остается одно: вздохнув, нести в Сбербанк жуткую сумму - 34 доллара. (RC: В. А. Александр. Битва с обмылками (1997) / / «Столица», 1997.09.29)

(1094') 'Es bleibt nur eins: seufzend in die Sberbank die furchtbare Summe zu bringen - 34 Dollar'.

(1095) В Индии жуткие налоги. (29.05.2015: http://alinalyamzi.tumblr.com / post/110627811807)

(1095') 'Indien hat furchtbare Steuern'.

Das Lexem entwickelte außerdem eine rein pejorative Bedeutung, die lediglich die persönliche Einstellung des Sprechers zu einem Sachverhalt ausdrückt, vgl.:

(1096) [...] забавно, что до конща жизни Айн Рэнд говорила по-английски с жстким акцентом [...] (RC: Сергей Голубицкий. Who is John Galt? (2004) / / «Бизнес-журнал», 2004.08.17) 
(1096') 'Es ist amüsant, dass Ain Rend auf Englisch bis zum Ende ihres Lebens mit einem furchtbaren Akzent redete'.

Die Belege (1091) - (1093), (1094) - (1095) und (1096) veranschaulichen den Gebrauch des Lexems jeweils in der intensivierenden, quantifizierenden und pejorativen Funktion, ohne auf die Bedeutung des Schreckens zu rekurrieren. Das GradAdjektiv bzw. -Adverb wird jedoch in dieser Phase im Kontext negativ konnotierter Bezugswörter verwendet. Darüber hinaus ist die Desemantisierung nicht komplett vollzogen. Zugleich bezeugen die Verwendungen einen Zuwachs an subjectivity, wodurch die Bedeutung auf die expressive Ebene im Rahmen von Traugott III (oben 3.6.3.4) übergeht.

Der Verlust des Zusammenhangs mit der Ausgangsbedeutung erfolgt durch die Verbindung des Lexems mit positiv konnotierten Kookkurrenzpartnern, vgl.:

(1097) Но я жуткий оптимист. [...] Декораций нет, актеров нет, а я все равно верю, что все будет. (RC: Таранда. На лету (2002) / / «Домовой», 2002.02.04)

(1097') 'Aber ich bin ein furchtbarer Optimist. Es gibt keine Dekorationen, keine Schauspieler, aber ich glaube trotzdem, dass es alles noch geben wird'.

(1098) [...] когда моя карьера только начиналась - начало 90-х, первые выборы, - все было жутко интересно. (RC: Эльвира Савкина. «У меня хищная жизненная позиция» (2002) / / «Дело» (Самара), 2002.05.11)

(1098') 'Als meine Karriere noch begann - Anfang der 90er, erste Wahlen war alles furchtbar interessant'.

\section{2 Кошмарный (košmarnyj)}

Das Lexem кошмарный (kоšmarnyj) wurde von dem Substantiv кошмар ('Alptraum') abgeleitet (Šapošnikov, s.v. кошмар) und findet sich im Korpus erst Ende des 19. Jh. mit der auf Alpträume und damit auch auf Schrecken bezogenen Bedeutung, vgl.:

(1099) Мерещилось ему что-то кошмарное и нелепое, казалось ему, что он не в своем уме. (RC: Ф. М. Достоевский. Братья Карамазовы (1880))

(1099') 'Es schien ihm etwas Schreckliches und Unsinniges, er kam sich vor, nicht richtig im Kopf zu sein'.

(1100) Отсюда реальная действительность в описании Блока [...] носит кошмарный оттенок. (RC: Андрей Белый. Апокалипсис в русской поэзии (1905))

(1100') 'Darüber hinaus hat die reale Wirklickeit in Bloks Beschreibung einen Hauch von Schrecklichkeit'.

Die Verwendung in (1099) bezieht sich auf eine mit Alp- bzw. Angsttraum verbundene Situation. In (1100) dagegen handelt es sich um eine metaphorische Verwendung des Wortes, durch die es eine äußerst schlimme Gegenwart charakterisiert, die mit einem Alptraum verglichen wird. 
Die metaphorische Verwendung wirkt expressiver und demonstriert damit das Auftreten von subjectivity und kündigt das Auftreten von Traugott I (oben 3.6.3.4) an.

Die übertragene Bedeutung dient ferner als Ausgangspunkt für das Einsetzen der Gradbedeutung. Intensivierung tritt in mehrdeutigen Kontexten durch pragmatic inferencing bzw. semantic reanalysis auf, vgl.:

(1101) Да что это - безумие больного человечества? Кошмарный бред, от которого нужно очнуться и расхохотаться? (RC: B. В. Вересаев. «Да здравствует весь мир!» (о Льве Толстом) (1909-1910))

(1101') 'Was ist es, der Wahnsinn der kranken Menschheit? Schrecklicher Wahn, von dem man aufwachen und loslachen soll?'

(1102) 'Жутко было слушать исповедь этого человека-зверя, с таким спокойствием излагавшего историю своего кошмарного преступления. (RC: А. Ф. Кошко. Очерки уголовного мира царской России. 1 (1926))

(1102') Es war unheimlich, das Bekenntnis dieses Menschentieres zu hören, der die Geschichte seines schrecklichen Verbrechens mit solcher Ruhe darlegte'.

(1103) И все же Самгин думал, глядя на парижскую жизнь: «Нужен дважды гениальный Босх, чтоб превратить вот такую действительность в кошмарный гротеск». (GB, 01.06.2015: https://books.google.de/books?id=Uc4MAQAAIAAJ\&q=\%D0\%BA\%D0\%BE\%D1\%88\%D0\%BC\%D0\%B0\%D1\%80\%D0\%BD\%D1\%8B\%D0\%B9\&dq=\%D0\%BA\%D0\%BE\%D1\%88\%D0\%BC\%D0\%B0\%D1\%80\%D0\%BD\%D1\%8B\%D0\%B9\&hl=de\&sa=X\&ei=Gk9sVafLCIOQsgHR_YFg\&ved=0CCYQ6AEwATiWAQ)

(1103') 'Und dennoch dachte Samgin, während er das Pariser Leben beobachtete: „Man braucht den zweimal genialen Bosch, um diese Wirklichkeit in eine schreckliche Groteske zu verwandeln"'.

Das Wort košmarnyj wird in (1101) - (1103) mit äußerst schlimmen Sachverhalten verbunden. Das in (1101) verwendete Bezugswort бред (bred) wird traditionell als unsinniges Gerede eines Kranken im bewusstlosen Zustand definiert (vgl. Ušakov, s.v. бред 1). In der Kollokation mit kоšmarnyj wird das Wort als ein Traum im metaphorischen Sinne verwendet, von dem die kranke Menschheit „erwachen" soll. Zugleich wird bred mit dem Unsinn der kranken Menschheit verglichen und damit auf die zweite Bedeutung des Substantivs bezogen (vgl. Ušakov, s.v. бред 2). Dadurch wird dem Adjektiv eine intensivierende Funktion zugeschrieben, sodass bred als großer Unsinn bzw. Wahnsinn verstanden wird. In (1102) handelt es sich um ein Verbrechen, welches alptraumhaft für die Zuhörer wirkt. Der Grund für solche Gefühle stellt der Grad von dessen Ausprägung dar. Die Verbindung zwischen der Realität und deren alptraumhafter Groteske wird in (1103) hergestellt.

Die in (1101) - (1103) verwendeten Bezugswörter stellen Extremwerte aufweisende Prädikate dar, sodass deren Verbindung mit košmarnyj zu der Intensivierung der Bedeutung des Adjektivs führt. 
Das Einsetzen der Gradbedeutung erfolgt außerdem durch die Wiederholung desselben Begriffs in der adjektivischen Zusammensetzung, i.e. durch semantic repetition, vgl.:

(1104) Если в центре творчества Ибсена чувствуется кошмарный страх поэта перед лищемерием, так ведь на это есть глубокие социальные, исторические причины. (RС: И. Ф. Анненский. Книга отражений (1906))

(1104') 'Wenn man im Zentrum der Kunst von Ibsen die schreckliche Angst des Dichters vor der Heuchelei spürt, dann gibt es dafür tiefe soziale, historische Gründe'.

(1105) Волны кошмарного ужаса перекатываются через них и оставляют за собою лишь бодрость и смех! (RC: В. В. Вересаев. К жизни (1908))

(1105') 'Die Wellen der schrecklichen Furcht rollen über sie hinweg und hinterlassen nur Munterkeit und Lachen'.

Die Mehrdeutigkeit aufweisenden Kontexte weisen noch mehr Expressivität auf als die metaphorisch gebrauchten Kontexte.

Die nächste Stufe der Entwicklung der Gradbedeutung bezieht sich auf das Auftreten des Lexems in den Kontexten, wo lediglich die intensivierende Funktion hervorgehoben wird. Der Bezug auf die Ausgangsbedeutung - bis auf negative Konnotationen - geht verloren, vgl.:

(1106) В его комнате царил кошмарный беспорядок. Одежда, тетради и учебники валялись на полу. (RC.: Дмитрий Емец. Таня Гроттер и магический контрабас (2002))

(1106') 'In seinem Zimmer herrschte furchtbare Unordnung. Kleidung, Hefte und Lehrbücher lagen auf dem Boden'.

(1107) Боря тоже кошмарно переживает, буквально плачет, слёзы крупные, как... как рюмки. (RC: И. Грекова. Перелом (1987))

(1107') 'Borja macht sich auch furchtbare Sorgen, er weint buchstäblich, die Tränen sind groß wie... wie Gläser'.

Das Lexem kann außerdem Funktionsverbgefüge intensivieren, vgl.:

(1108) Все это сопровождается крайне раздражающим миганием экрана, символизирующим вспышки сознания героя. [...] Мало того, что мигание кошмарно действует на нервы, этот прием еще и выглядит как [...] из другой киноэпохи.

(1108') 'Das alles wird durch das äußerst ärgerliche Blinken des Bildschirms begleitet, welches Geistesblitze der Person symbolisieren soll. [...] Nicht genug, daß das Blinken furchtbar auf die Nerven geht, diese Technik sieht dazu aus, als wäre sie aus einer anderen Kinoepoche.'

(1108") 'nervt sehr'

Das Lexem wird außerdem in quantitativer Funktion verwendet, vgl.: 
(1109) Они не могли вляпаться в какую-нибудь историю? В какую? Ну, например, продуть в казино кошмарную сумму денег? (RC.: Эдуард Володарский. Дневник самоубийцы (1997))

(1109') 'Konnten sie sich in eine unangenehme Geschichte einlassen? Welche? Nun, zum Beispiel in einem Kasino eine furchtbare Summe Geld verspielen?'

(1110) 'Трепалась она по полчаса с каждым, ия в панике представлял себе, какой кошмарный счет придет нам с Шурой в конще месяца за эти переговоры! (RC: Владимир Кунин. Кыся (1998-2000))

(1110') Sie quatschte mit jedem eine halbe Stunde, und ich stellte mir panikartig vor, was für eine furchtbare Rechnung wir mit Šura am Ende des Monats für diese Telefonate bekommen werden!'

Neben der intensivierenden und quantifizierenden Funktion hat sich eine pejorative Bedeutung entwickelt, vgl.:

(1111) Два святых человека - Любовь Сергеевна и педагог Галина Петровна. Они занимались со мной ночами, а утром мы шли в «Прагу», ждали до девяти утра, до открытия, и ели сосиски с капустой - кошмарный вкус, но это было грандиозно. (RC: Ольга Новикова. Мужской роман (1999))

(1111') 'Zwei heilige Menschen - Ljubov' Sergejevna und die Lehrerin Galina Petrovna. Sie unterrichteten mich nachts, und morgens gingen wir ins „Praga“, warteten bis neun Uhr, bis zur Eröffnung, und aßen Würstchen mit Kohl - furchtbarer Geschmack, aber es war großartig'.

(1112) Стив говорил по-русски кошмарно, путался в падежах и спряжениях и при этом вворачивал время от времени пословицы и поговорки, причем к месту. (RC: Марианна Баконина. Девять граммов пластита (2000))

(1112') 'Steve sprach furchtbares Russisch, verwechselte Kasus und Konjugation und flocht dabei ab und zu Sprichwörter ein, die aber passten'.

Die Belege (1006) - (1008), (1009) - (1010), (1111) - (1112) demonstrieren jeweils die intensivierende, quantifizierende und pejorative Bedeutung des Lexems. Zugleich heben sie den Zuwachs an subjectivity hervor, indem košmarnyj mehr einstellungsbezogen wirkt und auf der expressiven Ebene im Rahmen von Traugott III (oben 3.6.3.4) fungiert.

Die Desemantisierung und damit der endgültige Verlust des Zusammenhangs mit der Ausgangsbedeutung erfolgt durch die Verbindung des Lexems mit positiv konnotierten Bezugswörtern. Im russischen Korpus finden sich keine Belege, die diesen letzten Entwicklungsschritt des Lexems veranschaulichen können. Die Suche im Internet hat jedoch die folgenden Verbindungen ergeben, vgl.:

(1113) Дорогая, я ужасно рад, я кошмарно рад, что ты моя жена. (30.07.2014: http://l-a-m-p-a.livejournal.com/177784.html)

(1113') 'Schatz, ich bin schrecklich froh, furchtbar froh, dass du meine Ehefrau bist'. 
(1114) К черту это все, я сегодня просто жутко, кошмарно счастлив, даже не предполагал, что способен еще на такие чувства...(01.06.2015: http://fictionbook.in/ekaterina-vilmont-mimoletnosti-ili-podumaesh-binom-nyutona.html?page $=39$ )

(1114') 'Zum Teufel das alles, heute bin ich schrecklich, furchtbar glücklich, ich habe sogar nicht vermutet, dass ich zu solchen Gefühlen fähig bin'.

\section{3 Страшный (strašnyj)}

Das Wort страшный (strašnyj) tritt bereits im Altrussischen auf. Die Ausgangsbedeutung bezieht sich auf Furcht hervorrufende bzw. mit Angst erfüllende Sachverhalte (siehe Sreznevskij 1912, s.v. страшьныи), vgl. die folgenden Verwendungen:

(1115) Страшное слово сердце мужа првднна смущаетъ, въсть же блага я веселитъ єго. (RС: Библия. Притчи)

(1115') 'Das furchtbare Wort verwirrt das Herz eines frommen Mannes, eine gute Nachricht erfreut ihn'.

Das Auftreten der Gradbedeutung lässt sich in mehrdeutigen Verwendungen erkennen. Die Suche im Korpus, das in der zu untersuchenden Zeitspanne vorwiegend aus kirchlichen Texten besteht, legt nahe, dass Intensivierung zunächst in den auf schlimme Sünden und Qualen bezogenen Kontexten auftritt und in Sreznevskij (1912, s.v. страшьныи) als groß und schwer bezeichnet wird, vgl.:

(1116) Пришедши же въ сознаніе страшнагш грпха, [...] да плачетъ онъ, стоя у дверей молитвенныхъ домшвъ, и прося входящихъ на молитву, дабы каждый съ состраданіемъ приносилъ $w$ немъ усердныя молитвы ко гдссу. (RC: Книга Правил святых апостолов, святых соборов, вселенских и поместных, и святых отцов. Все правила и послания)

(1116') Nachdem er sich seiner schrecklichen Sünde bewusst geworden ist, weint er an den Türen von Gebetsstätten stehend und bittet die Eintretenden um ein Gebet, damit jeder sich mitfühlend mit einem fleißigen Gebet für ihn zu Gott hinwendet.

(1117) Помышляю судъ и боюся истязанія, трепешу страшныя $и$ ужасныя муки, огня, болпзни [...]. (RC: Минея. 11 ноября: Великомученика Стефана, царя сербского, Дечанского)

(1117') 'Ich denke an das (jüngste) Gericht und fürchte mich vor der Folterung, ich zittere vor den furchtbaren und schrecklichen Qualen, Feuer, Krankheit.'

(1118) Тьмы кромпшнія, и страшнагш мученія, раба твоего избави мя [...]. (RC: Октоих. Глас 5-й, вторник)

(1118') 'Erlöse deinen Gottesknecht von der völligen Finsternis und der furchtbaren Qual.'

Aus den Belegen (1116) - (1118) geht hervor, dass страшный ('strašnyj') mehrdeutig gebraucht wird. Zunächst bezieht sich das Lexem auf die Bedeutung des Schreckens, wobei die Begriffe ,Sünde' und ,Qual' als furchterregend wahrgenommen werden. Zugleich fungiert страшный ('strašnyj') als ein Gradadjektiv, das die Intensität der Sachverhalte als Grund für die hervorgerufene Furcht ausdrückt. 
Intensivierung tritt außerdem in heftigen Wettererscheinungen beschreibenden Kontexten auf, vgl. Unwetter und Sturm in (1119) - (1120), Wind in (1121). Die Intensität der Erscheinungen ist wiederum mit Angstgefühlen verbunden und verkörpert den Grund für ihr Auftreten, vgl.:

(1119) [...] єгда страшная оная и неудержимая буря, и волненіе въ нощи воздвижеся, и вси бывшіи въ корабли купншсъ корабленниками, и съ самою матерію твоею погибоша во глубинп [...] (RC: Добротолюбие. Часть первая. Послание Марка Подвижника к Николаю иноку)

$(1119 ')^{\prime}$ ' [...] wenn sich ein schrecklicher und unwiderstehlicher Sturm und Seegang in der Nacht erhebt und alle sich auf dem Schiff befindenden Seeleute samt deiner Mutter in der Tiefe ums Leben kommen [...]'

(1120) Искусный мореплаватель не токмо в страшное волнение и бурю, но и во время кратчашия тишины бодрствует, укрепляет орудия, готовит парусы, наблюдает звезды, примечает перемены воздуха, смотрит на восстающия тучи, исчисляет расстояние от берегов, мерит глубину моря и от потаенных водою камней блюдется. (RC: М. В. Ломоносов. Слово похвальное Ея величеству Государыне императрице Елисавете Петровне, самодержице Всероссийской, говоренное ноября 26 дня 1749 года (1749))

(1120') 'Ein geschickter Seemann bleibt wach, befestigt Geschütze, bereitet die Segel vor, beobachtet die Sterne, bemerkt Luftveränderungen, schaut die sich zusammenziehenden Wolken an, berechnet die Entfernung bis zu der Küste und zu den sich unter dem Wasser befindenden Steinen nicht nur während eines schrecklichen Seegangs und Sturms, sondern auch während der kürzesten Stille.'

(1121) [...] время было ужасное, возбуждающее отчаяние: западный ветер завывал, ударяя со страшным стремлением в стены дома, дождь сильный, подобный стремлению морских волн, разливался, беспрестанная молния мелькала, громовые удары один другого преследовали со страшным треском: казалось, настал час всеобщего разрушения, казалось, вся природа [...] желает исчезнуть вместе с несчастным М-м. (RC: А. И. Клушин. Несчастный М-в (1793))

(1121') '[...] die Zeit war schrecklich, Verzweiflung hervorrufend: der Westwind heulte, mit furchtbarem Drang auf die Hauswände schlagend, der starke Regen ähnelte dem Drang von Meereswellen, unaufhörlicher Blitz schimmerte, Donnerschläge folgten mit einem furchtbaren Krach: die Stunde der totalen Zerstörung schien gekommen zu sein, die ganze Natur schien zusammen mit unglücklichem M-m verschwinden zu wollen.'

Aus (1121) geht außerdem hervor, dass страшный ('strašnyj') die mehrdeutige Verwendung auch in Verbindung mit Geräuschen aufweist. In der Zusammensetzung страшный треск громовых ударов ('furchtbarer Knall von Donnerschlägen') bezieht sich das Adjektiv auf die Bedeutung des Schreckens sowie die Intensität 
der Geräusche, wobei Angstgefühle als Folge der hohen Gradausprägung angesehen werden können. Weitere Kollokationen werden in den adjektivischen (1122) - (1124) und adverbialen Verwendungen demonstriert, vgl.:

(1122) [...] громы свой страшный треск вдруг остановляют, облака на самый край горизонта утекают, вихри в пещеры свои низвергаются, и волны, до небес восходящие и ревом своим небесные круги устрашающие, опадают и текут наподобие тихого источника. (RC: Д. И. Фонвизин. Иосиф (1769))

(1122') ' [...] die Donner halten ihren schrecklichen Knall plötzlich an, die Wolken ziehen zum Rand des Horizonts, die Wirbelwinde strömen in ihre Höhlen und die Wellen, die sich bis zum Himmel erheben und mit ihrem Tosen die Himmelskreise erschrecken und fließen wie eine ruhige Quelle.'

(1123) Под частыми ударами два пальмовых древа потряслися, скрипнули и с страшным упали шумом; трепешущая земля от того восстенала; птищы, [...] испустив скорбный глас свой, отлетают. (RС: Д. И. Фонвизин. Иосиф (1769))

(1123') 'Wegen der häufigen Schläge schüttelten sich zwei Palmen, knarrten und fielen mit schrecklichem Lärm; die bebende Erde stöhnte deswegen; Vögel stoßen einen trauervollen Schrei aus und fliegen weg.'

(1124) [...] в сие мгновение страшно зашумел ветер [...] (RC: Н. М. Карамзин. Наталья, боярская дочь (1792))

(1124') '[...] in dem Moment fing der Wind an, schrecklich zu rauschen [...]'

Semantic repetition tritt als weitere Möglichkeit der Herausbildung der Gradbedeutung auf. Страшный ('strašnyj') wird mit synonymischen Begriffen verwendet, was die Wiederholung der Bedeutung des Schreckens und die Intensivierung des Bezugsworts zu Folge hat, vgl.:

(1125) Лекарь наш о сем уведомлен и в страшном испуге прибежал $к$ батюшке. (RC: И. М. Долгоруков. Повесть о рождении моем, происхождении и всей моей жизни, писанная мной самим и начатая в Москве, 1788-го года в августе месяце, на 25-ом году моей жизни / Части 1-2 (1788-1822))

(1125') 'Unser Arzt war darüber informiert und kam in schrecklicher Angst zu dem Vater.'

(1126) Он напоминал собою Макбета более, чем все современные актеры, терзающие Шекспира, и это ему было тем легче, что тут он [...] действительно был объят страшным ужасом [...]. (RC: Н. С. Лесков. Некуда (1864))

(1126') 'Er ähnelte Macbeth mehr als alle gegenwärtigen, Shakespeare quälenden Schauspieler, und es war für ihn um so leichter, weil er hier tatsächlich von der schrecklichen Angst erfaßt war.'

(1127) Например, сижу и вдруг чувствую, что уже давно не дышу! Минут пять точно! Мне так страшно стало, представить себе не можете! (RC: Ярослав Кудлак. Симбиоз / / «Наука и жизнь», 2009) 
(1127') 'Beispielsweise sitze ich und fühle plötzlich, dass ich schon längst nicht mehr atme! Seit fünf Minuten bestimmt! Ich habe mich so furchtbar erschreckt, Sie können es sich gar nicht vorstellen!'

Die mehrdeutigen Verwendungen, die die Bedeutung des Schreckens sowie der Intensivierung demonstrieren, verweisen außerdem auf das Auftreten von subjectivity, indem sie die Einstellung des Sprechers bzw. Schreibers zum Ausdruck bringen und die erste semantisch-pragmatische Tendenz (Traugott I, oben 3.6.3.4) andeuten.

Die nächste Entwicklungsetappe bezieht sich auf die Konventionalisierung der Implikatur und das Auftreten der ausgebildeten Gradbedeutung, ohne einen Bezug auf die Ausgangsbedeutung zu zeigen. Das Lexem findet sich zunächst in negativ konnotierten Kontexten, vgl. die adverbialen (1128) - (1129) und adjektivischen (1130) - (1132) Verwendungen:

(1128) Я страшно расстроен. (RC: А. Н. Островский. Письма (1853))

(1128') 'Ich bin furchtbar verstimmt.'

(1129) А на улице было холодно, ноябрь, он страшно замерз. (RC: Алексей Слаповский. Большая Книга Перемен / / «Волга», 2010)

(1129') 'Es war kalt draußen, November, er fror furchtbar.'

(1130) Страшная зубная боль, у меня всегда сопровождаемая нервным расстройством, в продолжение двух недель совершенно одолела меня. (RC:А. Н. Островский. Письма (1850))

$\left(1130^{\prime}\right)^{\prime}$ Furchtbare Zahnschmerzen, die bei mir immer mit einer Nervenverstimmung ablaufen, machten mich fertig innerhalb von zwei Wochen.'

(1131) Она была страшная плакса. (RC: Л. Н. Толстой. Детство (1852))

(1131') 'Sie war eine furchtbare Heulsuse.'

(1132) Говорят, он был страшный бабник? - спросила журналистка. (RC: Дина Рубина. Окна (2011))

(1132') 'Man sagt, er war ein furchtbarer Weiberheld? - fragte die Journalistin.'

Das Lexem kann außerdem Funktionsverbgefüge intensivieren, vgl.:

(1133) Сегодня к вечеру мошкара опять появилась. [...] Она лазит по всему телу [...], страшно действуя на нервы. (RC: В. Н. Гельфанд. Дневники 1941-1943 гг. (1941-1943))

(1133') 'Heute gegen Abend tauchten wieder Mücken auf. [...] Sie krabbeln auf dem ganzen Körper [...] und gehen furchtbar auf die Nerven.'

(1133") 'nerven sehr'

Das Lexem kann außerdem in quantifizierender Funktion auftreten, vgl.:

(1134) Все эти затруднения преодолелись, но поглотили страшные суммы, требовали много сил и времени. (В.А. Слепцов. Владимирка и Клязьма (1860-1861))

(1134')‘All diese Schwierigkeiten wurden überwunden, verschlangen aber furchtbare Summen, erforderten viele Kräfte und Zeit.' 
Die Belege (1128) - (1133), (1134) demonstrieren jeweils die intensivierende und quantifizierende Bedeutung des Lexems. Zugleich heben sie den Zuwachs an subjectivity hervor, indem strašnyj' mehr einstellungsbezogen wirkt und auf der expressiven Ebene im Rahmen von Traugott III (oben 3.6.3.4) fungiert.

Die anschließende Etappe der Herausbildung der Gradbedeutung ist gekennzeichnet durch das Auftreten des Lexems mit positiv konnotierten Kookkurrenzpartnern. Damit wird der Bezug auf die Ausgangsbedeutung vollkommen beseitigt, vgl.:

(1135) Но всё-таки говорю: рад я, что встретился с Вами, страшно pad! (RC: Максим Горький. Письма (1889-1906))

(1135') 'Aber ich sage trotzdem: ich bin froh, Sie getroffen zu haben, furchtbar froh!'

(1136) Вячик нашел в себе мужество не показать, что он страшно обрадовался. (RC: Алексей Слаповский. Большая Книга Перемен / / «Волга», 2010)

(1136') 'Vjačik fand den Mut, nicht zu zeigen, dass er sich furchtbar gefreut hat.'

(1137) Таким и запомнился он мне, мой отец [...]. Страшный добряк, очень деликатный, он никогда громко не разговаривал, не кричал и не ругался. (RC: Леонид Утесов. «Спасибо, сердце!» (1982))

(1137') 'So habe ich meinen Vater in Erinnerung behalten [...]. Ein furchtbar gutmütiger Mensch, sehr rücksichtsvoll, er hat nie laut geredet, nicht geschrien und nicht geschimpft.'

\section{4 Ужасный (užasnyj)}

Die Ausgangsbedeutung von ужасный (иžasnyj) geht auf užas zurück und bezieht sich auf Furcht hervorrufende Sachverhalte, siehe Sreznevskij (1912, s.v. 8жaсьныи), vgl.:

(1138) и се, sвпрь четвертый страшенъ и ужасенъ и крппокъ излиха [...] (RC: Библия. Даниил)

(1138') 'und dieses vierte Tier ist furchterregend und schrecklich und zu stark'

(1139) Въ часъ ужасный, въ часъ страшный, въ часъ шсужденія [...]. (RC: Октоих. Глас 3-й, вторник)

(1139') 'In schrecklicher Stunde, in furchtbarer Stunde, in der Stunde des Tadels $[\ldots]^{\prime}$

Das Auftreten der Gradbedeutung findet sich in mehrdeutigen Kontexten. Die ersten Belege lassen sich im Korpus des Altrussischen in kirchlichen Texten feststellen, wie es auch bei страшный ('strašnyj') der Fall war. In (1140) bezieht sich das Adjektiv auf Sünden, die als furchterregend sowie sehr schwer bzw. schlimm charakterisiert werden können, vgl.:

(1140) Пріидутъ бо въ помышленіе согрпшеній своихъ ужасни, и шбличитъ ихъ сопротивъ беззаконія ихъ. (RC: Минея, август. 24 августа: Святого священномученика Евтиха. Святителя Петра, митрополита всея России, чудотворца) 
(1140')'Sie kommen in Gedanken über ihre furchtbaren Sünden, und er entlarvt ihre Gesetzlosigkeit'

Die Verwendungen in (1141) - (1442) demonstrieren weitere ambige Verwendungen:

(1141) [...] Евангельская историа описывает ужсасное страдание одного беснуемаго человека, который кроме того что глухотою и немотою был связан, разбивал себя на всяком месте, изо рта точил пену, скрежетал зубами, вдруг окаменевал, много раз бросался в огонь, много раз в воду. (RC: архиепископ Платон (Левшин). Слово в неделю четвертую Великаго Поста (1765))

(1141') 'Die [...] biblisch Geschichte beschreibt die furchtbare Qual eines wahnsinnigen Menschen, der abgesehen von seiner Taub- und Stummheit, sich überall verletzte, Schaum vor dem Mund hatte, mit den Zähnen knirschte, sich plötzlich versteinerte, sich mehrmals ins Feuer und mehrmals ins Wasser warf.'

(1142) Иулиа 23 и 24-го был в Киеве великой и ужасной пожар, едва не весь град сгорел, церквей погорело 30 и людей множество. (RC: В.Н.Татищев. История российская в семи томах. Том второй (1750))

(1142') 'Am 23. und 24. Juli gab es in Kiew einen großen und furchtbaren Brand, fast die ganze Stadt wurde niedergebrannt, 30 Kirchen wurden durch das Feuer zerstört und mehrere Leute starben.'

Der Gebrauch von страшный ('strašnyj') in (1141) - (1442) bezieht sich jeweils auf stark ausgeprägte Sachverhalte, d.h. Qual und Brand, die durch ihren hohen Grad furchterregend wirken.

Die Belege (1143) - (1144) exemplifizieren semantic repetition, wobei ужасный ('užasnyj') in Verbindung mit synonymen Begriffen auftritt und diese verstärkt:

(1143) Выговорив сие, он ушел и оставил меня в ужасном страхе. (RC: М. Д. Чулков. Пригожая повариха, или Похождение развратной женщины (1770))

(1143') 'Nachdem er das gesagt hat, ging er und ließ mich in furchtbarer Angst.'

(1144) Я была в ужасном испуге и крепко обняла батюшку, чтоб заслонить его собою. (RC: Ф. М. Достоевский. Неточка Незванова (1849))

(1144') 'Ich war in furchtbarer Angst und umarmte mein Väterchen, um ihn mit sich zuzudecken.'

Die mehrdeutigen Verwendungen (1141) - (1144) demonstrieren das Auftreten der Intensivierung sowie das von subjectivity. Der Gebrauch wirkt mehr einstellungsbezogen im Rahmen von Traugott I (oben 3.6.3.4).

Die Konventionalisierung der Implikatur geht mit dem Verlust des direkten Zusammenhangs des Lexems mit der Ausgangsbedeutung einher und führt zu dem Auftreten der Gradbedeutung in eindeutigen Kontexten, vgl. die adverbialen (1145) - (1146) und adjektivischen (1147) - (1148) Verwendungen: 
(1145) На дороге сделалась мне ужасная тоска. (Д. И. Фонвизин. Отрывки из дневника четвертого заграничного путешествия (1786-1787))

(1145') 'Unterwegs empfand ich furchtbare Trauer.'

(1146) Температура воздуха и, соответственно, воды редко поднималась выше пятнадцати градусов. Да еще дул ужасный ветер... (RC: А. Муразова. Земля людей. Потомки короля Градлона / / «Вокруг света», 1997])

(1146') 'Die Temperatur der Luft und dementsprechend des Wassers stieg selten höher als fünfzehn Grad. Dabei blies ein furchtbarer Wind.'

(1147) Здесь ужсасно жарко! - сказал он. (RC: В. А. Соллогуб. Большой свет (1840))

(1147') 'Es ist furchtbar warm hier! - sagte er.'

(1148) В ее голосе мне почудилась какая-то странная интонация, будто она сомневается в его рассказах, и мне стало ужсасно обидно. (RC: Михаил Шишкин. Письмовник (2009) / / «Знамя», 2010)

(1148') 'Es kam mir vor, als hörte ich in ihrer Stimme eine seltsame Intonation, als würde sie seine Erzählungen bezweifeln, und ich fand es furchtbar ärgerlich.'

Das Lexem kann außerdem Funktionsverbgefüge intensivieren, vgl.:

(1149) Вот такая обстановка. Уэсасно действует на нервы. (RC: Аркадий Хайт. Монологи, миниатюры, воспоминания (1991-2000))

(1149') 'So ist die Lage. Es geht furchtbar auf die Nerven.'

(1149') 'nervt sehr'

Das Lexem kann in quantifizierender Funktion auftreten, vgl.:

(1150) Что же касается до безопасности в Париже, то я внутренне уверен, что всеведение полициймейстера не весьма действительно и польза от полицейских шпионов отнюдь не соответствует той ужасной сумме, которую полиция на них употребляет. (RC: Д. И. Фонвизин. К П. И. Панину (1777-1778))

(1150') 'Was die Sicherheit in Paris betrifft, da bin ich innerlich überzeugt, dass die totale Informiertheit des Polizeimeisters nicht immer wirksam ist und der Nutzen von polizeilichen Spionen keineswegs der furchtbaren Summe entspricht, die die Polizei für sie ausgibt.'

Neben der intensivierenden Bedeutung entwickelte sich eine pejorative, die lediglich die persönliche Einstellung zum Ausdruck bringt, vgl.:

(1151) Женские общества есть; только от них небольшое утешение: они играют в вист, одеваются дурно и ужасно говорят по-франиузски. (RC: М. Ю. Лермонтов. Герой нашего времени (1839-1841))

(1151') 'Frauengesellschaften gibt es; sie bieten jedoch geringen Trost: sie spielen Whist, kleiden sich schlecht und sprechen furchtbar Französisch.'

(1152) Но несмотря на ужасный наряд, выглядела Лика не так уж плохо, на щеках играл румянец. (RC: Дарья Донцова. Уха из золотой рыбки (2004)) 
(1152') 'Ungeachtet de furchtbaren Kleidung sah Lika gar nicht so schlecht aus, eine Röte färbte ihre Wangen.'

Die Belege (1145) - (1149), (1150) und (1151) - (1152) demonstrieren den Gebrauch von ужасный (иžasnyj) jeweils in intensivierender, quantifizierender und pejorativer Funktion. Die Zunahme an subjectivity in den Verwendungen legt nahe, dass die Verwendung des Lexems auf die expressive Ebene übergeht und dem Ausdruck der Einstellung des Sprechers bzw. Schreibers dient. Dies geschiet im Rahmen von Traugott III (oben 3.6.3.4).

Anschließend findet sich ужасный (иžasnyj) in Verbindung mit positiv konnotierten Bezugswörtern und verliert damit endgültig den Zusammenhang mit der Ausgangsbedeutung, vgl.:

(1153) Алексею я был ужасно рад. Мы помянули университетскую жизнь. (RC: Н. В. Станкевич. Письма К.А. Беру (1835))

(1153') 'Ich war schrecklich froh, Aleksej zu sehen. Wir gedachten des Universitätslebens.

(1154) Все тут дело заключалось в том, что им действительно ужасно нравились в Петербурге модные магазины, торцовая мостовая, прекрасные тротуары и газовое освещение, чего, как известно, нет в Москве [...] (RC: А. Ф. Писемский. Тысяча душ (1858))

(1154') 'Die Sache war die, dass sie tatsächlich Modegeschäfte, Uferpromenaden, schöne Gehwege und die Gasbeleuchtung in Petersburg furchtbar mochten - alles, was, wie bekannt ist, es in Moskau nicht gibt.'

(1155) Он был ужасно веселый, все время шутил, смеялся [...]. (RC: Марина Москвина. Небесные тихоходы: путешествие в Индию (2003))

$(1155$ ') 'Er war furchtbar fröhlich, machte Scherze die ganze Zeit, lachte [...]'

\subsection{Zusammenfassung}

Die dargestellte Analyse der Entwicklung von Gradbedeutung in den russischen Lexemen aus der Wortgruppe des Schreckens demonstriert, dass die Herausbildung der Intensivierung in den untersuchten Wörtern ähnliche Entwicklungsschritte aufweist, wie sie oben anhand des Materials der deutschen Lexeme aus derselben Wortgruppe (oben Kap. 4.1) beschrieben worden sind, vgl.:

1. Die Quelle der Gradbedeutung ist die Bedeutung 'Schrecken hervorrufend'.

2. Die Herausbildung der Gradbedeutung beginnt mit pragmatic enrichment bzw. strengthening der Kontexte und hat daher pragmatic overload und Ambiguität der Verwendungen zu Folge. Die Intensivierungsbedeutung tritt durch pragmatic inferencing sowie semantic repetition im Rahmen der semantischen Reanalyse in Erscheinung. Dabei entsteht die Gradbedeutung in einer früheren Phase der Entwicklung als Nebenbedeutung, wobei die Ausgangsbedeutung noch die primäre Rolle spielt.

3. Die Zunahme an Intensität in dem Bedeutungsgehalt der Lexeme korreliert mit dem Verlust ihrer Ausgangsbedeutung und dem Zuwachs an subjectivity. Zunächst kollokiert die Gradbedeutung mit negativ konnotierten Kookkurrenzpartnern, ohne einen direkten Zusammenhang mit der 
Ausgangsbedeutung zu zeigen. Außerdem können Lexeme Funktionsverbgefüge intensivieren. In dieser Etappe wird der Prozess der Desemantisierung noch nicht vollkommen vollzogen.

4. Die intensivierende Funktion der Lexeme kann in die quantifizierende übergehen und eine große Menge des durch das Bezugswort ausgedrückten Sachverhalts bezeichnen.

5. Neben der Gradbedeutung tritt gelegentlich die pejorative Sinnrichtung in Erscheinung, die den Verlust des direkten Zusammenhangs mit der Ausgangsbedeutung des Lexems sowie den Übergang auf die expressive Ebene und damit die Entwicklung von subjectivity demonstriert.

6. Die reine Gradbedeutung demonstriert die letzte Stufe der Entwicklung und modifiziert positiv konnotierte Kookkurrenzpartner. Der Bezug auf die frühere lexikalische Bedeutung geht damit vollkommen verloren.

7. Der Verlust der Ausgangsbedeutung in dem Bedeutungsgehalt des Lexems korreliert mit der Zunahme an subjectivity. Zunächst setzt die erste semantisch-pragmatische Tendenz im Sinne Traugotts ein, die im weiteren Verlauf in die dritte Tendenz übergeht. 


\section{Relevanz der deutschen Wortfelder für das Russische}

In diesem Kapitel werde ich die Wortgruppen untersuchen, deren Lexeme ihre Gradbedeutung aus einer ursprünglich negativ konnotierten Bedeutung entwickelt haben. Manche Wörter haben ein stärkeres Ausbleichen der Ausgangsbedeutung erfahren als andere und können von daher im gegenwärtigen Russischen in positiv konnotierten Kontexten auftreten.

\subsection{Wortgruppe 'Übel wollend / verursachend'}

Die Gruppe von russischen Grad-Adjektiven und -Adverbien mit der Ausgangsbedeutung 'Übel wollend / verursachend' enthält die Lexeme грубый (grubyj 'grob'), жестокий (žestokij 'grausam'), лютый (ljutyj 'grausam'), немилосердный (nетіloserdnyj 'unbarmherzig'), нещадный (пеšс̌adnyj 'erbarmungslos') und свирепый (svirepyj 'wütend'). Aus der Analyse wurden aus verschiedenen Gründen die Leхете беспощадный (bespoščadnyj 'unerbittlich'), злой (zloj 'böse'), сердитый (serdityj 'böse'), яростный (jarostnyj 'wütend') und ярый (jaryj 'böse') ausgeschlossen. Беспощадный (bespoš̌̌ plett ausgebildet zu haben, denn das Lexem zeigt noch einen Zusammenhang mit der Ausgangsbedeutung und wird oft metaphorisch und in doppeldeutigen Kontexten gebraucht. Ярый (jaryj) wirkt dagegen voll entwickelt, tritt jedoch in der Gegenwartssprache lediglich in wenigen Phraseologismen mit dem Sinn „sich ganz einer Tätigkeit widmen“ auf, z.B. ярый сторонник ('einfriger Anhänger') so Vinogradov (1977: 171). Sporadische Verwendungen finden sich auch bei злой (zloj) und сердитый (serdityj), die entweder die Intensität von Wettererscheinungen wie Frost und Wind oder Geschmackseigenschaften von Senf, Tabak u.a. charakterisieren. Demzufolge werden diese Lexeme in dem folgenden Kapitel nicht berücksichtigt.

Der Bedeutungswandel von der Ausgangsbedeutung zu einer intensivierenden Bedeutung kann durch die folgenden Belege exemplifiziert werden:

6.1а грубо ответить (grubo otvetit' 'grob antworten') - грубо ошибиться (grubo ošibit'sja 'sich stark irren')

6.1b жестокие слова (žestokie slova 'grausame Worte') - жестокий сладкоежка (žestokij sladkoežka 'großes Leckermaul')

6.1с лютый зверь (ljutyj zver' 'grausames Tier') - люто пить (ljuto pit' 'heftig saufen')

6.1d немилосердно поступать (nemiloserdno postupat' 'unbarmherzig handeln') - немилосердно помятый пиджак (nemiloserdno pomjatyj pidžak 'stark zerknitteres Sakko')

6.1e нещадное наказание (nеščadnoe nakazanie 'schonungslose Strafe') нещадно мерзнуть (пеšс̌аdno mjorznut' 'stark frieren')

6.1f свирепый нрав (svirepyj nrav 'grausamer Charakter') - свирепый ветер (svirepyj veter 'starker Wind')

6.1g яростный взгляд (jarostnyj vzgljad 'wütender Blick') - яростный энтузиазм (jarostnyj entuziazm 'großer Enthusiasmus') 


\subsection{1 Грубый (grubyj)}

Die intensivierende Funktion von грубый kann in den folgenden Wortverbindungen auftreten: грубая ошибка (grubaja оšibka 'großer Fehler'), грубая ложь (grubaja lož' 'große Lüge'), грубая тоска (grubaja toska 'große Sehnsucht'), грубая бедность (grubaja bednost' 'große Armut'), грубые противоречия (grubyje protivorečija 'große Einwände') u.a. Der Gebrauch wird durch die folgenden Belege demonstriert:

(1156) Это будет чересчур грубое нарушение закона. (RC: Григорий Пунанов: «Известия», 2001.11.29)

(1156') 'Das wird eine viel zu große Verletzung des Gesetzes.'

(1157) Конечно, Трубников в аэропорту мог грубо ошибиться, приняв за русскоговорящую гидессу Наташу всего лишь похожую на нее туристку, но теперь уже Семин настаивал на осторожности. (RC: Геннадий Прашкевич, Александр Богдан. Человек «Ч» (2001))

(1157') 'Trubnikov hätte sich natürlich im Flughafen stark irren können, indem er eine Touristin für die russischsprechende Führerin Nataša hielt, aber jetzt bestand Sjomin auf Vorsicht.'

Die Korpusanalyse hat keine Verwendungen feststellen können, die den Gebrauch des Lexems in Verbindung mit positiv konnotierten Kookkurrenzpartnern demonstrieren.

\subsection{2 Жестокий (žestokij)}

Die Gradbedeutung von žestokij findet sich beispielsweise in den folgenden Verbindungen: жестокая депрессия (žestokaja depressija 'große Depression'), жестокая боль (žestokaja bol' 'großer Schmerz'), жестокий сnор (žestokij spor 'großer Streit'), жестокая простуда (žestokaja prostuda 'starke Erkältung'), жестокое заблуждение (žestokoe zabluždenie 'großer Irrtum'), жестокий голод (žestokij golod 'großer Hunger'). Die Verwendung des Lexems lässt sich durch die folgenden Belege veranschaulichen:

(1158) Такого жестокого гриппа у меня еще никогда не было. (RC: Лидия Вертинская. Синяя птица любви (2004))

(1158') 'So eine starke Grippe hatte ich noch nie.'

(1159) Фаддей Титович решил, что, присоединяясь ко мне, он прославит свое имя в веках. Он жестоко ошибался, тем не менее, предложил даже издавать некоторые мои труды за его счет, но я отказался от этой сделки [...]. (RC: А. Л. Чижевский. Вся жизнь (1959-1961))

(1159') 'Faddej Titovič hat sich entschlossen, dass er meinen Namen in den Jahrzehnten berühmt machen wird, indem er sich mir anschließt. Er hat sich stark geirrt, hat mir jedoch sogar angeboten, meine Werke auf seine Rechnung zu verkaufen, ich habe diesen Deal aber abgelehnt.'

Im Korpus finden sich keine Belege, die den Gebrauch von žestokij mit positiv konnotierten Bezugswörtern demonstrieren. Die Suche im Internet hat jedoch eine Tendenz erkennen lassen: 
(1160) Я по своей сущности жестокий сладкоежка. (22.05.2015: http://ask.fm/KatherineBilan/answer/33332112252)

(1160') 'Ich bin von Natur aus ein großes Leckermaul.'

Die Verwendung in (1158) demonstriert den eindeutigen Verlust der Verbindung mit der Ausgangsbedeutung des menschlichen Verhaltens. Das Adjektiv žestokij bezieht sich lediglich auf das Ausprägungsgrad der menschlichen Eigenschaft, vgl. dagegen die Verwendung in (1159), wo žestokij in der Ausgangsbedeutung gebraucht wird:

(1161) зайчик катился с горки на скейтборде, при этом съедая мороженное. На его пути встретился жестокий сладкоежка, который захотел отобрать это лакомство... (22.05.2015: http://upmult.$\mathrm{ru} / \mathrm{cruel} /$ multwyx.html)

(1159') 'Das Häschen rollte mit dem Skateboard den Berg herunter und aß ein Eis dabei. Unterwegs begegnete ihm ein grausames Leckermaul, das dieses Naschwerk wegnehmen wollte.'

\subsection{3 Лютый (ljutyj)}

Die intensivierende Funktion von лютый kann durch die folgenden Wortverbindungen exemplifiziert werden: лютый мороз (ljutyj moroz 'starker Frost'), лютое мучение (ljutoe тис̌еnie 'starke Qual'), лютый ветер (ljutyj veter 'starker Wind'), лютая боль (ljutaja bol' 'starke Schmerzen'), лютый голод (ljutyj golod 'starker Hunger'), люто тосковать (ljuto toskovat' 'sehr traurig sein'). Die Belege (1162) - (1164) sollen den Gebrauch des Lexems in negativ konnotierten Kontexten veranschaulichen, vgl.:

(1162) Люто пили ремесленники, мастеровые: выражение «пьян, как сапожник» родилось не случайно. (RC: И. С. Шкловский. Новеллы и популярные статьи (1982)])

(1162') 'Die Handwerker tranken sehr viel: die Redewendung „betrunken wie ein Schuhmacher" ist nicht einfach so entstanden.'

(1163) Стоял лютый мороз, они совсем окоченели и едва удерживались, когда неожиданно пришла помощь. (RC: Константин Ваншенкин. Писательский клуб (1998))

(1163') 'Es war sehr starker Frost, sie waren erstarrt und konnten sich kaum halten, als unerwartet Hilfe kam.'

(1164) Но дело люто ответственное: журналистов будет вагон и тележка. (RC: Сергей Шаргунов. Чародей (2008))

(1164') 'Die Angelegenheit ist sehr verantwortungsvoll: es wird eine Menge Journalisten geben.'

Die Verwendung des Lexems in positiv konnotierten Kontexten folgt in (1165):

(1165) Лютая радость распирала грудь Хопрова, он кидал слова, словно со стороны, как чужую речь, слышал свой голос. (RC: М. A. Шолохов. Поднятая целина. Книга 1 (1958))

(1165')‘Choprov konnte sich vor großer Freude nicht halten, er redete hastig und undeutlich und hörte seine Stimme wie eine fremde Rede.' 


\subsection{4 Немилосердный (nemiloserdnyj)}

Немилосердный bezieht sich ursprünglich auf grausame Personen. Die Verwendung des Lexems mit intensivierender Bedeutung kommt beispielsweise in den folgenden Verbindungen vor: немилосердная скука (nemiloserdnaja skuka 'starke Langweile'), немилосердная тряска (nemiloserdnaja trjaska 'starkes Schütteln').

Die Belege (1166) - (1167) sollen den Gebrauch des Lexems in negativ konnotierten Wortverbindungen demonstrieren:

(1166) Голова ныла давно, теперь еще и ноги не слушались, и немилосердно клонило в сон. (RC: Дарья Симонова. Без Россини (2002))

(1166') 'Der Kopf schmerzte seit langem, jetzt wollten die Beine nicht mehr, und ich wurde sehr stark von Müdigkeit befallen.'

(1167) Пьяные, усталые, в дорогих, но немилосердно измятых пиджаках, сжимая в зубах сигареты, потирая пальцами лбы и щеки, мы подошли к столу и задумались. (RC: Андрей Рубанов. Сажайте, и вырастет (2005))

(1167') 'Betrunken, müde, gekleidet in teuere aber stark zerknitterte Jacken, Zigaretten in dem Mund zusammenpressend, kamen wir an den Tisch heran und versanken in Gedanken.'

In (1168) tritt das Lexem mit positiven Konnotationen auf:

(1168) Накануне отъезда в Курск ночевал со мной на одной койке Евг. Долматовский. По обыкновению он немилосердно хохмил, а затем рассказал, что написал большую поэму о плене на основании личных наблюдений. (RC: Л. К. Бронтман. Дневники и письма (1943-1946))

(1168') 'Vor der Abreise nach Kursk übernachtete mit mir auf einem Bett Jevg. Dolmatovskij. Gewöhnlich scherzte er sehr viel, dann erzählte er, dass er ein großes Poem über die Gefangenschaft aufgrund seiner persönlichen Beobachtungen gedichtet hat.'

Die Korpusanalyse hat keine Kollokationen feststellen können, die das Auftreten des Lexems mit positiv konnotierten Bezugswörtern demonstrieren.

\subsection{5 Нещадный (пеščadnyj)}

Die intensivierende Funktion von нещадный findet sich in Verbindungen wie нещадно потеть (nеščadno potet' 'stark schwitzen'), нещадно курить (nеščadno kurit' 'sehr viel rauchen'), нещадно фальшивить (nеščadno fal'šivit' 'sehr unrein singen') usw., vgl. auch die folgenden Belege:

(1169) Наступила холодная осень, мы нещадно мерзли. (RC: Александр Яковлев. Омут памяти. Т.1 (2001))

(1169') 'Es wurde kalter Herbst, wir froren sehr stark.'

(1170) Не слышны в саду даже шорохи!. - нещадно фальшивя, забасил вдруг Астахов. (RC: Семен Данилюк. Рублевая зона (2004))

(1170') 'Man hört im Garten nicht mal Geräusche! - Astachov hat angefangen, im $B a ß$ und sehr unrein zu singen.' 
(1171) Появился проводник. Его нещадно шатало и носило. (RC: Михаил Гиголашвили. Чертово колесо (2007))

(1171') 'Der Schaffner kam. Es schaukelte ihn sehr stark.'

Die Korpusanalyse hat keine Verwendungen des Lexems mit positiv konnotierten Bezugswörtern feststellen können. Die Suche im Internet hat jedoch welche ergeben, vgl.:

(1172) И все же есть одно событие, которое меня нещадно радует. Это наш потрясающий, феерический переезд. (04.06.2015: http://darkdiary.ru / users/anna_baker/3208534/comment/)

(1172') 'Es gibt jedoch ein Ereignis, welches mich sehr freut. Das ist unser großartiger Umzug.'

(1173) [...] заходил мужик и спрашивал, сколько лет детке, и услышав ответ из уст еле говорящего ребенка, нещадно веселился [...] (04.06.2015: http://dnevniki.ykt.ru/Really_Apelsinka/702754)

(1173') ' [...] kam ein Mann und fragte, wie alt das Baby war und freute sich sehr über die Antwort aus dem Mund des kaum sprechenden Kinds.'

\subsection{6 Свирепый (svirepyj)}

Свирепый fungiert als Intensivierer in Wortverbindungen wie свирепый мороз (svirepyj moroz 'starker Frost'), свирепый голод (svirepyj golod 'starker Hunger'), свирепая нищета (svirepaja niščeta 'große Armut'), свирепая скорость (svirepaja skorost' 'große Geschwindigkeit'), vgl. hierzu die Belege:

(1174) Да и о двадцати килограммах столового маргарина он думал теперь со свирепым урчанием в желудке. (RC: Владимир Орлов. Альтист Данилов (1980))

(1174') 'Über zwanzig Kilo Margarine dachte er jetzt mit starkem Magenknurren.'

(1175) [...] Пекалов нанял и очень дорожил этим малорослым беловолосым мужичком, которого отовсюду гнали за свирепое пьянcmво. (RC: Владимир Маканин. Утрата (1984)])

(1175') ' [...] Pekalov stellte [ihn] ein und schätzte diesen untersetzten weißhaarigen Kerl, der von überall wegen seiner starken Trunksucht vertrieben wurde.'

(1176) Меня засыпало снежной крупой и невыносимо хлестало свирепым зимним ветром. (RC: Эдуард Лимонов. Книга воды (2002))

(1176') 'Es hat mich mit Schnee überschüttet und unerträglich mit dem starken winterlichen Wind gepeitscht.'

Die Korpusanalyse hat keine Belege feststellen können, die den Gebrauch des Lexems in positiv konnotierten Kontexten demonstrieren.

\subsection{7 Яростный (jarostnyj)}

Das Auftreten von яростный als Intensivierer erfolgt in Verbindungen wie яростный ливень (jarostnyj liven' 'starker Regenschauer'), яростный ветер (jarostnyj veter 'starker Wind'), яростный энтузиазм (jarostnyj entuziazm 'großer 
Enthusiasmus'), яростное сопротивление (jarostnoe soprotivlenie 'starker Widerstand'), яростно бороться (jarostno borot'sja 'stark kämpfen'), яростно ненавидеть (jarostno nenavidet' 'sehr hassen'), яростно любить (jarostno ljubit' 'stark lieben'), vgl. auch die Belege:

(1177) Татарский акцент, с которым Зина яростно боролась, проступил с особой силой в первых же сказанных ею словах [...] (RC: Е. C. Гинзбург. Крутой маршрут: Часть 1 (1967))

(1177') 'Tatarischer Akzent, mit dem Sina stark kämpfte, kam zum Vorschein besonders stark in den ersten von ihr gesprochenen Worten.'

(1178) Тут же в магазин кинулись с улицы моментально промокшие под внезапным и яростным ливнем люди. (RC: Алексей Грачев. Ярый-3. Ордер на смерть (2000))

(1178') 'Sofort stürzten sich von der Straße momentan im plötzlichen und starken Regenschauer naß gewordene Leute.'

Die folgenden Belege sollen den Gebrauch des Lexems in Verbindung mit positiv konnotierten Kookkurrenzpartnern demonstrieren:

(1179) Яростный гром аплодисментов под управлением жюри. (RC: Дина Рубина. На солнечной стороне улицы (1980-2006))

$(1179$ ') 'Großer Beifallssturm unter der Leitung von der Jury.'

(1180) Ведь он так яростно любил жизнь. (RC: Семейные ценности (2002) / / «Культура», 2002.04.08])

(1180') 'Er liebte das Leben doch so stark.'

(1181) матушка Серафима разбила огород на месте цветника, учила ухаживать за ним, и Руфина Эрастовна с яростным энтузиазмом сражалась за урожай. (RC: Борис Васильев. Дом, который построил Дед (1990-2000))

(1181') 'Mutter Serafima legte einen Gemüsegarten auf dem Platz des Blumengartens an, brachte Rufina Erastovna die Pflege [des Gemüsegartens] bei, und Rufina Erastovna kämpfte mit großem Enthusiasmus für die Ernte.'

\subsection{Wortgruppe 'Sich wie ein Tier verhaltend'}

Die Gruppe von russischen Grad-Adjektiven und -Adverbien mit der Ausgangsbedeutung 'Sich wie ein Tier verhaltend' enthält die Lexeme звериный zverinyj ('tierisch') und зверский zverskij ('tierisch'). Die intensivierende Funktion der Wörter kann durch die folgenden Verwendungen exemplifiziert werden:

6.2а звериная шкура (zverinaja škura 'tierisches Fell') - звериная тоска (zverinaja toska 'starke Sehnsucht')

6.2с зверское мясо (zverskoe mjaso 'tierisches Fleisch') - зверски голоден (zverski goloden 'sehr hungrig')

Die Analyse der deutschen äquivalenten Adjektive bestialisch, tierisch und viehisch in Kapitelabschnitt 4.3 legte nahe, dass die metaphorisch gewonnene Bedeutung 
des rohen, brutalen menschlichen Verhaltens als Basis für die weitere Herausbildung der Gradbedeutung diente. In Bezug auf russische Adjektive mit „tierischer" Bedeutung fällt ebenso auf, dass eine ähnliche Entwicklung bei звериный zverinyj ('tierisch') und зверский zverskij ('tierisch') festzustellen ist.

Es gibt jedoch auch andere Intensivierer mit „tierischer" Ausgangsbedeutung, z.В. бычий (byčij 'bullig'), собачий (sobačij 'Hunde-') und волчий (volčij 'Wolfs-'). Bei diesen Lexemen basiert die Herausbildung der Gradbedeutung ebenso auf einer Metapher, jedoch ohne einen Zusammenhang mit dem übel wollenden Verhalten.

\subsection{1 Звериный (zverinyj)}

Die ursprüngliche Bedeutung des Lexems bezieht sich auf Tiere und wurde metaphorisch auf rohes, grausames menschliches Verhalten übertragen, z.B. звериные обычаи (zverinye obyčai 'tierische bzw. grausame Bräuche'), звериные законы (zverinye zakony 'tierische bzw. grausame Gesetze') (vgl. MAS, s.v. звериный).

Die intensivierende Bedeutung des Lexems lässt sich in Verbindungen wie звериная тоска (zverinaja toska 'große Sehnsucht'), звериная серьезность (zverinaja serjoznost' 'großer Ernst') finden, vgl. die Belege:

(1182) Звериная тоска охватила Сергея Сергеевича. (RC: Николай Дежнев. В концертном исполнении (1993))

(1182') 'Große Trauer erfasste Sergej Sergeevič.'

(1183) Честно говоря, не понимаю, почему товарищ Шурьев подходит $\kappa$ этой игре с такой звериной серьезностью? (RC: Василий Аксенов. Таинственная страсть (2007))

(1183') 'Ich kann es ehrlich gesagt nicht verstehen, wieso Genosse Šur'jev an dieses Spiel so tierisch ernst herangeht.'

Das Lexem findet sich außerdem in positiv konnotierten Kontexten, vgl.:

(1184) Лицо его выражало звериную радость. (RC: П. Н. Краснов. От Двуглавого Орла к красному знамени (книга 2) (1922))

(1184') 'Sein Gesicht drückte große Freude aus.'

\subsection{2 Зверский (zverskij)}

Die Ausgangsbedeutung bezieht sich auf Tiere, wie es in 6.2.1 bei звериный (zverinyj) demonstriert wurde, z.B. зверское обличье (zverskoe oblič'e 'tierische Gestalt'). Sie wurde metaphorisch auf grausame Personen und deren Verhalten übertragen, vgl. зверский поступок (zverskij postupok 'tierische bzw. grausame Tat'), зверское убийство (zverskoe ubijstvo 'tierischer bzw. grausamer Mord').

Der Gebrauch des Lexems in der Gradbedeutung findet sich in negativ sowie positiv konnotierten Wortverbindungen wiе зверский холод (zverskij cholod 'große Kälte'), зверский голод (zverskij golod 'großer Hunger'), зверская скука (zverskaja skuka 'große Langweile'), зверская аллергия (zverskaja allergija 'starke Allergie'), зверски пьяный (zverski p'janyj 'sehr betrunken'), зверски довольный (zverski dovol'nyj 'sehr zufrieden'), зверски вкусный (zverski vkusnyj 'sehr lecker'), vgl. auch die folgenden negativ (1185) - (1186) und positiv (1187) konnotierten Verbindungen: 
(1185) Тут я понял, что она, как всегда, зверски пьяна. (RC: Эдвард Радзинский. Наш Декамерон (1980-1990))

(1185') 'Da habe ich verstanden, dass sie wie immer sehr betrunken war.'

(1186) На дворе стоял зверский холод. (RC: Бернар Виоле. Тайны Делона (2003) / / «Искусство кино», 2003.06.30)

(1186') 'Es war sehr kalt draußen.'

(1187) Я сосредоточенно приканчиваю свои колбаски, очень жирные и зверски вкусные. (RC: Ольга Сульчинская. От мира до кругозора / / «Октябрь», 2003)

(1187') 'Ich esse konzentriert meine Würstchen auf, sehr fettig und sehr lecker.'

\subsection{Wortgruppe 'Geistig gestört'}

Die Wortgruppe 'geistig gestört' enthält Lexeme, welche sich in ihrer Ausgangsbedeutung auf geistig kranke Personen, d.h. безумный (bеzитпуj) und сумасшедший (sumasšedšij) sowie tollwütige Tiere und mit Tollwut befallene Personen wie бешеный (bеšеnyj) beziehen und anschließend eine intensivierende Bedeutung entwickelt haben. Der Übergang kann folgendermaßen demonstriert werden:

6.3а сумасшедший человек (sumasšedšij čelovek 'wahnsinniger Mensch') сумасшедшая радость (sumasšedšaja radost' 'große Freude')

6.3b бешеный человек (bešenyj čelovek 'tollwütiger Mensch') - бешеная скорость (bešenaja skorost' 'große Geschwindigkeit')

6.3с безумный человек (bezumnyj čelovek 'wahnsinniger Mensch') безумное счастье (bezumnoе sčast'e 'großes Glück').

\subsection{1 Безумный (bezumnyj)}

Die Verwendung des Lexems mit intensivierender Bedeutung kann durch Wortverbindungen wie безумный страх (bezumnyj strach 'große Angst'), безумное одиночество (bezumnoе odinočestvo 'große Einsamkeit'), безумная усталость (bezumnaja ustalost' 'große Müdigkeit'), безумно глупый (bezuтnо glupyj 'sehr dumm'), безумно теплый (bezumno tjoplyj 'sehr warm'), безумно приятный (bezumno prijatnyj 'sehr angenehm'), безумно красивый (bezumno krasivyj 'sehr schön') exemplifiziert werden.

Die Belege (1188) - (1189) demonstrieren den Gebrauch des Lexems in negativ konnotierten Kontexten:

(1188) Вдруг я почувствовал безумную усталость. (RC: Сергей Довлатов. Наши (1983))

(1188') 'Plötzlich spürte ich wahnsinnige Müdigkeit.'

(1189) Платье было безумно дорогое - во всяком случае, Илья никогда бы не подарил такое Вале. (RC: Татьяна Тронина. Русалка для интимных встреч (2004))

(1189') 'Das Kleid war wahnsinnig teuer - in jedem Fall hätte Ilja ein solches Valja nie geschenkt.'

In (1190) - (1191) tritt das Lexem in positiv konnotierten Verbindungen auf: 
(1190) В ее комнате из украшений стояли лишь медные, безумной красоты, турецкий кувшин и чайник. (RC: Алексей Щеглов. Фаина Раневская: вся жизнь (2003))

(1190') 'In ihrem Zimmer standen als Dekoration nur, wahnsinnig schön, ein türkischer Krug und Teekessel aus Kupfer.'

(1191) Скажу сразу, что фильм мне безумно понравился. (RC: Любовь и голуби (2007-2011))

(1191') Ich sage sofort, dass der Film mir wahnsinnig gut gefallen hat.'

\subsection{2 Бешеный (bešenyj)}

Der Gebrauch des Lexems in der Gradbedeutung lässt sich in Wortverbindungen wie бешеное сопротивление (bešenoe soprotivlenie 'großer Widerstand'), бешеный рост цен (bešenyj' rost cen 'starke Preissteigerung'), бешеный темп (bešenyj' temp 'großes Tempo'), бешеный восторг (bešenyj' vostorg 'große Begeisterung'), бешеный успех (bešenyj' иspech 'großer Erfolg'), бешено гордый (bešenyj' gordyj 'sehr stolz'), бешено популярный (bešепо populjarnyj 'sehr populär') erkennen.

Die Belege (1192) - (1193) demonstrieren das Auftreten des Lexems in negativ konnotierten Kontexten:

(1192) От страха у девушки бешено заколотилось сердце. (RС: Лев Дворецкий. Шакалы (2000)])

(1192') 'Das Herz des Mädchens fing an vor Angst wahnsinnig zu pochen.'

(1193) В 02.30 ночи [...] «Форд» на бешеной скорости врезался в придорожное дерево. (RC: Девять трупов за семь дней... (2004) / / «Калининградские Новые колеса», 2004.11.11)

(1193') 'Um 2.30 nachts ist ein „Ford“ in wahnsinnig rasendem Tempo gegen einen an der Straße stehenden Baum gerast.'

In (1194) - (1195) wird das Lexem in positiv konnotierten Verwendungen gebraucht:

(1194) Никита Михалков - бешено талантливый человек. (RC: Юрий Башмет. Вокзал мечты (2003))

(1194') 'Nikita Michalkow ist ein wahnsinnig talentierter Mensch.'

(1195) Мы объездили полмира, везде был бешеный успех! (RC: Лидия Вертинская. Синяя птица любви (2004))

(1195') 'Wir sind durch die Hälfte des Globus gereist, überall hatten wir wahnsinnigen Erfolg.'

\subsection{3 Сумасшедший (sumasšedšij)}

Die Verwendung des Lexems in intensivierender Funktion lässt sich in Wortverbindungen wie сумасшедшая ревность (sumasšedšaja revnost' 'große Eifersucht'), сумасшедшая скорость (sumasšedšaja skorost' 'große Geschwindigkeit'), сумасшедшая радость (sumasšedšaja radost' 'große Freude'), сумасшедшеe обаяние (suтаsร̌edšee obajanie 'großer Charme'), сумасшедшая популярность (sumasšedšaja populjarnost' 'große Popularität') erkennen. 
Die Belege (1196) - (1197) veranschaulichen den Gebrauch des Lexems in negativ konnotierten Kontexten:

(1196) Наша встреча много раз откладывалась из-за ее сумасшедшей загруженности. (RC: Светлана Мазурова. Людмила Шувалова: «Я прожила с ним красивую жизнь!» (2003) / / «Восточно-Сибирская правда» (Иркутск), 2003.06.07)

$(1196 ')^{\prime}$ 'Unser Treffen wurde mehrmals wegen ihres wahnsinnigen Beschäftigtseins verschoben.'

(1197) В свое время противники железных дорог доказывали, что сумасшедшая скорость $40 \mathrm{kм} /$ ч неминуемо разрушит человеческий организм. [Николай Антонов. Только их и видели... (2003) / / «Эксперт: Вещь», 2003.12.22]

(1197') 'Zu seiner Zeit bewiesen die Gegner der Bahn, dass die wahnsinnige Geschwindigkeit $40 \mathrm{~km} / \mathrm{h}$ unvermeidlich den menschlichen Körper zerstört.'

Die Belege (1198) - (1199) demonstrieren das Auftreten des Lexems in positiv konnotierten Kontexten:

(1198) А новая хозяйка, узнав, что здесь жил Блок, была в сумасшедшем восторге. (RC: Вячеслав Недошивин: «Чтение стихов, на мой взгляд, -дело интимное» (2002) / / «Известия», 2002.11.03)

(1197')'Die neue Vermieterin war wahnsinnig begeistert, nachdem sie erfahren hat, dass hier Block gelebt hatte.'

(1199) Всякий раз в это жаркое, сумасшедшее красивое парижское лето, проделывая путь до её больницы за рулём автомобиля, я мысленно говорила с Лизой, понимая, что никогда не произнесу этих слов вслух. (RC: Сати Спивакова. Не всё (2002))

(1198')'Jedes Mal in diesem warmen, wahnsinnig schönen Pariser Sommer auf dem Weg von ihrem Krankenhaus bis zum Auto sprach ich in meinen Gedanken mit Lisa, begreifend, dass ich diese Worte nie laut sagen werde.'

\subsection{Wortgruppe 'Religion'}

Die Gruppe 'Religion' verbindet Lexeme, die sich auf die religiösen Begriffe Gott, Teufel und Hölle beziehen: адский (adskij 'höllisch'), адовый (adovyj 'höllisch'), безбожный (bezbožnyj 'gottlos'), дьявольский (d'javol'skij 'teuflisch'), camaнинский (sataninskij 'satanisch'). Die Entwicklung der Gradbedeutung lässt sich durch die folgenden Verwendungen veranschaulichen:

6.4a адская пропасть (adskaja propast' 'höllischer Abgrund') - адски интересный (adski interesnyj 'sehr interessant')

6.4b адовые ворота (adovyje vorota 'höllische Pforte') - адски скучно (adski skučno 'sehr langweilig')

6.4c безбожные мысли (bezbožnyje mysli 'gottlose Gedanken') - безбожная клевета (bezbožnaja kleveta 'große Verleumdnung')

$6.4 \mathrm{~d}$ дьявольское наваждение (d'javol'skoe navaždenie 'teuflischer Zauber') - дьявольский холод (d'javol'skij cholod 'große Kälte') 
6.4е сатанинский вид (sataninskij vid 'satanisches Aussehen') - camaнинская гордость (sataninskaja gordost' 'großer Stolz')

6.5f чертовское обличье (čertovskoe oblič'e 'teuflisches Aussehen') - чертовски хороший фильм (čertovski chorošij fil'm 'ein verdammt guter Film')

\subsection{1 Адский (adskij)}

Das Lexem адский (adskij) wird ursprünglich in Bezug auf die Hölle verwendet, so Sreznevskij (1893, s.v. адьскыu), vgl.:

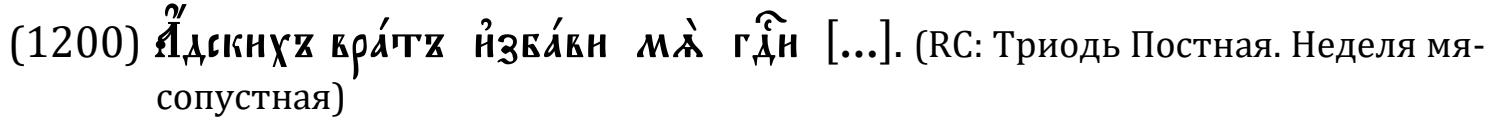

(1200') 'Erlöse mich von der höllischen Pforte.'

Die intensivierende Bedeutung des Lexems findet sich in den negativ konnotierten Verwendungen wiе адская боль (adskaja bol' 'starker Schmerz'), адская клевета (adskaja kleveta 'große Verleumdung'), адская скорость (adskaja skorost' 'große Geschwindigkeit'), адски вонять (adski vonjat' 'stark stinken'), адски сложно (adski složno 'sehr schwer') und positiv konnotierten Verbindungen wie адски интересен (adski interesen 'sehr interessant'), адски счастлив (adski sčastliv 'sehr glücklich'), адски соблазнительный (adski sooblaznitel'nyj 'sehr verführerisch'), адски шикарный (adski šikarnyj 'sehr schick').

Die Belege (1201) - (1202) demonstrieren das Auftreten des Lexems in negativ konnotierten Kontexten:

(1201) Знаю, знаю, что вам было адски скучно в воскресенье. (RC: П.Д. Боборыкин. Жертва вечерняя (1868))

(1201') 'Ich weiß, ich weiß, dass Sie sich sehr gelangweilt haben am Sonntag.'

(1202) Какой адский холод, - сказал, кутаясь в старую шинель, полковник. (RC: П. Н. Краснов. От Двуглавого Орла к красному знамени (книга 2) (1922))

(1202') 'Was für eine starke Kälte, - sagte der Oberst, indem er sich in den alten Mantel einhüllte.'

In (1203) - (1204) wird das Lexem in positiv konnotierten Kontexten gebraucht:

(1203) Дивно красив Париж и адски интересен! (RC: В. А. Каверин. Перед зеркалом (1965-1970))

(1203') 'Paris ist wunderbar schön und sehr interessant.'

(1204) Ибо магазин у него начал сверкать - в ночные эти и утренние часы ухитрялся довести его до адской чистоты и блеска. (RC: Евгений Попов. Пространственный эффект многомерного пространства (1970-2000))

(1204') 'Denn der Laden hat angefangen bei ihm zu blitzen - er schaffte es, ihn in den Nacht- und Morgenstunden sehr sauber und glänzend zu machen.' 


\subsection{2 Адовый (adovyj)}

Адовый (adovyj) wird in Bezug auf die Gradbedeutung als Synonym zu адский (adskij) gebraucht, so MAS (s.v. aдовый). Die Ausgangsbedeutung geht jedoch auch wie bei адский auf ад (ad 'Hölle') zurück (Sreznevskij 1893, s.v. адьскыи), z.B. врата адова (vrata adova 'höllische Pforte').

Die Gradbedeutung findet sich in der Gegenwartssprache in negativ sowie positiv konnotierten Verbindungen wiе адовая жара (adovaja žara 'große Hitze'), адовая скука (adovaja skuka 'große Langweile'), адовый интерес (adovyj interes 'großes Interesse'), адовая простота и изобретательность (adovaja prostota $i$ izobretatel'nost' 'große Einfachheit und Erfindungsgabe'), vgl. den Gebrauch des Lexems in negativ (1205) und positiv (1206) konnotierten Kontexten:

(1205) Если бы не было молодежи, то нам и жить было бы незачем. Скука была бы адовая. (MAS: s.v. адовый)

(1205') 'Wenn es die Jugend nicht gäbe, dann gäbe es ja auch keinen Grund zu leben. Die Langweile wäre dann sehr groß.

(1206) Увидев действие этой огромной крутящейся машины изнутри, Сеня поразился ее адовой простоте и изобретательности, какому-то беспрерывно громыхающему взрыву, раскидывающему полосатые тюки. (RC: Валентин Распутин. Нежданно-негаданно (1997))

(1206') 'Nachdem er die Wirkung dieser riesigen, sich drehenden Maschine von innen gesehen hat, hat Senja ihre große Einfachheit und Erfindungskunst bewundert, sowie die unaufhörlich polternde Explosion, die gestreifte Säcke umherwarf.'

\subsection{3 Безбожный (bezbožnyj)}

Die Ausgangsbedeutung dieses Lexems charakterisiert Gott leugnende Sachverhalte und Personen, z.В. безбожные мысли (bezbožnye mysli 'gottlose Gedanken'). Intensivierend tritt das Wort in Verbindungen wie безбожный цинизм (bezbožnyj cinizm 'großer Zynismus'), безбожно отставать (bezbožno otstavat' 'stark zurückbleiben'), безбожно коверкать (bezbožno koverkat' 'stark radebrechen'), безбожно устареть (bezbožno ustaret' 'stark veraltet sein'), безбожно пить (bezbožno pit' 'stark saufen') auf, vgl. die Belege, in denen das Lexem in negativ konnotierten Wortverbindungen erscheint:

(1207) На бегу, безбожно коверкая мелодию, напевал он из новомодной комической оперы [...] (RC: Борис Евсеев. Евстигней / / «Октябрь», 2010)

(1207') 'Er sang eine Melodie vor sich hin aus der neumodischen komischen Oper und verunstaltete sie sehr.'

(1208) Фильм устарел безбожно. (RC: Давид Карапетян. Владимир Высоцкий. Воспоминания. 2000-2002)

(1208') 'Der Film ist sehr veraltet.'

Die Korpusanalyse hat keine Kollokationen feststellen können, die das Auftreten des Lexems mit positiv konnotierten Bezugswörtern demonstrieren. 


\subsection{4 Дьявольский (d'javol'skij)}

Die Ausgangsbedeutung des Lexems bezieht sich auf den Teufel und charakterisiert teuflische Pläne (дьявольские планы d'javol'skie plany), teuflischen Charakter (дьявольский характер d'javol'skij charakter), teuflisches Vorhaben (дьявольское намерение d'javol'skoe namerenie). Als Intensivierer findet sich das Lexem in negativ konnotierten Wortverbindungen wie дьявольски неудобный ( $d^{\prime} j a$ vol'ski neudobnyj 'sehr unbequem'), дьявольски трудный (d'javol'ski trudnyj 'sehr schwierig'), дьявольски дорогой (d'javol'ski dorogoj 'sehr teuer') und positiv konnotierten Zusammensetzungen wiе дьявольски удачный (d'javol'ski udačnyj 'sehr erfolgreich'), дьявольски красивый (d'javol'ski krasivyj 'sehr schön'), дьявольски талантливый (d'javol'ski talantlivyj 'sehr begabt'), дьявольски вкусный (d'javol'ski vkusnyj 'sehr lecker'), vgl.:

(1209) У Бредихина дьявольски ныл зуб. (RC: В. Я. Шишков. Емельян Пугачев. Книга первая. Ч. 1-2 (1934-1939))

$(1209$ ') 'Bredichin tat sein Zahn sehr weh.'

(1210) Но задача, представлявшаяся мне поначалу элементарной, оказалась дьявольски сложной. (RC: Дуня Смирнова. Рыбий жир (1997) / / «Столица», 1997.10.28)

(1210') 'Aber die Aufgabe, die mir zuerst elementar zu sein schien, erwies sich als sehr schwierig.'

(1211) Кроме всего прочего, Амстер - дьявольски красивый город, словно созданный для пеших или велосипедных прогулок. (RC: Саша Грибоедова. Амстердам Тrip (2004) / / «Хулиган», 2004.07.15)

(1211') 'Unter anderem ist Amster eine sehr schöne Stadt, als wäre sie für Wandern und Radfahren geschaffen.'

\subsection{5 Сатанинский (sataninskij)}

Die Ausgangsbedeutung des Lexems geht auf ,Satan' zurück und bezieht sich auf die mit ihm verbundenen Sachverhalte, z.В. сатанинские замыслы (sataninskie zamysly 'satanisches Vorhaben'), сатанинский хохот (sataninskij chochot 'satanisches Lachen'), сатанинское колдовство (sataninskoe koldovstvo 'satanische Zauberei'). In der Gegenwartssprache tritt das Lexem als Intensivierer in negativ konnotierten Wortverbindungen wie сатанинская гордость (sataninskaja gordost' 'großer Stolz'), сатанинская ревность (sataninskaja revnost' 'starke Eifersucht'), сатанинское упрямство (sataninskoe uprjamstvo 'starker Trotz') auf, vgl.:

(1212) А вы дарите, но не хотите, чтобы вас отдаривали. Поэтому он обвиняет вас в сатанинской гордости ... (RC: В. В. Шульгин. Последний очевидец (1971))

(1212') 'Und Sie schenken, wollen aber keine Geschenke bekommen. Deshalb wirft er Ihnen großen Stolz vor.'

(1213) Когда Шилейко женился на мне, он почти перестал из-за своей сатанинской ревности видеться с Лозинским. (RC: А. А. Ахматова. Лозинский (1966))

(1213') 'Als Šilejko mich geheiratet hat, hat er wegen seiner großen Eifersucht beinahe aufgehört, sich mit Lozinskij zu treffen.' 
Die Korpusanalyse hat keine Belege ergeben, welche das Auftreten des Lexems in positiv konnotierten Kontexten veranschaulichen.

\subsection{6 Чертовский (čertovskij)}

Das Lexem bezieht sich in der Ausgangsbedeutung auf den Teufel und tritt in Wortverbindungen auf wie чертовский шабаш (čertovskij šabaš 'teuflischer Sabbat'), чертовское обличье (čertovskoe oblič'e 'teuflische Äußere') auf. Intensivierend findet sich das Lexem in negativ und positiv konnotierten Wortverbindungen, z.B. чертовски зол (čertovski zol 'sehr böse'), чертовски устать (čertovski ustat' 'sehr müde sein'), чертовски милый (čertovski milyj 'sehr nett'), чертовски интересный (čertovski interesnyj 'sehr interessant').

Die Belege (1214) - (1215) sollen den Gebrauch des Lexems in der intensivierenden Funktion veranschaulichen:

(1214) Все их действия можно объяснить, но чертовски трудно эти действия предсказать. (RC: Аркадий Стругацкий, Борис Стругацкий. Трудно быть богом (1963))

(1214')'Man kann all ihre Handlungen erklären, diese Handlungen sind jedoch sehr schwer vorherzusagen.'

(1215) Пою много и чувствую, что уже чертовски устала. (RC.: Геннадий Алексеев. Зеленые берега (1983-1984))

$(1215 ')$ 'Ich singe viel und spüre, dass ich sehr müde geworden bin.'

In (1216) - (1217) tritt das Lexem in positiv konnotierten Kontexten auf:

(1216) Вы сделали превосходную операцию, и я вам чертовски благодаpeн. (RC: Василий Аксенов. Новый сладостный стиль (2005))

$\left(1216^{\prime}\right)$ 'Sie haben eine ausgezeichnete Operation durchgeführt und ich bin Ihnen sehr dankbar.'

(1217) [...] этот молодой человек имел чертовский успех у женщин [...] (RC: 3.Н. Гиппиус. Без талисмана (1896))

$\left(1217^{\prime}\right)^{\prime}[\ldots]$ dieser junge Mann hatte einen großen Erfolg bei Frauen [...]'.

\subsection{Wortgruppe 'Fluch'}

Die Wortgruppe 'Fluch', die in der deutschen Sprache die Wörter verflucht und verdammt enthält, wird im gegenwärtigen Russischen nicht einschlägig benutzt. Die Wörter проклятый und треклятый, die, ihrer Bedeutung nach, dieser Gruppe entsprechen, haben bis jetzt keine intensivierende Funktion entwickelt und werden in dem Sinn 'in höchstem Maße unangenehm' verwendet. Dagegen sind in dieser Funktion die Lexeme aus der Gruppe 'Religion' gebräuchlich. 


\section{Zusammenfassung}

Die vorliegende Arbeit bietet eine ausführliche Analyse der Natur von 40 deutschen Grad-Adjektiven und -Adverbien. Im Rahmen meiner Studie habe ich die der Intensivierung zugrunde liegenden Mechanismen untersucht, die für dieses Phänomen charakteristischen Entwicklungsschritte dargestellt sowie deren Relevanz für die russische Sprache demonstriert.

Die Materialbasis meiner Untersuchung bilden vorwiegend Lexeme ursprünglich negativen Gehalts, die in der Gegenwartssprache positiv konnotierte Bezugswörter intensivieren können, z.B. arg gut, brutal lehrreich, furchtbar froh, unverschämt lecker. Sie wurden von mir aus verschiedenen Quellen gesammelt und bringen damit die in der Literatur vorhandenen Listen der Intensivierungsmittel auf den neuesten Stand.

Die untersuchten Lexeme wurden in 6 Bedeutungsfelder bzw. Wortgruppen je nach der für das Phänomen relevanten Ausgangsbedeutung eingeteilt: 'Furcht erregend', 'Übel wollend / verursachend', 'Sich wie ein Tier verhaltend', 'Geistig gestört' und 'Religion' und 'Fluch'. Die aus ursprünglich 33 Wörtern bestehende Liste wurde durch 7 weitere Wörter aus den oben genannten Begriffsfeldern ergänzt. Diese Lexeme können als Intensivierer zwar keine positiv konnotierten Kollokationspartner modifizieren, treten jedoch in intensivierender Funktion auf. Dieses Verfahren ermöglichte eine präzisere und umfangreichere Beschreibung des Mechanismus des Phänomens. Es wurde u.a. festgestellt, dass die einem Wortfeld angehörenden Lexeme einen ähnlichen Entwicklungsgang der Gradbedeutung einschlagen, obgleich sie in verschiedenem Grad von Desemantisierung betroffen sind.

Jedes zu untersuchende Lexem ist einer ausführlichen Analyse unterzogen worden, die ich mithilfe von zahlreichen, auf den historischen sowie den gegenwärtigen Sprachzustand bezogenen Korpora und Wörterbüchern durchgeführt habe. Zusammenfassungen, die ich für jedes behandelte Lexem in Kapitel 4 skizziert habe, legen nahe, dass die Herausbildung von allen untersuchten Intensivierern die Wirkung eines ähnlichen Mechanismus voraussetzt. Die Entwicklung der Gradbedeutung ergibt sich als ein gradueller Prozess und lässt verschiedene Stufen unterscheiden:

Auf der Stufe I wird ein Lexem in der Ausgangsbedeutung gebraucht.

Die Stufe II ist mit der Anreicherung des Bedeutungsgehalts des Lexems durch das Auftreten der Gradbedeutung verbunden. Das Lexem tritt in bridging contexts bzw. critical contexts auf, welche neben der primären Bedeutung eine intensitätsbezogene Inferenz ableiten lassen. Darüber hinaus geht die Ausgangsbedeutung zunächst nicht verloren. Im Gegenteil: das Lexem unterliegt dem Prozess von pragmatic enrichment, d.h. die Kontexte weisen die Mehrdeutigkeit des Gebrauchs und damit dessen pragmatic strengthening bzw. strengthening of informativeness auf.

Pragmatic enrichment kann aufgrund von zwei Mechanismen erfolgen: pragmatic inferencing und semantic repetition. Bei pragmatic inferencing handelt es sich um invited inferences, die im Kontext spontan auftreten und damit situationsspezifisch sind. Bei semantic repetition wiederholt das Adjektiv bzw. Adverb die Inhaltszüge des Kookkurrenzpartners, sodass es zu einer Verstärkung des Ausdrucks 
kommt, z.B. furchtbare Angst, wütender Zorn. Infolgedessen wird dem Lexem eine intensivierende Interpretation zugeschrieben. Semantic repetition kann außerdem dadurch erklärt werden, dass Adjektive und Adverbien meistens mit Extremwerte aufweisenden Prädikaten kollokieren (z.B. Tyrannei, Sturm, Gefecht). Solche Bezugswörter demonstrieren einen hohen Grad der Intensivierung und in einer Wortverbindung mit einem ebenso superlativischen Adjektiv bzw. Adverb, zu denen die von mir untersuchten Lexeme gehören, intensiviert wirken.

In dieser Phase kommt außerdem gelegentlich die Metapher ins Spiel. Dadurch wirkt der Gebrauch abstrakter und verliert den direkten Bezug zur Ausgangsbedeutung. Das ist beispielsweise bei böse der Fall: Das Lexem wird in Wortverbindungen mit personifizierten Wettererscheinungen gebraucht, z.B. böser Wind, sodass diese als Übel wollende lebende Wesen und zugleich als in hohem Maße ausgeprägte Sachverhalte dargestellt werden.

In der Stufe III kommt der Prozess der semantischen Reanalyse zur Geltung, sodass die Gradbedeutung in ausgebildeter Form in Erscheinung tritt. Die durch pragmatic enrichment der Kontexte entstandenen Inferenzen rufen neben der Ambiguität der Verwendungen deren pragmatic overload hervor. Dies hat die Umverteilung des semantischen Gehalts zu Folge, sodass die Inferenz als eine neue Bedeutung verwendet wird, während die Ausgangsbedeutung nicht mehr zu erkennen ist. Das Lexem tritt zunächst mit negativ konnotierten Kookkurrenzpartnern auf - damit bleibt der Bezug auf den ursprünglichen negativen Sinn erhalten.

Die Stufe IV verweist auf actualization der neu gewonnenen Bedeutung durch die Erweiterung von Kookkurrenzmöglichkeiten des Lexems. So kann ein Gradadverb neben Adjektiven, Adverbien, Verben und Substantiven auch Funktionsverbgefüge intensivieren, z.B. furchtbar unter Druck stehen vs. unter furchtbarem Druck stehen.

Neben der intensivierenden Funktion treten gelegentlich die quantifizierende und die pejorative Funktion auf. Die quantifizierende Rolle des Adjektivs besteht darin, Sammelbegriffe zu intensivieren und damit die Bedeutung 'groß, viel' zum Ausdruck zu bringen, z.B. fürchterliche Summe 'eine große Summe'. Die pejorative Bedeutung demonstriert die Verschlechterung des semantischen Gehalts und bezeichnet die Sachverhalte als 'sehr schlimm', z.B. furchtbarer Urlaub 'sehr schlimmer Urlaub'.

Auf der anschließenden Stufe tritt das Lexem in Wortverbindungen mit positiv konnotierten Kookkurrenzpartnern auf und demonstriert damit den kompletten Verlust des Bezugs auf die Ausgangsbedeutung. Wenige Lexeme weisen außerdem eine meliorative Bedeutungsentwicklung auf, z.B. tolles Wetter 'sehr gutes Wetter'.

Der dargestellte Entwicklungsgang lässt darauf schließen, dass Intensivierung ein komplexes und mehrstufiges Phänomen darstellt. Metaphorische sowie metonymische Prozesse können in einem früheren Stadium der Herausbildung eines Gradadverbs mitwirken, sind jedoch für die Erklärung der Natur der Erscheinung nicht ausreichend. Der Mechanismus der Intensivierung lässt sich dagegen sehr wohl im Rahmen der Grammatikalisierungstheorie beschreiben. Zu den markantesten Merkmalen des Phänomens gehört die Desemantisierung bzw. das Ausbleichen der ursprünglichen Bedeutung. Ein zukünftiger Intensivierer startet seinen Entwicklungsgang als ein qualitatives Adverb bzw. Adjektiv mit voller lexikalischer 
Ausgangsbedeutung, die im Laufe der Herausbildung der Gradbedeutung allmählich verloren geht. Je nach der Herausbildungsstufe sowie dem Lexem kann bleaching in verschiedenem Maße ausgeprägt sein.

Desemantisierung ist zugleich mit dem Erwerb einer expressiven Bedeutungskomponente bzw. subjectivity verbunden. Es handelt sich um den Übergang der Bedeutung aus der propositionalen auf die expressive Ebene. Je mehr die Ausgangsbedeutung in einem Intensivierer verdrängt wird, desto mehr einstellungsbezogen wirkt der Gebrauch. Das wird mittels semantic-pragmatic tendencies beschrieben. Durch metaphorische Übertragungen sowie das Auftreten der Gradbedeutung im Anfangsstadium der Bedeutungsentwicklung geht die zunächst auf externe Situationen bezogene Bedeutung in den Bereich persönlicher Einstellungen bzw. die evaluative Sphäre über und verweist auf die erste Tendenz. Der Verlust der Ausgangsbedeutung trägt zur Stärkung der wertenden Bedeutung bei und kündigt den Übergang zu der dritten Tendenz an.

Die Entwicklung der Gradbedeutung durch pragmatic inferencing sowie semantic reanalysis stellt eine weitere für Intensivierung und Grammatikalisierung charakteristische Eigenschaft dar.

Aus dem oben Gesagten lässt sich Folgendes schließen:

- $\quad$ Grammatikalisierung bei Intensivierern bezieht sich auf die Entwicklung von Grad-Adjektiven und -Adverbien aus deren qualitativen Äquivalenten;

- $\quad$ Die Lexeme unterliegen in einem früheren Stadium dem Prozess von pragmatic enrichment durch pragmatic inferencing bzw. semantic repetition;

- $\quad$ Die Konventionalisierung der Gradbedeutung erfolgt durch semantic reanalysis von pragmatic overload und zwar in ambigen Kontexten;

- Die Entwicklung von Gradbedeutung geht mit dem Verlust bzw. bleaching der Ausgangsbedeutung einher;

- $\quad$ Desemantisierung ist direkt mit einer Zunahme an subjectivity verbunden. Der Gebrauch erfährt einen Übergang aus der propositionalen auf die expressive Ebene im Rahmen von semantic-pragmatic tendencies;

- Intensivierer lassen sich je nach der Ausgangsbedeutung in Bedeutungsfelder einteilen und zeigen innerhalb eines Felds einen ähnlichen Entwicklungsgang.

Das dargestellte Schema lässt sich, wie oben gezeigt wurde, auch auf das russische Material anwenden. Fünf von sechs im Deutschen analysierten Wortfeldern wurden im gegenwärtigen Russischen festgestellt. Die ausführliche historische Analyse der Lexeme aus der Gruppe 'Furcht' legte nahe, dass die skizzierten Herausbildungsschritte auch für das Russische relevant sind. Ferner lässt sich schließen, dass die deutschen Bedeutungsfelder reichlicher belegt sind als die russischen. Beispielsweise enthält die deutsche Gruppe 'Furcht erregend' 14 Lexeme, die russische dagegen nur 4. Dies ist jedoch in Bezug auf die deutsche Gruppe 'Religion' nicht der Fall. Denn sie ist mit 2 Lexemen spärlicher repräsentiert als die 5 Lexeme enthaltende russische Gruppe. Die Gruppe 'Fluch', die im Deutschen als eine weitere Quelle von Intensivierern gebraucht wird, findet sich im Russischen nicht. Die 
zu diesem Feld gehörenden Lexeme dienen ausschließlich dem Ausdruck von höchst unangenehmen Sachverhalten. Es wäre in diesem Zusammenhang von besonderem Interesse, weitere deutsche und russische Felder zu analysieren, um historische sowie kulturell bedingte Gründe für die festgestellten Unterschiede erklären zu können.

\subsection{Resümee - Summary - Резюме}

Den Gegenstand der vorliegenden Arbeit bilden Grad-Adjektive und Grad-Adverbien, die eine hohe Ausprägung eines durch das Bezugswort ausgedrückten Begriffs bezeichnen. Im Fokus der Untersuchung steht der Entwicklungsgang von Intensivierern, die ursprünglich eine negative Konnotation haben und anschließend als bloße Verstärkungen auftreten, z.B. furchtbar nett, arg gut, tierisch froh, wahnsinnig interessant, verdammt lecker. Im Rahmen der Arbeit werden die Mechanismen des damit verbundenen Sprachwandelphänomens untersucht und die für diesen Prozess einschlägigen Stufen ermittelt. Dabei werden durchweg zahlreiche Belege zur exemplarischen Dokumentation herangezogen. Außerdem wird die Relevanz der mit den deutschen Daten gewonnenen Verallgemeinerungen anhand ausgewählter Daten des Russischen überprüft.

Die untersuchten Grad-Adjektive und Grad-Adverbien bilden Wortgruppen, die sich nach der Ausgangsbedeutung klassifizieren lassen, wobei die Lexeme innerhalb einer Gruppe einen ähnlichen Entwicklungsgang hin zu einer Intensitätsbedeutung einschlagen. Das Auftreten der Gradbedeutung beginnt mit pragmatischer Anreicherung der Ausgangsbedeutung eines Lexems und einer entsprechenden Inferenz und hat die Möglichkeit mehrdeutiger Verwendungen zu Folge. Konventionalisierung der Gradbedeutung führt zu allmählichem Ausbleichen der lexikalischen Ausgangsbedeutung, sodass in den untersuchten Verwendungen anschließend der Bezug auf diese Ausgangsbedeutung vollständig schwindet.

Der Vergleich mit den russischen Daten zeigt, dass die aus dem deutschen Material gewonnenen Entwicklungsschritte der untersuchten Intensivierer gleichermaßen für russische Grad-Adjektive und Grad-Adverbien gelten.

\section{Summary}

The object of the present work are intensifiers, i.e. degree adjectives and degree adverbs which express a high level of a reference word's quality. The research focusses on the development of the lexemes which originally have a qualitative meaning with negative connotations and have eventually lost connection with their initial meaning and appear as pure reinforcements, e.g. furchtbar nett, arg gut, tierisch froh, wahnsinnig interessant, verdammt lecker. The framework includes the analysis of this language change phenomenon as well as the description of the steps for this process including numerous examples from dictionaries, corpora and databanks. In addition, the relevance of the generalizations based on selected German data for corresponding data of the Russian language is checked.

The examined degree adjectives and degree adverbs form groups of words, i.e. semantic fields, which can be classified according to their initial meaning. In addition, all the lexemes within the same group usually undergo a similar development 
to intensifiers. The development of the intensifying meaning begins with the pragmatic enrichment of the initial meaning of a lexeme as well as with the process of pragmatic inferencing and leads to an ambiguous use. Once the inference is conventionalized, the initial meaning gradually fades out so that the connection with the original meaning can disappear completely.

The comparison with the Russian data shows that the development steps of the analyzed German intensifiers are similar to those of Russian degree adjectives and degree adverbs.

\section{Резюме}

Данная работа посвящена прилагательным и наречиям с семантикой интенсивности - признаковым лексемам, выражающим высокую степень обозначаемого ими объекта. В фокусе исследования находятся прилагательные и наречия, выступающие в современном немецком языке в усилительной функции и исходная семантика которых имеет негативные коннотации, например furchtbar: Tier vs. nett 'ужасный: животное vs. милый', wahnsinnig: Patient vs. interessant 'безумный: пациент vs. интересный'. В рамках диссертации проведен анализ данного языкового феномена и исследованы характерные для него ступени развития. В качестве доказательной базы приведены многочисленные примеры из словарей, немецкоязычных корпусов, баз данных и интернета. Основу исследования составляет немецкий материал, релевантность затронутой проблематики проверена и на материале русского языка.

Исследуемые прилагательные и наречия были распределены в группы, объединенные исходной семантикой. Было установлено, что внутри выделенных групп происходят похожие семантические процессы, ведущие к образованию значения интенсивности. К этим процессам относится, в частности, обогащение исходного семантического наполнения лексемы и появление импликатуры, связанной со значением интенсивности. Усилительное значение возникает, как правило, в определенных контекстах: например, при дублировании семантики прилагательного / наречия и семантики слова-референта (например, ужасно испугаться) или при сочетании лексемы со словами-референтами, семантика которых также предполагает высокую степень выраженности того или иного признака (сражаться, кричать, реветь и т.д.). Как следствие, лексема появляется в многозначных контекстах, которые имеют двойную интерпретацию, то есть указывают на употребление как в исходном, так и значении интенсивности. После конвенционализации импликатуры исходная семантика постепенно уходит на задний план и стирается. В результате лексема теряет исходные негативные коннотации и может употребляться в положительных контекстах (например, ужасно рад). 


\section{Literatur- und Abkürzungsverzeichnis}

\subsection{Primärquellen}

\subsubsection{Korpora und Wortlisten}

C.II: = COSMAS II = Corpus Search, Management and Analysis System (2011ff.). Institut für Deutsche Sprache, Programmbereich Korpuslinguistik, Mannheim, Deutschland.

DeReWo $=$ Korpusbasierte Grundformenliste DeReWo (2011), v-ww-bll-250000g2011-12-31-0.1, mit Benutzerdokumentation, <http://www.ids-mannheim.de/kl/derewo/>, Institut für Deutsche Sprache, Programmbereich Korpuslinguistik, Mannheim, Deutschland.

DRQEdit = Deutschsprachige Rechtsquellen in digitaler Edition; Projekt der Heidelberger Akademie der Wissenschaften, des Max-Planck-Instituts für europäische Rechtsgeschichte und der Professur für Historisch-Kulturwissenschaftliche Informationsverarbeitung der Universität zu Köln <http://drwwww.adw.uni-heidelberg.de/drqedit/ >.

DTA $=$ Deutsches Textarchiv; Berlin-Brandenburgische Akademie der Wissenschaften <http://www.deutschestextarchiv.de/>.

DWDS = das Projekt Digitales Wörterbuch der deutschen Sprache, Berlin-Brandenburgische Akademie der Wissenschaften <http://www.dwds.de/>.

FnhdC $=$ das Bonner Frühneuhochdeutschkorpus <http://www.korpora.org/Fnhd/>.

FWBD = die Frühneuhochdeutsche Datenbank der Arbeitsstelle von FWB, Akademie der Wissenschaften zu Göttingen.

MHDBDB = die Mittelhochdeutsche Begriffsdatenbank der Universität Salzburg $<$ http://mhdbdb.sbg.ac.at:8000/>.

$\mathrm{RC}=$ National Russian Corpus $<$ www.ruscorpora.ru $>$.

\subsubsection{Wörterbücher und Grammatiken}

AWB = Althochdeutsches Wörterbuch. Auf Grund der von Elias von Steinmeyer hinterlassenen Sammlungen im Auftrag der Sächsischen Akademie zu Leipzig bearbeitet und herausgegeben von Karg-Gasterstädt, Elisabeth; Frings, Theodor, (später) von Große, Rudolf. Bd. I ff. Berlin, $1968 \mathrm{ff}$.

BMZ = Benecke, Georg Friedrich; Müller, Wilhelm; Zarncke, Friedrich. Mittelhochdeutsches Wörterbuch (1854-1866). Mit Benutzung des Nachlasses von Georg Friedrich Benecke ausgearbeitet von Wilhelm Müller und Friedrich Zarncke. 3 Bde. Leipzig: Hirzel. <http://woerterbuchnetz.de/BMZ/>.

CGEL = A Comprehensive Grammar of the English Language (1985). Randolph Quirk, Greenbaum, Sidney; Leech, Geoffrey; Svartvik, Jan (Hrsgg.). London \& New York: Longman.

Dornseiff, Franz (2004). Der deutsche Wortschatz nach Sachgruppen. ${ }^{8}$ Berlin: de Gruyter.

DRW = Deutsches Rechtswörterbuch (1932ff.) Heidelberger Akademie der Wissenschaften. <http://drw-www.adw.uni-heidelberg.de/drw-cgi/zeige>. 
DUDEN (2012). Das große Wörterbuch der deutschen Sprache. ${ }^{4}$ Mannheim, CDROM.

DUDEN (1995). Die Grammatik: unentbehrlich für richtiges Deutsch. Bd. 4., 5 Mannheim etc.: Dudenverlag.

DUDEN (2009). Die Grammatik: unentbehrlich für richtiges Deutsch. Bd. 4., 8Mannheim: Dudenverlag.

DWB = Deutsches Wörterbuch von Jacob und Wilhelm Grimm (1854ff.) Grimm, Jakob; Grimm, Wilhelm (Hrsgg.). http://www.woerterbuchnetz.de/DWB/. Neubearbeitung (1983ff.): Berlin-Brandenburgische Akademie der Wissenschaften, Akademie der Wissenschaften zu Göttingen; Stuttgart: Hirzel.

FWB = Frühneuhochdeutsches Wörterbuch (1988ff.) Anderson, Robert R.; Goebel, Ulrich; Reichmann, Oskar (Hrsgg.). Berlin etc.: de Gruyter.

GWB = Goethe Wörterbuch (1978ff.). Akademie der Wissenschaften der DDR, Akademie der Wissenschaften zu Göttingen und Heidelberger Akademie der Wissenschaften. Stuttgart: Kohlhammer < http://woerterbuchnetz.de/GWB/>.

Kluge, Friedrich (2011). Etymologisches Wörterbuch der deutschen Sprache. ${ }^{25} \mathrm{Ber}-$ lin: de Gruyter.

Köbler, Gerhard (2014). Althochdeutsches Wörterbuch. 6. Auflage <http://www.koeblergerhard.de/ahdwbhin.html>

Lexer $=$ Matthias Lexer, Mittelhochdeutsches Handwörterbuch. Bd. 1-3. Leipzig 1872-1878. <http://woerterbuchnetz.de/Lexer/>

Paul, Herrmann (2002). Deutsches Wörterbuch: Bedeutungsgeschichte und Aufbau unseres Wortschatzes. ${ }^{10}$ Tübingen: Niemeyer.

Pfeifer, Wolfgang (1993). Etymologisches Wörterbuch des Deutschen. Berlin: Akademie Verlag <www.dwds.de>.

RhWB = Rheinisches Wörterbuch. Bearbeitet und herausgeben von Müller, Josef; ab Bd. VII von Meisen, Karl; Dittmaier, Heinrich; Zender, Matthias. Bonn \& Berlin, 1928-1971. <http://woerterbuchnetz.de/RhWB/>.

Splett $=$ Splett, Jochen. Althochdeutsches Wörterbuch: Analyse der Wortfamilienstrukturen des Althochdeutschen, zugleich Grundlegung einer zukünftigen Strukturgeschichte des deutschen Wortschatzes (1993). Berlin etc.: de Gruyter.

MAS = Jevgen'eva, Anastasija Petrovna (1999). Malyj akademičeskij slovar', ${ }^{4}$ Rossijskaja Akademija Nauk, Institut lingvističeskich issledovanij. Moskva: Russkij jazyk \& Poligrafresursy <http://www.feb-web.ru/feb/mas/mas-abc/default.asp>

Ožegov, Sergej Ivanovič; Švedova, Natalija Jul'evna (2010). Tolkovyj slovar' russkogo jazyka. Moskva <http://ozhegov.textologia.ru/>

Šapošnikov, A.K. (2010). Étimologičeskij slovar' sovremennogo russkogo jazyka. Moskva: Flinta: Nauka.

Semjonov, Anton Vjačeslavovič (2003). Étimologičeskij slovar' russkogo jazyka: Russkij jazyk ot A do Ja. Moskva: Junves.

Slovar' Akademii rossijskoj (1822), Bd. 6. Sankt-Peterburg <https://books.google.de/books ?id=XxBaAAAAcAAJ\&printsec=frontcover\&hl=de\&source=gbs_ge_summary_r\&cad $=0 \# v=$ onepage $\& q \& f=$ false $>$. 
Sorokin, Jurij Sergeevič. Slovar' russkogo jazyka XVIII veka. Leningrad: Nauka <http://www.feb-web.ru/feb/sl18/slov-abc/>

Sreznevskij, Izmail Ivanovič (1893). Materialy dlja drevne-russkogo jayzka po pis'mennym pamjatnikam. Sankt-Peterburg: Tipografija imperatorskoj akademii nauk. <http://etymolog.ruslang.ru/index.php?act=sreznevskij>

Ušakov, Dmitrij Nikolaevič (1935-1940). Tolkovyj slovar' russkogo jazyka. <http://feb-web.ru/feb/ushakov/ush-abc/default.asp>

Vasmer, Max. Étimologičeskij onlain-slovar' russkogo jazyka Maksa Fasmera. $<$ www.vasmer.info>

\subsection{Sekundärliteratur}

Aarts, Jan M.G.; Calbert, Joseph P. (1979). Metaphor and non-Metaphor: the Semantics of Adjective-Noun Combinations. Tübingen: Niemeyer.

Allerton, David J. (1987). „English intensifiers and their idiosynchrasies“. In: Steele, Ross: Threadgold, Terry (Hrsgg.). Language Topics. Essays in Honour of Michael Halliday. Bd. 2, Amsterdam: Benjamins, 15-31.

Andersen, Henning (2001). „Actualization and the (uni)directionality of change“. In: Andersen, Henning (Hrsg.) Actualization. Amsterdam \& Philadelphia: Benjamins, 225-248.

Autenrieth, Tanja (2002). Heterosemie und Grammatikalisierung bei Modalpartikeln: eine synchrone und diachrone Studie anhand von „eben“, „halt", „e(cher)t „einfach", schlicht" und „glatt“. Tübingen: Niemeyer.

Autenrieth, Tanja (2005). „Grammatikalisierung bei Modalpartikeln. Das Beispiel eben." In: Leuschner, Torsten; Mortelmans, Tanja; De Groodt, Sarah (Hrsgg.). Grammatikalisierung im Deutschen. Berlin \& New York: de Gruyter, 310-344.

Bäcklund, Ulf (1973). The Collocations of Adverbs of Degree in English. Acta Universitatis Upsaliensis, Studia Anglistica Upsaliensia 13, Uppsala.

Ballmer, Thomas T; Brennenstuhl, Waltraud (1986). Deutsche Verben. Eine sprachanalytische Untersuchung des deutschen Verbwortschatzes. Tübingen: Narr.

Barcelona, Antonio (2003a). „Clarifying and applying the notions of metaphor and metonymy within cognitive linguistics: An update“. In: Dirven, René (Hrsg.). Metaphor and Metonymy in Comparison and Contrast. Berlin \& New York: Mouton de Gruyter, 207-277.

Barcelona, Antonio (2003b). „The case for a metonymic basis of pragmatic inferencing: Evidence from jokes and funny anecdotes". In: Panther, Klaus-Uwe; Thornburg, Linda L. (Hrsgg.). Metonymy and Pragmatic Inferencing: (revisions of papers presented in the Workshop Metonymy and Pragmatic Inferencing organized for the $7^{\text {th }}$ International Pragmatics Conference held in Budapest, Hungary, July 7 - 14, 2000). Amsterdam \& Philadelphia: Benjamins, 81102.

Becher, [Wilhelm] (1907). „Vom deutschen Superlativ und seinen Verwandten“. Zeitschrift für den deutschen Unterricht 21, 262-273.

Berz, Fabian (1953). Der Kompositionstypus steinreich. Immensee: Calendaria.

Bickel, Hans (2006). „Das Internet als linguistisches Korpus“. Linguistik online: 28: 3 < https://bop.unibe.ch/linguistik-online/article/view/612/1052>.

Biedermann, Reinhard (1969). Die deutschen Gradadverbien. Diss. Heidelberg. 
Biener, Clemens (1940). „Die Steigerungsadverbia bei Adjektiven“. Beiträge zur Geschichte der deutschen Sprache und Literatur 64, 165-204.

Bierwisch, Manfred (1987a). „Bedeutung der Graduierung“. In: Bierwisch, Manfred; Lang, Ewald (Hrsgg.). Grammatische und konzeptuelle Aspekte von Dimensionsadjektiven. Studia Grammatica. Band XXVI/XXVII. Berlin: Akademie-Verlag, 91-286.

Bierwisch, Manfred (1987b). „Dimensionsadjektive als strukturierender Ausschnitt des Sprachverhaltens". In: Bierwisch, Manfred; Lang, Ewald (Hrsgg.). Grammatische und konzeptuelle Aspekte von Dimensionsadjektiven. Studia Grammatica. Band XXVI/XXVII. Berlin: Akademie-Verlag, 1-28.

Bolinger, Dwight (1972). Degree Words. The Hague \& Paris: Mouton.

Borst, Eugen (1902). Die Gradadverbien im Englischen. Heidelberg: Winter.

Bréal, Michel (1900). Semantics: Studies in the Science of Meaning. London: Heinemann.

Brugman, Claudi; Lakoff, George (1988). „Cognitive topology and lexical networks“. In: Small, Steven L. (Hrsg.). Lexical Ambiguity Resolution: Perspectives from Psycholinguistics, Neuropsychology, and Artificial Intelligence. San Mateo \& California: Kaufmann, 477-508.

Burger, Harald (2007). Phraseologie: Eine Einführung am Beispiel des Deutschen. Berlin: Erich Schmidt Verlag.

Bybee, Joan L.; Pagliuca, William (1985). „Cross-linguistic comparison and the development of grammatical meaning". Trends in linguistics 29, 59-83.

Bybee, Joan L.; Perkins, Revere; Pagliuca, William (1994). The Evolution of Grammar: Tense, Aspect, and Modality in the Languages of the World. Chicago \& London: University of Chicago Press.

Campbell, Lyle; Janda, Richard D. (2000a). „Introduction: conceptions of grammaticalization and their problems". Language Sciences 23: 2-3, 93-112.

Campbell, Lyle; Janda, Richard D. (2000b). „What's wrong with grammaticalization?" Language Sciences 23: 2-3, 113-161.

Claudi, Ulrike; Heine, Bernd (1986). „On the metaphorical base of grammar“. Studies in language 10, 297-335.

Croft, William (1993). „The role of domains in the interpretation of metaphors and metonymies". Cognitive Linguistics 4: 4, 335-370.

Defour, Tine (2010). „The semantic-pragmatic development of well from the viewpoint of (inter)subjectification". In: Davidse, Kristin; Vandelanotte, Lieven; Cuyckens, Hubert (Hrsgg.). Subjectification, Intersubjectification and Grammaticalization. Berlin \& New York: de Gruyter, 155-195.

Diewald, Gabriele (1997). Grammatikalisierung: eine Einführung in Sein und Werden grammatischer Formen. Tübingen: Niemeyer.

Diewald, Gabriele (1999). Die Modalverben im Deutschen: Grammatikalisierung und Polyfunktionalität. Tübingen: Niemeyer.

Diewald, Gabriele (2006). „Context types in grammaticalization as constructions." In: Schönefeld, Doris (Hrsg.). Constructions. Special Volume 1: Constructions all over - Case Studies and Theoretical Implications. <http://elanguage.net/journals/constructions/article/view/24>. 
Diewald, Gabriele (2009). „Konstruktionen und Paradigmen“. Zeitschrift für germanistische Linguistik 37, 445-468.

Eckardt, Regine (2002). „Semantic change in grammaticalization“ In: Katz, Graham (Hrsg.). Sinn \& Bedeutung VI, Proceedings of the Sixth Annual Meeting of the Gesellschaft für Bedeutung. Osnabrück: Universität Osnabrück, 53-67.

Eckardt, Regine (2006). Meaning Change in Grammaticalization: an Enquiry into Semantic Reanalysis. Oxford: Oxford University Press.

Eckardt, Regine (2010). „Grammaticalization and semantic reanalysis“. In: von Heusinger, Klaus et al. (Hrsgg.) Handbook Semantics. Berlin: Moutin de Gruyter.

Eckardt, Regine (2011). „Semantic reanalysis and language change“. Language and Linguistics Compass 5: 1, 33-46.

Eisenberg, Peter (1979). Grundriss der deutschen Grammatik. Bd. 2: Der Satz., ${ }^{3}$ Stuttgart, Weimar: Metzler.

Fettig, Adolf (1934). Die Gradadverbien im Mittelenglischen. Heidelberg: Winter.

Finegan, Edward (1995). „Subjectivity and subjectivisation: an introduction“. In: Stein, Dieter; Wright, Susan (Hrsgg.). Subjectivity and Subjectivisation: Linguistic Perspectives. Cambridge etc.: Cambridge University Press, 1-15.

Fleischer, Wolfgang (1982). Phraseologie der deutschen Gegenwartssprache. Leipzig: VEB Bibliographisches Institut.

Fritz, Leonore (1934). Die Steigerungsadverbia in den Denkmälern der mittelhochdeutschen Literatur von der Blütezeit bis zum 15. Jahrhundert. Diss. München: Val. Höfling.

Gabelentz, Georg von der (1891). Die Sprachwissenschaft, ihre Aufgaben, Methoden und bisherigen Ergebnisse. Leipzig: Weigel.

Gehweiler, Elke (2010). „The grammaticalization of the German adjectives lauter (and eitel)". In: Stathi, Katerina; Gehweiler, Elke; König, Ekkehard (Hrsgg.). Grammaticalization: Current Views and Issues. Amsterdam \& Philadelphia: Benjamins, 101-121.

Geis, Michael L.; Zwicky, Arnold M. (1971). „On invited inferences“. Linguistic Inquiry 2, 561-566.

Ghesquière, Lobke (2010). „On the subjectification und intersubjectification paths followed by the adjectives of completeness". In: Davidse, Kristin; Vandelanotte, Lieven; Cuyckens, Hubert (Hrsgg.). Subjectification, Intersubjectification and Grammaticalization. Berlin \& New York: de Gruyter.

Ghesquière, Lobke; Davidse, Kristin (2011). „The development of intensification scales in noun-intensifying uses of adjectives: sources, paths and mechanisms of change“. English Language and Linguistics 15:2, 251-277.

Greenbaum, Sidney (1970). Verb-Intensifier Collocations in English: an Experimental Approach. The Hague: Mouton.

Grice, Paul (1975). „Logic and conversation“. In: Cole, Peter; Morgan, Jerry L. (Hrsgg.). Syntax and Semantics, Bd. 3, Speech acts, New York: Academic Press, 41-58.

Guillaume, Gustave (1964). Langage et science du langage. Paris etc.: Presses de l'Université Laval, 73-86. 
Guimier, Claude (1985). „On the origin of the suffix -ly“. In: Fisiak, Jacek (Hrsg.). Historical Semantics, Word Formation (papers prepared for the international conference on historical semantics and historical word-formation held at at Błażejewko near Poznań from march 28 to 31, 1984). Berlin: de Gruyter Mouton, 155-170.

Heine, Bernd (2002). „On the role of context in grammaticalization“. In: Wischer, Ilse; Diewald, Gabriele (Hrsgg.) New Reflections on Grammaticalization. Amsterdam \& Philadelphia: Benjamins, 83-101.

Heine, Bernd; Claudi, Ulrike; Hünnemeyer, Friederike (1991a). Grammaticalization: A Conceptual Framework. Chicago \& London: University of Chicago Press.

Heine, Bernd; Claudi, Ulrike; Hünnemeyer, Friederike (1991b). „From cognition to grammar - evidence from African languages“. Studies in Language 19: 1, 149-187.

Heine, Bernd; Kuteva, Tania (2002). World Lexikon of Grammaticalization. Cambridge: Cambridge University Press.

Heine, Bernd; Reh, Mechthild (1984). Grammaticalization and Reanalysis in African Languages. Hamburg: Buske.

Helbig, Gerhard (1979). „Probleme der Beschreibung von Funktionsverbgefügen im Deutschen". Deutsch als Fremdsprache: Zeitschrift zur Theorie und Praxis des Deutschunterrichts für Ausländer 16: 5, 273-285.

Helbig, Gerhard; Buscha, Joachim (2005). Deutsche Grammatik: ein Handbuch für den Ausländerunterricht. Berlin etc.: Langenscheidt.

Hentschel, Elke (1998). „Die Emphase des Schreckens: furchtbar nett und schrecklich freundlich". In: Harden, Theo; Hentschel, Elke (Hrsgg.) Particulae particularum. Festschrift für Harald Weydt zum 60. Geburtstag. Tübingen: Stauffenburg, 119-132.

Hewson, John (1997). „Tense and aspect in modern Germanic“. In: Hewson, John; Bubeník, Vít (Hrsgg.). Tense and Aspect in Indo-European Languages: Theory, Typology, Diachrony. Amsterdam \& Philadelphia: Benjamins, 331-350.

Hofmann, Erich (1930). Ausdrucksverstärkung: Untersuchung zur etymologischen Verstärkung und zum Gebrauch der Steigerungsadverbia im Balto-Slawischen und in anderen indogermanischen Sprachen. Göttingen: Vandenhoek \& Ruprecht.

Hopper, Paul J.; Traugott, Elizabeth Closs (2003). Grammaticalization. Cambridge: Cambridge University Press.

Horn, Laurence L. (1984). „Toward a new taxonomy for pragmatic inference; Qbased and R-based implicature". In: Schiffrin, Deborah (Hrsg.). Meaning, Form, and Use in Context: Linguistic Applications; Georgetown University Round Table '84. Washington DC: Georgetown University Press, 11-42.

Ipsen, Gunther (1924). „Der alte Orient und die Indogermanen“. In: Friedrich, Johannes et al. (Hrsgg.) Stand und Aufgaben der Sprachwissenschaft. Festschrift für Wilhelm Streitberg. Heidelberg: Winter.

Ipsen, Gunther (1932). „Der neue Sprachbegriff“. Zeitschrift für Deutschkunde 46: 1-18.

Isitt, David (1984). „Rather impossible and totally difficult - an examination of the behaviour of some adverbial modifiers in English“. Moderna Språk 78, 13-17. 
Jahr, Silke (2000). Emotionen und Emotionsstrukturen in Sachtexten: ein interdisziplinärer Ansatz zur qualitativen und quantitativen Beschreibung der Emotionalität von Texten. Berlin \& New York: de Gruyter.

Kenny, Anthony (2003 [1963]). Action, emotion and will. London: Routledge \& Kegan Paul.

Karpova, Olga (2012). „Metonymical shifts in Russian Adjectives and Adverbs (on the Material of the Database of Semantic Shifts in Russian Adjectives and Adverbs)“. In: Heer, Dagmar (Hrsg.). $m^{*}$ OST 2010. Österreichische Studierendentagung für SlawistInnen. München \& Berlin: Sagner, 16-23.

Karpova, Olga (2012). „Expressive Adjektiv- und Adverbiallexik im Fremdsprachenunterricht". In: Kolesnik, Vitaliy et al. (Hrsgg.). Russischunterricht in Deutschland: Neue Perspektiven und Potenziale. Dresden [o.V.], 97-107.

Karpova, Olga (2013). „Non-Standard Models of Polysemy in German Adjectives and Adverbs". In: Rundblad, Gabriela et al. (Hrsgg.). Selected Papers from the $4^{\text {th }}$ UK Cognitive Linguistics Conference. London: UK Cognitive Linguistics Association, 172-194.

Kip, Herbert Z. (1901) . „Zur Geschichte der Steigerungsadverbien in der Deutschen Geistlichen Dichtung des 11. und 12. Jahrhunderts“. The Journal of Germanic Philology 3: 2, 143-237.

Kirchner, Gustav (1955). Gradadverbien im heutigen Englisch. Halle: VEB Max Niemeyer.

Kirschbaum, Ilja (2002a). Schrecklich nett und voll verrückt: Muster der AdjektivIntensivierung im Deutschen. <http://docserv.uni-duesseldorf.de/servlets/Derivate Servlet/Derivate-2650/650.pdf>.

Kirschbaum, Ilja (2002b). „Metaphorische und metonymische Muster der Adjektivintensivierung". In: Katz, Graham; Reinhard, Sabine; Reuter, Philip (Hrsgg.). Sinn \& Bedeutung VI, Proceedings of the Sixth Annual Meeting of the Gesellschaft für Bedeutung. University of Osnabrück, 201-215.

Kortmann, Bernd (1985). The Interaction of Imperfective Aspect and Situation Class in Present-Day British English. Duisburg: L.A.U.D.T.

Kövecses, Zoltán; Radden, Günter (1998). „Metonymy; Developing a cognitive linguistic view". Cognitive Linguistics 9: 1, 37-77.

Kranich, Svenja (2010). „Grammaticalization, subjectification and objectification“. In: Stathi, Katerina; Gehweiler, Elke; König, Ekkehard (Hrsgg.). Grammaticalization: Current Views and Issues. Amsterdam \& Philadelphia: Benjamins, 101-121.

Kühner, Gertrud (1934). Die Intensiv-Adverbien des Frühneuenglischen. Ludwigshafen: König \& Lieb.

Lakoff, George; Johnson, Mark (1980). Metaphors We Live by. Chicago: University of Chicago Press.

Lakoff, George (1987). Women, Fire and Dangerous Things: What Categories Reveal about Mind. Chicago: University of Chicago Press.

Langacker, Ronald W. (1987). Foundations of Cognitive Grammar. Bd. 1, Theoretical prerequisites. Stanford: Stanford University Press. 
Lee, Anthony van der; Reichmann, Oskar (1973). „Einführung in die Geschichte der Wortfeldtheorie". In: Lee, Anthony van der; Reichmann, Oskar (Hrsgg.). Aufsätze und Vorträge zur Wortfeldtheorie von Jost Trier. The Hague etc.: Mouton. Leech, Geoffrey Neil (1974). Semantics. Harmondsworth: Penguin Books.

Leech, Geoffrey Neil (2004). Meaning and the English verb. ${ }^{2}$ London: Longman.

Leech, Geoffrey Neil; Svartvik, Jan (2013). A Communicative Grammar of English. London: Longman.

Lehmann, Christian (2002). Thoughts on Grammaticalization. Erfurt: Seminar für Sprachwissenschaft der Universität.

Lehmann, Christian (2009). Grammatikalisierung. <http://www.christianlehmann.eu / ling/ling_theo/index.html?http://www.christianlehmann.eu/ling/ling_theo/grammatikalisierung.php $>$.

Lehrer, Adrienne (1974). Semantic Fields and Lexical Structure. Amsterdam \& London \& New-York: North-Holland Publishing Company etc.

Leuschner, Eduard L. (1918). Beiträge zur Geschichte von Steigerungsadverbia im Alt- und Mittelhochdeutschen. Diss. Göttingen: Univ. Press.

Levinson, Stephen C. (1983). Pragmatics. Cambridge: Cambridge University Press.

Levinson, Stephen C. (2000). Presumptive Meanings: the Theory of Generalized Conversational Implicature. Cambridge, Mass.: M.I.T. Press.

Lichtenberk, Frantisek (1991). „Semantic change and heterosemy in grammaticalization". Language 67, 475-509.

Lightfoot (1979). Principles of Diachronic Syntax. Cambridge: Cambridge University Press.

Lyons, John (1963). Structural semantics. Oxford: Blackwell.

Lyons, John (1977). Semantics. Bd. 2. Cambridge etc.: Cambridge University Press

Meillet, Antoine (1912). „L'évolution des formes grammaticales“. Scientia (Rivista di Scienza) 12: 26 (6), 384-400.

Meyer, Richard M. (1910a). „Bedeutungssysteme“. Zeitschrift für Vergleichende Sprachforschung 43, 352-368.

Meyer, Richard M. (1910b). „Die militärischen Titel“. Zeitschrift für Deutsche Wortforschung 12: 145-156.

Min-Jae, Kwon (2005). Modalpartikeln und Satzmodus: Untersuchungen zur Syntax, Bedeutung und Pragmatik der deutschen Modalpartikeln. <http://edoc.ub.uni-muenchen.de/4877/1/Kwon_Min-Jae.pdf>.

Motsch, Wolfgang (1971). Syntax des deutschen Adjektivs. Berlin: Akademie-Verlag. Müller, Karl (1899). „Die Verstärkung des sprachlichen Ausdrucks“. Zeitschrift des Allgemeinen Deutschen Sprachvereins 14, 6-13.

Narrog, Heiko; Heine, Bernd (2011). „Einleitung“. In: Narrog, Heiko; Heine, Bernd (Hrsgg.). The Oxford Handbook of Grammaticalization. Oxford: Oxford University Press, 1-16.

Nübling, Damaris (2010). Historische Sprachwissenschaft des Deutschen: eine Einführung in die Prinzipien des Sprachwandels. Tübingen: Narr.

Nunberg, Geoffrey (1979). „The non-uniqueness of semantic solutions: polysemy“. Linguistics and philosophy 3, 143-184.

Os, Charles van (1989). Aspekte der Intensivierung im Deutschen. Tübingen: Narr. 
Panther, Klaus-Uwe; Thornburg, Linda L. (2003). „Introduction: on the nature of conceptual metonymy“. In: Panther, Klaus-Uwe; Thornburg, Linda L. (Hrsgg.). Metonymy and Pragmatic Inferencing (revisions of papers presented in the Workshop Metonymy and Pragmatic Inferencing organized for the $7^{\text {th }}$ International Pragmatics Conference held in Budapest, Hungary, July 7 - 14, 2000). Amsterdam \& Philadelphia: Benjamins.

Paradis, Carita (1997). Degree Modifiers of Adjectives in Spoken British English. Lund: Lund University Press.

Paradis, Carita (2000). „Reinforcing adjectives: A cognitive semantic perspective on grammaticalization". In: Bermúdez-Otero, Ricardo et al. (Hrsgg.) Generative Theory and Corpus Studies: A Dialogue from 10 ICEHL. Berlin: Mouton de Gruyter, 233-258.

Paradis, Carita (2008). „Configurations, construals and change: expressions of degree". English Language and Linguistics 12: 2, 317-343.

Paul, Hermann (1880). Principien der Sprachgeschichte. Halle: Niemeyer.

Peltola, Niilo (1969). „Contributions to the study of intensives, I-II“. Neuphilologische Mitteilungen 70, 33-53.

Peters, Hans (1993). Die englischen Gradadverbien der Kategorie booster. Tübingen: Narr.

Pilz, Klaus Dieter (1981). Phraseologie: Redensartenforschung. Stuttgart: Metzler.

Portz, Renate (2009). „Adjektiv-Intensivierung: Linguistische und sprachdidaktische Aspekte der Graduierung". In: Aleksandris, Christina et al. (Hrsg.) Schnittstellen von Linguistik und Sprachdidaktik in der Auslandsgermanistik (SL\&SD 2009). Athen: Universität Athen, 144-158 <http://www.gs.uoa.gr/fileadmin/gs.uoa.gr/uploads / synedria/Schnittstellen_Linguistik_und_Didaktik_2009.pdf> .

Pottelberge, Jeroen van (1996). Verbnominale Konstruktionen als Vorläufer der Funktionsverbgefüge: Einige diachronische Beobachtungen anhand deutscher Evangelienübersetzungen aus dem Mittelalter. Gent: Studia Germanica Gandensia.

Pusch, Luise F. (1981). „Ganz“. In: Weydt, Harald (Hrsg.). Partikeln und Deutschunterricht: Abtönungspartikeln für Lerner des Deutschen. Heidelberg: Groos, 3143.

Quirk, Randolph; Greenbaum, Sidney; Leech, Geoffrey; Svartvik, Jan (1979). A Grammar of Contemporary English. London: Longman.

Reznikova, Tatiana; Rakhilina, Ekaterina; Karpova, Olga; Kyuseva, Maria; Ryzhova, Daria; Arkhangelskiy, Timofey. (2013). „Polysemy patterns in Russian adjectives and adverbs: A corpus-oriented database". In: Chahine, Irina Kor (Hrsg.). Current Studies in Slavic Languages 146, 313-322.

Rolf, Eckard (2005). Metaphertheorien: Typologie, Darstellung, Bibliographie. Berlin: De Gruyter.

Ruoff, Arno (1981). Häufigkeitswörterbuch gesprochener Sprache: gesondert nach Wortarten, alphabetisch, rückläufig alphabetisch und nach Häufigkeit geordnet. Tübingen: Niemeyer.

Saussure, Ferdinand de (1922). Cours de linguistique générale. Paris: Payot. 
Seiler, Hansjakob (1983). Possession as an Operational Dimension of Language. Tübingen: Narr.

Sperber, Hans (1914). Über den Affekt als Ursache der Sprachveränderung: Versuch einer dynamologischen Betrachtung des Sprachlebens. Halle: Niemeyer.

Sperber, Hans (1923). Einführung in die Bedeutungslehre. Bonn: Schroeder.

Spitzbardt, Harry (1954). Die modernen Gradadverbien (ein Beitrag zum englischen Sprachgebrauch des 20. Jahrhunderts). Unveröffentlichte Diss., Jena.

Stoffel, Cornelis (1901). Intensives and Down-Toners: A Study in English Adverbs. Heidelberg: Winter.

Sweetser, Eve E. (1988). „Grammaticalization and semantic bleaching“. In: Proceedings of the Fourteenth Annual Meeting of the Berkeley Linguistics Society, 389405.

Taylor, John R. (1989). Linguistic Categorization: Prototypes in Linguistic Theory. Oxford: Clarendon Press.

Timberlake, Alan (1977). „Reanalysis and actualization in syntactic change“. In: Charles N. Li (Hrsg.). Mechanisms of Syntactic Change. Austin: University of Texas Press, 141-177.

Traugott, Elizabeth Closs (1982). „From propositional to textual and expressive meanings: Some semantic-pragmatic aspects of grammaticalization". Current Issues in Linguistic Theory 4: 24, 245-271.

Traugott, Elizabeth Closs (1988). „Pragmatic strengthening and grammaticalization". Proceedings of the Fourteenth Annual Meeting of the Berkeley Linguistics Society 14, 406-416.

Traugott, Elizabeth Closs (1989). „On the rise of epistemic meanings in English: An example of subjectification in semantic change". Language 65: 1, 31-55.

Traugott, Elizabeth Closs (1990). „From less to more situated in language: The unidirectionality of semantic change". In: Adamson, Sylvia et al. (Hrsgg.). Papers of the $5^{\text {th }}$ International Conference on English Historical Linguistics. Amsterdam \& Philadelphia: Benjamins, 497-517.

Traugott, Elizabeth Closs (1995). „Subjectification in grammaticalization“. In: Stein, Dieter; Wright, Susan (Hrsgg.). Subjectivity and Subjectivisation. Cambridge: Cambridge University Press, 37-54.

Traugott, Elizabeth Closs (2010). „Revisiting subjectification and intersubjectification". In: Davidse, Kristin; Vandelanotte, Lieven; Cuyckens, Hubert (Hrsgg.). Subjectification, Intersubjectification and Grammaticalization. Berlin \& New York: de Gruyter, 29-70.

Traugott, Elizabeth Closs; Dasher, Richard B. (2002). Regularity in Semantic Change. Cambridge: Cambridge University Press.

Traugott, Elizabeth Closs; König, Ekkehard (1991). „The semantics-pragmatics of grammaticalization revisited“. In: Traugott, Elizabeth Closs; Heine, Bernd (Hrsgg.). Approaches to Grammaticalization. Bd. 1, Focus on theoretical and methodological issues, Amsterdam: Benjamins,189-218.

Trier, Jost (1931). „Über Wort- und Begriffsfelder“. In: Trier, Jost. Der deutsche Wortschatz im Sinnbezirk des Verstandes. Die Geschichte eines sprachlichen Feldes: Von den Anfängen bis zum Beginn des 13. Jahrhunderts. Bd.1, Heidelberg: Winter, 1-26. 
Trier, Jost (1932a). „Sprachliche Felder“. Zeitschrift für deutsche Bildung 8, 417-427. Trier, Jost (1932b). „Die Idee der Klugheit in ihrer sprachlichen Entfaltung“. Zeitschrift für Deutschkunde 46: 625-635.

Trier, Jost (1934). „Deutsche Bedeutungsforschung“. Germanische Philologie, Ergebnisse und Aufgaben. Festschrift für Otto Behaghel. Heidelberg: Winter, 173-200.

Trost, Igor (2006). Das deutsche Adjektiv: Untersuchungen zur Bedeutung, Komparation, Wortbildung und Syntax. Hamburg: Buske.

Ullmann, Stephen (1967). Grundzüge der Bedeutung: die Bedeutung in sprachwissenschaftlicher Sicht. Berlin: de Gruyter.

Vandewinkel, Sigi; Davidse, Kristin (2008). „The interlocking paths of development to emphasizer adjective pure“. Journal of Historical Pragmatics 9: 2, 255-287.

Vendler, Zeno (1967). Linguistics in Philosophy. Ithaca: Cornell University Press.

Vermeire, Antoine R. (1979). Intensifying Adverbs: a Syntactic, Semantic and Lexical Study of Fifteen Degree Intensifiers, Based on an Analysis of Two Computer Corpuses of Modern English. Diss. University of Lancaster.

Warren, Beatrice (1984). Classifying Adjectives. Göteborg: Acta.

Warren, Beatrice (1992). Sense Developments: A Contrastive Study of the Development of Slang Senses and Novel Standard Sense in English. Stockholm: Almqvist \& Wiksel.

Weinreich, Uriel (1963). „On the semantic structure of language“. In: Greenberg, Joseph Harold (Hrsg.). Universals of language. Report of a conference held at Dobbs Ferry, New York, April 13-15, 1961. Cambridge, Mass.: M.I.T. Press.

Werner, Jürgen (1960). „Zu Gradadverbien negativen Bedeutungsgehalts“. Forschungen und Fortschritte 34: 8, 244.

Zifonun, Gisela; Hoffmann, Ludger; Strecker, Bruno (1997). Grammatik der deutschen Sprache. Bd. 3, Berlin \& New York: de Gruyter.

Karpova, Ol'ga Sergeevna (2011a). „Baza dannych po mnogoznačnym kačestvennym prilagatel'nym i narečijam russkogo jazyka: pervye rezul'taty raboty“. Problemy kompjuternoj lingvistiki 5, 79-86.

Karpova, Ol'ga Sergeevna (2011b). „Russkie i nemeckie prilagatel'nye razmera: tipologičeskoe issledovanie semantičeskich sdvigov". In: Kretov, Aleksej A. (Hrsg.). Problemy leksiko-semantičeskoj tipologii. Voronež: Izdat. Voronežskogo Gosudarstvennogo Univ., 294-309.

Koleva-Zlateva, Živka (2010-2011). „Etimologičeskie zametki o slavjanskom *grozьнъ(jb) 'strašnyj; urodlivyj, nekrasivyj' i litovskom gražus 'krasivyj'. <https://www.academia.edu/4051069/On_the_etymology_of_Slav._groz\%D1\%8Cn\%D1\%8A_j\%D1\%8C_terrible_ugly_and_Lith._gra\%C5\%BEus_beautiful_in_Russian>

Kustova, Galina Ivanovna (2004). Tipy proizvodnych značenij i mechanizmy jazykovogo rasširenija. Moskva: Jazyki slavjanskich kul'tur.

Ljaševskaja, Ol'ga N., Šarov, Sergej A. (2009). Častotnyj slovar' sovremennogo russkogo jazyka (na materialach Nacional'nogo korpusa russkogo jazyka). Moskva: Azbukovnik http://dict.ruslang.ru/freq.php 
Rachilina, Ekaterina Vladimirovna; Reznikova, Tat'jana Isidorovna; Karpova, Ol'ga Sergeevna (2009). „Modeli semantičeskoj derivacii mnogoznačnych kačestvennych prilagatel'nych: metafora, metonimija i ich vzaimodejstvie“. Kompjuternaja lingvistika i intellektual'nye technologii: Po materialam ežegodnoj meždunarodnoj konferencii „Dialog 2009“ (Bekasovo 27-31 maja 2009g) 8: 15, 420-425.

Rachilina, Ekaterina Vladimirovna; Reznikova, Tat'jana Isidorovna; Karpova, Ol'ga Sergeevna (2010). „Semantičeskie perechody v attributivnych konstrukcijach: metafora, metonimija i rebranding". In: Rachilina, Ekaterina Vladimirovna (Hrsg.). Lingvistika Konstrukcij. Moskva: Azbukovnik, 396-455.

Švedova, Natalija Jul'evna. „Časticy“. In: Russkaja Grammatika. 15.05.2015 <http://rusgram.narod.ru/1689-1705.html>.

Vinogradov, Viktor Vladimirovič 1977. „Osnovnye tipy leksičeskich značenij slova”. In: Vinogradov, Viktor Vladimirovič. Izbrannye trudy. Leksikologija i leksikografija. Moskva: Nauka, 162-189.

\subsection{Weitere Abkürzungen}

Traugott I, II, III: Semantisch-pragmatische Tendenzen nach E. Traugott, dargestellt in Kap. 3.6.3.4 (Tendency I, II, III). 